Bandamycin as New Antifungal Agent and further Secondary

Metabolites from Terrestrial and Marine Microorganisms<smiles>NC(=O)NC(=O)c1cccc(O)c1O</smiles><smiles>CCCCOC1OC(CO)C(O)C(O)C1O</smiles><smiles>COc1c(O)cccc1O</smiles><smiles>CC1CC(=O)c2c(cc(O)c3c2C(=O)c2ccc(Cl)c(O)c2C3=O)C1O</smiles>

Dissertation 
Bandamycin as New Antifungal Agent and further Secondary Metabolites from Terrestrial and Marine Microorganisms

\author{
Dissertation \\ zur Erlangung des Doktorgrades \\ der Mathematisch-Naturwissenschaftlichen Fakultäten \\ der Georg-August-Universität zu Göttingen
}

vorgelegt von

\title{
Muhammad Bahi
}

aus

Banda Aceh (Indonesien)

Göttingen 2012 
D7

Referent:

Prof. Dr. H. Laatsch

Korreferent:

Prof. Dr. A. Zeeck

Tag der mündlichen Prüfung: $\quad$ 17.04.2012 
Die vorliegende Arbeit wurde in der Zeit von März 2007 bis November 2011 im Institut für Organische Chemie der Georg-August-Universität zu Göttingen unter der Leitung von Herrn Prof. Dr. H. Laatsch angefertigt.

Herrn Prof. Dr. H. Laatsch danke ich für die Möglichkeit zur Durchführung dieser Arbeit sowie die ständige Bereitschaft, auftretende Probleme zu diskutieren. 
For my parents, my wife (Anizar)

and my sons (Fiqhan, Amirul, Syauqal) 



\section{Table of Content}

$1 \quad$ Introduction ...................................................................................................... 1

$1.1 \quad$ Natural products in modern therapeutic use .......................................... 1

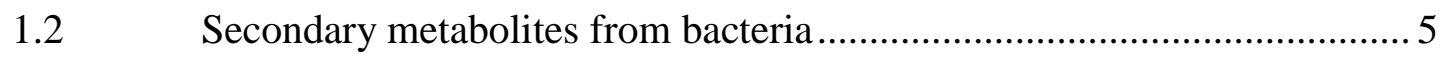

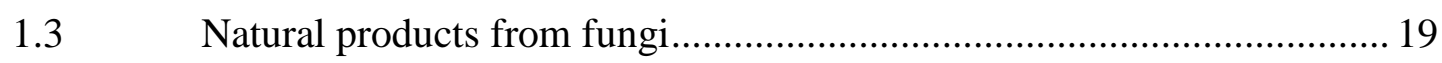

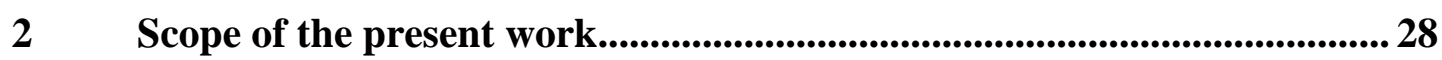

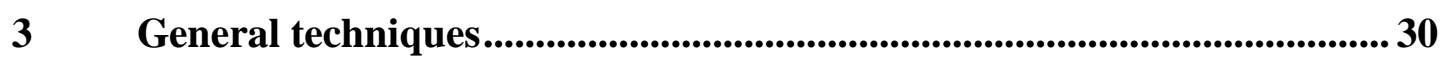

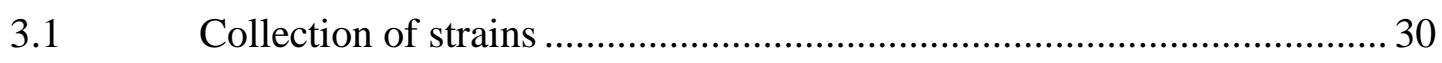

3.2 Strategic procedure in evaluating the selected strains ............................. 30

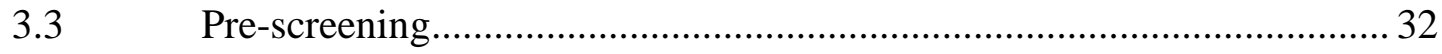

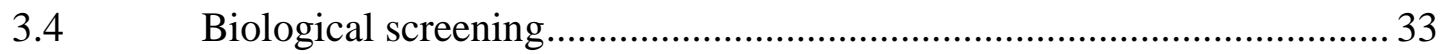

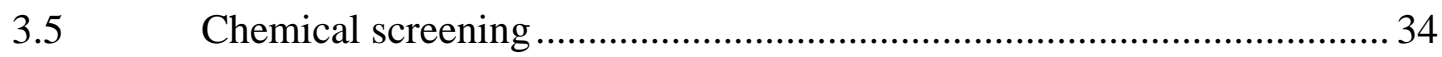

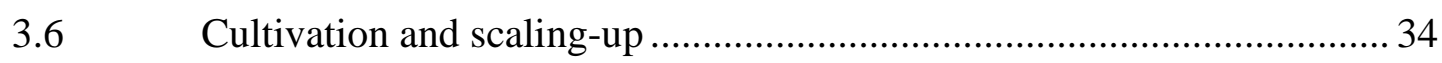

Isolation and purification work-up.................................................... 35

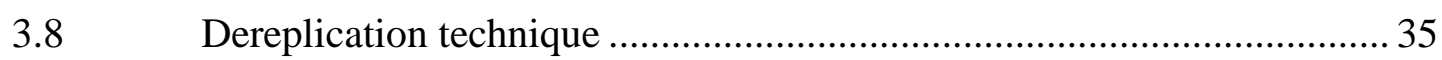

4 Investigation of selected bacterial strains ........................................................ 36

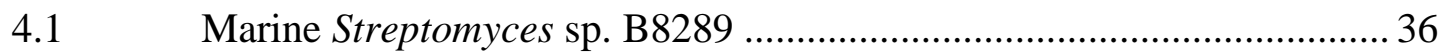

4.1.1 2-[5-(2-Oxo-propyl)-tetrahydrofuran-2-yl]-propionic acid ..................... 37

4.1.2 2-[5-(2-Oxo-propyl)-tetrahydrofuran-2-yl]propionic acid methyl ester .. 39

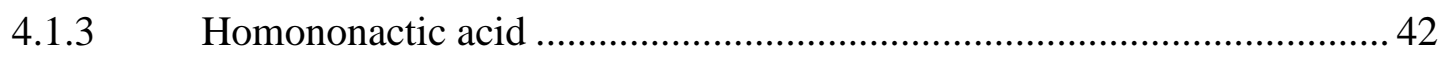

4.1.4 Homononactic acid methyl ester....................................................... 44

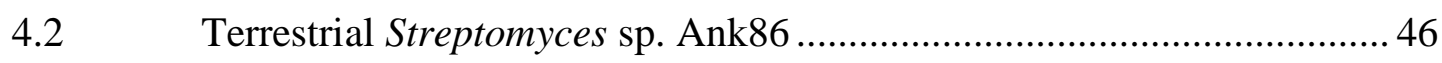

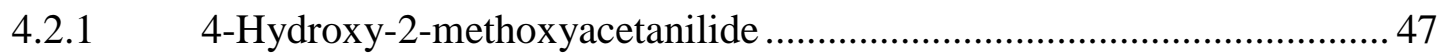

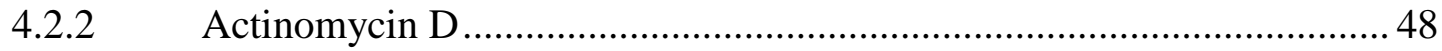

4.3 Terrestrial Streptomyces sp. Ank68 ................................................. 51

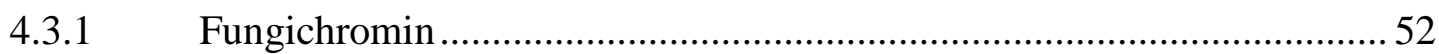

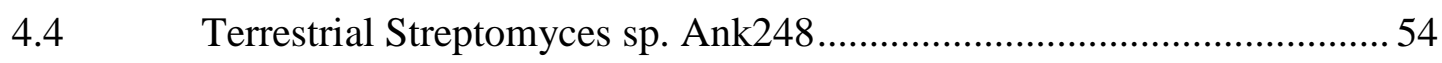

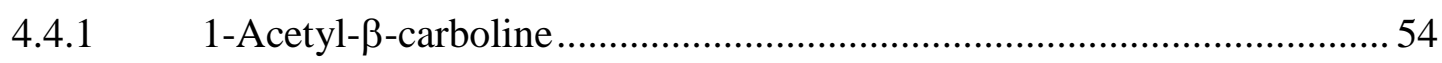

4.5 Terrestrial Streptomyces sp. Ank181 .................................................56

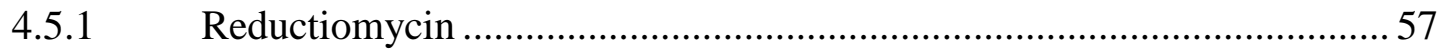

4.6 Ruminal bacterium Enterobacter amnigenus ZIA................................ 59

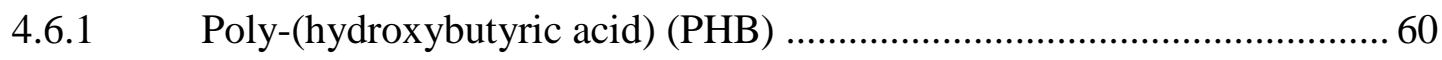




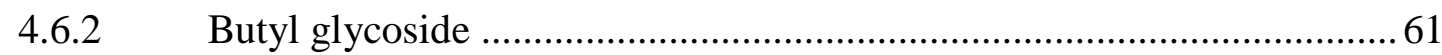

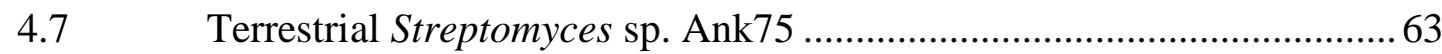

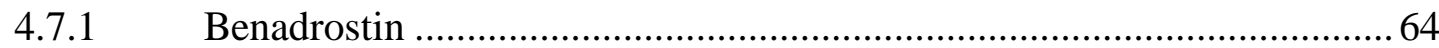

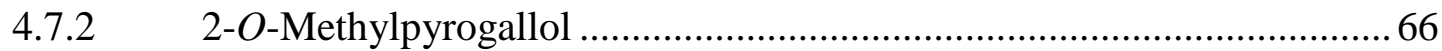

4.7.3 N-Aminocarbonyl-2,3-dihydroxybenzamide ........................................ 67

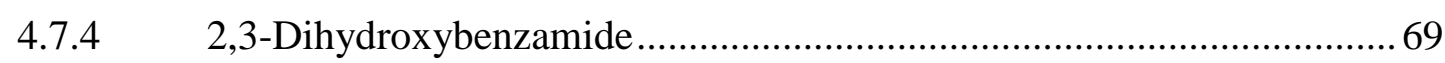

4.7.5 3-[(1Z)-1-Hexenyl-ONN-azoxyl]-1,2-butanediol ................................... 71

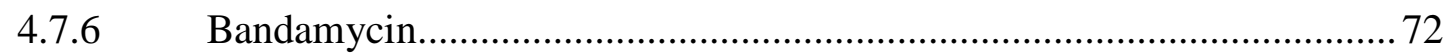

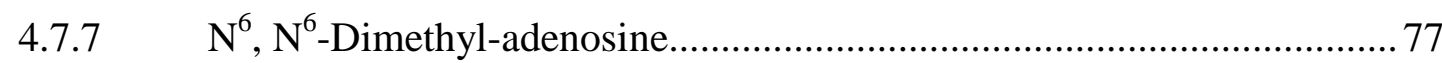

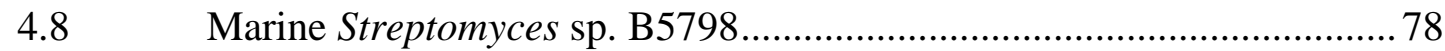

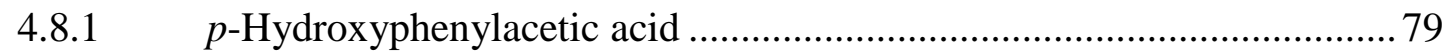

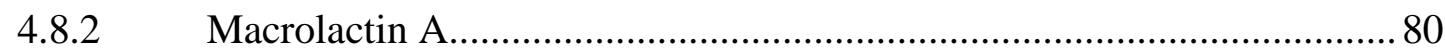

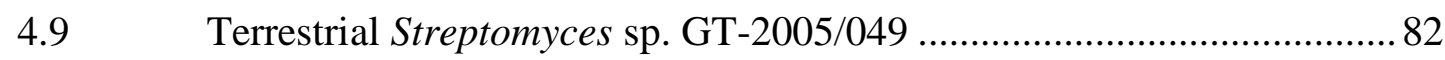

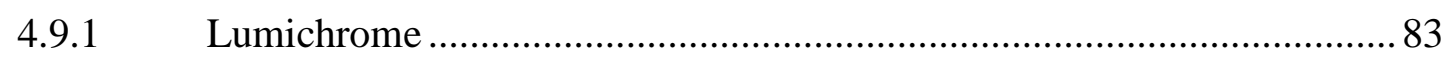

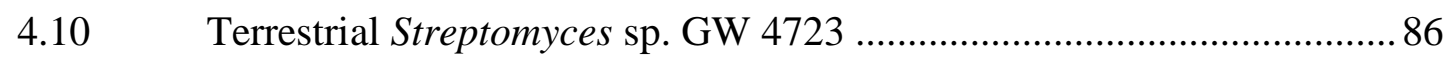

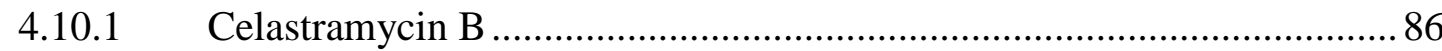

$5 \quad$ Metabolites from selected endophytic fungi .........................................91

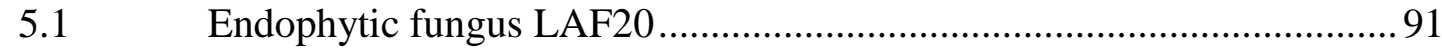

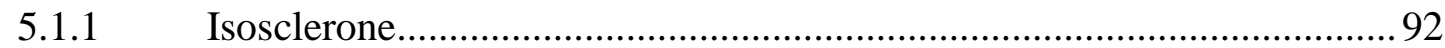

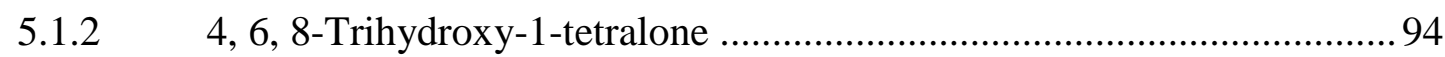

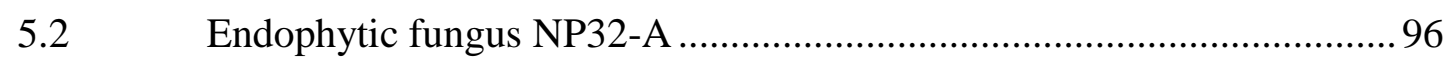

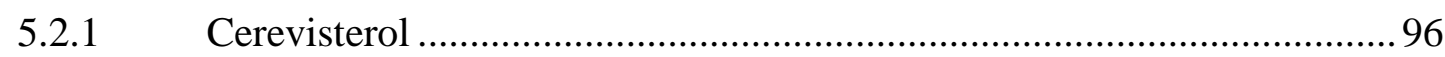

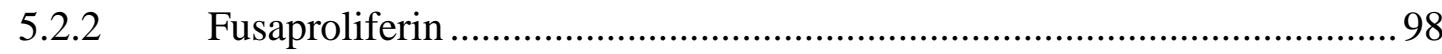

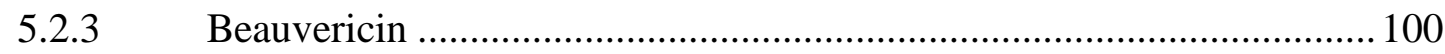

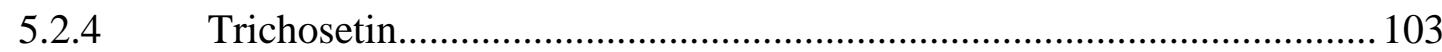

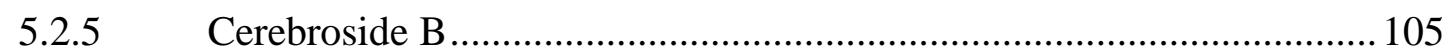

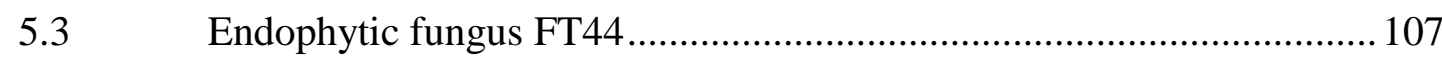

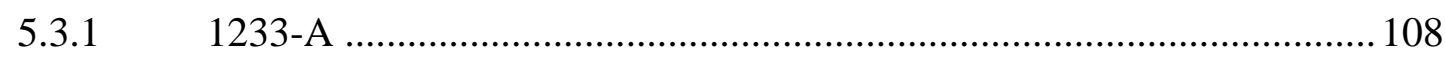

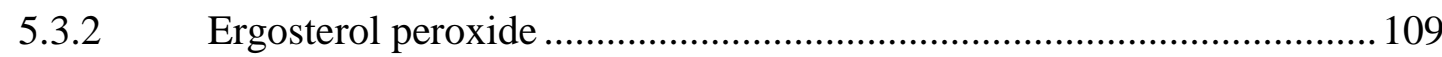

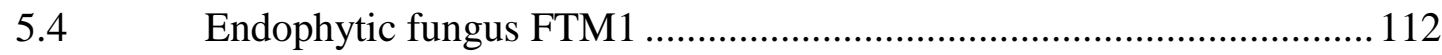

5.4.1 8-Hydroxy-6,7-dimethoxy-3-methylisocoumarin ...............................113

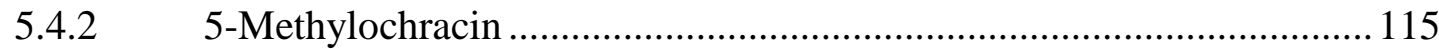


5.4.3 Alternariol 5-methyl ether (AME) …................................................. 117

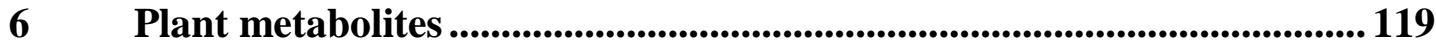

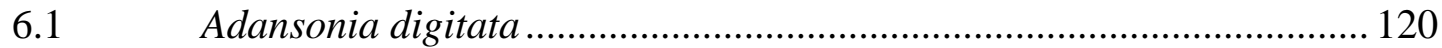

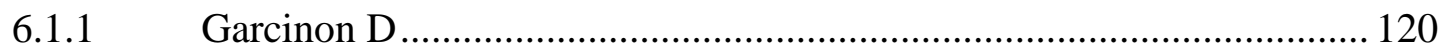

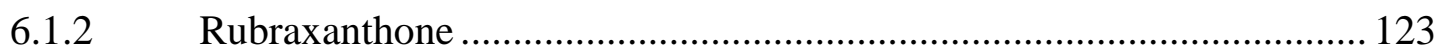

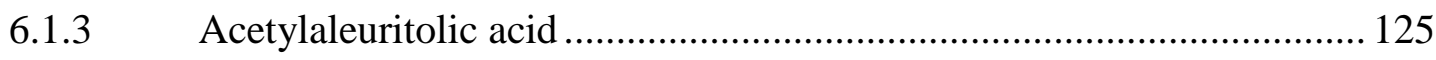

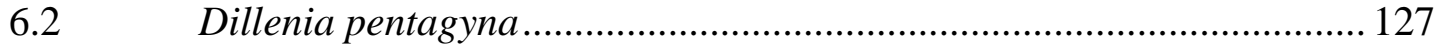

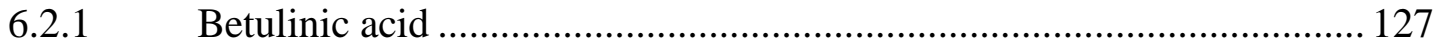

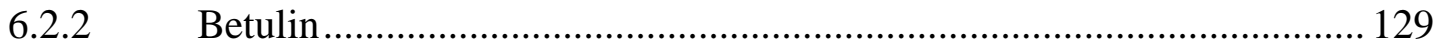

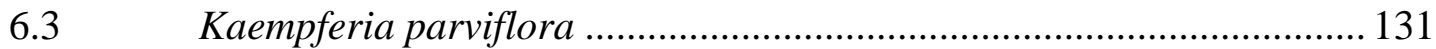

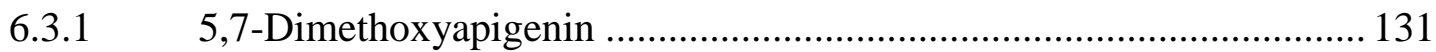

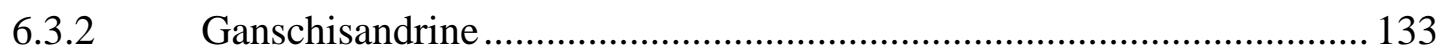

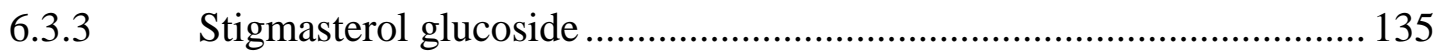

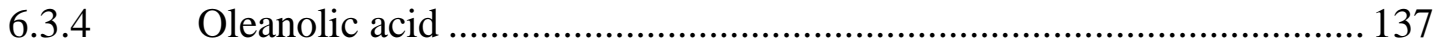

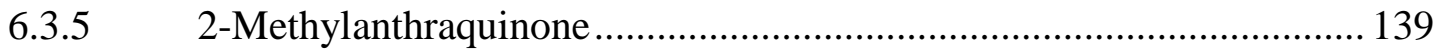

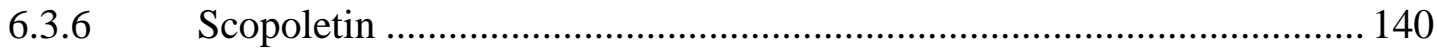

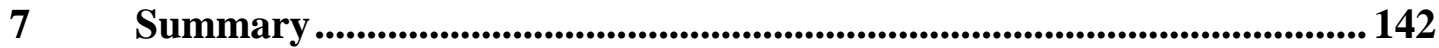

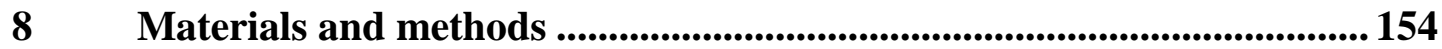

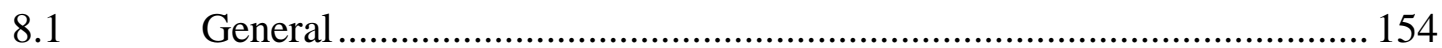

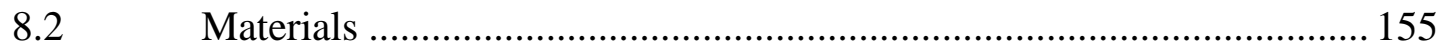

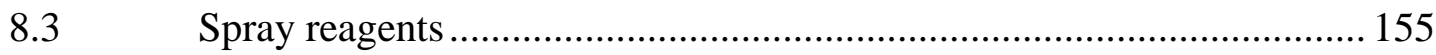

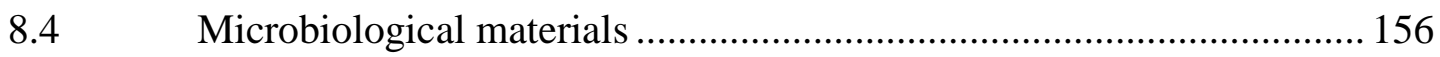

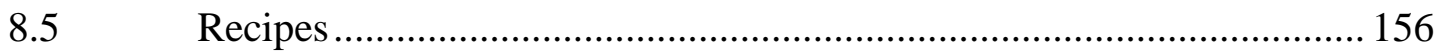

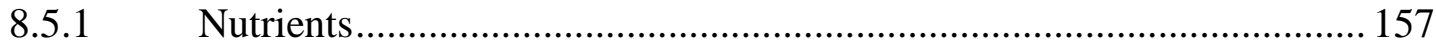

8.6 Stock solutions and media for cultivation of algae ............................... 160

8.7 Microbiological and analytical methods ........................................... 161

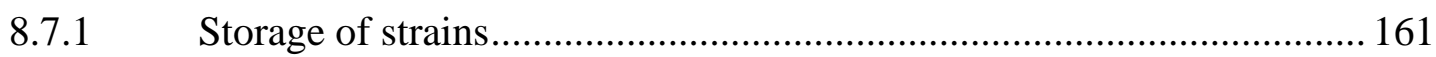

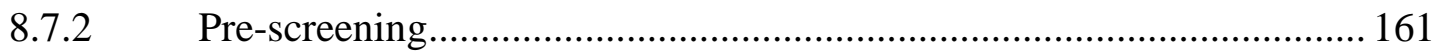

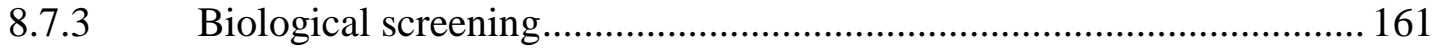

8.7.4 Chemical and pharmacological screening ........................................... 161

8.7.5 Brine shrimp microwell cytotoxicity assay....................................... 162

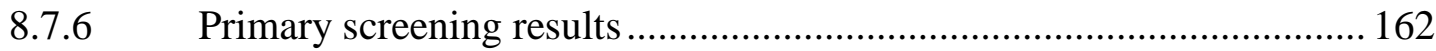


$9 \quad$ Metabolites from selected bacterial strains ................................................ 164

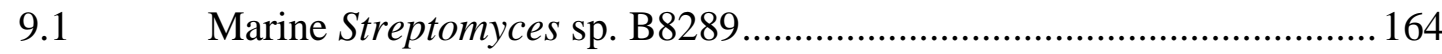

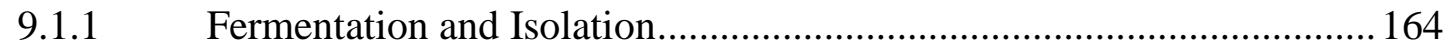

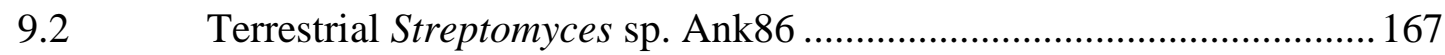

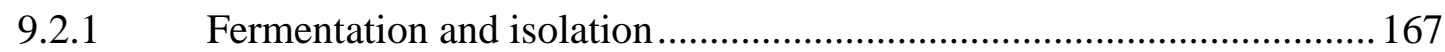

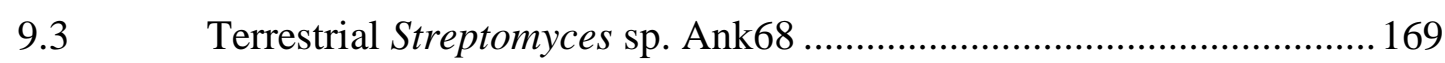

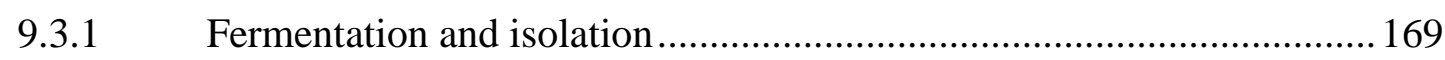

$9.4 \quad$ Terrestrial Streptomyces sp. Ank248 .............................................. 170

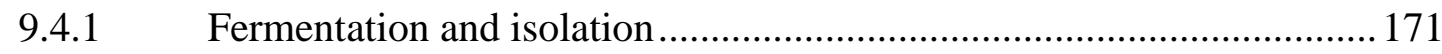

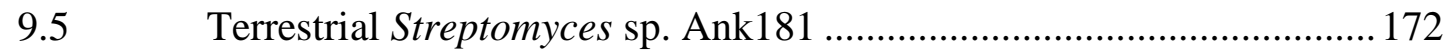

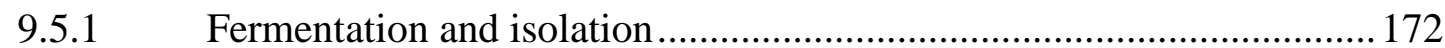

9.6 Ruminal Bacterium Enterobacter amnigenus ZIA …............................ 173

9.6.1 Fermentation and isolation............................................................. 173

9.7 Terrestrial Streptomyces sp. Ank75 ............................................... 175

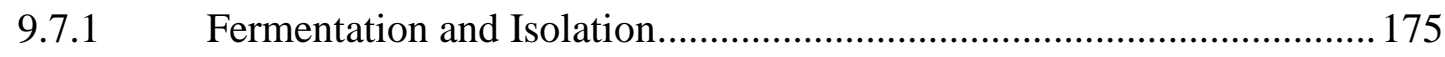

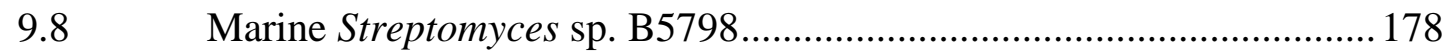

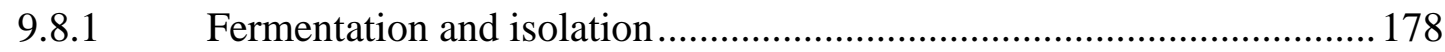

9.9 Terrestrial Streptomyces sp. GT-2005/049 _.......................................... 179

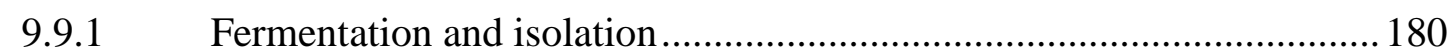

9.10 Terrestrial Streptomyces sp. GW 4723 .............................................. 181

9.10.1 Fermentation and isolation............................................................... 181

10 Metabolites from selected endophytic fungi ..............................................182

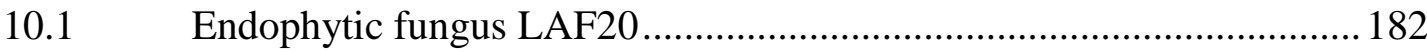

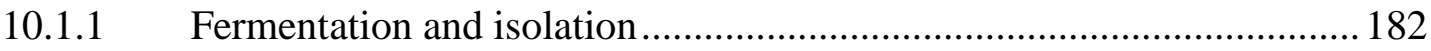

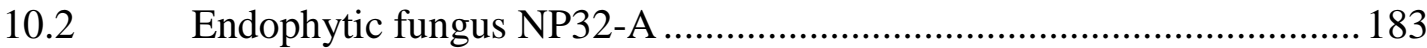

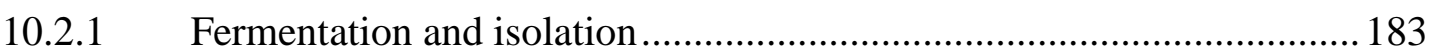

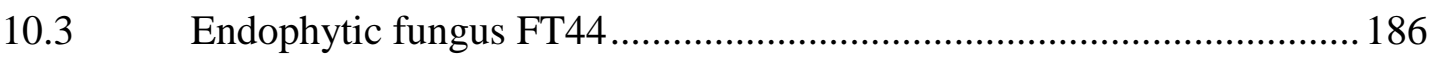

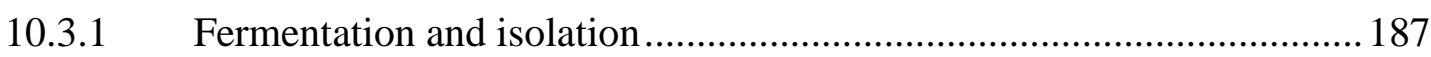

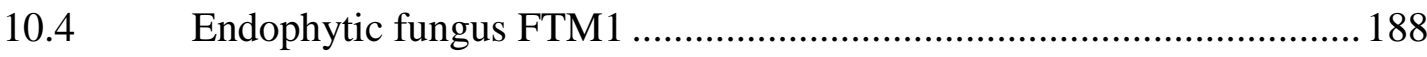

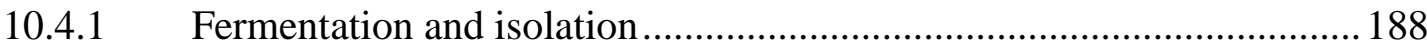

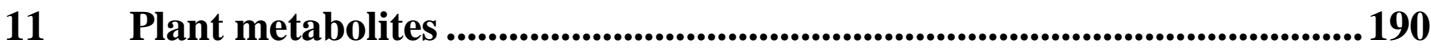

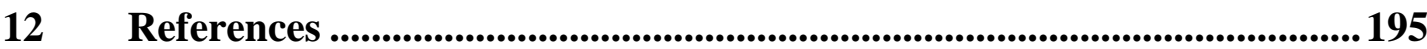




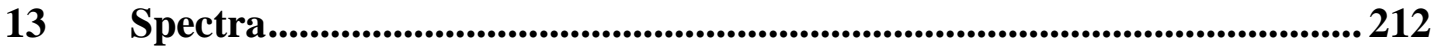





\section{Introduction}

For thousands of years, natural products have been continuously applied for human use. Also in pharmaceutical industries, natural products play an important role due to their chemical diversity and various bioactivities against diseases. Additionally, crude natural products have become an important source of drugs in developing countries due to their low cost and ready availability. According to the World Health Organization, about $80 \%$ of the world's population rely on traditional medicines for their health care. ${ }^{[1,2]}$

A number of drug classes derived from natural products plays an important role in therapeutic areas of oncology, immunosuppression, infectious and metabolic diseases. ${ }^{[3]}$ The development of resistance in infectious microorganisms to the available pharmaceuticals and the increase in diseases such as Ebola, SARS, and AIDS need the discovery and development of new drugs. ${ }^{[4]}$

Recently, seas and oceans have drawn attention as potential natural resources of many bioactive compounds. ${ }^{[5]}$ As the habitats for marine organisms considerably differ from terrestrial ones, it can be expected that their secondary metabolites will be different. ${ }^{[5,6]}$ Microorganisms have continued to be a productive and successful spotlight for marine natural products study. ${ }^{[7]}$

The study on natural products chemistry derived from marine microorganisms has increased remarkably in recent years. The demand of compounds with potential pharmaceutical and economic applications as cosmetics, drugs, personal-care products and fine chemicals is the reasons for this. ${ }^{[8]}$ Until now, from the many species which have been assayed for their activities, a treasure of bioactive molecules having unique structural features has been isolated. ${ }^{[4,6]}$

\subsection{Natural products in modern therapeutic use}

Despite successes in controlling some previously thought incurable diseases, recent increase in epidemic viral infections such as AIDS, hepatitis, Ebola, SARS have been reported in developing countries (Asia, Africa). ${ }^{[9]}$ Therefore, the intention to search for new resources of bioactive metabolites still gains worldwide attention, as 
most medicinal plants $(\sim 97 \%)$ have been investigated. As a result, many other natural sources have already been taken into consideration, especially those located in marine habitats, e.g. soft corals, sponges, jellyfishes, algae, and microorganisms. ${ }^{[10]}$ Microorganisms which are living in different biological ecosystems represent a huge source of diverse bioactive secondary metabolites. ${ }^{[11,12]}$ The discovery of penicillin (1) from microorganisms had initiated the pharmaceutical research in screening new antibiotic source, and a massive screening of microorganism started after Second World War. ${ }^{[13]}$ The antibiotic activity of penicillin (1) is due to the $\beta$-lactam ring. Cephalosporin $\mathrm{C}(\mathbf{2})$ is another example of $\beta$-lactam antibiotics derived from microorganisms. ${ }^{[14]}$<smiles>CC1(C)S[C@@H](C(=O)O)[C@H](C(=O)NC(=O)Cc2ccccc2)C1=O</smiles><smiles>CC(=O)OCC1=C(C(=O)O)N2C(=O)[C@H](NC(=O)CCCOC(=O)CN)[C@H]2SC1</smiles>

2

Mycophenolic acid (MPA) (3) is a natural product-based medicine used in human-organ transplantation as an immunosuppressant. Mycophenolic acid (3) was first isolated by Italian physician Bortolomeo Gosio as early as 1893. In addition, it is the first purified antibiotic from microorganism. ${ }^{[15]}$ 
<smiles>COc1c(C)c2c(c(O)c1C/C=C(\C)CCC(=O)O)C(=O)OC2</smiles>

Daunorubicin (DNR) (4) and doxorubicin (DOX) (5) were the first effective antitumor drugs developed in pharmaceutical industry in the 1960s. They were isolated from Streptomyces peucetius. DOX (5) and DNR (4) belong to the group of anthracyclines consisting of a tetracyclic ring system and sugar moieties. However, their use is limited by cardiotoxic side effects. ${ }^{[16]}$<smiles>COc1cccc2c1C(=O)c1c(O)c3c(c(O)c1C2=O)C[C@@](O)(C(C)=O)C[C@@H]3OC1CC(N)C(C)C(C)(O)O1</smiles>

4<smiles>COc1cccc2c1C(=O)c1c(O)c3c(c(O)c1C2=O)C[C@@](O)(C(=O)CO)C[C@@H]3OC1CC(N)C(C)C(C)O1</smiles>

5

By 1990, about $80 \%$ of drugs have been derived from microorganisms. Lengthening life expectancy of world population to over 77 years today is one of the most significant impacts of natural product-based drugs. However, there was about $30 \%$ drop in natural product-based drugs in clinical studies between 2001 and 2008. ${ }^{[13]}$ The increase of resistant pathogen indicates an urgent demand to continue antibiotics discovery and development. ${ }^{[17]}$ On the other hand, genetic sequencing and biosynthetic pathway manipulation maybe a solution for the discovery of next generation pharmaceutical agents. ${ }^{[13,18]}$ 


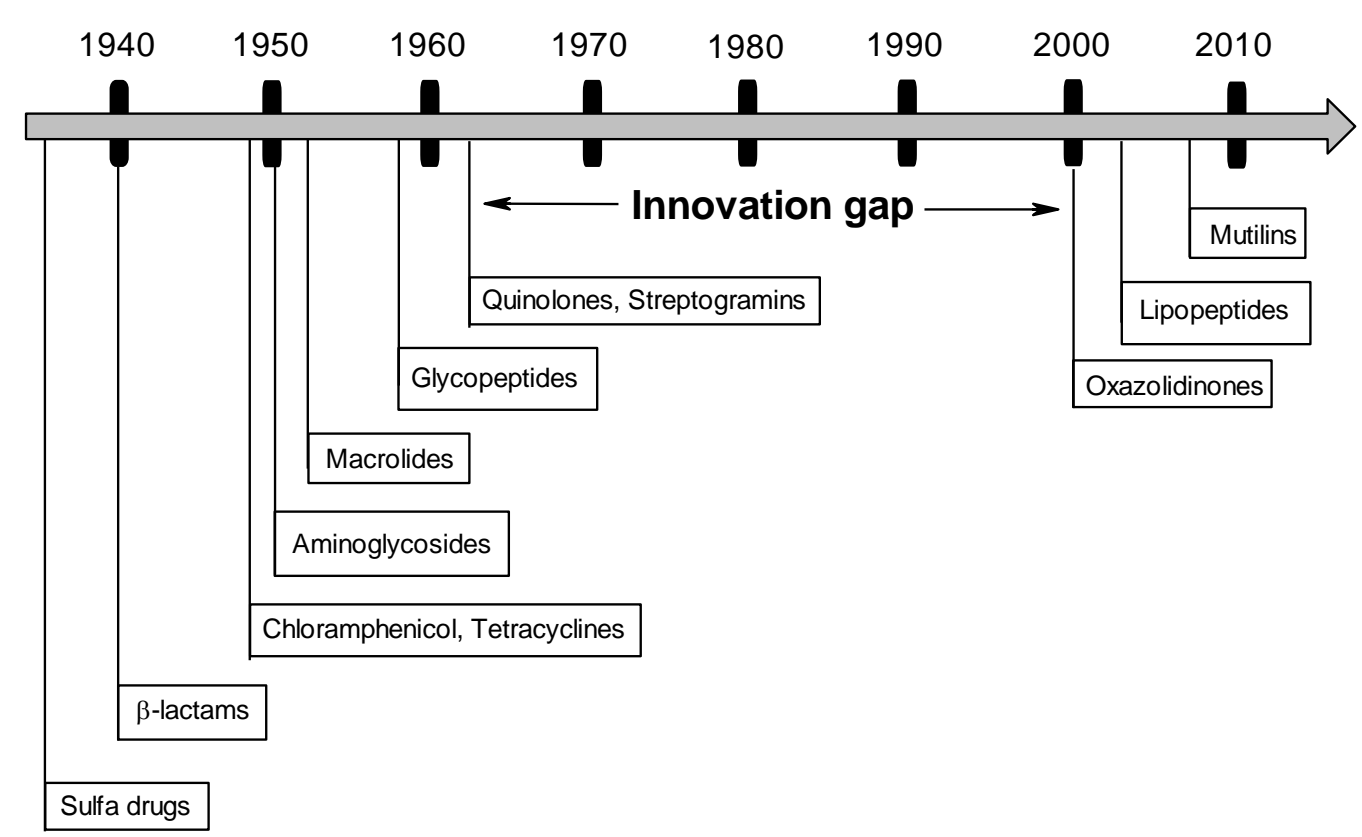

Figure 1: The major groups of antibiotics between 1940 and 2010 (Ref. ${ }^{[17]}$ modified)

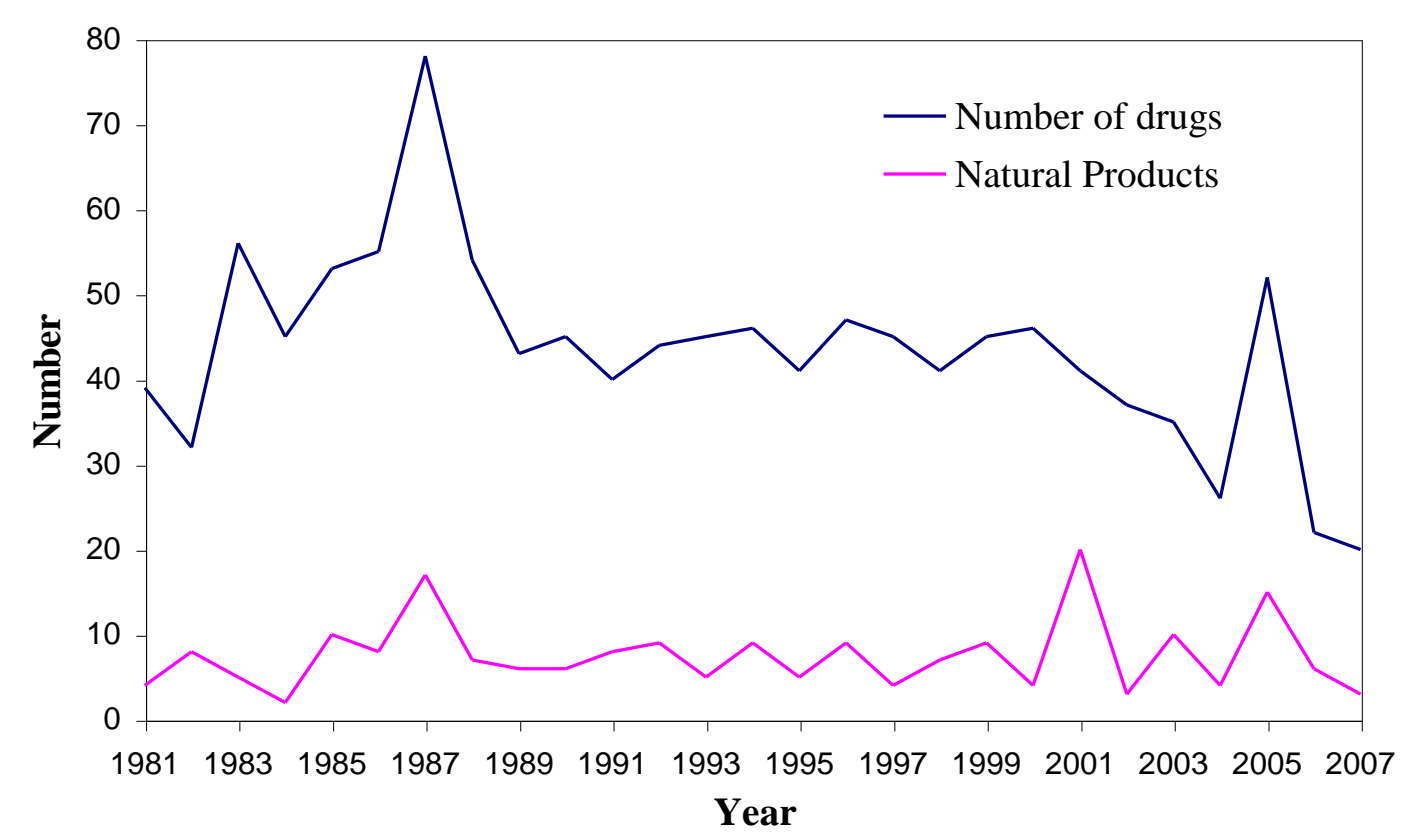

Figure 2: $\quad$ Numbers of drugs approved in the United States between 1981 and 2007 (according to ${ }^{[13]}$ ) 


\subsection{Secondary metabolites from bacteria}

The history of natural products from bacteria started about 60 years ago. In 1943, Selman Waksman and co-workers first reported the production of streptomycin (6) from Streptomyces griseus. ${ }^{[19]}$ This antibiotic had been used successfully in the treatment of tuberculosis. ${ }^{[20]}$

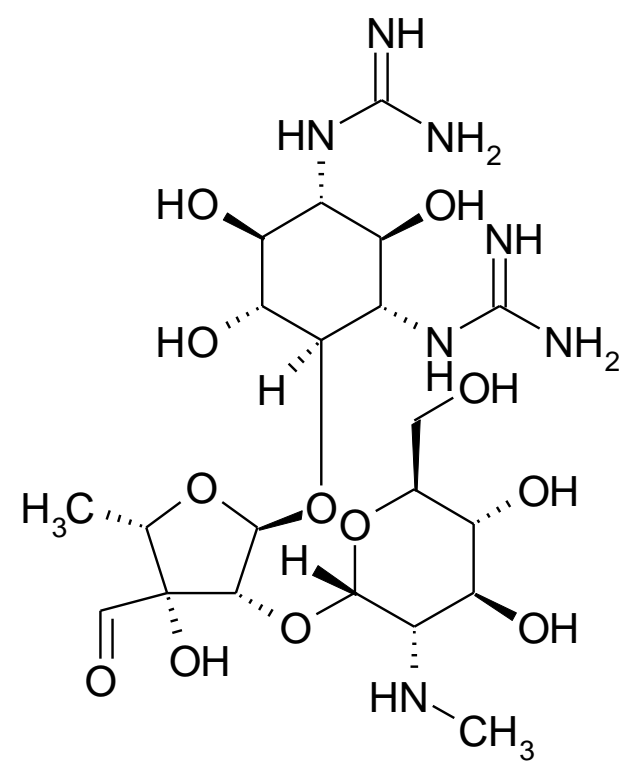

6

The genus of Streptomyces is the largest source of novel antibiotics from bacteria since the first report of streptomycin (6) was published in 1942. Currently, many microbial-derived antibiotics are reported from species of this genus, and this has increased exponentially for the last two decades. ${ }^{[21,22]}$ In 2001 , about $70 \%$ of all known antibiotics had been isolated from streptomycetes, and $4 \%$ of them were used clinically as human drugs. ${ }^{[23]}$

Erythromycin A (7) is a macrolide antibiotic derived from marine Streptomyces, and widely used as antimicrobial agent. The structure of erythromycin A (7) consists of a glycosidic 14-membered ring macrolide. Macrolides are one group of secondary metabolites produced by Streptomyces. Woodward firstly introduced the term "macrolide" for macrocyclic lactone antibiotics. The size of monolactone macrolides can be up to 62-membered cyclic systems. ${ }^{[24]}$ Additionally, most macrolide antibiotics have been reported to exhibit strong anti-inflammatory activity. ${ }^{[25]}$ 


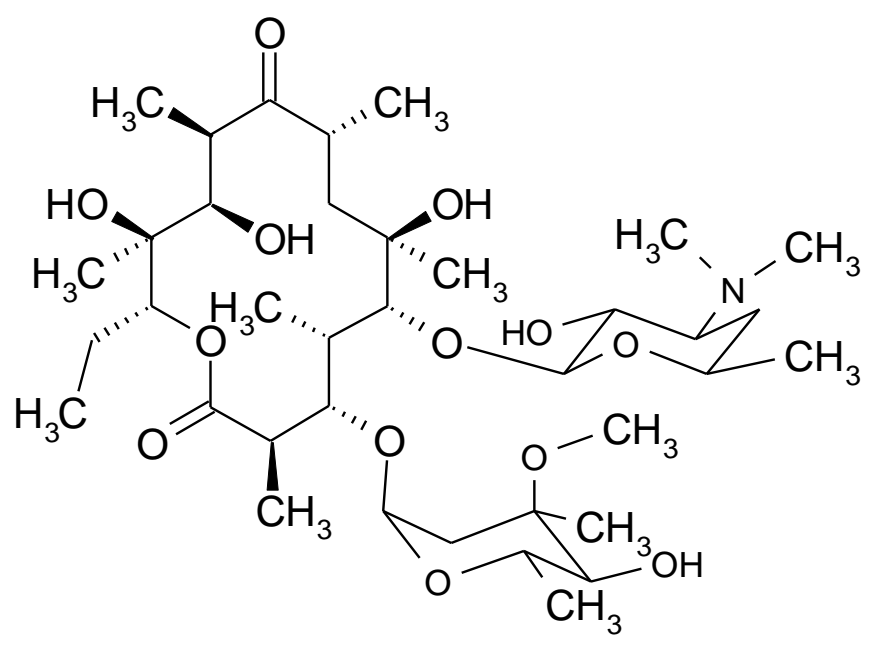

7

Neopeltolide (8 ) had been isolated from a deep-water organism Daedalopelta sp. in north Jamaican coast. It had been also reported to show antifungal activity against Candida albicans having MIC of $0.62 \mu \mathrm{g} / \mathrm{mL}^{\left[{ }^{[26]}\right.}$<smiles>CCC[C@H](C[C@@H](C[C@@H](C)C[C@H]1C[C@@H](OC(=O)/C=C\CCc2coc(/C=C\CNC(=O)OC)n2)C[C@H](OC)CC(=O)O1)OC)OC</smiles>
8

Arenicolides A-C (9-11) are 26-membered ring macrolides isolated from marine bacteria Salinispora arenicola. Among these macrolides, arenicolide A (9) was reported to exhibit moderate activity against the human colon adenocarcinoma cell line HCT-116. ${ }^{[27]}$ 


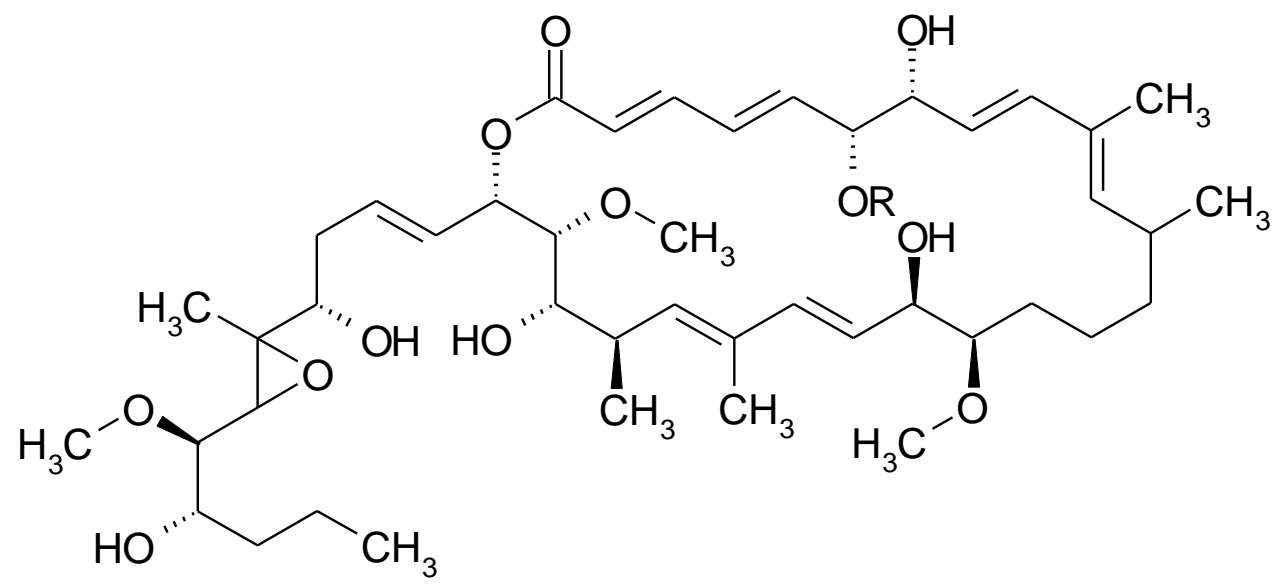

9: $\mathrm{R}=\mathrm{CH}_{3}$

$10 \mathrm{R}=\mathrm{H}$

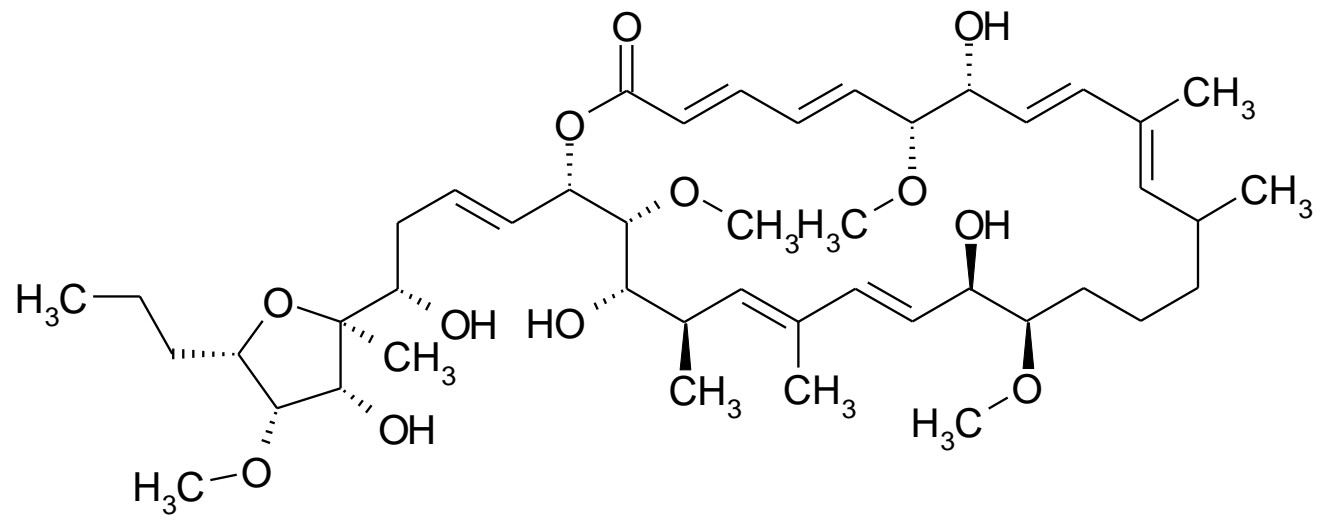

11

Pimaricin (12) belongs to the group of polyene macrolide with 26-membered ring possessing antifungal activity. ${ }^{[28]}$ The pimaricin analogue JBIR-13 (13) isolated from Streptomyces bicolor NBRC 12746 has been reported to show antifungal activity against Candida albicans with a minimum inhibitory concentration (MIC) value of $3.13 \mu \mathrm{g} / \mathrm{mL}{ }^{[29]}$ 


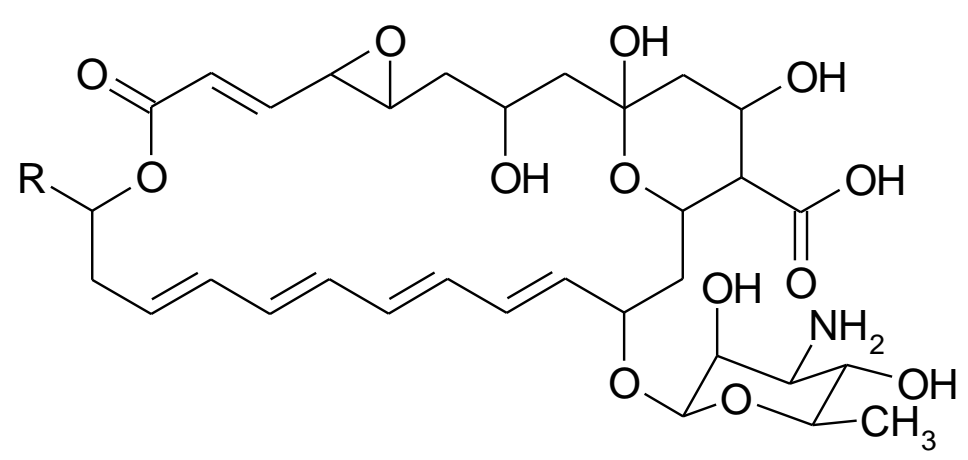

12: $\mathrm{R}=\mathrm{CH}_{3}$

13: $\mathrm{R}=\mathrm{C}_{3} \mathrm{H}_{7}$

Fiedler and co-workers have recently reported three new atacamycins A (14), B (15), and C (16), 22-membered macrolatones, as bioactive metabolites from Streptomyces sp. C38. Moderate antitumor activity had been exhibited by atacamycins A (14) and B (15) against tumor cell lines. ${ }^{[30]}$

Some antimicrobial agents have been reported as inhibitors of $\beta$-ketoacyl-(acylcarrier-protein (ACP)-synthase I/II (FabF/B), which plays an important role in bacterial fatty acid biosynthesis. ${ }^{[31]}$ Platensimycin (17) is an example of these inhibitors and was isolated independently in our group. ${ }^{[32]}$ Interestingly, it has been reported to demonstrate no cross-resistance to methicillin resistant Staphylococcus aureus (MRSA), vancomycin-resistant Enterococcus (VRE) and other tested antibioticresistant strains. ${ }^{[31,33]}$ 


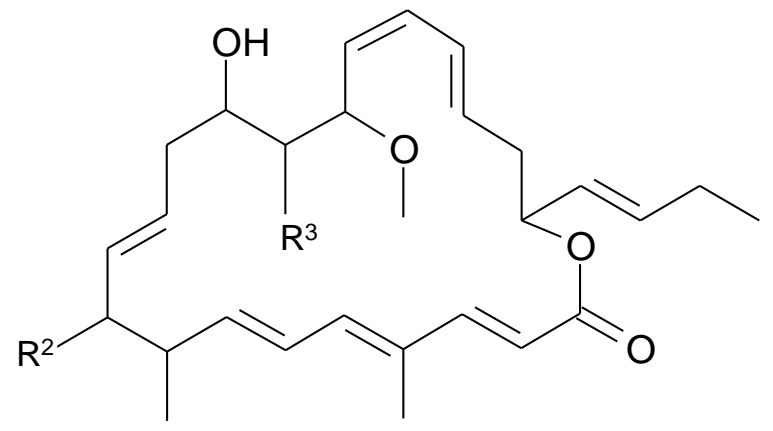

14: $\mathrm{R}^{2}=\mathrm{OMe}, \mathrm{R}^{3}=\mathrm{OH}$

15: $\mathrm{R}^{2}=\mathrm{H}, \mathrm{R}^{3}=\mathrm{OH}$

16: $\mathrm{R}^{2}=\mathrm{R}^{3}=\mathrm{H}$<smiles>CC1C[C@@]23C=CC(=O)C(C)(CCC(=O)Nc4c(O)ccc(C(=O)O)c4O)C1[C@H]1CC[C@@H]2O[C@@H]13</smiles>

17

Ionophore antibiotics are another type of antibiotics of bacterial origin, which can act as mobile ion carriers in complex form within cell membranes. ${ }^{[34]}$ Nonactin (18) is an example with antitumor activity: it was firstly isolated and reported from Streptomyces griseus; in addition to (-)-nonactic acid (19), the monomer of nonactin (18). ${ }^{[35,36]}$ 


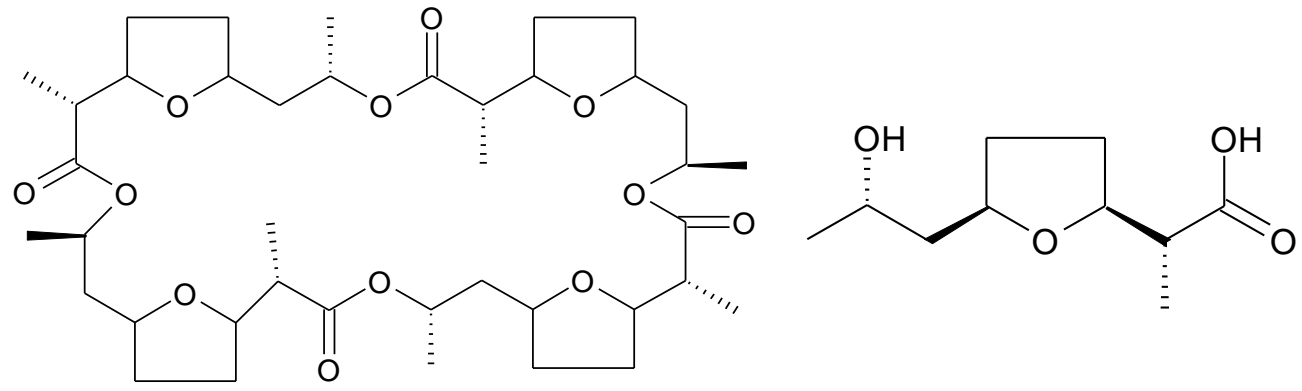

Alkaloids as another class of natural products are synthesized by both marine and terrestrial organisms in all evolutionary phases. Indole alkaloids in particular are present in a number of drugs which are currently on the market. ${ }^{[37]} \beta$-Hydroxy-Nacetyltryptamine (20) and staurosporine (21) are two indole alkaloids isolated from Streptomyces staurosporeus. ${ }^{[37,38]}$<smiles>CC(=O)NCC(O)c1c[nH]c2ccccc12</smiles>

20<smiles>CN[C@H]1C[C@H]2O[C@@](C)([C@@H]1OC)n1c3ccccc3c3c4c(c5c6ccccc6n2c5c31)C(=O)NC4</smiles>

21

A new catechol derivative, vanchrobactin (22), has been isolated from the bacterial fish pathogen Vibrio anguillarum serotype O2. Vanchrobactin (22) belongs to the siderophore group produced by bacteria as iron chelator. ${ }^{[39,40]}$ 
<smiles>NC(=[NH2+])NCCC[C@H](NC(=O)c1cccc(O)c1O)C(=O)N[C@@H](CO)C(=O)[O-]</smiles>

22

Vitamin B2 or riboflavine (23) is one of vitamins required in human nutrition and is found in both plants and bacteria. In bacteria, riboflavine (23) is used as an intercellular signalling agent to regulate the expression of genes in local populations. This feature is responsible for the coordination behaviour of gene or protein in motility, mating, stress responses and biofilm formation. ${ }^{[41]}$ Currently, it has been reported that a deficiency of riboflavine (23) may increase the risk of cancer and cardiovascular diseases. ${ }^{[42]}$<smiles>Cc1cc2nc3c(=O)[nH]c(=O)nc-3n(C[C@H](O)C(O)[C@H](O)CO)c2cc1C</smiles>

23

Six new chromophenazines A (24), B (26), C (25), D (27), E (28) and F (29) were isolated recently in our group from the terrestrial Streptomyces sp. Ank 315. Phenazine derivatives have been reported to exhibit broad-range activities such as antioxidant, neuroprotectant, antimicrobial, antiviral, antiparacitic, antitumor and antimalaria. Among the six chromophenazines, only chromophenazine D (27) 
showed moderate antimicrobial activity against Bacillus subtilis, Escherichia coli, and Mucor miehei (Tü 284). ${ }^{[43]}$<smiles>[R]c1cccc2c1nc1c3ccc(C)cc3c(=O)cc-1n2CC=C(C)C</smiles>

24: $\mathrm{R}=\mathrm{H}$

26: $\mathrm{R}=\mathrm{COOH}$<smiles>CC(C)=CCn1c2cc(=O)ccc-2nc2c(C(N)=O)cccc21</smiles>

25<smiles>[R]C(=O)c1cc(C(=O)c2ccccc2)cc2c1Nc1ccc([R])cc1N2CC=C(C)C</smiles>

27: $\mathrm{R}^{1}=\mathrm{OH}, \mathrm{R}^{2}=\mathrm{H}$

28: $\mathrm{R}^{1}=\mathrm{OH}, \mathrm{R}^{2}=\mathrm{C} 6 \mathrm{H} 5 \mathrm{CO}-$

29: $\mathrm{R}^{1}=\mathrm{NH}_{2}, \mathrm{R}^{2}=\mathrm{C} 6 \mathrm{H} 5 \mathrm{CO}-$

Three new angucycline antibiotics N05WA963A (30), B (31) and D (32) have been isolated from the culture broth of Streptomyces sp. N05WA963. In addition, they have been also reported to show potent antitumor activity against several cancer cell lines such as SW620, K-562, MDA-MB-231, YES-4, T-98 and U251SP. ${ }^{[44]}$ 


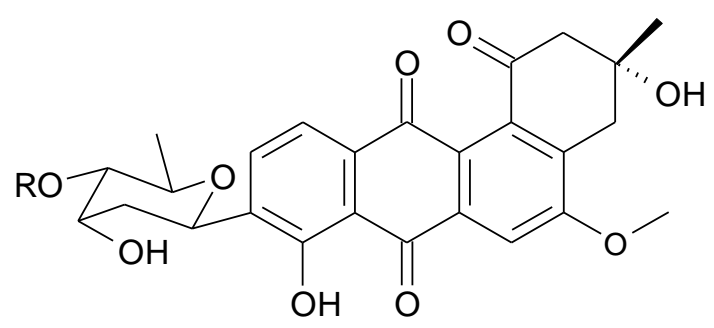

30: $\mathrm{R}=$<smiles>CC(C)COC1CCC(C)OC1C</smiles>

31: $\mathrm{R}=\mathrm{H}$

32: $\mathrm{R}=$<smiles>CC1CCC(COC2CCC(C)C(=O)C2)C(C)O1</smiles>

Recently, leinamycin (33), a new hybrid peptide-polyketide antibiotic with antitumor activity, has been isolated from Streptomyces atroolivaceus S-140. The biosynthesis of leinamycin (33) is derived from the polyketide pathway. Microbial polyketides are a large group of natural products and many of them are in clinical use as drugs. ${ }^{[45]}$ Most microbial peptide antibiotics are biosynthetically formed as nonribosomal metabolites produced in a multi-functional enzymatic process. ${ }^{[46]}$

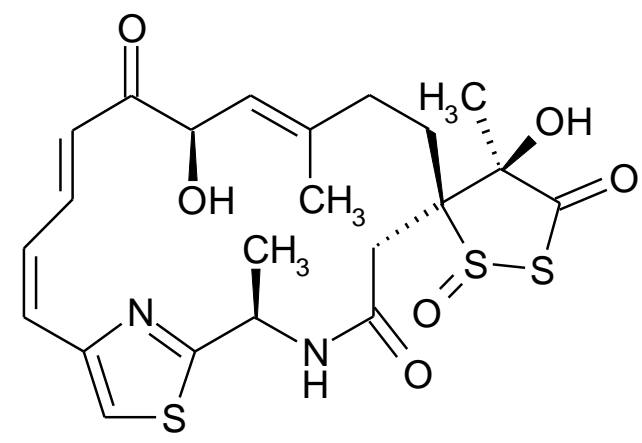


Two cyclic octapeptides, thalassospiramides A (34) and B (35) were isolated recently from a new marine-derived $\alpha$-proteobacterium of the Thalassospira genus. They exhibited immunosuppressive activity in an interleukin-5 production inhibition assay. $^{[47]}$<smiles>CCCCCC/C=C\CC(=O)NC(CO)C(=O)NC(C(=O)NC(CO)C(C)C)C(=O)NC(CO)[C@@H](O)CC(=O)NC(C(=O)N[C@H](/C=C/C(=O)N[C@@H](CC)C(=O)N(C)C(Cc1ccc(O)cc1)C(C)C)COC(=O)C(C)C)C(C)C</smiles>

34

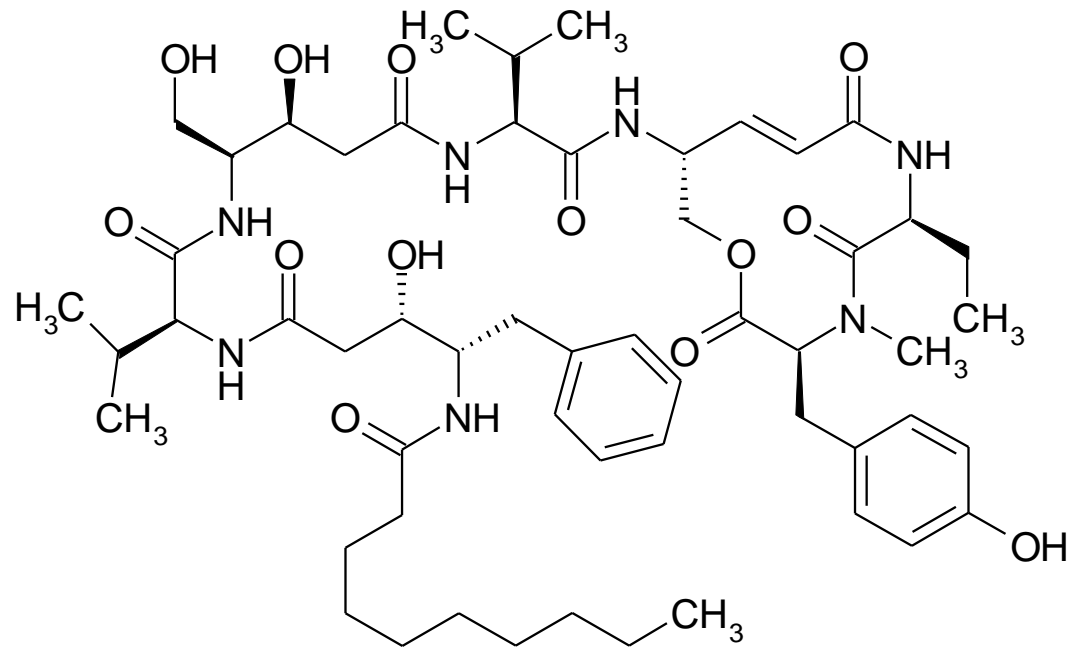

35

Thiopeptides or thiazolylpeptides are natural bacterial antibiotics, which are produced by filamentous prokaryotic organisms. Philipimycin (36) is an example of this group isolated from Actinoplanes philippinensis MA7347, with strong antibacterial activity against Gram-positive bacteria with MIC values ranging from 0.015 to 1 $\mu \mathrm{g} / \mathrm{mL}{ }^{[48]}$ 


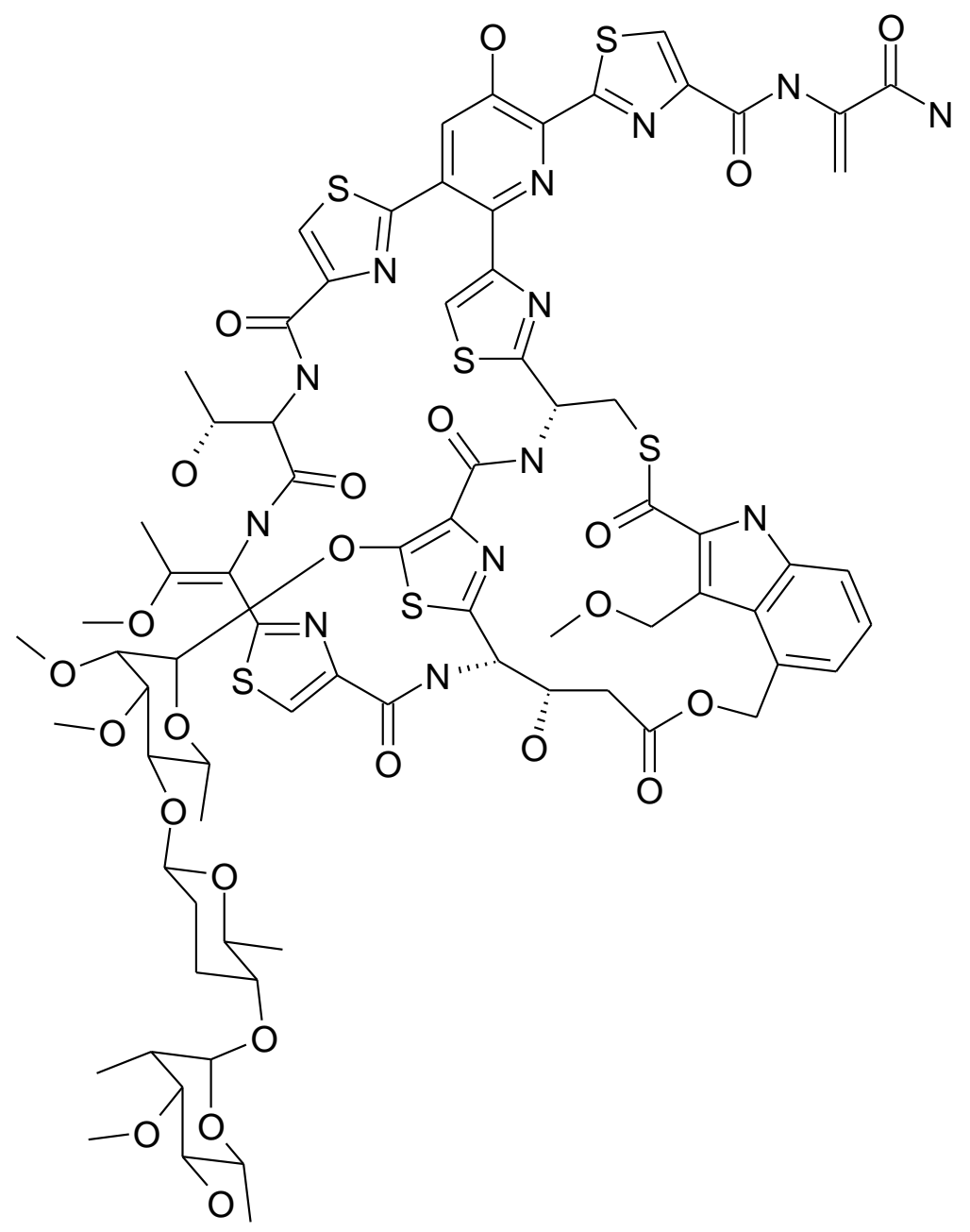

36

Val-geninthiocin (37) is another thiopeptide antibiotic, which was recently isolated in our group from the terrestrial Streptomyces sp. RSF18. ${ }^{[4]}$ This type of antibiotics is also strongly active as antimalarial agent against Plasmodium falciparum. ${ }^{[50]}$ 


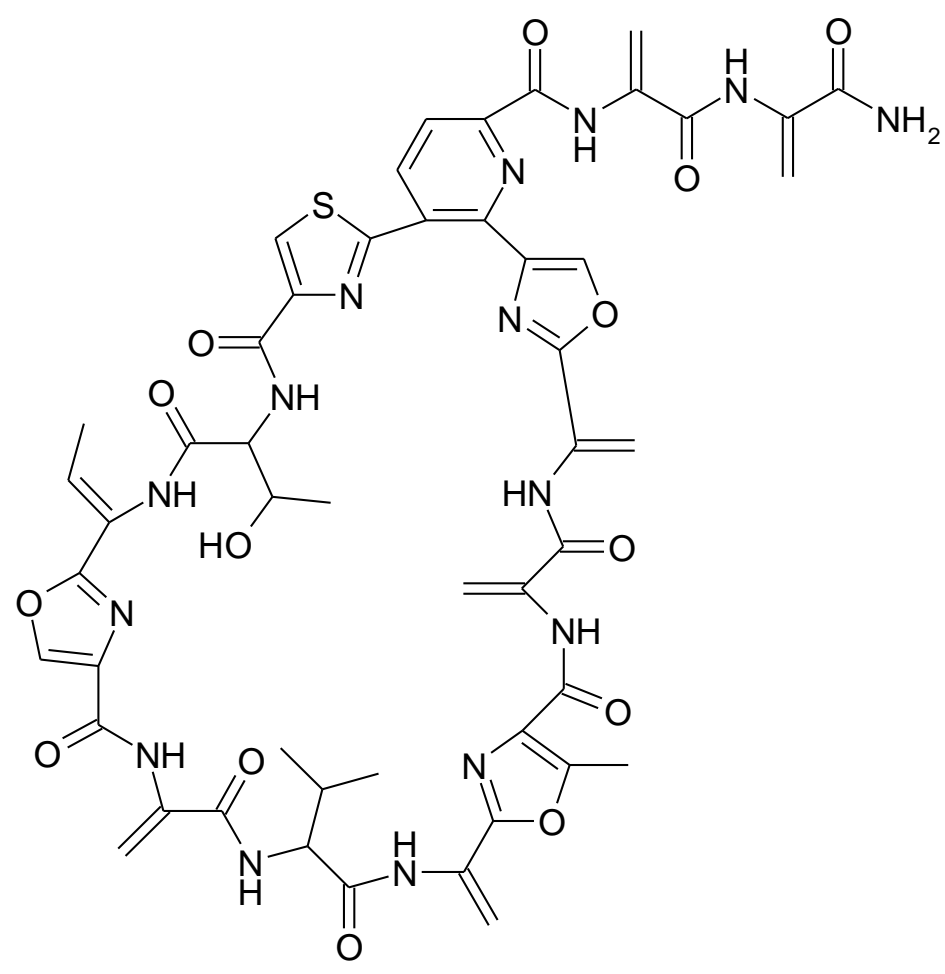

37

Kettapeptin (38), a new cyclic hexadepsipeptide antibiotic, had been isolated and characterized in our group from the terrestrial Streptomyces sp. GW 99/1572 by Maskey. It showed antibacterial and antitumor activities against Bacillus subtilis, Streptomyces viridochromogenes (Tü 57), Staphylococcus aureus, Escherichia coli and several human cancer cell lines. ${ }^{[51]}$

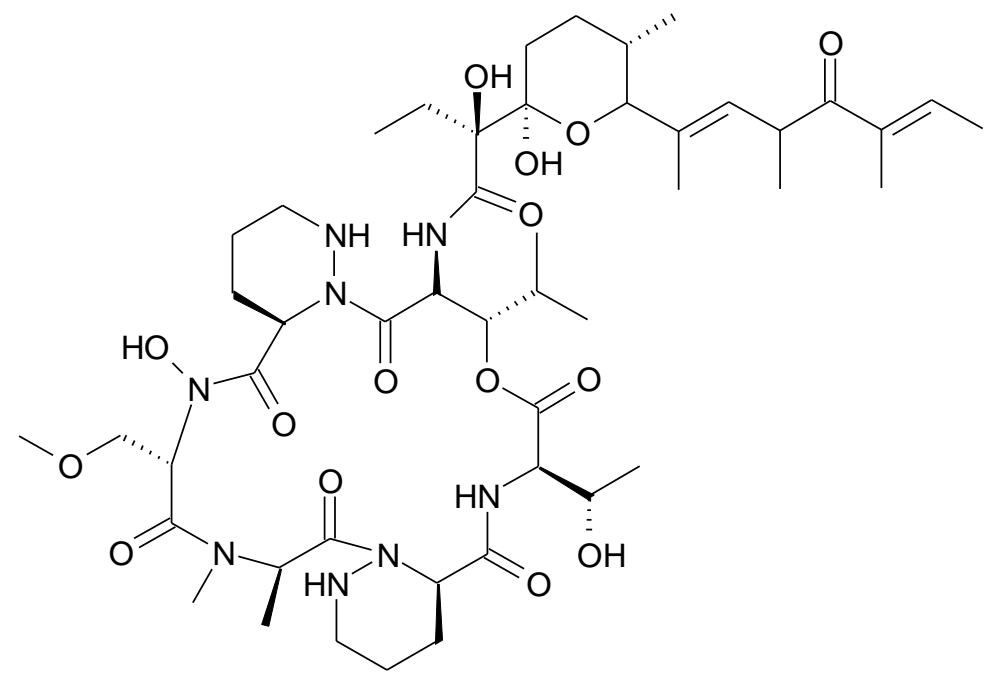


Azoxy compounds are another type of microbial-origin antibiotics exhibiting several biological activities: They are e.g. antiviral, antitumor, antifungal and antinematodal. ${ }^{[52]}$ Valanimycin $(39)^{[53]}$ and elaiomycin (40) are examples with anticancer activity among the azoxy derivatives isolated from Streptomyces sp. $^{[52,54]}$<smiles>C=C(N=[N+]([O-])CC(C)C)C(=O)O</smiles>

39<smiles>CCCCCC/C=C/[N+]([O-])=N/[C@H](COC)[C@H](C)O</smiles>

40

Maniwamycins A (41) and B (42) are further antifungal azoxy antibiotics. ${ }^{[55]}$ They were isolated from the terrestrial Streptomyces prasinopilosus. ${ }^{[56]}$<smiles>CCCC/C=C/[N+]([O-])=NC(C)C(C)=O</smiles>

41<smiles>CCCCC=C[N+]([O-])=NC(C)C(C)O</smiles>

42

Two new derivatives of elaiomycins $B(\mathbf{4 3})$ and $C(\mathbf{4 4})$ have been isolated recently from Streptomyces sp. BK 190. They have been reported to show slight antimicrobial activity against Staphylococcus lentus DSM 6672. ${ }^{[57]}$ Further azoxy antibiotics have been reported recently. ${ }^{[58]}$

Nucleobase-containing natural products e.g. purine and adenosine analogues have been of interest due to their significant pharmacological properties. ${ }^{[59,60]}$ Sinefungin (45) is a natural nucleoside first reported from Streptomyces griseolus in 1973. It demonstrated potent antifungal, antiviral, and antiparasitic activities. ${ }^{[61]}$ (-)Aristeromycin (46) is another example of natural nucleoside analogues and was first isolated from Streptomyces citricolor. It showed antiviral and inhibitory activities against cellular enzyme $S$-adenosyl-L-homocysteine hydrolase (SAH). ${ }^{[60]}$ 
<smiles>[R]N(NC(=O)COC)C(=O)CCCCCCCCC</smiles>

43: $\mathrm{R}=$<smiles>[R]=C=CCCCCC=CCCCCCCCC</smiles>

44: $\mathrm{R}=$<smiles>[R]=C=CCCCCC=CCC=CCCCC</smiles>

$\mathrm{HO}$<smiles>CC(=O)C(N)CCC(N)C[C@@H]1O[C@@H](n2cnc3c(C)ncnc32)[C@H](O)[C@@H]1O</smiles>

45<smiles>Nc1ncnc2c1ncn2[C@@H]1O[C@H](CO)[C@@H](O)[C@H]1O</smiles>

Cyanobacteria are an alternative and also prolific microbial source of natural products. ${ }^{[62]}$ Recently, two credneramides A (47) and B (48) containing a vinyl chloride unit have been reported from a Papua New Guinea cyanobacterium, cf. Trichodesmium sp. nov. They were reported additionally as inhibitor of calcium oscillations in cerebrocortical neurons of mice. ${ }^{[63]}$ 
<smiles>[R]C(=O)CC/C=C/C/C(=C/Cl)CCC</smiles>

47: $\mathrm{R}=$<smiles>CNCCc1ccccc1</smiles><smiles>[R]#CNCCC(C)C</smiles>

\subsection{Natural products from fungi}

After the discovery of penicillin (1) from Penicillium notatum in 1928 by Alexander Fleming, fungi have contributed a highly significant amount of drugs in the pharmaceutical industry for the treatment of human diseases. Currently, terrestrial and marine fungi were reported to produce many important and useful substances for medicines and plant protections. ${ }^{[64,65]}$

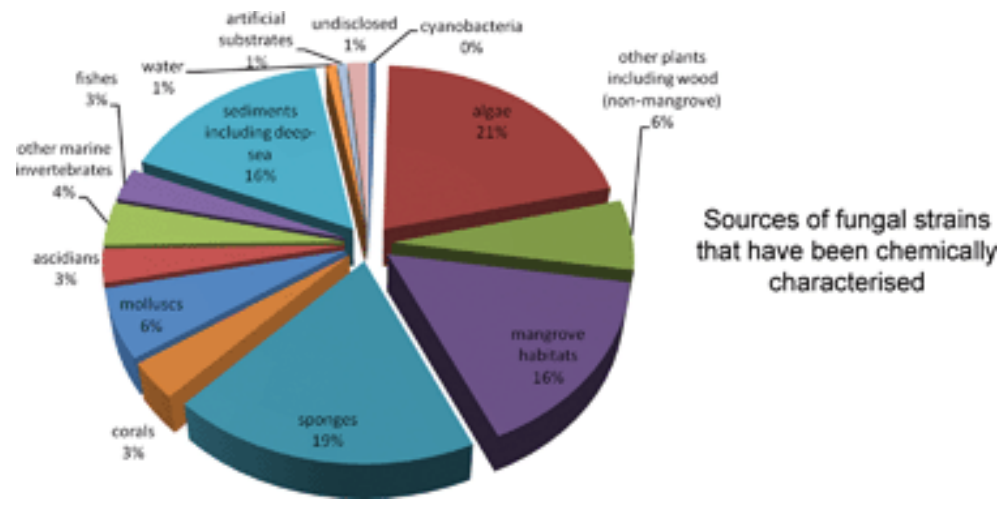

Figure 3: $\quad$ Percentages of new metabolites based on fungi sources until mid $2010 .^{[65]}$

Enniatin B (49) and bassianolide (50) are examples of fungi-originated cyclodepsipeptides. Enniatins act as ionophores in biological membranes, inhibitors of the ABC-transporter Pdr5p and topical agent for the treatment of respiratory infec- 
tions. Bassianolide (50) was toxic to insect larvae and some cancer cell lines in vitro. ${ }^{[66]}$

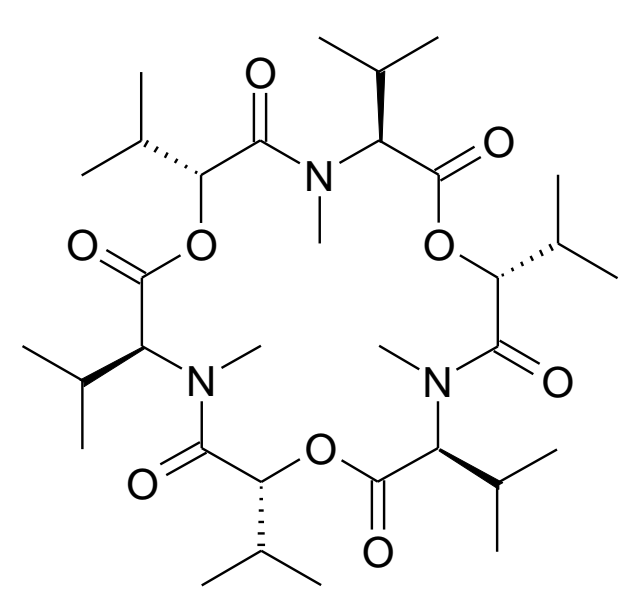

49

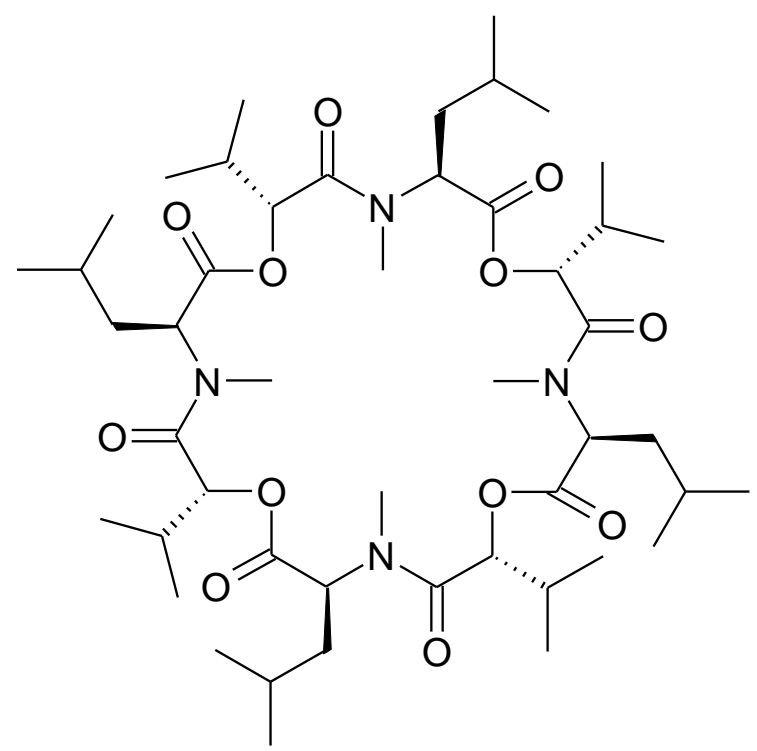

50

Fusaristatins A (51) and B (52) are two new cyclic lipopeptides isolated in our group from the endophytic fungus Fusarium sp. YG-45 in rice medium. Both fusaristatins A (51) and B (52) were active as anticancer agent against lung cancer cells LU 65. Additionally, fusaristatin B (52) showed moderate activity against topoisomerases I and II, respectively. ${ }^{[67]}$

Two known xanthones, sterigmatocystin (53) and secosterigmatocystin (54), were recently re-isolated in our group in a screening for insecticidal compounds from the endophytic fungus Podospora sp. Sterigmatocystin (53) revealed strong larvacidal activity against Anopheles gambiae indicating potential insecticidal use in order to control the vectors of malaria, dengue fever, yellow fever, and several other diseases caused by mosquitoes. ${ }^{[68]}$ 
<smiles>C=C(NC(=O)C(C)C(CCC(C)C(=O)/C=C/C(C)=C/CCC(C)C)OC(=O)C(CCC(N)=O)NC(=O)C(C)CNC(=O)C(=O)NC)C(=O)NCC(C)C</smiles>

51<smiles>C=C(C)C(=O)NCC(C)C(=O)NC(CCCC(=O)O)C(=O)OC(CCC(C)C(=O)/C=C/C(C)=C/CCC(C)C)C(C)C(=O)NC(=C)C(=O)NC</smiles>

52<smiles>COc1cc2c(c3oc4cccc(O)c4c(=O)c13)C1C=COC1O2</smiles>

53<smiles>COc1cc(O)c(C(CO)C(O)CO)c2oc3cccc(O)c3c(=O)c12</smiles>

54 
Recently, four new pyridine and tetramic acid alkaloids namely torrobiellones A (55), B (56), C (57) and D (58) have been isolated from the fungus Torrobiella sp. BCC 2165. Torrobiellone A (55) has been reported to show antimalarial activity against Plasmodium falciparum. ${ }^{[69]}$ In addition, a unique tetramic acid derivative, cryptocin (59), has been isolated from the endophytic fungus Cryptosporiopsis quercina. It exhibited antifungal activity against the plant pathogenic fungus Pyricularia oryzae. ${ }^{[11]}$<smiles>CCC(/C=C/C=C/C=C/C(=O)c1c(O)c([C@]2(O)CC[C@H](O)CC2)cn(O)c1=O)CO</smiles>

55<smiles>CCC(/C=C/C=C/C=C/C(=O)c1c(O)c([C@]2(O)CC[C@H](O)CC2)c[nH]c1=O)CO</smiles>

56<smiles>CCC(/C=C/C=C/C=C/C(=O)c1c(O)c(-c2ccc(O)cc2)c[nH]c1=O)CO</smiles>

57<smiles>CCC(/C=C/C=C/C=C/C(O)=C1\C(=O)NC(Cc2ccc(O)cc2)C1=O)CO</smiles> 


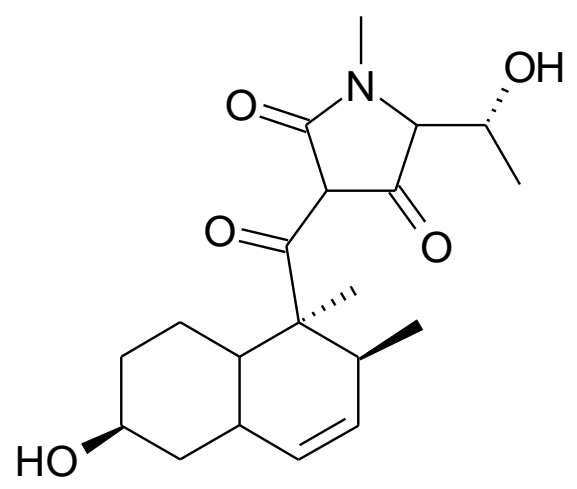

59

Humicolone (60) had been isolated from the terrestrial fungus Humicola grisea Traaen. It exhibited cytotoxicity against $\mathrm{KB}$ cell line with $\mathrm{IC}_{50}$ values ranging between 1 and 5 ppm. ${ }^{[70]}$<smiles>C[C@@H]1Oc2cccc3c2[C@@H](O1)[C@H](O)CC3=O</smiles>

60

Two new cyclohexanone epoxide derivatives, jesterone (61) and hydroxyjesterone (62) were isolated from the endophytic fungus Pestalotiopsis jesteri found in Papua New Guinea. Jesterone (61) specifically showed antimycotic activity against oomyceteous plant pathogenic fungi Pythium ultimum, Aphanomyces sp., Phytophthora citrophthora, and Phytophthora cinnamomi ${ }^{[71,72]}$ 
<smiles>C/C=C/C1=C(CO)[C@@H](O)[C@H]2O[C@@]2(CC=C(C)C)C1=O</smiles>

61<smiles>C/C=C/C1=C(CO)[C@@H](O)[C@H]2O[C@]2([C@H](O)C=C(C)C)C1=O</smiles>

62

The marine endophytic fungus Gymnascella dankaliensis from the Japanese sponge Halichondria japonica produced an unusual steroid derivative, dankasterone (63). Dankasterone (63) displayed cytotoxic activity against human cancer lines. ${ }^{[65,73]}$<smiles></smiles>

63

Recently, three new cerebroside derivatives, alternarosides A (64), B (65) and C (66), were reported from the marine fungus Alternaria raphani. They displayed weak antimicrobial activity against bacteria (Escherichia coli, Bacillus subtilis) and the fungus Candida albicans. ${ }^{[74]}$ 
<smiles>CCCCCCCCCCCCCC/C=C/[C@@H](O)C(=O)NC(COC1OC(CO)C(O)C(O)C1O)[C@H](O)/C=C/C(C)(C)C</smiles>

A (64)<smiles>C=C(CCCCCCCCC)C(=O)CCC(C)(C)C</smiles>

$\mathrm{B}(\mathbf{6 5})$<smiles>CCCCCCCC/C(C)=C/CCC(C)(C)C</smiles>

C (66)<smiles>CCCCCCCCC/C=C/CCC(C)(C)C</smiles>

Two new lactone derivatives, 1893 A (67 ) and B (68), have been reported from an unidentified endophytic fungus No. 1893. The fungus was isolated from an estuarine mangrove Kandelia candel. ${ }^{[75]}$

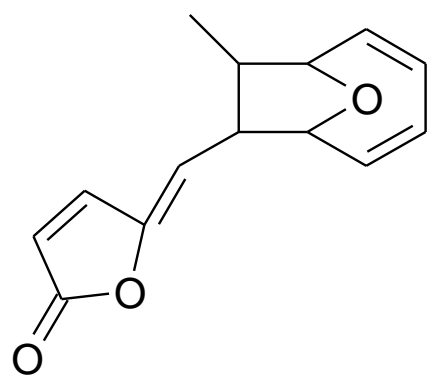

67

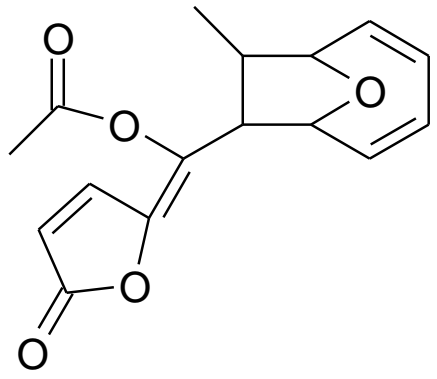

68

Recently, four new 10-membered lactones A (69), B (70), C (71) and D (72) have been reported from marine-derived endophytic fungus Curvularia sp. The fungus was isolated from the marine red alga Acanthophora spicifera. ${ }^{[76]}$ 
<smiles>C[C@@H]1CCC(O)C2OC2/C=C\C(=O)O1</smiles>

69<smiles></smiles>

71<smiles></smiles>

70<smiles>C[C@H]1OC(=O)/C=C\[C@H](O)/C=C/[C@@H](Cl)[C@@H]1O</smiles>

72

The endophytic fungus Hormonema sp. (ATCC 74360) has been reported to produce a new antifungal triterpenoid glycoside, enfumafungin (73). Its activity against Aspergillus fumigatus is an interesting feature. ${ }^{[75]}$

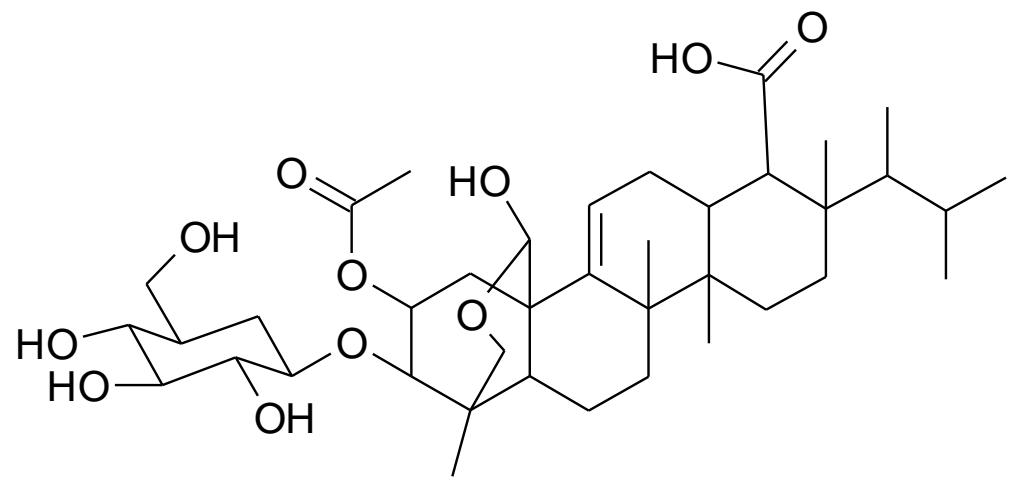

73

Recently, Verma and coworkers have isolated piperine (74) from an endophytic fungus Periconia sp. Piperine (74) is the alkaloid and pungent principle of black pepper and used in traditional medicine as antioxidant, analgesic, antidepressant and antibacterial. In addition, piperine (74) has shown strong antimycobacterial activity against Mycobacterium tuberculosis and Mycobacterium smegmatis. ${ }^{[77]}$ 
<smiles>O=C(/C=C/C=C/c1ccc2c(c1)OCO2)N1CCCCC1</smiles><smiles>COc1cc(O)c2c(=O)oc3cc(OC(C)=O)cc(C)c3c2c1</smiles>

75

Lin and co-workers have reported the isolation of a new alternariol derivative, 2240B (75) from Chinese endophytic fungus No. 2240. Alternariol has been reported as a mycotoxin contaminating fruits and cereals. In addition, alternariol 2240B (75) showed weak activities against two tumor cell lines, $\mathrm{KB}$ and $\mathrm{KB} v 200 .{ }^{[78]}$

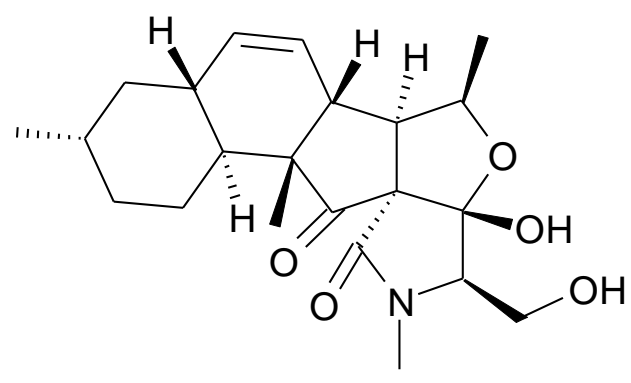


Jang and co-workers have reported a new pentacyclic ring system, fusarisetin A (76), from Fusarium sp. FN080326. It had shown inhibitory activity against MDAMB-231 cells. ${ }^{[79]}$

\section{Scope of the present work}

The current development of antibiotic resistance has become a major problem in modern medical therapy. In responding to this challenge, there is an urgent need to search for new sources and leads. Especially microorganisms are still a potent source of bioactive compounds with wide-ranging applications as antibiotics, agrochemicals, immunosuppressant, antiparasitic, and anticancer agents. ${ }^{[75]}$

Despite of the short period of research on marine and terrestrial natural products, more than 230,000 compounds have been published within 80 years. ${ }^{[80]}$ Moreover, many of them possess unique functional groups or rare skeletons as well as potent biological activities. It is noteworthy that presently published new compounds with novel or rare skeletons are almost exclusively found in marine organisms. ${ }^{[81]}$ Although, soil has been a common source to isolate microorganisms, living plants, leaf litter and dung have been reported as other promising environment of organisms. ${ }^{[82]}$

Accordingly, the main objective of the present investigation is concerned with the isolation and structure elucidation of biologically active and preferably new secondary metabolites from bacteria and endophytic fungi. This study was focussed mainly on the genus Streptomyces collected from terrestrial and marine sources, and terrestrial endophytic fungi.

To achieve this goal, chemical and biological screenings should be applied in such a way that minimizes the time and gets a maximum of results. For this reason, a 'horizontal screening' was selected to be applied: having few biological tests against Gram-positive and Gram-negative bacteria, fungi and yeasts, microalgae and brine shrimp, the antibacterial, antifungal, phytotoxic and cytotoxic activities are covered, and results can be used as a guide for further detailed investigations.

Microorganisms such as bacteria and endophytic fungi have proven as bioactive metabolite producers. Therefore, there are logical approaches to investigate selected terrestrial and marine-derived bacteria and endophytic fungi. 
- After selecting the strains based on the chemical and biological screenings, the fermentation on large scale is performed in order to isolate the metabolites.

- The crude extracts obtained from fermentation are then separated and purified via different chromatographic techniques i.e. silica gel, Sephadex LH-20, RP18 columns, HPLC, PTLC.

- Pure components are identified spectroscopically e.g. by NMR and MS techniques, and guided by dereplication database software such as AntiBase, Chemical Abstracts (SciFinder), and the Dictionary of Natural Products (Chapman \& Hall).

- Finally, the isolated pure metabolites are subjected to bioassays in order to characterize the biological activity against bacteria, fungi, algae, and brine shrimps (Artemia salina). 


\section{General techniques}

\subsection{Collection of strains}

All the strains (bacteria and fungi) in this research project were obtained via cooperations with various microbiological groups, summarized as follows:

- The terrestrial Streptomyces spp. (code beginning with Ank) were obtained from and taxonomically determined by Prof. Dr. H. Anke, Institute for Biotechnology and Drug Research, Kaiserslautern, Germany. The terrestrial Streptomyces spp. (code beginning with GT) were received from the Hans-Knöll-Institute, Germany. The terrestrial Streptomyces spp. (code beginning with GW) were obtained from the laboratory of Dr. Iris Grün-Wollny, Giessen, Germany.

- The marine Streptomyces spp. (code beginning with B) were obtained from the collection of Dr. E. Helmke, Alfred-Wegener Institute for Polar and Marine Research, Bremerhaven, Germany.

- Some endophytic fungal strains were isolated from medicinal plants collected in the Botanical Garden in Göttingen, in Cameroon and in Kenya and will also be discussed here.

\subsection{Strategic procedure in evaluating the selected strains}

It was essential to perform a well-defined strategy for a better evaluation of the samples in order to select the most suitable one from a large number of strains. This purpose can be achieved via the combination of simple bioassays and chemical screening techniques. Then, the cultivation of the selected strains was scaled up followed by the isolation of the metabolites. Finally, the activity tests for the pure compounds were carried out after the structure elucidation was performed. 


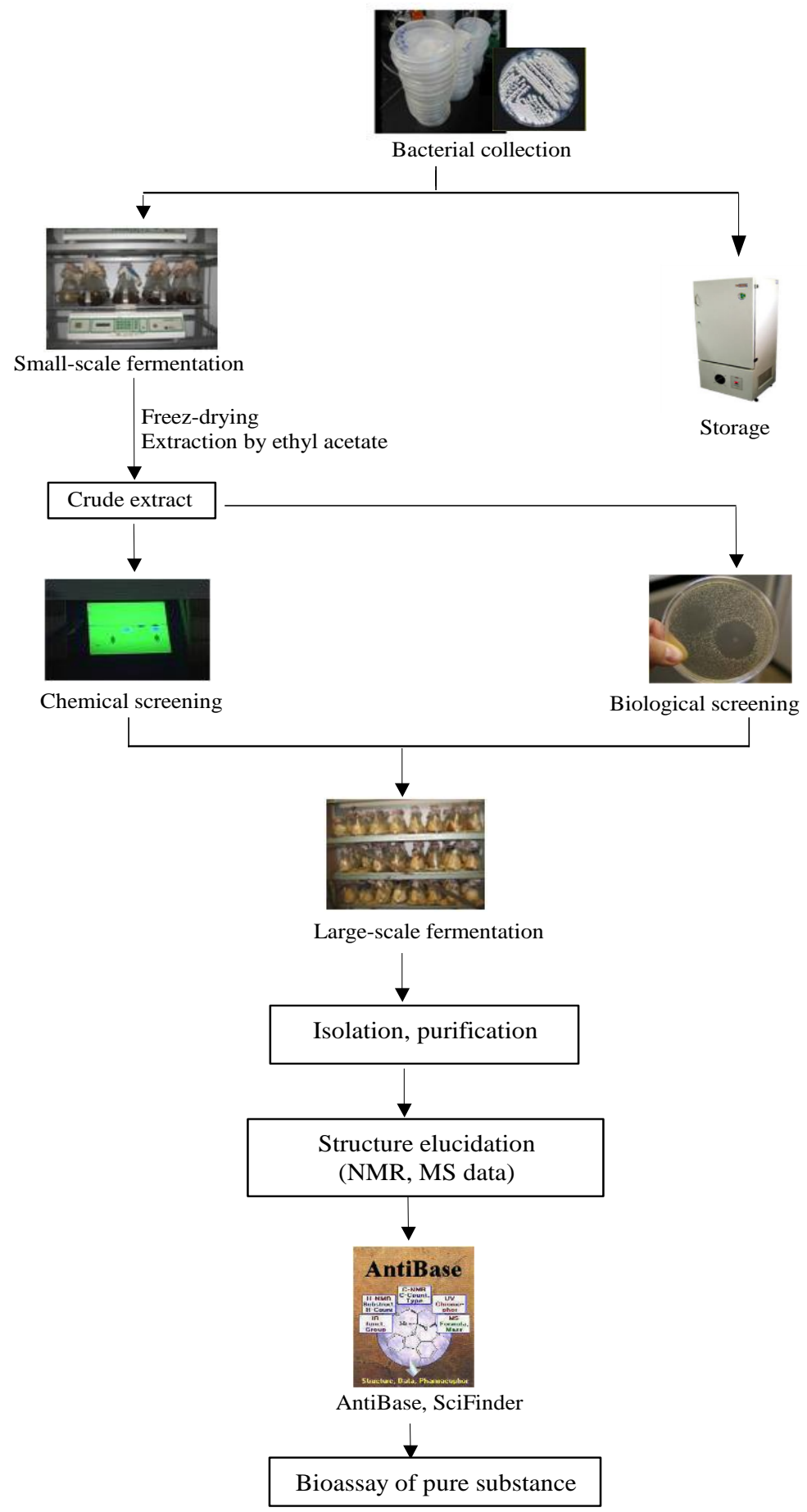

Figure 4: General screening steps for the selected strains. ${ }^{[83]}$ 


\subsection{Pre-screening}

For identification of the most promising candidates among the available cultures for further investigation, two different approaches can be applied, namely the biological screening and the chemical screening.

At the university level, the biological screening is performed in a 'horizontal' way with tests of low selectivity, to target a broad range of indications and to get rapidly activity results. In our group, agar diffusion tests were used in the screening of the crude extracts against Gram-positive and Gram-negative bacteria, fungi, microalgae (plants representatives), and brine shrimps (Artemia salina), to cover microorganisms, plants and higher organisms. The bio-autography method on TLC plates gave simultaneously additional information of bioactive components in the crude extracts. In case of interesting and positive results, the samples were sent to industrial partners for a more comprehensive 'vertical' screening e.g. for cytotoxicity tests (Oncotest GmbH, Freiburg) and agricultural application (BASF AG, Ludwigshafen). ${ }^{[84]}$
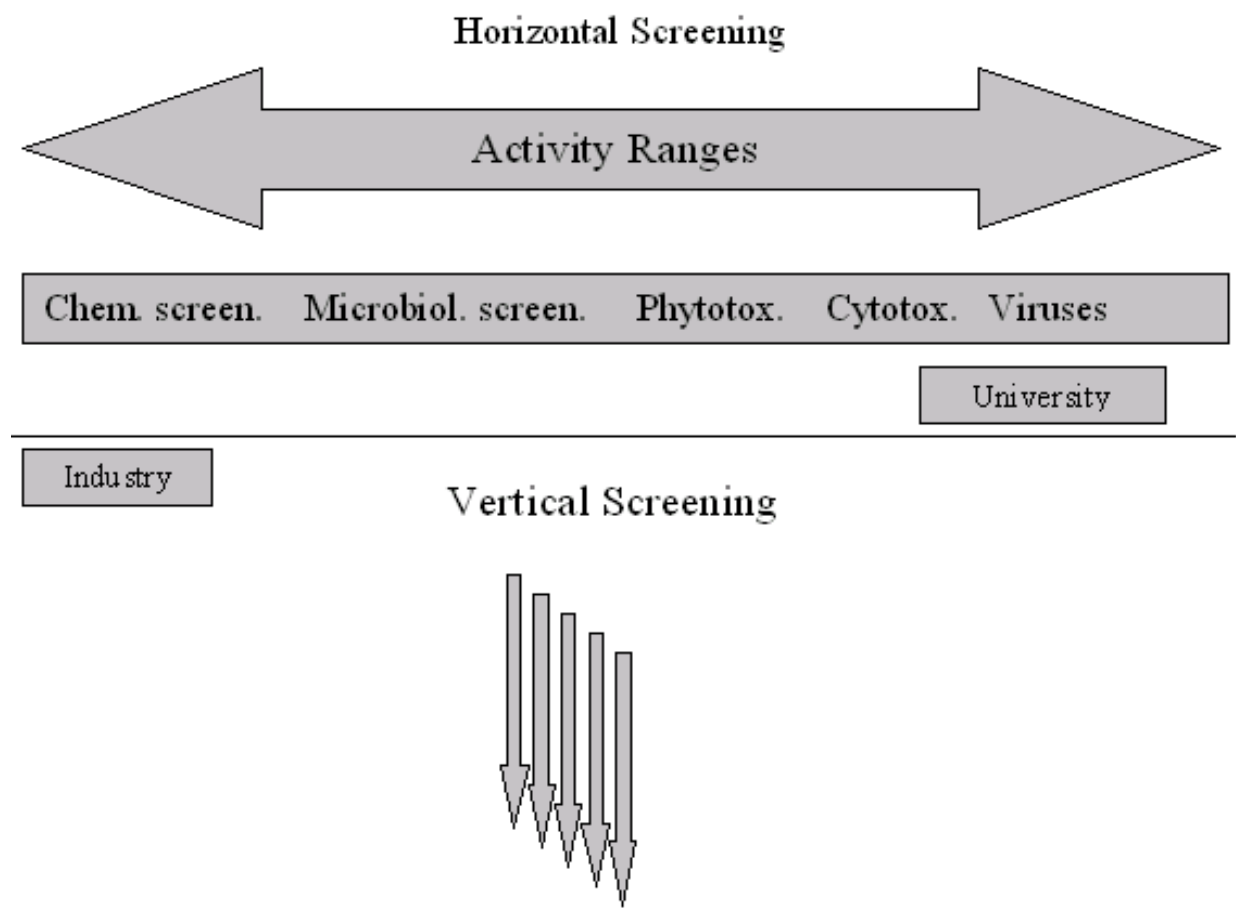

Single In dications

Figure 5: Principles of screening for activities of bioactive metabolites on university and industry levels. ${ }^{[84]}$ 
Pharmaceutical or medical industries, on the other hand, conduct a 'vertical' screening. Their biological screening is performed by selective tests using receptors or enzymes as sites of actions with a large number of samples. Mainly due to time and resources constraints, horizontal screening was conducted at university level in order to retrieve a quick results of biological activities in a sample using a limited number of tested-indicator organisms. ${ }^{[84]}$

\subsection{Biological screening}

The strains were sub-cultured on agar plates for 3-7 days and microscopically controlled for contaminations. Then, small pieces of the agar culture were inoculated into $1 \mathrm{~L}$ Erlenmeyer flasks containing $250 \mathrm{~mL}$ of a suitable medium followed by the incubation on a rotary shaker at $28{ }^{\circ} \mathrm{C}$. The resulting culture broth was lyophilised and the dried residue was extracted with ethyl acetate, which was then evaporated under reduced pressure. The crude extract was used for biological, chemical, and pharmacological screenings. For the biological activity tests, the agar diffusion technique was used with microorganisms and microalgae. In parallel, the cytotoxicity was evaluated against brine shrimps (Artemia salina).

Microorganisms used for biological activity tests

Bacillus subtilis

Staphylococcus aureus

Streptomyces viridochromogenes (Tü 57)

bacteria

Escherichia coli

Candida albicans

Mucor miehei (Tü 284)

fungi

Chlorella vulgaris

Chlorella sorokiniana

algae

Scenedesmus subspicatus 


\subsection{Chemical screening}

The isolation and structure elucidation of secondary metabolites from microorganisms are time consuming, multiple-step and expensive processes. Therefore, identification of known metabolites at the earliest stage from crude extracts or partially purified-fractions became an important issue. To achieve this, chemical screening via thin layer chromatography is one of the simplest and cheapest methods applied for the detection of chemical constituents in the crude extracts. After developing the TLC of the crude extract in a suitable solvent system (usually $\mathrm{CH}_{2} \mathrm{Cl}_{2} / \mathrm{MeOH}$ ), the UV-lamp and spray reagents were used to visualize and localize the interesting zones. In our group, we use regularly the following spray reagents:

- Anisaldehyde/sulphuric acid gives different colour reactions with many structural features.

- Ehrlich's reagent is used for the specific detection of indoles, which turn to pink, orange, blue, or violet. Other heterocycles and anthranilic acid may turn yellow.

- Concentrated sulphuric acid is applied for polyenes. Short conjugated chains show a brown or black colour while carotenoids turn to blue or green.

- Peri-hydroxy-quinones turn to red, blue, or violet with sodium hydroxide. Deep red prodigiosins stain to yellow with base.

- Chlorine $/ o, o^{\prime}$-dianisidine is a universal reagent for the detection of peptides.

\subsection{Cultivation and scaling-up}

After pre-screening of the strains, cultivating and scaling up steps of selected strains were performed for further investigation. Under certain circumstances, the optimisation of the culture conditions was needed in order to improve the microbial production of interesting natural metabolites.

Well-grown agar cultures were used to inoculate 100 of 1 L Erlenmeyer flasks containing $250 \mathrm{~mL}$ medium at $\mathrm{pH}$ 7.8. Then, the cultures were cultivated on a linear shaker at $28{ }^{\circ} \mathrm{C}$ for 4 or 5 days. After harvesting, the culture broth was mixed with Celite (diatomaceous earth) and filtered over the filter press to separate the water phase and the biomass. The water phase was passed through an Amberlite XAD-16 resin column followed by extraction with methanol. The biomass was exhaustively 
extracted with ethyl acetate and acetone. The organic phases were dried under reduced pressure and the dried-residue was used for the next separation steps.

\subsection{Isolation and purification work-up}

The technique applied for isolation and purification was dependent on the properties of the bioactive metabolites of interest e.g. the polarity and the amount of crude extracts. In general, the crude extract was subjected to silica gel column chromatography with a gradient of dichloromethane/methanol. After fractionation by column chromatography, the separated fractions were further subjected to size exclusion chromatography using Sephadex LH-20. High recovery rate and minimum decompositions of the chemical constituents are the advantages of Sephadex LH-20. The next isolation methods may include preparative thin layer chromatography (PTLC), and column chromatography e.g. on RP-18, or again Sephadex LH-20 and silica gel columns.

\subsection{Dereplication technique}

Dereplication has been originally defined as the analysis of natural products, a fraction, or an extract using bioassays, spectroscopic and structural information, and comparing the information with internal and/or commercial databases, ascertaining whether the present metabolites are new or known compounds. The importance of dereplication in drug discovery continues to rise and became essential in rapid identification of compounds due to more natural products being isolated from a wider range of sources. ${ }^{[85]}$

Currently, it has been reported that over 230,000 natural products have been isolated as metabolites from plants and microorganisms. Therefore, it is essential to develop a method to identify structures of isolated metabolites rapidly. ${ }^{\left[{ }^{[6]}\right.}$ Previously, biological activity and resistance patterns were used as the procedure of dereplication in searching for new bioactive natural product. ${ }^{[87]}$ Nowadays, there are a number of databases being developed for dereplication of natural products. ChemIDplus is one among them, which provides some important features such as the structure, molecular formula, physicochemical properties, names, synonyms, CAS Registry Numbers, classification and locator codes. ${ }^{[86]}$ In our group, AntiBase has been used intensively for the dereplication of microbial products and limited secondary metabolites from 
plants. It offers fast structural identification of natural compounds based on substructures, high-resolution mass spectra, chemical shifts of ${ }^{1} \mathrm{H}$ and ${ }^{13} \mathrm{C}$ NMR spectra in ISIS/Base and ChemFinder formats. Currently, it covers over 39,000 natural products from microorganisms and higher fungi, which is very useful in identifying related structures of natural compounds. ${ }^{[88]}$ The Dictionary of Natural Products (Chapman \& Hall) database is another comprehensive dereplication tool of natural products covering over 230,000 compounds. ${ }^{[86]}$ However, the difficult sub-structure search and limited spectral information are the disadvantages of the Dictionary of Natural Products compared with AntiBase.

\section{Investigation of selected bacterial strains}

\subsection{Marine Streptomyces sp. B8289}

TLC of the crude extract of the marine Streptomyces sp. B8289 showed some UV absorbing bands, which became blue, brown and red with anisaldehyde/sulphuric acid. The bioassay revealed weak activity against bacteria and fungi.

The sub-culture on agar plates showed colonies with a white aerial mycelium after incubation on $\mathrm{M}_{2}{ }^{+}$agar medium for 3 days at $28{ }^{\circ} \mathrm{C}$. The strain was cultivated in 100 of $1 \mathrm{~L}$ Erlenmeyer flasks each containing $250 \mathrm{~mL} \mathrm{M}_{2}^{+}$medium. The culture was fermented on a linear shaker $(110 \mathrm{rpm})$ for 5 days at $28{ }^{\circ} \mathrm{C}$ and the well-grown culture broth was mixed with Celite and filtered through a pressure filter. The filtered culture broth was passed through Amberlite resin XAD-16. The resin was washed with demineralised water and then eluted with methanol, whereas the biomass was extracted with ethyl acetate and acetone. Then, the organic extracts were evaporated to dryness under vacuum.

The brown crude extract was then subjected to silica gel column chromatography using dichloromethane and methanol, which gave four fractions. 


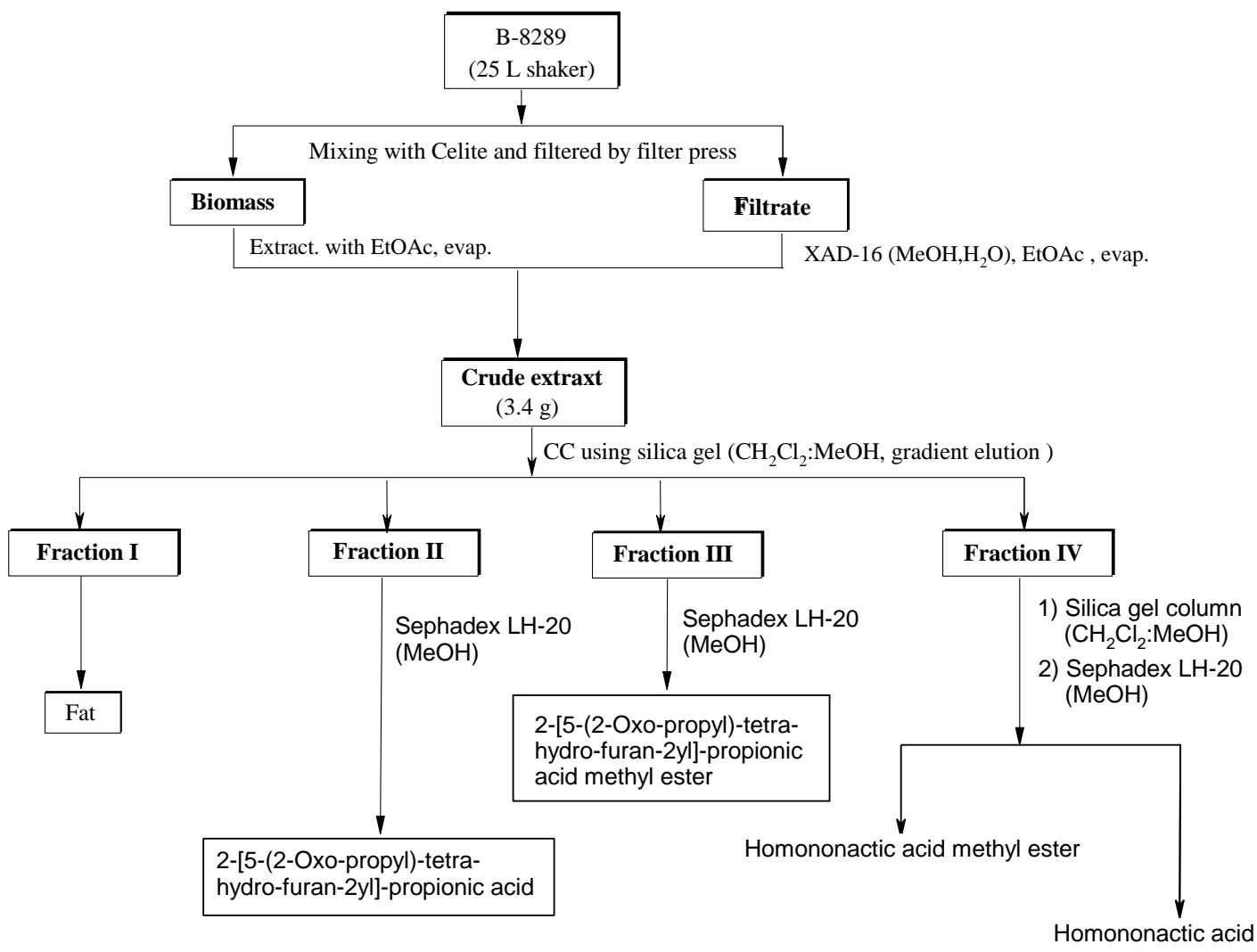

Figure 6: Work-up scheme for marine Streptomyces sp. B8289

\subsubsection{2-[5-(2-Oxo-propyl)-tetrahydrofuran-2-yl]-propionic acid}

2-[5-(2-Oxo-propyl)-tetrahydrofuran-2-yl]-propionic acid (77) was obtained from fraction III as a 2:1 mixture of two diastereomers in form of a yellowish oil. It did not show a UV absorbing band, but turned reddish violet with anisaldehyde/sulphuric acid. The ESI mass spectrum delivered the molecular weight of 200 Dalton and the HRESI mass analysis deduced the molecular formula as $\mathrm{C}_{10} \mathrm{H}_{16} \mathrm{O}_{4}$.

The ${ }^{1} \mathrm{H}$ NMR spectrum of the major isomer displayed the signal of an acidic proton as broad singlet at $\delta 8.84$. Two oxy-methine multiplets were observed at $\delta$ 4.27 and 4.04, and two methyl groups resonated at $\delta 2.16$ and 1.14 as singlet and doublet, respectively. The remaining multiplets in the region of $\delta 2.77 \sim 1.54$ comprising of seven protons corresponded to three methylene groups and one methine ( $\delta$ 2.50). 


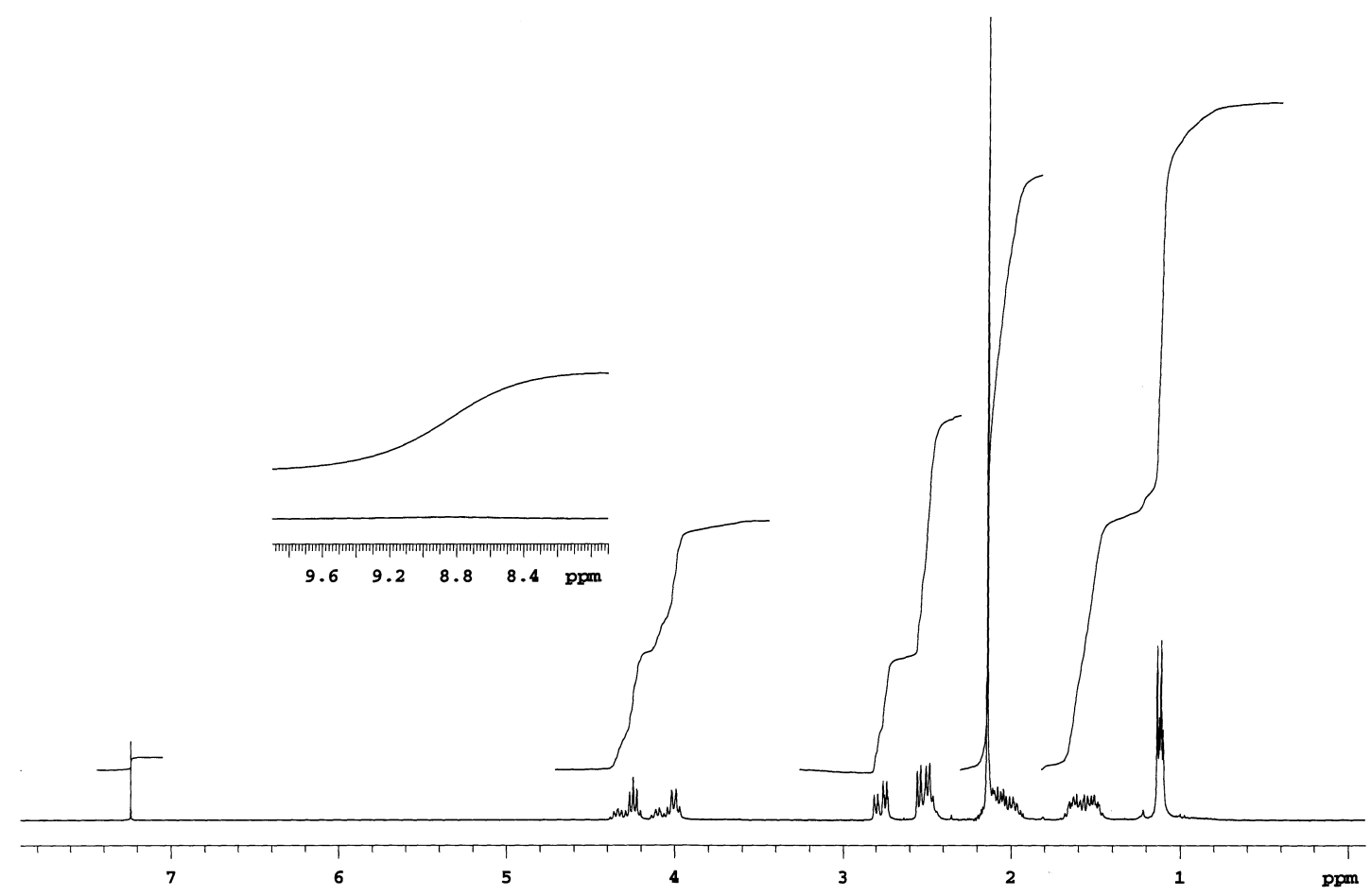

Figure 7: $\quad{ }^{1} \mathrm{H} \quad \mathrm{NMR}$ spectrum $\left(\mathrm{CDCl}_{3}, 300 \mathrm{MHz}\right)$ of 2-[5-(2-oxo-propyl)tetrahydrofuran-2-yl]propionic acid (77)

The ${ }^{13} \mathrm{C}$ NMR spectrum displayed two sets of each ten carbon signals, two of which were assigned as carbonyl groups at $\delta_{C} 207.4$ (ketone) and 179.5 (carboxylic acid), respectively. In addition, two oxycarbons and three methylene carbon signals appeared at $\delta_{C} 80.4,75.6,49.6,30.9,28.5$, respectively. A sub-structure search in AntiBase based on these spectroscopic data resulted in 2-[5-(2-oxo-propyl)tetrahydrofuran-2-yl]propionic acid (77) as suggested structure, which was further confirmed by comparison with authentic spectra and the literature. ${ }^{\left[{ }^{89]}\right.}$ Other than in the sample of Shaaban, ${ }^{[89]}$ the ${ }^{13} \mathrm{C}$ NMR spectrum of the sample isolated here showed most signal in duplicate, and also the protons H-2,5 showed four multiplets instead of two as in the reference sample. Zhang and Yadav in our group had also isolated compound 77 from Streptomyces sp. as a similar mixture of epimers. ${ }^{\text {[90] }}$ 


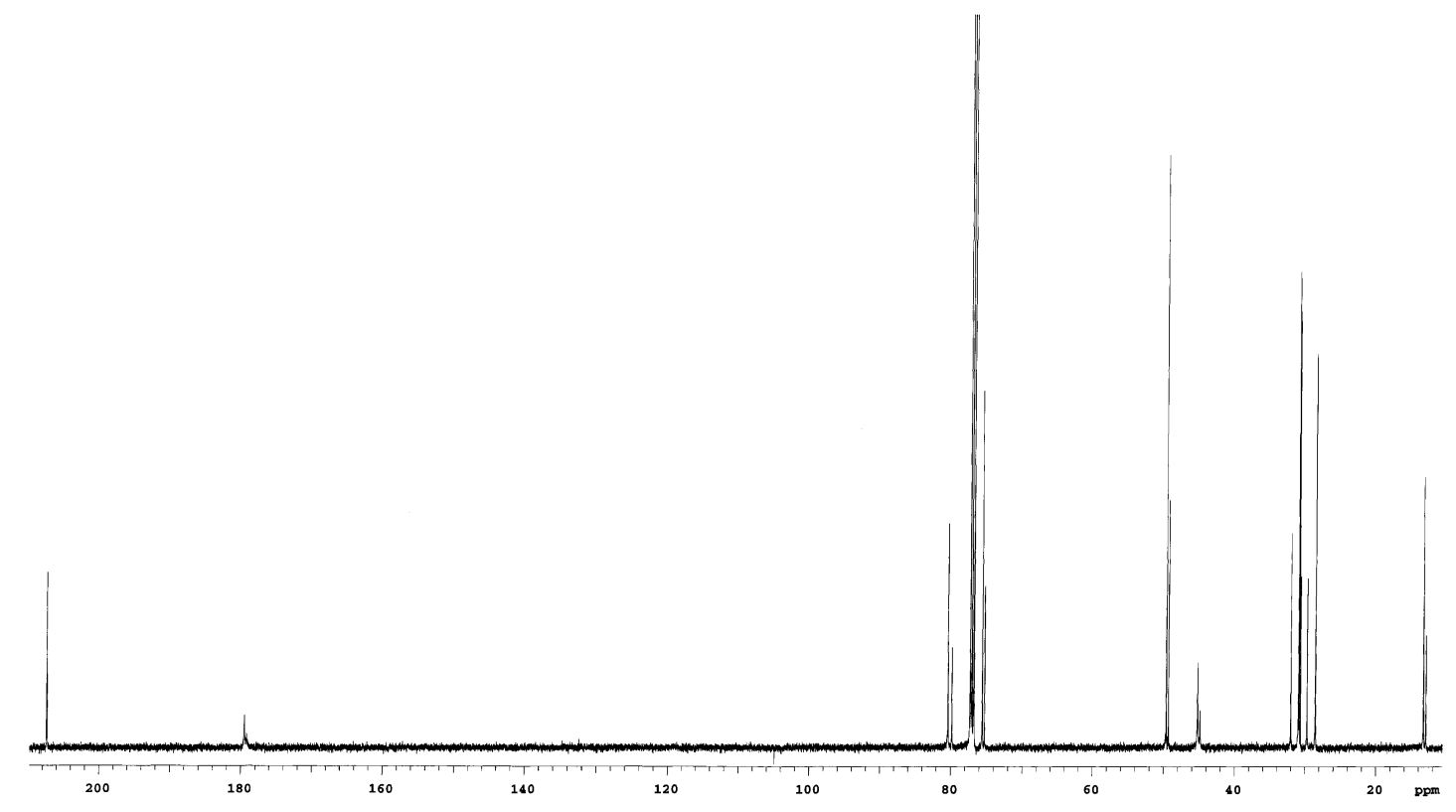

Figure 8: ${ }^{13} \mathrm{C} \quad \mathrm{NMR}$ spectrum $\left(\mathrm{CDCl}_{3}, 125 \mathrm{MHz}\right)$ of 2-[5-(2-oxo-propyl)tetrahydrofuran-2-yl]propionic acid (77)<smiles>[CH2]C(=O)[C@H]1CC[C@@H]([C+](C)C(=O)O)O1</smiles>

77

Compound $\mathbf{7 7}$ had been isolated previously in our group by Shaaban from Streptomyces sp. Act8970; the cis-configuration at the tetrahydrofurane ring of $\mathbf{7 7}$ had been established on the basis of NOESY experiments. ${ }^{[89]}$ 2-[5-(2-Oxo-propyl)tetrahydrofuran-2-yl]-propionic acid (77) showed weak biological activity against bacteria and fungi.

\subsubsection{2-[5-(2-Oxo-propyl)-tetrahydrofuran-2-yl]propionic acid methyl ester}

Compound 78 was separated from fraction II as yellowish oil by using Sephadex LH-20 column chromatography. It showed no UV absorbing bands but stained to violet with anisaldehyde/sulphuric acid. The high-resolution ESI mass analysis delivered a molecular formula of $\mathrm{C}_{11} \mathrm{H}_{18} \mathrm{O}_{4}$, which corresponded to a molecular weight of 214 Dalton. 
The ${ }^{1} \mathrm{H}$ NMR spectrum of $\mathbf{7 8}$ exhibited similar signals as 2-[5-(2-oxo-propyl)tetrahydrofuran-2-yl]-propionic acid (77), except for an additional singlet at $\delta 3.65$ $(3 \mathrm{H})$, suggesting the presence of a methyl ester group. A methyl ester was further supported by the presence of a carbon signal at $\delta_{C} 52.1$ as well as by the difference of $\Delta \mathrm{m}=14$ amu between $\mathbf{7 7}$ and $\mathbf{7 8}$, given by the mass spectrum. In addition, the ${ }^{13} \mathrm{C}$ NMR spectrum of compound $\mathbf{7 8}$ was very similar to compound $\mathbf{7 7}$, but the protons H-2,5 showed four multiplets instead of two as in Shaaban's sample, indicating a 1:1 sample of two diastereomers.

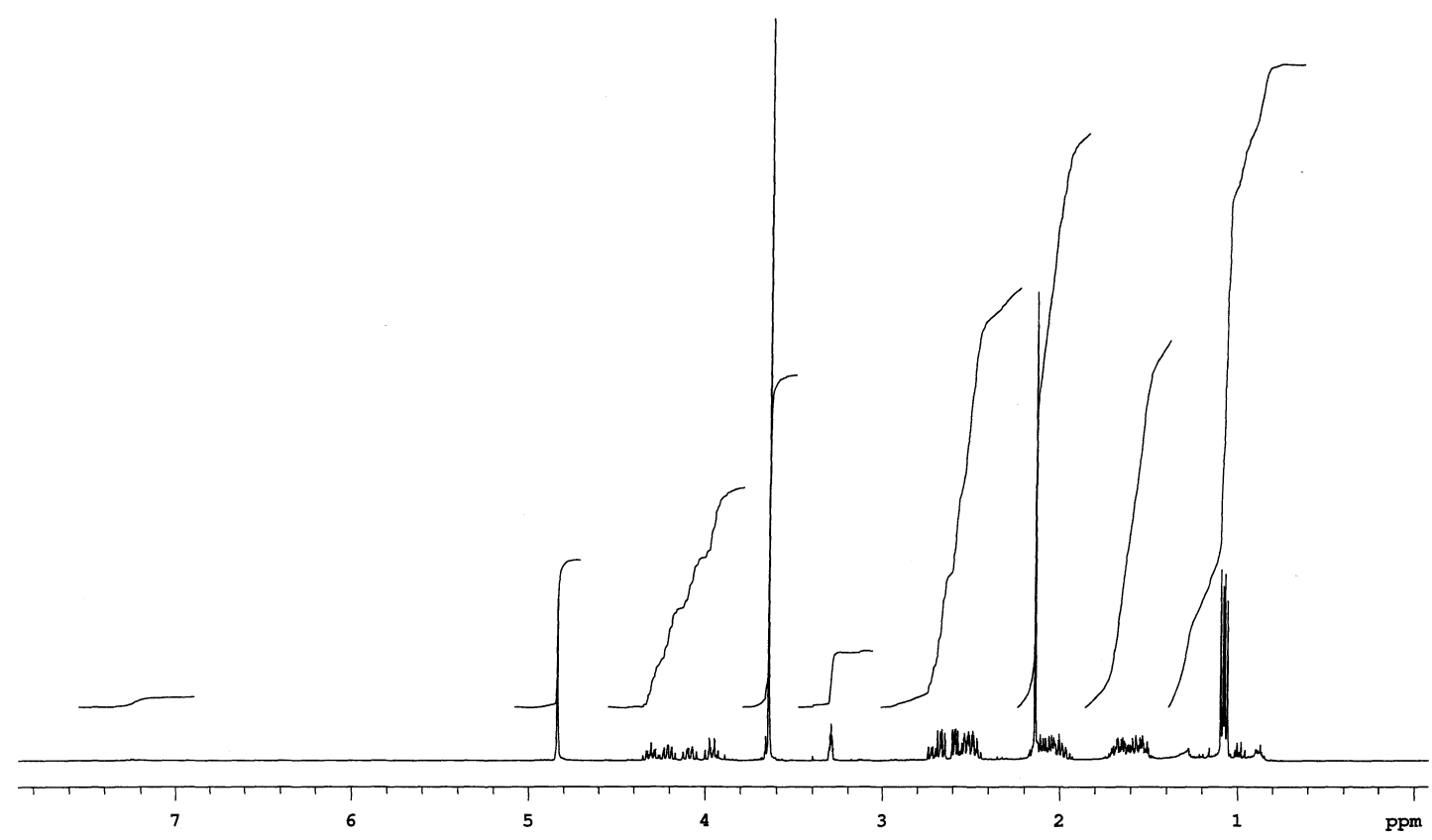

Figure 9: $\quad{ }^{1} \mathrm{H}$ NMR spectrum $\left(\mathrm{CD}_{3} \mathrm{OD}, 300 \mathrm{MHz}\right)$ of 2-[5-(2-oxo-propyl)tetrahydrofuran-2-yl]-propionic acid methyl ester (78) 


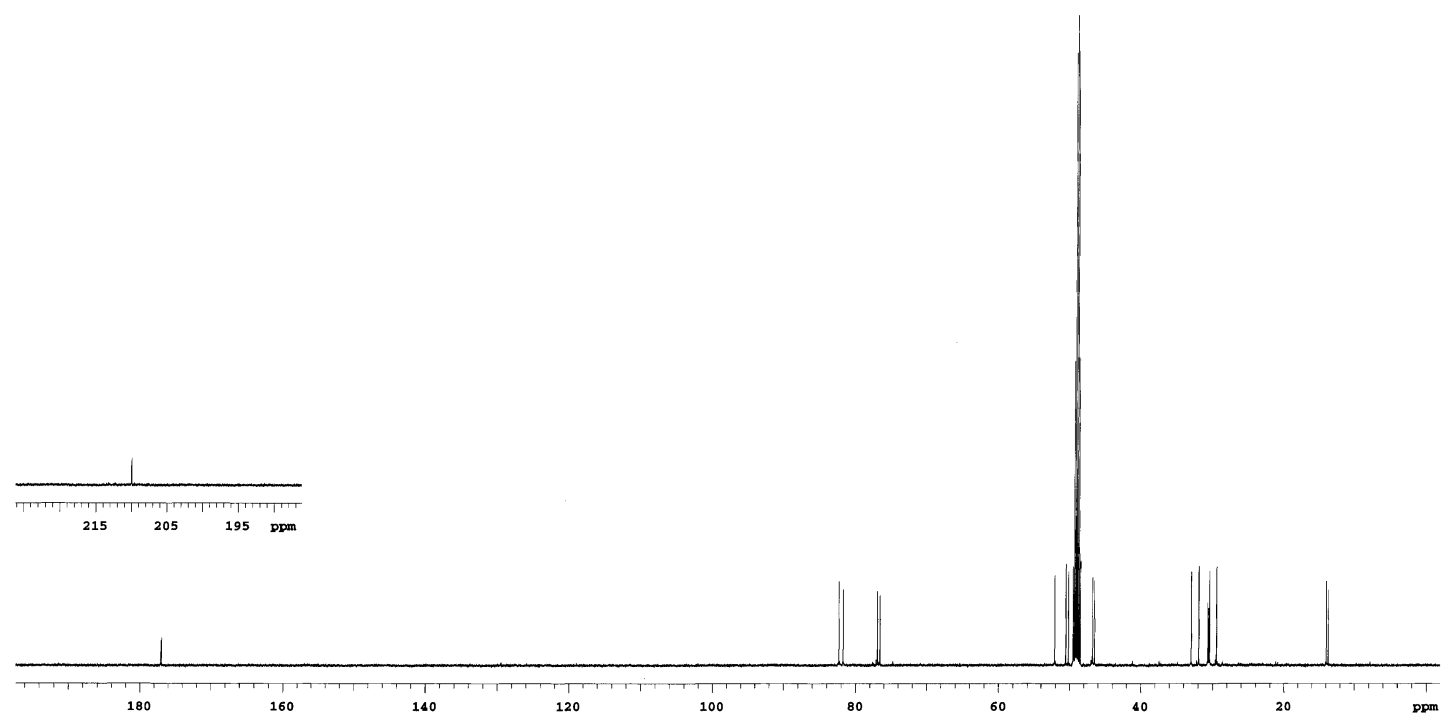

Figure 10: ${ }^{13} \mathrm{C}$ NMR spectrum $\left(\mathrm{CD}_{3} \mathrm{OD}, 125 \mathrm{MHz}\right)$ of 2-[5-(2-oxo-propyl)tetrahydrofuran-2-yl]-propionic acid methyl ester (78)

According to the HMBC spectrum of $\mathbf{7 8}$, the position of the methoxy group $\left(\delta_{C}\right.$ $52.1)$ was established by a ${ }^{3} J$ cross-coupling signal from $\mathrm{H}_{3}-4$ " $(\delta 3.65)$ to $\mathrm{C}-1$ " $\left(\delta_{C}\right.$ 177.1). The acetyl group was further confirmed by the ${ }^{2} J$ cross signal between $\mathrm{H}_{3}-1^{\prime}$ $(\delta 2.15)$ and $\mathrm{C}-2^{\prime}\left(\delta_{C} 210.0\right)$. This acetyl is attached directly to the methylene carbon C-3' $\left(\delta_{C} 50.5\right)$ shown by ${ }^{3} J$ coupling from $\mathrm{H}_{3}-1$ ' to $\mathrm{C}-3$ '. Furthermore, the signal of $\mathrm{H}$ $3 "(\delta 1.10)$ showed ${ }^{2} J$ and ${ }^{3} J$ couplings to the ester carbonyl $\left(\mathrm{C}-1 ", \delta_{C} 177.1\right)$, the methine (C-2", $\left.\delta_{C} 46.8\right)$, and the oxymethine (C-2, $\left.\delta_{C} 82.3\right)$.

Trials to determine the relative configuration were only partially successful: Diagnostic couplings in the NOESY experiment were the contacts between $\mathrm{H}-2$ and $\mathrm{H}$ 5 , which were seen for both isomers; additionally, there was a weak cross signal between 1'-methyl and the methoxy group, indicating a cis-disubstitution at the tetrahydrofurane ring in both isomers and therefore a mixture of C-2" epimers. This agrees well with the assumption of (-)-nonactic acid (19) as precursor, which also occurs in form of the 2 "-epimer.

2-[5-(2-Oxo-propyl)-tetrahydrofuran-2-yl]-propionic acid methyl ester (78) has been obtained previously by esterification of 2-[5-(2-oxo-propyl)-tetrahydrofuran-2yl]-propionic acid (77) with diazomethane; ${ }^{[89]}$ it is reported here as new natural derivative of nonactic acid (19) from bacteria. 


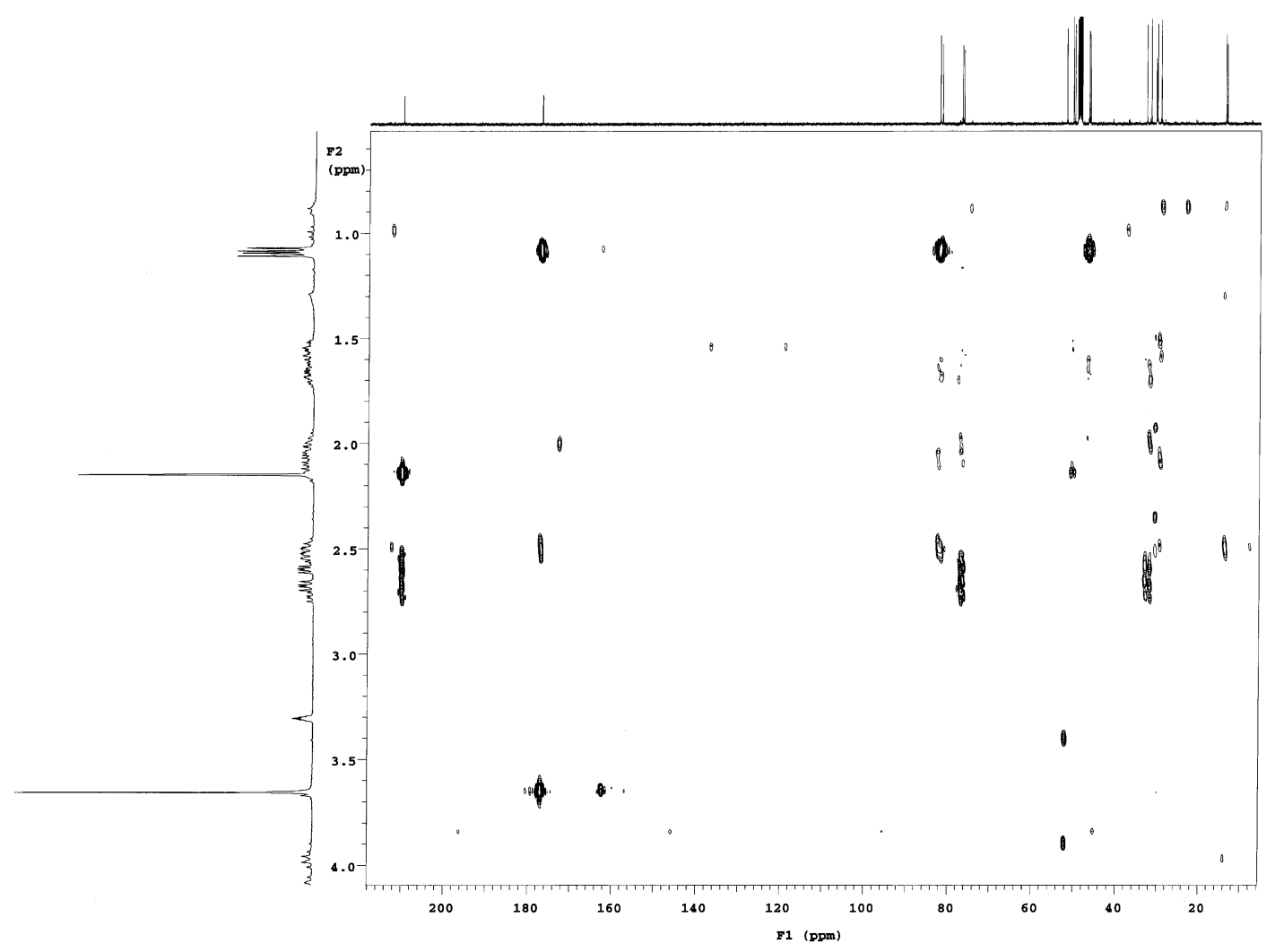

Figure 11: $\mathrm{HMBC}$ spectrum $\left(\mathrm{CD}_{3} \mathrm{OD}, 600 \mathrm{MHz}\right)$ of 2-[5-(2-oxo-propyl)tetrahydrofuran-2-yl]-propionic acid methyl ester (78)

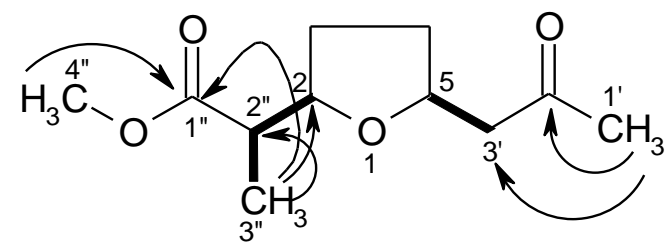

78

Figure 12: $\quad$ Selected ${ }^{1} \mathrm{H}_{-}{ }^{1} \mathrm{H}$ COSY $(-)$ and $\mathrm{HMBC}(\longrightarrow)$ correlations of 2-[5(2-oxo-propyl)-tetrahydrofuran-2-yl]-propionic acid methyl ester (78)

Compound 78 is a nonactic acid derivative, which may be biosynthesized from acetate, propionate or succinate. Nonactic acid is a building block of macrotetrolides. $^{[91]}$

\subsubsection{Homononactic acid}

Compound 79 was isolated as yellowish oil; the HRESI mass analysis gave the molecular formula $\mathrm{C}_{11} \mathrm{H}_{20} \mathrm{O}_{4}$, corresponding to a molecular weight of 216 Dalton. 
The ${ }^{1} \mathrm{H}$ NMR spectrum revealed no aromatic or olefinic signals. Three oxygenated methine signals were observed at $\delta 4.06,3.91,3.64$. In the region of $\delta 2.39 \sim 1.36$ with integration of nine protons, a series of multiplets was present, which corresponded to the protons of three methylene and one methine groups. Two methyl signals appeared at $\delta 1.01$ and 0.79 as doublet and triplet, respectively.

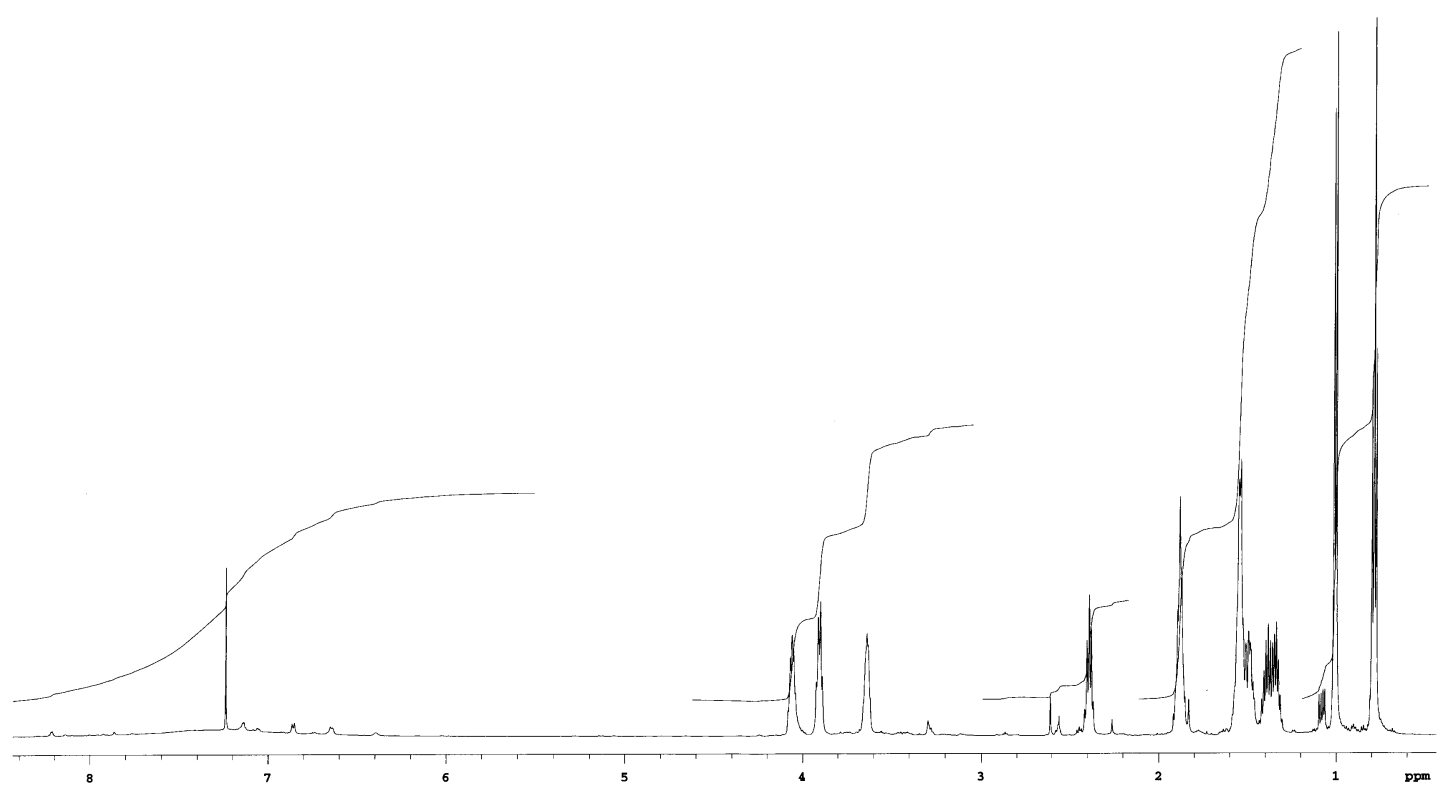

Figure 13: $\quad{ }^{1} \mathrm{H}$ NMR spectrum $\left(\mathrm{CDCl}_{3}, 300 \mathrm{MHz}\right)$ of homononactic acid (79)

The ${ }^{13} \mathrm{C}$ NMR spectrum displayed 13 carbon signals, and one signal at $\delta_{C} 177.8$ suggested the presence of a carboxylic acid carbonyl. Three oxygenated carbon atoms showed resonances at $\delta_{C} 80.1,76.6$, and 70.1. The remaining carbon signals appeared in the aliphatic region comprising of one methine, four methylene and two methyl groups between $\delta_{C}$ 44.9-9.7. A sub-structure search in AntiBase and comparison with an authentic spectrum led to homononactic acid (79) as the confirmed structure. ${ }^{[89]}$ 


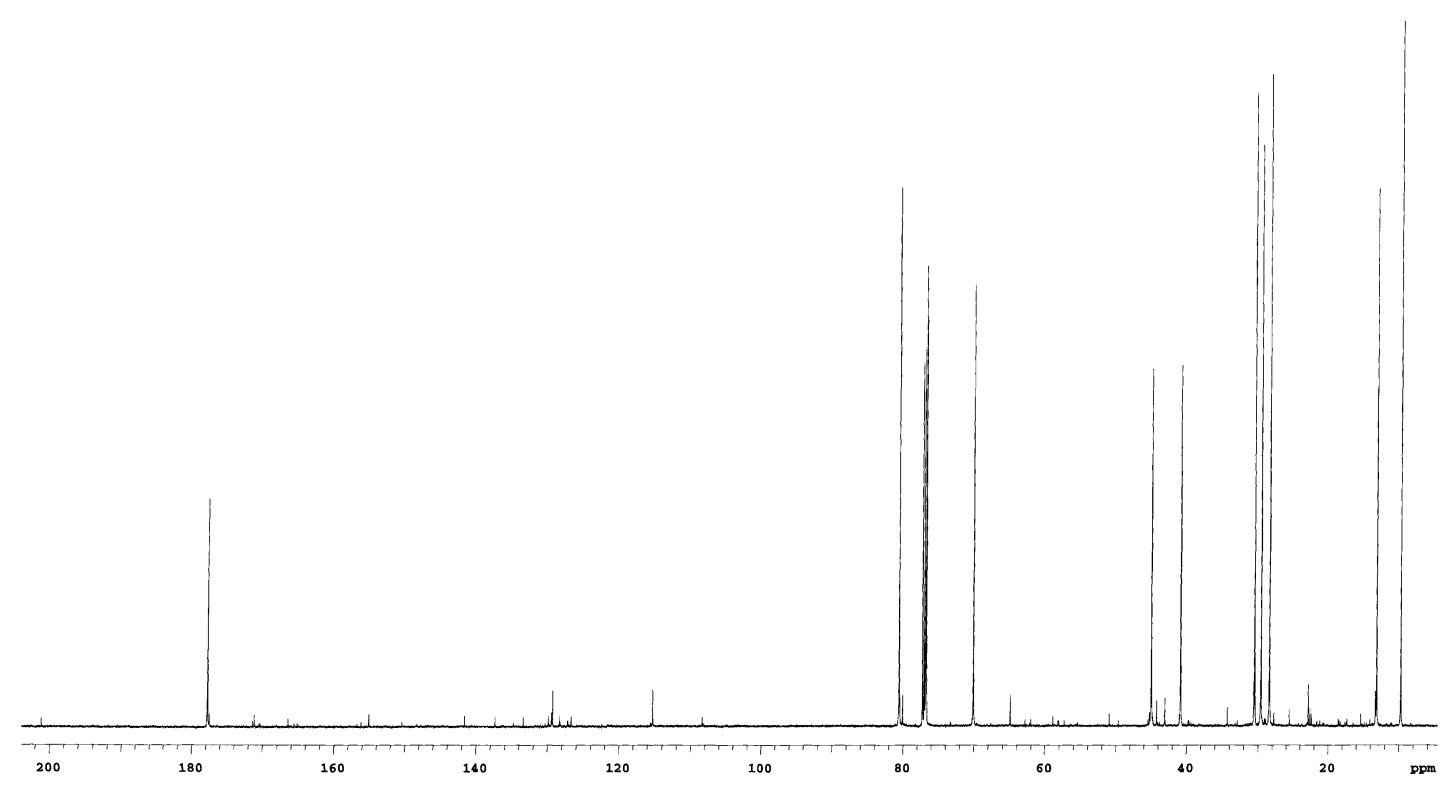

Figure 14: ${ }^{13} \mathrm{C}$ NMR spectrum $\left(\mathrm{CDCl}_{3}, 125 \mathrm{MHz}\right)$ of homononactic acid (79)<smiles>CCC(O)C[C@@H]1CC[C@H](C(C)C(=O)O)O1</smiles>

79

Homononactic acid (79) had been reported first time as a microbial natural product by Prikrylova and co-workers from Streptomyces griseus. Compound $\mathbf{7 9}$ was isolated in mixture with nonactic acid (19) from Streptomyces griseus. ${ }^{[92]}$

\subsubsection{Homononactic acid methyl ester}

Homononactic acid methyl ester (80) was obtained as light yellow and non-UV absorbing oil. It stained to brown with anisaldehyde/sulphuric acid. The molecular formula of 80 was deduced from the (+)-HRESI mass analysis as $\mathrm{C}_{12} \mathrm{H}_{22} \mathrm{O}_{4}$, corresponding to a molecular weight of 230 Dalton. Compound 80 exhibited ${ }^{1} \mathrm{H}$ and ${ }^{13} \mathrm{C}$ NMR spectra similar to homononactic acid (79), with three oxygenated methine, one non-oxygenated methine, two methyl, and four methylene groups. The only major difference in the NMR spectra was the presence of an additional singlet at $\delta 3.60\left(\delta_{\mathrm{C}}\right.$ 51.5) assigned for a methoxy group. 


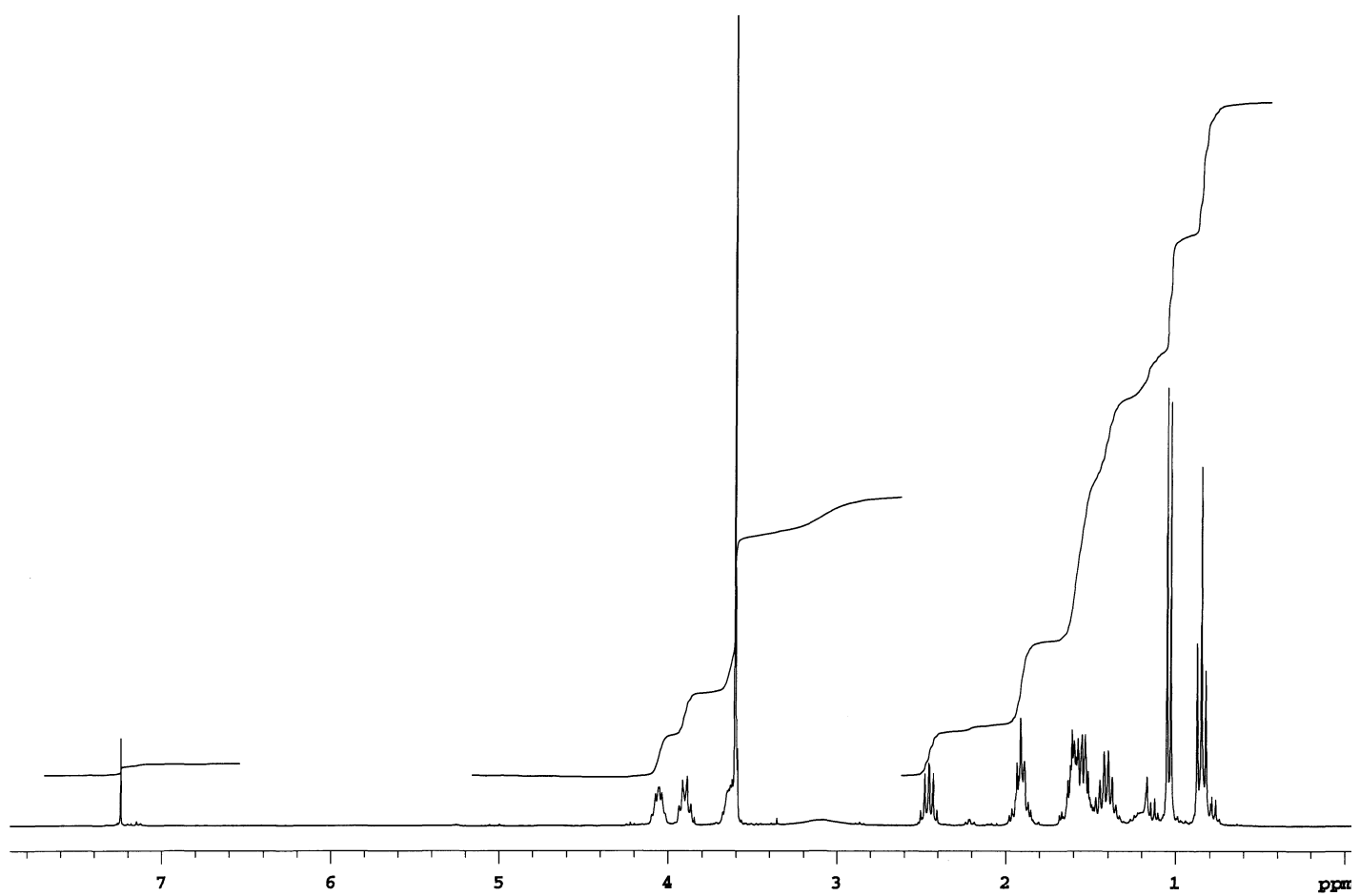

Figure 15: $\quad{ }^{1} \mathrm{H}$ NMR spectrum $\left(\mathrm{CDCl}_{3}, 300 \mathrm{MHz}\right)$ of homononactic acid methyl ester (80)

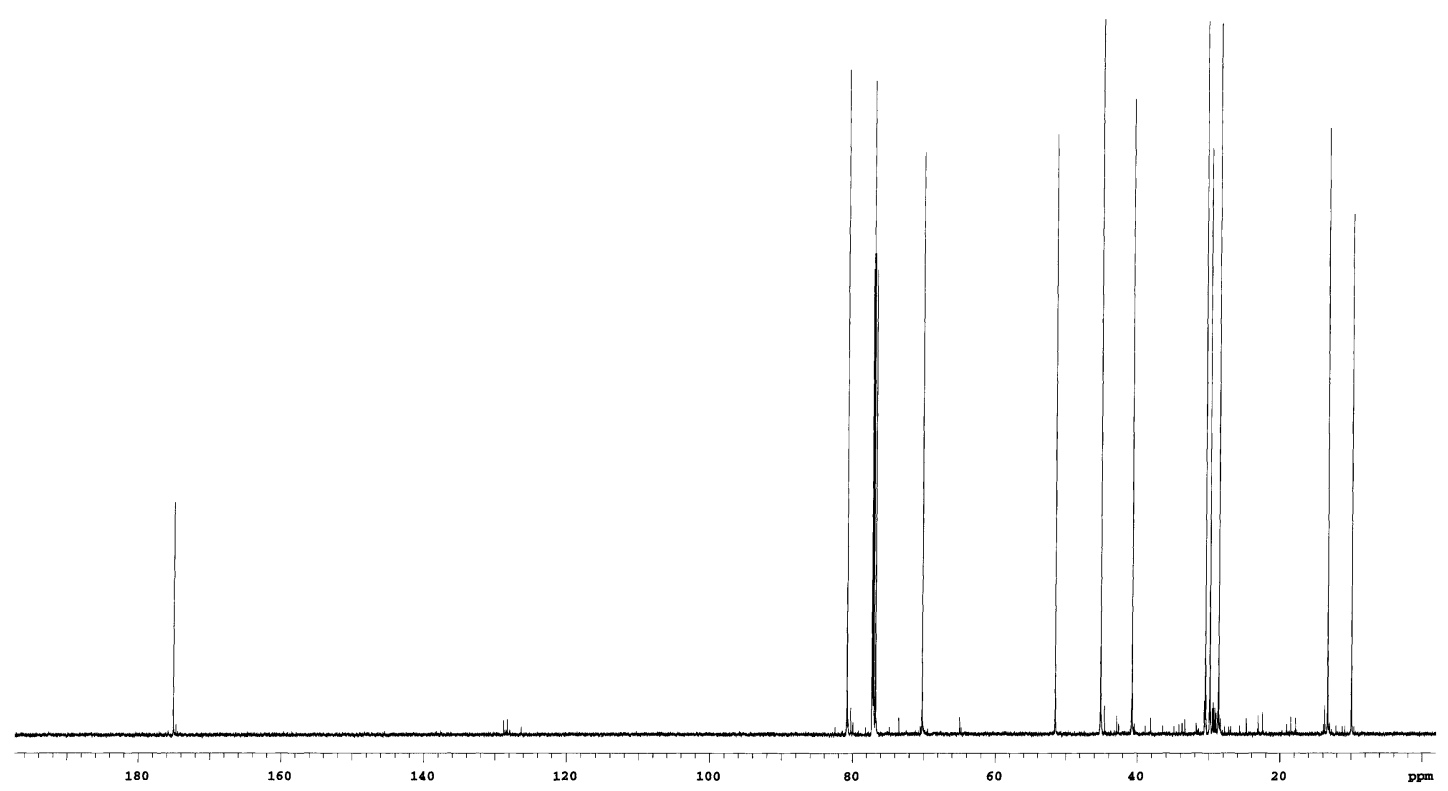

Figure 16: ${ }^{13} \mathrm{C}$ NMR spectrum $\left(\mathrm{CDCl}_{3}, 125 \mathrm{MHz}\right)$ of homononactic acid methyl ester (80) 


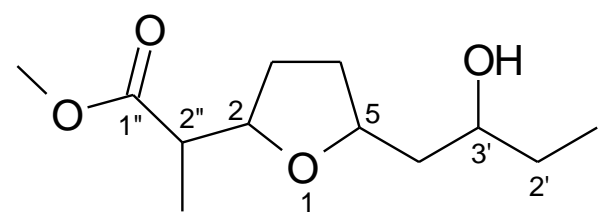

80

Homononactic acid methyl ester (80) was synthesized from homononactic acid (79) by treating with diazomethane at $25{ }^{\circ} \mathrm{C} .{ }^{[92]}$ Compound 80 had also been isolated first time in our group as a natural product from the marine-derived Streptomyces sp. Act8970. ${ }^{[89]}$

\subsection{Terrestrial Streptomyces sp. Ank86}

The antimicrobial assay of Streptomyces sp. Ank86 showed moderate activity against Streptomyces viridochromogenes (Tü 57), Bacillus subtilis, and Staphylococcus aureus. TLC of the crude extract showed UV absorbing bands in the middle polar region, which changed to blue and violet by spraying with anisaldehyde/sulphuric acid solution after heating. The TLC also exhibited orange polar zones, which changed to deep red by moistening with concentrated sulphuric acid hinting to the presence of an actinomycin.

Well grown agar plates of the marine Streptomyces sp. Ank86 were used to inoculate a $25 \mathrm{~L}$ shaker culture on $\mathrm{M}_{2}$ medium. After 6 days at $28^{\circ} \mathrm{C}$, the culture was harvested and mixed with Celite prior to filtering using a filter press. The water phase was passed through Amberlite XAD-16 resin followed by elution with methanol. The biomass and filtrate were extracted separately with ethyl acetate. The combined organic phases were evaporated to afford $2.4 \mathrm{~g}$ of crude extract, which was then subjected to column chromatography on silica gel to give four fractions. The fractions were further purified by using different chromatographic methods to get the pure components. 


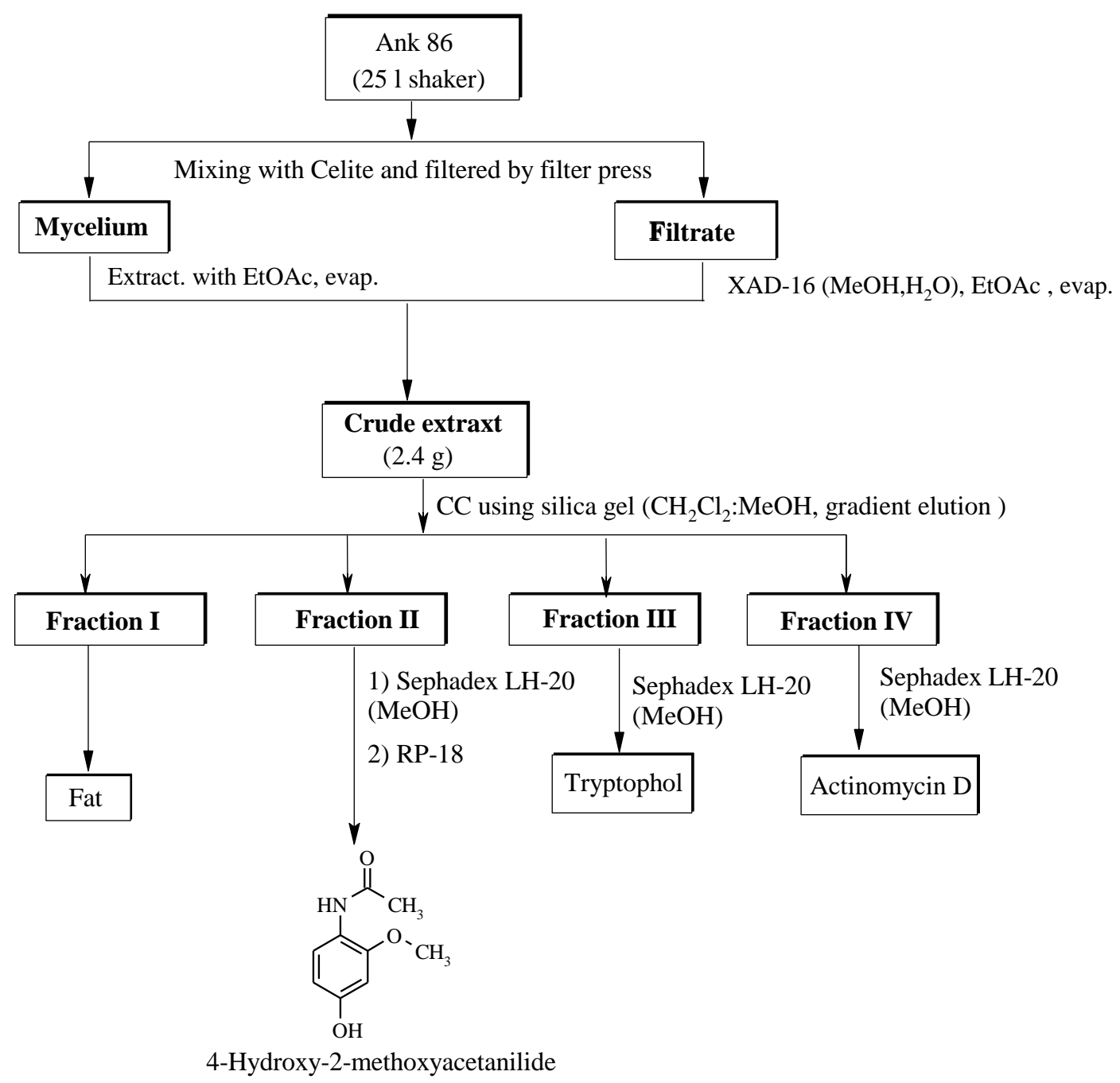

Figure 17: Work-up scheme for terrestrial Streptomyces sp. Ank86

\subsubsection{4-Hydroxy-2-methoxyacetanilide}

4-Hydroxy-2-methoxyacetanilide (81) was separated from fraction II by using Sephadex LH-20 and RP-18 columns as white solid. It was UV absorbing at $254 \mathrm{~nm}$ and gave no reaction with anisaldehyde/sulphuric acid.

The ${ }^{1} \mathrm{H}$ NMR spectrum demonstrated three aromatic protons at $\delta 7.56,7.52$, and 6.85. The ABX spin pattern showed two ortho-coupled $1 \mathrm{H}$ doublets at $\delta 7.56$ and 6.85 , along with one meta-coupled proton at $\delta 7.52(J=1.96)$. Additionally, the spectrum displayed two methyl singlets at $\delta 3.90$ and 2.53, corresponding to methoxy and acyl $\left(\mathrm{CH}_{3} \mathrm{CO}\right)$ groups, respectively.

EIMS showed the molecular ion peak at $m / z$ 181. Additionally, the spectrum displayed fragment ions at $\mathrm{m} / \mathrm{z}, 166$ and 151 (base peak) due to the loss of two methyl 
fragments. A sub-structure search in AntiBase based on ${ }^{1} \mathrm{H}$ NMR and mass spectra gave structure 81. Comparison with authentic spectra from our spectra collection confirmed this assignment.

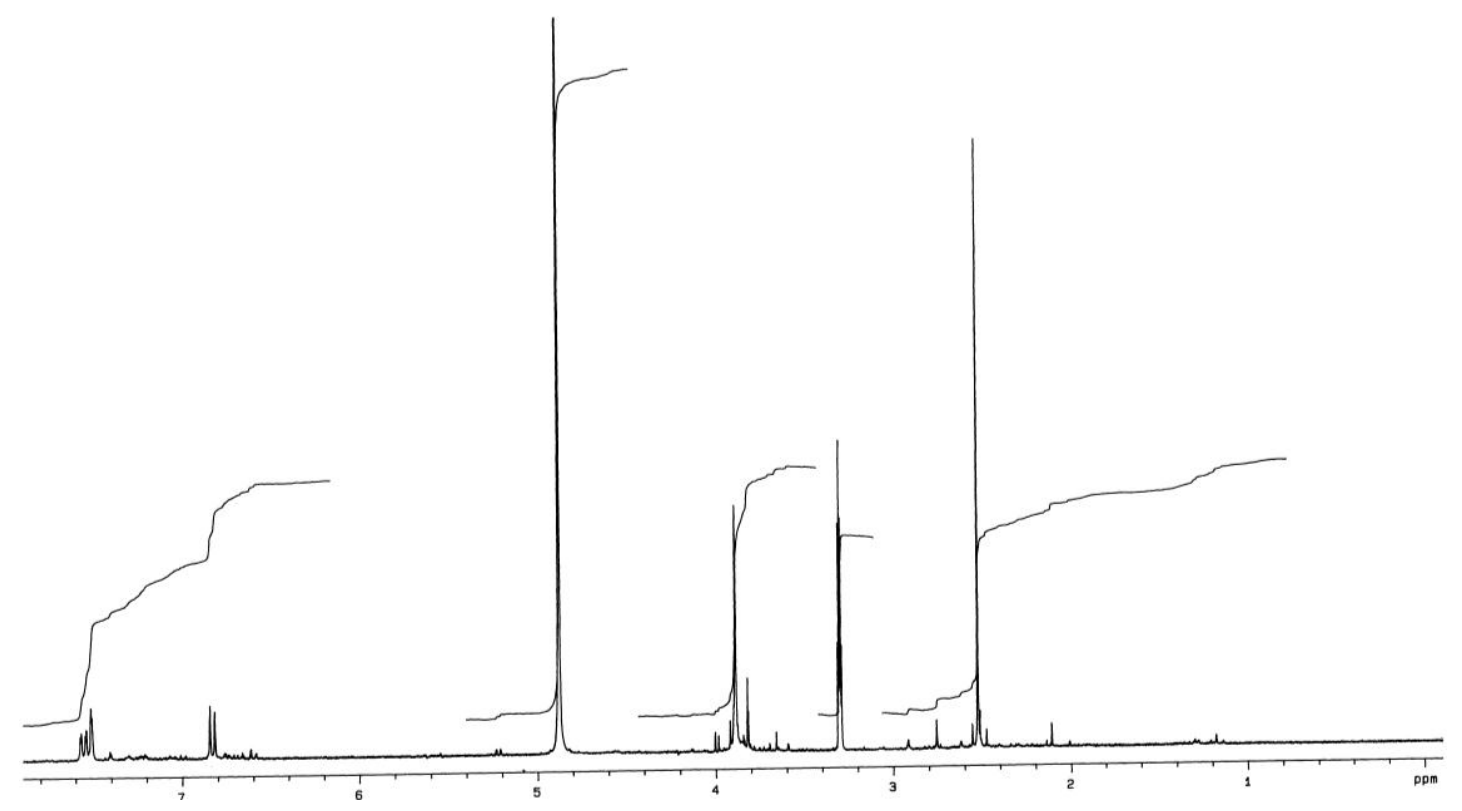

Figure 18: $\quad{ }^{1} \mathrm{H} \quad \mathrm{NMR} \quad$ spectrum $\quad\left(\mathrm{CD}_{3} \mathrm{OD}, \quad 300 \quad \mathrm{MHz}\right) \quad$ of 4 4-hydroxy-2methoxyacetanilide $(\mathbf{8 1})$<smiles>COc1cc(O)ccc1NC(C)=O</smiles>

81

Compound $\mathbf{8 1}$ had been reported previously as a fungal metabolite ${ }^{[93]}$ and was reported here as a bacterial product. A trivial compound, tryptophol, was also isolated from this strain; it was often isolated in our group from bacteria.

\subsubsection{Actinomycin D}

Actinomycin D (82) was separated from fraction IV as reddish-orange solid using Sephadex LH-20 column chromatography; it gave an orange colour after staining 
with anisaldehyde/sulphuric acid and heating. It was UV absorbing and showed no colour change with sodium hydroxide, but turned red with concentrated sulphuric acid. Actinomycins belong to a family of chromopeptide antitumor antibiotics and were isolated from many streptomycetes. The natural actinomycins all share the same phenoxazinone chromophore, varying only in the amino acid content of their two depsipentapeptide moieties. ${ }^{[94,95]}$

The ${ }^{1} \mathrm{H}$ NMR spectrum showed two ortho protons at $\delta 7.21$ and 7.42 of a 1,2,3,4-tetrasubstituted aromatic ring, and two $3 \mathrm{H}$ singlets at 2.25 and 2.57 for methyl groups attached to an aromatic system. This is characteristic of the phenoxazinone chromophore in actinomycins. In addition, it depicted the signals of $4 \mathrm{NH}$ groups as doublets between $\delta 7.60-8.15$. It also exhibited eight hydrogen signals of oxygenated methines or $\alpha$-amino acid protons at $\delta 6.03$ (d), 5.96 (d), 5.25-5.15 (m, 2 H), 4.78 (d), 4.73 (d), 4.61 (dd) and 4.49 (dd). The signals between $\delta$ 4.01-3.48 exhibited many methylene and methine groups. Six methyl singlets were observed between $\delta$ 2.98-2.20, four of them for $\mathrm{N}$-methyl groups and two for methyl groups attached to an aromatic ring. Methylene groups appeared between $\delta 1.80-2.24$ as multiplets with intensity of $6 \mathrm{H}$. Additionally, the spectrum showed 5 signals between $\delta$ 0.75 and 1.41 for five methyl groups. At highest field, the doublet of a methyl group was observed at $\delta 0.75$.

The ESI mass spectrum revealed the molecular ion peak at $m / z 1255[\mathrm{M}+\mathrm{H}]^{+}$, which corresponded to the molecular weight of 1254 Dalton. A sub-structure search in AntiBase and comparison the spectroscopic data with the authentic spectrum confirmed the structure as actinomycin D (82). ${ }^{[94]}$ 


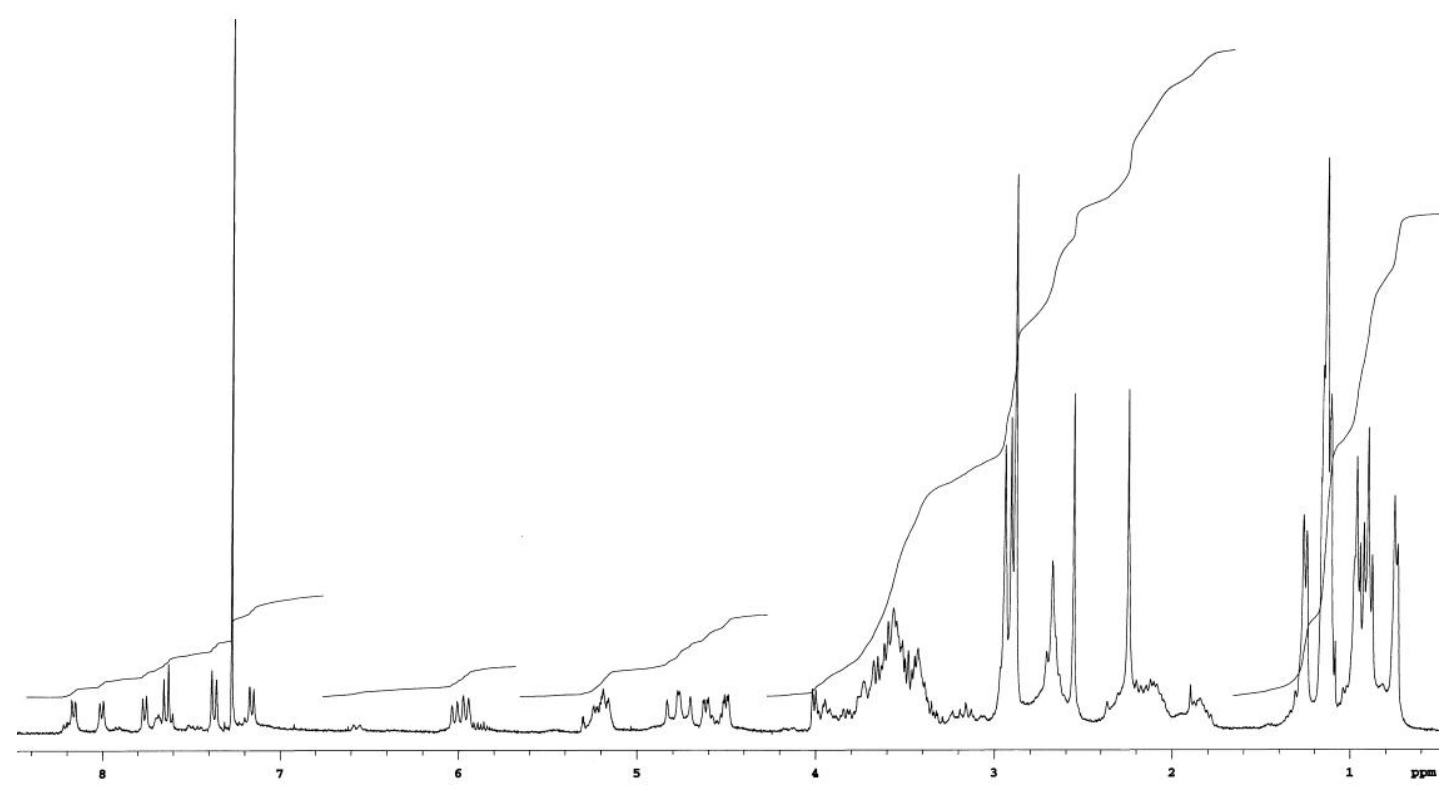

Figure 19: $\quad{ }^{1} \mathrm{H}$ NMR spectrum $\left(\mathrm{CDCl}_{3}, 300 \mathrm{MHz}\right)$ of actinomycin $\mathrm{D}(\mathbf{8 2})$

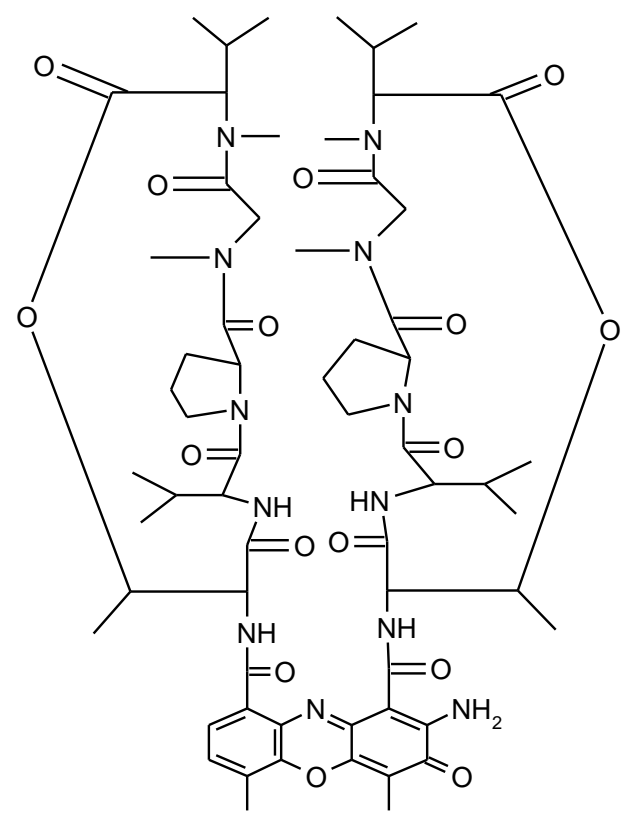

82

Actinomycin D (82) had been first isolated from Streptomyces chrysomallus in 1949 and was synthesized by Brockmann and Lackner. ${ }^{[94]}$ Actinomycin D (82) has been used in clinical application as anticancer agent in treatments of Wilm's tumor and soft tissue sarcoma in children, and has anti-HIV activity. ${ }^{[96-98]}$ 


\subsection{Terrestrial Streptomyces sp. Ank68}

Pre-screening of the crude extract from Streptomyces sp. Ank68 revealed strong antibacterial activity against Bacillus subtilis, Staphylococcus aureus, and Streptomyces viridochromogenes (Tü 57), and moderate antifungal activity against Candida albicans and Mucor miehei (Tü 284). TLC of crude extract displayed UV absorbing zones at 254 and $366 \mathrm{~nm}$, which changed to blue, red and black with anisaldehyde/sulphuric acid.

The strain was cultivated on $\mathrm{M}_{2}$ medium in $25 \mathrm{~L}$ scale as shaker culture for five days at $28{ }^{\circ} \mathrm{C}$. The brown culture broth was mixed with Celite and filtered with the aid of a filter press. The water phase was passed through Amberlite XAD-16 column and eluted with methanol, while the mycelial residue was extracted with ethyl acetate and acetone. The crude extract was subjected to silica gel chromatography column using a dichloromethane/methanol gradient.

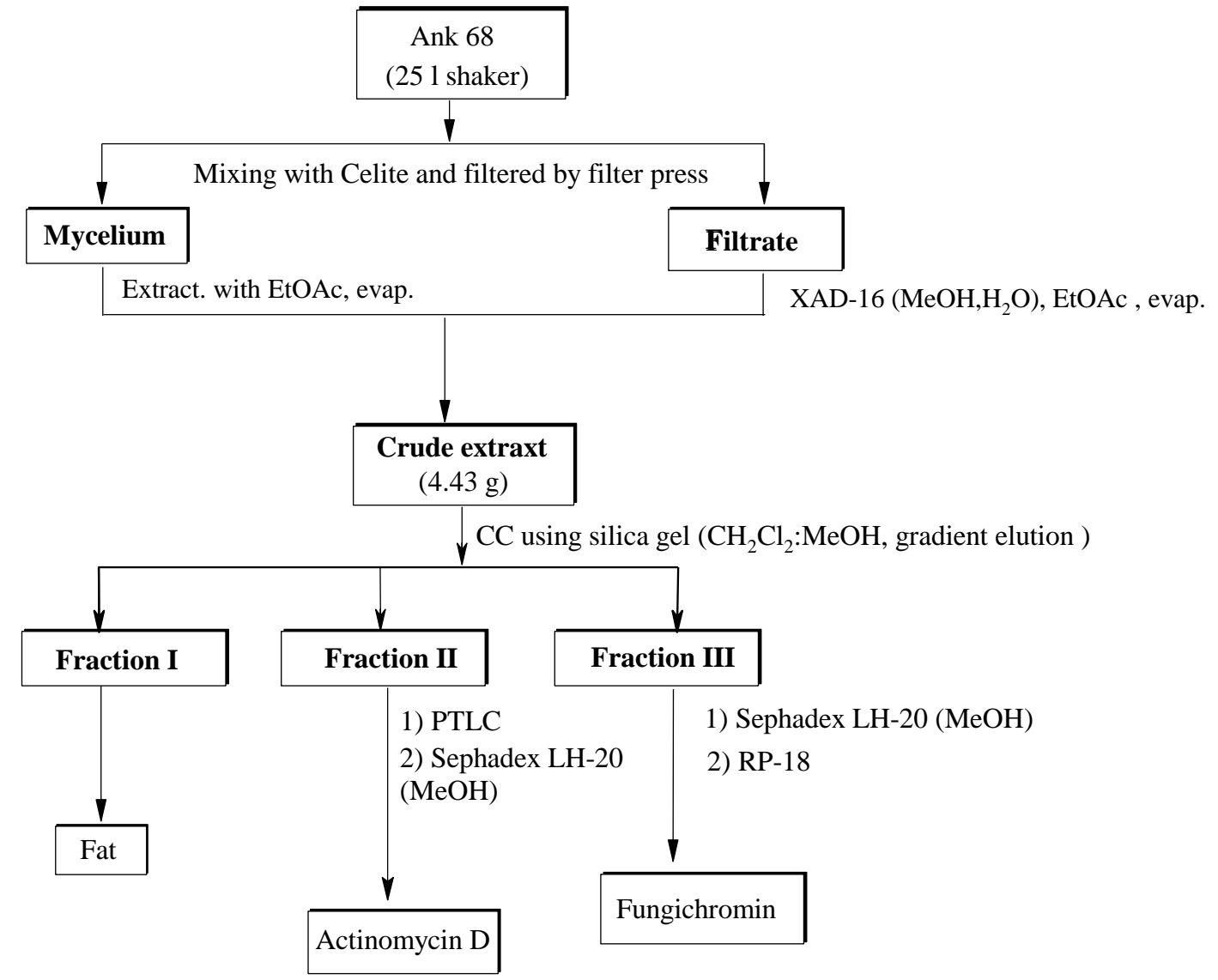

Figure 20: Work-up scheme for terrestrial Streptomyces sp. Ank68 


\subsubsection{Fungichromin}

Fraction III was worked up with Sephadex and RP-18 columns to isolate compound $\mathbf{8 3}$ as yellow solid. It exhibited a highly polar blue UV fluorescent band (366 $\mathrm{nm})$, which turned green-blue with anisaldehyde/sulphuric acid.

The ${ }^{1} \mathrm{H}$ NMR spectrum revealed resonances of nine protons for five olefinic double bonds between $\delta 6.49-5.94$ and eleven oxygenated methine groups between 5.23-3.48, respectively. The multiplet of a methine proton attached to an $s p^{2}$ carbon atom was observed at $\delta 2.45$. In addition, the signals between $\delta 1.69-0.85$ were assigned for methylene and three methyl groups. The ${ }^{13} \mathrm{C}$ NMR spectrum revealed 35 carbon signals, and one carbon atom signal at $\delta_{C} 170.4$ suggested the presence of a carbonyl group. The remaining carbon signals were one quaternary $s p^{2}$ carbon at $\delta_{C}$ 138.7, nine $s p^{2}$ methines, eleven $s p^{3}$ methines, nine $s p^{3}$ methylenes, and three methyl signals between $\delta_{C}$ 134.9-11.6.

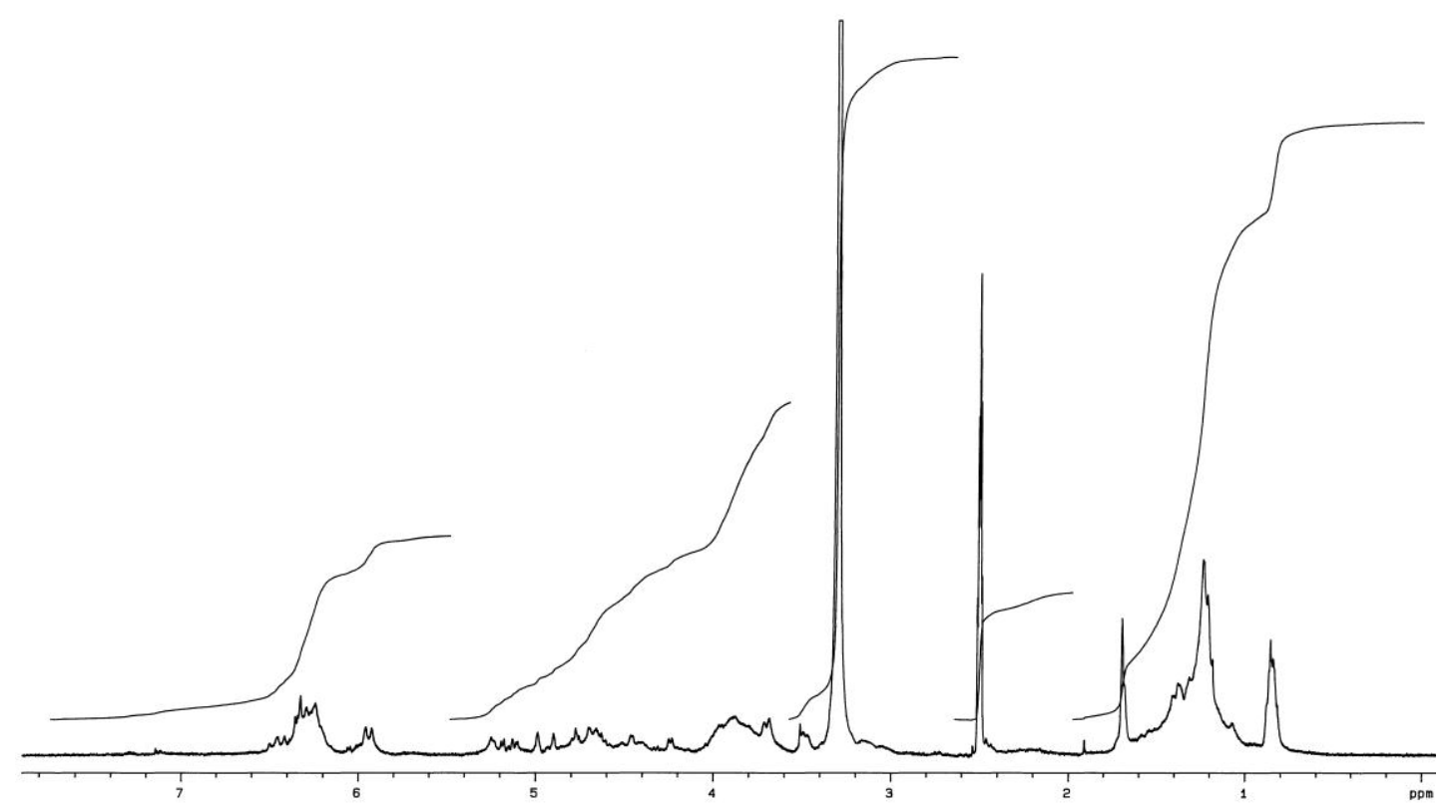

Figure 21: $\quad{ }^{1} \mathrm{H}$ NMR spectrum (DMSO- $d_{6}, 300 \mathrm{MHz}$ ) of fungichromin $(\mathbf{8 3})$ 


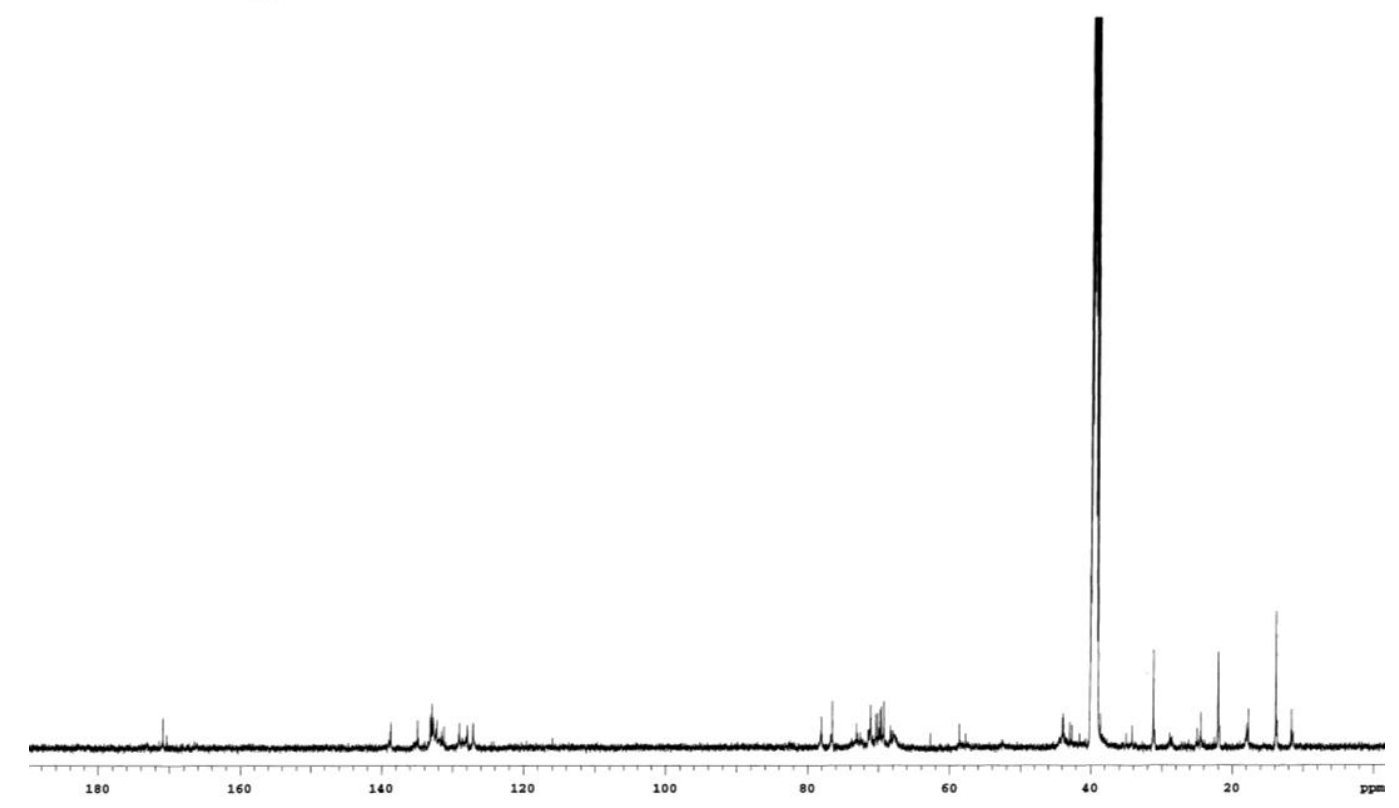

Figure 22: $\quad{ }^{13} \mathrm{C}$ NMR spectrum (DMSO- $d_{6}, 125 \mathrm{MHz}$ ) of fungichromin $(\mathbf{8 3})$

The ESI mass spectrum supplied a pseudo-molecular ion at $m / z, 671.4[\mathrm{M}+\mathrm{H}]^{+}$ and the high-resolution ESI mass analysis gave $\mathrm{C}_{35} \mathrm{H}_{59} \mathrm{O}_{12}$ as the molecular formula. The sub-structure search in AntiBase based on the ${ }^{1} \mathrm{H},{ }^{13} \mathrm{C}$ NMR and mass spectra afforded structure $\mathbf{8 3}$, which was confirmed by comparison with authentic spectra from our group and literature data. ${ }^{[99]}$<smiles>CC=CC=CC=CC=CC=C(C)C(O)C(O)C(O)CC(O)CC(O)CC(O)CC(O)CC(O)C(O)C(C(=O)OC(C)C(C)O)=C(O)CCCCC</smiles>

83

Fungichromin (83) has been reported as one of several macrocyclic polyene antibiotics, which showed antifungal, antiprotozoal and antinematodal activities. ${ }^{[99]}$ The biosynthesis of fungichromin $(\mathbf{8 3})$ is derived from the polyketide pathway with 12 acetate, one propionate and one intact octanoate units. ${ }^{[100]}$ 


\subsection{Terrestrial Streptomyces sp. Ank248}

The chromatogram of the crude extract from Streptomyces sp. Ank248 showed several UV absorbing bands under the UV lamp, which turned blue, yellow, violet and red colours with anisaldehyde/sulphuric acid after heating. The biological activity test revealed activities against bacteria and Artemia salina, respectively.

The well grown agar culture of the strain was fermented on LB-medium on a 10 $\mathrm{L}$ scale for 4 days at $28^{\circ} \mathrm{C}$ and worked up to obtain $1.23 \mathrm{~g}$ of crude extract.

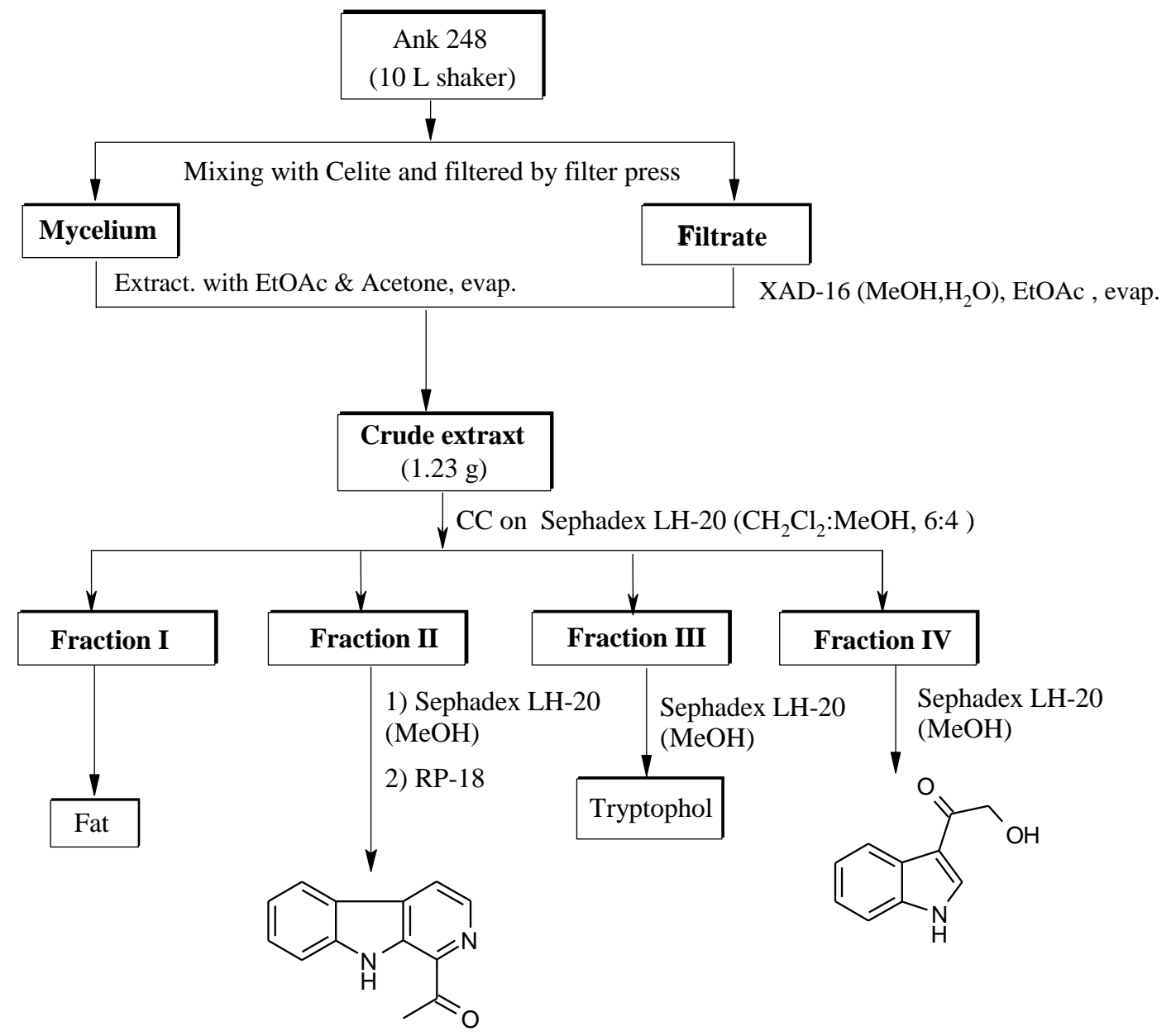

Figure 23: Work-up scheme for terrestrial Streptomyces sp. Ank248

\subsubsection{1-Acetyl- $\beta$-carboline}

Compound $\mathbf{8 4}$ was isolated as brownish solid from fraction II, which turned yellow by staining with anisaldehyde/sulphuric acid. 
The ${ }^{1} \mathrm{H}$ NMR spectrum of 84 exhibited a broad signal at $\delta 10.28(\mathrm{NH}$ or $\mathrm{OH})$, a doublet at $\delta 8.53$ with a coupling constant of $4.9 \mathrm{~Hz}$, which was less than ortho coupling but bigger than the meta coupling of aromatic systems, suggesting a heteroaromatic ring. The resonances of a multiplet between $\delta 8.15-8.13(2 \mathrm{H})$, a doublet of doublet at $\delta 7.58(2 \mathrm{H})$, and a multiplet at $\delta 7.32$ demonstrated a 1,2-disubstituted benzene ring. Furthermore, a $3 \mathrm{H}$ singlet at $\delta 2.88$ indicated an acetyl group.

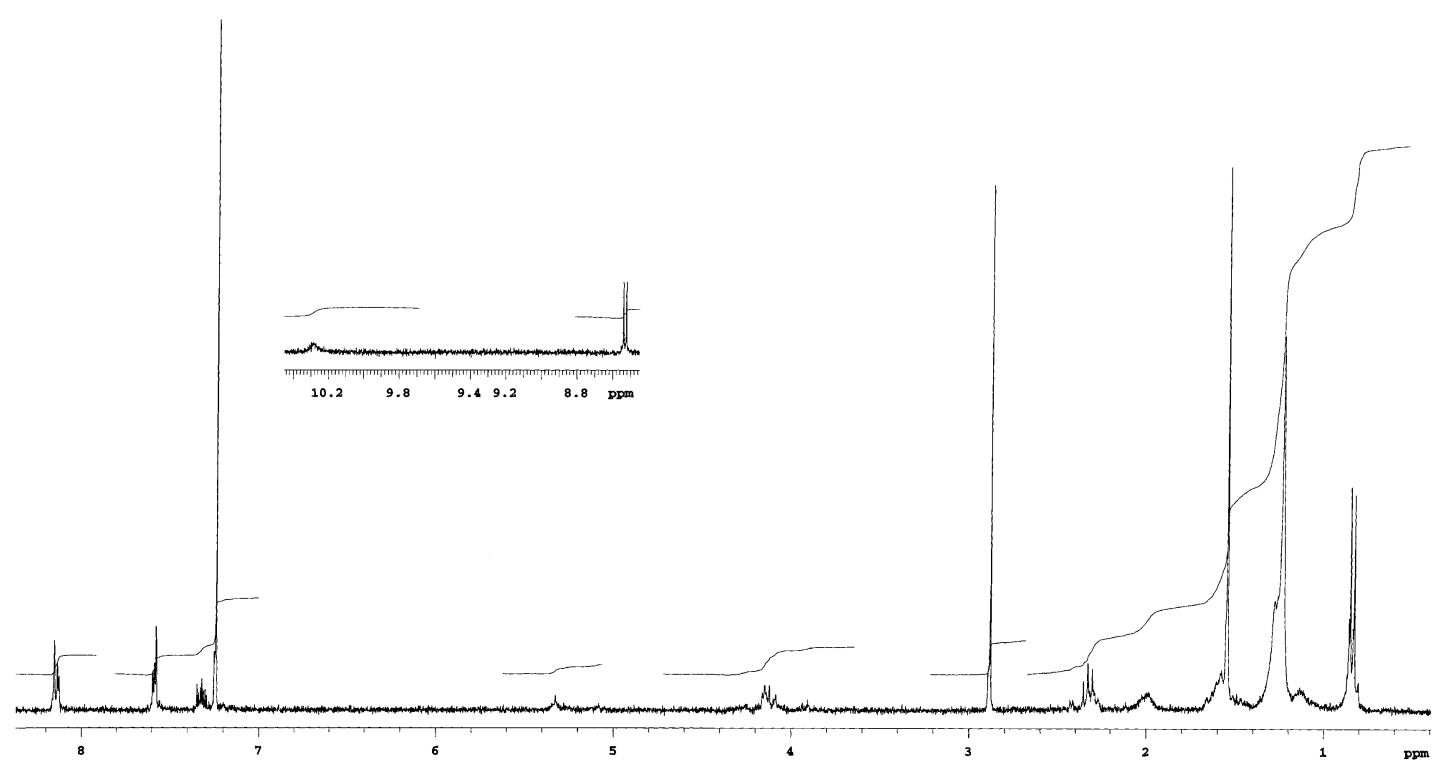

Figure 24: $\quad{ }^{1} \mathrm{H}$ NMR spectrum $\left(\mathrm{CDCl}_{3}, 300 \mathrm{MHz}\right)$ of 1 -acetyl- $\beta$-carboline (84)<smiles>CC(=O)c1nccc2c1[nH]c1ccccc12</smiles>

The sub-structure search in AntiBase with the ${ }^{1} \mathrm{H}$ NMR data and comparing with the authentic spectra as well as literature data ${ }^{[101,102]}$ led to structure 84. This $\beta$ carboline alkaloid was previously reported as plant metabolite. ${ }^{[103,104]}$ Interestingly, bacteria and fungi produced also this type of compounds, which exhibited several pharmacological activities as enzyme inhibitors for monoamineoxidase and cAMPphosphodiesterase. ${ }^{[105,106]} \beta$-Carboline derivatives have also been reported to be used 
as herbicides, fungicides and anti-tumor agents. ${ }^{[107,108]}$ In addition, 3-(hydroxyacetyl)-indole and tryptophol were also isolated from this strain. Ikeda and coworkers had reported that 3-(hydroxyacetyl)-indole showed activities as antifungal and anticancer agent. ${ }^{[108]}$

\subsection{Terrestrial Streptomyces sp. Ank181}

In the primary screening, the thin layer chromatogram of the crude extract from Streptomyces sp. Ank181 showed UV absorbing bands at $254 \mathrm{~nm}$, which stained to blue, deep green and violet in the less polar and middle regions, respectively, by spraying with anisaldehyde/sulphuric acid after heating.

The antimicrobial assay exhibited a high activity against Candida albicans, moderate activity against $E$. coli, Streptomyces viridochromogenes (Tü 57), and Mucor miehei (Tü 284). It showed a weak activity against Bacillus subtilis, and no activity against the Gram positive bacteria, Staphylococcus aureus and the algae, Chlorella vulgaris, Chlorella sorokiniana and Scenedesmus subspicatus.

A well grown agar culture of the strain was used to inoculate a $25 \mathrm{~L}$ jar fermentor on $\mathrm{M}_{2}$ medium ( $\mathrm{pH}$ 7.8). After 6 days at $28^{\circ} \mathrm{C}$, the culture broth was filtered. The resulting filtrate and mycelial residue were extracted separately using ethyl acetate and acetone giving a brown crude extract after evaporation under vacuum. The resulting extracts showed a similar TLC pattern and were therefore combined. The crude extract was then chromatographed on silica gel (column) with a dichloromethane/methanol gradient as shown in the work up scheme. 


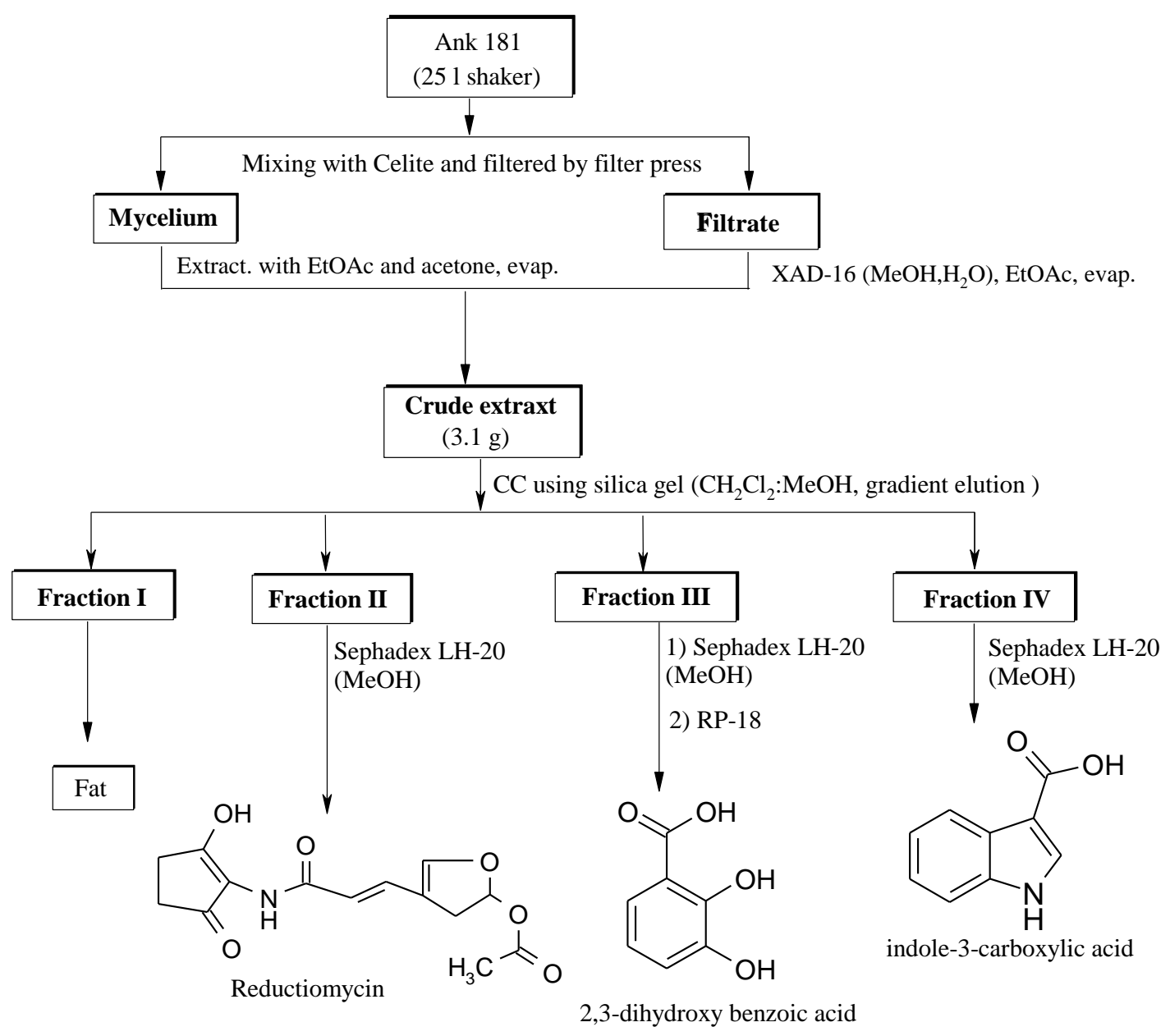

Figure 25: Work-up scheme for terrestrial Streptomyces sp. Ank181

\subsubsection{Reductiomycin}

Fraction II was subjected to a Sephadex LH-20 column to isolate reductiomycin (85) as yellow needle-shaped crystals. It showed a UV absorbing band and a deep green colour reaction by spraying with anisaldehyde/sulphuric acid.

The ${ }^{1} \mathrm{H}$ NMR spectrum showed a broad singlet of an H/D exchangeable proton at $\delta 13.76$ and a further broad NH singlet at $\delta 7.68$. The two doublets at $\delta 7.50\left({ }^{3} \mathrm{~J}=\right.$ $15.1 \mathrm{~Hz})$ and $\delta 6.01\left({ }^{3} J=15.1 \mathrm{~Hz}\right)$ each with intensity $1 \mathrm{H}$ were due of a transdisubstituted $\alpha, \beta$-unsaturated carbonyl derivative. The $3 \mathrm{H}$ singlet at $\delta 2.10$ indicated a methyl group connected with an $s p^{2}$ carbon atom. One of the two diastereotopic protons of a methylene group (H-3') showed doublets of a doublet at $\delta 3.05$. The two methylene groups $\mathrm{CH}_{2}-4, \mathrm{CH}_{2}-5$ and one proton of $\mathrm{CH}_{2}-3^{\prime}$ showed a multiplet at $\delta$ 
2.60. The ${ }^{1} \mathrm{H}$ NMR spectrum also showed a singlet at $\delta 6.90$ for the $s p^{2}$ attached proton $\mathrm{H}-5^{\prime}$ and doublets of a doublet at $\delta 6.70$ for the anomeric proton $\mathrm{H}-2$ '.

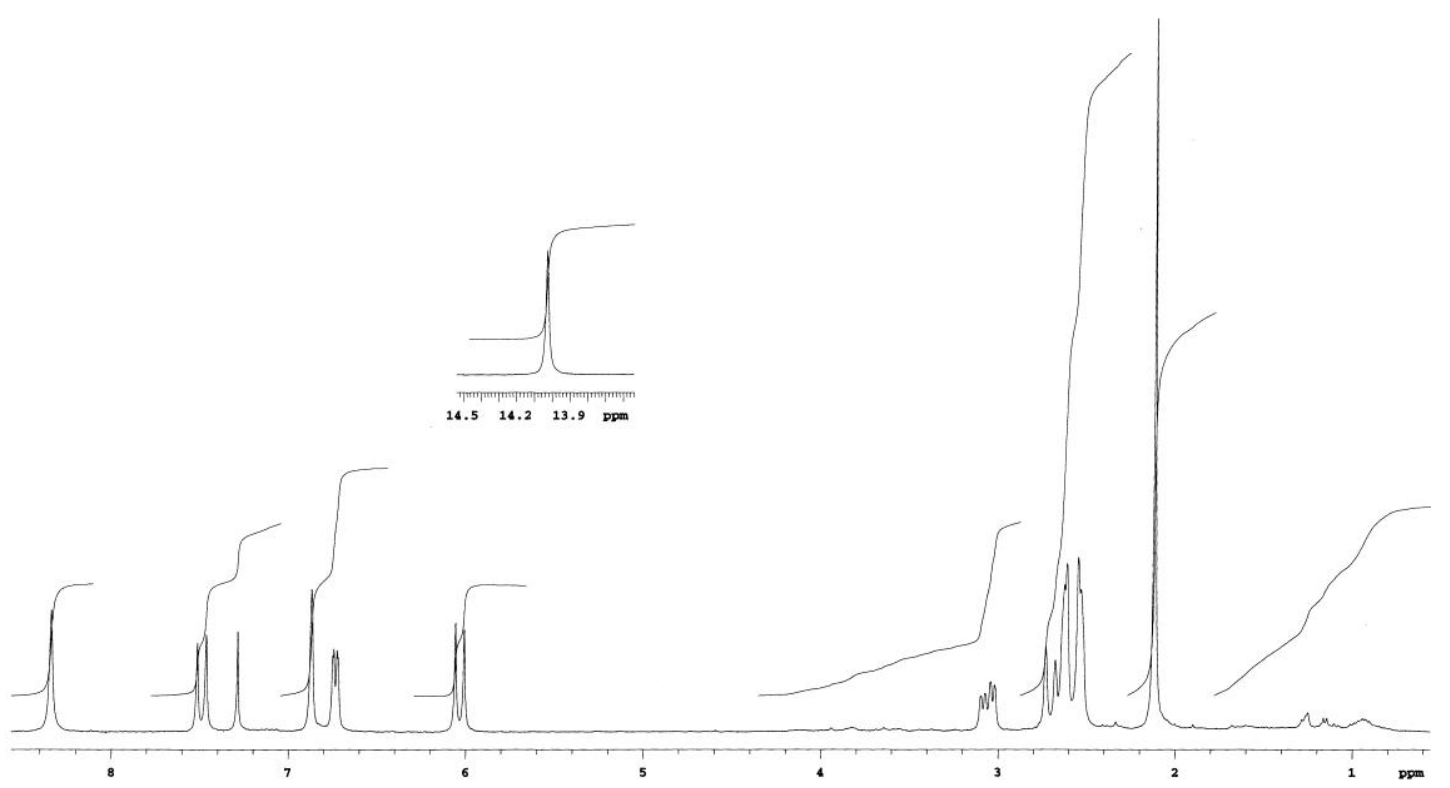

Figure 26: $\quad{ }^{1} \mathrm{H}$ NMR spectrum $\left(\mathrm{CDCl}_{3}, 300 \mathrm{MHz}\right)$ of reductiomycin $(\mathbf{8 5})$

The ESI mass analysis delivered an odd molecular weight of 293 Dalton indicating the presence of nitrogen. The substructure search in AntiBase using these spectral data resulted in reductiomycin (85) which was further confirmed by comparing with authentic spectra as well as literature data. ${ }^{[101]}$<smiles>CC(=O)OC1CC(C=CC(=O)NC2=C(O)CCC2=O)=CO1</smiles>

85

Reductiomycin (85) is classified as antibiotic active against Gram-positive bacteria, fungi, the Newcastle disease virus and certain yeasts. Reductiomycin (85) showed a low acute toxicity $\left(\mathrm{LD}_{50}\right)$ intraperitoneally in mice of about $80 \mathrm{mg} / \mathrm{kg} .{ }^{[109]}$ Chemically, reductiomycin (85) consists of two unique moieties, an unusual acetoxydihydrofuran and 2-amino-3-hydroxycyclopent-2-enone. ${ }^{[110]}$ The acetoxydihydrofuran is also present in other compounds such as oudenone (an inhibitor for tyrosine 
hydroxylase) and reductic acid (a reducing agent). The acetoxydihydrofuran moiety seems to play an important role in their biological activities. The 2-amino-3hydroxycyclopent-2-enone moiety has shown to be present in various antitumor antibiotics including pyrrolo[1,4]benzodiazepine antibiotics, mitomycin, ansamitocin and naphthyridinomycin. ${ }^{[111]}$ In addition, 2,3-dihydroxybenzoic acid and indole-3carboxylic acid were also isolated from this strain.

\subsection{Ruminal bacterium Enterobacter amnigenus ZIA}

Ruminants such as cattles, sheep, buffaloes, deer, goats, and camels have a unique digestive system. They have a stomach with four compartments, and the rumen is one of them. The rumen contains microorganisms that are able to digest cellulose. ${ }^{[112]}$ Enterobacter is one genus among them, Gram-negative, facultatively anaerobic and rod-shaped bacteria. ${ }^{[113]}$

Enterobacter amnigenus ZIA was isolated from the rumen of a Tunisian cow. Pre-screening of crude extract showed no biological activity against different microorganisms, but TLC of the crude extract showed UV absorbing bands at $254 \mathrm{~nm}$, which stained to blue, red, brown and green with anisaldehyde/sulphuric acid.

The strain was fermented on a $10 \mathrm{~L}$ scale in LB medium as shaker culture for 4 days at $28{ }^{\circ} \mathrm{C}$. The culture broth was harvested and filtered to separate the cell mass, which was later extracted with ethyl acetate and acetone, respectively. The culture filtrate was then passed through Amberlite XAD-16 and eluted with $10 \mathrm{~L}$ methanol. The methanol extract was concentrated by using a rotary evaporator under reduced pressure. The organic phases were then mixed prior to monitoring by TLC to yield $3.3 \mathrm{~g}$ of crude extract. TLC of the crude extract on a silica gel column using a $\mathrm{CH}_{2} \mathrm{Cl}_{2}-\mathrm{MeOH}$ gradient with increasing polarity resulted in four sub-fractions (I-IV), as monitored by TLC. 


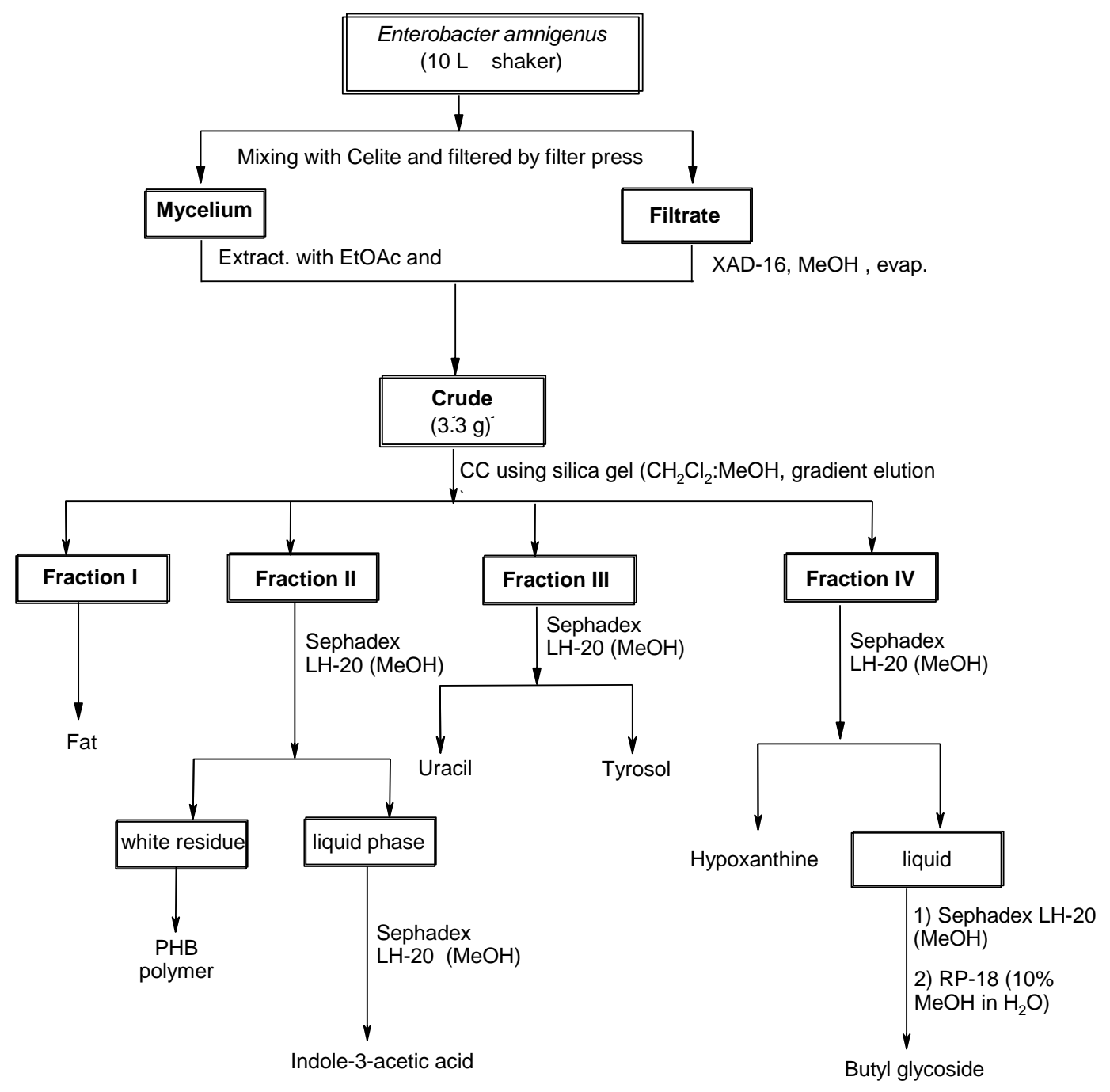

Figure 27: Work-up scheme for ruminal bacterium Enterobacter amnigenus ZIA

\subsubsection{Poly-(hydroxybutyric acid) (PHB)}

Compound 86 was obtained as white precipitate from sub-fraction II after being subjected to Sephadex chromatography. The ${ }^{1} \mathrm{H}$ NMR spectrum showed three signals in the aliphatic region at $\delta 5.20(\mathrm{~m}, 1 \mathrm{H}), 2.45(\mathrm{dd}, 2 \mathrm{H})$ and $1.22(\mathrm{~d}, 3 \mathrm{H})$. A substructure search in AntiBase and comparison with the authentic spectra ${ }^{[114]}$ as well as the literature data ${ }^{[115]}$ suggested poly-(hydroxybutyric acid) $(\mathbf{8 6})$ as the structure. Poly-(hydroxybutiric acid) (86) has been isolated in our group from both terrestrial and marine-derived Streptomyces sp. ${ }^{[115]}$ The high-polymeric variety is used as biologically degradable and environmentally safe plastic material from microorganisms. $^{[115]}$ 


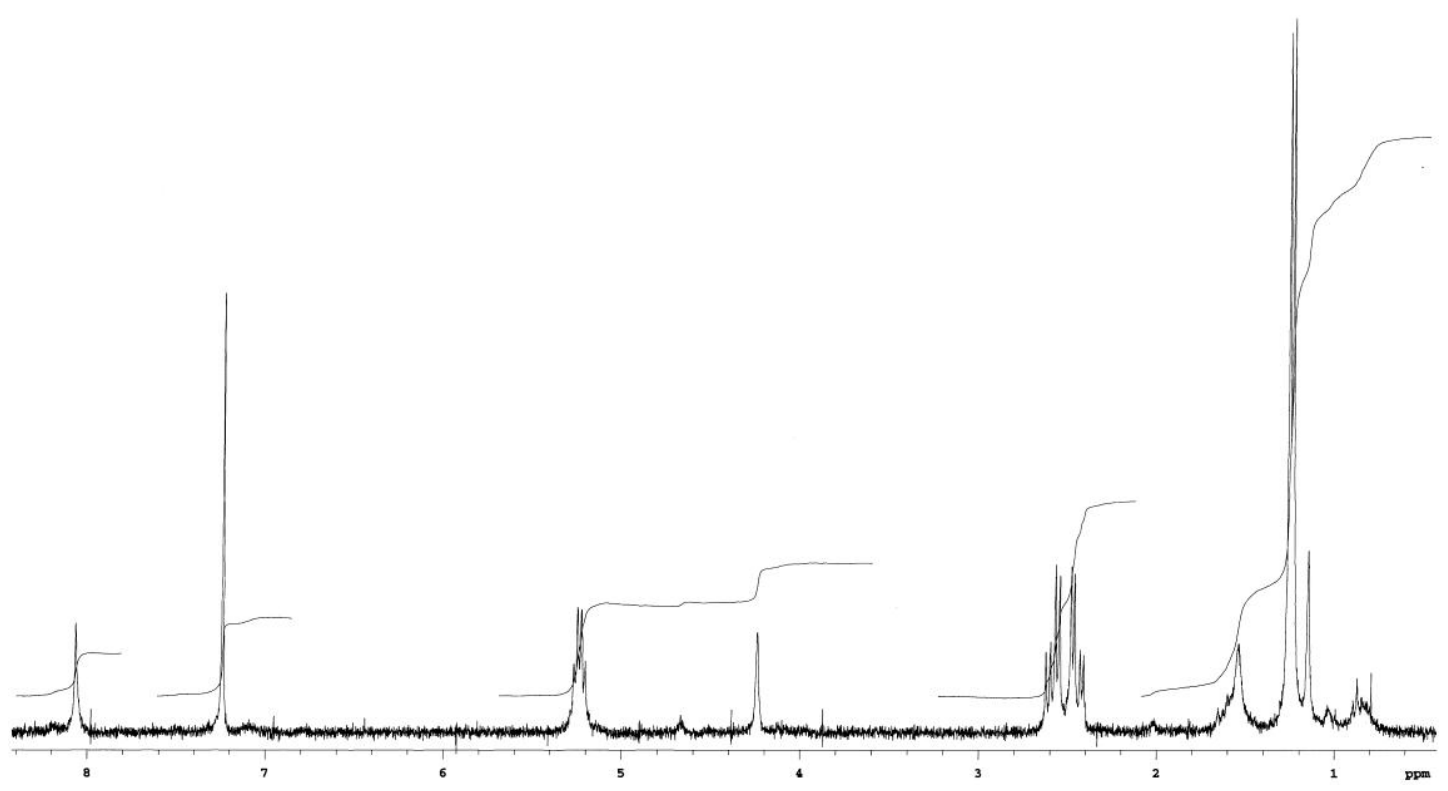

Figure 28: $\quad{ }^{1} \mathrm{H}$ NMR spectrum $\left(\mathrm{CDCl}_{3}, 300 \mathrm{MHz}\right)$ of poly-(hydroxybutyric acid) (86)

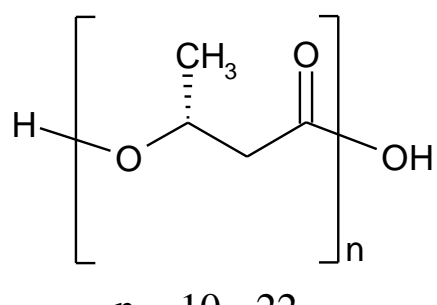

86

In addition, indole-3-acetic acid, uracil, tyrosol and hypoxanthine were isolated separately from different fractions during the work-up of the strain. They are often isolated as trivial metabolites from bacteria.

\subsubsection{Butyl glycoside}

Compound 87 showed no UV absorption and stained to green with anisaldehyde/sulphuric acid. It was isolated as oily compound from sub-fraction IV after purification by using Sephadex and RP-18 chromatography. The high-resolution ESI mass analysis delivered a quasi-molecular ion peak at $\mathrm{m} / \mathrm{z} 259.11536[\mathrm{M}+\mathrm{Na}]^{+}$calculated for $\mathrm{C}_{10} \mathrm{H}_{20} \mathrm{O}_{6} \mathrm{Na}$ as molecular formula. 
The ${ }^{1} \mathrm{H}$ NMR spectrum displayed resonances of a doublet of an anomeric proton at $\delta 4.72(\mathrm{~d}, J=1.5 \mathrm{~Hz})$, and multiplets of five oxygenated methines and two oxygenated methylene groups between $\delta 3.81-3.39$, which suggested the presence of a sugar moiety. In addition, it showed two multiplets and one triplet of two methylenes and a methyl group at $\delta 1.56,1.41$ and 0.93 , respectively.

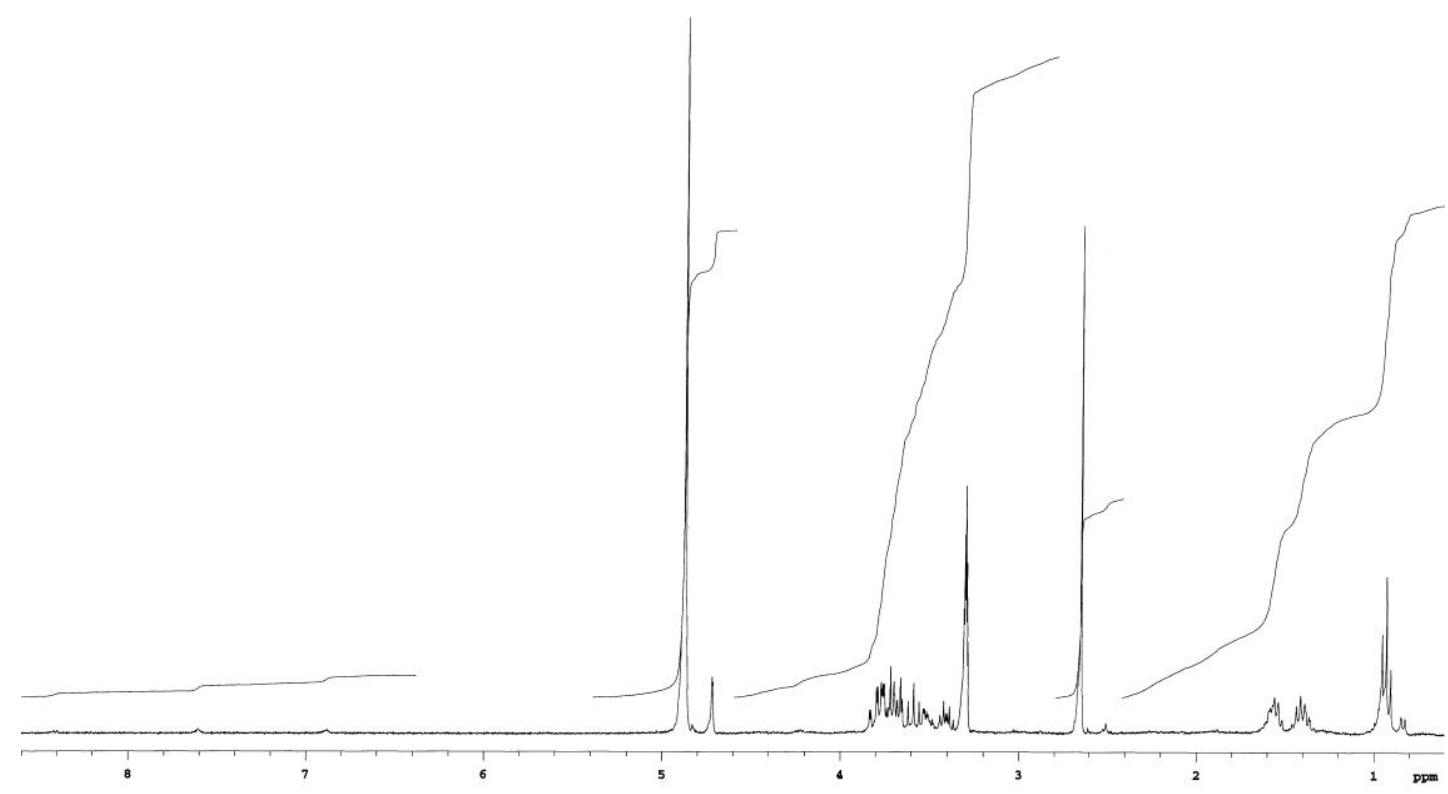

Figure 29: $\quad{ }^{1} \mathrm{H}$ NMR spectrum $\left(\mathrm{CD}_{3} \mathrm{OD}, 300 \mathrm{MHz}\right)$ of butyl glycoside (87)

The ${ }^{13} \mathrm{C}$ NMR spectrum revealed ten signals. Seven of them were due to oxygenated carbon atoms. The resonance at $\delta_{C} 101.5$ was characteristic of the acetal carbon in a sugar skeleton. The other oxygenated carbon signals were observed at $\delta_{C}$ 74.5 (CH-2'), $72.6(\mathrm{CH}-3 '), 72.3\left(\mathrm{CH}-4{ }^{\prime}\right), 68.6(\mathrm{CH}-5 '), 68.2\left(\mathrm{CH}_{2}-1\right)$, and $62.9\left(\mathrm{CH}_{2-}\right.$ 6'), respectively. Additionally, three signals at $\delta_{C} 32.7,20.5$, and 14.3 were assigned as two methylenes and one methyl group of an aliphatic chain. The sub-structure search in AntiBase and the Chemical Abstracts based on these spectroscopic data of 87 resulted in no hits, so that the compound was identified as a new $n$-butyl hexopyranoside. The sugar part was not determined due to limited amount of the compound, but an identical butyl glycoside (87) has been isolated in parallel from another ruminal bacterium Enterobacter amnigenus ZIH and was fully characterised by 2D NMR. ${ }^{[116]}$ 


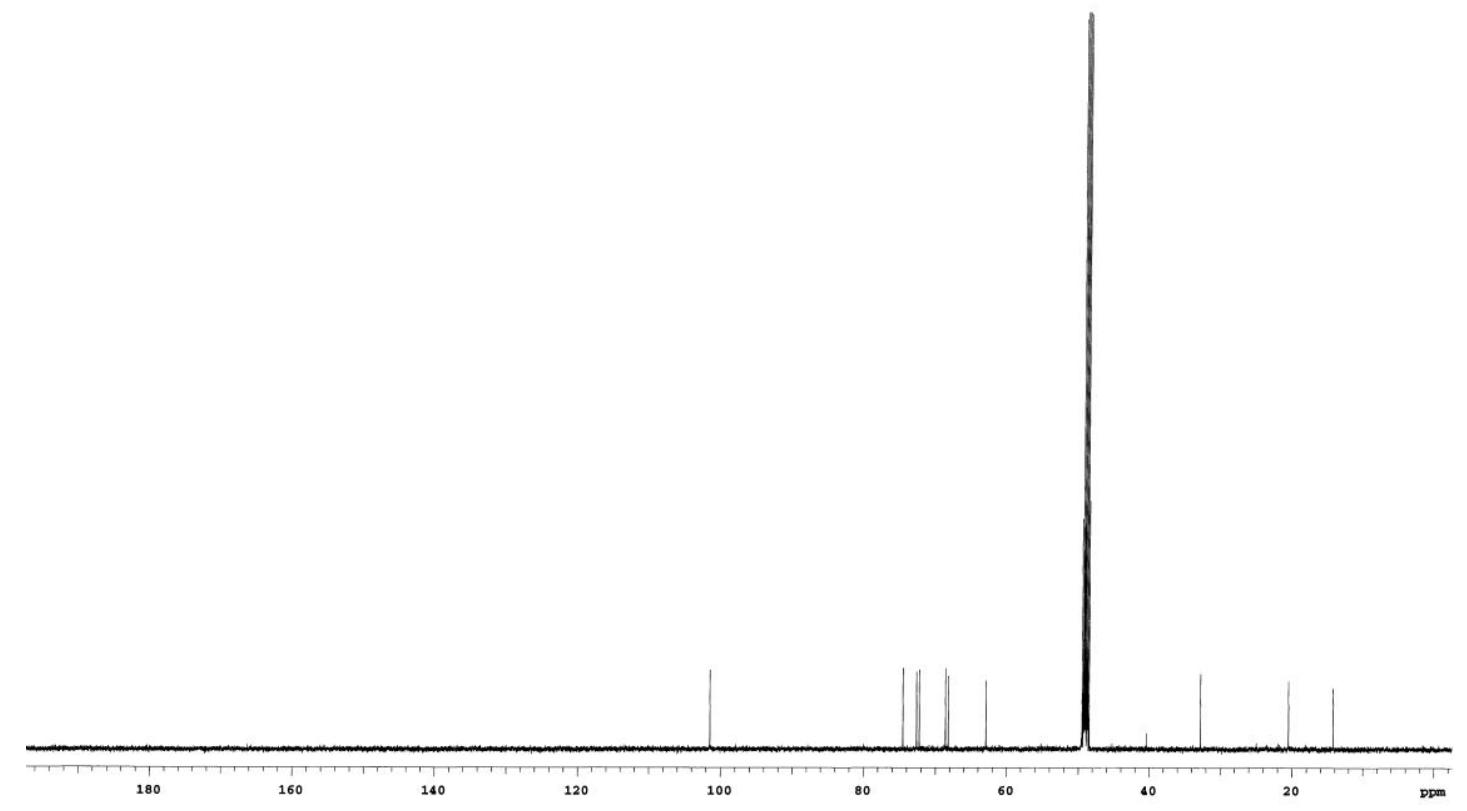

Figure 30: $\quad{ }^{13} \mathrm{C}$ NMR spectrum $\left(\mathrm{CD}_{3} \mathrm{OD}, 125 \mathrm{MHz}\right)$ of butyl glycoside 87<smiles>CCCCO[C]1O[C@H](CO)C(O)C(O)C1O</smiles>

87

Alkyl glycosides are present in nature as glycolipids with rhamnose, sophorose and trehalose as carbohydrate units. They show surfactant behaviour when the alkyl chain consists of at least four carbon atoms and are biodegradable and less toxic. ${ }^{[117-}$ 119]

\subsection{Terrestrial Streptomyces sp. Ank75}

The terrestrial Streptomyces sp. Ank75 formed on $\mathrm{M}_{2}$ agar medium a white aerial mycelium within 3 days at $28^{\circ} \mathrm{C}$. The crude extract of the strain showed activities against Candida albicans, Mucor miehei (Tü 284), Chlorella vulgaris, Chlorella sorokiniana, but was inactive against bacteria and Artemia salina (brine shrimp test).

The Ank75 strain was fermented in $25 \mathrm{~L}$ scale on $\mathrm{M}_{2}$ medium for 5 days at 28 ${ }^{\circ} \mathrm{C}$. The culture broth was filtered over Celite and the water phase was adsorbed on Amberlite XAD-16 resin and eluted with methanol after washing with demineralised 
water prior to the elution with methanol. The biomass was exhaustively extracted with ethyl acetate and acetone. The thin layer chromatograms of crude extract from both organic residues were identical, thus the extracts were combined for further work-up procedure. The crude extract was subjected to silica gel column chromatography using a dichloromethane/methanol gradient to afford four fractions, which were further purified via different chromatography methods to obtain the pure metabolites (see work-up scheme).

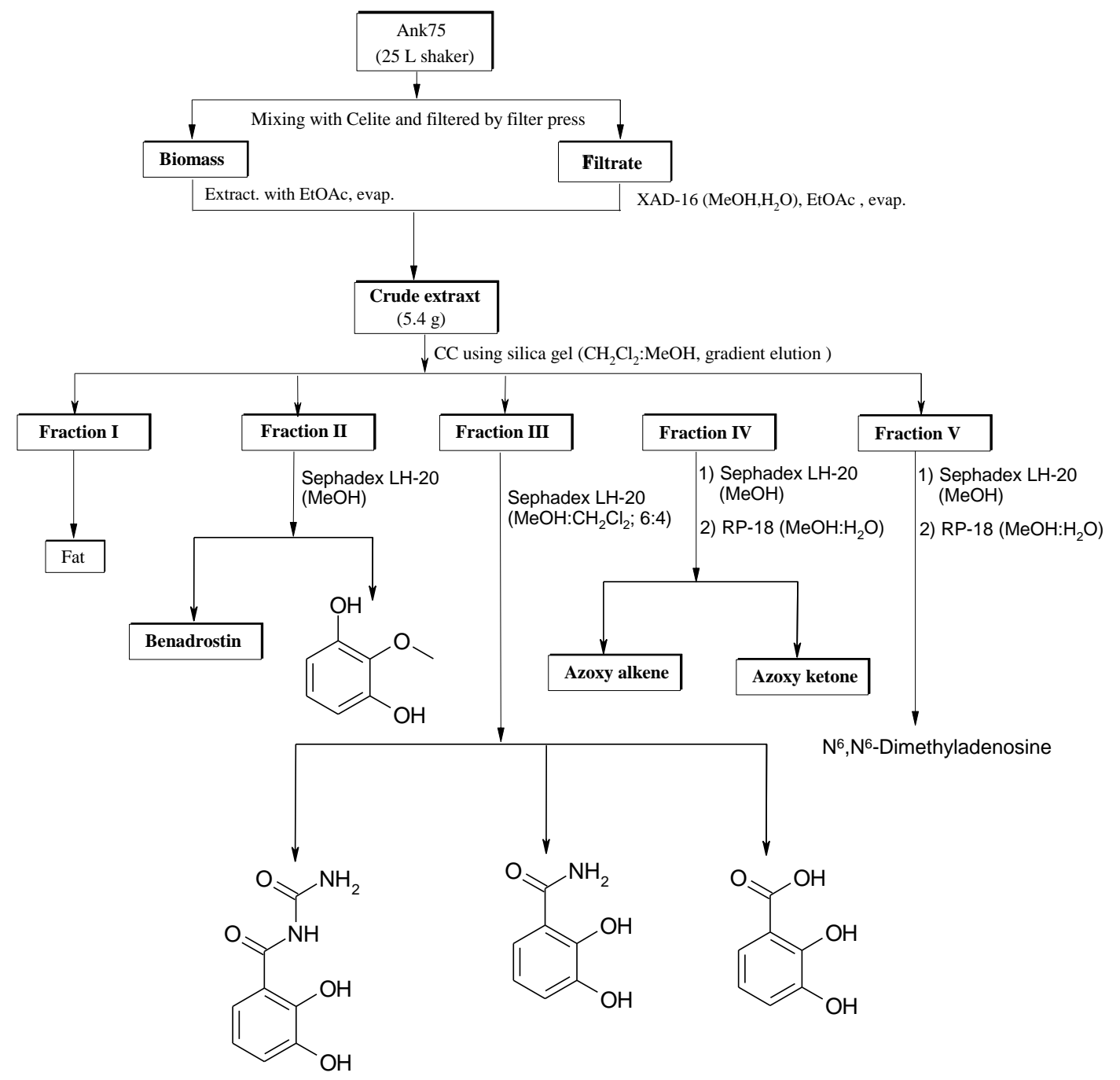

Figure 31: Work-up scheme for terrestrial Streptomyces sp. Ank75

\subsubsection{Benadrostin}

Compound $\mathbf{8 8}$ was isolated as white solid and showed an UV absorbing spot, which turned violet with anisaldehyde/sulphuric acid. The HRESI mass analysis delivered $\mathrm{C}_{8} \mathrm{H}_{5} \mathrm{NO}_{4}$ as the molecular formula. The ${ }^{1} \mathrm{H}$ NMR spectrum displayed two 
doublets of doublets and one triplet at $\delta 7.35(J=7.5$ and $1.7 \mathrm{~Hz}), 7.24(J=7.9$ and $1.7 \mathrm{~Hz})$, and $7.18(J=7.7 \mathrm{~Hz})$. Furthermore, a broad singlet of an H/D exchangeable proton was observed at $\delta 11.17$. The sub-structure search in AntiBase based on these data resulted in benadrostin $(\mathbf{8 8})$ as the coincident structure to the spectroscopic data. This was further confirmed by comparison with authentic spectra and literature data. ${ }^{[101,120]}$

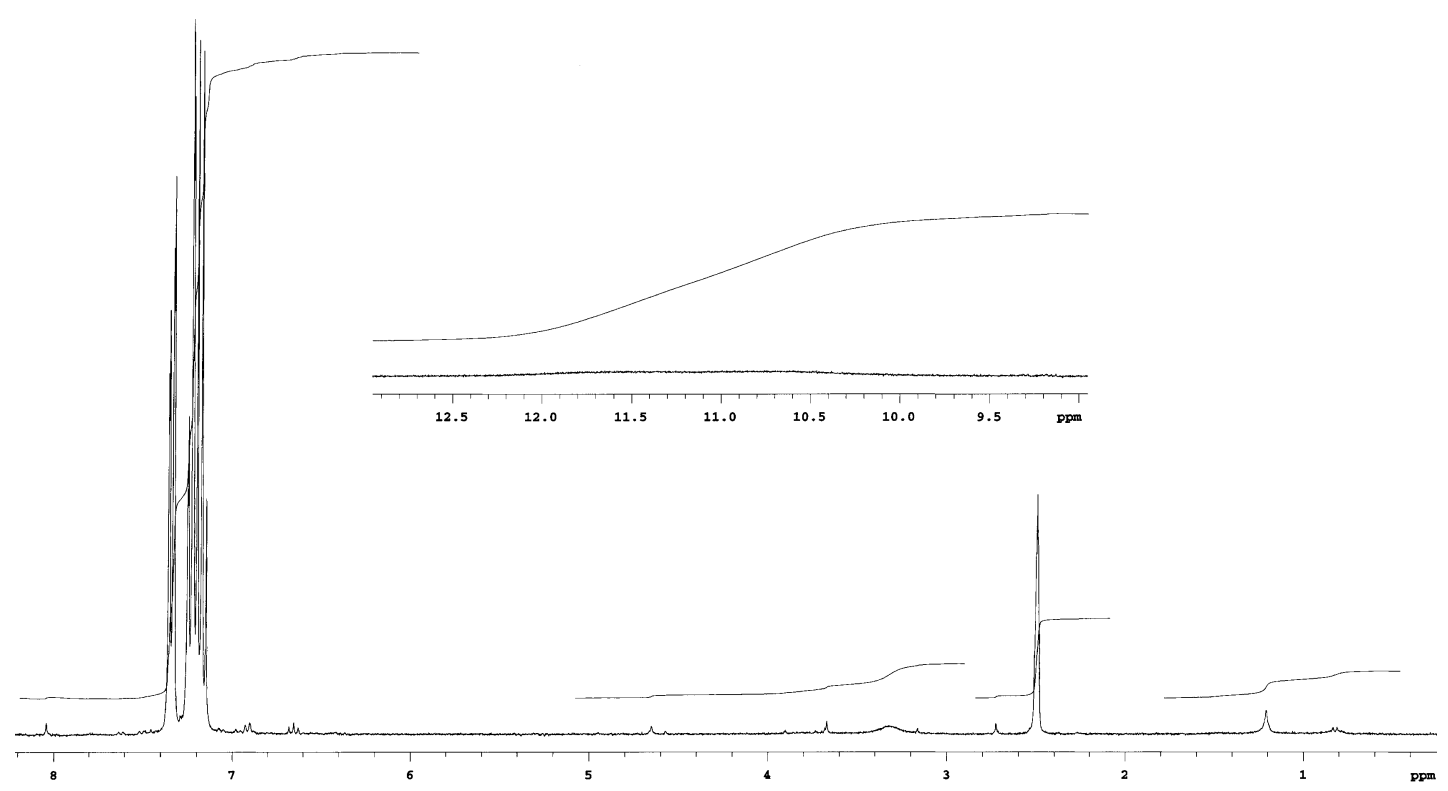

Figure 32: $\quad{ }^{1} \mathrm{H}$ NMR spectrum (DMSO- $d_{6}, 300 \mathrm{MHz}$ ) of benadrostin (88)<smiles>O=c1[nH]c(=O)c2cccc(O)c2o1</smiles>

\section{8}

Benadrostin (88) had been reported as an inhibitor of poly(ADP-ribose)synthetase. ${ }^{[121]}$ This enzyme plays an importance role in the biosynthesis of the protein-bound homopolymer, poly(ADP-ribose) from nicotinamide adenine dinucleotide (NAD). ${ }^{[121]}$ Several nuclear proteins, i.e. histone and non-histone proteins, had been reported as the acceptors of these polymers. ${ }^{[122-125]}$ Hence, the biosynthesis of poly(ADP-ribose) has been suggested to be required for the efficient DNA repair. ${ }^{[125]}$ Additionally, poly(ADP-ribose) synthetase had been also reported to play an im- 
portant role in some pathological conditions such as systemic lupus erythematodes, myasthenia gravis, and diabetes mellitus. ${ }^{[126,127]}$

\subsubsection{2-O-Methylpyrogallol}

Compound $\mathbf{8 9}$ was isolated from fraction II as white solid, which stained to red with anisaldehyde/sulphuric acid. The HRESI mass analysis delivered a pseudomolecular ion peak $[\mathrm{M}]^{+}(\mathrm{m} / \mathrm{z}$ 140.4730) corresponding to the molecular formula $\mathrm{C}_{7} \mathrm{H}_{8} \mathrm{O}_{3}$. The ${ }^{1} \mathrm{H}$ NMR spectrum showed three proton signals in the aromatic region at $\delta 6.72(\mathrm{t}, 1 \mathrm{H})$ and $6.37(\mathrm{~d}, 2 \mathrm{H})$ suggesting a 1,2,3-trisubstituted aromatic system, and one methoxy signal at $\delta 3.77$. The ${ }^{13} \mathrm{C}$ NMR spectrum exhibited four carbon signals at $\delta_{C} 151.5,124.5,108.3$ and 60.5, respectively. The sub-structure search in AntiBase based on the NMR and mass spectra did not give any appropriate structure, while the Chemical Abstracts suggested the synthetic 2-O-methylpyrogallol (89) as the most plausible candidate. The latter structure was confirmed indeed by comparison with the literature. ${ }^{[128,129]}$

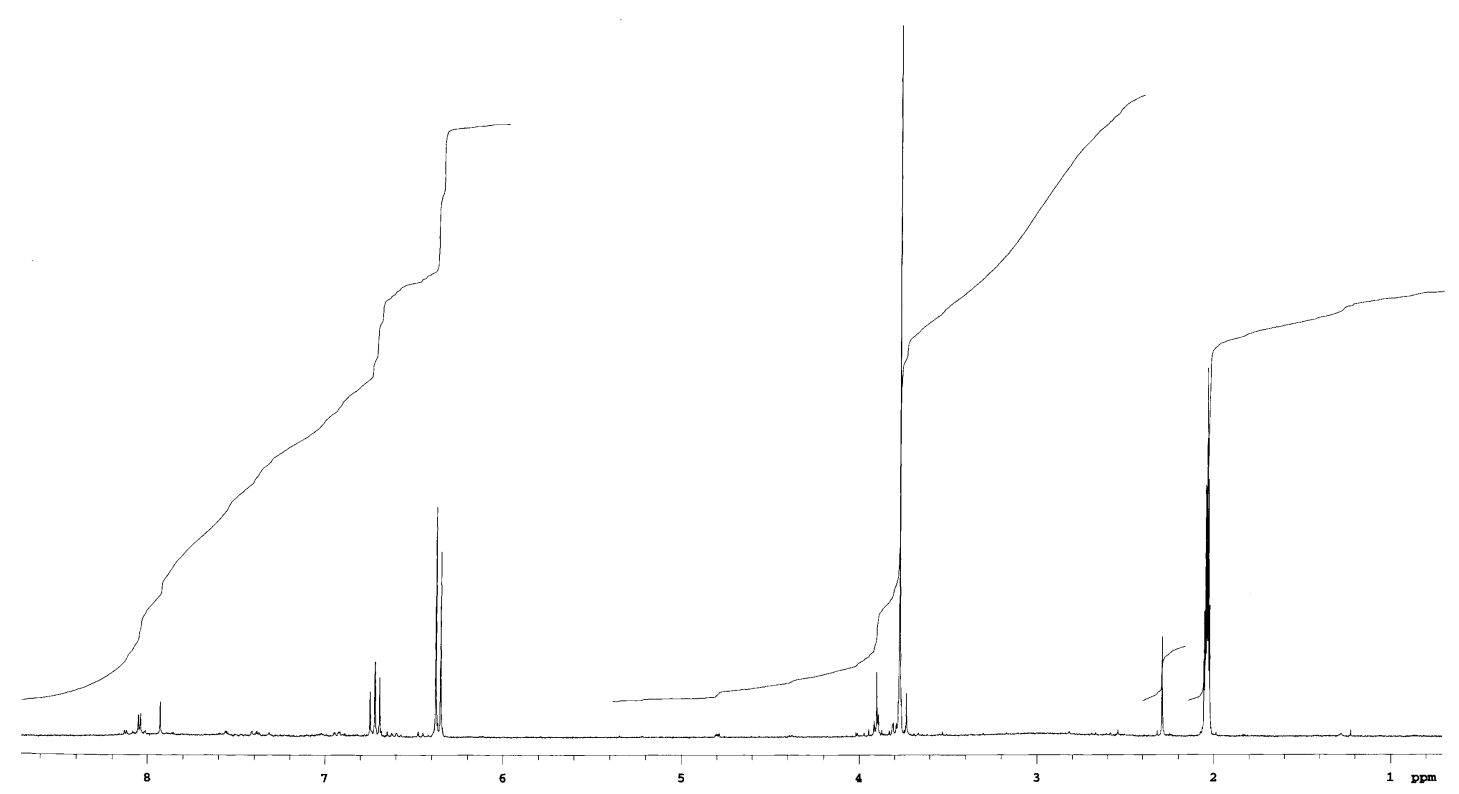

Figure 33: $\quad{ }^{1} \mathrm{H}$ NMR spectrum (acetone- $d_{6}, 300 \mathrm{MHz}$ ) of 2-O-methylpyrogallol (89) 


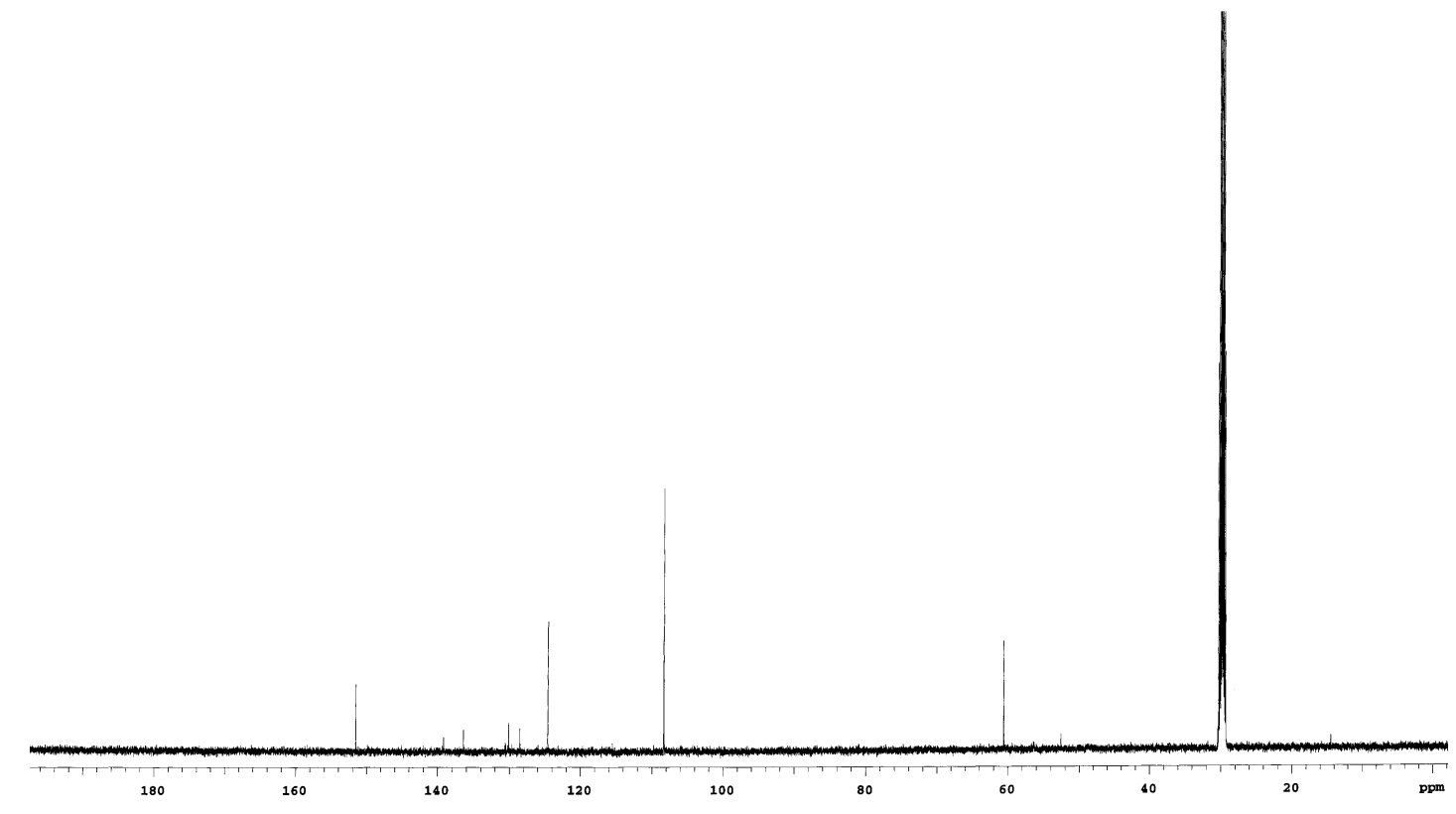

Figure 34: ${ }^{13} \mathrm{C}$ NMR spectrum (acetone- $d_{6}, 125 \mathrm{MHz}$ ) of 2-O-methylpyrogallol (89)<smiles>Oc1cccc(O)c1O</smiles>

89<smiles>O=C(O)c1cc(O)c(O)c(O)c1</smiles>

90

Compound 89 had been obtained by synthesis and had been also reported as a metabolite from gallic acid (90). ${ }^{[129]}$ It is for the first time isolated here from bacteria as natural product.

\subsubsection{N-Aminocarbonyl-2,3-dihydroxybenzamide}

Compound 91 was purified from fraction III by Sephadex LH-20 chromatography using methanol as eluent. The ${ }^{1} \mathrm{H}$ NMR spectrum exhibited $\mathrm{ABC}$ signals of a 1,2,3-trisubstituted benzene ring at $\delta 7.45(\mathrm{dd}), 7.00(\mathrm{dd})$ and $6.79(\mathrm{t})$, respectively. The HRESI mass analysis delivered a pseudomolecular ion peak $[\mathrm{M}+\mathrm{H}]^{+}(\mathrm{m} / \mathrm{z}$ 197.05573) corresponding to the molecular formula $\mathrm{C}_{8} \mathrm{H}_{9} \mathrm{~N}_{2} \mathrm{O}_{4}$. The sub-structure search in AntiBase based on the ${ }^{1} \mathrm{H}$ NMR and mass spectra afforded $\mathrm{N}$ aminocarbonyl-2,3-dihydroxybenzamide (91) as the suggested structure. The struc- 
ture was then confirmed by comparing with authentic spectra and literature data. ${ }^{[101]}$ Compound 91 had been isolated for the first time in our group from Streptomyces sp. Ank132. ${ }^{[101]}$

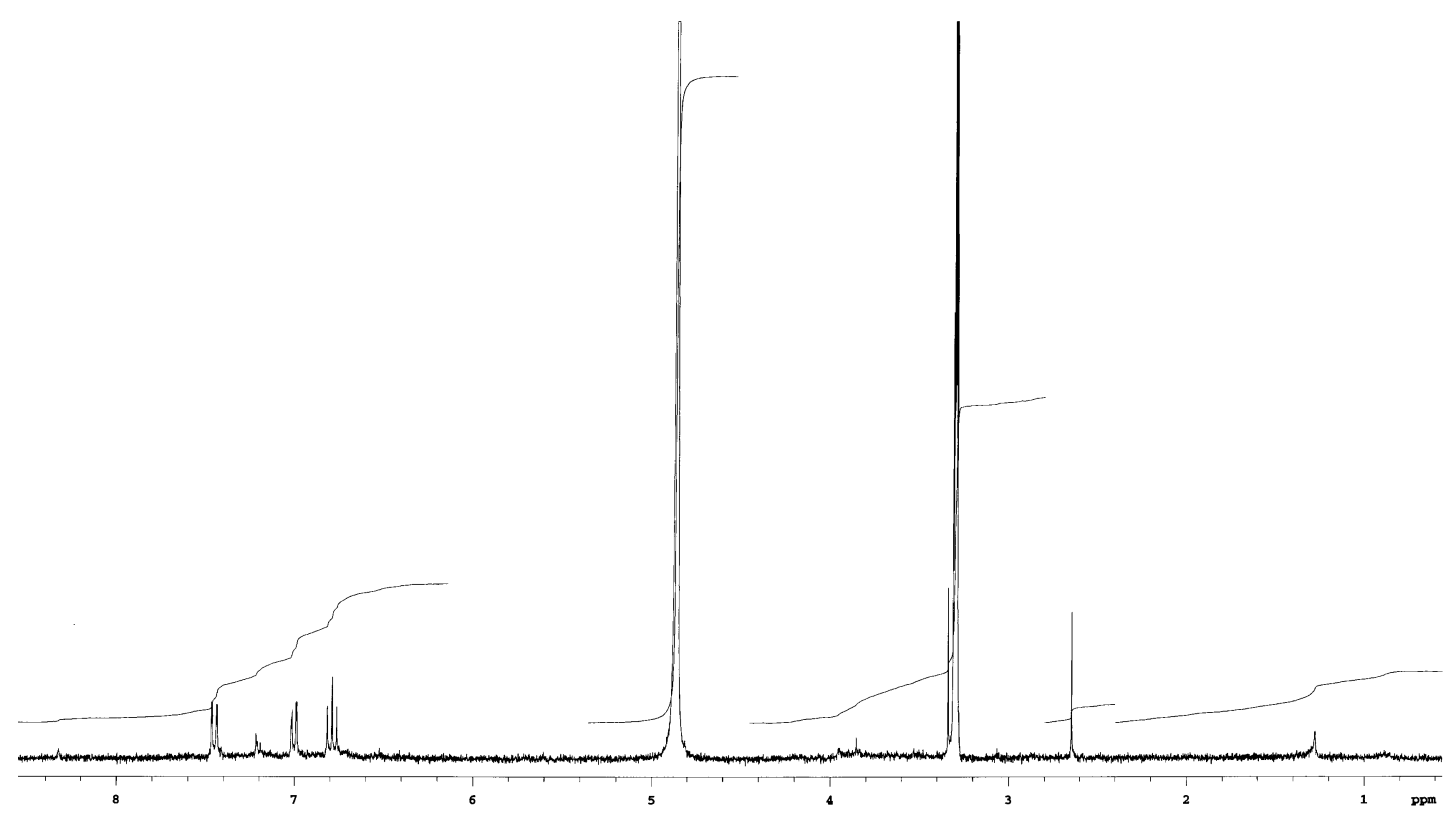

Figure 35: $\quad{ }^{1} \mathrm{H}$ NMR spectrum $\left(\mathrm{CD}_{3} \mathrm{OD}, 300 \mathrm{MHz}\right)$ of $\mathrm{N}$-aminocarbonyl-2,3dihydroxybenzamide (91)<smiles>NC(=O)NC(=O)c1cccc(O)c1O</smiles>

91

Compound 91 is a salicylurea derivative and has been used in food industry to preserve residues with high water content. The ability of acetylurea in preserving residues of grapes and apples can promote the growth of lactic bacteria and inhibit the growth of putrid bacteria. ${ }^{[130]}$ 


\subsubsection{2,3-Dihydroxybenzamide}

Compound 92 was obtained as brownish UV active solid from fraction III after separation on Sephadex LH-20 using methanol. It showed no colour reaction with anisaldehyde/sulphuric acid.

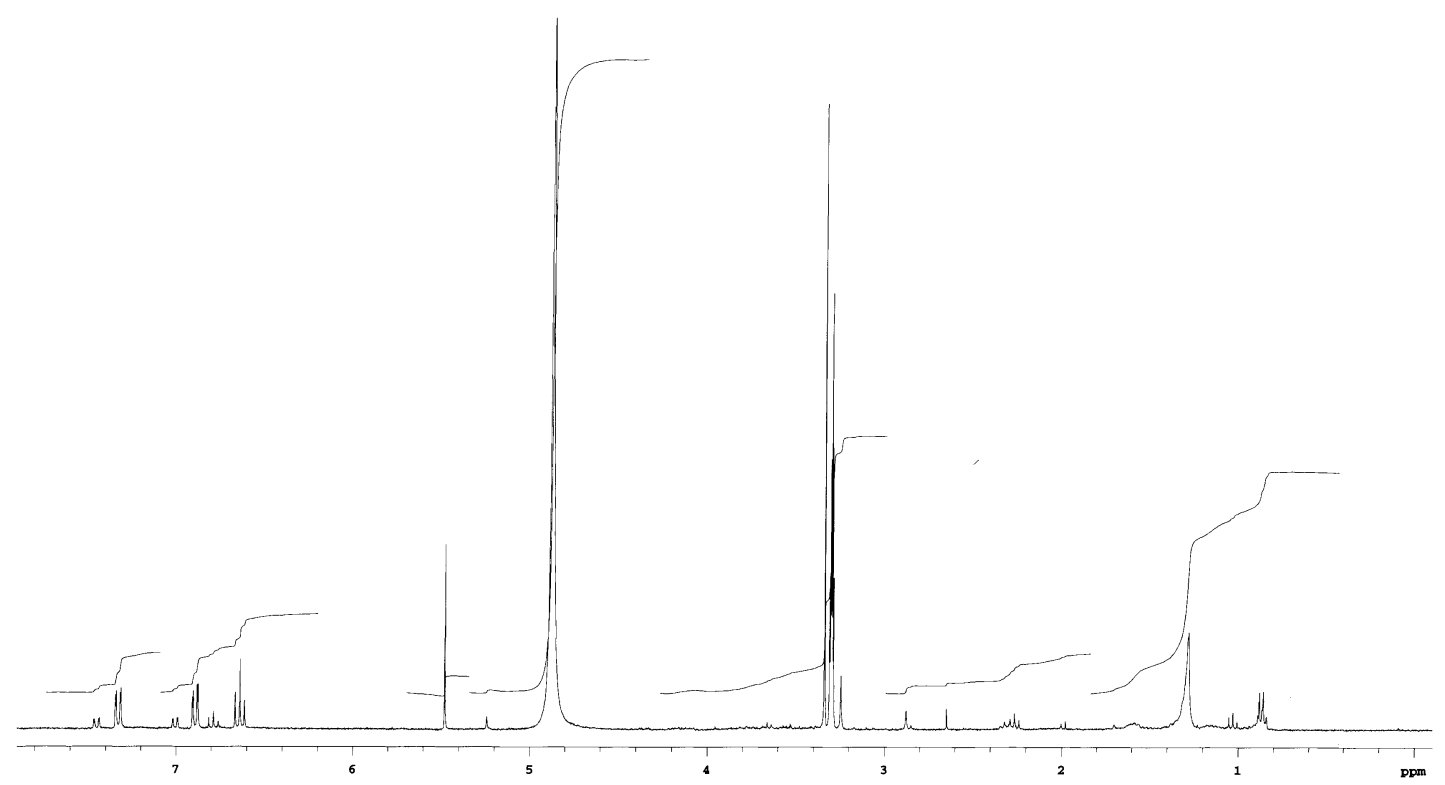

Figure 36: $\quad{ }^{1} \mathrm{H}$ NMR spectrum $\left(\mathrm{CD}_{3} \mathrm{OD}, 300 \mathrm{MHz}\right)$ of 2,3-dihydroxybenzamide (92)

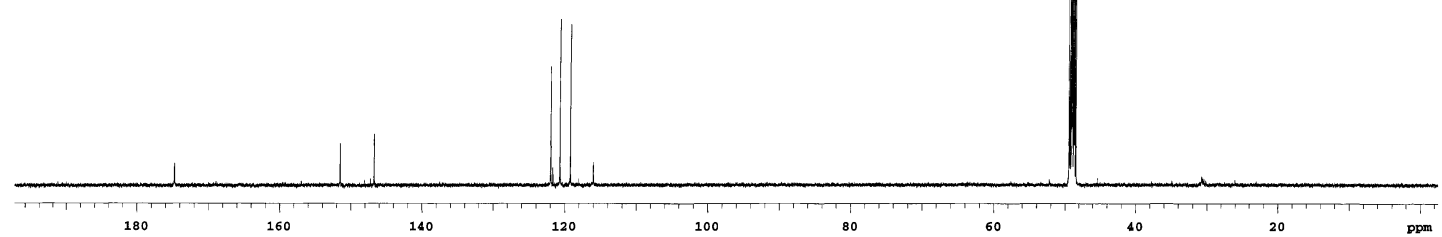

Figure 37: $\quad{ }^{13} \mathrm{C}$ NMR spectrum $\left(\mathrm{CD}_{3} \mathrm{OD}, 125 \mathrm{MHz}\right)$ of 2,3-dihydroxybenzamide (92) 


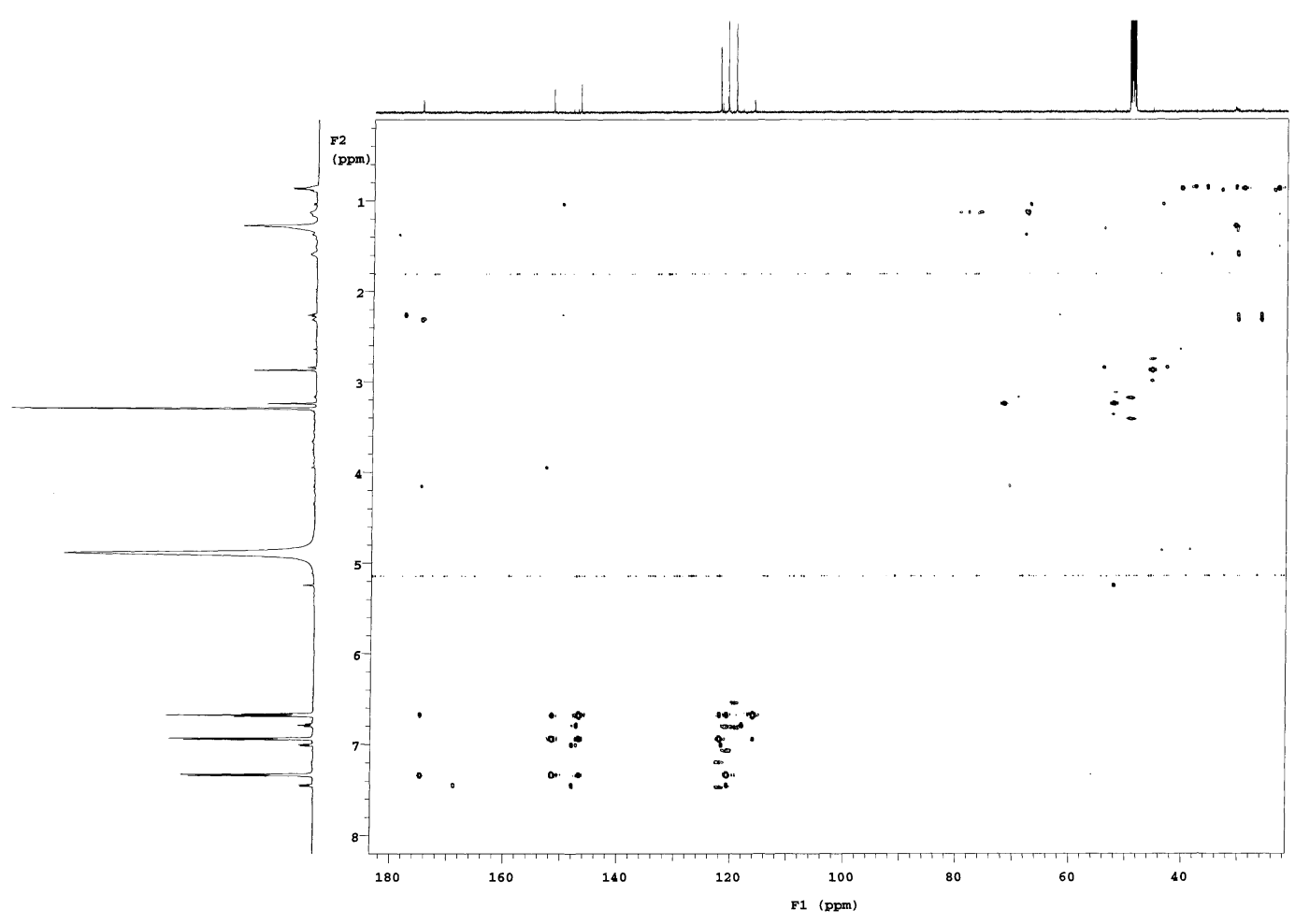

Figure 38: $\quad \mathrm{HMBC}$ spectrum $\left(\mathrm{CD}_{3} \mathrm{OD}, 600 \mathrm{MHz}\right)$ of 2,3-dihydroxybenzamide (92)

The ${ }^{1} \mathrm{H}$ NMR spectrum displayed the ABC system of a 1,2,3-trisubstituted benzene ring in the aromatic region. In the ${ }^{13} \mathrm{C}$ NMR spectrum, it revealed seven signals, of four quaternary carbon atoms and three $s p^{2}$ methines. One of the four quaternary carbon signals at $\delta_{C} 174.8$ was identified as carbonyl group, and two carbon signals in the aromatic region at $\delta_{C} 151.6$ and 146.8 were due to oxygenated quaternary $s p^{2}$ carbons. The remaining signals at $\delta_{C} 121.9,121.6,119.2$, and 116.0 were assigned as three $s p^{2}$ methines and a quaternary $s p^{2}$ carbon, respectively. According to HMBC correlations of $\mathbf{9 2}$, the positions of the carbonyl group were assigned through the ${ }^{3} J$ cross-signal between H-6 $(\delta 7.33)$ and C-1' $\left(\delta_{C} 174.8\right)$, and the ${ }^{3} J$ cross-signal between H-5 $(\delta 6.64)$ and C-1 $\left(\delta_{C} 116.0\right)$. The HRESI mass analysis delivered the molecular formula $\mathrm{C}_{7} \mathrm{H}_{7} \mathrm{NO}_{2}$. Based on the ${ }^{1} \mathrm{H}$ NMR, ${ }^{13} \mathrm{C}$ NMR, HMBC spectroscopic data and mass measurement, the isolated compound was assigned as 2,3dihydroxybenzamide $\mathbf{( 9 2 )}$, which was isolated here for the first time from a Streptomyces sp. 


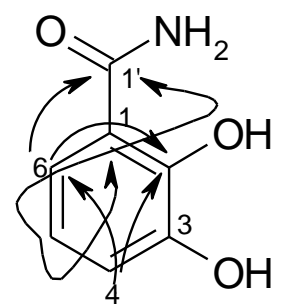

92

Figure 39: $\quad \operatorname{HMBC}(\longrightarrow)$ ) correlations of 2,3-dihydroxybenzamide (92)

Similarly, compound $\mathbf{9 2}$ is also a salicylic acid derivative as well as compound 91. It is known as sub-structure in various iron chelators of the catechol type.

\subsubsection{3-[(1Z)-1-Hexenyl-ONN-azoxyl]-1,2-butanediol}

Compound 93 was isolated as oil from fraction IV after purification on $\mathrm{Se}$ phadex LH-20 and RP-18 columns. It revealed UV absorption at $254 \mathrm{~nm}$ and stained to green with anisaldehyde/sulphuric acid. The molecular formula was deduced as $\mathrm{C}_{10} \mathrm{H}_{20} \mathrm{~N}_{2} \mathrm{O}_{3}$ by high-resolution ESI MS of the pseudomolecular $[\mathrm{M}+\mathrm{Na}]^{+}$ion at $\mathrm{m} / \mathrm{z}$ 239.13680 .

The ${ }^{1} \mathrm{H}$ NMR spectrum exhibited signals of olefinic protons at $\delta 6.78$ and 5.78 as doublet and multiplet, respectively. In the aliphatic region, the spectrum showed two multiplets of methine groups at $\delta 4.16$ and 3.76, which may be attached to oxygen or nitrogen. The remaining signals between $\delta 3.56-0.92$ were assigned as four methylenes, and two methyl groups. The sub-structure search in AntiBase based on the ${ }^{1} \mathrm{H}$ NMR and mass spectra resulted in 3-[(1Z)-1-hexenyl-ONN-azoxyl]-1,2butanediol (93). The structure was further confirmed by comparison with literature data. ${ }^{[131]}$ It has been reported that compound 93 was isolated from an "Actinomadura-like fungus" and showed antifungal activity, ${ }^{[131]}$ but it is for the first time here isolated from a terrestrial Streptomyces sp. It is the reduction product of LL-BH872a, and an isomer of 3-[(hex-1-enyl)-NNO-azoxy]-butane-1,2-diol. 


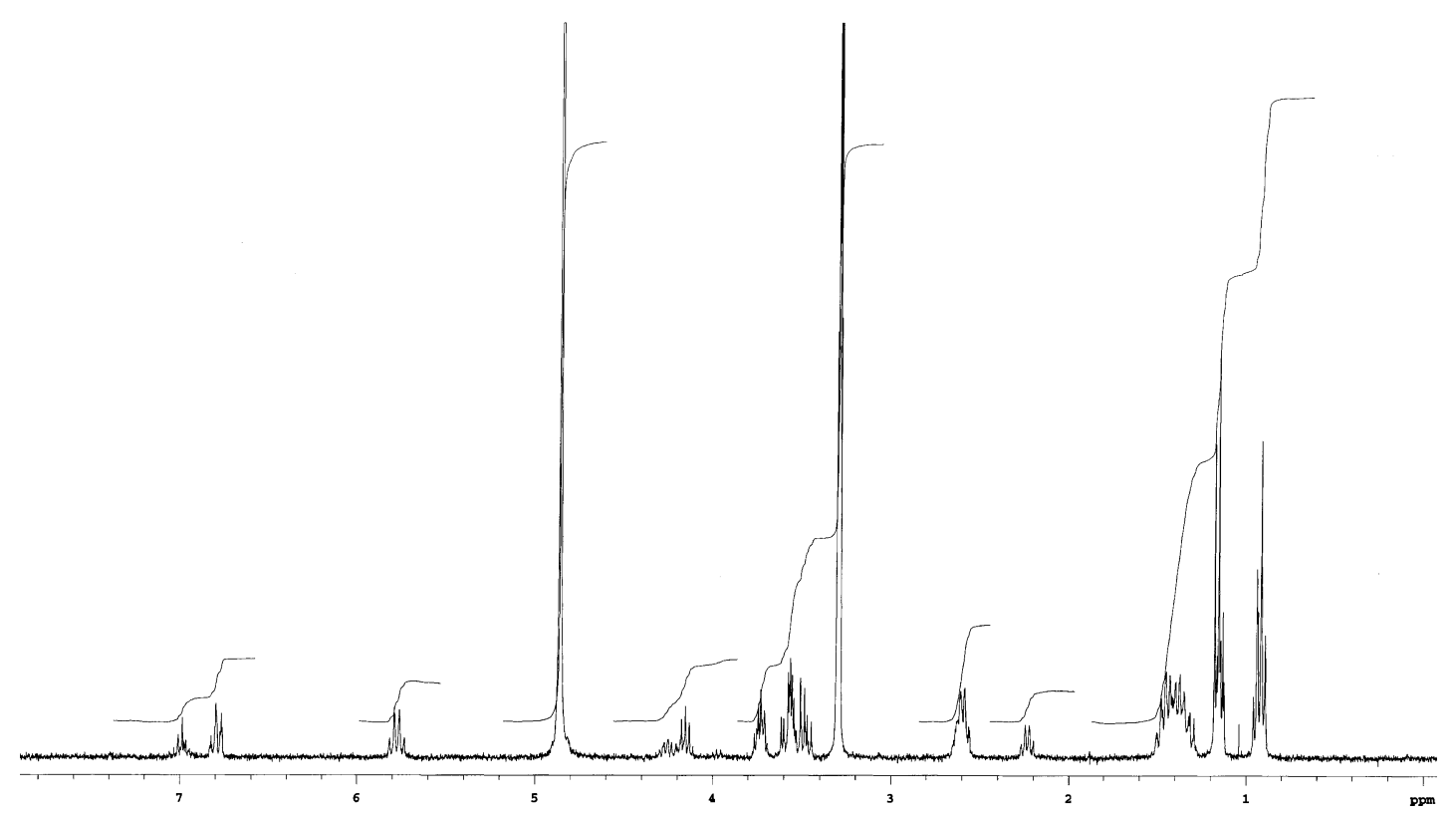

Figure 40: $\quad{ }^{1} \mathrm{H}$ NMR spectrum $\left(\mathrm{CD}_{3} \mathrm{OD}, 300 \mathrm{MHz}\right)$ of 3-[(1Z)-1-hexenyl-ONNazoxyl]-1,2-butanediol (93)<smiles>CCCCC=C[N+]([O-])=NC(C)C(O)CO</smiles>

93

\subsubsection{Bandamycin}

Working up fraction IV with Sephadex LH-20 and RP-18 columns afforded bandamycin (94) and additionally 3-[(1Z)-1-hexenyl-ONN-azoxyl]-1,2-butanediol (93) as yellow oils. It showed UV absorption at $254 \mathrm{~nm}$ and stained to green with anisaldehyde/sulphuric acid. 


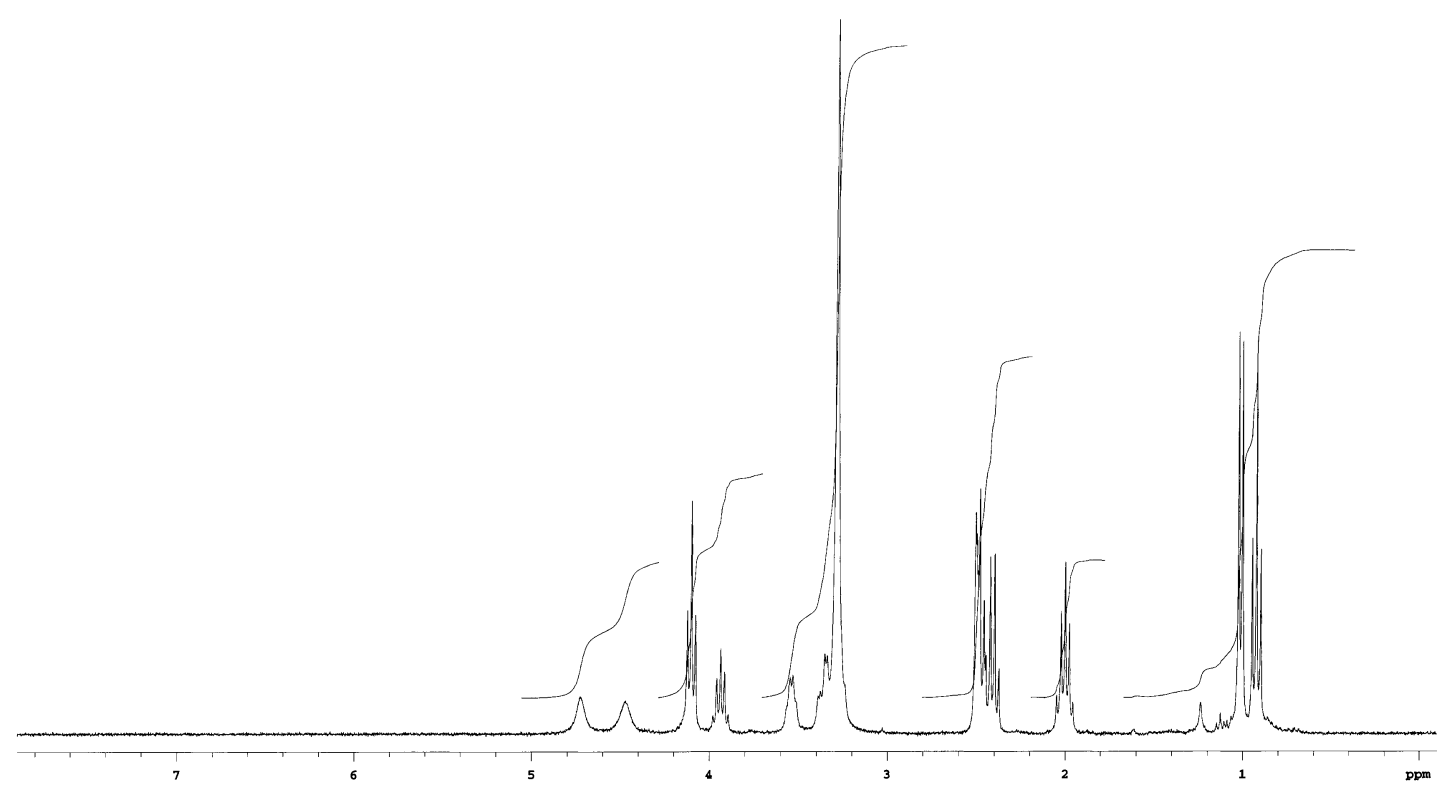

Figure 41: $\quad{ }^{1} \mathrm{H}$ NMR spectrum $\left(\mathrm{DMSO}-d_{6}, 300 \mathrm{MHz}\right)$ of bandamycin (94)

Based on the ${ }^{1} \mathrm{H}$ NMR spectra, both bandamycin (94) and 3-[(1Z)-1-hexenylONN-azoxyl]-1,2-butanediol (93) showed similar proton signals in the aliphatic region except of the olefinic protons. The ${ }^{1} \mathrm{H}$ NMR spectrum of bandamycin (94) exhibited two broad singlets at $\delta 4.73$ and 4.48 , which might be due to the presence of two hydroxyl groups. Two methylene and two methine groups bearing oxygen or nitrogen were observed at $\delta 4.10(2 \mathrm{H}), 3.94(1 \mathrm{H}), 3.55(1 \mathrm{H})$, and $3.34(2 \mathrm{H})$, respectively. The remaining signals at $\delta 2.49,2.40,2.00,1.01$, and 0.92 were assigned for three methylene and two methyl groups. The carbon spectrum indicated the presence of ten carbon atoms: The carbon atom types were analysed by the HSQC spectrum implying the presence of four aliphatic carbons connected to heteroatoms, three aliphatic methylenes, and two methyls. 


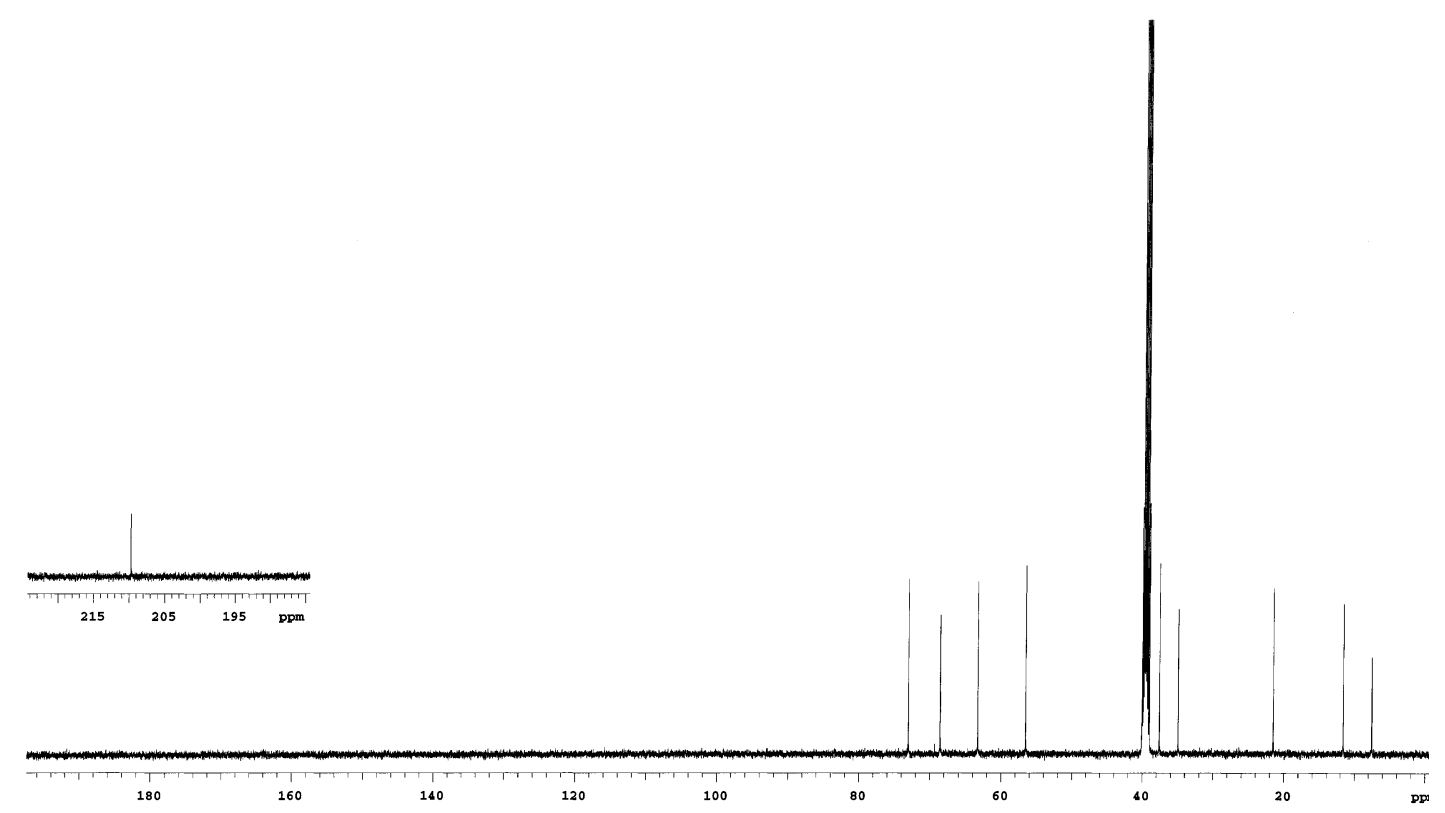

Figure 42: $\quad{ }^{13} \mathrm{C}$ NMR spectrum (DMSO- $d_{6}, 125 \mathrm{MHz}$ ) of bandamycin (94)

In the HMBC spectrum, the position of the ketone carbonyl $\left(\mathrm{C} 4 ', \delta_{C} 209.7\right)$ was assigned through correlations of ${ }^{2} J$ cross-signals between H-5' $(\delta 2.40)$ and H-3' $(\delta$ 2.49 ) to C-4' $\left(\delta_{C} 209.7\right)$, and ${ }^{3} J$ cross-signals between $\mathrm{H}_{2}-2^{\prime}(\delta 2.00)$ and $\mathrm{H}_{3}-6$ ' $(0.92)$ to $\mathrm{C}-4^{\prime}\left(\delta_{C} 209.7\right)$. The methyl doublet at $\delta 1.01\left(\delta_{C} 11.6\right)$ correlated to C-3 ( ${ }^{2} J$ coupling), and C-2 ( ${ }^{3} J$ coupling). In addition, ${ }^{2} J$ correlations of $\mathrm{H}-3(\delta 3.94)$ and $\mathrm{H}_{2}-1(\delta$ $3.34)$ to an oxygenated methine $\left(\mathrm{C}-2, \delta_{C} 73.0\right)$ were observed. Surprisingly, there was no COSY correlation between C-2,3, which indicated an orthogonal orientation due to steric hindrance or a hydrogen bridge. The HRESI mass analysis gave $\mathrm{C}_{10} \mathrm{H}_{20} \mathrm{~N}_{2} \mathrm{O}_{4}$ as the molecular formula, which required two double bond equivalents. One of the double bond equivalents has been assigned to the keto carbonyl group and the other one could be due to an azoxy group. 


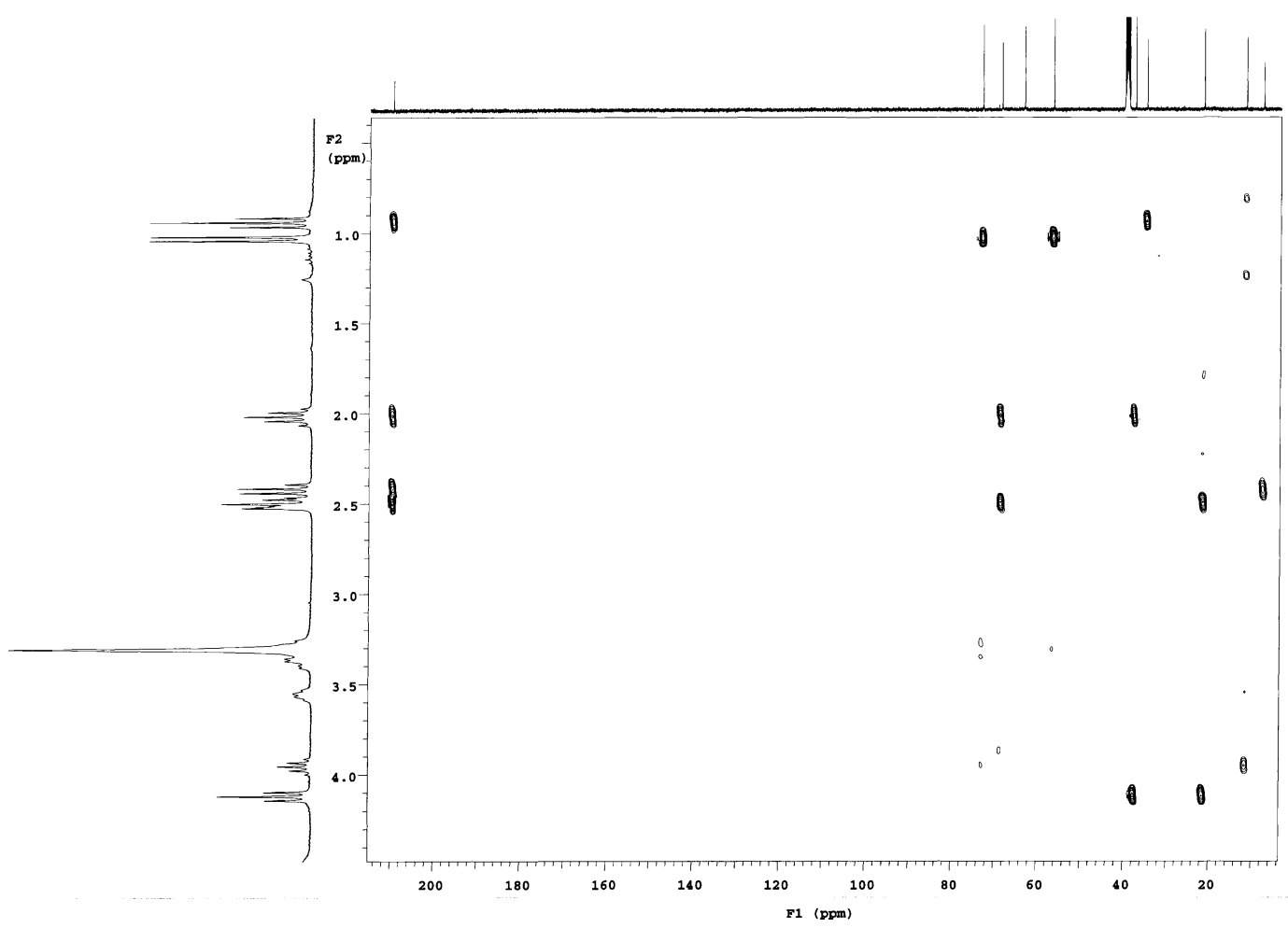

Figure 43: $\quad$ HMBC spectrum (DMSO- $d_{6}, 600 \mathrm{MHz}$ ) of bandamycin (94)

The presence of an azoxy group was further supported by an absorption band in the IR spectrum at $1500 \mathrm{~cm}^{-1}$, which is characteristic for the azoxy moiety. ${ }^{[132]} \mathrm{In}$ addition, the absorption bands of carbonyl and hydroxyl groups appeared at 1710 and $3420 \mathrm{~cm}^{-1}$, respectively.

Two isomeric azoxy derivatives are possible: compound 94, or an isomer with the oxygen atom at the other nitrogen. According to shift calculations, ${ }^{[133]}$ the $\mathrm{N}^{+}-\mathrm{O}^{-}$ unit causes a strong downfield shift both in the ${ }^{1} \mathrm{H}$ and ${ }^{13} \mathrm{C}$ spectra with respect to the other side. As this is the case for the compound investigated here, the structure 94 shown in Figure 45 was the most plausible one for bandamycin. The cyclic oxadiaziridine isomers ${ }^{[134,135]}$ of azoxybenzenes have been described, but are instable and can be excluded here. Bandamycin is a new antifungal agent from bacteria.

According to AntiBase, there were about 20 compounds isolated from microorganisms containing the azoxy unit. ${ }^{[88]}$ Helaly and co-workers reported the biosynthesis of an aliphatic azoxy compound, elaiomycin (40), which is derived from $n$ octylamine, L-serine, and acetate units. ${ }^{[136]}$ L-Serine and L-valine are the biosynthetic precursors of a related azoxy antibiotic, valanimycin $(\mathbf{3 9}){ }^{[53,136]}$ 


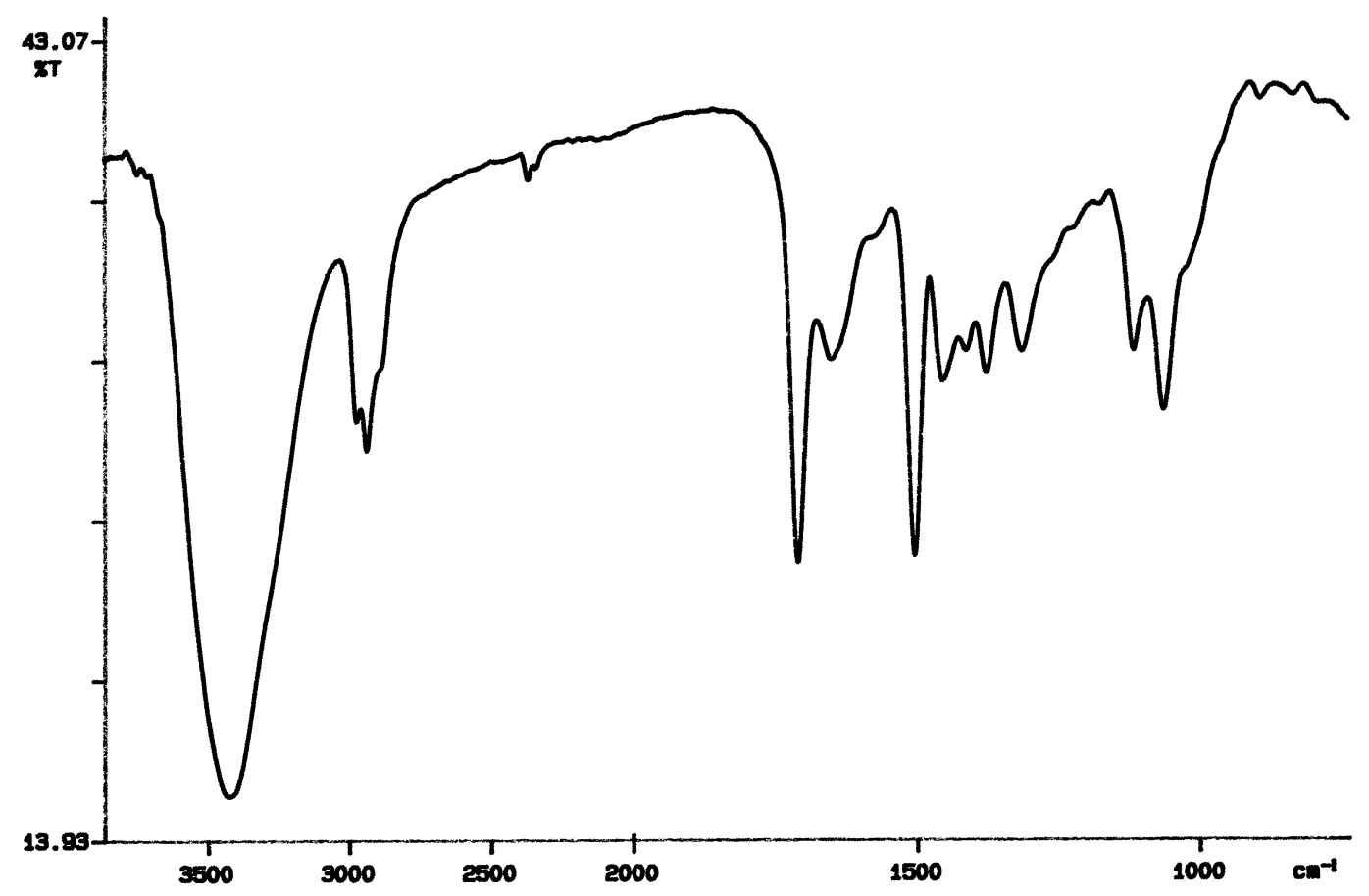

Figure 44: IR spectrum ( $\mathrm{KBr}$ disc) of bandamycin (94)

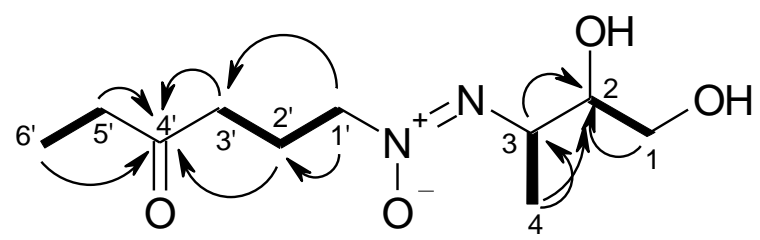

94

Figure 45: $\quad$ Selected ${ }^{1} \mathrm{H}-{ }^{1} \mathrm{H}$ COSY $(-)$ and $\operatorname{HMBC}(\longrightarrow)$ correlations of bandamycin (94)

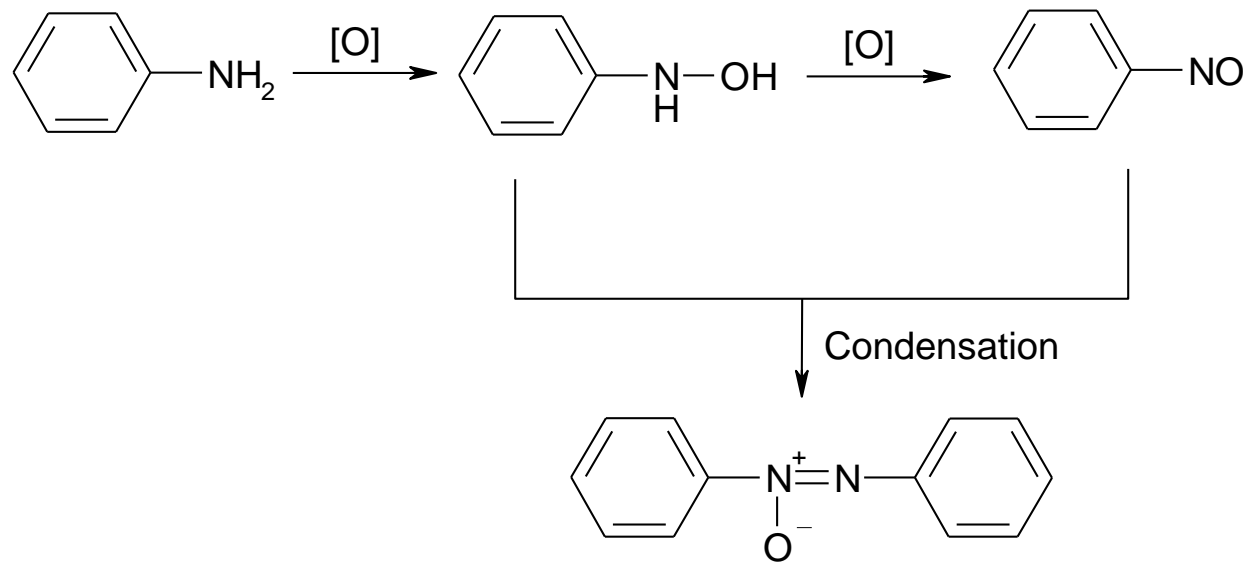

Figure 46: Formation of azoxybenzene by oxidation of aniline. ${ }^{[137]}$ 
The synthesis of azoxy compounds had been reported for the first time by Zinin in 1841. Zinin prepared azoxybenzene by the reduction of nitrobenzene under alkaline conditions. ${ }^{[138]}$ Chang and Liu had synthesized azoxybenzenes via catalytic oxidation of anilines with hydrogen peroxide. ${ }^{[137]}$

\subsection{7 $\mathbf{N}^{6}, \mathrm{~N}^{6}$-Dimethyl-adenosine}

$\mathrm{N}^{6}, \mathrm{~N}^{6}$-Dimethyl-adenosine (95) was obtained as pale yellow oil showing UV absorption at $254 \mathrm{~nm}$. It stained to greenish yellow with anisaldehyde/sulphuric acid on the thin layer chromatogram. The ${ }^{1} \mathrm{H}$ NMR spectrum revealed two singlets at $\delta$ 8.18 and 8.16, one signal of an anomeric proton $(\delta 5.94, \mathrm{~d})$, four proton signals of three oxygenated methines and one oxymethylene group between $\delta 4.74$ and 3.87, and one singlet at $\delta 3.34$ with the intensity of six protons. The signals at $\delta 3.34$ and 3.47 were later assigned as two methyl groups attached to a nitrogen atom. The ESI mass spectrum delivered a pseudo-molecular ion peak at $m / z 296[\mathrm{M}+\mathrm{H}]^{+}$corresponding to $\mathrm{C}_{12} \mathrm{H}_{18} \mathrm{~N}_{5} \mathrm{O}_{4}$ as the molecular formula from HRMS analysis. A search in AntiBase based on these spectroscopic data as well as by comparison with literature data resulted in $\mathrm{N}^{6}, \mathrm{~N}^{6}$-dimethyl-adenosine $(\mathbf{9 5})$ as the confirmed structure. ${ }^{[139]}$

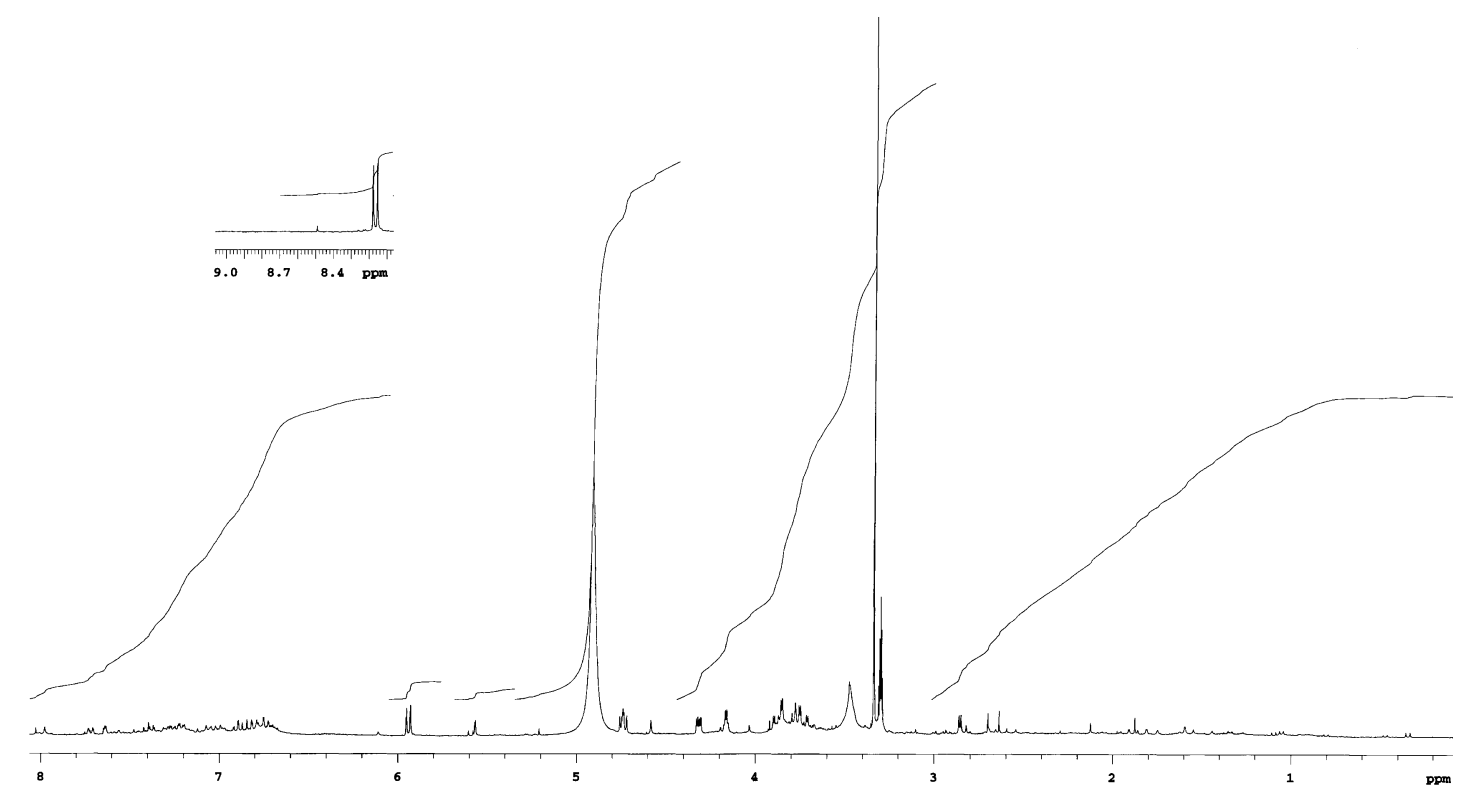

Figure 47: $\quad{ }^{1} \mathrm{H}$ NMR spectrum $\left(\mathrm{CD}_{3} \mathrm{OD}, 300 \mathrm{MHz}\right)$ of $\mathrm{N}^{6}, \mathrm{~N}^{6}$-dimethyl-adenosine (95) 


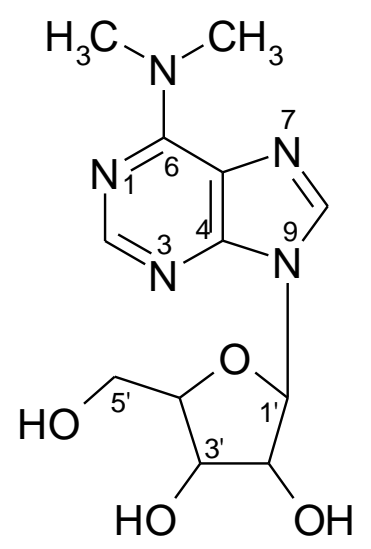

95

Compound 95 was isolated in our group as microbial metabolite from both terrestrial and marine-derived Streptomyces sp. According to AntiBase ${ }^{[88]}$, there are at least 62 adenosine derivatives which have been isolated from microorganisms.

\subsection{Marine Streptomyces sp. B5798}

In the biological pre-screening, the crude extract of the marine Streptomyces $\mathrm{sp}$. B5798 showed no activity against bacteria, fungi, and algae, but it was active against brine shrimps. The TLC of the crude extract revealed four UV absorbing bands at $254 \mathrm{~nm}$, which stained to violet, red, and deep brown with anisaldehyde/sulfuric acid.

The strain Streptomyces sp. B5798 formed white mycelial colonies after incubation on $\mathrm{M}_{2}{ }^{+}$agar medium for 3 days at $28^{\circ} \mathrm{C}$. This agar culture was then used to inoculate $25 \mathrm{~L}$ of $\mathrm{M}_{2}^{+}$medium with $50 \%$ artificial seawater. After 5 days, the culture broth was filtered over Celite to afford the water phase and the biomass. The water phase was passed through Amberlite XAD-16, which was then washed with water followed by elution with methanol. The biomass was extracted thoroughly with ethyl acetate and acetone. As the organic extracts of both water phase and biomass showed identical chromatograms, they were combined. This crude extract was fractionated on a silica gel column to obtain four fractions, which were further purified by various chromatographic techniques. 


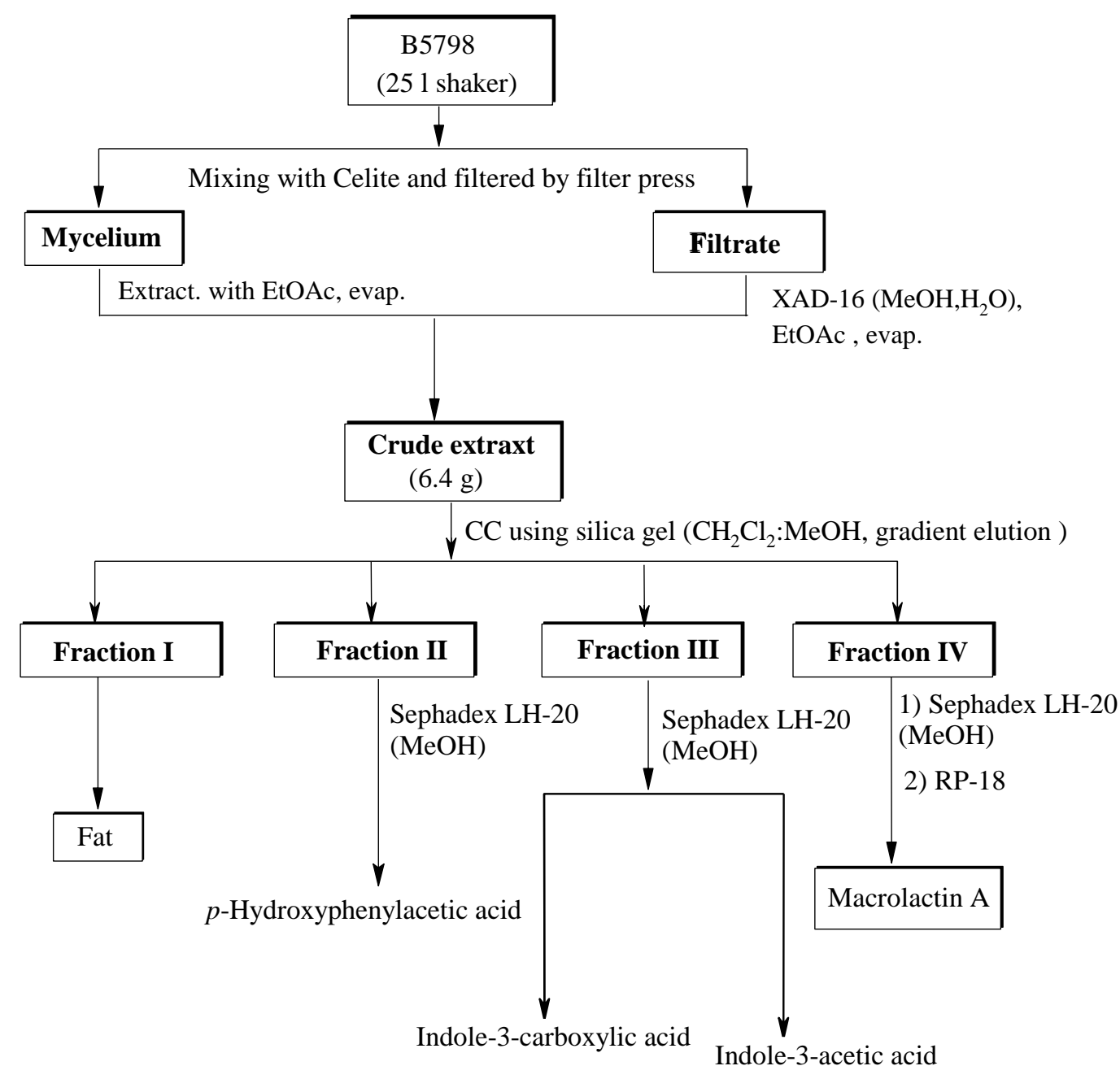

Figure 48: $\quad$ Work-up scheme for marine Streptomyces sp. B5798

\subsection{1 $p$-Hydroxyphenylacetic acid}

Working up fraction II by Sephadex column chromatography resulted in compound 96 as white solid. It showed a UV absorbing zone at $254 \mathrm{~nm}$ and stained to pink with anisaldehyde/sulphuric acid. The ${ }^{1} \mathrm{H}$ NMR spectrum exhibited two doublets and one singlet with intensity of each two protons in the aromatic and aliphatic regions, respectively. The two doublets at $\delta 7.08$ and 6.72 with a coupling constant $8.6 \mathrm{~Hz}$ suggested a 1,4-disubstituted benzene system. The resonance at $\delta 3.47(2 \mathrm{H})$ was assumed to be due to a methylene group situated between benzene ring and carbonyl group. The sub-structure search in AntiBase using ${ }^{1} \mathrm{H}$ NMR data gave $p$ hydroxyphenylacetic acid (96). The structure was further confirmed by comparison with the authentic spectrum and the literature. ${ }^{[140]}$ 


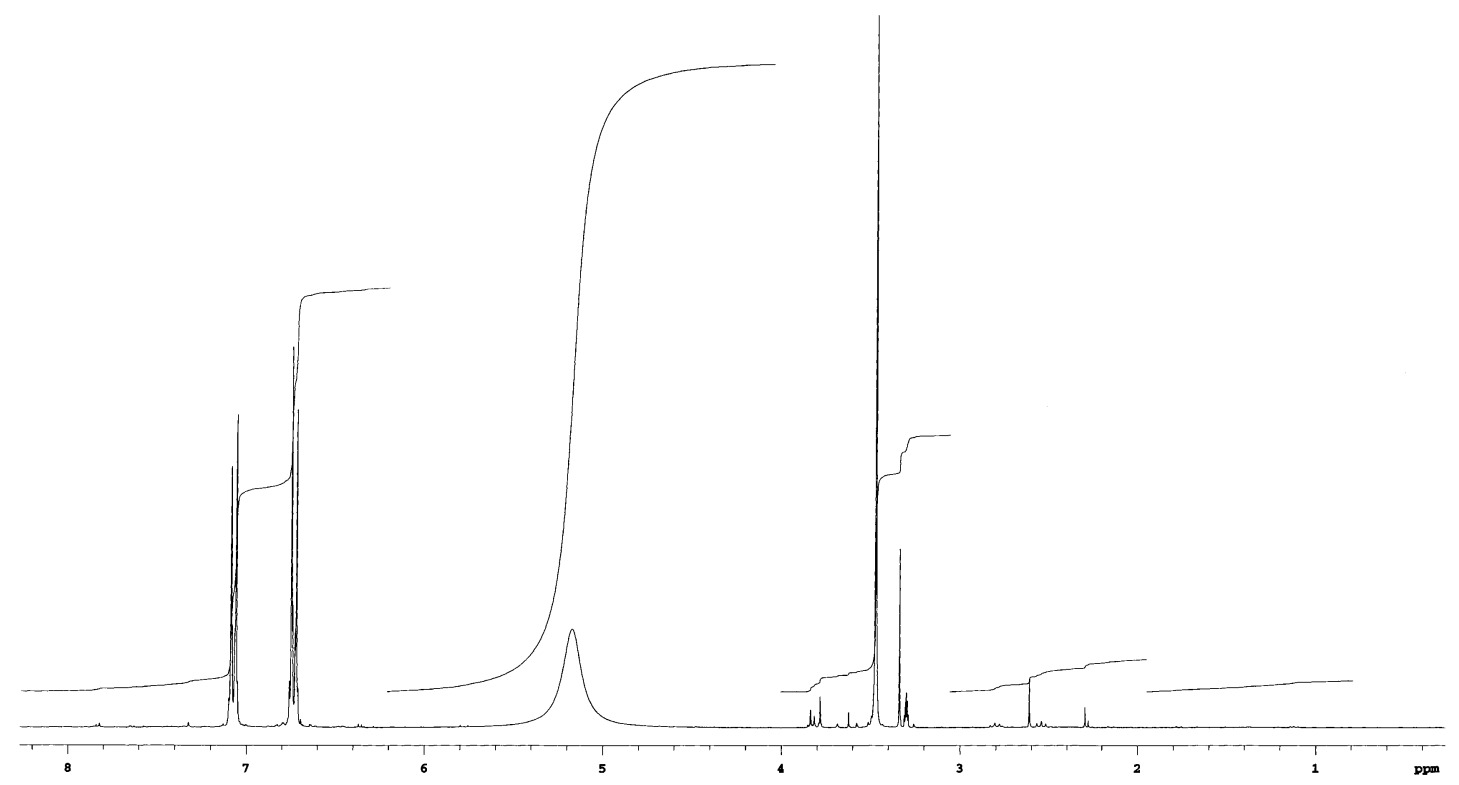

Figure 49: $\quad{ }^{1} \mathrm{H}$ NMR spectrum $\left(\mathrm{CD}_{3} \mathrm{OD}, 300 \mathrm{MHz}\right)$ of $p$-hydroxyphenylacetic acid (96)<smiles>O=C(O)Cc1ccc(O)cc1</smiles>

96

p-Hydroxyphenylacetic acid (96) is an intermediate in the microbial degradation of phenylalanine and tyrosine. A degradation product of $p$-hydroxyphenylacetic acid (96) is 3,4-dihydroxybenzaldehyde, a precursor of the anti-Parkinson drug Levodopa. ${ }^{[141]}$

\subsubsection{Macrolactin A}

Working up of sub-fraction IV on Sephadex LH-20 and RP18 columns delivered the oily compound 97, which showed a UV absorbing band at $254 \mathrm{~nm}$ and stained to black with anisaldehyde/sulphuric acid. The ${ }^{1} \mathrm{H}$ NMR spectrum revealed twelve olefinic proton signals between $\delta 7.23-5.53$. In addition, four oxymethine signals were observed at $\delta 5.02,4.48,4.33$, and 3.98. The remaining signals between $\delta 2.47-1.26$ were assigned to six methylenes and one methyl group, respectively. 


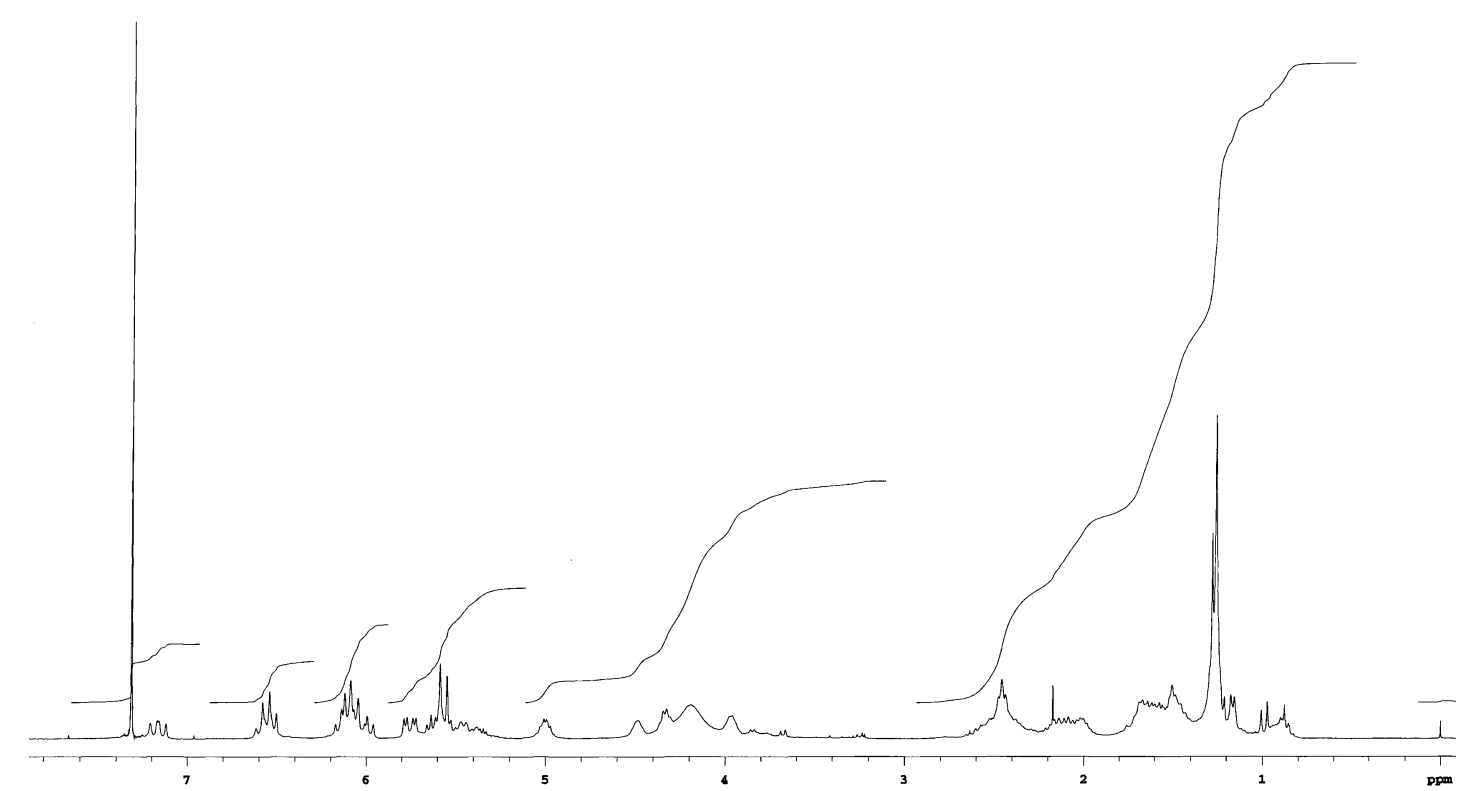

Figure 50: $\quad{ }^{1} \mathrm{H}$ NMR spectrum $\left(\mathrm{CDCl}_{3}, 300 \mathrm{MHz}\right)$ of macrolactin A (97)

The ${ }^{13} \mathrm{C}$ NMR spectrum showed 24 carbon signals consisting of one lactone carbonyl at $\delta_{\mathrm{C}} 167.6,12$ olefinic carbons between $\delta_{\mathrm{C}} 144.4-117.6$, four oxymethine carbon signals at $\delta_{\mathrm{C}} 71.8,71.7,69.5$ and 68.9 . The remaining signals were attributed to seven $s p^{3}$ carbon atoms, which were six methylenes at $\delta_{\mathrm{C}} 42.4,42.2,32.5,30.2,25.1$, 20.1 , and one methyl at $\delta_{\mathrm{C}} 17.40$, respectively. The high-resolution ESI mass analysis gave $\mathrm{C}_{24} \mathrm{H}_{34} \mathrm{O}_{5}$ as molecular formula. The sub-structure search in AntiBase using these spectroscopic data and comparison with authentic spectra in literature led to macrolactin A (97). ${ }^{[83]}$ 


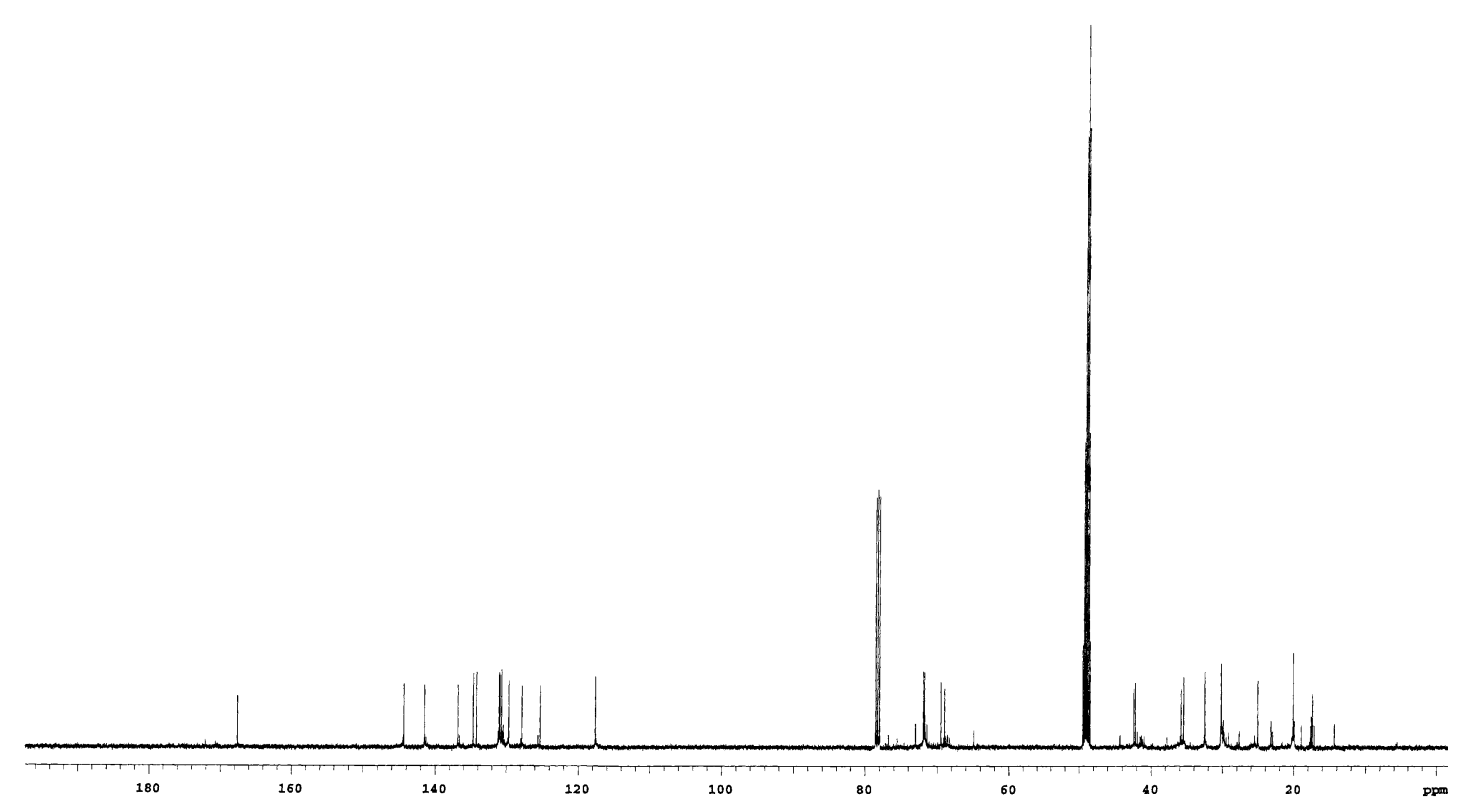

Figure 51: $\quad{ }^{13} \mathrm{C}$ NMR spectrum $\left(\mathrm{CD}_{3} \mathrm{OD} / \mathrm{CDCl}_{3}, 125 \mathrm{MHz}\right)$ of macrolactin $\mathrm{A}(\mathbf{9 7})$<smiles></smiles>

97

Macrolactin A (97) has been reported to exhibit antibacterial properties, anticancer activity against B16-F-10 murine melanoma cells, antiviral activity against Herpes simplex and to inhibit the squalene synthase. ${ }^{[142,143]}$ Macrolactin A (97) had also been reported as one of the macrolide derivatives biosynthetically following the polyketide route. ${ }^{[144,145]}$ Two trivial metabolites, indole-3-carboxylic acid and indole3-acetic acid, had been also isolated from this strain.

\subsection{Terrestrial Streptomyces sp. GT-2005/049}

In pre-screening, the crude extract of Streptomyces sp. GT-2005/049 afforded a strong activity against bacteria, fungi and brine shrimps, and showed UV absorbing bands at $254 \mathrm{~nm}$. A $30 \mathrm{~L}$ culture in $\mathrm{M}_{2}$ medium and extraction with organic solvents 
afforded a yellow oily crude extract. Fractionation and purification by different chromatographic methods delivered seven constituents, namely lumichrome (98), 1methyluracil, thymine, indole-3-carboxylic acid, 1-acetyl- $\beta$-carboline (84), uracil and hypoxanthine (Figure 60).

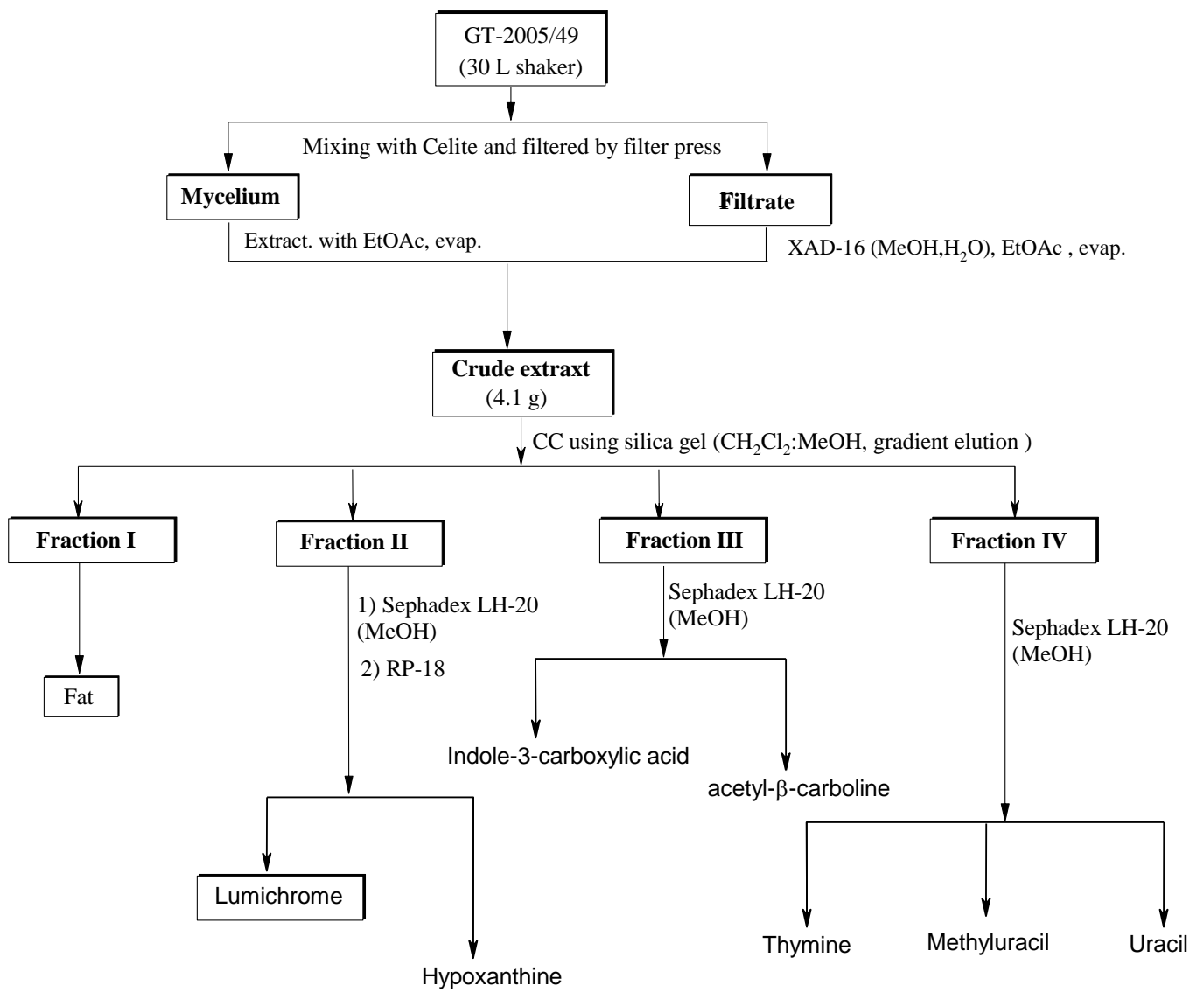

Figure 52: Work-up scheme for terrestrial Streptomyces sp. GT-2005/049

\subsubsection{Lumichrome}

Lumichrome (98) was obtained as pale yellow and UV-fluorescence absorbing solid. It was isolated from fraction II by subjecting to Sephadex L-20 and RP-18 columns. The ${ }^{1} \mathrm{H}$ NMR spectrum exhibited two singlets in the aromatic region at $\delta 8.03$ $(1 \mathrm{H})$ and $7.85(1 \mathrm{H})$, and two $3 \mathrm{H}$ singlets at $\delta 2.33$ and 2.27 . 


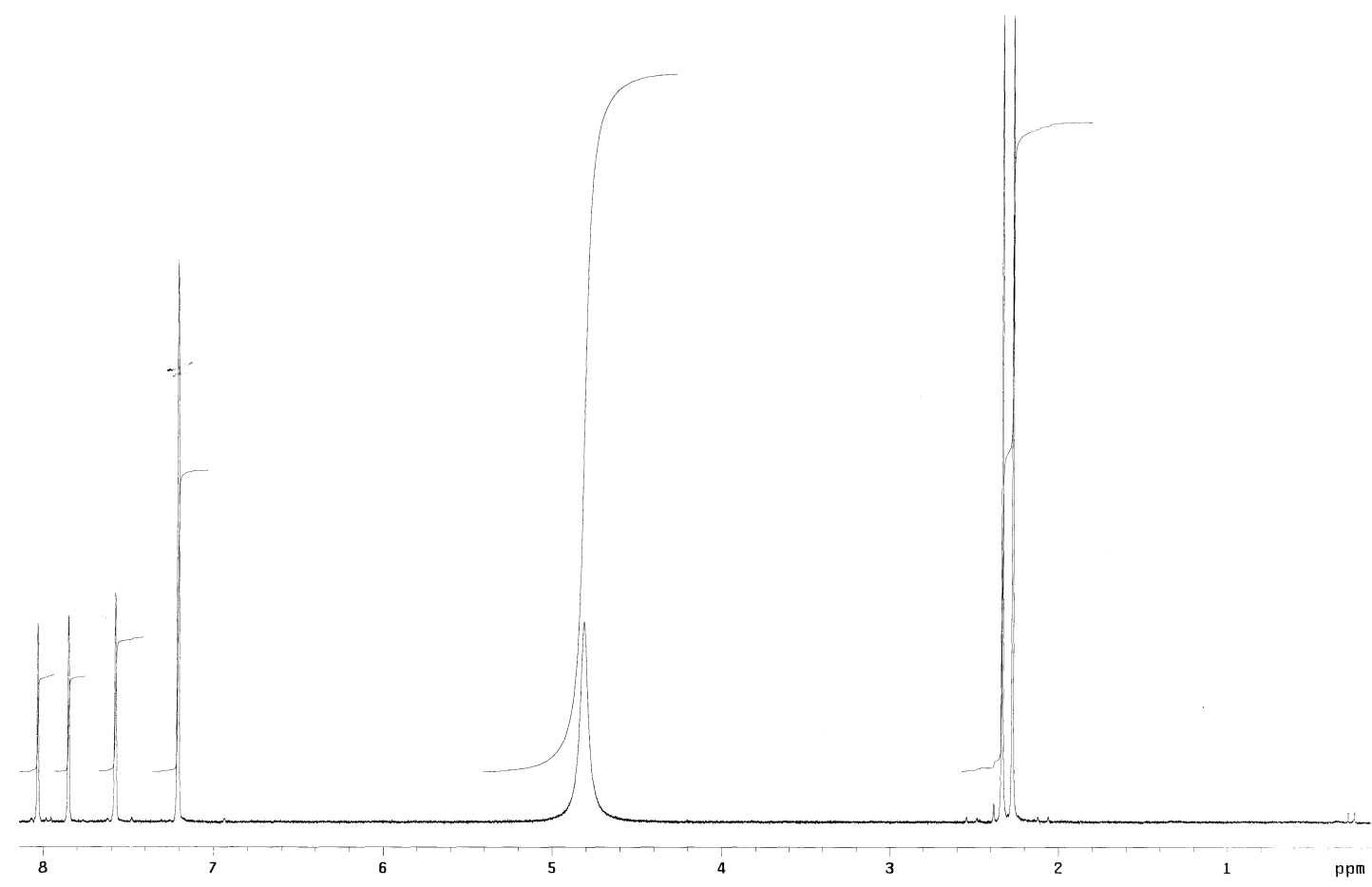

Figure 53: $\quad{ }^{1} \mathrm{H}$ NMR spectrum (pyridine- $d_{5}, 300 \mathrm{MHz}$ ) of lumichrome (98)

The ${ }^{13} \mathrm{C}$ NMR spectrum showed twelve carbon signals, eight of them were quaternary carbons, two methines $s p^{2}$, and two methyl groups. Two quaternary carbon signals at $\delta_{C} 161.9$ and 151.7 were assigned to carboxamides. The HRESI mass analysis showed a molecular ion peak at $m / z 265.07014\left([\mathrm{M}+\mathrm{Na}]^{+}\right)$corresponding to the molecular formula $\mathrm{C}_{12} \mathrm{H}_{10} \mathrm{~N}_{4} \mathrm{O}_{2} \mathrm{Na}$. A sub-structure search in AntiBase by applying these spectroscopic data gave lumichrome (98). The structure was further confirmed via comparison with authentic spectra and literature data. ${ }^{[89]}$ 


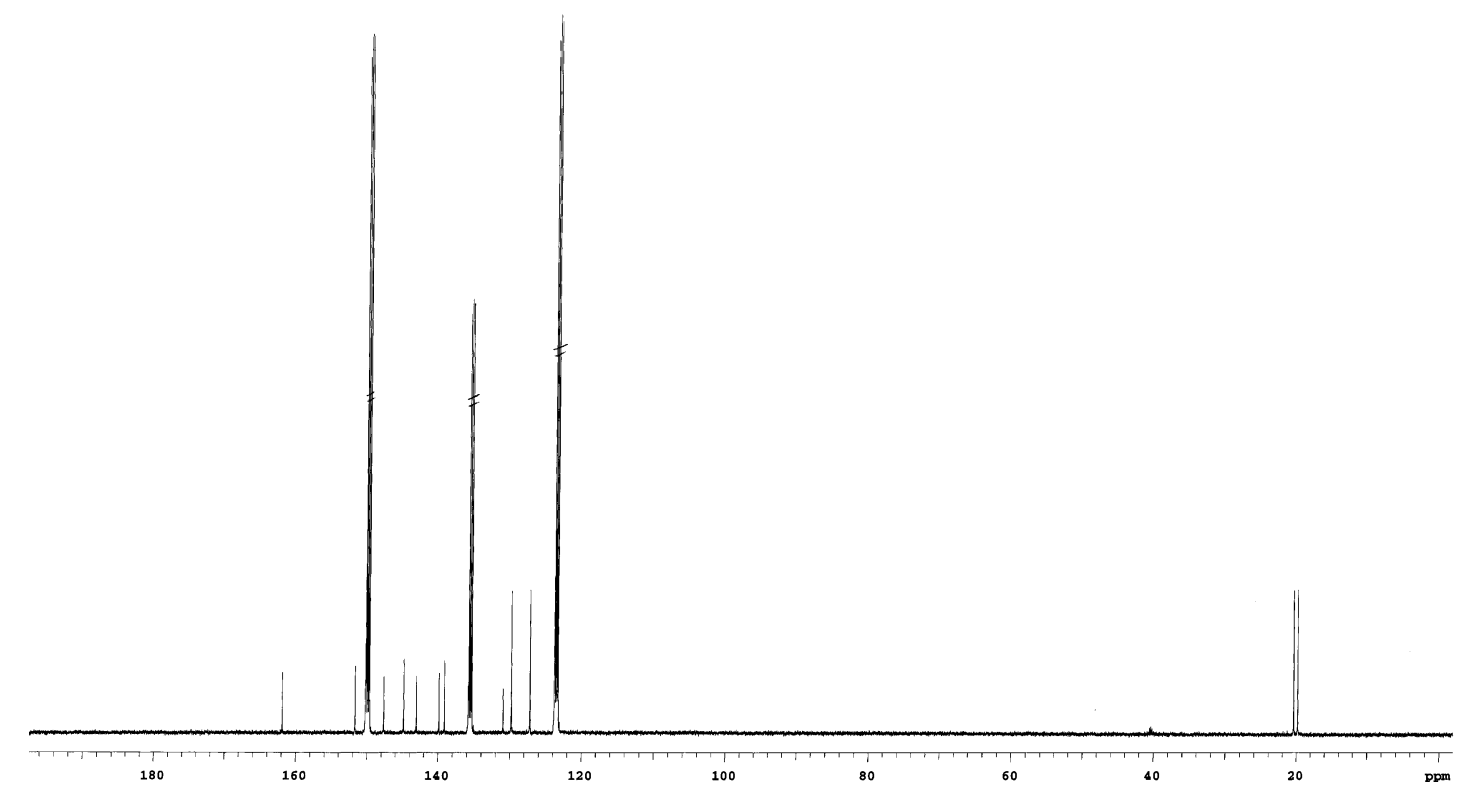

Figure 54: $\quad{ }^{13} \mathrm{C}$ NMR spectrum (pyridine- $d_{5}, 125 \mathrm{MHz}$ ) of lumichrome (98)<smiles>Cc1cc2nc3[nH]c(=O)[nH]c(=O)c3nc2cc1C</smiles>

98<smiles>Cc1cc2nc3c(=O)[nH]c(=O)nc-3n(C)c2cc1C</smiles>

100<smiles>Clc1cc2c(cc1Cl)Oc1cc(Cl)c(Cl)cc1O2</smiles>

99<smiles>Cc1cc2nc3c(=O)[nH]c(=O)nc-3n([C@@H]3OC(CO)[C@@H](O)[C@H]3O)c2cc1C</smiles>

101

Lumichrome (98) is considered as a metabolite of riboflavine (23) and competed with tetrachlorodibenzodioxin (99) for binding to the cytosolic TCCD receptor in 
rodent tissues. ${ }^{[146]}$ Lumichrome (98) and two lumichrome analogs, namely 1methyllumichrome (100) and 1-( $\alpha$-ribofuranosyl)-lumichrome (101), were isolated from Micromonospora sp. Tü 6368 (actinomycetes). ${ }^{[147]}$

\subsection{Terrestrial Streptomyces sp. GW 4723}

The terrestrial Streptomyces sp. GW 4723 grew on agar with a white aerial mycelium and dark green pigmented agar. The scale-up of this strain had been carried out in $\mathrm{M}_{2}$ medium by Fotso Fondja Yao during her Ph.D study: The crude extract of this strain showed antibacterial, antialgal and antifungal activities against Bacillus subtilis, Staphylococcus aureus, Escherichia coli, Streptomyces viridochromogenes (Tü 57), Chlorella vulgaris, Chlorella sorokiniana, Scenedesmus subspicatus, and Mucor miehei. ${ }^{[148]}$ Three new angucyclinones, celastramycin C, celastramycin $\mathrm{D}$, and celastramycin $\mathrm{D}$ had been previously isolated from this strain in $\mathrm{M}_{2}$ medium. In the current investigation, the strain was re-fermented as $60-\mathrm{L}$ shaker culture in $\mathrm{LB}^{+}$medium for 10 days at $28^{\circ} \mathrm{C}$. Chromatography of crude extract on different stationary phases delivered celastramycin B (103) and four trivial compounds such as acetyl- $\beta$-carboline, uracil, tyrosol, and adenosine. Aim of this refermentation was the structure re-assignment of celastramycin B (103).

\subsubsection{Celastramycin B}

In a previous study, Fondja had isolated three new angucyclinones along with a small amount of the known metabolite celastramycin B (103). ${ }^{[148]}$ The ${ }^{13} \mathrm{C}$ NMR CO signals at $\delta_{C} 181.6$ and 192.3 were an indication of a double chelated and a nonchelated quinone carbonyl, i.e. a 1,11-dihydroxylated tetracenequinone. However, as the other celastramycins described by Fondja were clearly angucyclinones, a respective skeleton was also plausible in the case of celastramycin B, i.e. a 1,4-benzo[a]anthracenequinone ring system should be present. ${ }^{[149]}$ Due to limited amount of the sample, the HMBC correlations of hydroxyl groups were not found by Fondja. Therefore, the intention of this re-isolation was to confirm the old structure $\mathbf{1 0 2}$ or to suggest a new one on the basis of improved measurements.

In this study, we have refermented the Streptomyces sp. GW 4723 in $\mathrm{LB}^{+}$medium and pbtainewd $1.4 \mathrm{mg}$ of celastramycin $\mathrm{B}$, while the other three new quinones were not found. The ${ }^{1} \mathrm{H}$ NMR spectrum exhibited two singlets of two chelated $\mathrm{OH}$ 
protons at $\delta 12.23(8-\mathrm{OH})$ and $12.18(6-\mathrm{OH})$. In addition, the signals of aromatic, oxymethine, methine, methylene and methyl protons were also observed in the ${ }^{1} \mathrm{H}$ NMR spectrum. The ${ }^{13} \mathrm{C}$ NMR indicated 19 carbon signals, and three signals at $\delta_{C}$ 197.2, 192.3 and 181.5 suggested the presence of carbonyl groups. The (+)-ESI mass spectrum showed peak at $\mathrm{m} / \mathrm{z} 767\left([2 \mathrm{M}+\mathrm{Na}]^{+}\right)$and an isotope peak at $\mathrm{m} / \mathrm{z} 769$, indicating the presence of a chlorine atom. The sub-structure search in AntiBase based on spectroscopic data and comparison with a reference sample confirmed celastramycin B, a compound, which was first reported from Streptomyces MaB-QuH-8. ${ }^{[150]}$

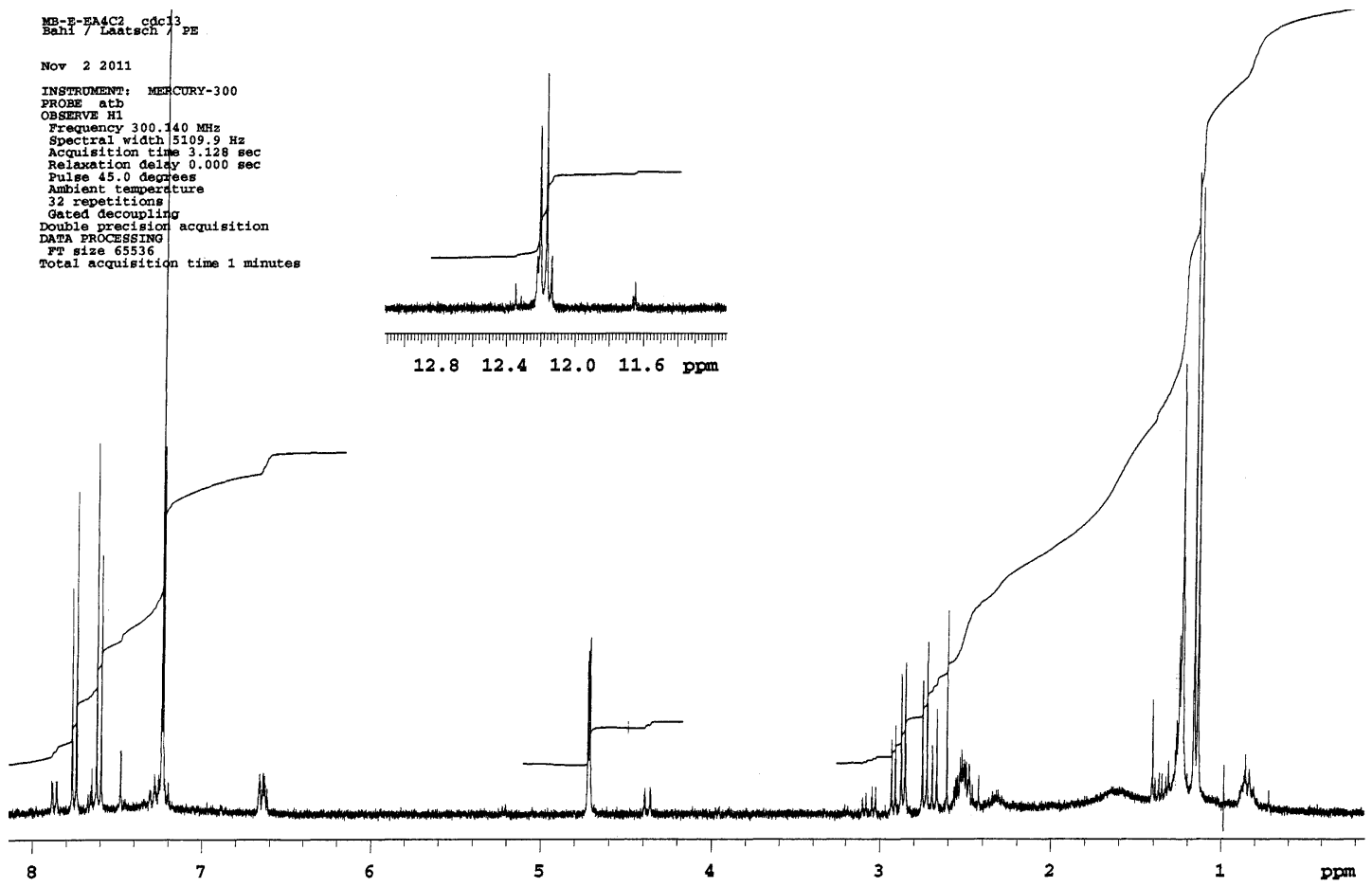

Figure 55: $\quad{ }^{1} \mathrm{H}$ NMR spectrum $\left(\mathrm{CDCl}_{3}, 300 \mathrm{MHz}\right)$ of celastramycin $\mathrm{B}(\mathbf{1 0 3})$<smiles>CC1CC(=O)c2c(cc3c(c2O)C(=O)c2ccc(Cl)c(O)c2C3=O)C1O</smiles><smiles>CC1CC(=O)c2c(cc(O)c3c2C(=O)c2ccc(Cl)c(O)c2C3=O)C1O</smiles> 


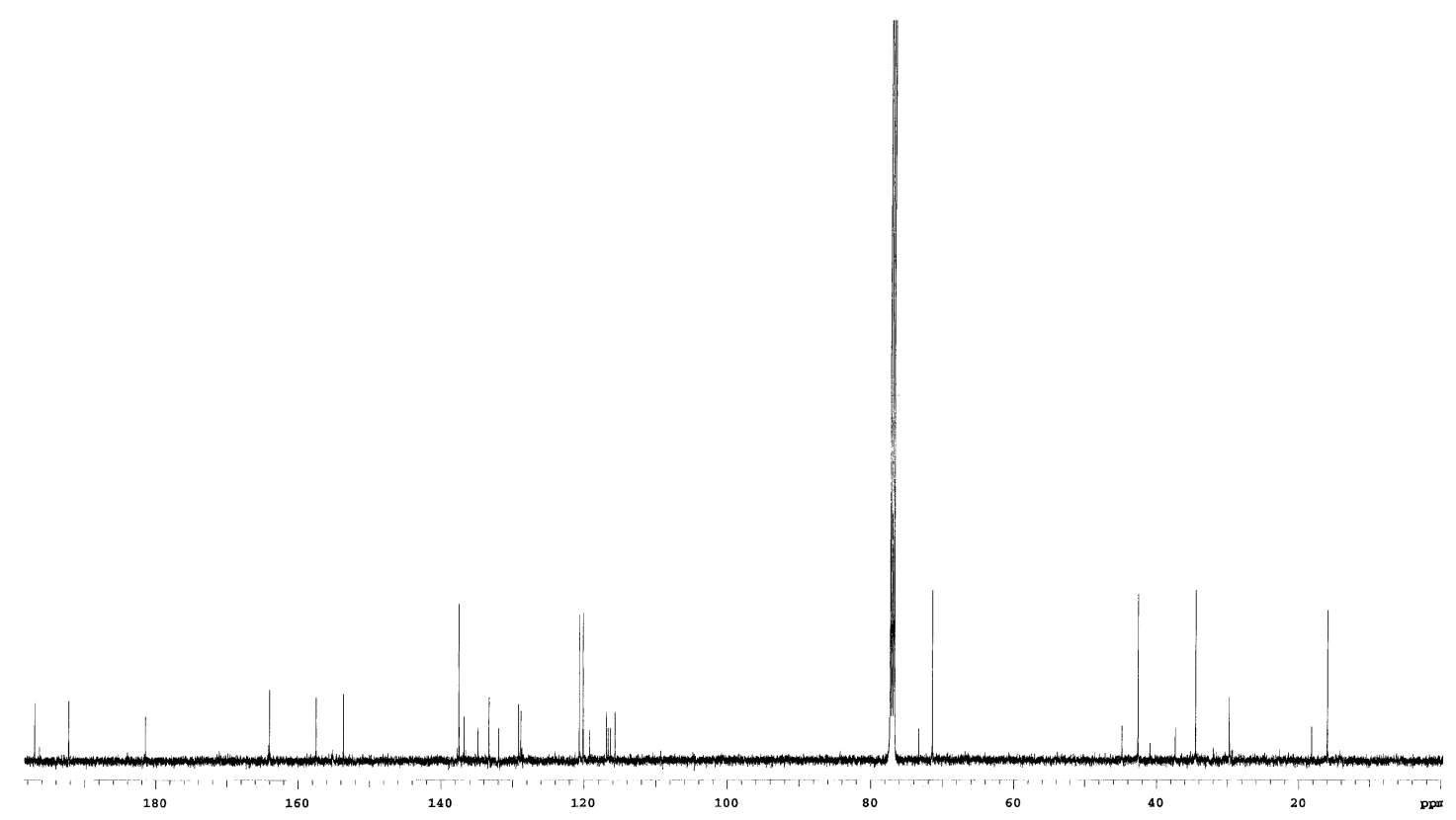

Figure 56: ${ }^{13} \mathrm{C}$ NMR spectrum $\left(\mathrm{CDCl}_{3}, 125 \mathrm{MHz}\right)$ of celastramycin $\mathrm{B}(\mathbf{1 0 3})$

According to the literature, the signals of non-chelated and one chelated quinone carbonyls appear in the region of $\delta_{\mathrm{C}} 180 \pm 3$, and 186; doubly chelated carbonyls show signals at $\delta_{\mathrm{C}} 190-192$, and $195-200$, respectively. ${ }^{[149]}$ Hence, the carbon signal at $\delta_{\mathrm{C}} 192.3$ (C-7) was assigned to a double chelated quinone carbonyl, while the carbon signal at $\delta_{\mathrm{C}} 181.5(\mathrm{C}-12)$ should be a non-chelated $\mathrm{C}=\mathrm{O}$.

Furthermore, the proton signal at $\delta 7.25$ (H-11 in 102) appeared at an unusually high field for a peri-proton. A cross-coupling correlation of H-11 $(\delta 7.25)$ with the phenolic carbon at $\delta_{\mathrm{C}} 164.0$, which corresponded to a ${ }^{4} J$ coupling would be very unlikely for structure 102. On the other hand, the singlet signal at $\delta 7.25$ in structure 103 showed the expected cross-signal in the HMBC spectrum with the carbonyl C-7 but not with the carbonyl C-12. In addition, the hydroxyl singlet at $\delta 12.18$ exhibited strong coupling with carbon at $\delta_{\mathrm{C}} 120.7$, which was very unusual for a ${ }^{5} J$ correlation between 6-OH and $\mathrm{C}-11$ in structure 102. To sum up, celastramycin B is not an anthracyclinone 102, but an angucyclinone $\mathbf{1 0 3}$.

This was confirmed in the HMBC spectrum by weak correlations of H-5 to the carbonyl carbons at $\delta_{\mathrm{C}} 192.3(\mathrm{C}-7)$ and $197.2(\mathrm{C}-1)$. The proton signal of 8-OH exhibited strong correlations to C-9 $\left(\delta_{\mathrm{C}} 129.1\right)$ and C-7a $\left(\delta_{\mathrm{C}} 115.7\right)$. Similarly, the proton signal of 6-OH exhibited strong correlations to C-5 ( $\left.\delta_{\mathrm{C}} 120.7\right)$, C-6 $\left(\delta_{\mathrm{C}} 164.0\right), \mathrm{C}-$ 
$6 \mathrm{a}\left(\delta_{\mathrm{C}} 116.8\right)$ and weak correlation to $\mathrm{C}-4 \mathrm{a}\left(\delta_{\mathrm{C}} 153.7\right)$, respectively. Based on these HMBC correlations and literature, structure $\mathbf{1 0 2}$ can be revised to an angular frame and the structure $\mathbf{1 0 3}$ is confirmed for celastramycin B.

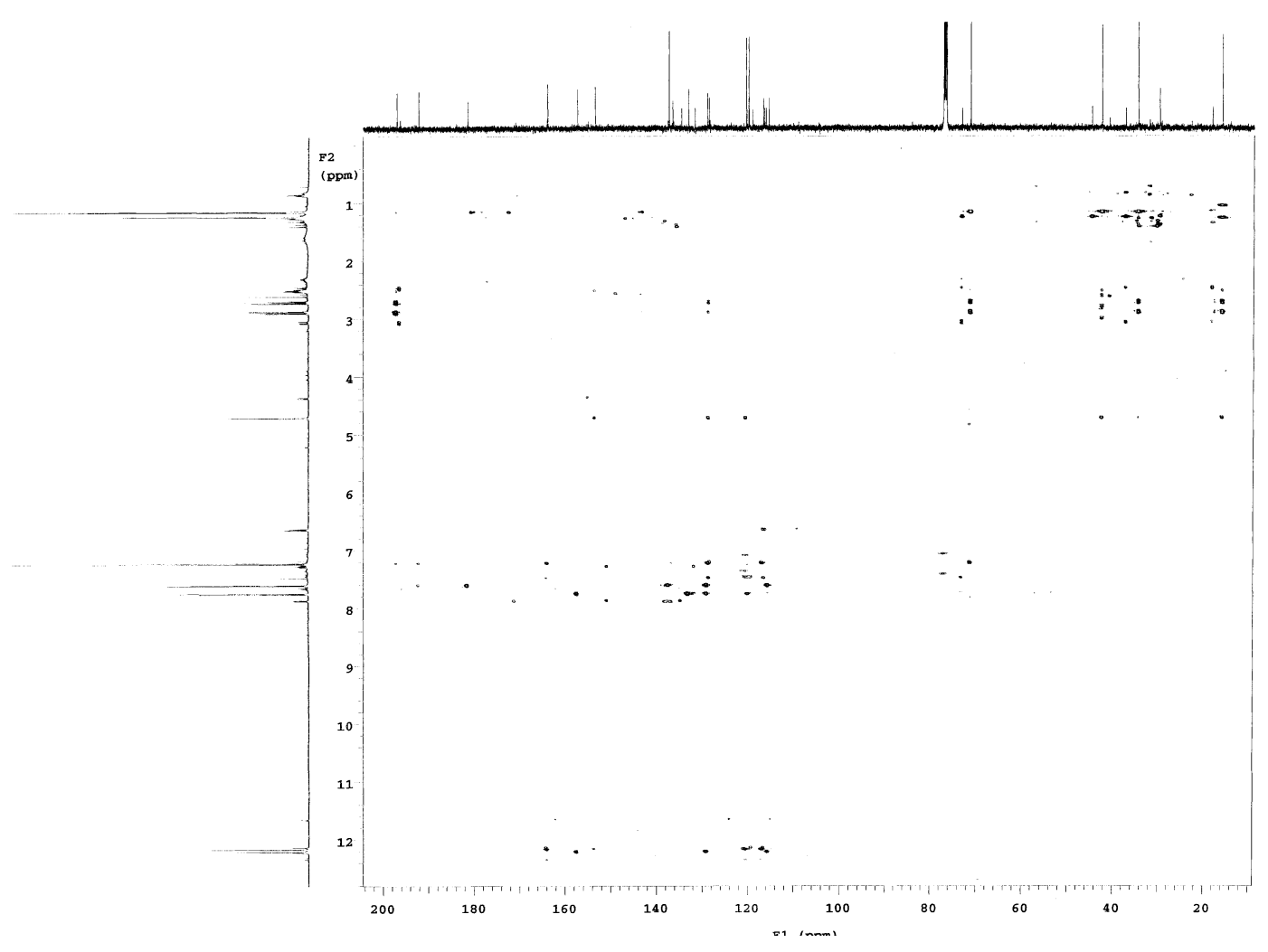

Figure 57: $\quad \mathrm{HMBC}$ spectrum of celastramycin B (103).

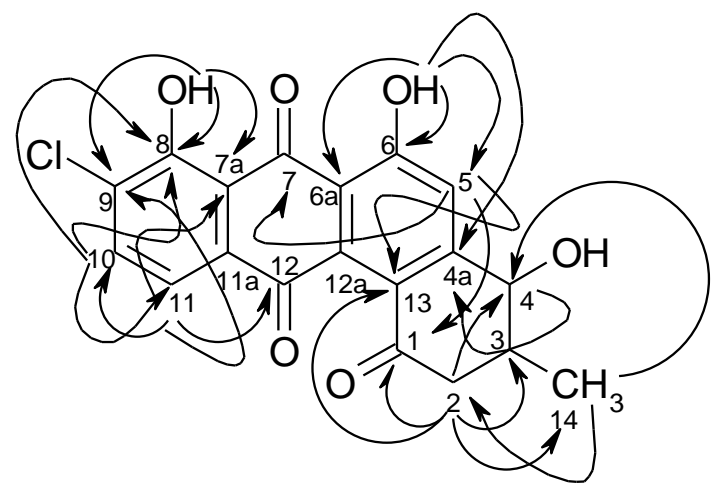

Figure 58: $\quad$ Selected HMBC $(\longrightarrow)$ correlations of celastramycin B (103) 
Table 1: $\quad{ }^{1} \mathrm{H}$ NMR $\left(\mathrm{CDCl}_{3}, 300 \mathrm{MHz}\right)$ and ${ }^{13} \mathrm{C} \mathrm{NMR}\left(\mathrm{CDCl}_{3}, 125 \mathrm{MHz}\right)$ data of celastramycin B (103)

\begin{tabular}{|c|c|c|c|}
\hline Position & $\delta_{\mathrm{C}}$ & $\delta_{\mathrm{H}}($ mult.; $J$ in $\mathrm{Hz})$ & HMBC \\
\hline 8 & 157.5 & - & - \\
\hline $7 \mathrm{a}$ & 115.7 & - & - \\
\hline 9 & 129.1 & - & - \\
\hline 10 & 137.5 & $7.77(\mathrm{~d}, 8.1 \mathrm{~Hz})$ & $8,9,11,11 \mathrm{a}$ \\
\hline 11 & 120.1 & $7.63(\mathrm{~d}, 8.1 \mathrm{~Hz})$ & $7 \mathrm{a}, 9,10,12,7^{*}$ \\
\hline $11 \mathrm{a}$ & 133.3 & - & - \\
\hline 12 & 181.5 & - & - \\
\hline $12 \mathrm{a}$ & 136.8 & - & - \\
\hline 13 & 128.8 & - & - \\
\hline $4 a$ & 153.7 & - & - \\
\hline 5 & 120.7 & $7.25(\mathrm{~d}, 0.5 \mathrm{~Hz})$ & $13,6,6 \mathrm{a}, 7^{*}, 1^{*}$ \\
\hline 6 & 164.0 & - & - \\
\hline $6 a$ & 116.8 & - & - \\
\hline 7 & 192.3 & - & - \\
\hline 1 & 197.2 & - & - \\
\hline \multirow[t]{2}{*}{2} & 42.6 & $2.91(\mathrm{dd}, 6.9,17.1 \mathrm{~Hz})$ & $13,1,3,4,14$ \\
\hline & & $2.73(\mathrm{dd}, 7.8,17.1 \mathrm{~Hz})$ & \\
\hline 3 & 34.5 & $2.54(\mathrm{~m})$ & $2^{*}, 4^{*}, 14^{*}$ \\
\hline 4 & 71.5 & $4.73(\mathrm{~d}, 2.8 \mathrm{~Hz})$ & $13,4 \mathrm{a}, 5,2,14$ \\
\hline 14 & 15.9 & $1.17(\mathrm{~d}, 6.9 \mathrm{~Hz})$ & $2,3,4$ \\
\hline $8-\mathrm{OH}$ & & 12.23 & $8,7 a, 9$ \\
\hline $6-\mathrm{OH}$ & & 12.18 & $4 a^{*}, 5,6,6 a$ \\
\hline
\end{tabular}

* weak correlation 


\section{Metabolites from selected endophytic fungi}

\subsection{Endophytic fungus LAF20}

The endophytic fungus LAF20 was isolated from the medicinal plant Laggera alata (Asteraceae), which was collected from the edges of the Mau forest in the Rift Valley Province of Kenya. Laggera alata is used as traditional medicine for the treatment of inflammatory diseases including hepatitis and as insecticide. ${ }^{[68,151]}$ Plant endophytic fungi have been defined as symbiotic microorganisms living within plant tissues for a mutual benefit, e.g. by exhibiting ecological effects such as insecticidal, anti-nematodal, antibacterial properties. They even help the host plant to be resistant against external biotic and abiotic stress. Phenols, lactones, steroids, terpenoids, and alkaloids have been reported as secondary metabolites from endophytic fungi. ${ }^{[152]}$

The fungus LAF20 was incubated on $\mathrm{M}_{2}$ agar medium at $28^{\circ} \mathrm{C}$ for 6 days. The pre-screening of the crude extract did not exhibit antimicrobial activities against different microorganisms, but TLC of crude extract showed blue, red, grey colour reactions with anisaldehyde/sulphuric acid.

The strain was cultivated on rice medium for one month at $25^{\circ} \mathrm{C}$ in resting culture. The fungal culture was then extracted with ethyl acetate and the solvent was evaporated under vacuum. The crude extract was subjected to column chromatography on silica gel with $\mathrm{CH}_{2} \mathrm{Cl}_{2} / \mathrm{MeOH}$. 


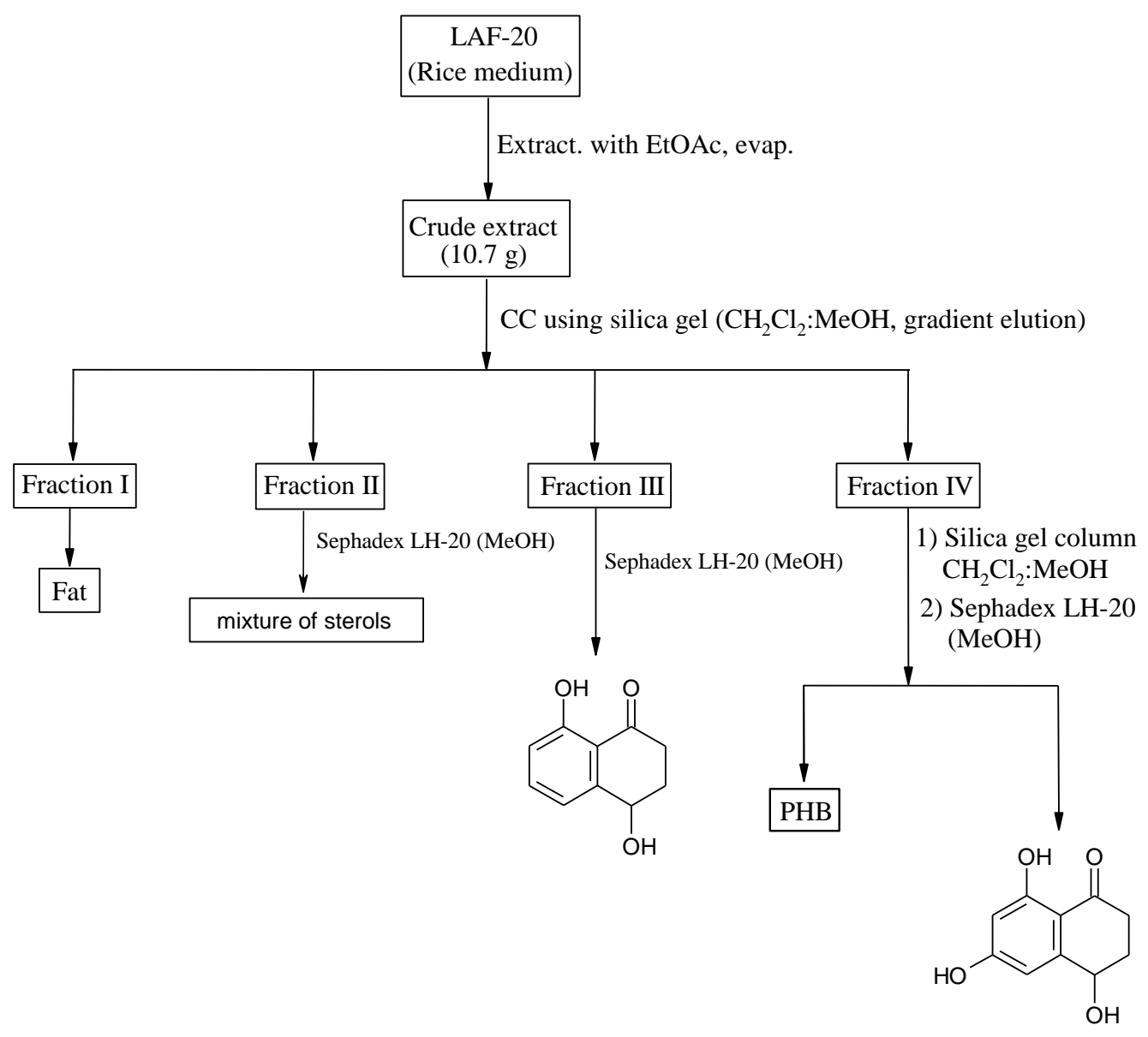

Figure 59: Work-up scheme for the fungus LAF-20

\subsubsection{Isosclerone}

Isoclerone 104 was isolated as white solid from a UV absorbing zone, which turned brown with anisaldehyde/sulphuric acid. The EI mass spectrum gave a molecular weight of $\mathrm{m} / \mathrm{z} 178$ corresponding to a molecular formula $\mathrm{C}_{10} \mathrm{H}_{10} \mathrm{O}_{3}$. The ${ }^{1} \mathrm{H}$ NMR spectrum revealed two doublets and one doublet of doublet at $\delta 7.00,6.91$ and 7.48, respectively, with coupling constants of 7.7 and $8.2 \mathrm{~Hz}$, suggesting a 1,2,3trisubstituted aromatic ring. A singlet at $\delta 12.4$ indicated the presence of a chelated hydroxyl group. The resonance of an oxygenated methine was observed at $\delta 4.90$, and signals of two methylenes appeared between $\delta 2.96$ and 2.17. 


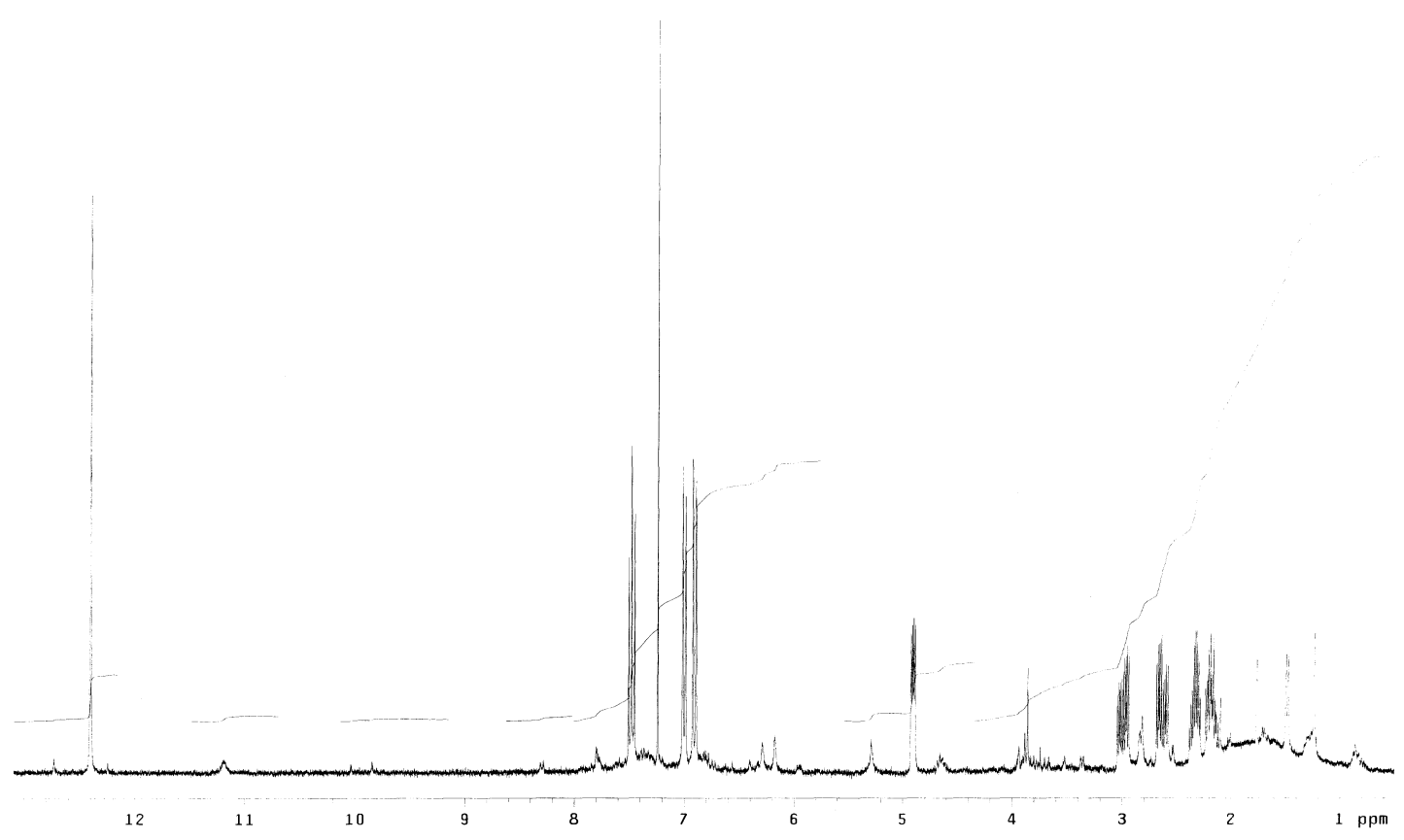

Figure 60: $\quad{ }^{1} \mathrm{H}$ NMR spectrum $\left(\mathrm{CDCl}_{3}, 300 \mathrm{MHz}\right)$ of isosclerone (104)

The ${ }^{13} \mathrm{C}$ NMR spectrum showed 10 carbon signals, one carbonyl at $\delta 204.1$, six $s p^{2}$ carbons (three methines and three quaternary), one oxygenated $s p^{3}$ carbon at $\delta$ 67.7 and two methylene signals at $\delta 34.6$ and 31.3, respectively. A sub-structure search in AntiBase based on the molecular formula delivered 25 hits. Further substructure searches in AntiBase based on the ${ }^{1} \mathrm{H}$ and ${ }^{13} \mathrm{C}$ NMR spectroscopic data led to isosclerone as the suggested structure for 104. The naphthalenone derivative isosclerone ${ }^{[153]}(\mathbf{1 0 4})$ was further confirmed by comparison of the spectroscopic data with the literature. ${ }^{[154]}$

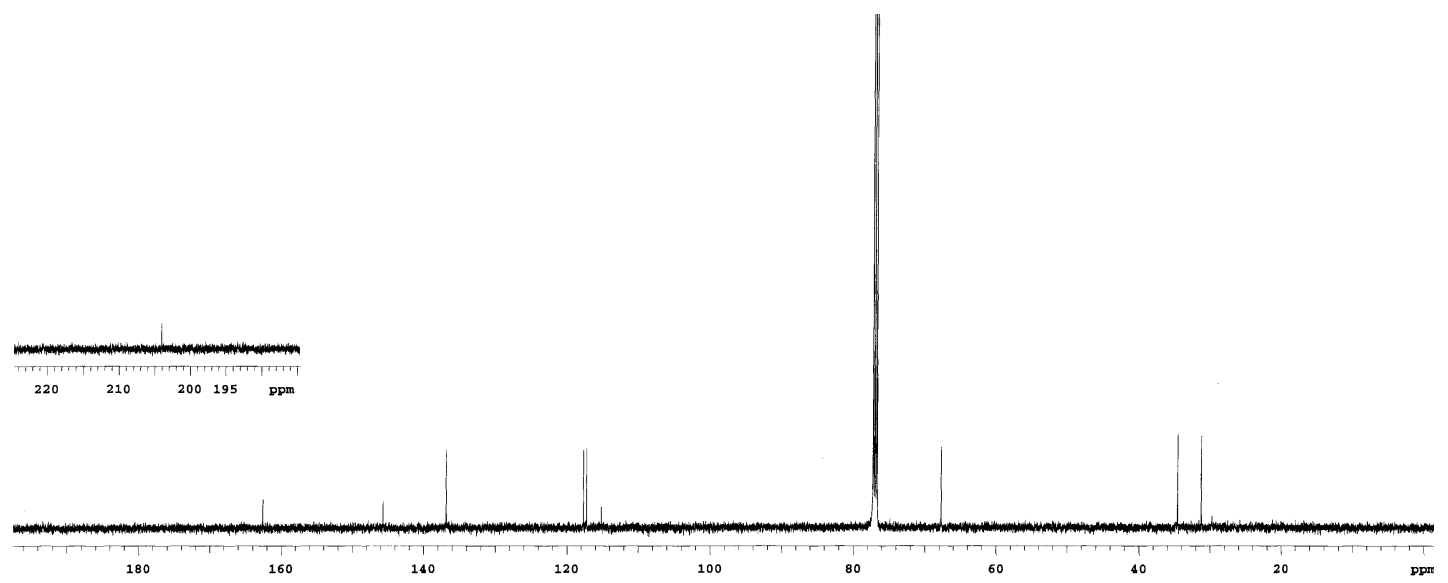

Figure 61: $\quad{ }^{13} \mathrm{C}$ NMR spectrum $\left(\mathrm{CDCl}_{3}, 125 \mathrm{MHz}\right)$ of isosclerone (104) 


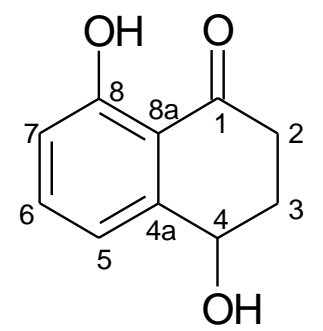

104

Isosclerone (104) is a phytotoxic phenol, previously isolated from the fungus Discula sp. ${ }^{[155]}$ It has been reported as nematocidal agent against Bursaphelenchus xylophilus. ${ }^{[156]}$

\subsubsection{4, 6, 8-Trihydroxy-1-tetralone}

Compound 105 was isolated as brownish solid; the molecular formula was deduced as $\mathrm{C}_{10} \mathrm{H}_{10} \mathrm{O}_{4}$ from the HRESI mass analysis. The ${ }^{1} \mathrm{H}$ NMR spectrum depicted two doublets at $\delta 6.52$ and 6.15 with a coupling constant of $2.3 \mathrm{~Hz}$, suggesting meta coupling of protons within an aromatic ring. In addition, signals of one oxygenated methine and multiplets for two diastereotopic $\mathrm{CH}_{2}$ groups were revealed at $\delta 4.71$ and between 2.75 and 2.03.

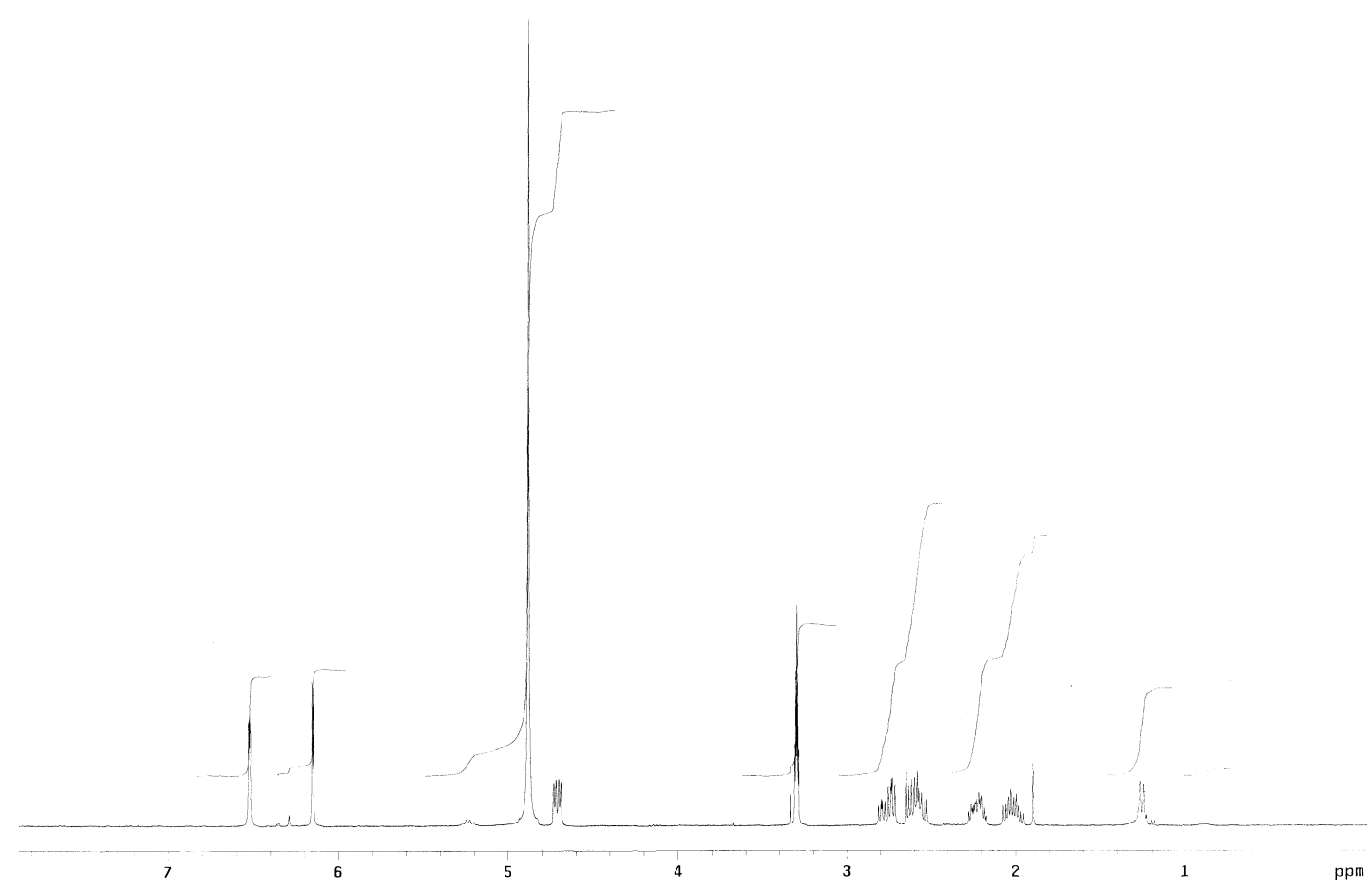

Figure 62: $\quad{ }^{1} \mathrm{H}$ NMR spectrum $\left(\mathrm{CD}_{3} \mathrm{OD}, 300 \mathrm{MHz}\right)$ of 4,6,8-trihydroxy-1tetralone (105) 
The ${ }^{13} \mathrm{C}$ NMR spectrum exhibited the signals of a ketone carbonyl at $\delta 203.5$, two $s p^{2}$ methine signals at $\delta 107.6$ and 102.5, and four quaternary carbons at $\delta 167.3$, $166.7,150.9$, and 110.0 suggesting a tetrasubstituted aromatic system. In the aliphatic region, the ${ }^{13} \mathrm{C}$ NMR spectrum showed signals of one oxymethine and two methylene groups at $\delta 68.5,35.7$, and 32.6, respectively. A sub-structure search in AntiBase based on the molecular formula delivered 44 hits. After filtering with the ${ }^{1} \mathrm{H}$ and ${ }^{13} \mathrm{C}$ NMR data, only 3,4-dihydro-4,6,8-trihydroxy-1(2H)-naphthalenone and 6hydroxyisosclerone were left. The structure 4,6,8-trihydroxy-1-tetralone (105) was finally confirmed by comparison with authentic spectra and the literature data. ${ }^{[156]}$

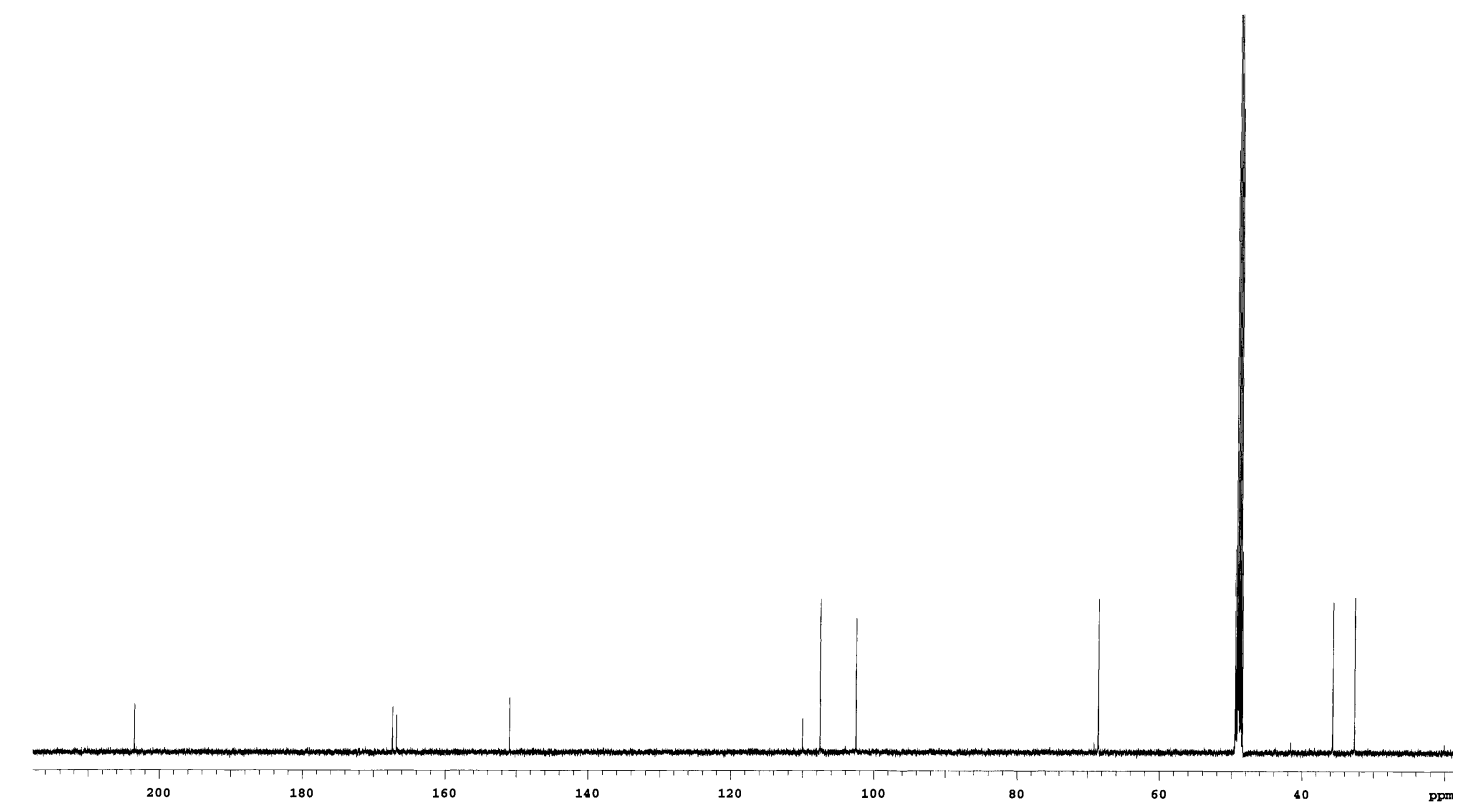

Figure 63: ${ }^{13} \mathrm{C}$ NMR spectrum $\left(\mathrm{CD}_{3} \mathrm{OD}, 125 \mathrm{MHz}\right)$ of 4,6,8-trihydroxy-1tetralone (105)<smiles>O=C1CCC(O)c2cc(O)cc(O)c21</smiles> 
4,6,8-Trihydroxy-1-tetralone (105) was active against the nematode Bursaphelenchus xylophilus. ${ }^{[156]}$ It had also been reported as one of the phytotoxic phenols isolated from the fungus Tubakia dryina (Sacc.) Sutton. ${ }^{[155]}$

\subsection{Endophytic fungus NP32-A}

The fungal strain NP32-A was isolated from the leaves of Campyloneurum phyllitidis, which was collected in the Botanical Garden, Germany of the University of Göttingen. It formed white fluffy cotton-like mycelial colonies after incubation on $\mathrm{M}_{2}$ agar medium for 5 days at $28^{\circ} \mathrm{C}$. This strain was selected for chemical investigation considering its biological activities against E. coli, B. subtilis, Streptomyces viridochromogenes Tü 57, Staphylococcus aureus, and Rhizoctonia solani. On thin layer chromatography, it showed several UV active zones and blue, red, yellow, or violet colour reactions with anisaldehyde/sulphuric acid after heating.

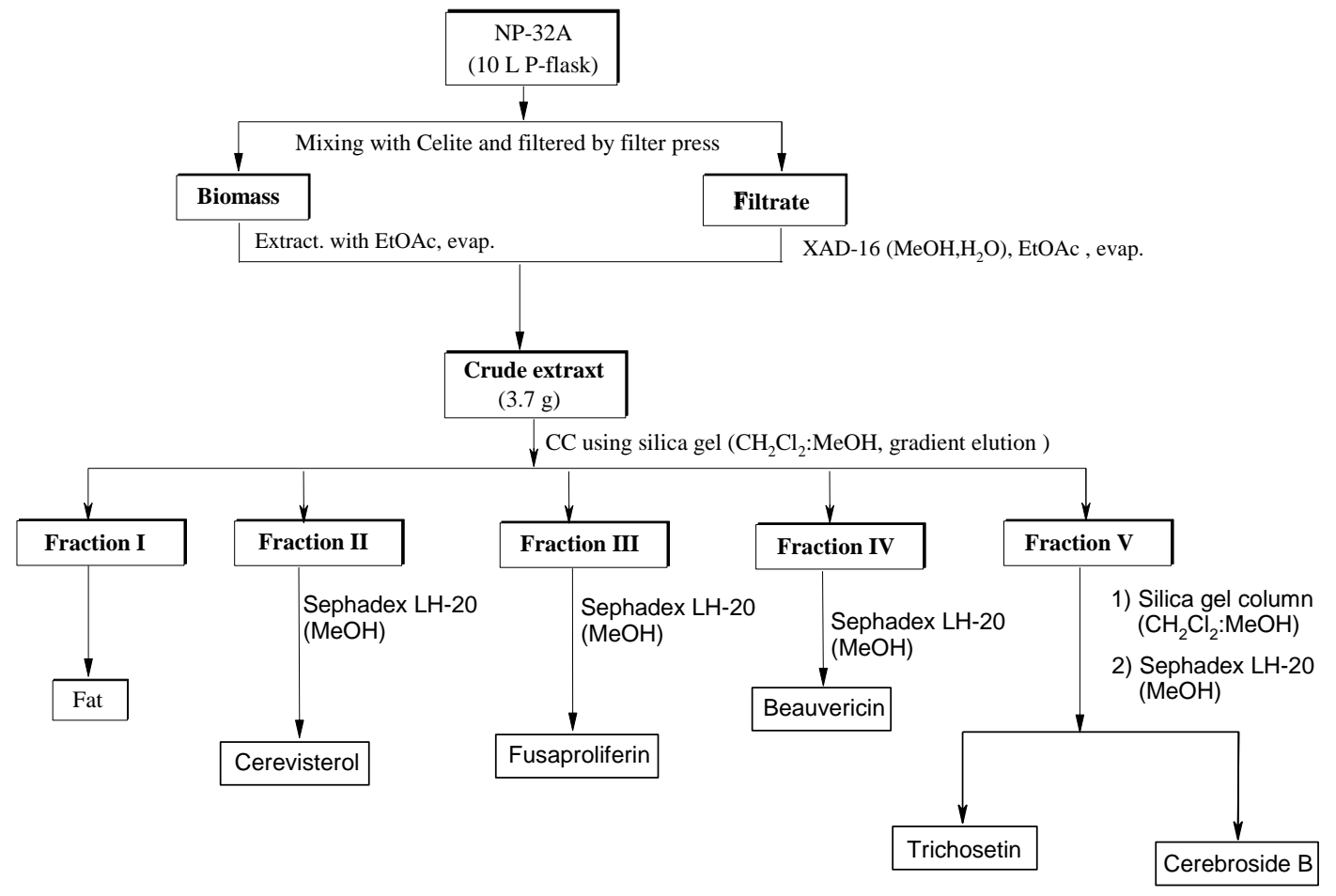

Figure 64: Work-up scheme for endophytic fungus NP32-A

\subsubsection{Cerevisterol}

Cerevisterol (106) was obtained as white solid from subfraction II and was not UV-active. The ${ }^{1} \mathrm{H}$ NMR spectrum exhibited signals of three $s p^{2}$ and two oxygenated 
$s p^{3}$ methines. In the aliphatic region between $\delta 3.37$ and 0.55 , the spectrum revealed the resonances of protons for seven methylene, five methine, and six methyl groups.

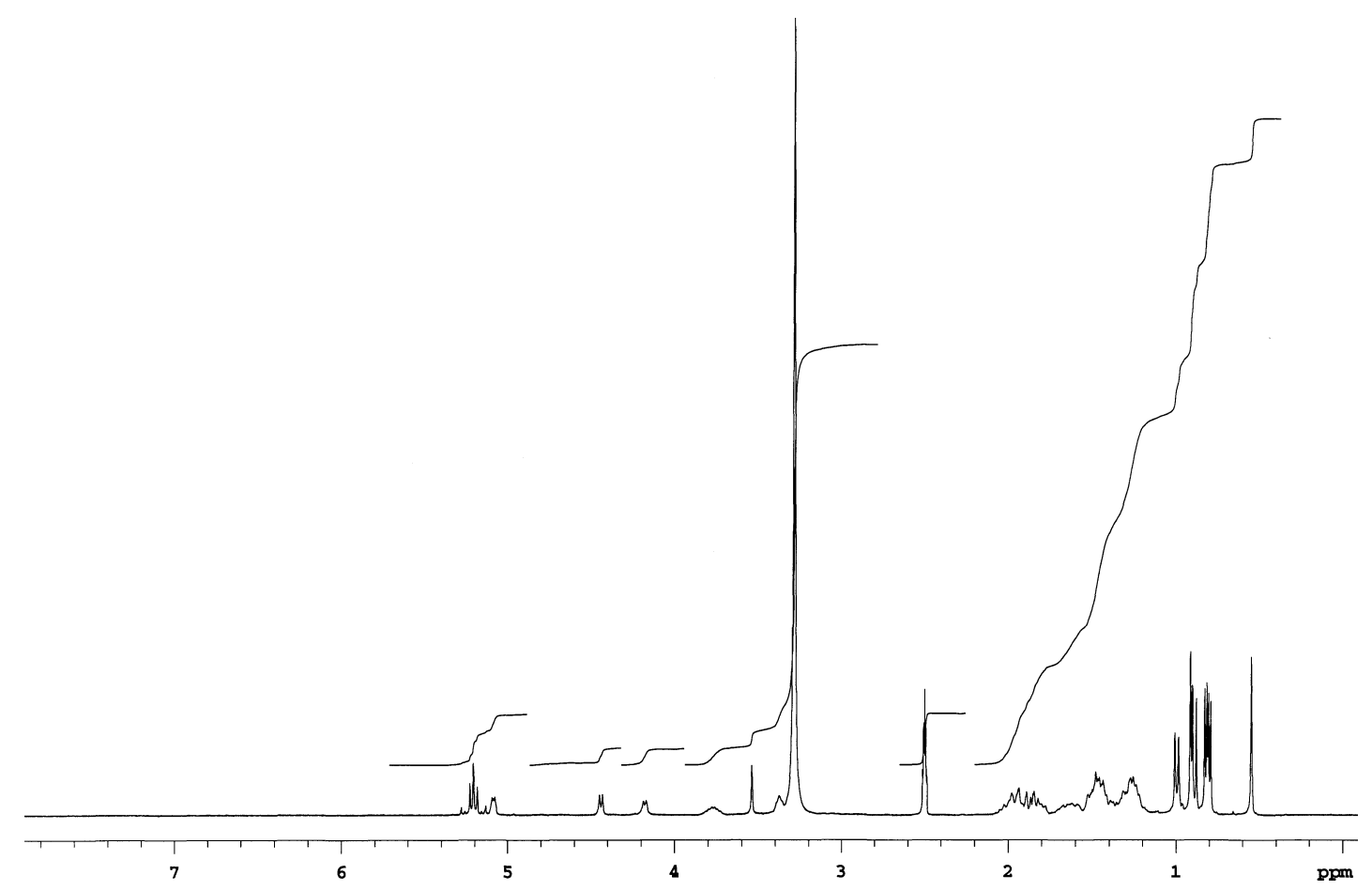

Figure 65: $\quad{ }^{1} \mathrm{H}$ NMR spectrum (DMSO- $d_{6}, 300 \mathrm{MHz}$ ) of cerevisterol (106)

The HRESI mass analysis delivered $\mathrm{C}_{28} \mathrm{H}_{46} \mathrm{O}_{3}$ as the molecular formula, and the ${ }^{13} \mathrm{C}$ NMR spectrum showed signals of 28 carbon atoms; four of them were due to $s p^{2}$ carbon atoms, and three to oxygenated $s p^{3}$ carbons. A sub-structure search in AntiBase based on the molecular formula delivered 11 hits. After filtering with the ${ }^{1} \mathrm{H}$ and ${ }^{13} \mathrm{C}$ NMR data, six structures were left. The structure $\mathbf{1 0 6}$ was finally assigned as cerevisterol and confirmed by comparison with authentic spectra and the literature data. ${ }^{[157,158]}$ 


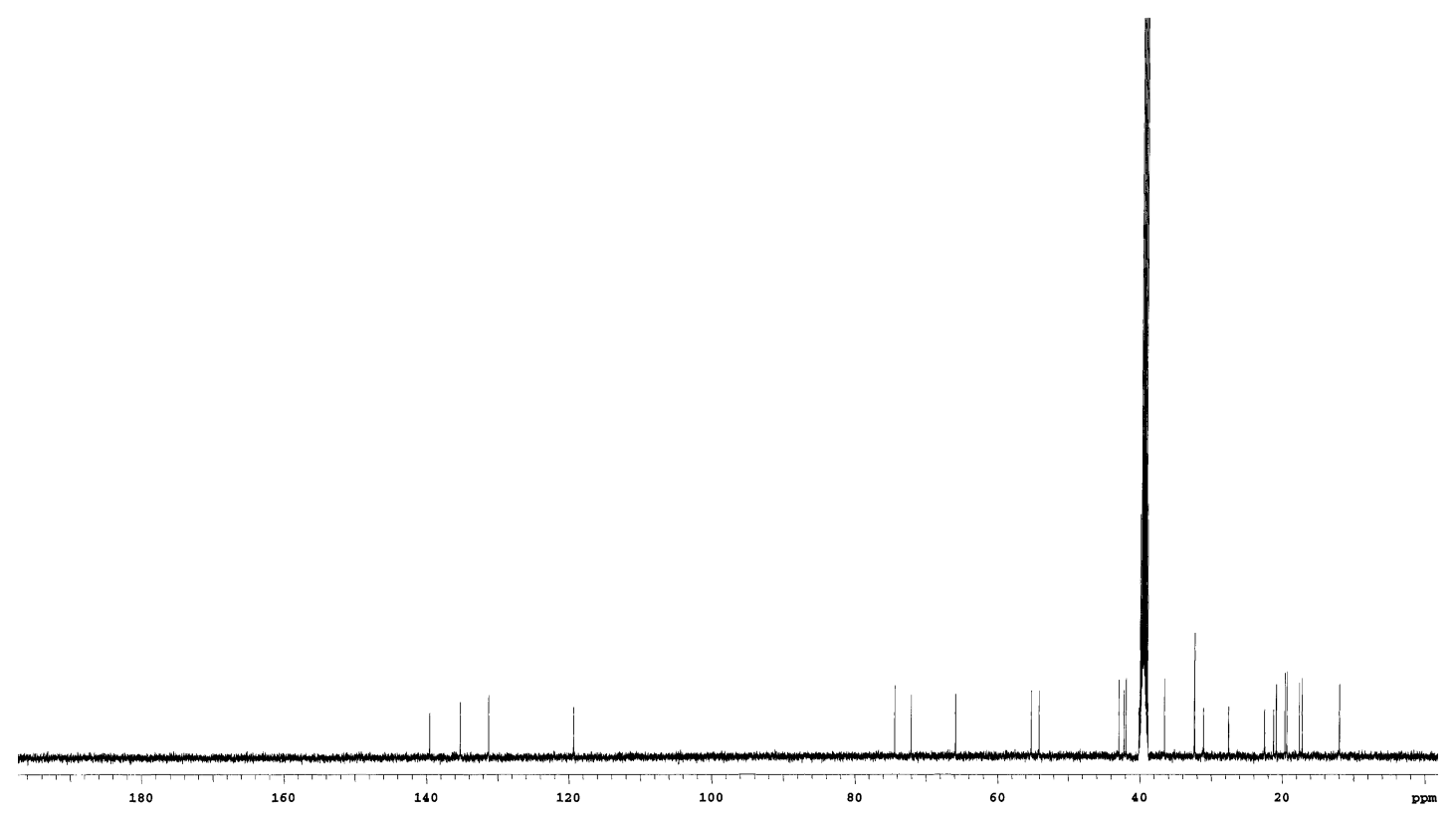

Figure 66: $\quad{ }^{13} \mathrm{C}$ NMR spectrum (DMSO- $d_{6}, 125 \mathrm{MHz}$ ) of cerevisterol (106)

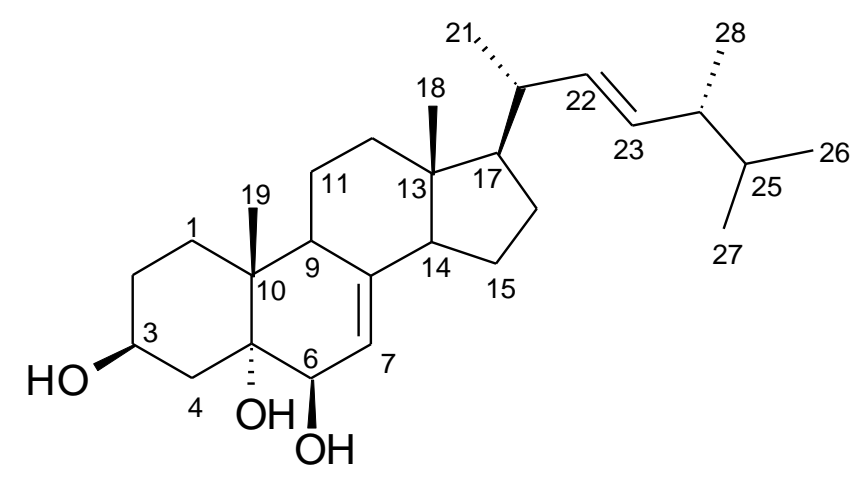

106

Cerevisterol (106) is an ergosterol derivative and was for the first time isolated as a natural secondary metabolite from yeast in $1932 .{ }^{[159]}$ Cerevisterol (106) was not active against HeLa cells, ${ }^{[160]}$ however, Mizushina and co-workers have reported a selective inhibition of eukaryotic DNA polymerase. ${ }^{[161]}$

\subsubsection{Fusaproliferin}

Fusaproliferin (107) was isolated as a UV absorbing white solid. The HRESI mass analysis delivered a pseudo-molecular ion at $m / z, 467.27680\left([\mathrm{M}+\mathrm{Na}]^{+}\right)$corresponding to a molecular formula of $\mathrm{C}_{27} \mathrm{H}_{40} \mathrm{O}_{5} \mathrm{Na}$. The ${ }^{1} \mathrm{H}$ NMR spectrum revealed signals of three $s p^{2}$ methines, one oxygenated methylene, one oxygenated $s p^{3}$ me- 
thine, and six methyl groups between $\delta$ 5.38 0.96. The remaining signals between $\delta$ 2.45-1.71 were assigned to two $s p^{3}$ methines and six $s p^{3}$ methylene groups.

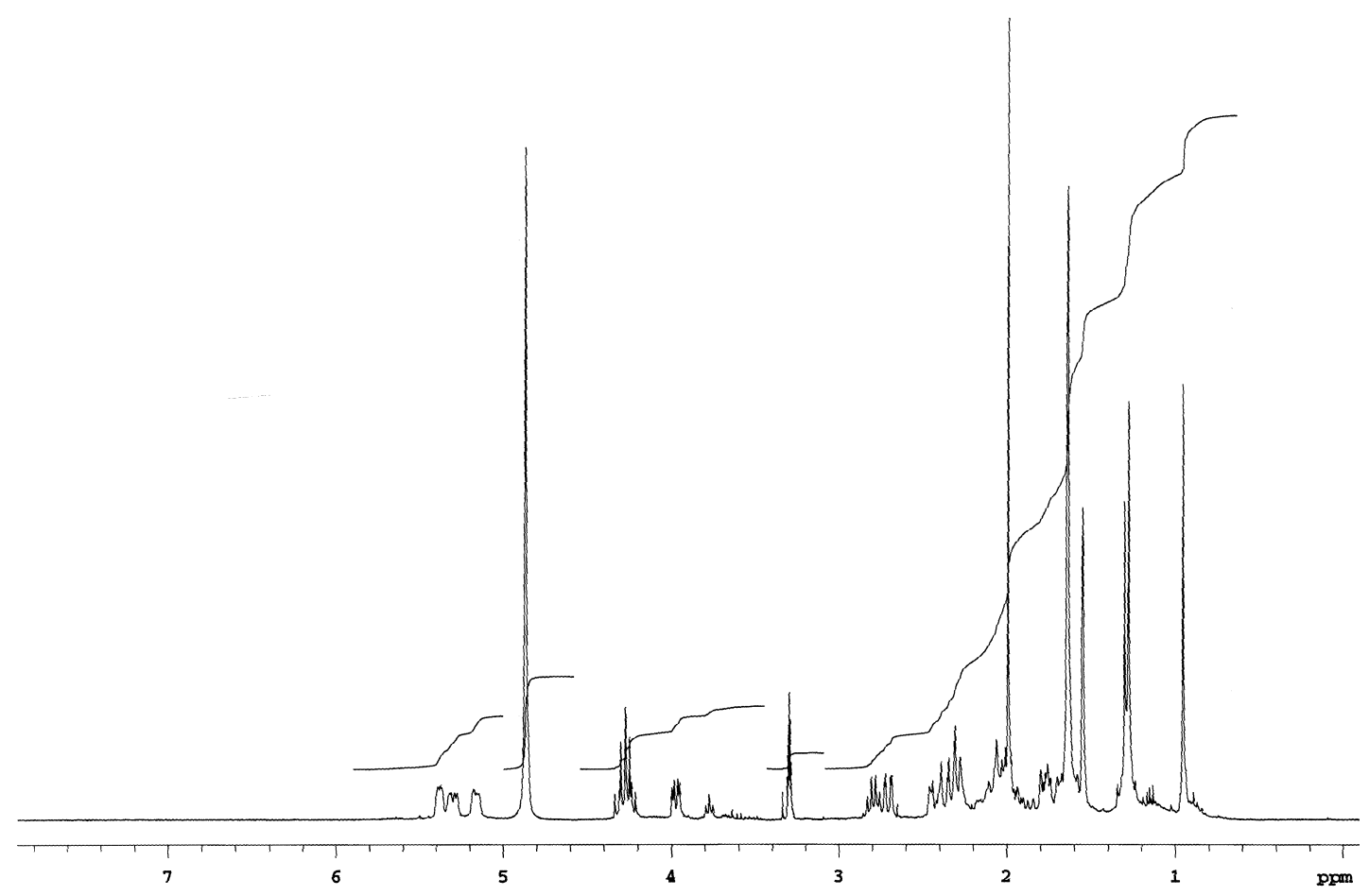

Figure 67: $\quad{ }^{1} \mathrm{H}$ NMR spectrum $\left(\mathrm{CD}_{3} \mathrm{OD}, 300 \mathrm{MHz}\right)$ of fusaproliferin (107)

The ${ }^{13} \mathrm{C}$ NMR spectrum exhibited resonances of two carbonyl groups at $\delta_{C}$ 209.0 and 172.7, eight $s p^{2}$ carbons (three methines and five quaternary carbons) between $\delta_{C} 149.9$ and 123.0 and two oxygenated carbon $s p^{3}$ atoms at $\delta_{C} 77.1$ and 67.1. A sub-structure search in AntiBase based on the molecular formula resulted in 6 hits. After filtering with the ${ }^{1} \mathrm{H}$ and ${ }^{13} \mathrm{C}$ NMR data, the structure 107 was finally confirmed as fusaproliferin by comparison with authentic spectra and the literature data. $^{[162]}$ 


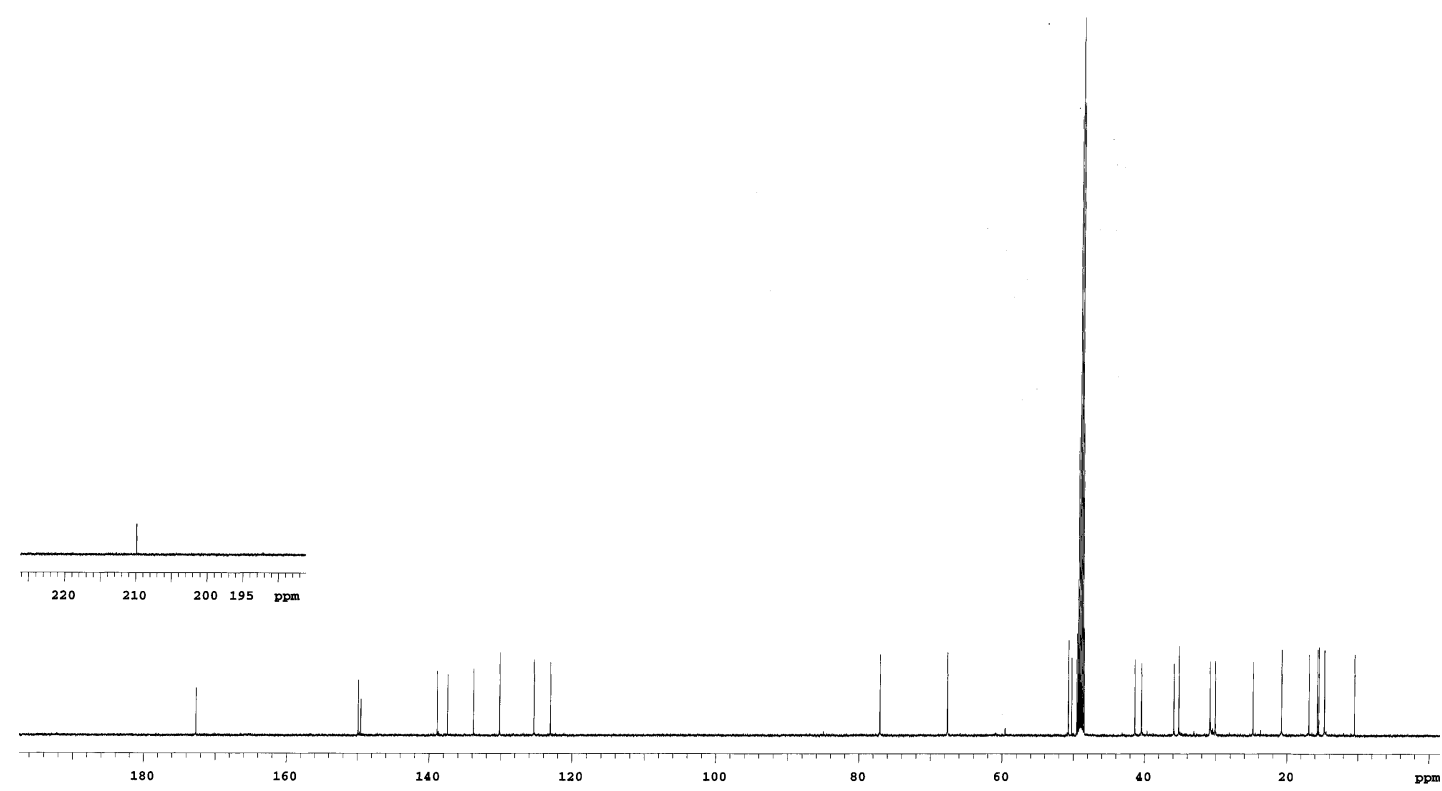

Figure 68: $\quad{ }^{13} \mathrm{C}$ NMR spectrum $\left(\mathrm{CD}_{3} \mathrm{OD}, 125 \mathrm{MHz}\right)$ of fusaproliferin $(\mathbf{1 0 7})$

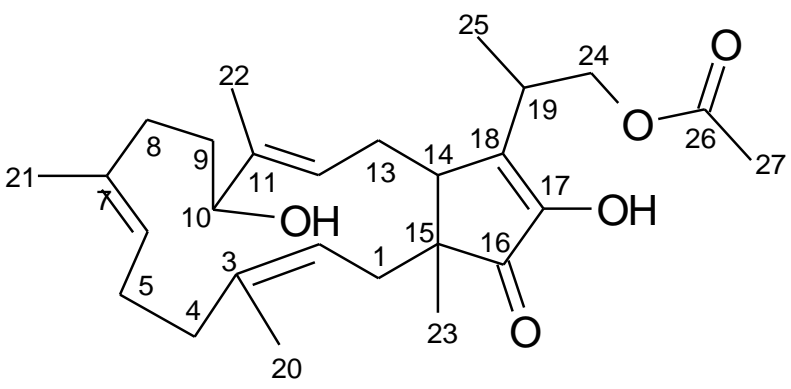

107

Fusaproliferin (107) was for the first time isolated from the fungus Fusarium proliferatum Nirenberg, which is worldwide reported to infect plants and a number of agricultural products. ${ }^{[163]}$ Fusaproliferin (107) is therefore one of the mycotoxins found in food such as maize. ${ }^{[164,165]}$ Fusaproliferin (107) showed also toxicity against Artemia salina and a lepidopteran cell line. ${ }^{[165]}$

\subsubsection{Beauvericin}

Beauvericin (108) was obtained as white solid from subfraction IV and showed UV absorption at $254 \mathrm{~nm}$. The ${ }^{1} \mathrm{H}$ NMR spectrum demonstrated resonances of five aromatic protons at $\delta 7.25$, three aliphatic methines, one methylene between $\delta 3.41$ and 3.36, and three methyl groups at $\delta 3.14,0.84$ and 0.24 . 


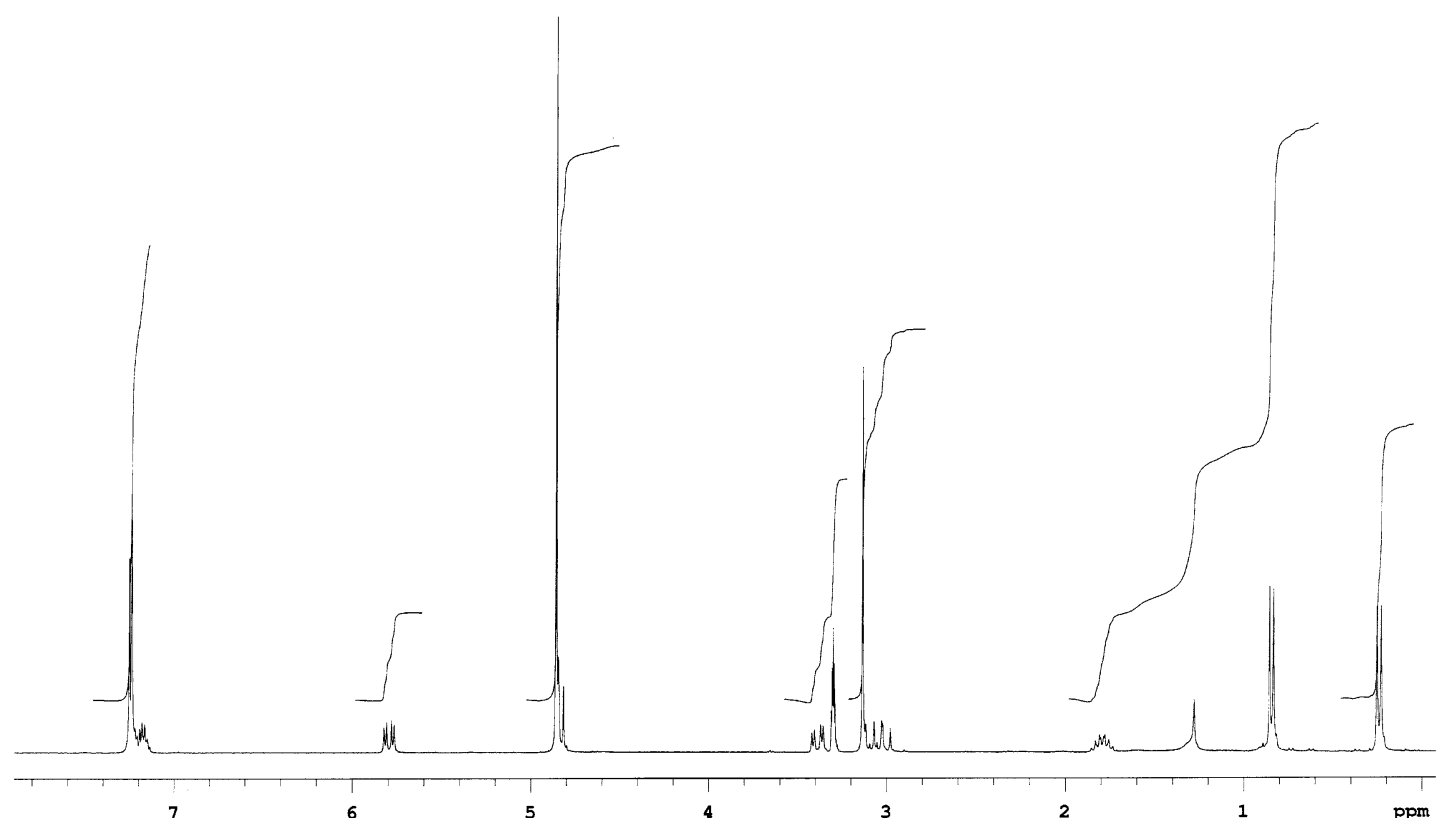

Figure 69: $\quad{ }^{1} \mathrm{H}$ NMR spectrum $\left(\mathrm{CD}_{3} \mathrm{OD}, 300 \mathrm{MHz}\right)$ of beauvericin (108)

The ${ }^{13} \mathrm{C}$ NMR spectrum indicated 13 signals assigned to two carbonyls and a mono-substituted aromatic benzene. The remaining signals represented one oxygenated (77.2) and six aliphatic carbons between $\delta$ 58-17. The HRESI mass analysis gave a pseudo-molecular ion peak at $\mathrm{m} / z$ 806.39831 $\left([\mathrm{M}+\mathrm{Na}]^{+}\right)$as calculated for $\mathrm{C}_{45} \mathrm{H}_{57} \mathrm{~N}_{3} \mathrm{O}_{9} \mathrm{Na}$. The blue colour reaction in the chlorine/dianisidine test indicated that the compound $\mathbf{1 0 8}$ was a peptide. A search in AntiBase using the molecular formula, $\mathrm{C}_{45} \mathrm{H}_{57} \mathrm{~N}_{3} \mathrm{O}_{9}$, delivered only one hit, which was beauvericin. The structure of beauvericin (108) was further confirmed by comparing ${ }^{1} \mathrm{H}$ and ${ }^{13} \mathrm{C}$ NMR spectral data with literature data. ${ }^{[166,167]}$ The simplicity of ${ }^{1} \mathrm{H}$ and ${ }^{13} \mathrm{C}$ NMR spectra of beauvericin (108) is explained by the symmetry in its molecule: It is a cyclotrimeric ester of $\mathrm{N}$-methylphenylalanine (NMePhe) and 2-hydroxyisovaleric acid (Hiv). ${ }^{[168]}$ Beauvericin (108) had been reported for the first time as a fungal cyclohexadepsipeptide antibiotic from fungus Beauveria bassiana. ${ }^{[169]}$ 


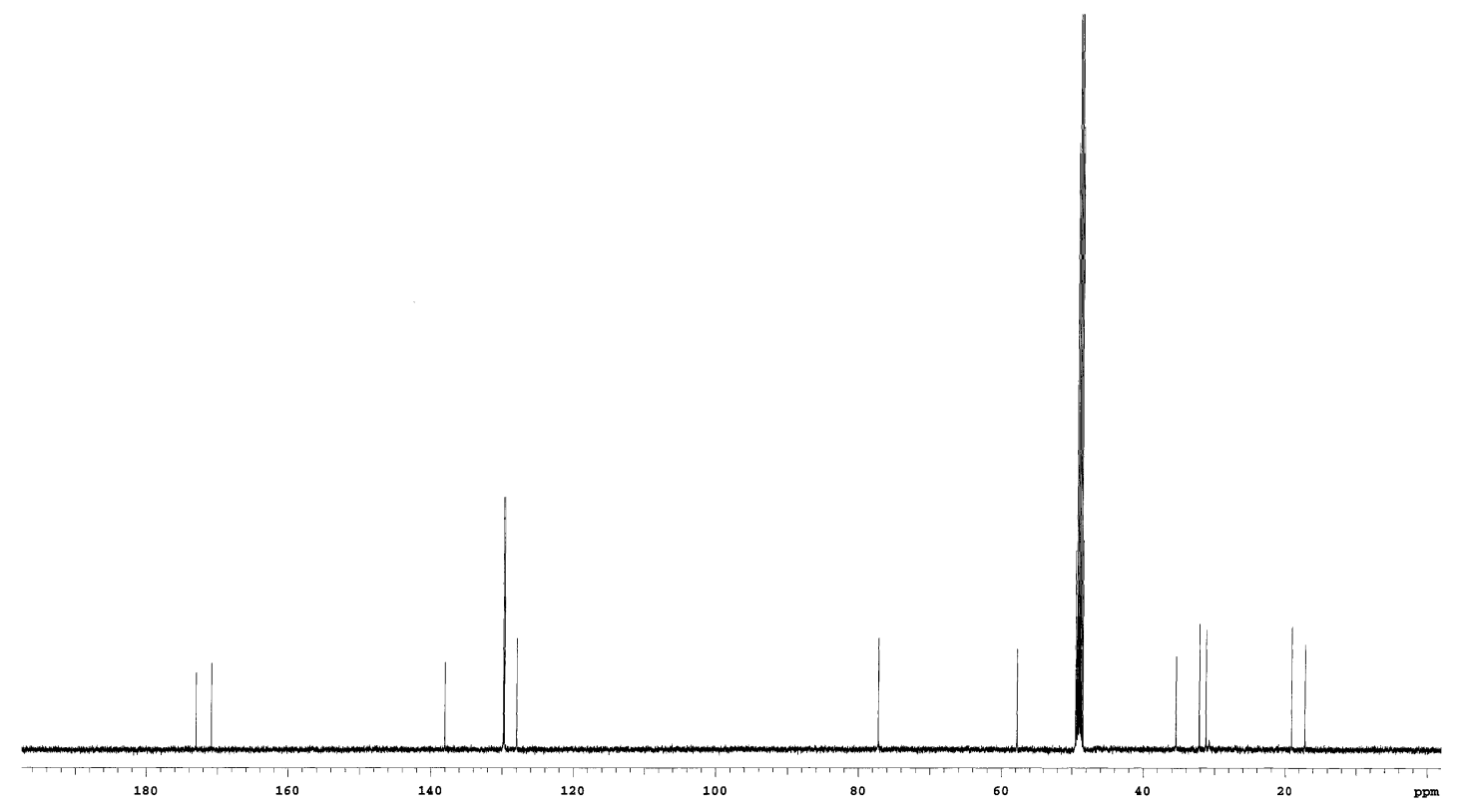

Figure 70: $\quad{ }^{13} \mathrm{C}$ NMR spectrum $\left(\mathrm{CD}_{3} \mathrm{OD}, 125 \mathrm{MHz}\right)$ of beauvericin (108)<smiles>CC(C)C(OC(=O)C(Cc1ccccc1)N(C)C(=O)[C@H](OC(=O)[C@H](Cc1ccccc1)N(C)C(=O)C(Cc1ccccc1)N(C)C(=O)[C@H](Cc1ccccc1)C(C)C)C(C)C)C(=O)OC(C(C)C)C(C)C</smiles>

\section{8}

In our biological tests, beauvericin (108) showed strong activity against brine shrimps. It exhibited also toxicity against mosquito larvae with an $\mathrm{LC}_{50}$ value of 26 ppm. ${ }^{[166]}$ It has also been reported that beauvericin (108 was cytotoxic against two human cell lines, namely hepatocellular carcinoma (Hep G2) and fibroblast-like fetal lung cells (MRC5). It has been further reported as an acyl-CoA inhibitor. ${ }^{[170]}$ 


\subsubsection{Trichosetin}

Trichosetin (109) was obtained as a UV absorbing white solid from sub-fraction V. It stained to yellow on heating after spraying with anisaldehyde/sulphuric acid. The HRESI mass analysis resulted in a pseudo-molecular ion peak at $\mathrm{m} / z 382.19880$ $\left([\mathrm{M}+\mathrm{Na}]^{+}\right)$calculated for $\mathrm{C}_{21} \mathrm{H}_{29} \mathrm{NO}_{4} \mathrm{Na}$. The ${ }^{1} \mathrm{H}$ NMR spectrum showed signals of four olefinic protons between $\delta 5.36$ and 5.18, four methylene, five $s p^{3}$ methines and three methyl groups.

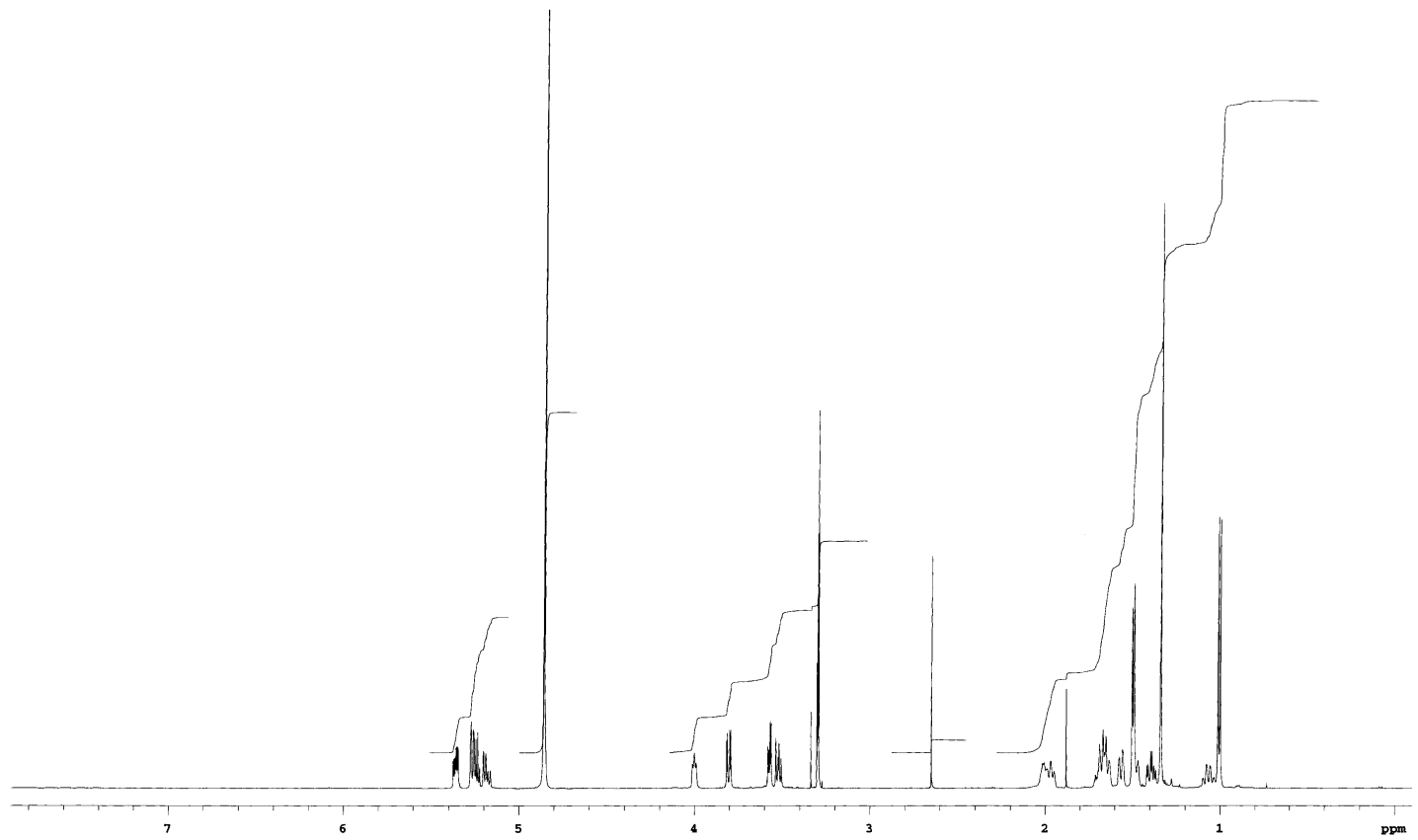

Figure 71: $\quad{ }^{1} \mathrm{H}$ NMR spectrum $\left(\mathrm{CD}_{3} \mathrm{OD}, 300 \mathrm{MHz}\right)$ of trichosetin (109)

The ${ }^{13} \mathrm{C}$ NMR spectrum revealed 21 carbon signals: two carbonyls at $\delta_{C} 192.7$ (ketone) and 180.3 (amide), four $s p^{2}$ methines, two quaternary carbons $\left(\delta_{C} 203.6\right.$ and 104.9), one quaternary $s p^{3}\left(\delta_{C} 52.7\right)$, four methylene, five $s p^{3}$ methine, and three methyl groups. In the HMBC spectrum (Figure 72), the methyl doublet ( $\delta 1.19)$ displayed a ${ }^{3} J$ coupling to methylene carbon C-7 $(\delta 41.1)$ and the methyl doublet $(\delta$ $1.49)$ showed ${ }^{2} J$ and ${ }^{3} J$ couplings to two methine carbons C-13 $(\delta 134.9)$ and C-14 $(\delta$ 129.7). The methyl singlet ( $\delta 1.34$ ) exhibited ${ }^{2} J$ and ${ }^{3} J$ couplings to a methine carbon C-11 $(\delta 43.2)$ and two quaternary carbons at $\delta 52.7(\mathrm{C}-2)$ and $203.6(\mathrm{C}-1)$. In addition, the methine proton at $\delta 5.36$ showed cross couplings to two methine carbons at $\delta 44.9$ (C-3) and 43.2 (C-11). A search in AntiBase based on the molecular formula, 
$\mathrm{C}_{21} \mathrm{H}_{29} \mathrm{NO}_{4}$, resulted in 4 hits. After filtering with the HMBC and COSY NMR correlations (Figure 72) as well as the ${ }^{1} \mathrm{H}$ and ${ }^{13} \mathrm{C}$ NMR spectra, trichosetin was assigned as the best fit for compound 109, and the structure was further confirmed by comparison with the literature data. ${ }^{[171,172]}$ Trichosetin (109) showed biological activities as phytotoxin and anti-HIV agent. ${ }^{[172]}$ In addition, it has been reported to show antimicrobial activity against Gram-positive bacteria. ${ }^{[172]}$

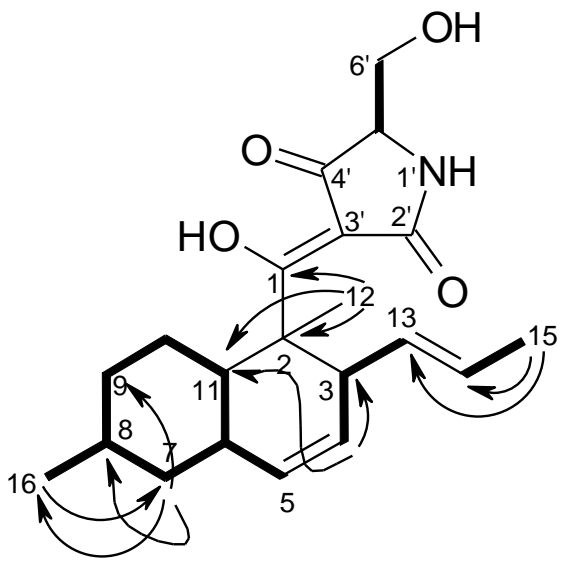

109

Figure 72: Selected ${ }^{1} \mathrm{H}^{1}{ }^{1} \mathrm{H} \operatorname{COSY}(-)$ and $\mathrm{HMBC}(\longrightarrow)$ correlations of $\mathbf{1 0 9}$

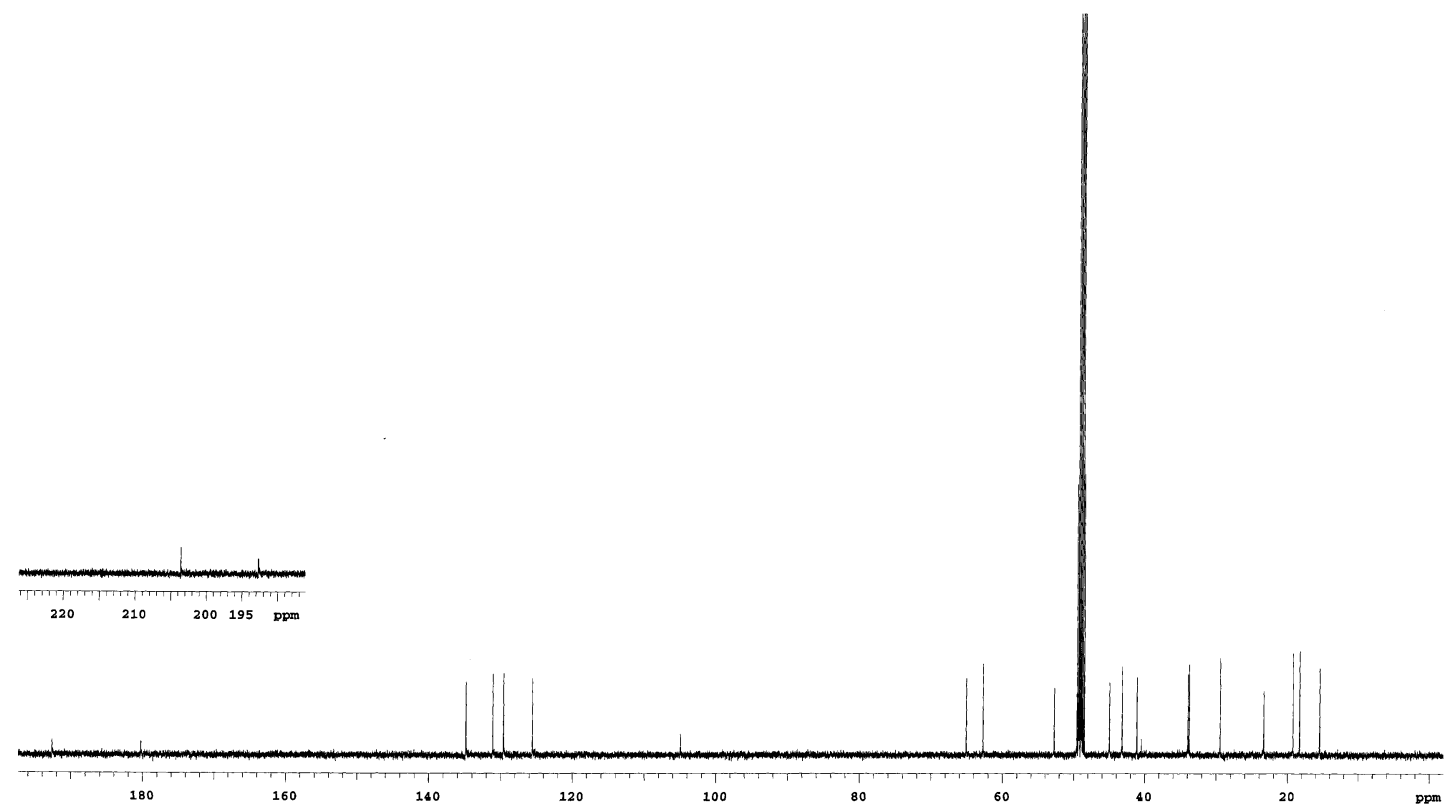

Figure 73: $\quad{ }^{13} \mathrm{C}$ NMR spectrum $\left(\mathrm{CD}_{3} \mathrm{OD}, 125 \mathrm{MHz}\right)$ of trichosetin (109) 
Trichosetin (109) is a member of the tetramic acid derivatives, which consist of two unique structural features, namely a 2,4-pyrrolidinedione and a bicyclic hydrocarbon unit. The biosynthesis of trichosetin (109) seems to occur via the cyclisation of a linear polyketide, of which acetate, serine and methionine were the precursors. ${ }^{[172]}$

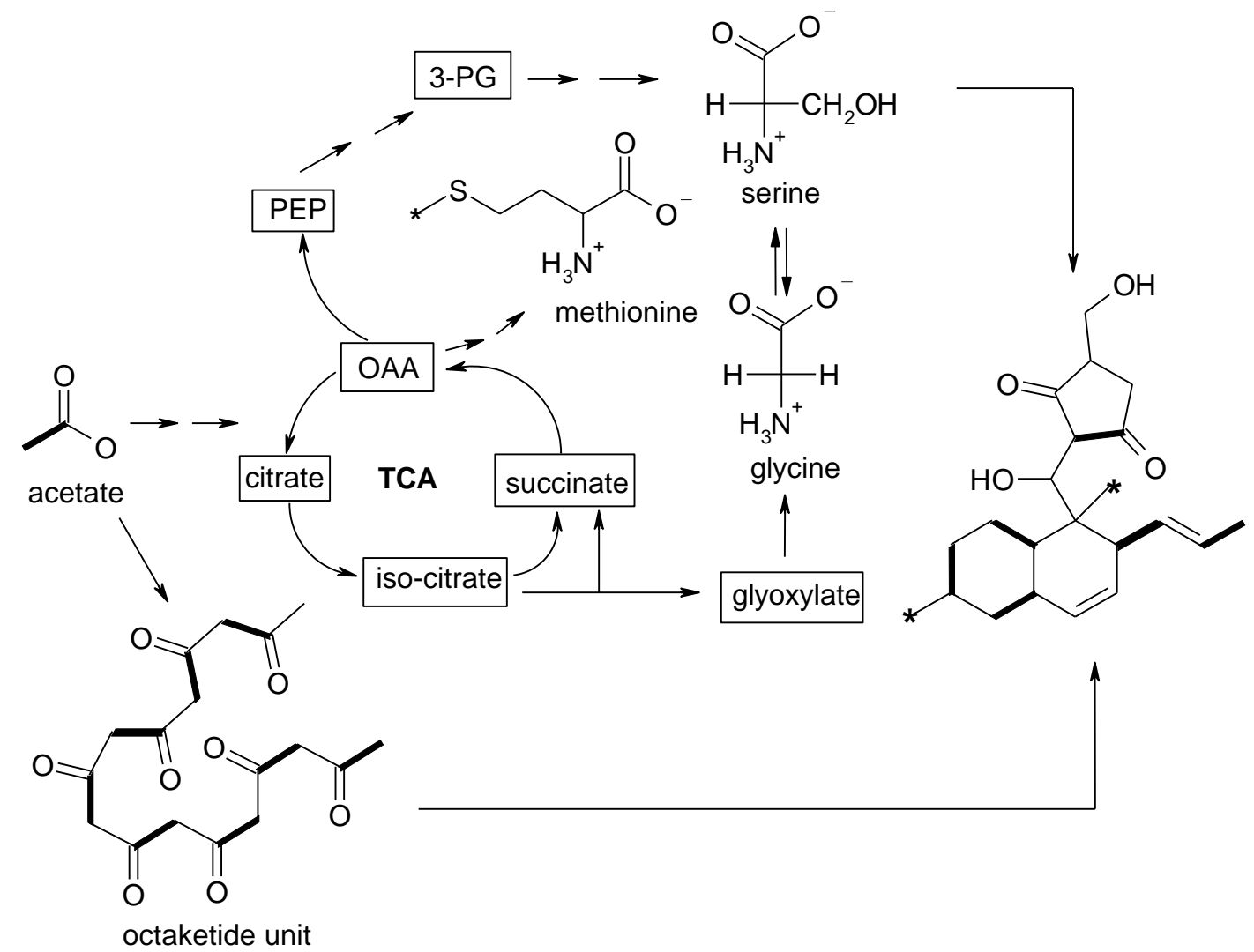

Figure 74: $\quad$ Proposed biosynthesis pathway of trichosetin (109). ${ }^{[172]}$

\subsubsection{Cerebroside B}

Compound 110 was isolated as white UV-inactive compound and stained to red on heating with anisaldehyde/sulphuric acid. The ${ }^{1} \mathrm{H}$ NMR spectrum exhibited resonances of three vinylic protons at $\delta 5.72,5.45$ and 5.10, one anomeric proton at $\delta$ 4.24 with a coupling constant of $J=7.8 \mathrm{~Hz}$, suggesting a sugar moiety in the molecule. The triplet and singlet at $\delta 1.56$ and 0.86 were assigned to methyl groups. A high broadened singlet at $\delta 1.25$ indicated a long aliphatic hydrocarbon chain. 


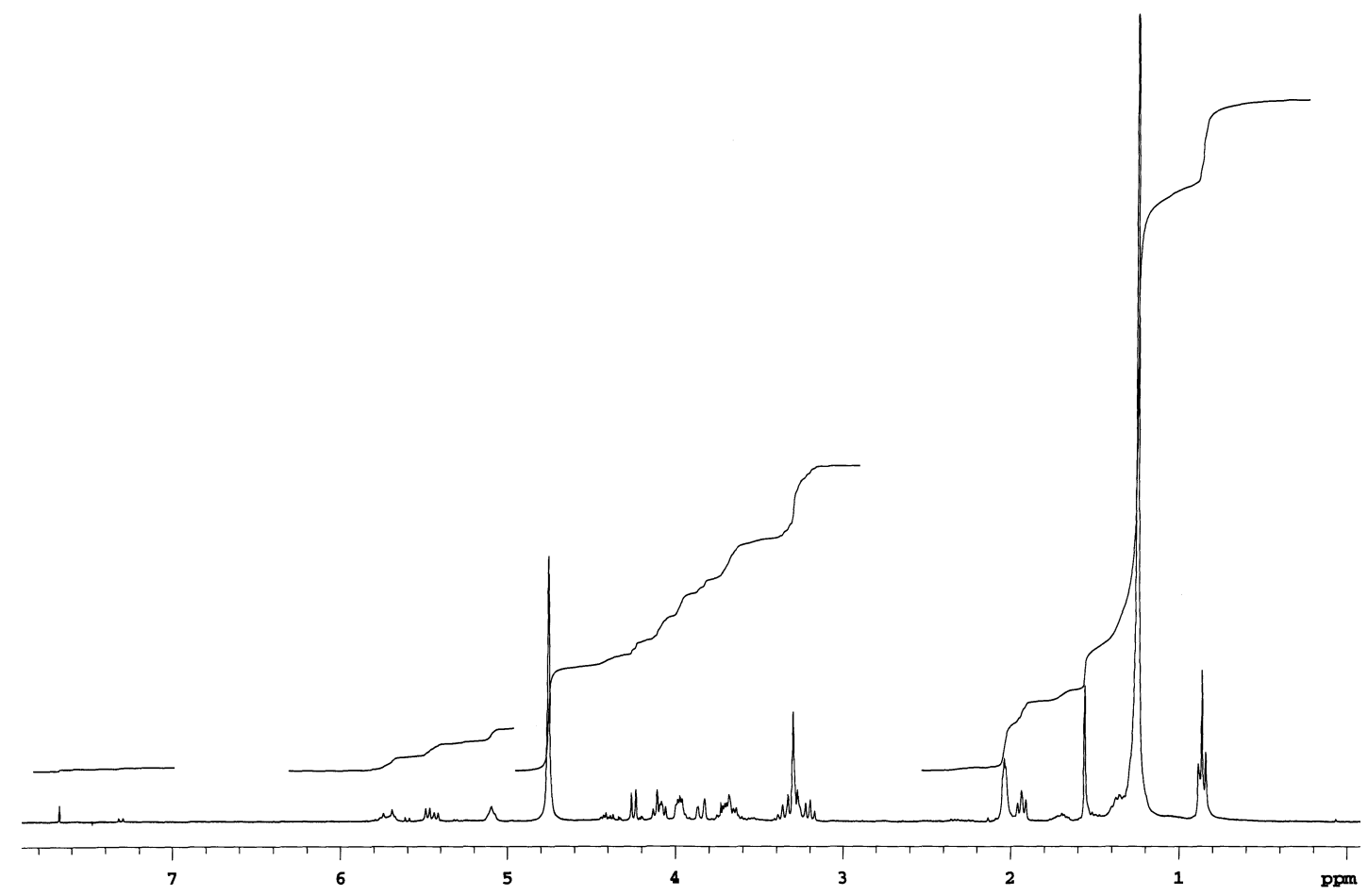

Figure 75: $\quad{ }^{1} \mathrm{H}$ NMR spectrum $\left(\mathrm{CD}_{3} \mathrm{OD} / \mathrm{CDCl}_{3}, 300 \mathrm{MHz}\right)$ of cerebroside $\mathrm{B}(\mathbf{1 1 0})$

The ${ }^{13} \mathrm{C}$ NMR spectrum exhibited four olefinic carbon signals at $\delta_{C} 136.5$, 134.6, 130.3, 124.3, and an amide carbonyl signal at $\delta_{C}$ 176.7. The ESI mass spectrum supplied a pseudo-molecular ion peak at $\mathrm{m} / \mathrm{z} 750\left([\mathrm{M}+\mathrm{Na}]^{+}\right)$corresponding to the molecular weight of 727 Dalton. The HRESI mass analysis revealed the molecular formula $\mathrm{C}_{41} \mathrm{H}_{77} \mathrm{NO}_{9}$, which indicated that a third double bond equivalent was contributed by a sugar ring. The anomeric carbon signal of the sugar unit appeared at $\delta_{C}$ 104.1. A sub-structure search in AntiBase based on the molecular formula, $\mathrm{C}_{41} \mathrm{H}_{77} \mathrm{NO}_{9}$, delivered 4 hits. Among them, cerebroside $\mathrm{B}(\mathbf{1 1 0})$ was proposed on the basis of the NMR data. The structure was further confirmed by comparing the spectroscopic data with the literature values. ${ }^{[173]}$ 


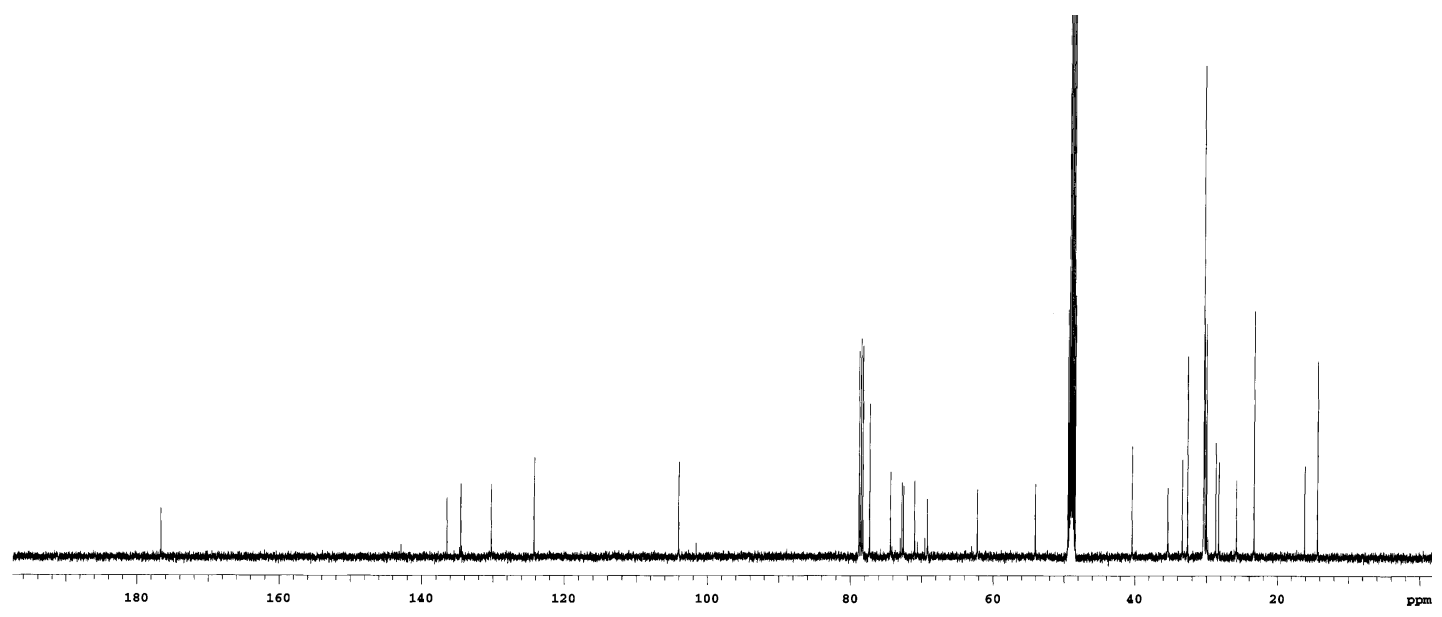

Figure 76: ${ }^{13} \mathrm{C}$ NMR spectrum $\left(\mathrm{CD}_{3} \mathrm{OD} / \mathrm{CDCl}_{3}, 125 \mathrm{MHz}\right)$ of cerebroside $\mathrm{B}$ (110)

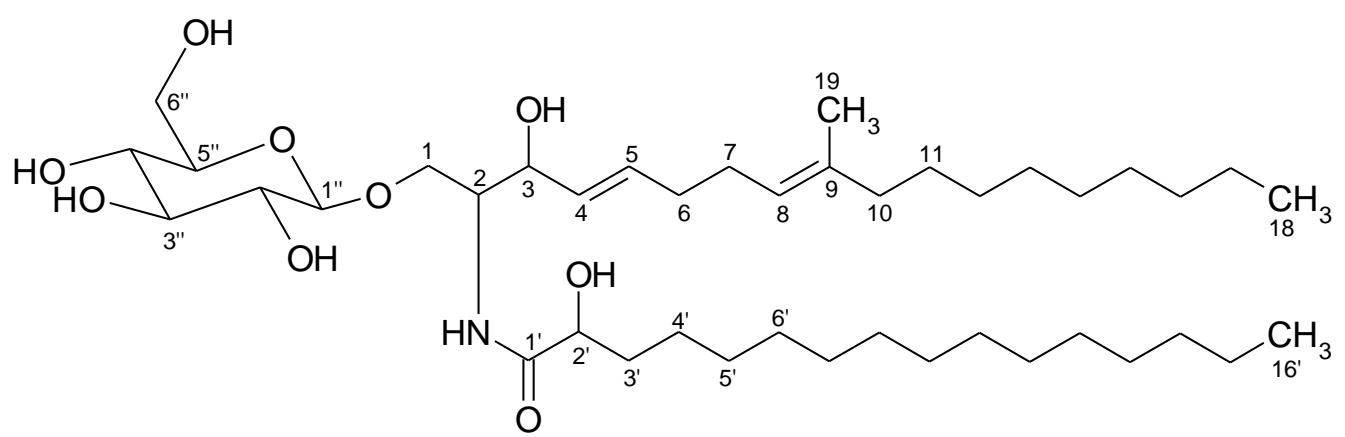

110

Cerebrosides are glycosphingolipids and were found in many phytopathogens as elicitors inducing e.g. the disease resistance in rice. ${ }^{[174]}$ Cerebroside B (110) has been reported as antifungal agent against Candida albicans. ${ }^{[175]}$ The sugar unit of cerebroside B (110) had been previously reported as $\beta$-D-glucose. ${ }^{[175]}$

\subsection{Endophytic fungus FT44}

The endophytic fungus FT44 was isolated from the Cameroonian medicinal plant Endodesmia calophylloides (Clusiaceae) collected from Balmayo in the Centre Province; the plant has also been found in Nigeria, Gabon and Angola. ${ }^{[176]} E$. calophylloides belongs to the family of Guttiferae or Clusiaceae and this family has been reported to produced a wide range of natural products including xanthones, biflavonoids, and triterpens.

The strain was cultivated in rice medium for one month at $28{ }^{\circ} \mathrm{C}$ in resting culture. The fungal culture was then extracted with ethyl acetate and the solvent was 
evaporated under vacuum. TLC of crude extract showed blue, red and green colour reactions with anisaldehyde/sulphuric acid. The crude extract was subjected to column chromatography on silica gel. This fungus produced two major bioactive compounds namely, 1233-A (111) and ergosterol peroxide (112), and a mixture of other sterols, which was not further separated.

\subsubsection{3-A}

Compound 111 was isolated as a UV absorbing colourless oil. The ${ }^{1} \mathrm{H}$ NMR spectrum exhibited two singlets of olefinic protons at $\delta 5.73$ and 5.68. The resonances at $\delta 4.59,3.41$ and 1.62 were assigned as three methine groups, and signals of an ABX system were observed at $\delta 4.05 / 3.88$ and 2.09. The remaining signals of five methylene and three methyl groups appeared between $\delta 2.24-0.84$.

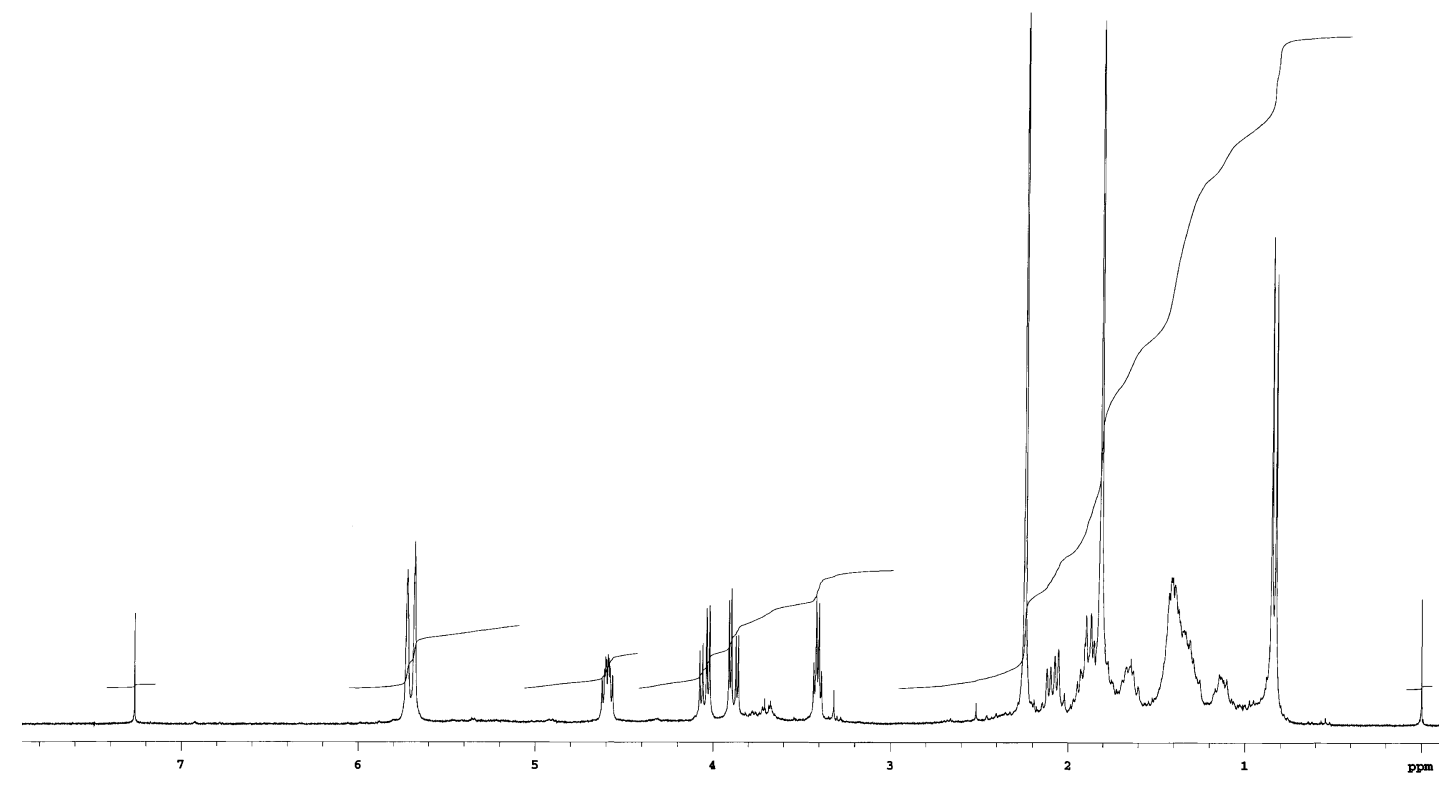

Figure 77: $\quad{ }^{1} \mathrm{H}$ NMR spectrum $\left(\mathrm{CDCl}_{3}, 300 \mathrm{MHz}\right)$ of 1233-A (111)

The ${ }^{13} \mathrm{C}$ NMR spectrum exhibited 18 carbon signals: Two of them at $\delta 171.6$ and 169.9 were due to acid carbonyls. Four olefinic carbon signals appeared at 156.8 $\left(\mathrm{C}_{\mathrm{q}}\right), 141.9\left(\mathrm{C}_{\mathrm{q}}\right), 129.4(\mathrm{CH})$, and $116.7(\mathrm{CH})$. The remaining carbon signals were assigned to three methine, six methylene and three methyl groups. The ESI mass spectrum delivered the molecular weight of 324 Dalton calculating for $\mathrm{C}_{18} \mathrm{H}_{28} \mathrm{O}_{5}$. A sub-structure search in AntiBase based on the molecular formula delivered 13 hits. After filtering with the ${ }^{1} \mathrm{H}$ and ${ }^{13} \mathrm{C}$ NMR data, only one structure was left. The struc- 
ture was finally confirmed as 1233-A (111) by comparison with authentic spectra and the literature data. ${ }^{[177]}$

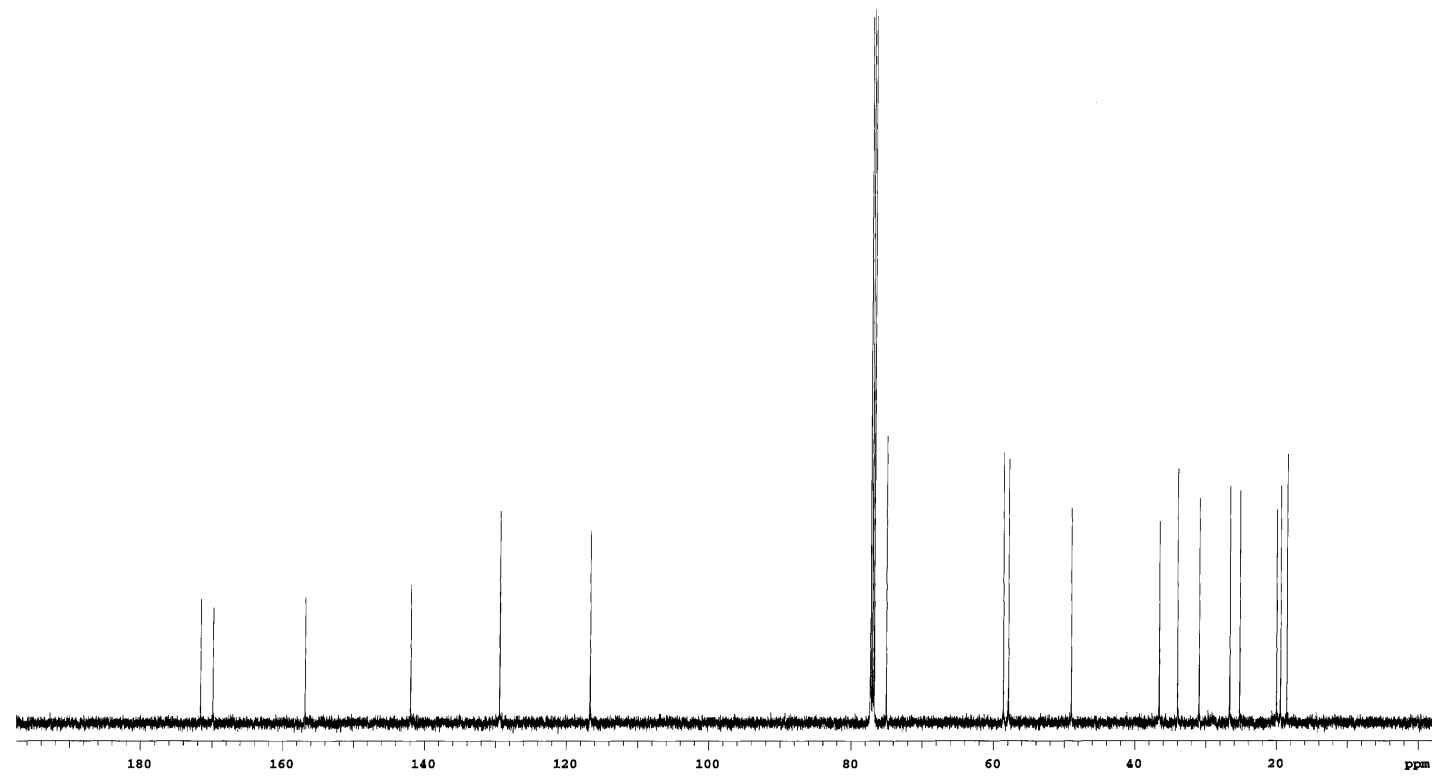

Figure 78: $\quad{ }^{13} \mathrm{C} \mathrm{NMR}$ spectrum $\left(\mathrm{CDCl}_{3}, 125 \mathrm{MHz}\right)$ of $1233-\mathrm{A}(\mathbf{1 1 1})$<smiles>CC(=CC(=O)O)C=C(C)CC(C[18OH])CCCCCC1OC(=O)C1CO</smiles>

111

Compound 111 has been reported as one of $43 \beta$-lactone antibiotics from nature. ${ }^{[88]}$ The $\beta$-lactone 1233-A (111) was isolated first time from the fungus Cephalosporium sp. ACC 1233. ${ }^{[178]}$ It exhibited activity as inhibitor of 3-hydroxy-3methylglutaryl coenzyme A synthase and cholesterol biosynthesis. ${ }^{[177,178]}$ Recently, Pascale and co-worker have reported that $\beta$-lactone derivatives are potent inactivators of homoserine transacetylase (HTA) in searching new antimicrobial agents. ${ }^{[179]}$ The biosynthesis of 1233-A (111) proceeds on the polyketide pathway via condensation of seven acetate units. ${ }^{[180]}$

\subsubsection{Ergosterol peroxide}

Ergosterol peroxide (112) was obtained as a UV active white solid, which turned blue on heating with anisaldehyde/sulphuric acid. It was isolated from the middle 
polar fraction and its molecular formula was established as $\mathrm{C}_{28} \mathrm{H}_{44} \mathrm{O}_{3} \mathrm{Na}$ by HRESI MS. In the ${ }^{1} \mathrm{H}$ NMR spectrum, four mutually coupled olefinic protons were assigned to $\mathrm{H}-6, \mathrm{H}-7, \mathrm{H}-22$ and $\mathrm{H}-23$, respectively. The multiplet at $\delta 3.97$ was characteristic for the oxygenated methine $\mathrm{H}-3$ of ergosterol derivatives.

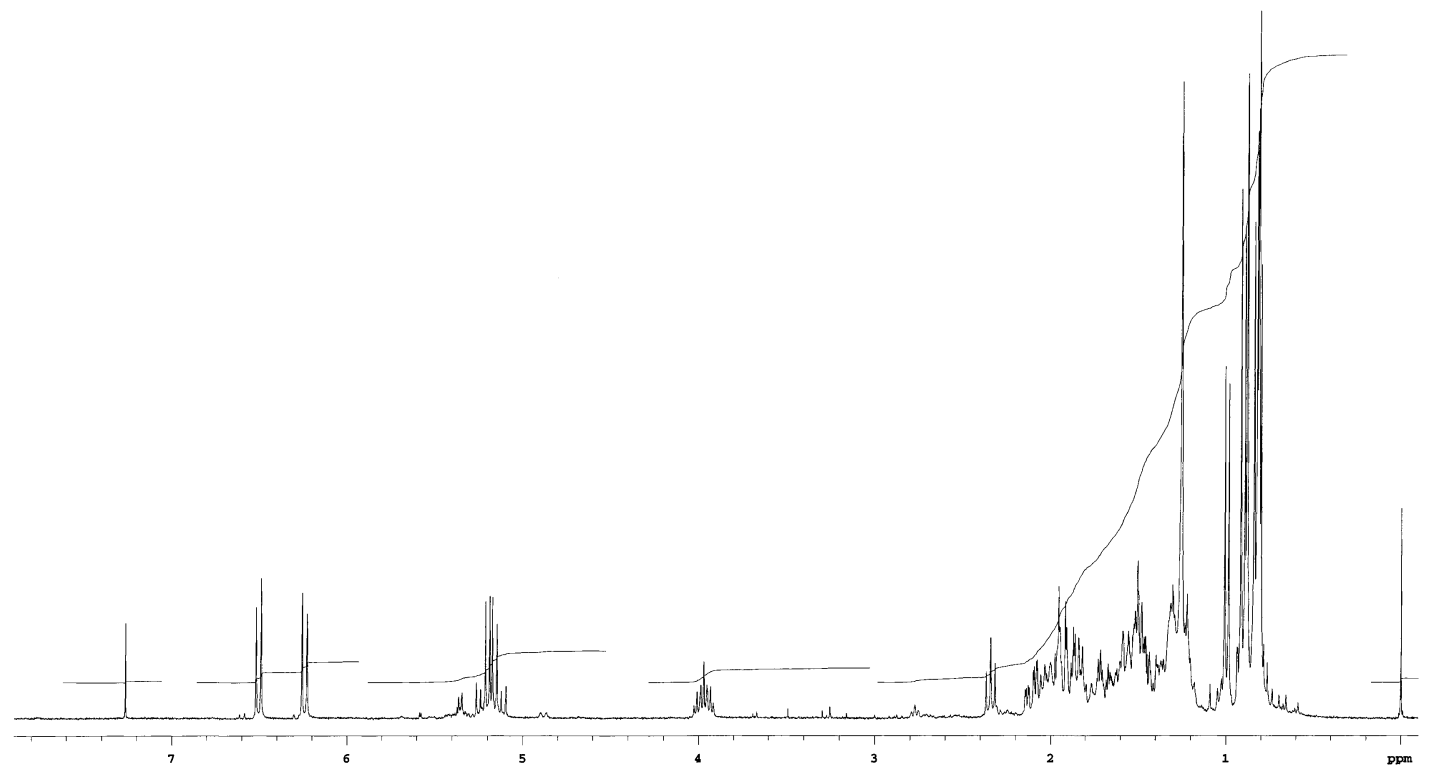

Figure 79: $\quad{ }^{1} \mathrm{H}$ NMR spectrum $\left(\mathrm{CDCl}_{3}, 300 \mathrm{MHz}\right)$ of ergosterol peroxide (112)

Twenty eight signals were observed in the ${ }^{13} \mathrm{C}$ NMR spectrum consisting of four olefinic carbons, two oxygenated quaternary, and one oxygenated methine $\left(\delta_{C} 66.5\right)$. The remaining signals between $\delta 56.2$ and 12.9, were assigned to seven methylene, six methine, six methyl and two quaternary carbon atoms. A search in AntiBase on the basis of the molecular formula, $\mathrm{C}_{28} \mathrm{H}_{44} \mathrm{O}_{3}$, delivered 15 hits. After filtering with the ${ }^{1} \mathrm{H}$ and ${ }^{13} \mathrm{C}$ NMR data, three structures were left. The structure was finally confirmed as ergosterol peroxide (112) by comparison with authentic spectra and the literature data. ${ }^{[181]}$ 


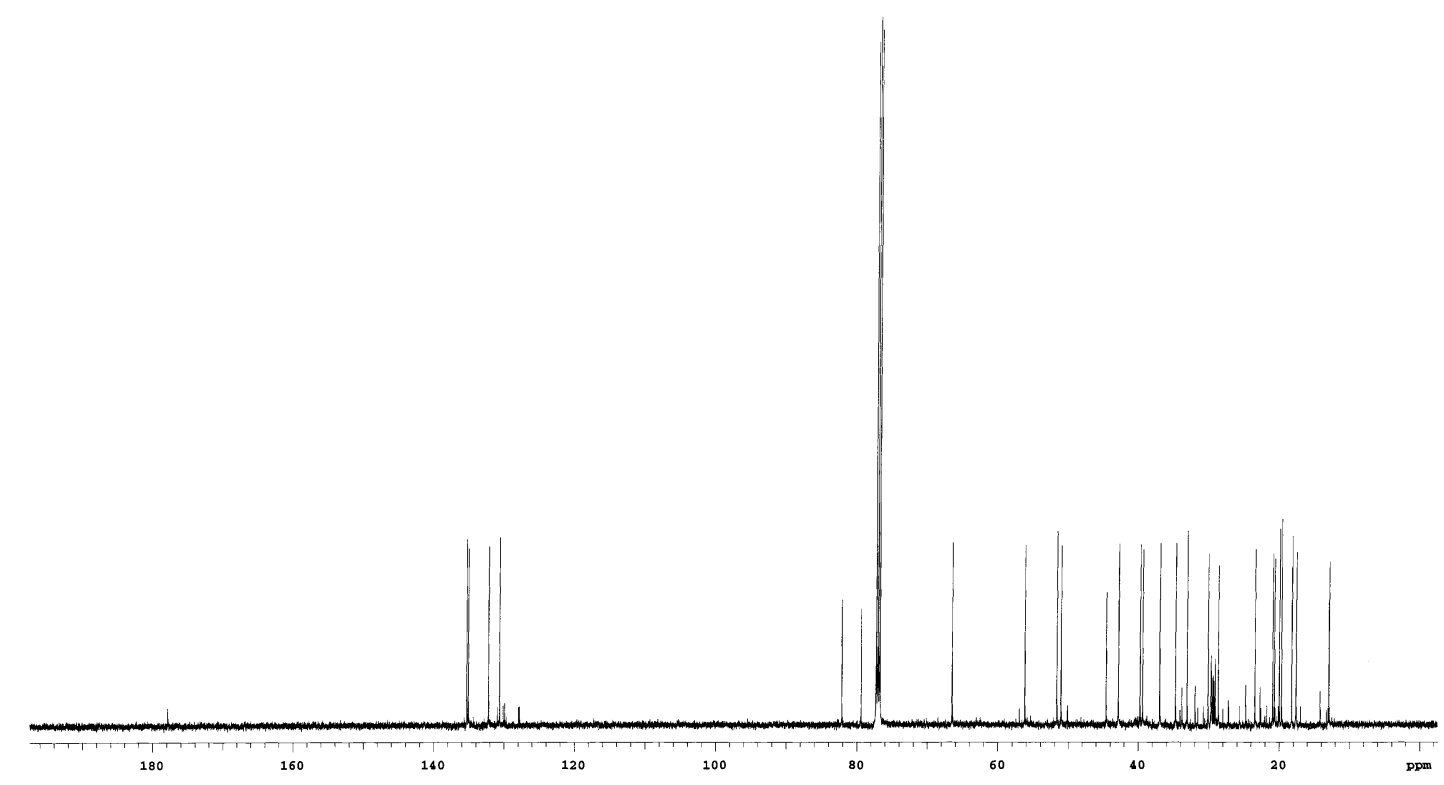

Figure 80: $\quad{ }^{13} \mathrm{C}$ NMR spectrum $\left(\mathrm{CDCl}_{3}, 125 \mathrm{MHz}\right)$ of ergosterol peroxide (112)

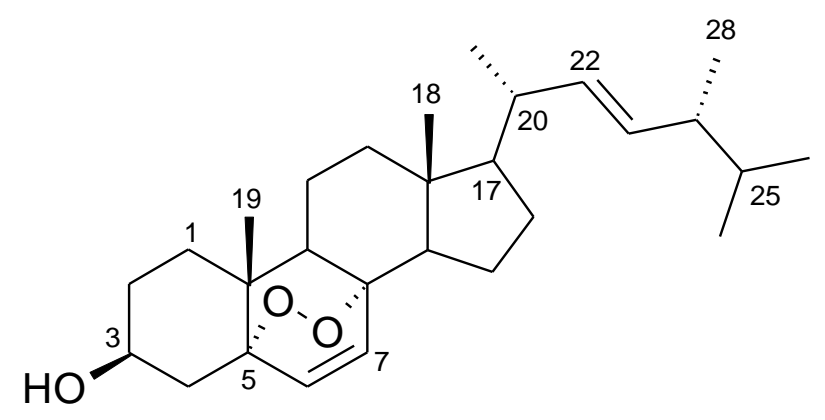

112

Ergosterol peroxide (112) has been reported to show cytotoxic activity against several human tumor cell lines and antibacterial activity against Mycobacterium tuberculosis. ${ }^{[182,183]}$ In addition, ergosterol peroxide (112) has been reported as a synthetic product by oxidation of ergosterol in the presence of ultraviolet light (Figure 81). ${ }^{[184]}$ 


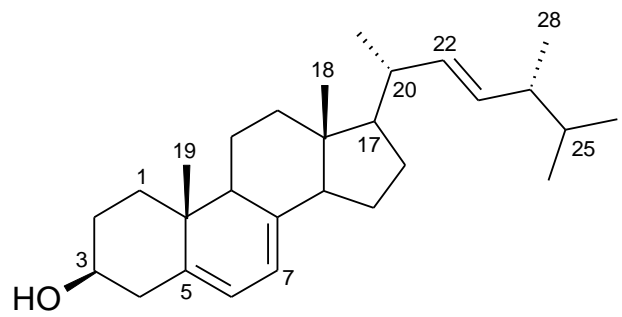

Ergosterol

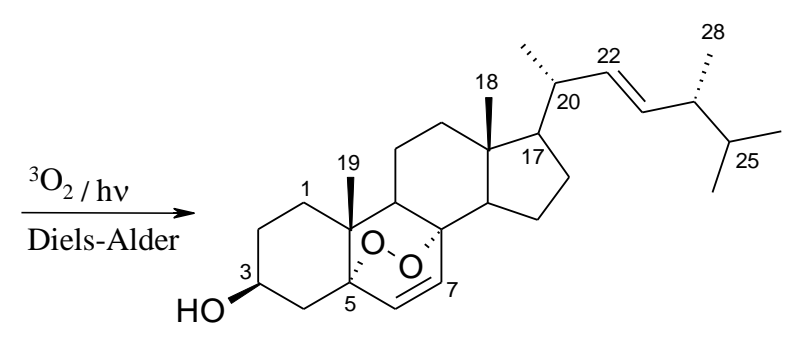

Ergosterol peroxide

Figure 81: $\quad$ Proposed reaction for synthesis of ergosterol peroxide (112)

\subsection{Endophytic fungus FTM1}

The endophytic fungus strain FTM1 was isolated from the Cameroonian medicinal plant Endodesmia calophylloides (Clusiaceae) collected from Balmayo in the Centre Province.

The strain was cultivated in rice medium for one month at $28{ }^{\circ} \mathrm{C}$ in resting culture. The fungal culture was then extracted with ethyl acetate and the solvent was evaporated under vacuum. TLC of the crude extract revealed UV absorbing bands, which turned blue, grey and violet with anisaldehyde/sulphuric acid. The crude extract was subjected to column chromatography on silica gel. Three compounds were isolated and purified by different chromatographic methods, namely 8-hydroxy-6,7dimethoxy-3-methylisocoumarin (113), 5-methylochracin (114) and alternariol monomethyl ether (115), respectively. 


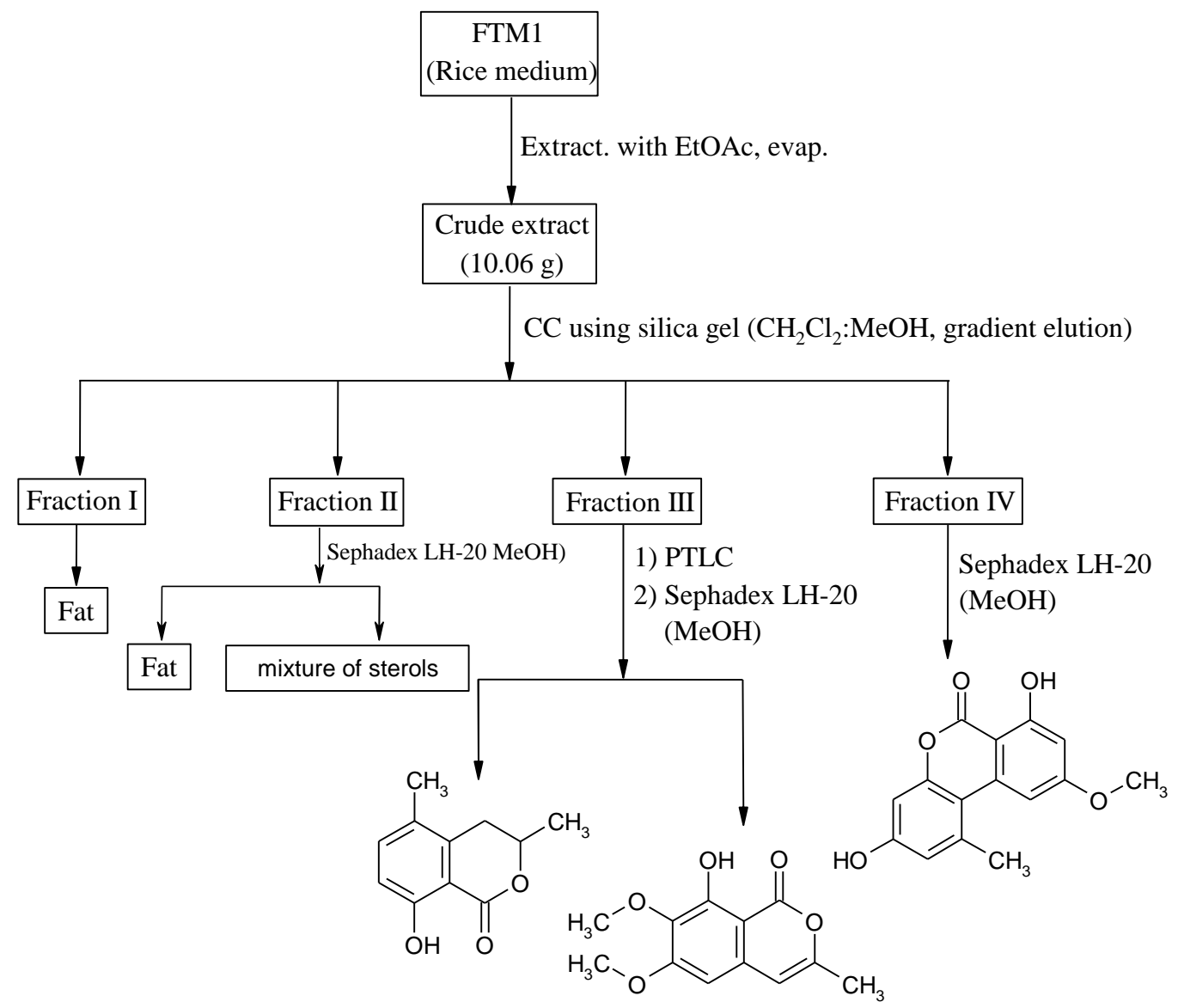

Figure 82: Work-up scheme for the endophytic fungus FTM1

\subsubsection{8-Hydroxy-6,7-dimethoxy-3-methylisocoumarin}

8-Hydroxy-6,7-dimethoxy-3-methylisocoumarin (113) was isolated as white solid, which showed a UV absorbing band at $254 \mathrm{~nm}$ on TLC. The ${ }^{1} \mathrm{H}$ NMR spectrum exhibited five singlets at $\delta 6.33,6.19,3.95,3.91$ and 2.27. Three singlets observed in the aliphatic region at $\delta 3.95,3.91$ and 2.27 were assigned for two methoxy and one methyl groups. In addition, an H/D exchangeable broad singlet of a hydroxyl group appeared at $\delta 11.07$. The ${ }^{13} \mathrm{C}$ NMR spectrum displayed two methoxy groups, one methyl, eight $s p^{2}$ carbons, and a carbonyl function. The high-resolution (+)-ESI mass analysis showed a pseudomolecular ion at $m / z 259.05780\left([\mathrm{M}+\mathrm{Na}]^{+}\right)$, indicative of a molecular formula $\mathrm{C}_{12} \mathrm{H}_{12} \mathrm{O}_{5} \mathrm{Na}$. A search in AntiBase based solely on the molecular formula delivered 20 hits. After filtering with the ${ }^{1} \mathrm{H}$ and ${ }^{13} \mathrm{C}$ NMR data, only 8-hydroxy-6,7-dimethoxy-3-methylisocoumarin and 8-O-methylreticulol were left. The structure was finally confirmed as 8-hydroxy-6,7-dimethoxy-3-methylisocoumarin (113) by comparison with authentic spectra and literature data. ${ }^{[185]}$ 


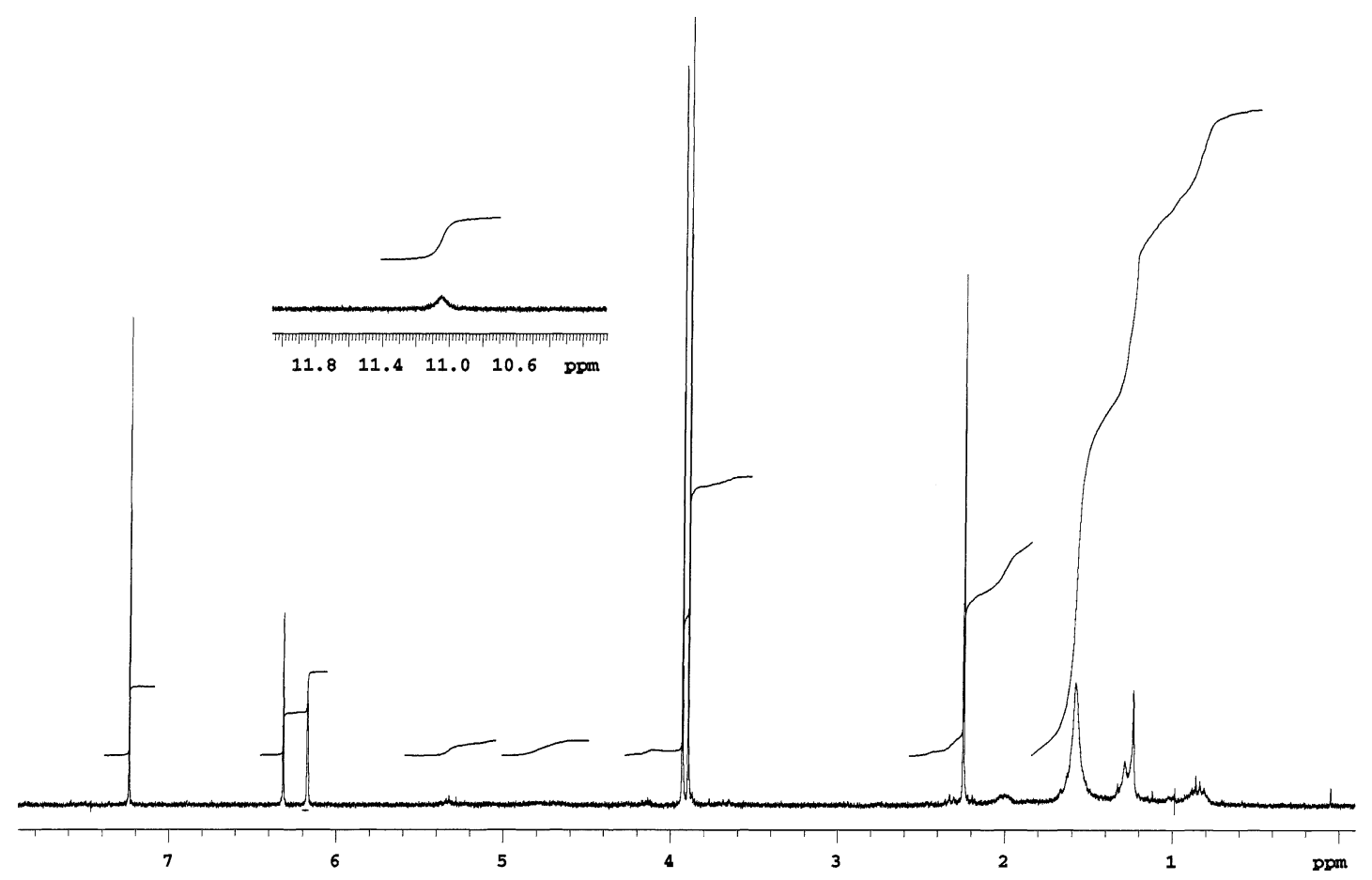

Figure 83: $\quad{ }^{1} \mathrm{H}$ NMR spectrum $\left(\mathrm{CDCl}_{3}, 300 \mathrm{MHz}\right)$ of 8-hydroxy-6,7-dimethoxy-3methylisocoumarin (113)

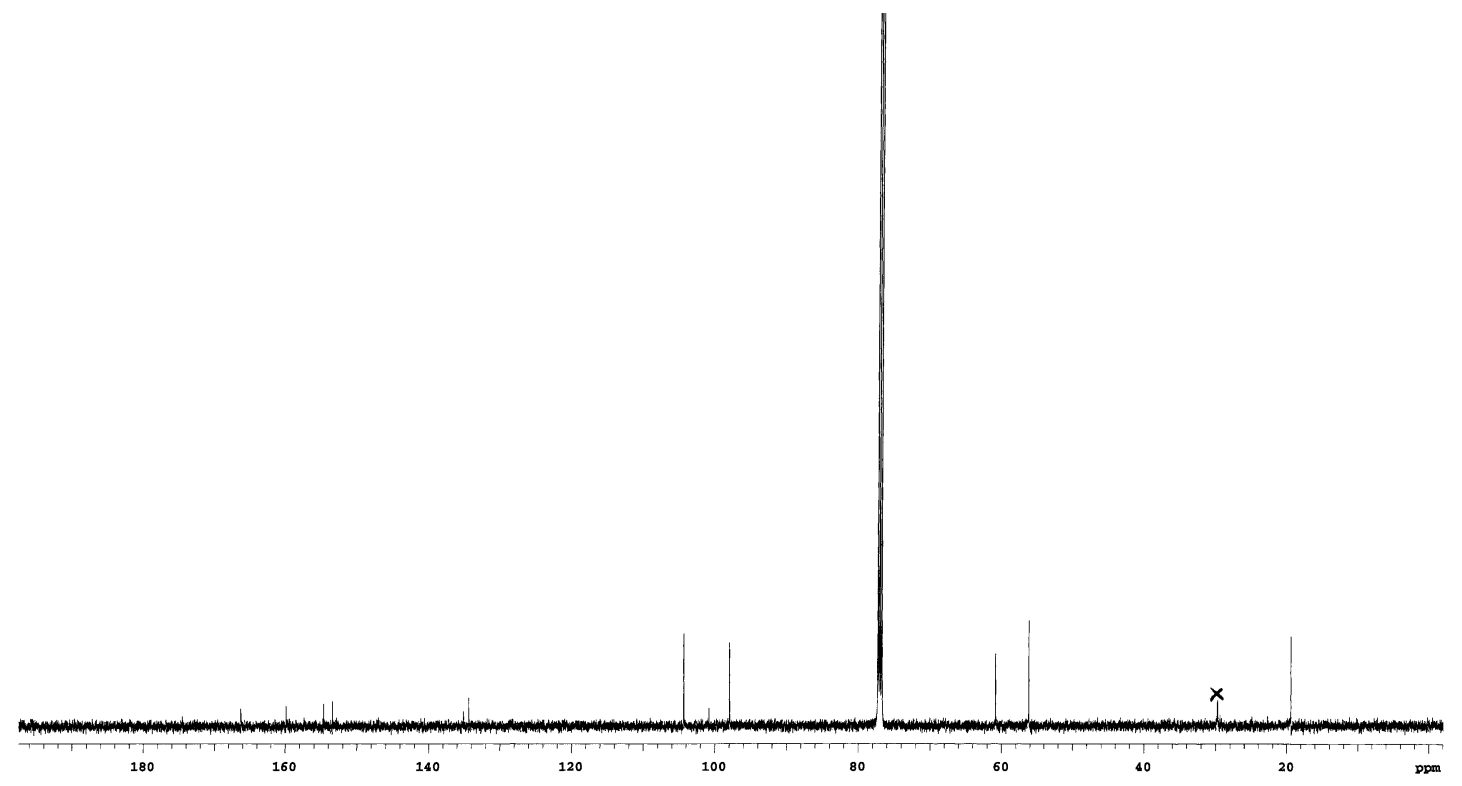

Figure 84: ${ }^{13} \mathrm{C}$ NMR spectrum $\left(\mathrm{CDCl}_{3}, 125 \mathrm{MHz}\right)$ of 8-hydroxy-6,7-dimethoxy3-methylisocoumarin (113) 
<smiles>COc1cc2cc(I)oc(=O)c2c(O)c1OC</smiles>

\section{3}

8-Hydroxy-6,7-dimethoxy-3-methylisocoumarin (113) has been isolated previously from Streptomyces mobaraensis. ${ }^{[186]}$ Recently, Boonlarppradab and coworkers have reported that 8-hydroxy-6,7-dimethoxy-3-methylisocoumarin (113) showed cytotoxic activities against MCF-7, KB and NCI-H187 cell lines. ${ }^{[187]} 8$ Hydroxy-6,7-dimethoxy-3-methylisocoumarin (113) has been reported also as inhibitor of phosphodiesterase and topoisomerase I. $^{[188]}$

\subsubsection{5-Methylochracin}

Compound 114 was isolated as white solid, which showed a UV absorbing zone at $254 \mathrm{~nm}$ and stained to grey on heating with anisaldehyde/sulphuric acid. The (+)-HRESI mass analysis showed a pseudo-molecular ion at $\mathrm{m} / z, 215.2010([\mathrm{M}+$ $\mathrm{Na}]^{+}$), indicating a molecular formula of $\mathrm{C}_{11} \mathrm{H}_{12} \mathrm{O}_{3} \mathrm{Na}$. 


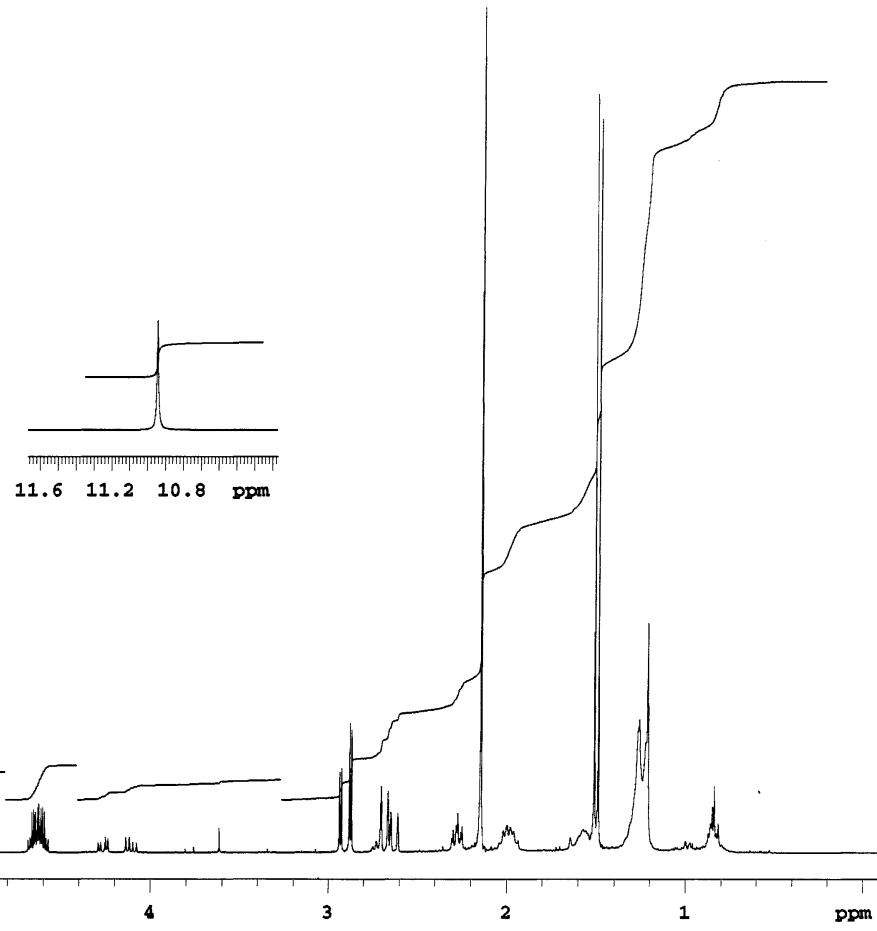

Figure 85: $\quad{ }^{1} \mathrm{H}$ NMR spectrum $\left(\mathrm{CDCl}_{3}, 300 \mathrm{MHz}\right)$ of 5-methylochracin (114)

In the ${ }^{1} \mathrm{H}$ NMR spectrum, two AB-type aromatic signals appeared at $\delta 7.24(\mathrm{~d}$, $\left.1 \mathrm{H},{ }^{3} J=8.5 \mathrm{~Hz}, \mathrm{H}-6\right)$ and $6.76\left(\mathrm{~d}, 1 \mathrm{H},{ }^{3} J=8.5 \mathrm{~Hz}, \mathrm{H}-7\right)$, suggesting a $1,2,3,4-$ tetrasubstituted benzene. In addition, signals of two methyls at $\delta 2.16$ and 1.51 , one methylene at $\delta 2.91$ and 2.67, and one methine at $\delta 4.64$ were observed. The ${ }^{13} \mathrm{C}$ NMR spectrum exhibited six signals for aromatic carbons including a phenolic one, the presence of an oxymethine and a methylene carbon at $\delta 75.4$ and 31.9 , two methyl groups at $\delta 20.9$ and 18.1, and a carbonyl group at $\delta 170.1$, respectively. A substructure search in AntiBase based on the molecular formula delivered 30 hits. After filtering with the ${ }^{1} \mathrm{H}$ and ${ }^{13} \mathrm{C}$ NMR data, only 5- and 7-methylmellein were left. The structure 5-methylochracin (5-methylmellein, 114) was finally confirmed by comparison with authentic spectra and the literature data. ${ }^{[189]}$ 


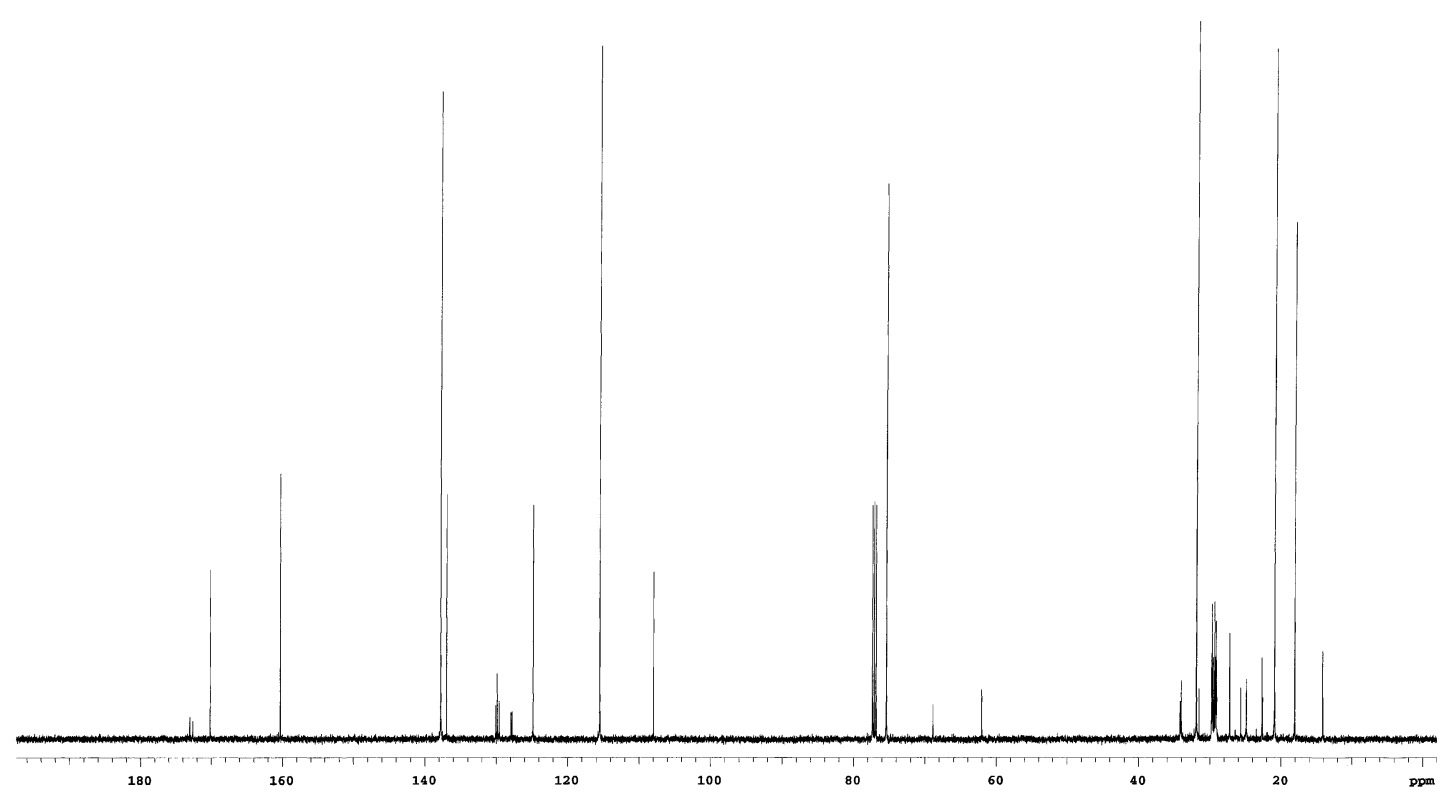

Figure 86: $\quad{ }^{13} \mathrm{C}$ NMR spectrum $\left(\mathrm{CDCl}_{3}, 125 \mathrm{MHz}\right)$ of 5-methylochracin (114)<smiles>Cc1ccc(O)c2c1CC(C)OC2=O</smiles>

\section{4}

5-Methylochracin (114) has been isolated first time from a plant pathogenic fungus Fusicoccum amygdali Del. ${ }^{[190,191]}$ It showed selective antiparasitic activity against Leishmania donovani ${ }^{[192]}$ and antifeedant activity against the cotton leafworm Spodoptera littoralis ${ }^{[193]}$ but it showed no phytotoxicity in vitro. ${ }^{[192]}$

\subsubsection{Alternariol 5-methyl ether (AME)}

Fraction IV was subjected to column chromatography on Sephadex LH-20 to obtain compound $\mathbf{1 1 5}$ as reddish-white solid. It revealed a UV absorbing band at 254 $\mathrm{nm}$, which turned violet with anisaldehyde/sulphuric acid. The HRESI mass analysis exhibited a peak at $m / z 271.06120[\mathrm{M}-\mathrm{H}]^{-}$, indicating a molecular formula of $\mathrm{C}_{15} \mathrm{H}_{11} \mathrm{O}_{5}$. 


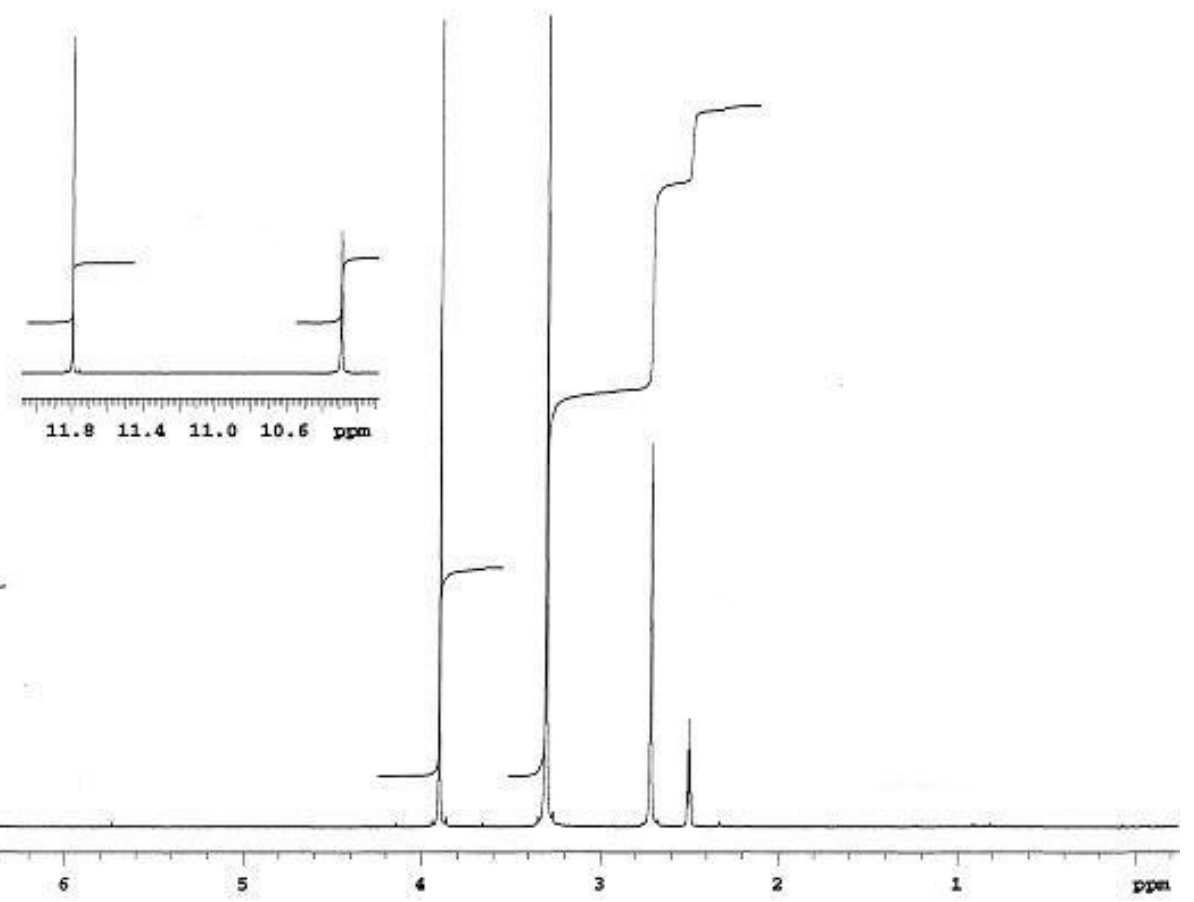

Figure 87: ${ }^{1} \mathrm{H}$ NMR spectrum (DMSO- $d_{6}, 300 \mathrm{MHz}$ ) of alternariol-5-methyl ether (115).

The ${ }^{1} \mathrm{H}$ NMR spectrum showed two pairs of meta-positioned aromatic protons at $\delta 7.19$ and $6.59(\mathrm{~d}, J=2.0 \mathrm{~Hz}), \delta 6.72$ and $6.63(\mathrm{~d}, J=2.5 \mathrm{~Hz})$, a signal of a chelated hydroxyl group at $\delta 11.80$ (s), and two singlet signals of methoxy and methyl groups at $\delta 3.90$ and 2.72. The ${ }^{13} \mathrm{C}$ NMR spectrum displayed 15 carbon atom signals, including a carbonyl group, twelve aromatic carbons, one methoxy, and one methyl group. A search in AntiBase based on the molecular formula delivered 28 hits. After filtering with the ${ }^{1} \mathrm{H}$ and ${ }^{13} \mathrm{C}$ NMR spectroscopic data, alternariol-monomethyl ether (115) was suggested. The structure $\mathbf{1 1 5}$ was finally confirmed by comparing with authentic spectra and literature data. ${ }^{[194]}$ 


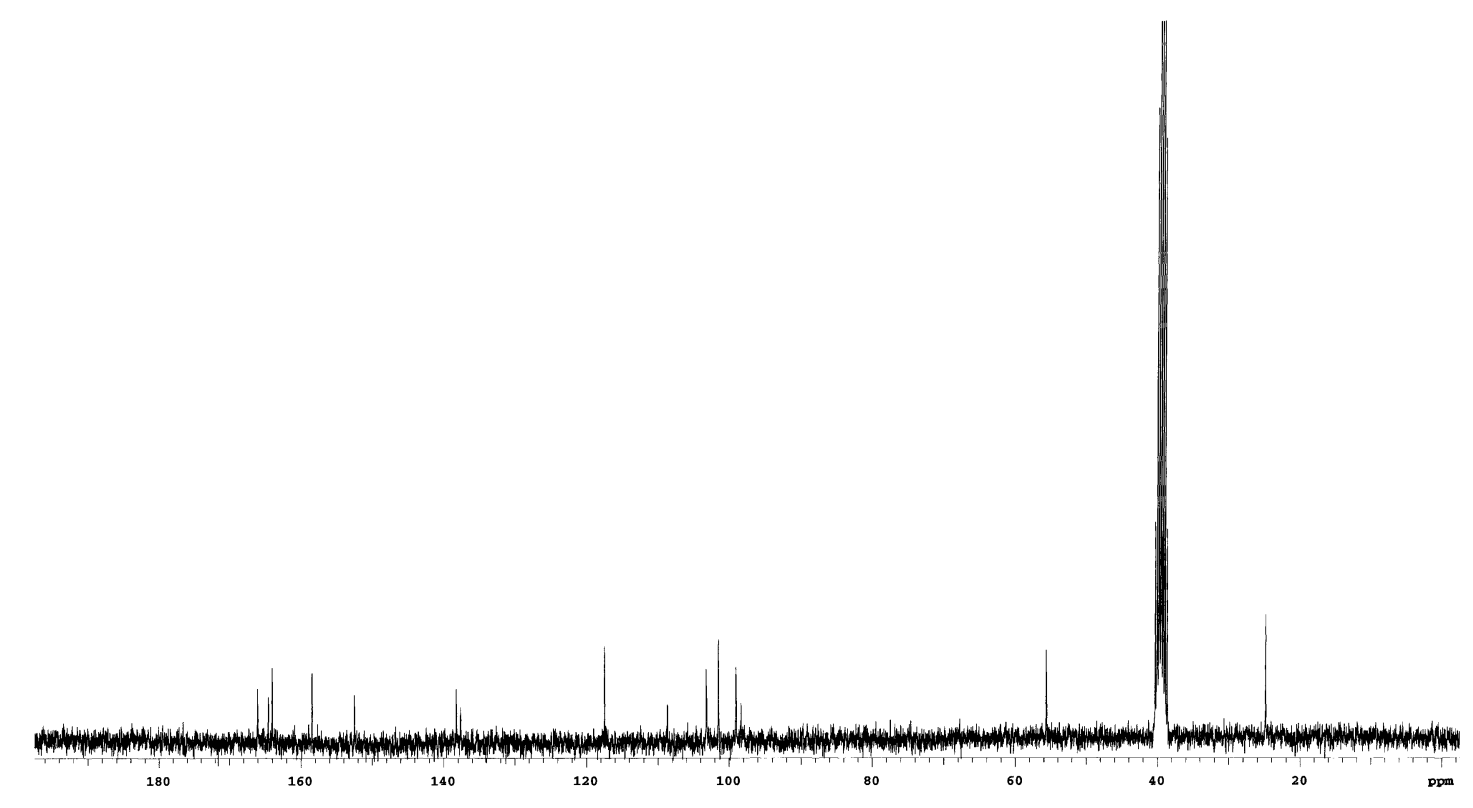

Figure 88: ${ }^{13} \mathrm{C}$ NMR spectrum (DMSO- $d_{6}, 75 \mathrm{MHz}$ ) of alternariol-5-methyl ether (115).<smiles>COc1cc(O)c2c(=O)oc3cc(O)cc(F)c3c2c1</smiles>

115

Alternariol-5-methyl ether (115) had been reported to show cytotoxic activity against KB, KBv200 and L5178Y mouse lymphoma cells. ${ }^{[194,195]}$ The compound showed also inhibitory activity against several protein kinases. ${ }^{[195]}$

\section{Plant metabolites}

Samples from plants were received from our research collaborators, Prof. S. Ganapaty (India) and Prof. Aye Aye Tun (Myanmar). Among others, five compounds were isolated from three different plants from Myanmar, namely Adansonia agitate, Dillenia pentagyna, Kaempferia parviflora; six further samples were isolated from an Indian plant. 


\subsection{Adansonia digitata}

Adansonia digitata belongs to the genus Adansonia in the family of Malvaceae. ${ }^{[196]}$ The genus Adansonia comprises eight species and is distributed in Australia, Africa and Asia. ${ }^{[197]}$ Adansonia digitata has been widely used as medicinal plant and alternative source of food in Africa, Asia and Australia. ${ }^{[197,198]}$ Flavonoids, tannins, steroids, lipids, vitamins, amino acids, carbohydrates and terpenoids have been reported as chemical constituents. ${ }^{[196,197]}$

In the current investigation, three compounds were isolated from this plant and identified by using spectroscopic methods, i.e. garcinon D (116), rubraxanthone (117), and acetyl aleuritolic acid (118).

\subsubsection{Garcinon D}

Compound 116 was obtained as yellow powder and revealed a molecular ion peak at $\mathrm{m} / z 451.17272[\mathrm{M}+\mathrm{Na}]^{+}$in HRESIMS, which corresponds to the sodiated molecular formula $\mathrm{C}_{24} \mathrm{H}_{28} \mathrm{O}_{7} \mathrm{Na}$. The ${ }^{1} \mathrm{H}$ NMR spectrum displayed two singlets of aromatic signals at $\delta 6.78$ and 6.34 and showed the presence of a methoxy group at $\delta$ 3.75. In the aliphatic region, the spectrum exhibited two multiplets at $\delta 3.32(16-\mathrm{H})$ and $1.57(15-\mathrm{H})$. The spectrum showed additionally four methyl groups at $\delta 1.73$, 1.62 and 1.22 , respectively. Furthermore, the signals at $\delta 5.18\left({ }^{3} J=7.1 \mathrm{~Hz}, 1 \mathrm{H}\right)$ and $3.21\left({ }^{3} \mathrm{~J}=7.1 \mathrm{~Hz}, 2 \mathrm{H}\right)$ demonstrated the presence of $s p^{2}$ protons adjacent to a methylene group, which indicated an isoprene unit. 


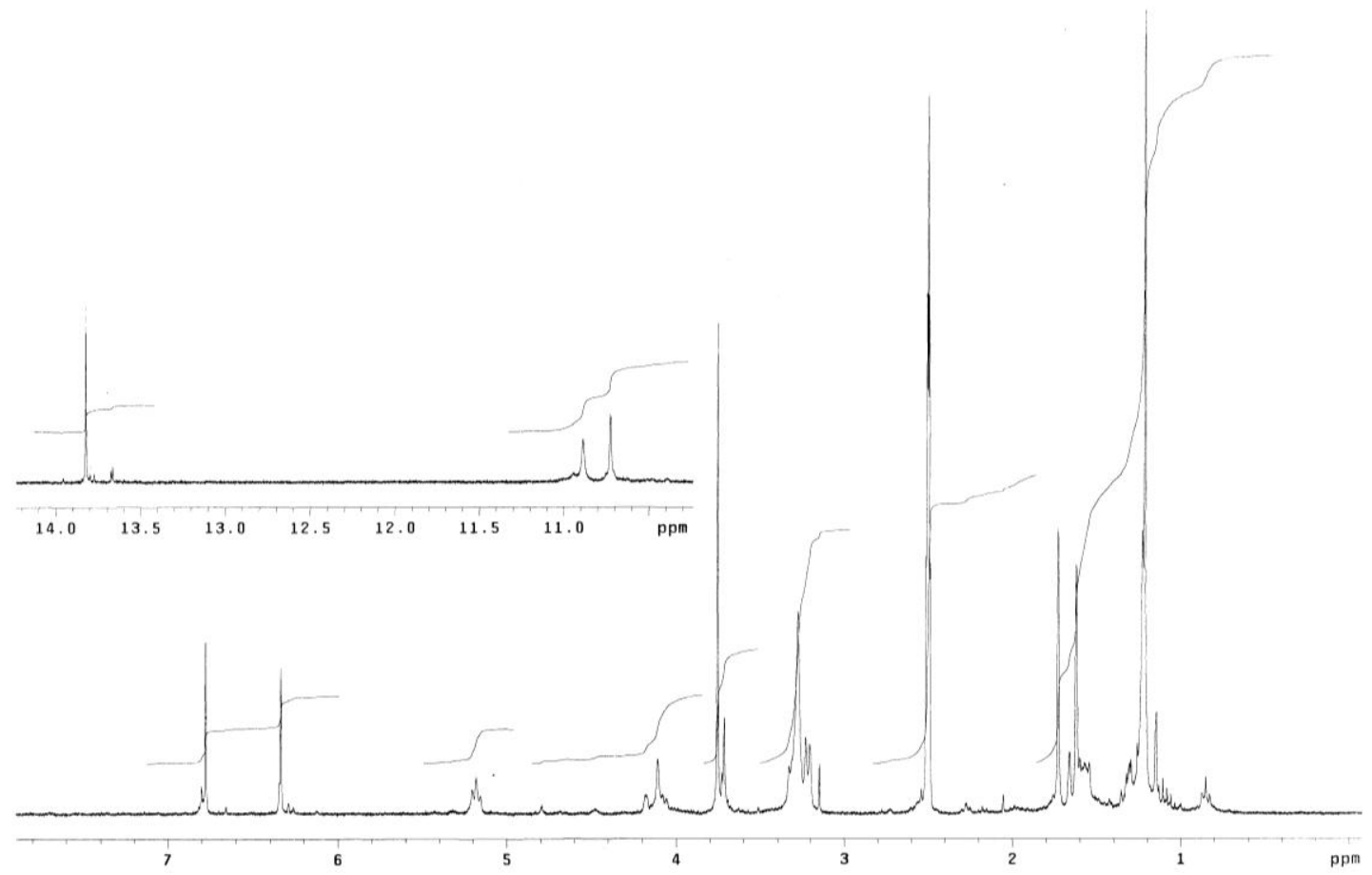

Figure 89: $\quad{ }^{1} \mathrm{H}$ NMR spectrum (DMSO- $d_{6}, 300 \mathrm{MHz}$ ) of garcinon D (116)

The ${ }^{13} \mathrm{C}$ NMR spectrum showed 23 carbon signals which comprised of an $\alpha, \beta-$ unsaturated ketone at $\delta 181.9$, six quaternary $s p^{2}$ carbons between $\delta 162 \sim 143.0$ most likely attached to heteroatoms, four quaternary $s p^{2}$ carbons between $\delta 139 \sim 101$, three $s p^{2}$ methine carbons at $\delta 122.2,101.3,92.0$ and nine $s p^{3}$ carbon atoms between $\delta$ 69.0 17.6. 


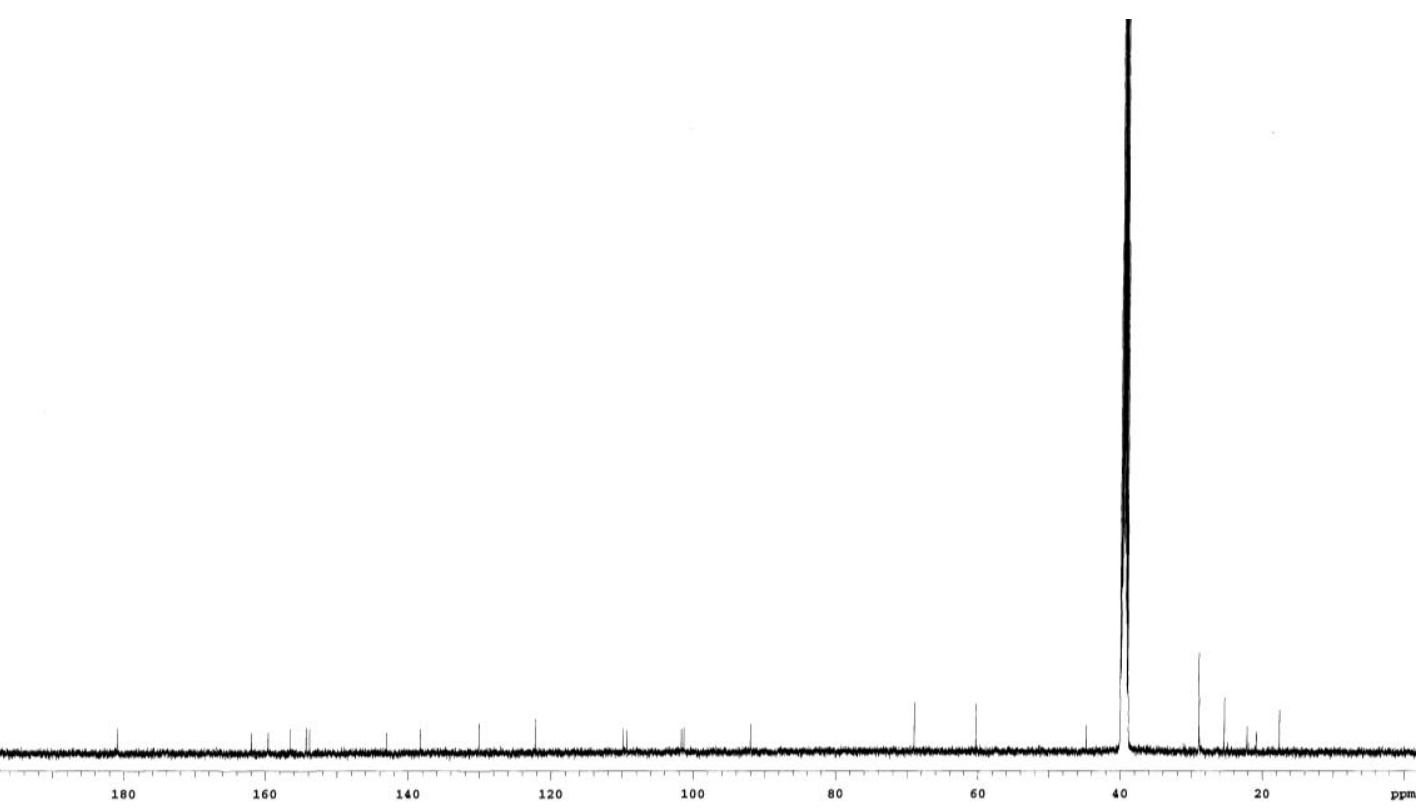

Figure 90: $\quad{ }^{13} \mathrm{C}$ NMR spectrum (DMSO- $d_{6}, 125 \mathrm{MHz}$ ) of garcinon D (116)

The structure was elucidated based on 2D NMR correlations. The HMBC spectrum revealed two correlations of protons at $\delta 6.78$ and 6.34 with the carbonyl carbon at $\delta_{C}$ 181.9. The $s p^{2}$ proton signal at $\delta 6.78$ showed further a strong ${ }^{3} J$ correlation with the quaternary $s p^{2}$ carbon at $\delta_{C} 109.8\left(\mathrm{C}_{\mathrm{q}}-8 \mathrm{a}\right)$; the methyl protons at $\delta 1.22\left(\delta_{C}\right.$ 28.7) exhibited strong ${ }^{2} J$ and ${ }^{3} J$ correlations with the quaternary $s p^{3}$ carbon at $\delta_{C} 69.0$ $\left(\mathrm{C}_{\mathrm{q}}-17\right)$ and a methylene carbon at $\delta_{C} 44.7\left(\mathrm{CH}_{2}-16\right)$. In addition, the $s p^{2}$ methine and methylene protons at $\delta 6.34$ and 3.21 correlated strongly with the quaternary $s p^{2}$ carbon at $\delta_{C} 109.3\left(\mathrm{C}_{\mathrm{q}}-2\right)$. The position of a methoxy group was assigned through the correlation of the proton signal at $\delta 3.75$ with the quaternary $s p^{2}$ carbon at $\delta_{C} 143.1$ $\left(\mathrm{C}_{\mathrm{q}}-7\right)$. A search in AntiBase based on its molecular formula, $\mathrm{C}_{24} \mathrm{H}_{28} \mathrm{O}_{7}$, delivered two hits, however, the spectroscopic data did not match these structures. A search in The Dictionary of Natural Product ${ }^{[80]}$ with the molecular formula delivered garcinon D (116) as a structure, which and was further confirmed by comparing the spectroscopic values with the literature data. ${ }^{[199]}$ 


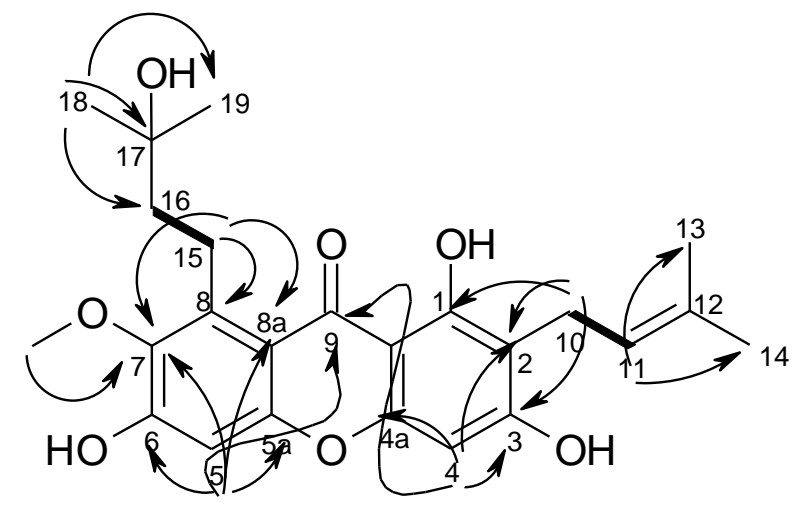

116

Figure 91: Selected ${ }^{1} \mathrm{H}-{ }^{1} \mathrm{H}$ COSY $(-)$ and $\operatorname{HMBC}(\longrightarrow)$ correlations of garcinon D (116)

Garcinon D (116) is a member of xanthone-related compounds isolated from Garcinia mangostana ${ }^{[200]}$ It showed cytotoxic activity against the HT-29 human colon cancer, epidermoid carcinoma (KB), and breast cancer (BC-1) cell lines. ${ }^{[199]}$

\subsubsection{Rubraxanthone}

Rubraxanthone (117) was obtained as yellow compound with a pseudomolecular ion peak at $\mathrm{m} / z 433.162196[\mathrm{M}+\mathrm{Na}]^{+}$in HRESIMS, which corresponds to the sodiated molecular formula $\mathrm{C}_{24} \mathrm{H}_{26} \mathrm{O}_{6} \mathrm{Na}$. In the aromatic region of the ${ }^{1} \mathrm{H} \mathrm{NMR}$ spectrum, it showed two meta-coupled aromatic protons at $\delta 6.03$ and $6.09(J=2.2$ $\mathrm{Hz}$ ) for $\mathrm{H}-2$ and $\mathrm{H}-4$, and a singlet at $\delta 6.60$ for $\mathrm{H}-5$. A resonance of a methoxy group appeared at $\delta 3.73$. Three singlets at $\delta 1.49,1.52$ and 1.78 corresponded to three methyl groups of a geranyl moiety. The presence of the geranyl skeleton was shown by the deshielded resonance of a methylene group $\left(\mathrm{CH}_{2}-10\right)$ at $\delta 4.00$, which is in a peri position to a carbonyl group. HMBC correlations (Figure 94) showed that the methoxy protons at $\delta 3.73$ gave a strong correlation to the quaternary $s p^{2}$ carbon at $\delta_{\mathrm{C}} 144.7$ (C-7). In addition, the methylene protons at $\delta 4.00$ revealed two ${ }^{3} J$ correlations with two quaternary $s p^{2}$ carbons at 112.1 (C-8a) and 144.7 (C-7). Finally, the structure was assigned as rubraxanthone (117) on the basis of spectroscopic and mass analysis data and was further confirmed by comparing with literature values. ${ }^{[201]}$ 


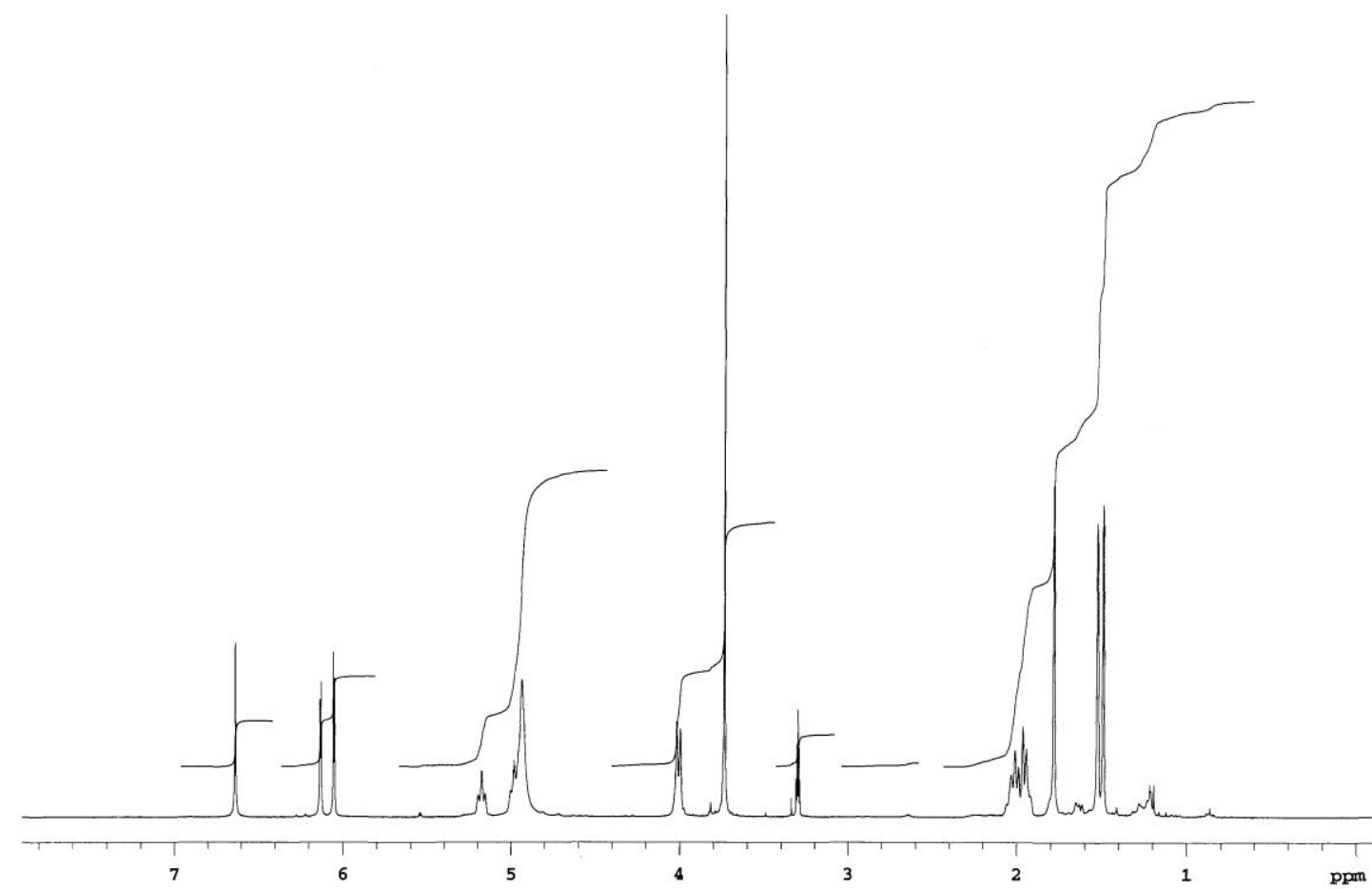

Figure 92: $\quad{ }^{1} \mathrm{H}$ NMR spectrum $\left(\mathrm{CD}_{3} \mathrm{OD}, 300 \mathrm{MHz}\right)$ of rubraxanthone (117)

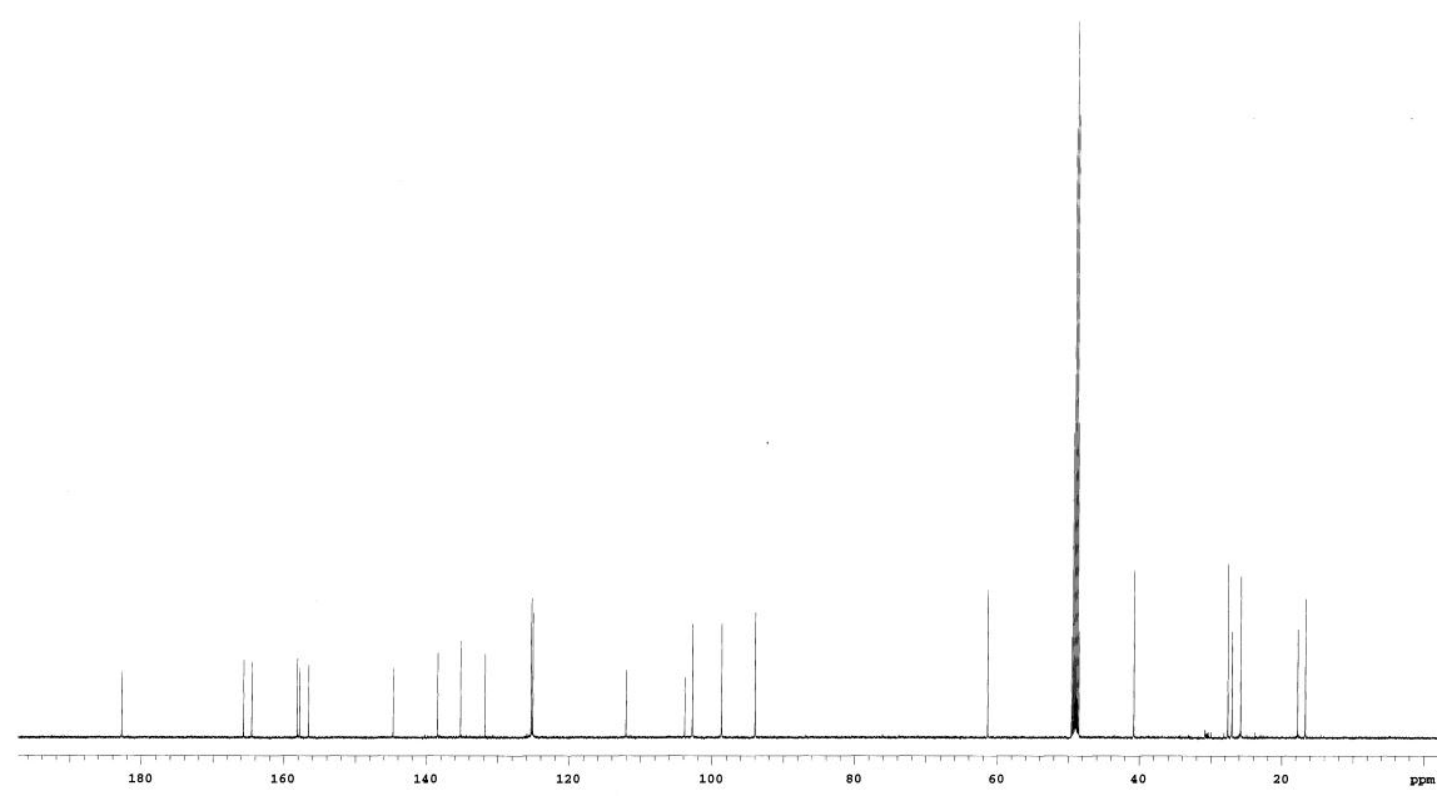

Figure 93: $\quad{ }^{13} \mathrm{C}$ NMR spectrum $\left(\mathrm{CD}_{3} \mathrm{OD}, 300 \mathrm{MHz}\right)$ of rubraxanthone (117) 


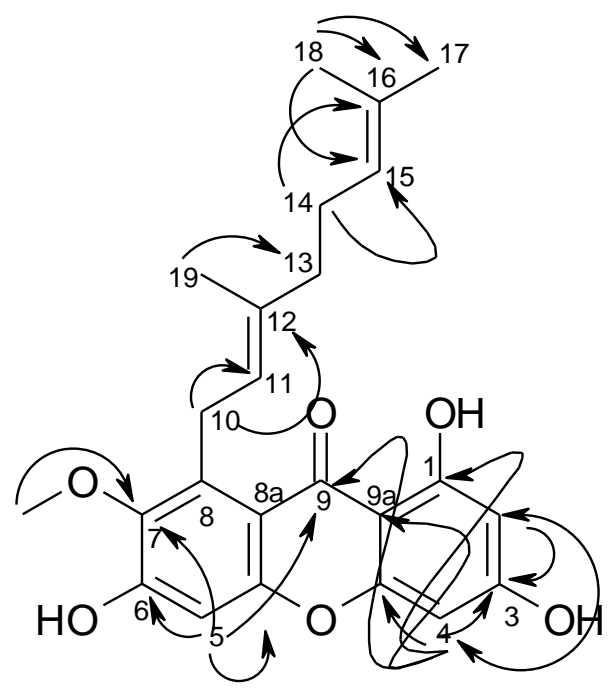

117

Figure 94: Selected ${ }^{1} \mathrm{H}_{-}{ }^{1} \mathrm{H} \operatorname{COSY}(-)$ and $\mathrm{HMBC}(\longrightarrow)$ correlations of rubraxanthone (117).

Rubraxanthone $^{[201]}$ (117) has been isolated firstly from Garcinia rubra. ${ }^{\text {[202]; }}$; reported to show cytotoxicity against HL-60 cells. ${ }^{[203]}$ In addition, Jantan and coworkers have reported that rubraxanthone (117) exhibited inhibitory effects on platelet-activating factor binding to rabbit platelets. ${ }^{[204]}$

\subsubsection{Acetylaleuritolic acid}

Acetyl aleuritolic acid (118) was isolated as white powder; the HRESI mass analysis gave an ion at $\mathrm{m} / z 521.36066\left([\mathrm{M}+\mathrm{Na}]^{+}\right)$, calculating for $\mathrm{C}_{32} \mathrm{H}_{50} \mathrm{O}_{4} \mathrm{Na}$. The ${ }^{1} \mathrm{H}$ NMR spectrum showed an olefinic proton at $\delta 5.20(15-\mathrm{H})$. A multiplet at $\delta 4.50$ was assigned to the proton signal of the oxygenated position at C-3 in a steroid skeleton; this was additionally supported by a ${ }^{13} \mathrm{C}$ NMR resonance at $\delta_{C} 80.8(\mathrm{CH}-3)$. A series of methylene and methyl signals was observed between $\delta 2.80-0.70$. 


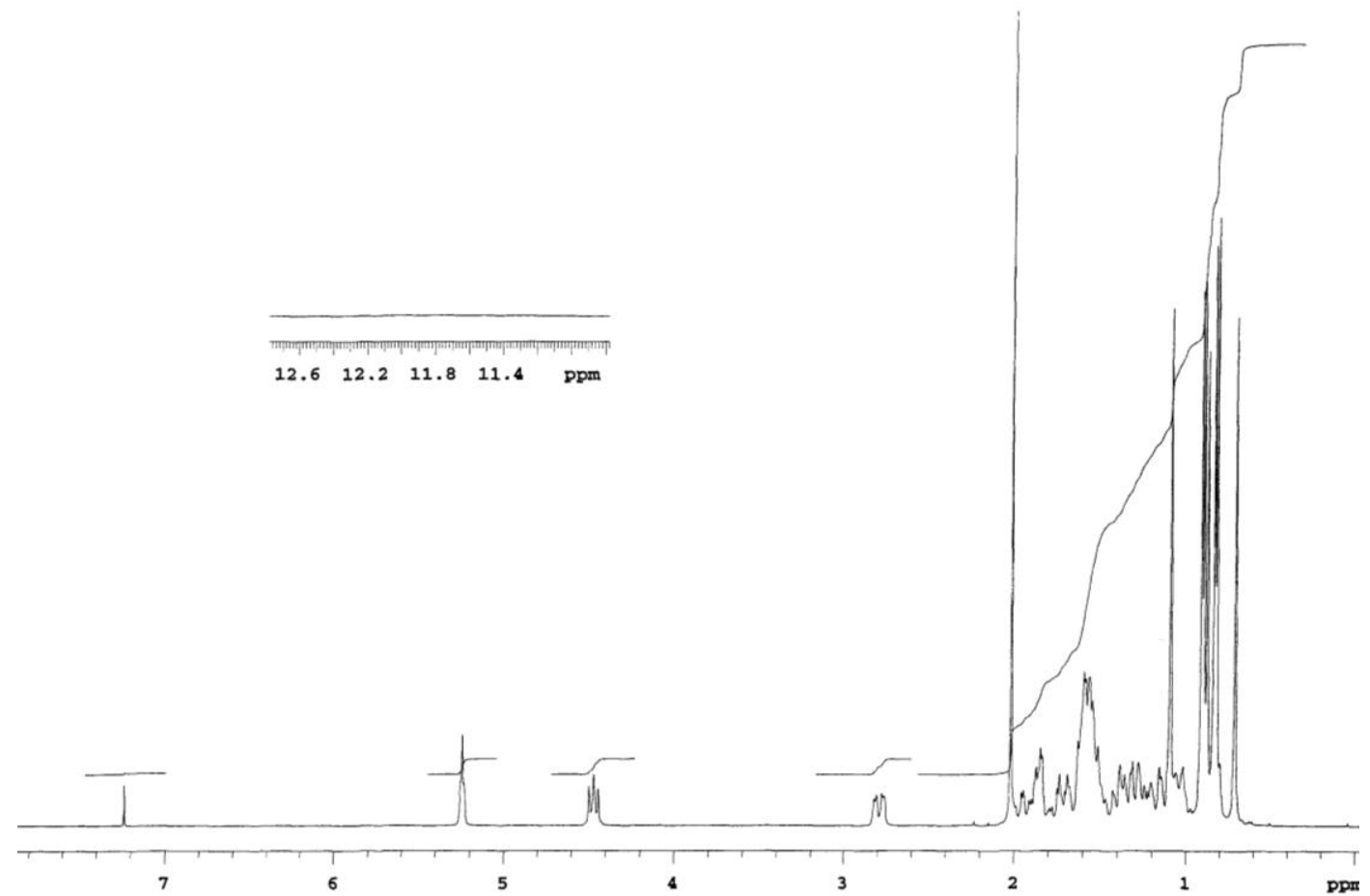

Figure 95: $\quad{ }^{1} \mathrm{H}$ NMR spectrum $\left(\mathrm{CDCl}_{3}, 300 \mathrm{MHz}\right)$ of acetylaleuritolic acid (118)

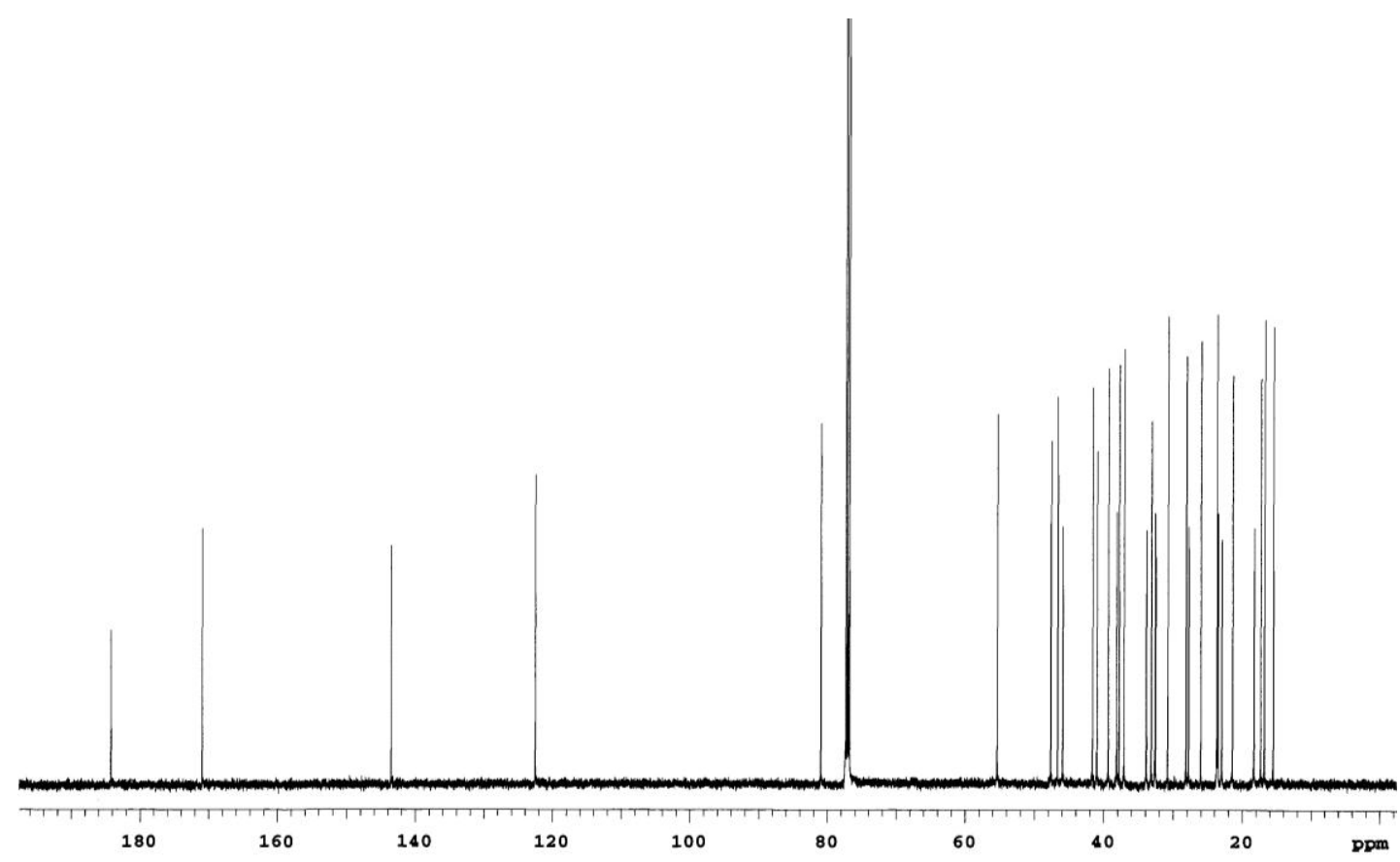

Figure 96: $\quad{ }^{13} \mathrm{C}$ NMR spectrum $\left(\mathrm{CDCl}_{3}, 125 \mathrm{MHz}\right)$ of acetylaleuritolic acid (118) 


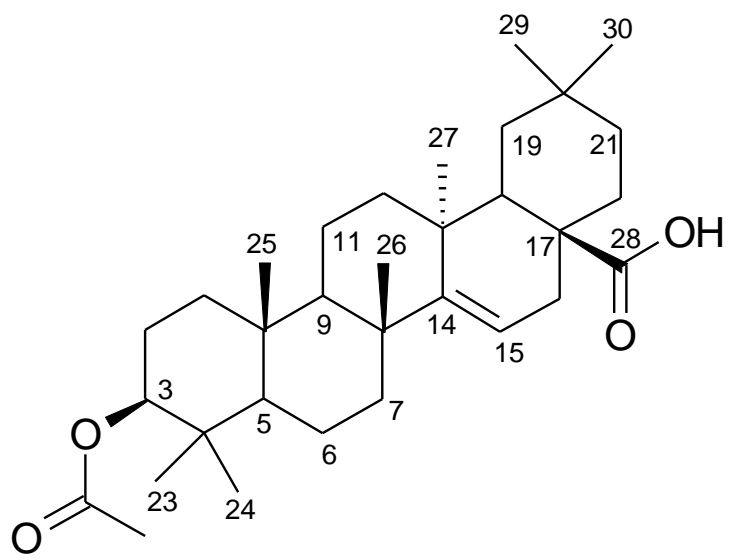

118

In addition, the ${ }^{13} \mathrm{C}$ NMR spectrum revealed two olefinic and two carbonyl carbons at $\delta 143.5\left(\mathrm{C}_{\mathrm{q}}-14\right), 122.4(\mathrm{CH}-15), 170.8\left(\mathrm{C}_{\mathrm{q}}\right.$-ester $)$ and $184.1\left(\mathrm{C}_{\mathrm{q}}-28\right)$, respectively. A search in AntiBase based on its molecular formula, $\mathrm{C}_{32} \mathrm{H}_{50} \mathrm{O}_{4}$, delivered 9 hits. The ${ }^{1} \mathrm{H}$ and ${ }^{13} \mathrm{C}$ NMR spectroscopic data suggested acetyl aleuritolic acid as the proposed structure for 118. In addition, all the spectral values were then compared with the authentic spectra in our group and found to be identical with acetylaleuritolic acid (118). The structure was finally confirmed by comparing the experimental data with the literature data. ${ }^{[205]}$

Acetyl aleuritolic acid (118) has been reported first time from the plant Aleurites montana (Euphorbiaceae) and belongs to a group of pentacyclic triterpene. ${ }^{[206,207]}$ Ramos and co-workers have reported that acetylaleuritolic acid (118) exhibited analgetic activity. ${ }^{[208]}$ In addition, $\mathbf{1 1 8}$ has shown trypanocidal activity against protozoan Trypanosoma cruzi. ${ }^{[209]}$

\subsection{Dillenia pentagyna}

Dillenia pentagyna belongs to the family of Dilleniaceae, including about 120 species. ${ }^{[210]}$ Many Dillenia species are native to southern Asia, Australia, and India and were used as medicinal plants. ${ }^{[210,211]}$ Terpenoids and flavonoids were reported as the major constituents. ${ }^{[211,212]}$

\subsubsection{Betulinic acid}

Betulinic acid (119) was obtained as white solid. The ${ }^{1} \mathrm{H}$ NMR spectrum showed many overlapping proton signals in the upfield region. Two olefinic protons ap- 
peared at $\delta 4.70$ and 4.56 and one methyl group at $\delta 1.65$ suggested the presence of an exomethylene group. The proton signals at $\delta 0.93,0.87,0.77,0.65$ were assigned to five tertiary methyl groups.

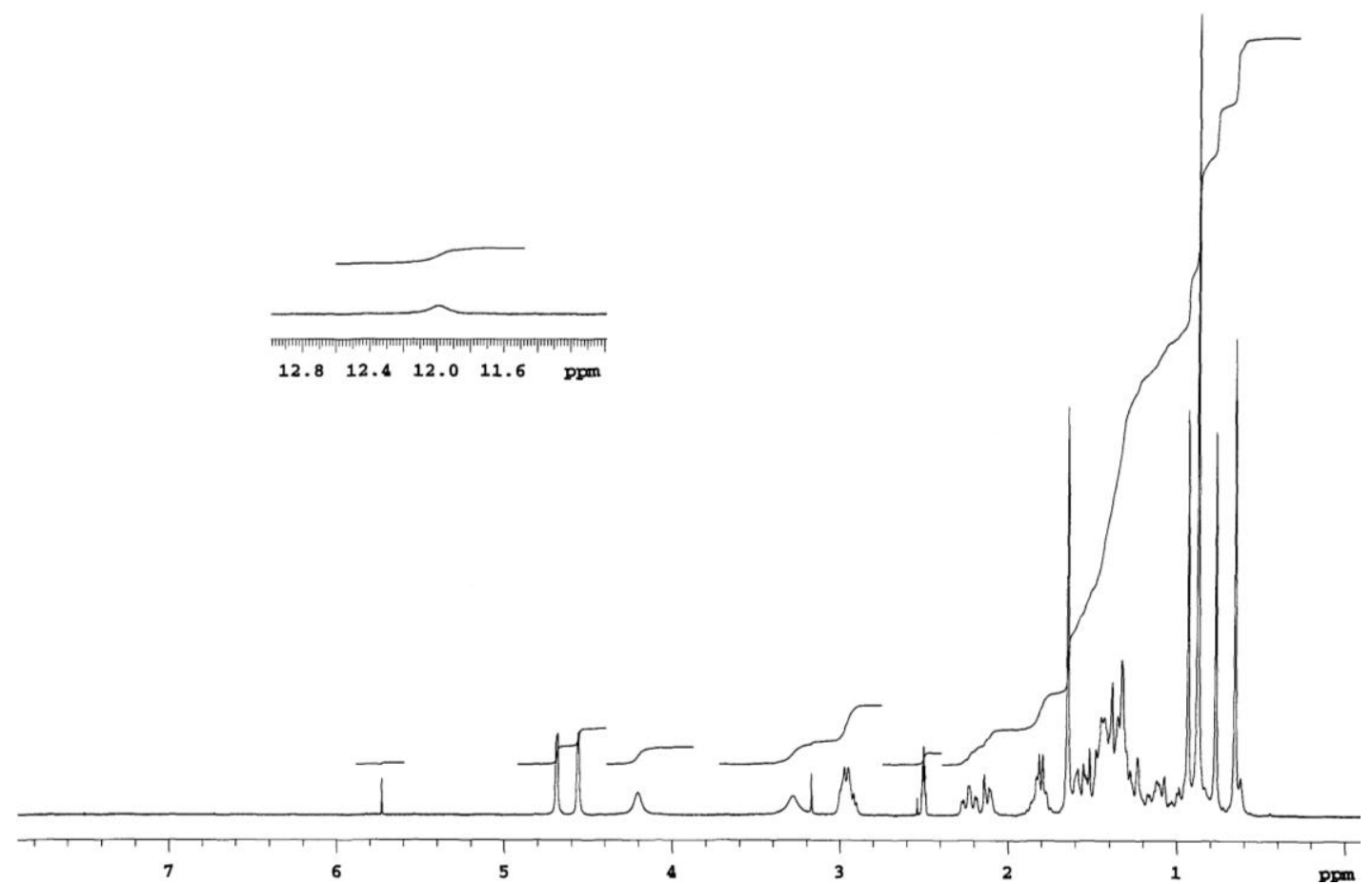

Figure 97: $\quad{ }^{1} \mathrm{H}$ NMR spectrum (DMSO- $d_{6}, 300 \mathrm{MHz}$ ) of betulinic acid (119)

The ${ }^{13} \mathrm{C}$ NMR spectrum showed three $s p^{2}$ carbon signals at $\delta 176.9,150.1$, 109.4 , and an oxygenated $s p^{3}$ carbon at $\delta 76.7$, respectively. The other carbon signals in the region of 55.3-14.4 were due to $s p^{3}$ carbons. The HRESI mass analysis of compound 119 showed a pseudo-molecular ion peak at $m / z 455.35309\left([\mathrm{M}-\mathrm{H}]^{-}\right)$, which was in agreement with the molecular formula $\mathrm{C}_{30} \mathrm{H}_{48} \mathrm{O}_{3}$. A search in AntiBase based on its molecular formula, $\mathrm{C}_{15} \mathrm{H}_{10} \mathrm{O}_{2}$, delivered 21 hits. A sub-structure search on the basis of the ${ }^{1} \mathrm{H}$ and ${ }^{13} \mathrm{C}$ NMR spectroscopic data suggested betulinic acid as the structure for 119. In addition, all the spectral values were then compared with the authentic spectra in our group and found to be identical with betulinic acid (119). ${ }^{[213]}$ 


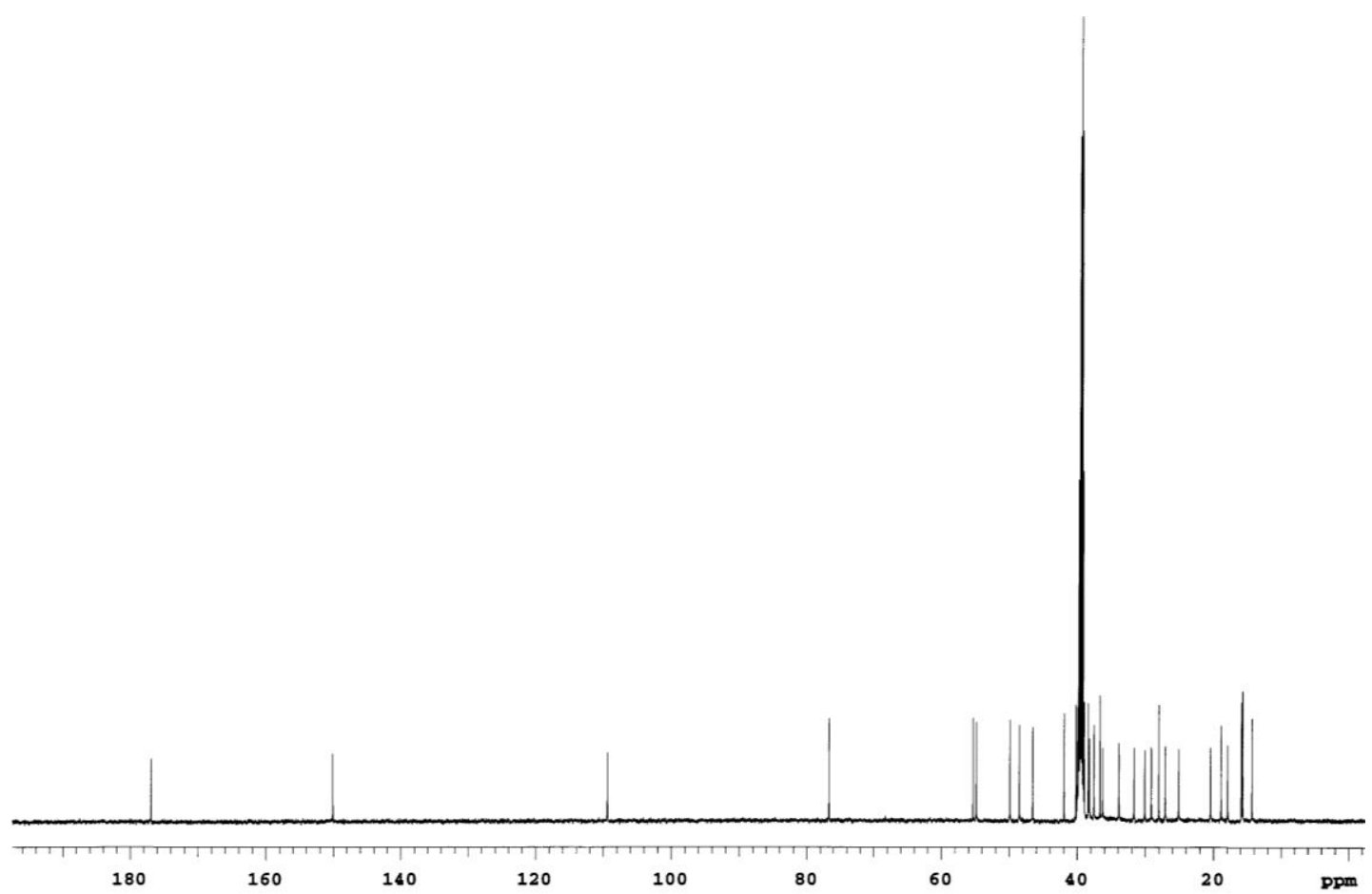

Figure 98: $\quad{ }^{13} \mathrm{C}$ NMR spectrum (DMSO- $d_{6}, 125 \mathrm{MHz}$ ) of betulinic acid (119)

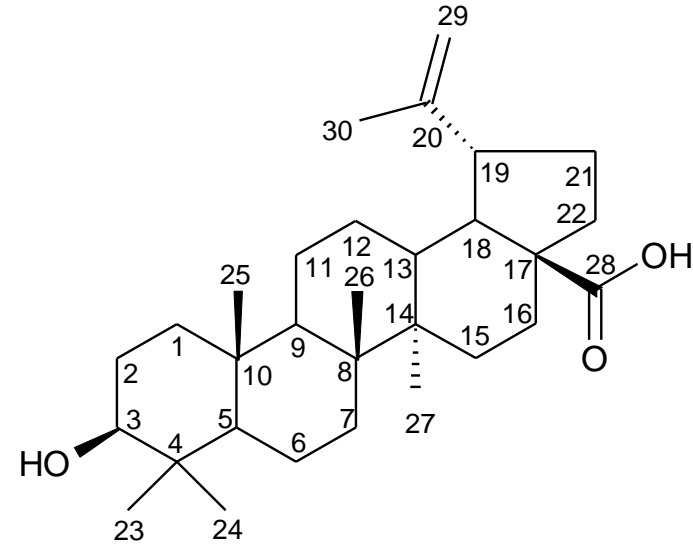

119

The pentacyclic triterpene betulinic acid ${ }^{[214]}(\mathbf{1 1 9})$ has antiinflammatory properties and show antiviral activity against HIV as well as cytotoxic properties. ${ }^{[212,215]}$

\subsubsection{Betulin}

Betulin (120) was obtained as white solid. The ${ }^{1} \mathrm{H}$ NMR spectrum had similar proton resonances as betulinic acid (119), except that the two doublets at $\delta 3.53$ and 3.78 were assigned as two protons at position 28. The structure of $\mathbf{1 2 0}$ was confirmed by comparing the ${ }^{1} \mathrm{H}$ NMR spectrum with the authentic spectrum in our group through AntiBase. 


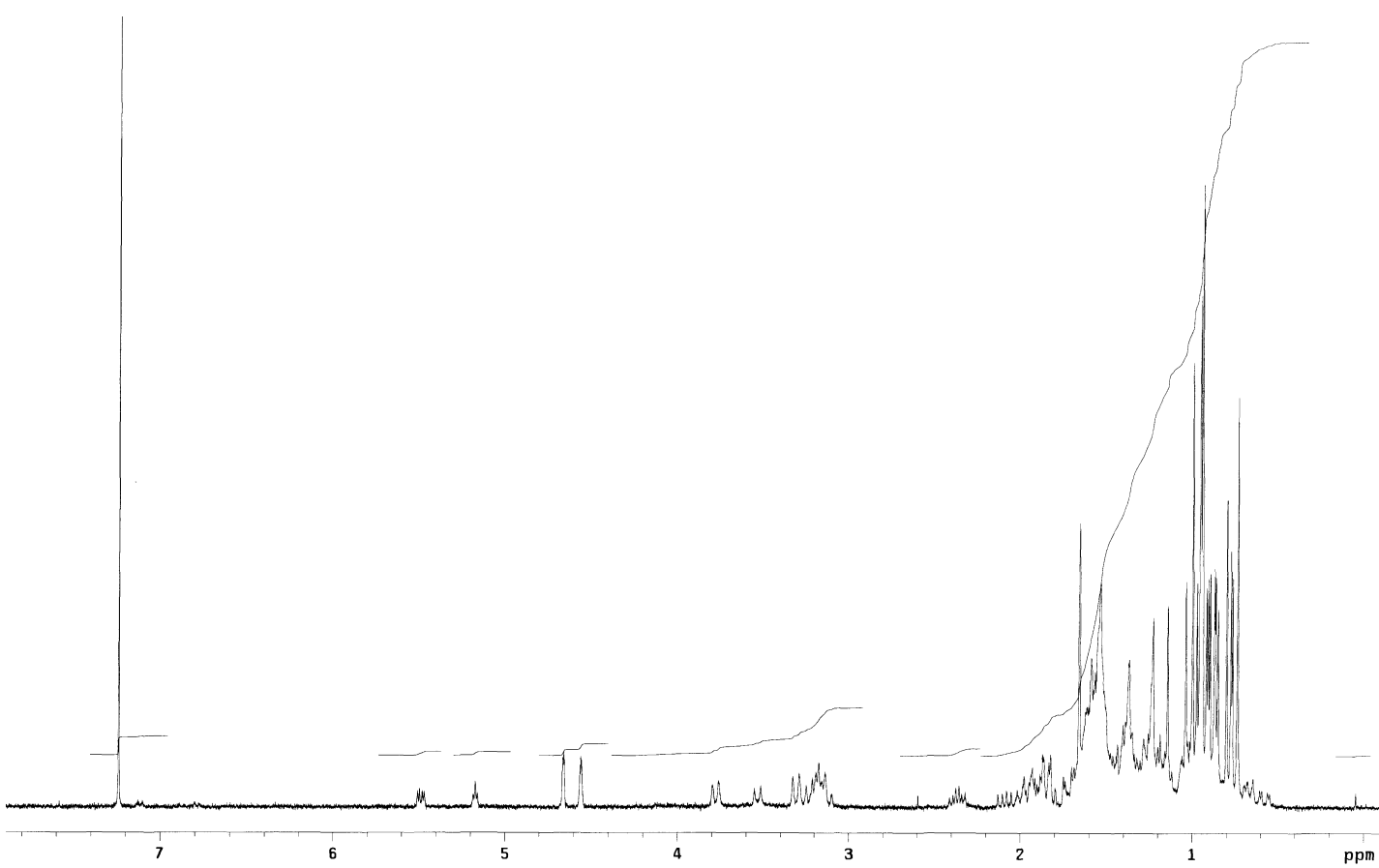

Figure 99: $\quad{ }^{1} \mathrm{H} \mathrm{NMR}$ spectrum $\left(\mathrm{CDCl}_{3}, 300 \mathrm{MHz}\right)$ of betulin (120)

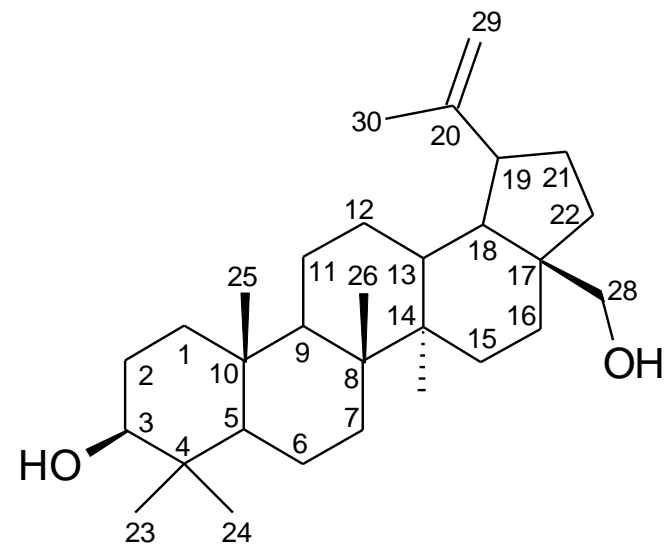

120

Both betulin (120) and betulinic acid (119) consist of a lupane skeleton. ${ }^{[216]}$ In addition, they have been reported to exhibit promising biological activities such as anti-HIV, anti-inflammatory, antiviral, and anticancer properties. ${ }^{[216,217]}$ 


\subsection{Kaempferia parviflora}

Kaempferia parviflora belongs to the family of Zingiberaceae and is used as folk medicin and food ingredient in southern Asia. Flavonoids have been reported as the major bioactive compounds from the Kaempferia genus. ${ }^{[218,219]}$ In addition, diterpenes, monoterpenes, cyclohexane oxides and cinnamates have been reported as phytochemicals of Kaempferia species. ${ }^{[220,221]}$

\subsubsection{5,7-Dimethoxyapigenin}

5,7-Dimethoxyapigenin (121) was obtained as white powder: The ESI mass spectrum indicated the pseudo-molecular ion peak at $\mathrm{m} / z 297\left([\mathrm{M}-\mathrm{H}]^{-}\right)$, which fixed the molecular weight to 298 Dalton. The HRESI mass analysis of $\mathrm{m} / z 299.09140$ $[\mathrm{M}+\mathrm{H}]^{+}$corresponded to the molecular formula $\mathrm{C}_{17} \mathrm{H}_{15} \mathrm{O}_{5}$. In the aromatic region, the ${ }^{1} \mathrm{H}$ NMR spectrum indicated seven protons. Two doublets of an $\mathrm{A}_{2} \mathrm{~B}_{2}$ system at $\delta$ 7.87 and $6.90(J=8.8 \mathrm{~Hz})$, and two meta-coupled proton signals at $\delta 6.82$ and 6.49 suggested a para- and a 1,2,3,5-tetrasubstituted benzene ring. A singlet at $\delta 6.56$ is characteristic of the 3-H position of flavones. 


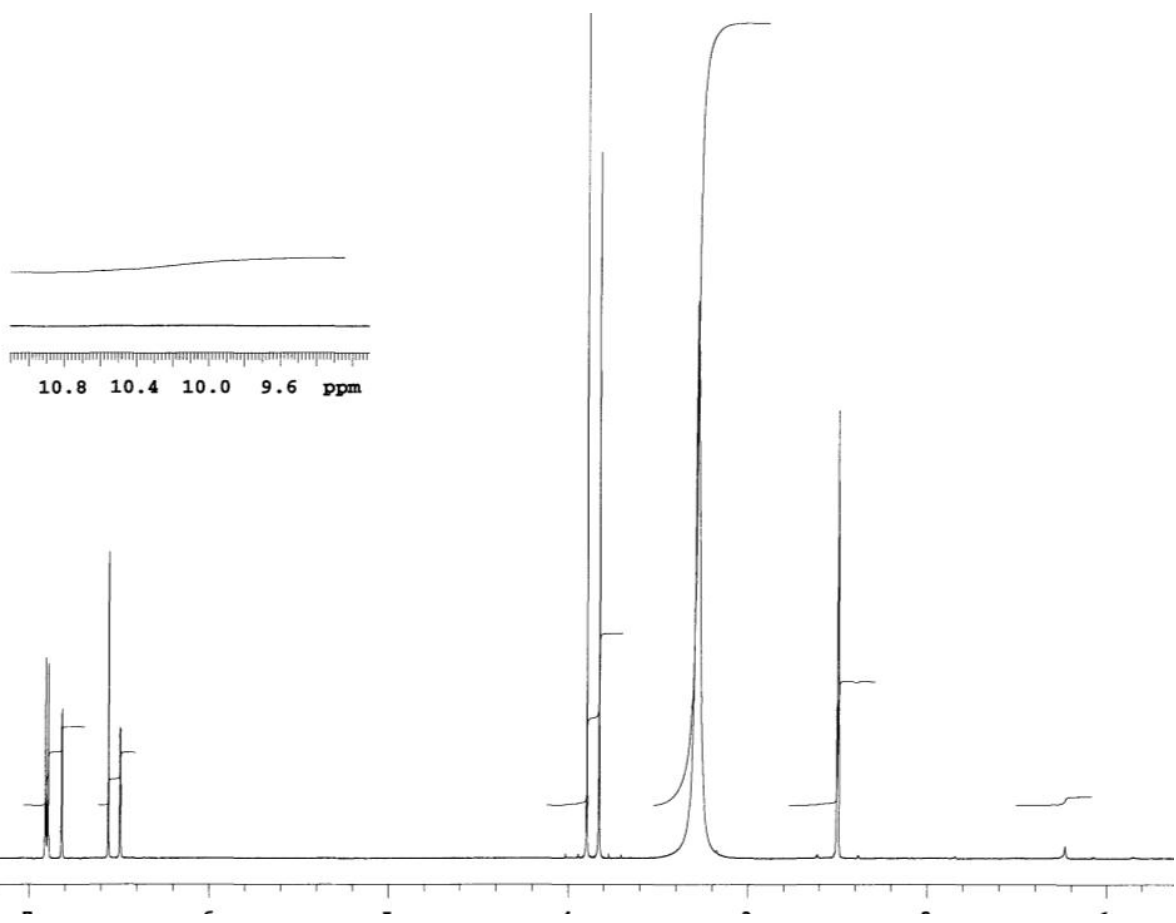

Figure 100: ${ }^{1} \mathrm{H}$ NMR spectrum (DMSO- $d_{6}, 300 \mathrm{MHz}$ ) of 5,7-dimethoxyapigenin (121)

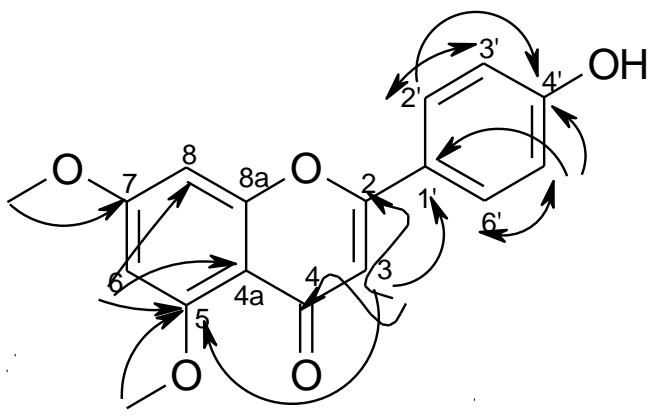

121

Figure 101: Selected ${ }^{1} \mathrm{H}-{ }^{1} \mathrm{H}$ COSY $(-)$ and $\mathrm{HMBC}(\longrightarrow)$ correlations of 5,7dimethoxyapigenin (121)

A sub-structure search in AntiBase with the molecular formula, $\mathrm{C}_{17} \mathrm{H}_{14} \mathrm{O}_{5}$, delivered 19 hits. The structure of compound $\mathbf{1 2 1}$ was confirmed by 1D and 2D NMR data as 5,7-dimethoxyapigenin and all spectral data were in agreement with the literature values. $^{[222]} 5,7$-Dimethoxyapigenin $(\mathbf{1 2 1})$ is a flavone derivative and has been reported to show antimutagenic activity. ${ }^{[223]}$ 


\subsubsection{Ganschisandrine}

Compound 122 was obtained as white powder and revealed a molecular ion peak at $m / z 395.18283[\mathrm{M}+\mathrm{Na}]^{+}$in HRESIMS analysis corresponding to the sodiated molecular formula $\mathrm{C}_{22} \mathrm{H}_{28} \mathrm{O}_{5} \mathrm{Na}$. In the aromatic region of the ${ }^{1} \mathrm{H}$ NMR spectrum, it showed a typical pattern of $\mathrm{o} / \mathrm{m}$-coupled protons in 1,2,4-position with two doublets at $\delta 6.94,6.83$ and one doublet of doublet at $\delta 6.95$. Furthermore, the ${ }^{1} \mathrm{H}$ NMR spectrum revealed a methyl, two methine and two methoxy groups.

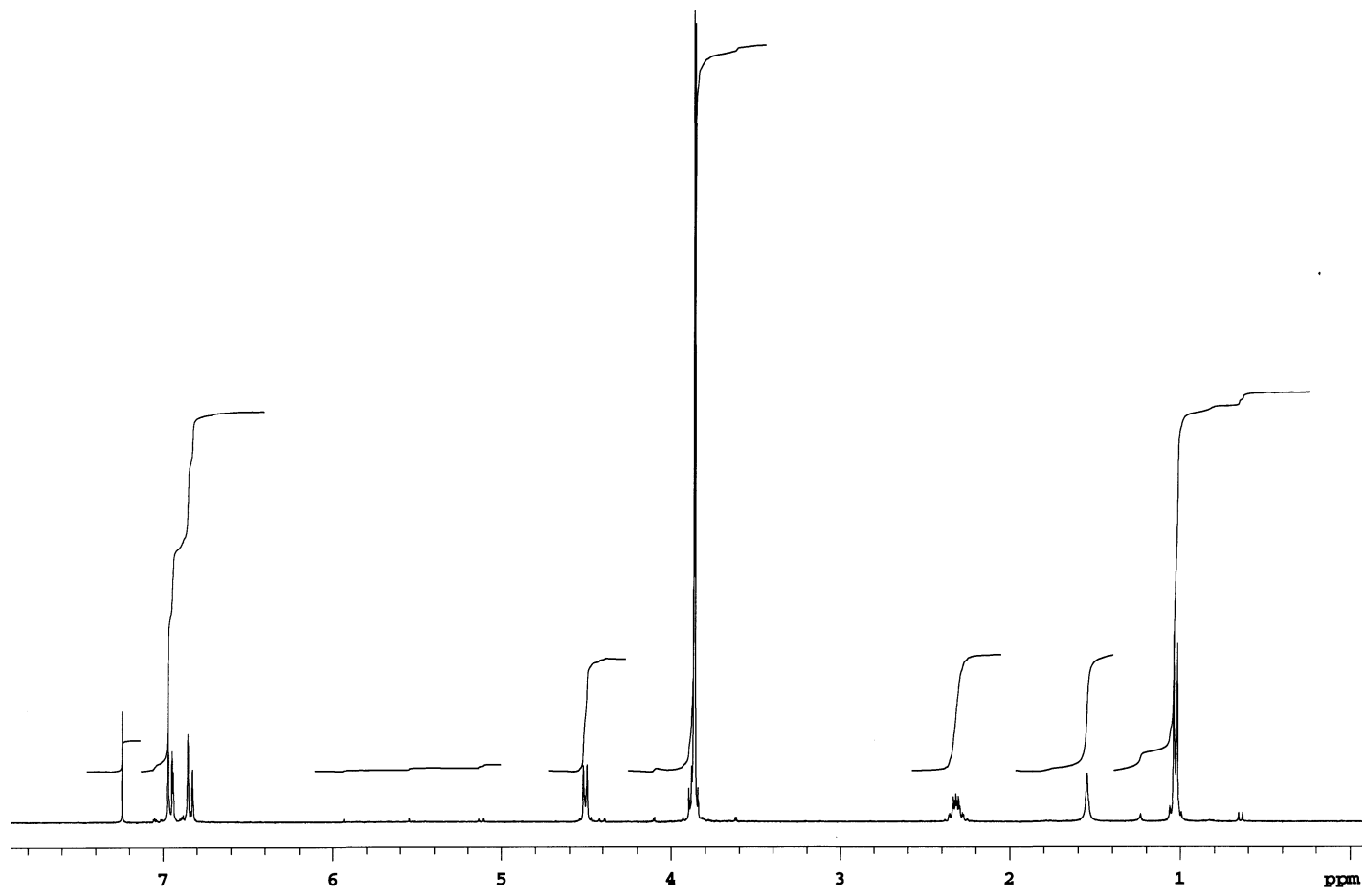

Figure 102: ${ }^{1} \mathrm{H}$ NMR spectrum $\left(\mathrm{CDCl}_{3}, 300 \mathrm{MHz}\right)$ of ganschisandrine (122)

The ${ }^{13} \mathrm{C}$ NMR spectrum showed only eleven carbon signals whereas the HRESI mass analysis has shown the presence of 22 carbon atoms in the molecule. It was assumed therefore that compound $\mathbf{1 2 2}$ had a symmetrical structure. The ${ }^{13} \mathrm{C}$ NMR spectrum exhibited five oxygenated carbon atoms at $\delta_{C} 148.8,148.3,87.2,55.9$ and 55.8, respectively. A search in AntiBase based on its molecular formula delivered eight hits. The HMBC spectrum exhibited cross coupling signals from H-6 $(\delta 6.95)$ and proton of $8-\mathrm{CH}_{3}\left(\delta\right.$ 1.02) towards the methine carbon $\mathrm{C}-7\left(\delta_{C} 87.2\right)$. A substructure search in AntiBase based on these HMBC correlations suggested ganschisandrine (122) as the structure for this compound, which was further confirmed by comparison with literature data. ${ }^{[224,225]}$ 


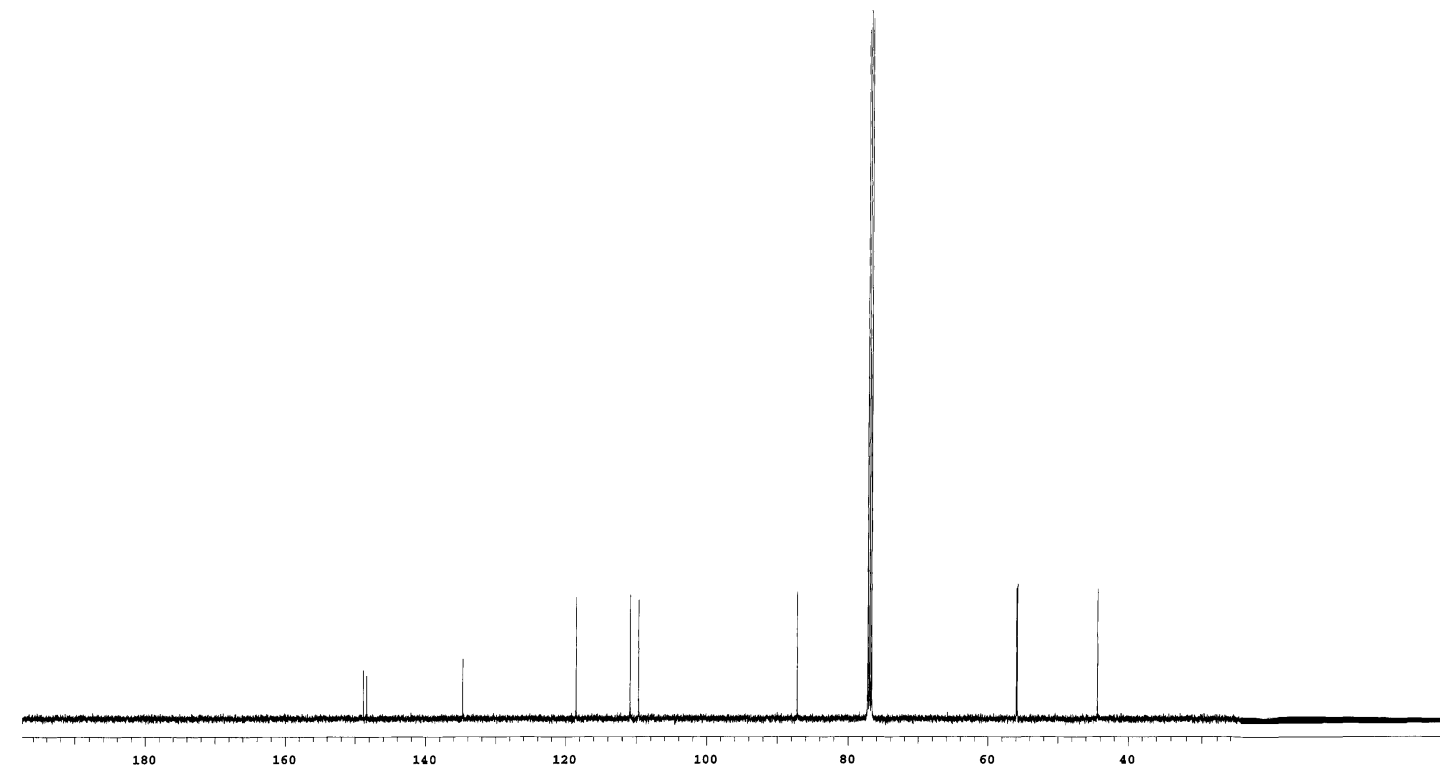

Figure 103: ${ }^{13} \mathrm{C} \mathrm{NMR} \mathrm{spectrum}\left(\mathrm{CDCl}_{3}, 125 \mathrm{MHz}\right)$ of ganschisandrine (122)

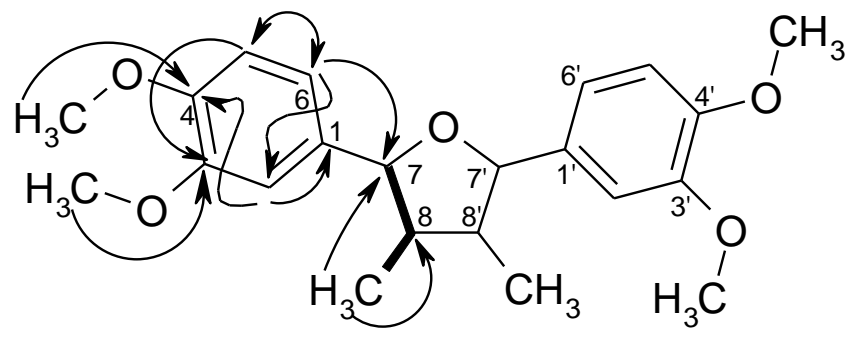

122

Figure 104: Selected ${ }^{1} \mathrm{H}_{-}{ }^{1} \mathrm{H} \operatorname{COSY}(-, \longleftrightarrow)$ and $\mathrm{HMBC}(\longrightarrow)$ correlations of ganschisandrine (122)

Ganschisandrine (122) has been isolated previously from a Chinese medicinal plant Schisandra sphenanthera ${ }^{[225]}$ It belongs to a group of tetrahydrofuran lignans and has been reported to demonstrate potent activities as antitumor, antiviral, antihepatotoxic, antioxidant and trypanocidal agent. ${ }^{[224,226]}$ Four natural stereoisomers of tetrahydrofuran lignans (structures II to V) have been reported so far from plants (Figure 105). ${ }^{[227]}$ Since the experimental analysis data were in agreement with the literature data, form IV was proposed as the relative configuration of the isolated ganschisandrine (122). 


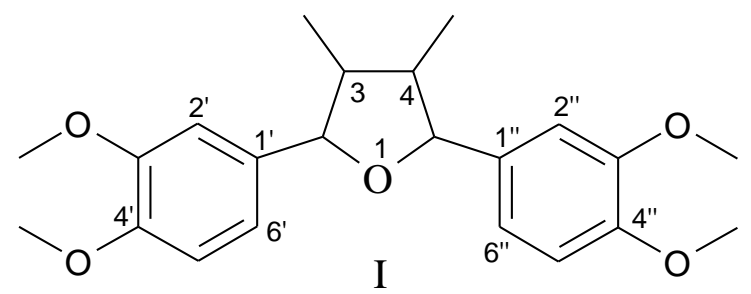

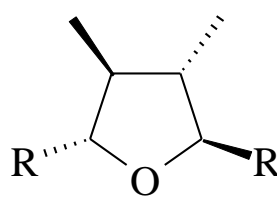

II

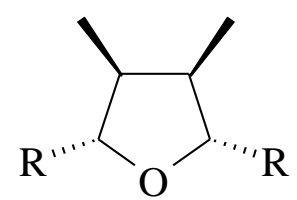

III

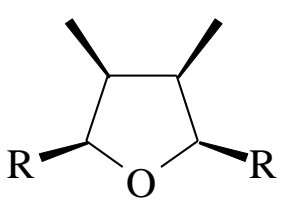

IV

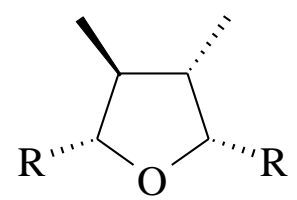

$\mathrm{V}$

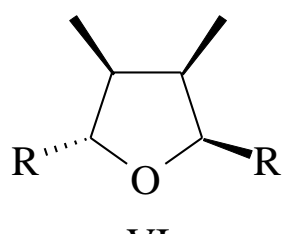

VI<smiles>[R]C1O[C@@H]([AlH])[C@@H](C)[C@@H]1C</smiles>

VII

$$
\mathrm{R}=\text { dimethoxyphenyl }
$$

Figure 105: The six natural stereoisomers of tetrahydrofuran lignans. ${ }^{[227]}$

\subsubsection{Stigmasterol glucoside}

Stigmasterol glucoside (123) was obtained as white powder. The ${ }^{1} \mathrm{H}$ NMR spectrum showed the typical pattern of a steroid with many overlapping proton signals in the upfield region. An olefinic proton appeared at $\delta 5.33$, six methyl groups were observed between $\delta$ 0.93-0.69, in addition to seven methine protons between $\delta$ 2.150.65 . Six oxymethines of a sugar moiety appeared between $\delta 4.27-3.11$ along with an oxymethylene at $\delta 3.49$ and 11 methylene protons. 


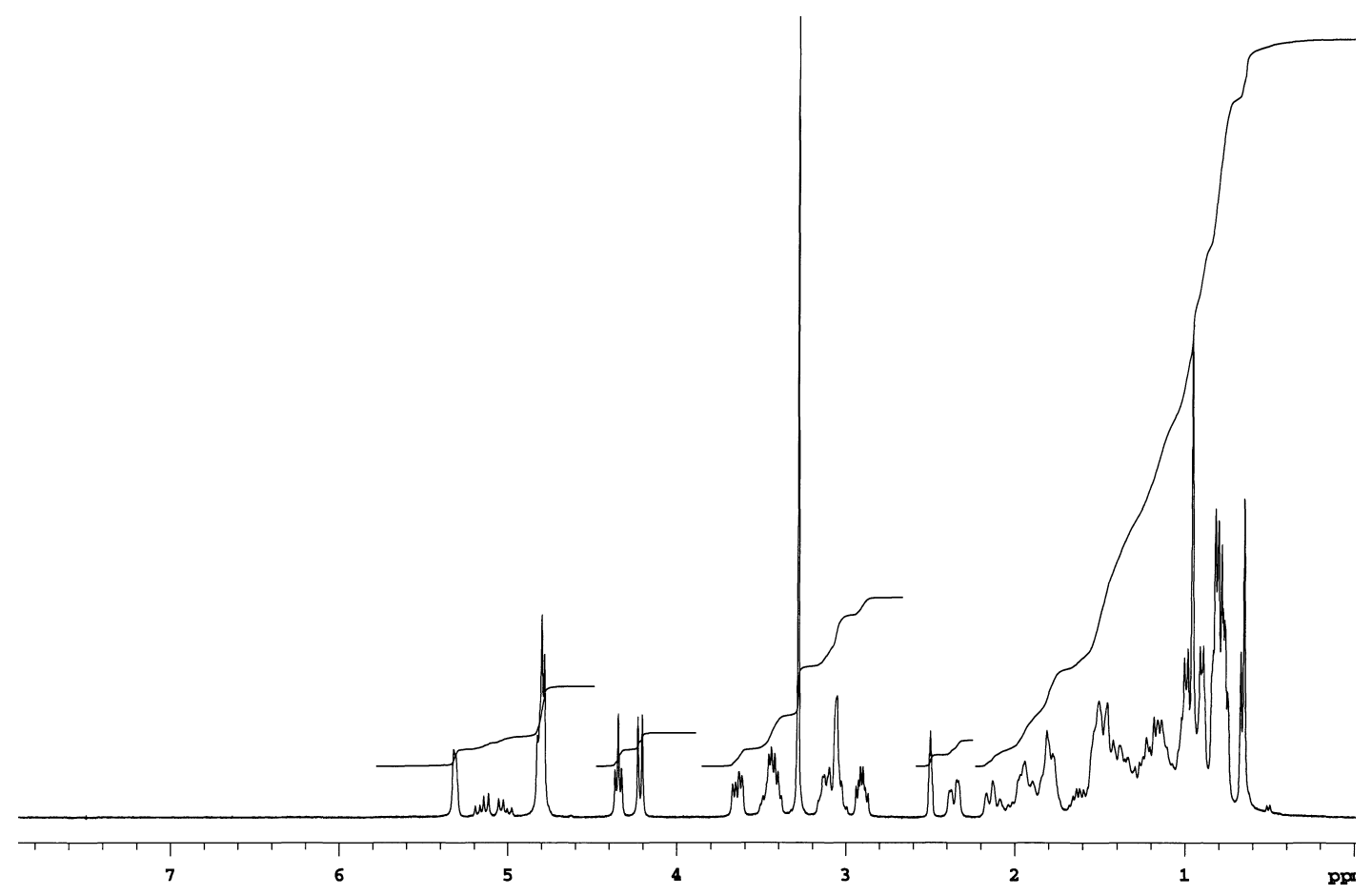

Figure 106: $\quad{ }^{1} \mathrm{H}$ NMR spectrum (DMSO- $d_{6}, 300 \mathrm{MHz}$ ) of stigmasterol glucoside (123)

The ${ }^{13} \mathrm{C}$ NMR spectrum exhibited 35 carbon signals constituted by three quaternary and three olefinic carbons, seven oxygenated carbons, 9 methylene, seven methine and six methyl carbons. The signal at $\delta_{C} 100.7$ was assigned to an anomeric carbon (C-1'). The four methine resonances at $\delta_{C} 76.8,76.7,76.6,73.3$ and one methylene resonance at $\delta_{C} 61.0$ were assigned to C-5', C-3', C-2', C-4' and C-6', respectively, of a sugar unit. 


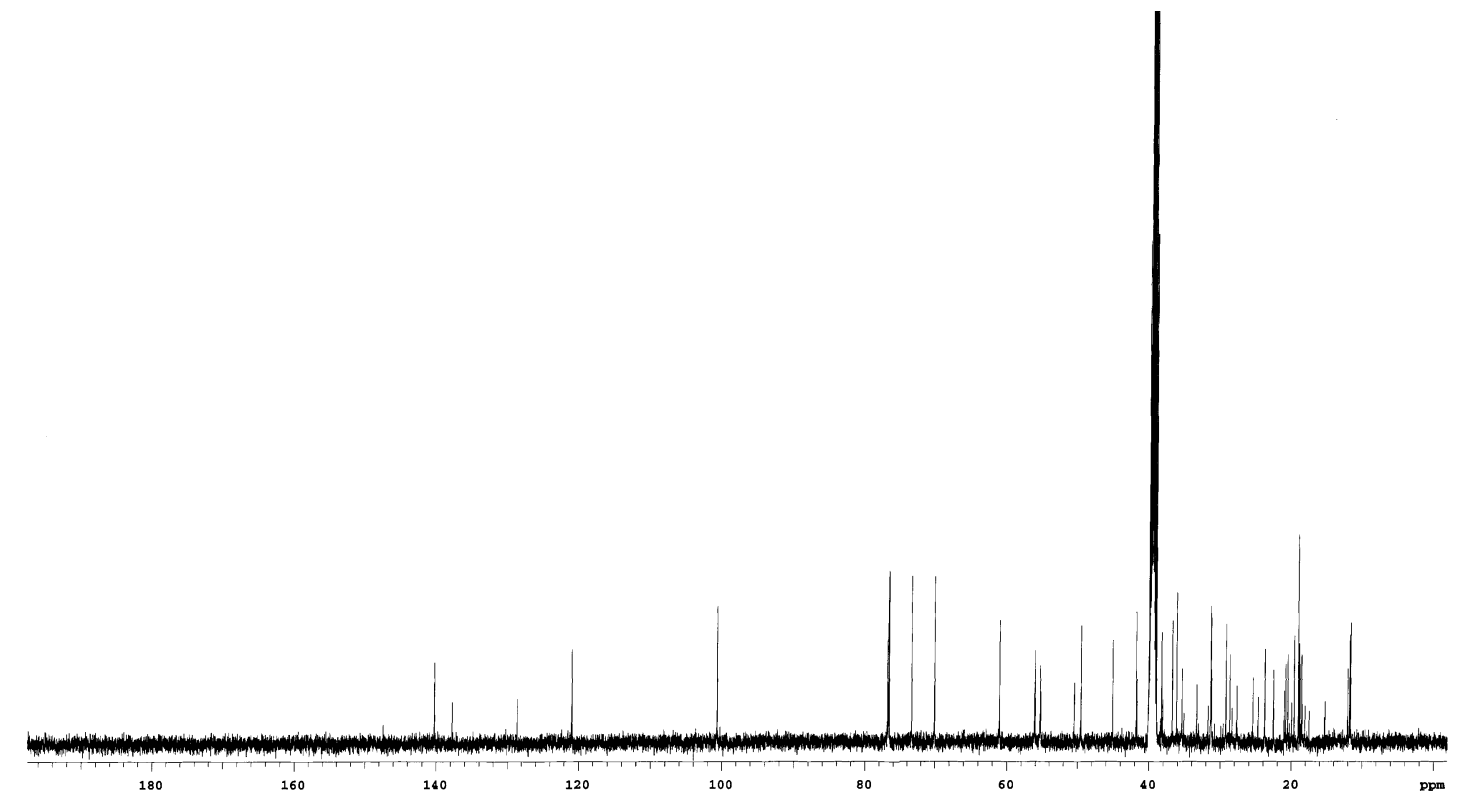

Figure 107: ${ }^{13} \mathrm{C}$ NMR spectrum (DMSO- $d_{6}, 125 \mathrm{MHz}$ ) of stigmasterol glucoside (123)

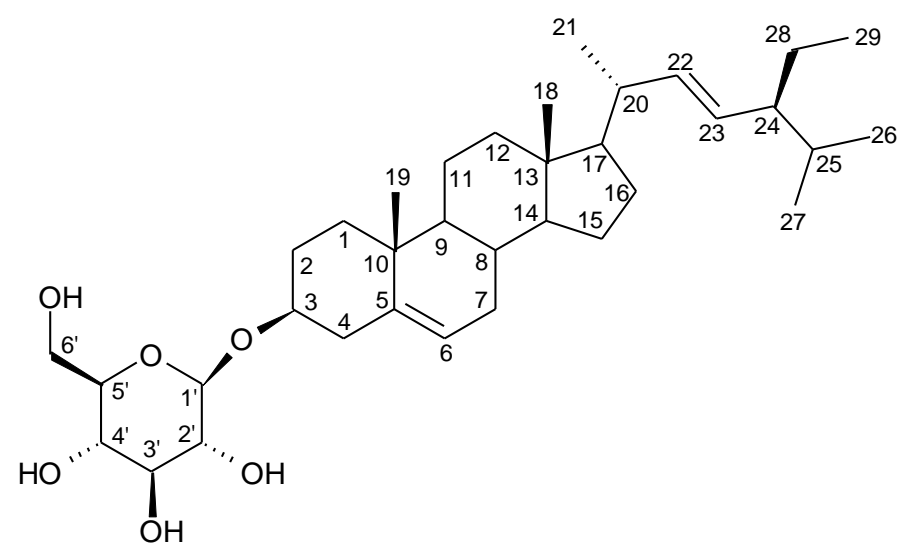

123

The ESI mass spectrum delivered the pseudomolecular ion peak at 597 $\left([\mathrm{M}+\mathrm{Na}]^{+}\right.$, which gave a molecular weight of 574 Dalton. The HRESI mass analysis gave the molecular formula $\mathrm{C}_{35} \mathrm{H}_{58} \mathrm{O}_{6}$. The structure of $\mathbf{1 2 3}$ was confirmed by comparing the NMR spectra with authentic spectra in AntiBase and was also in agreement with the literature data. ${ }^{[228]}$

\subsubsection{Oleanolic acid}

Oleanolic acid (124) was obtained as pale yellow solid. Its ESI mass spectrum showed the molecular weight of 456 Dalton and the HRESI MS analysis gave the molecular formula $\mathrm{C}_{30} \mathrm{H}_{48} \mathrm{O}_{3}$. The ${ }^{1} \mathrm{H}$ NMR spectrum exhibited one olefinic proton 
and one proton of an oxygenated methine at $\delta 5.23$ and 3.14 of triterpenoid derivatives. In addition, protons of methine, methylene and seven methyl groups appeared between $\delta$ 2.82-0.77.

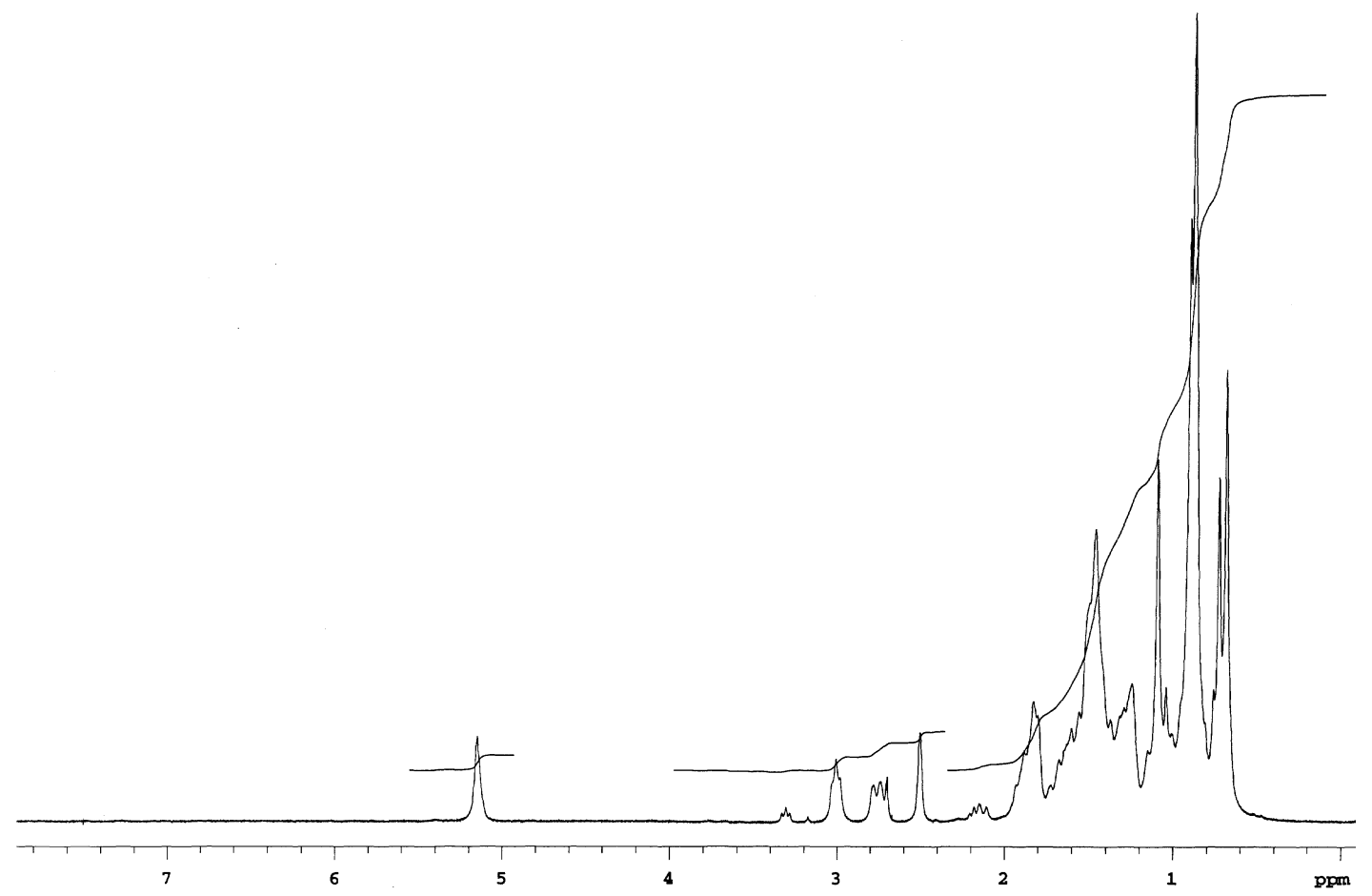

Figure 108: $\quad{ }^{1} \mathrm{H} \mathrm{NMR}$ spectrum $\left(\mathrm{CDCl}_{3}, 300 \mathrm{MHz}\right)$ of oleanolic acid (124)

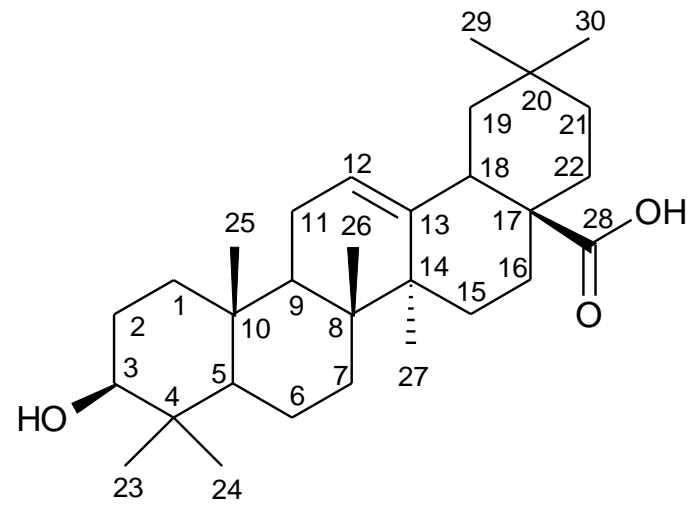

124

A sub-structure search in AntiBase delivered oleanolic acid (124) as the suggested structure, which was also confirmed by comparing the spectra with the literature data. ${ }^{[22]}$ Oleanolic acid (124) and its glycoside derivatives have been reported to show antitumor, antifungal, antiviral, anti-inflammatory, and anti-hyperlipidemic activities. $^{[229,230]}$ 


\subsubsection{2-Methylanthraquinone}

The ${ }^{1} \mathrm{H}$ NMR spectrum of a yellow compound (125) revealed five resonances of aromatic protons and one aromatic-bound methyl singlet at $\delta 2.52$. The multiplets at $\delta_{H} 8.28$ and 7.77 were assigned to an $o$-disubstituted benzene ring, and the signals at $\delta_{H} 8.18(\mathrm{~d}), 8.08$ (d) and $7.58(\mathrm{dd})$ suggested a second 1,2,4-trisubstituted benzene ring. HRESI mass analysis gave a pseudo-molecular ion at $\mathrm{m} / \mathrm{z} 245.05735$ $\left([\mathrm{M}+\mathrm{Na}]^{+}\right)$, which was consistent with a molecular formula of $\mathrm{C}_{15} \mathrm{H}_{10} \mathrm{O}_{2} \mathrm{Na}$.

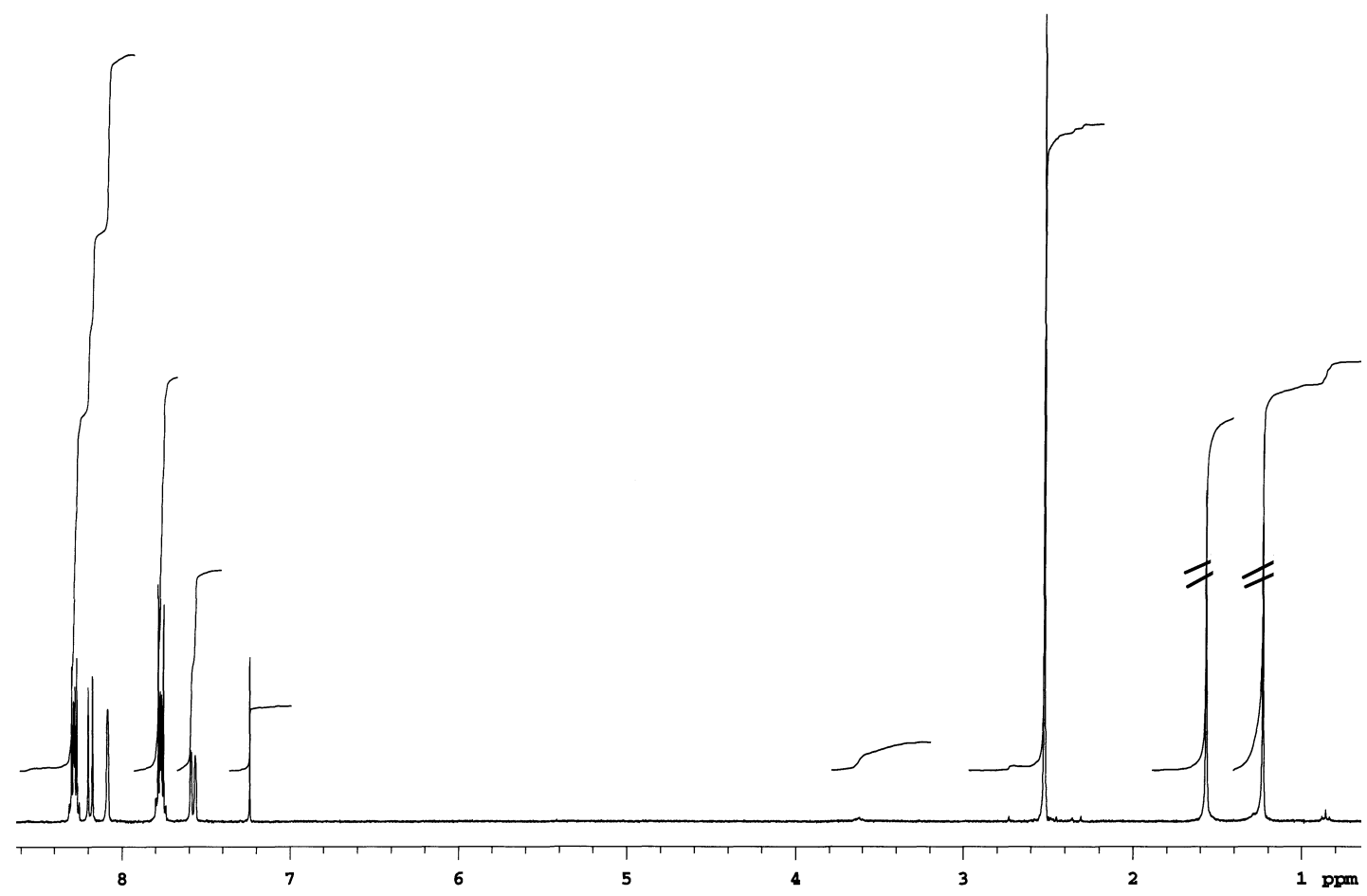

Figure 109: ${ }^{1} \mathrm{H}$ NMR spectrum $\left(\mathrm{CDCl}_{3}, 300 \mathrm{MHz}\right)$ of 2-methylanthraquinone (125)<smiles>Cc1ccc2c(c1)C(=O)c1ccccc1C2=O</smiles>

125

A search in AntiBase based on its molecular formula, $\mathrm{C}_{15} \mathrm{H}_{10} \mathrm{O}_{2}$, delivered only one hit, which was 2-methylanthraquinone. The structure of 2-methylanthraquinone (125) was further confirmed by comparing the spectroscopic data with the literature 
values. ${ }^{[231,232]}$ 2-Methylanthraquinone $(\mathbf{1 2 5})$ has been reported as one of the anthraquinones found in the family of Rubiaceae; it exhibited cytotoxicity against the LAC cell line. ${ }^{[233]}$

\subsubsection{Scopoletin}

Compound 126 was isolated as yellow solid in mixture with betulinic acid (119). High-resolution electrospray mass analysis (HRESIMS) showed an ion peak at $\mathrm{m} / \mathrm{z}$ $215.03147\left([\mathrm{M}+\mathrm{Na}]^{+}\right)$, which indicated a molecular formula of $\mathrm{C}_{10} \mathrm{H}_{8} \mathrm{O}_{4} \mathrm{Na}$. The ${ }^{1} \mathrm{H}$ NMR spectrum exhibited a methoxy singlet at $\delta 3.93$. The two doublets exhibited at $\delta 7.58$ and 6.25 with $J=9.5 \mathrm{~Hz}$, suggesting cis-protons of a double bond, and two singlet signals at $\delta 6.89$ and 6.82 attributed to an electron rich 1,2,4,5-tetrasubstituted benzene ring.

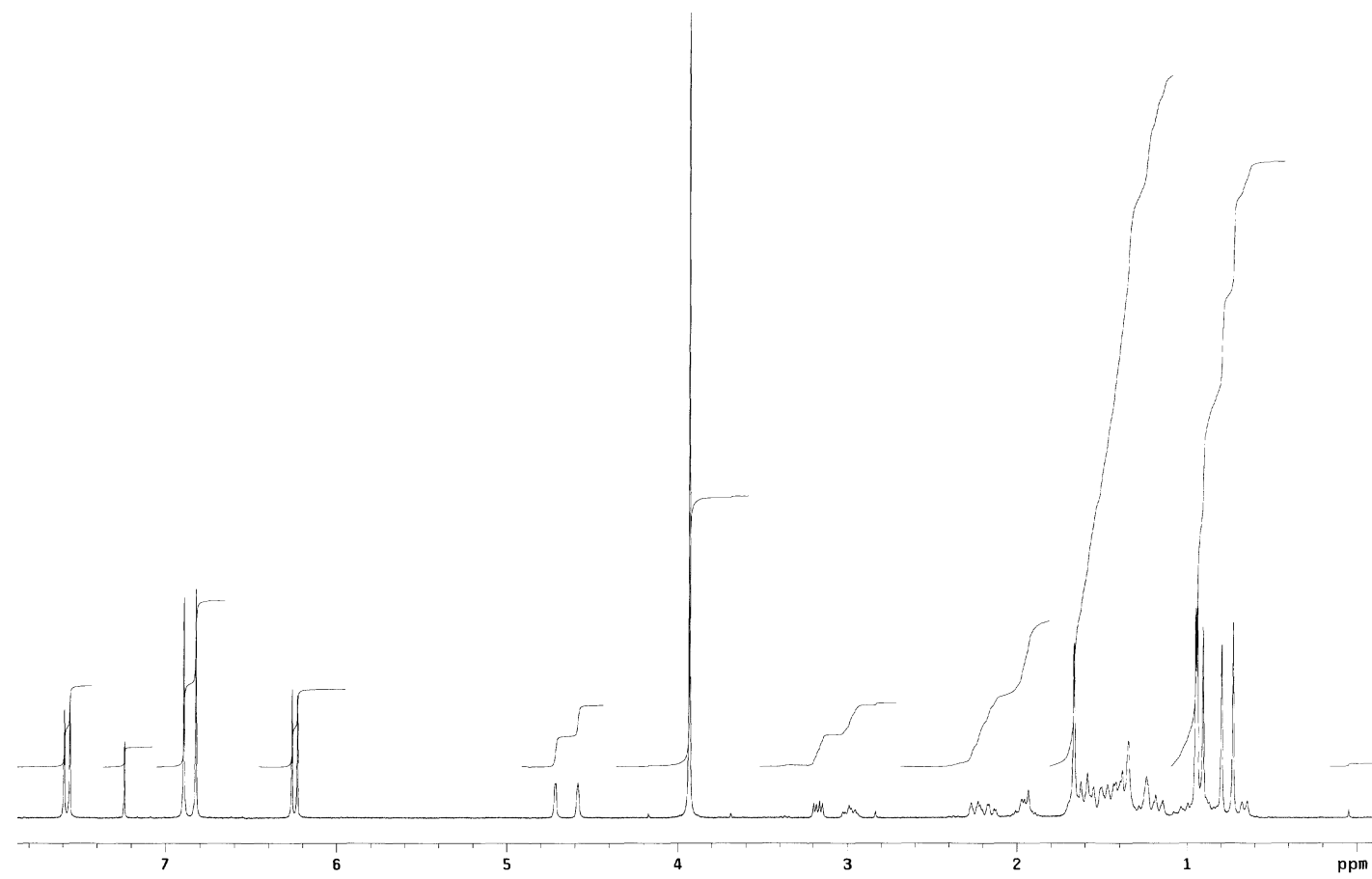

Figure 110: ${ }^{1} \mathrm{H} \mathrm{NMR}$ spectrum $\left(\mathrm{CDCl}_{3}, 300 \mathrm{MHz}\right)$ of scopoletin (126) 
<smiles>COc1cc2ccc(=O)oc2cc1O</smiles>

126

A sub-structure search in AntiBase delivered scopoletin (126) as the suggested structure, which was confirmed by comparison with literature data. ${ }^{[231]}$ Scopoletin (126) is a coumarin derivative and has been reported to have anticancer activity. ${ }^{[234]}$ 


\section{Summary}

The demand for the development of new drugs for the pharmaceutical market is still growing. Drug-resistant infectious diseases and disorders are the major reasons for natural product scientists to search in sponges, tunicates, and soft corals as alternative sources for bioactive metabolites. ${ }^{[235]}$ Additionally, microorganisms are still in the focus of interest. ${ }^{[236]}$ They are favoured over plants and animals due to the possibility to cultivate them in any quantity and at any time. ${ }^{[235]}$

In continuing our search for new and biologically active compounds, we are performing the screening of extracts from terrestrial and marine-derived microorganisms. In the present work, seven terrestrial and two marine Streptomyces spp., one ruminal bacterium, and four endophytic fungi were selected according to the results of chemical and biological screenings. Fermentation, extraction and purification were performed under standard procedures. The database AntiBase ${ }^{[88]}$ was used for dereplication of known metabolites, and NMR and MS measurements were carried out for structure elucidations. Additionally, antibacterial, antifungal and cytotoxicity assays were conducted with the pure compounds.

The marine-derived Streptomyces sp. B8289 was found to inhibit the growth of Staphylococcus aureus, Streptomyces viridochromogenes Tü 57, Escherichia coli, the fungus Mucor miehei Tü 284, and the microalgae Chlorella vulgaris, Chlorella sorokiniana, and Scenedesmus subspicatus in the agar diffusion test. From a $25 \mathrm{~L}$ shaker culture, four major constituents were isolated: 2-[5-(2-oxopropyl)-tetrahydrofuran-2-yl]-propionic acid (77), the new 2-[5-(2-oxopropyl)-tetrahydrofuran-2-yl]propionic acid methyl ester (78), homononactic acid (79), and homononactic acid methyl ester (80).

Homononactic acid (79), 2-[5-(2-oxopropyl)-tetrahydrofuran-2-yl]-propionic acid (77), 2-[5-(2-oxopropyl)-tetrahydrofuran-2-yl]-propionic acid methyl ester (78), and homononactic acid methyl ester $(\mathbf{8 0})$ are nonactic acid derivatives. 2-[5-(2Oxopropyl)-tetrahydrofuran-2-yl]-propionic acid (77) had been isolated previously in our group from Streptomyces sp. Act8970, and its relative stereochemistry was established partially on the basis of NOESY experiments by Shaaban. ${ }^{[89]}$ 
<smiles>[CH2]C(=O)C[C@@H]1CC[C@H]([C+](C)C(=O)O)O1</smiles>

77<smiles>CCC(O)CC1CCC(C(C)C(=O)O)O1</smiles>

79<smiles>[CH2-]C(=O)C[C@@H]1CC[C@H]([C@@H](C)C(=O)OC)O1</smiles>

78<smiles>CCC(O)CC1CCC(C(C)C(=O)OC)O1</smiles>

80

The terrestrial Streptomyces sp. Ank86 exhibited moderate activity against $B a$ cillus subtilis, Staphylococcus aureus, and Streptomyces viridochromogenes (Tü 57). From this strain, actinomycin D (82), tryptophol and 4-hydroxy-2-methoxyacetanilide (81) were obtained.<smiles>COc1cc(O)ccc1NC(C)=O</smiles>

81

The terrestrial Streptomyces sp. Ank68 exhibited strong activity against bacteria, fungi and brine shrimps, due to the content of actinomycin D (82) and fungichromin (83). Both these compounds are well-known antibiotics. ${ }^{\text {[98-102] }}$ 
<smiles>CCCCCC(O)C(C(=O)OC(C)C(O)C=CC=CC=CC=CC=C(C)C(O)C(O)C(O)CC(O)CC(O)CC(O)CC(O)CC(O)CC)C(O)C(C)O</smiles>

83

1-Acetyl- $\beta$-carboline (84) and 3-(hydroxyacetyl)-indole were isolated from the terrestrial Streptomyces sp. Ank248. Both compounds have been reported to exhibit weak antimicrobial and anticancer activities. ${ }^{[108]}$

The terrestrial Streptomyces sp. Ank181 delivered three known compounds, namely reductiomycin (85), 2,3-dihydroxybenzoic acid, and indole-3-carboxylic acid. Additionally, the antimicrobial assay exhibited a high activity against Candida albicans, moderate activity against E. coli, Streptomyces viridochromogenes (Tü 57), and Mucor miehei (Tü 284). It showed a weak activity against Bacillus subtilis. The activity of this strain was due to reductiomycin $(\mathbf{8 5})$.

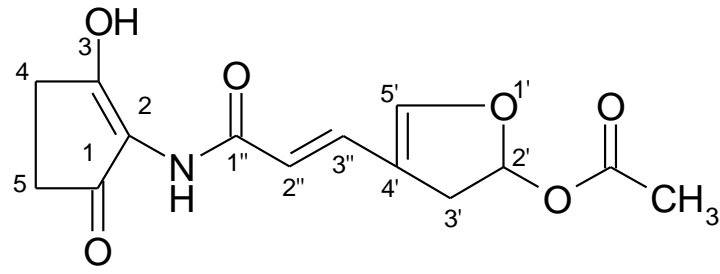

85

The terrestrial Streptomyces sp. Ank75 was selected due to its inhibition zones in the agar diffusion test against the fungi Candida albicans and Mucor miehei (Tü 284), and the algae Chlorella vulgaris and Chlorella sorokiniana. Seven constituents were isolated from this strain and three of them were new compounds, namely 2-Omethylpyrogallol (89), 2,3-dihydroxybenzamide (92), and bandamycin (94). Bandamycin (94) exhibited antifungal activity against Candida albicans and Mucor miehei (Tü 284). The other four known compounds were benadrostin (88), N-aminocarbonyl-2,3-dihydroxybenzamide (91), 3-[(1Z)-1-hexenyl-ONN]-1,2-butanediol (93), and $\mathrm{N}^{6}, \mathrm{~N}^{6}$-dimethyl-adenosine (95). 
<smiles>O=c1[nH]c(=O)c2cccc(O)c2o1</smiles>

88<smiles>NC(=O)NC(=O)c1cccc(O)c1O</smiles>

91<smiles>COc1c(O)cccc1O</smiles>

89<smiles>NC(=O)c1cccc(O)c1O</smiles>

92<smiles></smiles>

93<smiles>CCC(=O)CCC[N+]([O-])=NC(C)C(O)CO</smiles>

94

The crude extract of the terrestrial Streptomyces sp. GT-2005/049 exhibited strong activity against bacteria, fungi, and Artemia salina. From this strain, seven known compounds have been isolated. They were lumichrome (98), thymine, 1methyluracil, uracil, indole-3-carboxylic acid 1-acetyl- $\beta$-carboline (84), and hypoxanthine. Lumichrome (98) has been reported as the first endogenous substance in rats demonstrating affinity for the TCCD receptor. ${ }^{[89,146]}$ 
<smiles>Cc1cc2nc3[nH]c(=O)[nH]c(=O)c3nc2cc1C</smiles>

98

The marine Streptomyces sp. B5798 showed strong activity in the brine shrimp test. This strain produced macrolactin A (97), indole-3-carboxylic acid, indole-3acetic acid, and p-hydroxyphenylacetic acid (96). Among these compounds, macrolactin A (97) has been reported as an antibiotic, anticancer and antiviral agent. ${ }^{[142,143]}$<smiles></smiles>

97

The Streptomyces sp. GW 4723 had been fermented previously in $\mathrm{M}_{2}$ medium, and three new angucyclinone derivatives along with celastramycin B had been isolated ${ }^{[148]}$ for which the anthracyclinone structure $\mathbf{1 0 2}$ has been reported. ${ }^{[150]}$ As other by-products of celastramycin B found by Fondja had an angucyclinone skeleton, it was assumed that the celastramycin B structure may be wrong. Due to limited amount of the sample in the previous study, the HMBC correlations of hydroxyl groups were not found in the structure of celastramycin B. To finally answer the remaining questions, the strain GW 4723 was refermented in $\mathrm{LB}^{+}$medium to deliver five compounds, one of which was celastramycin B.

The structure $\mathbf{1 0 2}$ has been reported previously for celastramycin $\mathrm{B}$. The ${ }^{13} \mathrm{C}$ NMR spectrum would show similar signals at $\delta_{\mathrm{C}} \sim 186$ for two monochelated qui- 
none carbonyls ${ }^{[149]}$ in structure 102. However, the ${ }^{13} \mathrm{C}$ NMR spectrum exhibited signals at $\delta_{\mathrm{C}} 181.5$ and 192.3 indicating a non-chelated and a double chelated quinone carbonyl, which matched better for structure 103. A cross-coupling correlation of $\mathrm{H}$ $11(\delta 7.25)$ in $\mathbf{1 0 2}$ with the phenolic carbon at $\delta_{\mathrm{C}} 164.0$, which corresponded to a ${ }^{4} J$ coupling would be very unlikely for structure 102. On the other hand, the singlet at $\delta$ 7.25 in structure $\mathbf{1 0 3}$ showed the expected cross-signal in HMBC the spectrum with the carbonyl C-7 but not with the carbonyl C-12. In addition, the hydroxyl singlet at $\delta 12.18$ exhibited strong coupling with carbon at $\delta_{\mathrm{C}} 120.7$, which was very unusual for a ${ }^{5} J$ correlation between $6-\mathrm{OH}$ and $\mathrm{C}-11$ in structure 102. Finally, the structure of celastramycin B was revised into $\mathbf{1 0 3 .}$<smiles>CC1CC(=O)c2c(cc3c(c2O)C(=O)c2ccc(Cl)c(O)c2C3=O)C1O</smiles>

102<smiles>CC1CC(=O)c2c(cc(O)c3c2C(=O)c2ccc(Cl)c(O)c2C3=O)C1O</smiles>

103

The strain of Enterobacter amnigenus ZIA was isolated from the rumen of a Tunisian cow. In the biological test, the crude extract of E. amnigenus ZIA showed no activity against the tested microorganisms. Six compounds were isolated from this strain, namely poly-(hydroxybutyric acid) (PHB) (86), indole-3-acetic acid, uracil, tyrosol, hypoxanthine, and a new butyl glycoside (87).<smiles>CCCCO[C]1O[C@@H](CO)C(O)C(O)C1O</smiles> 
The endophytic fungus LAF20 showed no biological activity against bacteria, fungi, algae and Artemia salina, but TLC of crude extract showed UV absorbing bands and different colour reactions with anisaldehyde/sulphuric acid. This strain delivered three known chemical constituents, namely poly-(hydroxybutyric acid) (PHB) (86), isosclerone (104), 4,6,8-trihydroxy-1-tetralone (105), and a mixture of sterol derivatives.<smiles>O=C1CCC(O)c2cccc(O)c21</smiles>

104<smiles>O=C1CCC(O)c2cc(O)cc(O)c21</smiles>

105

The endophytic fungus NP32-A exhibited interesting results in the bioassay against Bacillus subtilis, Escherichia coli, Candida albicans, Mucor miehei (Tü 284), Rhizoctonia solani and Artemia salina. Fusaproliferin (107) and beauvericin (108), isolated from the strain, demonstrated strong activity against Artemia salina, while trichosetin (109) exhibited moderate activity against Mucor miehei (Tü 284) and Rhizoctonia solani. In addition, beauvericin (108) also showed weak activities against Bacillus subtilis, Escherichia coli, and Candida albicans. In contrast, cerevisterol (106) and cerebroside B (110) had no biological activities against bacteria, fungi, algae or in the brine shrimp test. 
<smiles>CC(C)C(C)/C=C/C(C)C1CCC2C3=CC(O)C4(C)CC(O)CCC4(C)C3CCC21C</smiles>

106<smiles>CC(C)C(=O)N(C)[C@@H](Cc1ccccc1)C(=O)O[C@@H](C(=O)N(C)C(Cc1ccccc1)C(=O)OC(C(=O)N(C)C(Cc1ccccc1)C(=O)OC(C(=O)O)C(C)C)C(C)C)C(C)C</smiles>

108

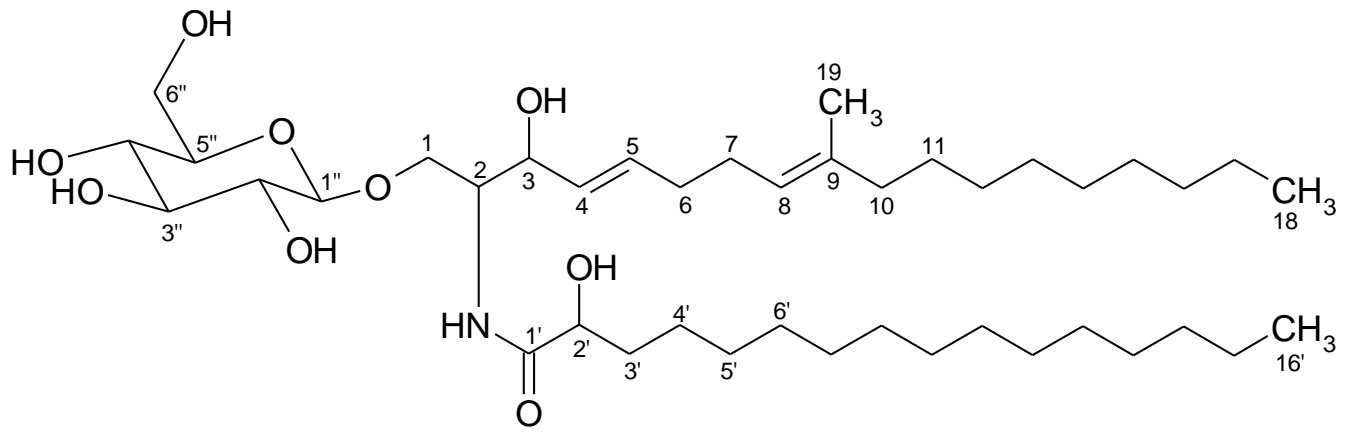

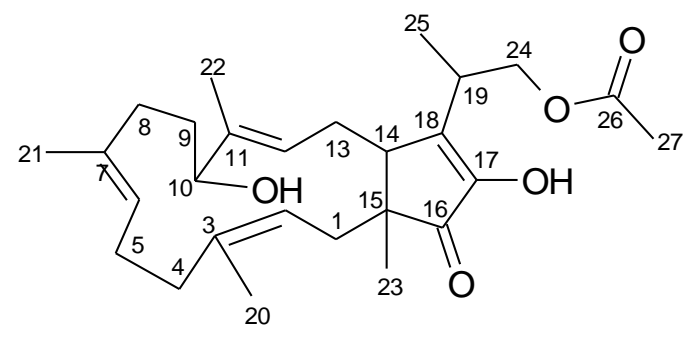

107<smiles>C/C=C/C1C=CC2CC(C)CCC2=C1C(O)=C1C(=O)NC(CO)C1=O</smiles>

109

TLC analysis of endophytic fungus FT44 showed UV absorbing bands and different colour reactions with anisaldehyde/sulphuric acid. Ergosterol peroxide (112) and 1233-A (111) were isolated. Both compounds have been reported as inhibitors of 3-hydroxy-3-methylglutaryl coenzyme A synthase. ${ }^{[177,178]}$ 


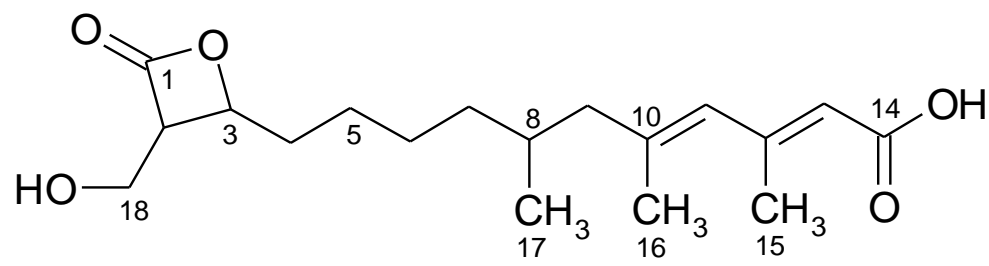

\section{1}

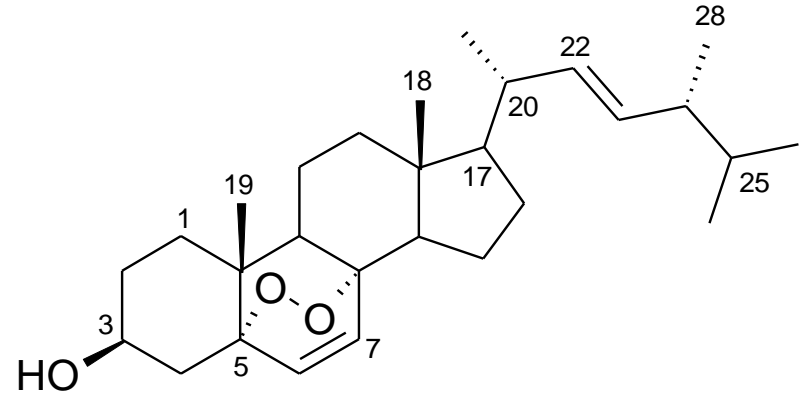

112

The fermentation of an endophytic fungus Alternaria sp. FTM1 in rice medium afforded 8-hydroxy-6,7-dimethoxy-3-methylisocoumarin (113), 5-methylochracin (114), and alternariol 5-methyl ether (115).<smiles>COc1cc2cc(C)oc(=O)c2c(O)c1OC</smiles><smiles>Cc1ccc(O)c2c1CC(C)OC2=O</smiles>

113 114<smiles>COc1cc(O)c2c(=O)oc3cc(O)cc(C)c3c2c1</smiles> 
In continuing research on plant-derived secondary metabolites, Prof. S. Ganapaty's group in India and Prof. Aye Aye Tun's group in Myanmar contributed eleven pure compounds. Their structures were elucidated as: garcinon D (116), rubraxanthone (117), acetyl aleuritolic acid (118), betulinic acid (119), 5,7dimethoxyapigenin (121), ganschisandrine (122), stigmasterol glucoside (123), oleanolic acid (124), betulin (120), 2-methylanthraquinone (125), scopoletin (126). Garcinon D (116), rubraxanthone (117), acetyl aleuritolic acid (118), betulinic acid (119) and 5,7-dimethoxyapigenin (121) were isolated from Myanmar medicinal plants, whereas stigmasterol glucoside $(\mathbf{1 2 3})$, oleanolic acid (124), betulin (120), 2 methylanthraquinone (125) and scopoletin (126) were isolated from Indian medicinal plant. In addition, all these plant secondary metabolites were reported in the literature to show a multitude of different biological activities.<smiles>COc1c(O)cc2oc3cc(O)c(CC=C(C)C)c(O)c3c(=O)c2c1CCC(C)(C)O</smiles>

116<smiles>COc1c(O)cc2oc3cc(O)cc(O)c3c(=O)c2c1C/C=C(\C)CCC=C(P)P</smiles>

117

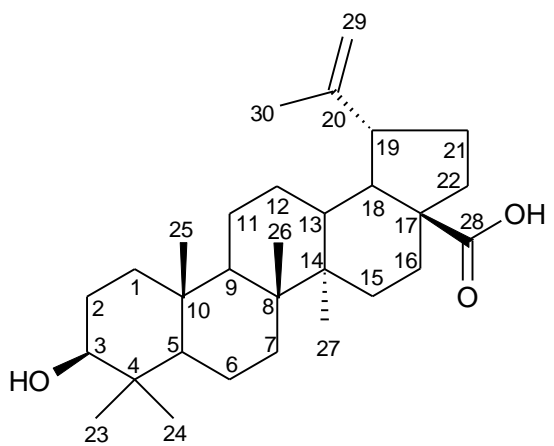

119 
<smiles>COc1cc(OC)c2c(=O)cc(-c3ccc(O)cc3)oc2c1</smiles>

121<smiles>COc1ccc(C2=C(C)[C@@H](C)C(c3ccc(OC)c(OC)c3)O2)cc1OC</smiles>

122

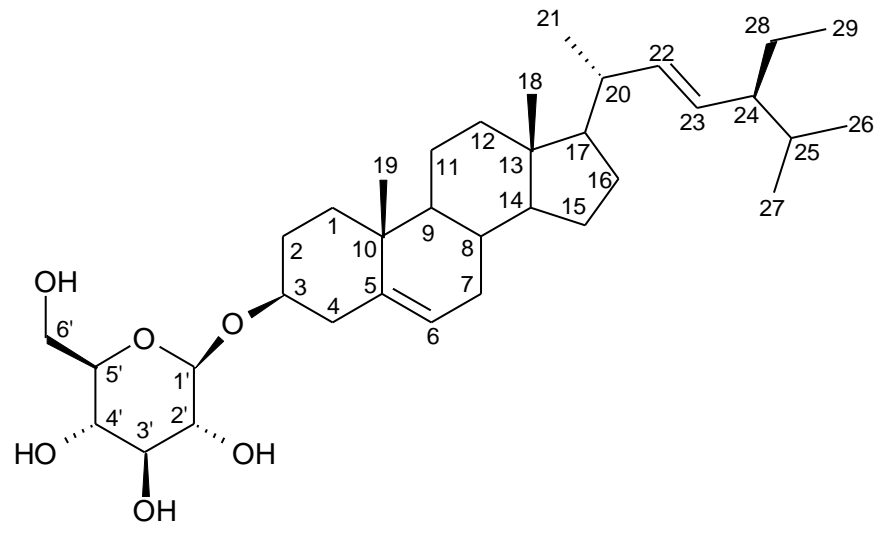

\section{3}

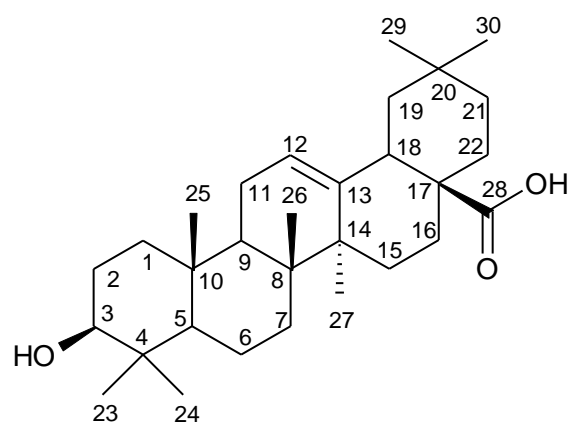

124<smiles>Cc1ccc2c(c1)C(=O)c1ccccc1C2=O</smiles>

125

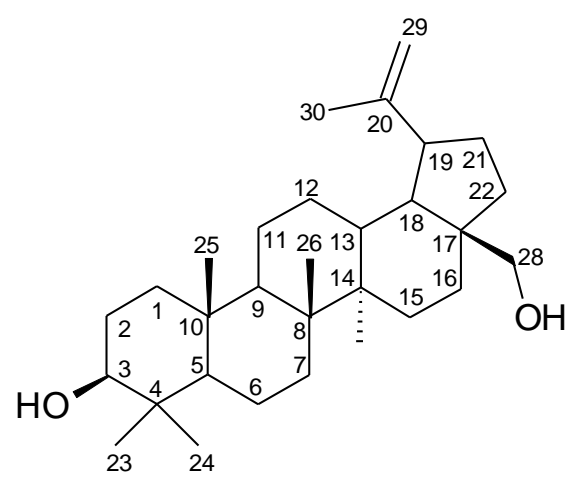

120<smiles>COc1cc2ccc(=O)oc2cc1O</smiles>

126 
Table 2: Total number of isolated compounds from microorganisms and plants in this thesis.

\begin{tabular}{lccc}
\hline Strains/Plants & $\begin{array}{l}\text { No. of } \\
\text { strains/plants }\end{array}$ & $\begin{array}{l}\text { Total no. of } \\
\text { compounds }\end{array}$ & $\begin{array}{l}\text { No. of new com- } \\
\text { pounds }\end{array}$ \\
\hline Marine Streptomyces sp. & 2 & 8 & 1 \\
Terrestrial Streptomyces sp. & 7 & 21 & 1 \\
Ruminal bacterium & 1 & 6 & - \\
Endophytic fungi & 4 & 13 & - \\
Medicinal plants & 4 & 11 & \\
\hline
\end{tabular}

Based on this study, natural sources such as microorganisms (bacteria and endophytic fungi), plants and animals are still of interest as producers of bioactive compounds in the field of natural product chemistry. Since nature does not produce secondary metabolites without reason, there is a demand to test the isolated products from microorganisms or plants against other biological systems and to determine the biological uses of the isolated compounds. 


\section{Materials and methods}

\subsection{General}

IR spectra: Perkin-Elmer 1600 Series FT-IR; Perkin-Elmer 297 infrared spectro-photometer; Beckman DU-640; Shimadzu FT-IR; ( $\mathrm{KBr}$ tablet and film). UV/VIS spectra: Perkin-Elmer Lambda 15 UV/VIS spectrometer. - Optical rotations: Polarimeter (Perkin-Elmer, model 243). - ${ }^{1}$ H NMR spectra: Varian Unity 300 (300.145 MHz), Bruker AMX 300 (300.135 MHz), Varian Inova 500 (499.8 MHz), Varian Inova 600 (600 MHz). Coupling constants $(J)$ in Hz. Abbreviations: s $=$ singlet, $\mathrm{d}=$ doublet, $\mathrm{dd}=$ doublet of doublet, $\mathrm{t}=$ triplet, $\mathrm{q}=$ quartet, $\mathrm{m}=$ multiplet , br = broad. $-{ }^{13}$ C NMR spectra: Varian Unity 300 (75.5 MHz), Varian Inova 500 (125.7 MHz), Varian Inova $600(150.7 \mathrm{MHz})$. Chemical shifts were measured relatively to tetramethylsilane as internal standard. Abbreviations: APT (Attached Proton Test): $\mathrm{CH} / \mathrm{CH}_{3}$ up and $\mathrm{C}_{\mathrm{q}} / \mathrm{CH}_{2}$ down. - 2D NMR spectra: $\mathrm{H}, \mathrm{H} \operatorname{COSY}\left({ }^{1} \mathrm{H},{ }^{1} \mathrm{H}-\right.$ Correlated Spectroscopy), HMBC (Heteronuclear Multiple Bond Connectivity), HMQC (Heteronuclear Multiple Quantum Coherence) and NOESY (Nuclear Overhauser Effect Spectroscopy). - Mass spectra: a) EIMS (70 eV) were recorded on a Finnigan MAT 95 or Jeol AccuTOF GC II spectrometer with perfluorokerosene as reference substance for EI HRMS. b) DCI-MS: Finnigan MAT 95, $200 \mathrm{eV}$, reactant gas $\mathrm{NH}_{3}$. c) ESIMS, HRESIMS, HPLC-MS and ESI-MS/MS were recorded on a Finnigan LCQ with quaternary pump Rheos 4000 (Flux Instrument). High-resolution mass spectra (HRMS) were recorded by ESI MS on an Apex IV 7 Tesla FourierTransform Ion Cyclotron Resonance Mass Spectrometer (Bruker Daltonics, Billerica, MA, USA). HPLC-MS using a Phenomenex Synergi Max-RP $150 \times 2.00 \mathrm{~mm} 4$ $\mu 80$ A RP-C12 column, solvent A: $\mathrm{MeOH}+0.05 \% \mathrm{HCOOH}$, solvent $\mathrm{B}: \mathrm{H}_{2} \mathrm{O}+$ $0.05 \% \mathrm{HCOOH}$, flow rate $300 \mu \mathrm{L} / \mathrm{min}$, gradient: start $10 \%$ or $20 \%$ of $\mathrm{A}, 0-20 \mathrm{~min}$ from $10 \%$ or $20 \%$ to $100 \%$ of $\mathrm{A}, 20-30 \mathrm{~min}$ at $100 \% \mathrm{~A}$, UV/VIS-Diode-ArrayDetector (200-800 nm ever $5 \mathrm{~nm}$ ). ESI MS/MS was performed with normalized collision energy of 35\%. d) GC-CI-MS were recorded by Thermo Finnigan TRACE GC Ultra with Varian FactorFour Capillary Column (VF-5ms $30 \mathrm{~m} \times 0.25 \mathrm{~mm}+5 \mathrm{~m}$ EZGuard, layer $0.25 \mu \mathrm{m}$ ) and DSQ detector. Isobutan was used as reactant gas. GC-EIMS were recorded by Thermo Finnigan TRACE with Varian GC Capillary Column 
(wcot fused silica coated CP-SIL 8CB for amines; $30 \mathrm{~m} \times 0.25 \mathrm{~mm}$, layer $0.25 \mu \mathrm{m}$ ).

- Filter press: Schenk Niro 212 B40.

\subsection{Materials}

Thin layer chromatography (TLC): DC-Folien Polygram SIL G/UV 254 (Macherey-Nagel \& Co.). - Glass plates for chemical screening: Merck silica gel 60 F254, $(10 \times 20 \mathrm{~cm}) .-$ Preparative thin layer chromatography (PTLC): $55 \mathrm{~g}$ Silica gel P/UV 254 (Macherey-Nagel \& Co., Düren, Germany) is added to $120 \mathrm{ml}$ of demineralised water and the suspension stirred for 60 minutes. Sixty $\mathrm{ml}$ of the homogenous suspension is poured on a horizontal held $20 \times 20 \mathrm{~cm}$ glass plate and the unfilled spaces are covered by evenly distributing the suspension. The plates are air dried for 24 hours and activated by heating for 3 hours at $130{ }^{\circ} \mathrm{C}$. - Column chromatography (CC): MN silica gel 60: 0.05-0.2 mm, 70-270 mesh (Macherey-Nagel \& Co); size exclusion chromatography was done on Sephadex LH-20 (Lipophilic Sephadex, Amersham Biosciences Ltd; purchased from Sigma-Aldrich Chemie, Steinheim, Germany). Amberlite XAD-16 resin was obtained from Rohm and Haas, France.

\subsection{Spray reagents}

Anisaldehyde/sulphuric acid: $1 \mathrm{ml}$ anisaldehyde was added to $100 \mathrm{ml}$ of a stock solution containing $85 \mathrm{ml}$ methanol, $14 \mathrm{ml}$ acetic acid and $1 \mathrm{ml}$ sulphuric acid. Ehrlich's reagent: $1 \mathrm{~g}$ 4-dimethylaminobenzaldehyde was dissolved in a mixture of $25 \mathrm{ml}$ hydrochloric acid (37\%) and $75 \mathrm{ml}$ methanol; it gives red colouration with indole and yellow for other N-heterocycles on heating. Chlorine/o-dianisidin reaction: The reagent was prepared from $100 \mathrm{ml}(0.032 \%) o$-dianisidin in $1 \mathrm{~N}$ acetic acid, $1.5 \mathrm{~g} \mathrm{Na}_{2} \mathrm{WO}_{4} \cdot 2 \mathrm{H}_{2} \mathrm{O}$ in $10 \mathrm{ml}$ water, $115 \mathrm{ml}$ acetone and $450 \mathrm{mg} \mathrm{KI}$. The moistened TLC plate was kept ca. $30 \mathrm{~min}$ in a chlorine atmosphere (from $0.5 \mathrm{~g} \mathrm{KClO}_{3}+2$ $\mathrm{ml}$ conc. $\mathrm{HCl}$ ) and then subjected to drying for ca. $1 \mathrm{~h}$, till the excess of chlorine was evaporated and then dipped into the reagent. The reagent is specific for peptides as universal spraying reagent: Peptides turn blue or bluish green; a brown colour indicates excess chlorine. $\mathbf{N a O H}$ or KOH: $2 \mathrm{~N} \mathrm{NaOH}$ or $\mathrm{KOH}$ solutions are used to identify peri-hydroxyquinones by deepening of the colour from orange to violet or blue. 


\subsection{Microbiological materials}

Fermenter: The $50 \mathrm{~L}$ fermenter type U20 (Biostat) consisted of a $70 \mathrm{~L}$ metallic container (50 L working volume), propeller stirrer, and culture container covered with thermostat for autoclaving, cooling and thermostating (Braun Melsungen, Germany). - Storage of strains: Deep-freeze storage in a Dewar vessel, 1'Air liquid type BT 37 A. - Capillaries for deep-freeze storage: diameter $1.75 \mathrm{~mm}$, length 80 mm, Hirschmann Laborgeräte Eberstadt. - Soil for soil culture: Luvos Heilerde LUVOS JUST GmbH \& Co. Friedrichshof (from the health shop). - Ultraturrax: Janke \& Munkel KG. - Shaker: Infors AG (CH 4103 Einbach) type ITE. - Laboratory shaker: IKA-shaker type S50 (max. 6000 Upm). - Autoclave: Albert Dargatz Autoclave, volume $119 \mathrm{~L}$, working temperature $121^{\circ} \mathrm{C}$, working pressure $1.2 \mathrm{~kg} / \mathrm{cm}^{2}$. Antibiotic assay discs: 9 mm diameter, Schleicher \& Schüll No. 321 261. - Culture media: glucose (Carl Roth GmbH \& Co. KG), agar (C. L. T. Intertrade Co., LTD), peptone (Fluka), yeast extract (Hartge Ingredients $\mathrm{GmbH} \& \mathrm{Co} . \mathrm{KG}$ ), malt extract (Aspera Brauerei Reise $\mathrm{GmbH}$ ), tryptone (Fluka). - Antifoam solution: Niax PPG 2025; Union Carbide Belgium N. V. (Zwiijndrecht). - Petri dishes: 94 mm diameter, $16 \mathrm{~mm}$ height, Fa. Greiner Labortechnik, Nürtingen, Germany. - Celite (diatomaceous earth): Celite France S. A., Rueil-Malmaison Cedex, France. - Sterile filters: Midisart 2000, $0.2 \mu \mathrm{m}$, PTFE-Filter, Sartorius, Göttingen. - Laminar-FlowBox: Kojar KR-125, Reinraumtechnik GmbH, Rielasingen-Worblingen 1. - Brine shrimp eggs (Artemia salina): SERA Artemia Salinenkrebseier, SERA Heinsberg (brine shrimp eggs and food can be obtained from aquaristic shops).

\subsection{Recipes}

All cultures were autoclaved at 1.2 bar and $120{ }^{\circ} \mathrm{C}$. Sterilisation time for $1 \mathrm{~L}$ shaker culture: 33 min, 2 L concentrated medium for fermenter: 50 min and fermenter containing $16 \mathrm{~L}$ water: $80 \mathrm{~min}$.

\section{Artificial seawater}

$\begin{array}{ll}\text { Iron citrate } & 2 \mathrm{~g} \text { (powder) } \\ \mathrm{NaCl} & 389 \mathrm{~g} \\ \mathrm{MgCl}_{2} \cdot 6 \mathrm{H}_{2} \mathrm{O} & 176 \mathrm{~g} \\ \mathrm{Na}_{2} \mathrm{SO}_{4} & 68.8 \mathrm{~g} \\ \mathrm{CaCl}_{2} & 36.0 \mathrm{~g}\end{array}$




$\begin{array}{ll}\mathrm{Na}_{2} \mathrm{HPO}_{4} & 0.16 \mathrm{~g} \\ \mathrm{SiO}_{2} & 0.30 \mathrm{~g} \\ \text { trace element stock soln. } & 20 \mathrm{~mL} \\ \text { stock soln. } & 200 \mathrm{~mL} \\ \text { tap water } & \text { add } 20 \mathrm{~L}\end{array}$

\section{Trace element stock solution}

\begin{tabular}{ll}
\hline $\mathrm{H}_{3} \mathrm{BO}_{3}$ & $0.611 \mathrm{~g}$ \\
$\mathrm{MnCl}_{2}$ & $0.389 \mathrm{~g}$ \\
$\mathrm{CuSO}_{4}$ & $0.056 \mathrm{~g}$ \\
$\mathrm{ZnSO}_{4} \cdot 7 \mathrm{H}_{2} \mathrm{O}$ & $0.056 \mathrm{~g}$ \\
$\mathrm{Al}_{2}\left(\mathrm{SO}_{4}\right)_{3} \cdot 18 \mathrm{H}_{2} \mathrm{O}$ & $0.056 \mathrm{~g}$ \\
$\mathrm{NiSO}_{4} \cdot 6 \mathrm{H}_{2} \mathrm{O}$ & $0.056 \mathrm{~g}$ \\
$\mathrm{CO}\left(\mathrm{NO}_{3}\right)_{3} \cdot 6 \mathrm{H}_{2} \mathrm{O}$ & $0.056 \mathrm{~g}$ \\
$\mathrm{TiO}_{2}$ & $0.056 \mathrm{~g}$ \\
$\left(\mathrm{NH}_{4}\right)_{6} \mathrm{Mo}_{7} \mathrm{O}_{24} \cdot 4 \mathrm{H}_{2} \mathrm{O}$ & $0.056 \mathrm{~g}$ \\
$\mathrm{LiCl}$ & $0.028 \mathrm{~g}$ \\
$\mathrm{SnCl}$ & $0.028 \mathrm{~g}$ \\
$\mathrm{KI}$ & $0.028 \mathrm{~g}$ \\
Tap water & $\mathrm{ad} 1 \mathrm{~L}$ \\
\hline
\end{tabular}

Stock solution

\begin{tabular}{ll}
\hline $\mathrm{KCl}$ & $110 \mathrm{~g}$ \\
$\mathrm{NaHCO}$ & $32 \mathrm{~g}$ \\
$\mathrm{KBr}$ & $16 \mathrm{~g}$ \\
$\mathrm{SrCl}_{2} \cdot 6 \mathrm{H}_{2} \mathrm{O}$ & $6.8 \mathrm{~g}$ (dissolved separately) \\
$\mathrm{H}_{3} \mathrm{BO}_{3}$ & $4.4 \mathrm{~g}$ \\
$\mathrm{NaF}$ & $0.48 \mathrm{~g}$ \\
$\mathrm{NH}_{4} \mathrm{NO}_{3}$ & $0.32 \mathrm{~g}$ \\
Tap water & add $2 \mathrm{~L}$ \\
\hline
\end{tabular}

\subsubsection{Nutrients}

\section{$M_{2}$ medium (without seawater)}

\begin{tabular}{ll}
\hline Malt extract & $10 \mathrm{~g}$ \\
Glucose & $4 \mathrm{~g}$ \\
Yeast extract & $4 \mathrm{~g}$ \\
Tap water & ad $1 \mathrm{~L}$ \\
\hline
\end{tabular}

The $\mathrm{pH}$ was adjusted to 7.8 using $2 \mathrm{~N} \mathrm{NaOH}$. Solid medium was prepared by adding $18 \mathrm{~g}$ of agar. 
$\mathrm{M}_{2}{ }^{+}$medium $\left(\mathrm{M}_{2}\right.$ medium with seawater)

\begin{tabular}{ll}
\hline Malt extract & $10 \mathrm{~g}$ \\
Glucose & $4 \mathrm{~g}$ \\
Yeast extract & $4 \mathrm{~g}$ \\
artificial sea water & $500 \mathrm{~mL}$ \\
Tap water & $500 \mathrm{~mL}$ \\
\hline
\end{tabular}

The $\mathrm{pH}$ was adjusted to 7.8 using $2 \mathrm{~N} \mathrm{NaOH}$. Solid medium was prepared by adding $18 \mathrm{~g}$ of agar.

\section{Luria-Bertani Medium (LB)}

\begin{tabular}{ll}
\hline Trypton & $10 \mathrm{~g}$ \\
Yeast extract & $5 \mathrm{~g}$ \\
$\mathrm{NaCl}$ & $10 \mathrm{~g}$ \\
Tap water & $1000 \mathrm{~mL}$ \\
\hline
\end{tabular}

The $\mathrm{pH}$ was adjusted to 7.8 using $2 \mathrm{~N} \mathrm{NaOH}$. Solid medium was prepared by adding $18 \mathrm{~g}$ of agar.

\section{$\mathrm{LB}^{+}$Medium (Luria-Bertani medium with glucose)}

\begin{tabular}{ll}
\hline Tryptone & $10 \mathrm{~g}$ \\
Yeast & $5 \mathrm{~g}$ \\
Glucose & $5 \mathrm{~g}$ \\
$\mathrm{NaCl}$ & $10 \mathrm{~g}$ \\
Tap water & $1000 \mathrm{~mL}$ \\
\hline
\end{tabular}

The $\mathrm{pH}$ was adjusted to 7.8 using $2 \mathrm{~N} \mathrm{NaOH}$. Solid medium was prepared by adding $18 \mathrm{~g}$ of agar.

\section{Rice Medium}

\begin{tabular}{ll}
\hline rice & $100 \mathrm{~g}$ \\
distilled water & $75 \mathrm{~mL}$ \\
\hline
\end{tabular}

The rice was kept in water overnight before autoclaving. 
M Test Agar (for test organisms Escherichia coli, Bacillus subtilis (ATCC 6051), Staphylococcus aureus, Mucor miehei (Tü 284):

\begin{tabular}{ll}
\hline Malt extract & $10 \mathrm{~g}$ \\
Yeast extract & $4 \mathrm{~g}$ \\
Glucose & $4 \mathrm{~g}$ \\
Agar & $20 \mathrm{~g}$ \\
Demineralised water & $1000 \mathrm{~mL}$ \\
\hline
\end{tabular}

The $\mathrm{pH}$ was adjusted to $7.8 \mathrm{using} 2 \mathrm{~N} \mathrm{NaOH}$.

\section{Sabouraud-Agar}

(for test organism Candida albicans)

\begin{tabular}{ll}
\hline Glucose & $40 \mathrm{~g}$ \\
Bacto peptone & $10 \mathrm{~g}$ \\
Agar & $20 \mathrm{~g}$ \\
Demineralised water & $1000 \mathrm{~mL}$ \\
\hline
\end{tabular}

The $\mathrm{pH}$ was adjusted to 7.8 using $2 \mathrm{~N} \mathrm{NaOH}$.

\section{Nutritional solution A}

\begin{tabular}{ll}
\hline Soybean meal (defatted) & $30 \mathrm{~g}$ \\
Glycerol & $30 \mathrm{~g}$ \\
$\mathrm{CaCO}_{3}$ & $2 \mathrm{~g}$ \\
Artificial sea water & $750 \mathrm{ml}$ \\
Demineralised water & $250 \mathrm{ml}$ \\
\hline
\end{tabular}

Nutritional solution B

\begin{tabular}{ll}
\hline Starch & $10 \mathrm{~g}$ \\
NZ-amine & $5 \mathrm{~g}$ \\
Soybean meal & $2 \mathrm{~g}$ \\
Yeast extract & $5 \mathrm{~g}$ \\
$\mathrm{KNO}_{3}$ & $3 \mathrm{~g}$ \\
Algal extract & $2.5 \mathrm{~mL}$ \\
Artificial sea water & $750 \mathrm{~mL}$ \\
Demineralised water & $250 \mathrm{~mL}$ \\
\hline
\end{tabular}




\subsection{Stock solutions and media for cultivation of algae}

\section{Fe-EDTA}

$0.7 \mathrm{~g}$ of $\mathrm{FeSO}_{4} 7 \mathrm{H}_{2} \mathrm{O}$ and $0.93 \mathrm{~g}$ EDTA (Titriplex III) are dissolved in $80 \mathrm{ml}$ of demineralised water at $60{ }^{\circ} \mathrm{C}$ and then diluted to $100 \mathrm{ml}$.

\section{Trace element Solution II:}

\section{Solution A:}

\begin{tabular}{ll}
\hline $\mathrm{MnSO}_{4} \cdot \mathrm{H}_{2} \mathrm{O}$ & $16.9 \mathrm{mg}$ \\
$\mathrm{Na}_{2} \mathrm{MoO}_{4} \cdot 2 \mathrm{H}_{2} \mathrm{O}$ & $13.0 \mathrm{mg}$ \\
$\mathrm{Co}\left(\mathrm{NO}_{3}\right)_{2} \cdot 6 \mathrm{H}_{2} \mathrm{O}$ & $10.0 \mathrm{mg}$ \\
\hline
\end{tabular}

Salts are dissolved in $10 \mathrm{ml}$ of demineralised water.

\section{Solution B:}

\begin{tabular}{ll}
\hline $\mathrm{CuSO}_{4} \cdot 5 \mathrm{H}_{2} \mathrm{O}$ & $5.0 \mathrm{mg}$ \\
$\mathrm{H}_{3} \mathrm{BO}_{3}$ & $10.0 \mathrm{mg}$ \\
$\mathrm{ZnSO}_{4} \cdot 7 \mathrm{H}_{2} \mathrm{O}$ & $10.0 \mathrm{mg}$ \\
\hline
\end{tabular}

Salts are dissolved each in $10 \mathrm{ml}$ of demineralised water. Solutions A is added to $\mathrm{B}$ and diluted to $100 \mathrm{ml}$ with demineralised water.

Bold's Basal medium (BBM): (for algae Chlorella vulgaris, Chlorella sorokiniana and Scenedesmus subspicatus.

\begin{tabular}{|c|c|}
\hline $\mathrm{NaNO}_{3}$ & $0.250 \mathrm{~g}$ \\
\hline $\mathrm{KH}_{2} \mathrm{PO}_{4}$ & $0.175 \mathrm{~g}$ \\
\hline $\mathrm{K}_{2} \mathrm{HPO}_{4}$ & $0.075 \mathrm{~g}$ \\
\hline $\mathrm{MgSO}_{4} \cdot 7 \mathrm{H}_{2} \mathrm{O}$ & $0.075 \mathrm{~g}$ \\
\hline $\mathrm{NaCl}$ & $0.025 \mathrm{~g}$ \\
\hline $\mathrm{CaCl}_{2} \cdot 2 \mathrm{H}_{2} \mathrm{O}$ & $0.025 \mathrm{~g}$ \\
\hline Fe-EDTA & $1.0 \mathrm{~mL}$ \\
\hline Trace element solution II & $0.1 \mathrm{~mL}$ \\
\hline
\end{tabular}

Salts are dissolved in $10 \mathrm{ml}$ of demineralised water and added to Fe-EDTA and trace element solution II. The mixture is filled up to one litre with demineralised water. Solid medium was prepared by adding $18 \mathrm{~g}$ of agar. 


\subsection{Microbiological and analytical methods}

\subsubsection{Storage of strains}

All bacteria strains were stored in liquid nitrogen for long time. The strains were used to inoculate agar plates with the suitable media at room temperature.

\subsubsection{Pre-screening}

The microbial isolates (obtained from culture collections) were cultured in a $1 \mathrm{~L}$ scale in 1 L-Erlenmeyer flasks each containing 200 250 $\mathrm{ml}$ of $\mathrm{M}_{2}$ or (for marine strains) $\mathrm{M}_{2}{ }^{+}$medium. The flasks were shaken for 3-5 days at $28^{\circ} \mathrm{C}$, after which the entire fermentation broth was freeze-dried and the residue extracted with ethyl acetate. The extracts were evaporated to dryness and used for the antimicrobial tests at $400 \mu \mathrm{g} /$ paper disk.

\subsubsection{Biological screening}

The crude extract was dissolved in $\mathrm{CHCl}_{3} / 10 \% \mathrm{MeOH}$ (400 $\mu \mathrm{g} /$ paper disk), in which the paper disks were dipped, dried under sterile conditions (flow box) and put on an agar plates inoculated with the Gram-positive bacteria Bacillus subtilis (ATCC6051), Staphylococcus aureus and Streptomyces viridochromogenes (Tü 57), the Gram-negative Escherichia coli, the yeast, Candida albicans, and the fungi $M u$ cor miehei (Tü 284) along with the three microalgae, Chlorella vulgaris, Chlorella sorokiniana, and Scenedesmus subspicatus.

The plates were incubated at $37{ }^{\circ} \mathrm{C}$ for bacteria (12 hours), $27{ }^{\circ} \mathrm{C}$ for fungi (24 hours), and $24-26{ }^{\circ} \mathrm{C}$ under daylight for micro-algae (96 hours). The diameters of the inhibition zones were measured by ruler.

\subsubsection{Chemical and pharmacological screening}

Samples of the extracts were separated on silica gel glass plates $(10 \times 20 \mathrm{~cm})$ with two solvent systems $\mathrm{CHCl}_{3} / 5 \% \mathrm{MeOH}$ and $\mathrm{CHCl}_{3} / 10 \% \mathrm{MeOH}$. After drying, the plates were photographed under UV light at $254 \mathrm{~nm}$ and marked at $366 \mathrm{~nm}$, and subsequently stained by anisaldehyde and Ehrlich's reagent. Finally, the plates were scanned for documentation. For the pharmacological investigations, approximately $25 \mathrm{mg}$ of the crude extract was sent to industrial partners. 


\subsubsection{Brine shrimp microwell cytotoxicity assay}

To a $500 \mathrm{ml}$ separating funnel, filled with $400 \mathrm{ml}$ of artificial seawater, $1 \mathrm{~g}$ of dried eggs of Artemia salina L. The suspension was aerated by bubbling air into the funnel and kept for 24 to 48 hours at room temperature. After aeration had been removed, the suspension was kept for $1 \mathrm{~h}$ undisturbed, whereby the remaining unhatched eggs dropped. In order to get a higher density of larvae, one side of the separating funnel was covered with aluminium foil and the other illuminated with a lamp, whereby the phototropic larvae were gathering at the illuminated side and could be collected by pipette. 30 to 40 shrimp larvae were transferred to a deep-well microtiter plate (wells diameter $1.8 \mathrm{~cm}$, depth $2 \mathrm{~cm}$ ) filled with $0.2 \mathrm{ml}$ of salt water and the dead larvae counted (number N). A solution of $20 \mu \mathrm{g}$ of the crude extract in 5 to 10 $\mu \mathrm{l}$ DMSO was added and the plate kept at room temperature in the dark. After $24 \mathrm{~h}$, the dead larvae were counted in each well under the microscope (number A). The still living larvae were killed by addition of $c a .0 .5 \mathrm{ml}$ methanol so that subsequently the total number of the animals could be determined (number $\mathrm{G}$ ). The mortality rate $\mathrm{M}$ was calculated in \%. Each test row was accompanied by a blind sample with pure DMSO (number B) and a control sample with $1 \mu \mathrm{g} /$ test actinomycin D. The mortality rate $\mathrm{M}$ was calculated using the following formula:

$$
M=\left[\frac{(A-B-N)}{(G-N)}\right] \cdot 100
$$

with

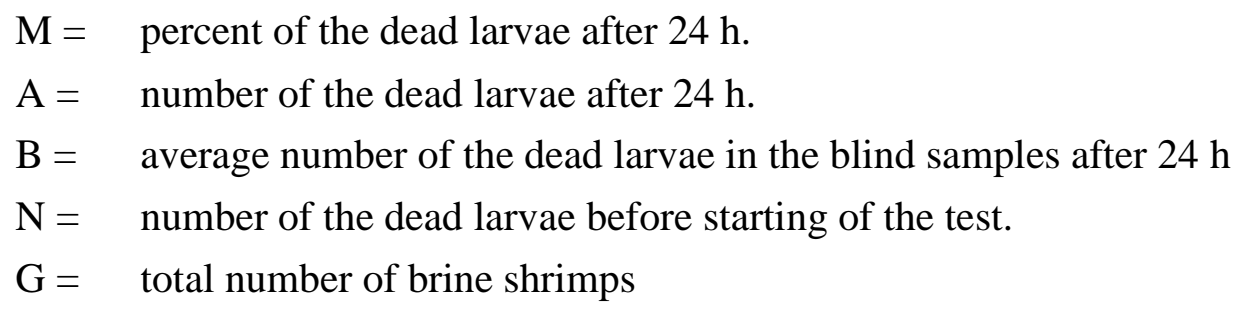

The mortality rate with actinomycin D must be $100 \%$.

\subsubsection{Primary screening results}

Antibiotic screening (disk diffusion test): The test is performed using paper discs with a diameter of $8 \mathrm{~mm}$ under standardized conditions (see above). If the inhibition zone is ranging from 11 to $20 \mathrm{~mm}$, the compound is considered to be weakly 
active (+), from 21 to $30 \mathrm{~mm}$ designated as active (++) and over $30 \mathrm{~mm}$ is highly active (+++). - Chemical screening: evaluation of the separated bands by the number, intensity and colour reactions with different staining reagents on TLC. - Toxicity test: By counting survivors after $24 \mathrm{hrs}$, the mortality of the extracts was calculated (see above). The extracts, fractions or isolated compounds were considered inactive when the mortality rate was lower than $10 \%$ (-), from 10 to $59 \%$ as weakly active (+), from 60 to $95 \%$ as active (++) and over $95 \%$ as strongly active (+++). 


\section{Metabolites from selected bacterial strains}

\subsection{Marine Streptomyces sp. B8289}

Culture of the marine Streptomyces sp. B8289 showed a white mycelium on agar plates (using $\mathrm{M}_{2}{ }^{+}$agar medium and incubation at $28{ }^{\circ} \mathrm{C}$ for four days). Fermentation of the strain as $1 \mathrm{~L}$ scale at $28{ }^{\circ} \mathrm{C}$ using $\mathrm{M}_{2}{ }^{+}$for three days afforded a brown culture broth. After extraction of the filtrate and biomass separately with ethyl acetate (3 times for each of filtrate and biomass), brown extracts were obtained.

The extracts were tested antimicrobially (see Table 3 ). The chemical screening on TLC showed four middle polar bands after spraying with anisaldehyde/sulphuric acid, which turned blue, red and brown.

Table 3: Antimicrobial activities of the crude extract of marine Streptomyces sp. B8289

\section{Tested microorganisms}

Inhibition zone $\varnothing[\mathrm{mm}]$

Bacillus subtilis 0

Staphylococcus aureus 13

Streptomyces viridochromogenes (Tü 57)

Escherichia coli

Candida albicans

Mucor miehei (Tü 284)

Chlorella vulgaris

\subsubsection{Fermentation and Isolation}

The marine Streptomyces sp. isolate B8289 was inoculated from its soil culture on three $\mathrm{M}_{2}{ }^{+}$agar plates prepared with tap water. After incubation for 72 hours at 28 ${ }^{\circ} \mathrm{C}$, the well-developed colonies were used for up-scaling culture. The up-scaling fermentation was carried out in $25 \mathrm{~L}_{\text {of }} \mathrm{M}_{2}^{+}$medium. The cultures were cultivated at $110 \mathrm{rpm}$ for 5 days at $28^{\circ} \mathrm{C}$. After 5 days, the cultures were harvested and the broth was mixed with $c a .1 \mathrm{~kg}$ Celite and filtered by means of a filter press. The biomass was extracted with ethyl acetate and acetone until the filtrate was colourless. The water phase was adsorbed on XAD-16 resin column and followed by elution with methanol. The acetone extract was concentrated to a small volume in vacuo and then 
extracted with EtOAc. As TLC of both extracts showed similar compositions, they were combined and evaporated under vacuum, to yield $3.4 \mathrm{~g}$ of brown crude extract.

The extract was subjected to column chromatography $(3 \times 60 \mathrm{~cm}, 150 \mathrm{~g}$ silica gel), the column being eluted with $\mathrm{CH}_{2} \mathrm{Cl}_{2}-\mathrm{MeOH}$ gradient (0 to $10 \% \mathrm{MeOH}$ ) and separated under TLC control into four fractions. The first fraction contained only fatty acids and was not further analysed. The second fraction afforded 2-[5-(2-oxopropyl)tetrahydrofuran-2-yl]-propionic acid methyl ester (78, $6.1 \mathrm{mg})$ after purification on Sephadex LH-20 column. The third fraction yielded 2-[5-(2-oxo-propyl)tetrahydrofuran-2-yl]-propionic acid (77, $6.3 \mathrm{mg}$ ) after purification on Sephadex LH20 column. The last fraction was subjected to silica gel column chromatography and followed by Sephadex LH-20 column to obtain homononactic acid (79, $100.5 \mathrm{mg}$ ) and homononactic acid methyl ester $(\mathbf{8 0}, 30.1 \mathrm{mg})$, respectively.

2-[5-(2-Oxo-propyl)-tetrahydrofuran-2-yl]propionic acid (mixture of epimers, 77): Colourless oil, non UV absorbing band, turned brown with anisaldehyde/sulphuric acid. $-\boldsymbol{R}_{f}=0.15\left(\mathrm{CH}_{2} \mathrm{Cl}_{2} / 5 \%\right.$<smiles></smiles>
$\left.\mathrm{CH}_{3} \mathrm{OH}\right)$. - ${ }^{1} \mathbf{H}$ NMR $\left(\mathbf{C D C l}_{3}, 300 \mathrm{MHz}\right.$, minor epimer I/major epimer II 1:2): $\delta=$ 8.84 (br s, 1H, COO마), 4.36/4.27 (m, 1H, H-5), 4.13/4.04 (m, 1H, H-2), 2.80 (dd, J $\left.=15.9,6.5 \mathrm{~Hz}, 1 \mathrm{H}, \mathrm{H}_{\mathrm{A}}-3^{\prime}\right), 2.55\left(\mathrm{dd}, J=15.8,6.4 \mathrm{~Hz}, 1 \mathrm{H}, \mathrm{H}_{\mathrm{B}}-3^{\prime}\right), 2.50(\mathrm{q}, J=7.0$ $\mathrm{Hz}, 1 \mathrm{H}, \mathrm{H}-2$ "), 2.17 (s, 3H, $\left.\mathrm{H}_{3}-1^{\prime}\right), 2.08\left(\mathrm{~m}, 1 \mathrm{H}, \mathrm{H}_{\mathrm{A}}-4\right), 2.00\left(\mathrm{~m}, 1 \mathrm{H}, \mathrm{H}_{\mathrm{A}}-3\right), 1.63(\mathrm{~m}$, $\left.1 \mathrm{H}, \mathrm{H}_{\mathrm{B}}-3\right), 1.54\left(\mathrm{~m}, 1 \mathrm{H}, \mathrm{H}_{\mathrm{B}}-4\right), 1.14 / 1.13\left(\mathrm{~d}, J=7.0 \mathrm{~Hz}, 3 \mathrm{H}, \mathrm{H}_{3}-3 "\right) .-{ }^{13} \mathbf{C}$ NMR $\left(\mathrm{CDCl}_{3}, \mathbf{1 2 5} \mathrm{MHz}\right.$, minor epimer I/major epimer II): $\delta=207.4 / 207.3\left(\mathrm{C}_{\mathrm{q}}{ }^{-}{ }^{\prime}\right)$, $179.5\left(\mathrm{C}_{\mathrm{q}}-1 "\right)$, 80.4/79.8 (CH-2), 75.6/75.3 (CH-5), 49.6/49.3 $\left(\mathrm{CH}_{2}-3{ }^{\prime}\right), 45.2 / 44.7$ (CH-2"), 32.0/30.9 ( $\left.\mathrm{CH}_{2}-4\right), 30.8 / 30.6\left(\mathrm{CH}_{3}-1^{\prime}\right), 29.7 / 28.5\left(\mathrm{CH}_{2}-3\right), 13.3 / 13.0\left(\mathrm{CH}_{3}-\right.$ 3"). - (+)-ESI MS: $m / z(\%)=223\left([\mathrm{M}+\mathrm{Na}]^{+}, 60\right), 423\left([2 \mathrm{M}+\mathrm{Na}]^{+}, 100\right) .-(-)$-ESI MS: $m / z(\%)=199\left([\mathrm{M}-\mathrm{H}]^{-}, 47\right), 399\left([2 \mathrm{M}-\mathrm{H}]^{-}, 100\right) .-(+)-$ HRESI MS: $m / z=$ 223.09510 [M+Na $]^{+}$(calcd. 223.09410 for $\mathrm{C}_{10} \mathrm{H}_{16} \mathrm{O}_{4} \mathrm{Na}$ ). 


\section{2-[5-(2-Oxo-propyl)-tetrahydrofuran-2-}

yl]-propionic acid methyl ester (mixture of ep-

imers, 78): Colourless oil, non UV absorbing band, stained to brown with anisalde-<smiles>[CH]C(=O)C[C@@H]1CC[C@H](C(C)C(=O)O[CH2])O1</smileshyde/sulphuric acid. $-\boldsymbol{R}_{\boldsymbol{f}}=0.33\left(\mathrm{CH}_{2} \mathrm{Cl}_{2} / 5 \% \mathrm{CH}_{3} \mathrm{OH}\right) .-{ }^{\mathbf{1}} \mathbf{H} \mathbf{N M R}\left(\mathbf{C D}_{\mathbf{3}} \mathbf{O D}, 300\right.$ MHz, epimer I/epimer II): $\delta=4.32 / 4.21$ (m, 1H, H-5), 4.09/3.97 (m, 1H, H-2), 3.65 (s, 3H, OMe), $2.67\left(\mathrm{~m}, 1 \mathrm{H}, \mathrm{H}_{\mathrm{A}}-3^{\prime}\right), 2.58$ (m, 1H, $\left.\mathrm{H}_{\mathrm{B}}-3^{\prime}\right), 2.50$ (m, 1H, H-2"), $2.15\left(\mathrm{~s}, 3 \mathrm{H}, \mathrm{H}_{3}-1^{\prime}\right), 2.09\left(\mathrm{~m}, 1 \mathrm{H}, \mathrm{H}_{\mathrm{A}^{-}}-4\right), 2.00\left(\mathrm{~m}, 1 \mathrm{H}, \mathrm{H}_{\mathrm{A}}-3\right), 1.65\left(\mathrm{~m}, 1 \mathrm{H}, \mathrm{H}_{\mathrm{B}}-3\right), 1.54$ $\left(\mathrm{m}, 1 \mathrm{H}, \mathrm{H}_{\mathrm{B}}-4\right), 1.10 / 1.08\left(\mathrm{~d}, J=7.0 \mathrm{~Hz}, 3 \mathrm{H}, \mathrm{H}_{3}-3 "\right) .-{ }^{13} \mathbf{C}$ NMR $\left(\mathbf{C D}_{3} \mathbf{O D}, 125\right.$ MHz, epimer I/epimer II 1:1): $\delta=210.02 / 209.95\left(\mathrm{C}_{\mathrm{q}}-2^{\prime}\right), 177.1 / 177.0\left(\mathrm{C}_{\mathrm{q}}-1^{\prime \prime}\right)$, 82.3/81.7 (CH-2), 76.9/76.6 (CH-5), 52.10/50.08 ( $\left.\mathrm{CH}_{3}-4 "\right)$, 50.5/50.2 $\left(\mathrm{CH}_{2}-3^{\prime}\right)$, 46.8/46.5 (CH-2"), 33.0/31.9 $\left(\mathrm{CH}_{2}-4\right), 30.7 / 30.5\left(\mathrm{CH}_{3}-1^{\prime}\right), 30.4 / 29.4\left(\mathrm{CH}_{2}-3\right)$, 14.0/13.7 $\left(\mathrm{CH}_{3}-3 "\right) .-(+)$-ESI MS: $m / z(\%)=237\left([\mathrm{M}+\mathrm{Na}]^{+}, 36\right), 451\left([2 \mathrm{M}+\mathrm{Na}]^{+}\right.$,

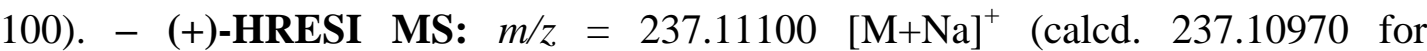
$\left.\mathrm{C}_{11} \mathrm{H}_{18} \mathrm{O}_{4} \mathrm{Na}\right)$.

Homononactic acid (79): Colourless oil, non UV absorbing band, stained to yellow with anisaldehyde/sulphuric acid. $-\boldsymbol{R}_{f}=0.19\left(\mathrm{CH}_{2} \mathrm{Cl}_{2} / 5 \%\right.$

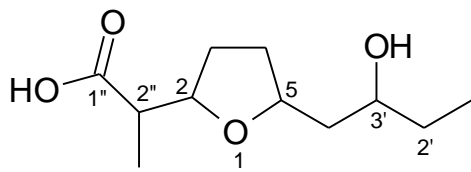
$\left.\mathrm{CH}_{3} \mathrm{OH}\right) .-{ }^{\mathbf{1}} \mathbf{H}$ NMR $\left(\mathbf{C D C l}_{\mathbf{3}}, \mathbf{3 0 0} \mathbf{M H z}\right): \delta=4.06$ (m, 1H, H-2), 3.91 (m, 1H, H-5), 3.64 (m, 1H, H-3'), 2.39 (m, 1H, H-2"), 1.89 (m, 2H, H-3), 1.54-1.36 (m, 6H, H-4,2',4'), 1.01 (d, $J=7.0$ Hz, 3H, 4"-CH $), 0.79$ (t, $J=$ $\left.7.5 \mathrm{~Hz}, 3 \mathrm{H}, \mathrm{H}-1^{\prime}\right) .-{ }^{13} \mathbf{C}$ NMR $\left(\mathbf{C D C l}_{3}, \mathbf{1 2 5} \mathbf{M H z}\right): \delta=177.8\left(\mathrm{C}_{\mathrm{q}}-1^{\prime \prime}\right), 80.1(\mathrm{CH}-5)$, 76.7 (CH-2), 70.1 (CH-3'), $44.9\left(\mathrm{CH}_{2}-4\right), 40.8\left(\mathrm{CH}_{2}-4\right)$, 30.5 (CH-2"), $29.6\left(\mathrm{CH}_{2}-2^{\prime}\right)$, $28.4\left(\mathrm{CH}_{2}-3\right), 13.3\left(\mathrm{CH}_{3}-4 "\right), 9.7\left(\mathrm{CH}_{3}-1\right)$. - (+)-ESI MS: $\mathrm{m} / z(\%)=239\left([\mathrm{M}+\mathrm{Na}]^{+}\right.$, 36), $455\left([2 \mathrm{M}+\mathrm{Na}]^{+}, 100\right) .-(+)$-HRESI MS: $m / z=239.12620[\mathrm{M}+\mathrm{Na}]^{+}$(calcd $^{-}$ 239.12540 for $\mathrm{C}_{11} \mathrm{H}_{20} \mathrm{O}_{4} \mathrm{Na}$ ).

Homononactic acid methyl ester (80): Colourless oil, UV non-absorbing band, turned yellow with anisaldehyde/sulphuric acid. $-\boldsymbol{R}_{\boldsymbol{f}}=0.34$

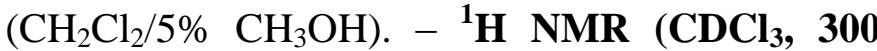
MHz): $\delta=4.06$ (m, 1H, H-2), 3.91 (m, 1H, H-5), 3.64 (m, 1H, H-3'), 3.60 (s, 3H, 1"$\left.\mathrm{COOCH}_{3}\right), 2.44(\mathrm{dq}, 1 \mathrm{H}, J=8.2,7.0 \mathrm{~Hz}, \mathrm{H}-2 "), 1.91(\mathrm{~m}, 2 \mathrm{H}, \mathrm{H}-3), 1.62-1.37$ (m, 
6H, H-4,2',4'), 1.04 (d, 3H, $J=7.0 \mathrm{~Hz}, \mathrm{H}-3 "), 0.85$ (t, 3H, $J=7.4 \mathrm{~Hz}, \mathrm{H}-1) .-(+)-$ ESI MS: $m / z(\%)=253\left([\mathrm{M}+\mathrm{Na}]^{+}, 36\right), 483\left([2 \mathrm{M}+\mathrm{Na}]^{+}, 100\right) .-(+)-$ HRESI MS: $m / z=253.14210[\mathrm{M}+\mathrm{Na}]^{+}$(calcd. 253.14100 for $\left.\mathrm{C}_{12} \mathrm{H}_{22} \mathrm{O}_{4} \mathrm{Na}\right)$.

\subsection{Terrestrial Streptomyces sp. Ank86}

The terrestrial Streptomyces sp. Ank86 was grown on $\mathrm{M}_{2}$ agar. After incubation at $28{ }^{\circ} \mathrm{C}$ for four days, colonies with a white aerial mycelium were formed. This culture was used for $1 \mathrm{~L}$ fermentation in $\mathrm{M}_{2}$ medium at $28^{\circ} \mathrm{C}$ for five days on the round shaker. The extract was used for the biological and chemical screening analysis.

On TLC, it showed several UV absorbing zones, which changed to violet, red, and blue with anisaldehyde/sulphuric acid.

Table 4: Antimicrobial activities of the crude extract of terrestrial Streptomyces sp. Ank86

\section{Tested microorganisms}

Inhibition zone $\varnothing[\mathrm{mm}]$

\begin{tabular}{ll} 
Bacillus subtilis & 13 \\
Staphylococcus aureus & 11 \\
Streptomyces viridochromogenes (Tü 57) & 13 \\
\hline
\end{tabular}

\subsubsection{Fermentation and isolation}

Well-grown plates were inoculated in a $25 \mathrm{~L}$ shaker culture on $\mathrm{M}_{2}$ medium and grown at $28{ }^{\circ} \mathrm{C}$ on linear shaker. After 6 days, the culture was harvested and the water phase and biomass were separated with the aid of a filter press. The water phase was subjected to XAD-16 resin column, followed by washing with demineralised water. Then, the XAD-16 resin was eluted with methanol. The biomass was extracted with ethyl acetate and acetone. The organic extracts were combined based on similar compositions of the extracts on TLC.

The crude extract $(2.4 \mathrm{~g})$ was chromatographed on a silica gel column $(3 \times 60$ $\mathrm{cm}, 150 \mathrm{~g}$ ) using gradient solvent system of dichloromethane and methanol (0 to $15 \% \mathrm{MeOH})$ to afford four fractions. Fraction I contained fats and was not further investigated. The purification of fraction II with Sephadex LH-20 (MeOH as eluent) and RP-18 columns delivered 4-hydroxy-2-methoxyacetanilide $(\mathbf{8 1}, 2.10 \mathrm{mg})$. The purification of fraction III with Sephadex LH-20 using methanol as solvent system 
afforded tryptophol. And finally, the fourth fraction was subjected to Sephadex LH$20(\mathrm{MeOH}$ as eluent) to isolate actinomycin D $(\mathbf{8 2}, 30.4 \mathrm{mg})$.

4-Hydroxy-2-methoxyacetanilide (81): Colourless solid, UV absorbing at $254 \mathrm{~nm}$, no colour reaction with anisaldehyde/sulphuric acid. $-\boldsymbol{R}_{f}=0.5\left(\mathrm{CH}_{2} \mathrm{Cl}_{2} / 5 \% \mathrm{MeOH}\right) .-{ }^{1} \mathbf{H}$ NMR (CD ${ }_{3}$ OD, 300 MHz): $\delta=7.56\left(\mathrm{dd},{ }^{3} J=8.2 \mathrm{~Hz},{ }^{4} J=1.9 \mathrm{~Hz}, 1 \mathrm{H}\right.$, 2-H), $7.52\left(\mathrm{~d},{ }^{4} \mathrm{~J}=1.9 \mathrm{~Hz}, 1 \mathrm{H}, 5-\mathrm{H}\right), 6.85\left(\mathrm{~d},{ }^{3} J=8.2 \mathrm{~Hz}, 1 \mathrm{H}, 3-\right.$ H), 3.89 (s, 3H, 9-H), 2.53 (s, 3H, 8-H). - (+)-ESI MS: $m / z(\%)$

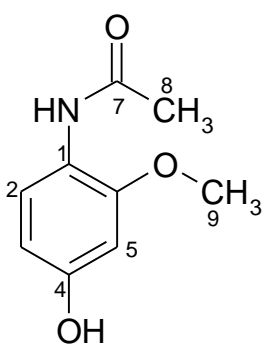
$=182\left([\mathrm{M}+\mathrm{H}]^{+}, 32\right), 204\left([\mathrm{M}+\mathrm{Na}]^{+}, 100\right) .-(+)$-HRESI MS: $m / z=182.08125$ $\left([\mathrm{M}+\mathrm{H}]^{+}\right), 204.06321\left([\mathrm{M}+\mathrm{Na}]^{+}\right)\left(\right.$calc. 204.06311 for $\left.\mathrm{C}_{9} \mathrm{H}_{11} \mathrm{NO}_{3} \mathrm{Na}\right)$.

Actinomycin D (82): Reddish-orange solid, UV absorbing zone at $254 \mathrm{~nm}$, and red with anisaldehyde/sulphuric acid, no colour change with $2 \mathrm{~N}$ $\mathrm{NaOH}$, deep red colour reaction with conc. $\mathrm{H}_{2} \mathrm{SO}_{4}$. - $\boldsymbol{R}_{\boldsymbol{f}}=0.37\left(\mathrm{CHCl}_{3} / \mathrm{MeOH}\right.$ 95:5). $\quad-{ }^{1} \mathrm{H} \quad \mathrm{NMR}$ $\left(\mathrm{CDCl}_{3}, 300 \mathrm{MHz}\right): \delta=8.13\left(\mathrm{~d} \mathrm{br},{ }^{3} J=6.4 \mathrm{~Hz}, 1\right.$ $\mathrm{H}, \mathrm{NH}-\mathrm{Val}_{\alpha}$ ), 7.97 (d br, ${ }^{3} \mathrm{~J}=6.4 \mathrm{~Hz}, 1 \mathrm{H}, \mathrm{NH}-$ $\left.\mathrm{Val}_{\beta}\right), 7.81\left(\mathrm{~d} \mathrm{br},{ }^{3} J=6.4 \mathrm{~Hz}, 1 \mathrm{H}, \mathrm{NH}-\mathrm{Thr}_{\beta}\right), 7.67$ $\left(\mathrm{d},{ }^{3} J=7.9 \mathrm{~Hz}, 1 \mathrm{H}, \mathrm{H}-8\right), 7.39\left(\mathrm{~d},{ }^{3} J=7.9 \mathrm{~Hz}, 1 \mathrm{H}\right.$, 7-H), $7.21\left(\mathrm{~d} \mathrm{br},{ }^{3} J=6.4 \mathrm{~Hz}, 1 \mathrm{H}, \mathrm{NH}-\mathrm{Thr}_{\alpha}\right.$ ), 6.03

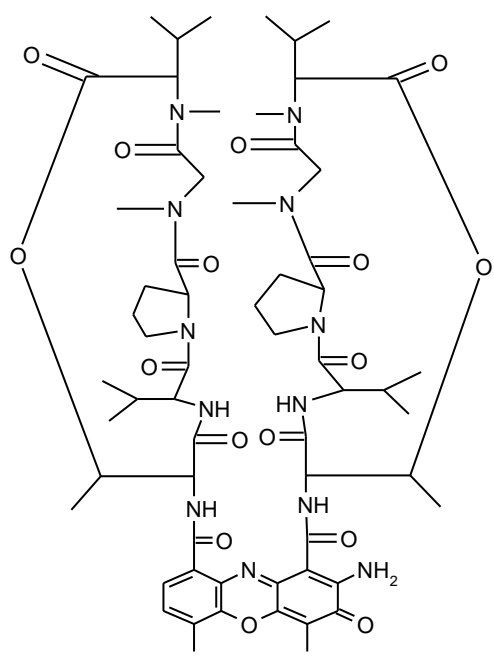
$\left(\mathrm{d},{ }^{3} J=9.1 \mathrm{~Hz}, 1 \mathrm{H}, \mathrm{H}-2, \operatorname{Pro}_{\alpha}\right), 5.96\left(\mathrm{~d},{ }^{3} J=9.1 \mathrm{~Hz}, 1 \mathrm{H}, \mathrm{H}-2 \operatorname{Pro}_{\beta}\right), 5.25-5.15$ (m, 2 H, H-3, Thr $\left.{ }_{\alpha, \beta}\right), 4.81\left(\mathrm{~d},{ }^{3} J=18.1 \mathrm{~Hz}, 1 \mathrm{H}, \mathrm{H}_{\mathrm{a}}-2, \operatorname{Sar}_{\alpha}\right), 4.73\left(\mathrm{~d},{ }^{3} J=18.8 \mathrm{~Hz}, 1 \mathrm{H}\right.$, $\left.\mathrm{H}_{\mathrm{a}}-2, \operatorname{Sar}_{\beta}\right), 4.61\left(\mathrm{dd},{ }^{3} J=6.4 \mathrm{~Hz},{ }^{3} J=2.3 \mathrm{~Hz}, 1 \mathrm{H}, \mathrm{H}-2, \mathrm{Thr}_{\alpha}\right), 4.49\left(\mathrm{dd},{ }^{3} J=6.4,{ }^{3} J\right.$ $\left.=2.3 \mathrm{~Hz}, 1 \mathrm{H}, \mathrm{H}-2, \mathrm{Thr}_{\beta}\right), 4.03-3.40\left(\mathrm{~m}, 8 \mathrm{H}, \mathrm{H}_{\mathrm{b}}-2 \operatorname{Sar}_{\alpha, \beta}, \mathrm{H}-2, \operatorname{Val}_{\alpha, \beta}, \mathrm{H}_{2}-5, \operatorname{Pro}_{\alpha, \beta}\right)$, 2.93, 2.90, $2.88\left(3 \mathrm{~s}, 3 \mathrm{H}+3 \mathrm{H}+6 \mathrm{H}, \mathrm{NCH}_{3} \mathrm{Me}-\mathrm{Val}_{\alpha, \beta}, \mathrm{NCH}_{3}, \mathrm{Sar}_{\alpha, \beta}\right), 2.67,2.57(\mathrm{~m}$ $\left.+\mathrm{s}, 6+3, \mathrm{H}_{\mathrm{a}}-3, \operatorname{Pro}_{\alpha, \beta}, \mathrm{H}-2,3, \mathrm{MeVal}_{\alpha, \beta}, \mathrm{CH}_{3}-6\right), 2.38-1.76+2.23(\mathrm{~m}+\mathrm{s}, 8+3 \mathrm{H}$, $\left.\mathrm{H}_{\mathrm{b}}-3, \mathrm{H}_{2}-4, \mathrm{Pro}_{\alpha, \beta} \mathrm{H}-3, \mathrm{Val}_{\alpha, \beta} \mathrm{CH}_{3}-4\right), 1.28$ (m, $\left.6 \mathrm{H}, \mathrm{CH}_{3}, \mathrm{Thr}_{\alpha, \beta}\right)$, 1.17-1.07 (m, $6 \mathrm{H}$, $\left.\mathrm{CH}_{3}, \mathrm{Val}_{\alpha, \beta}\right), 0.99-0.82$ (m, $\left.12 \mathrm{H}, \mathrm{CH}_{3} \mathrm{Val}_{\alpha, \beta}, \mathrm{CH}_{3}, \mathrm{Val}_{\alpha, \beta}\right), 0.75$ (d, ${ }^{3} \mathrm{~J}=4.9 \mathrm{~Hz}, 6 \mathrm{H}$, $\left.\mathrm{CH}_{3}, \mathrm{Val}_{\alpha, \beta}\right),-(+)$-ESI MS: $m / z(\%)=1277\left([\mathrm{M}+\mathrm{Na}]^{+}, 100\right), 1255\left([\mathrm{M}+\mathrm{H}]^{+}, 6\right)$. $(-)-$ ESI MS: $m / z(\%)=1254\left([\mathrm{M}-\mathrm{H}]^{\top}\right)$. 


\subsection{Terrestrial Streptomyces sp. Ank68}

The bioassay of the crude extract, of $1 \mathrm{~L}$ fermentation in $\mathrm{M}_{2}$ medium at $28{ }^{\circ} \mathrm{C}$ for three days, from strain Ank68 exhibited strong activities against bacteria, fungi, algae, and Artemia salina (brine shrimp).It showed strong UV absorbing zones at 254 and $366 \mathrm{~nm}$, strong red and black colour reactions with anisaldehyde/sulphuric acid.

Table 5: Antimicrobial activities of the crude extract of terrestrial Streptomyces sp. Ank68

Tested microorganisms

Inhibition zone $\varnothing[\mathrm{mm}]$

Bacillus subtilis $\quad 40$

Staphylococcus aureus $\quad 29$

Streptomyces viridochromogenes (Tü 57) 24

Escherichia coli 0

Candida albicans 20

Mucor miehei (Tü 284) 22

Chlorella vulgaris 19

Chlorella sorokiniana 19

Scenedesmus subspicatus 18

$\begin{array}{ll}\text { Artemia salina } & 100 \%\end{array}$

\subsubsection{Fermentation and isolation}

The terrestrial Streptomyces sp. Ank68 was grown on agar of $\mathrm{M}_{2}$ medium. After incubation at $28{ }^{\circ} \mathrm{C}$ for four days, white mycelium colonies were formed. These wellgrown plates of Streptomyces sp. Ank68 were inoculated and grown in a $25 \mathrm{~L}$ shaker culture of $\mathrm{M}_{2}$ medium at $28{ }^{\circ} \mathrm{C}$ on linear shaker. After 5 days, the cultures were harvested and mixed with Celite to separate water phase and biomass by using the filter press. The water phase was subjected to XAD-16 column, and followed by washing with demineralised water. After that, the XAD resin was eluted with methanol. The biomass was extracted with ethyl acetate and acetone. On the basis of TLC, these three organic extracts were combined.

The crude extract (4.43 g) was then chromatographed on a silica gel column $(3 \times$ $75 \mathrm{~cm}, 150 \mathrm{~g}$ ) using a gradient solvent systems of dichloromethane and methanol (100\% $\mathrm{CH}_{2} \mathrm{Cl}_{2}$ to $20 \%$ methanol in dichloromethane) to afford three fractions. From fraction II, actinomycin D $(\mathbf{8 2}, 20.30 \mathrm{mg})$ was isolated after purification steps with 
preparative thin layer chromatography (PTLC) and Sephadex LH-20 column $(\mathrm{MeOH})$. Fraction III was subjected subsequently to Sephadex LH-20 (MeOH) and $\mathrm{RP}-18\left(\mathrm{MeOH} / \mathrm{H}_{2} \mathrm{O}\right)$ columns to obtain fungichromin $(\mathbf{8 3}, 4.15 \mathrm{mg})$. Fraction I contained fats and revealed blue spots after staining with anisaldehyde/sulphuric acid; it was not further investigated.

\section{Fungichromin $\quad(83)$ :}

Highly polar yellow solid, UV fluorescent band at $366 \mathrm{~nm}$, black with anisaldehyde/sulphuric acid. $-\boldsymbol{R}_{f}=$ $0.17\left(\mathrm{CH}_{2} \mathrm{Cl}_{2} / 10 \% \mathrm{MeOH}\right)$. -

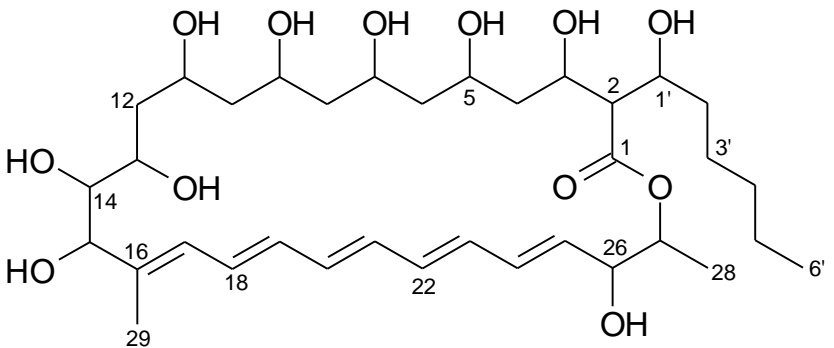

${ }^{1}$ H NMR (DMSO- $d_{6}, 300$ MHz): $\delta=6.49-6.23(\mathrm{~m}, 7 \mathrm{H}, 18,19,20,21,22,23,24-\mathrm{H})$, $5.94\left(\mathrm{~d},{ }^{3} \mathrm{~J}=10.9 \mathrm{~Hz}, 2 \mathrm{H}, 17,25-\mathrm{H}\right), 5.23-3.48$ (m, 11H, 1',3,5,7,9,11,13,14,15,26,27H), 2.45 (m, 1H, 2-H), 1.69 (s, 3H, 29-H), 1.40-1.07 (m, 21H, 2',3',4,4',5',6,8,10,12, 28-H), 0.85 (m, 3H, 6'-H). $-{ }^{13}$ C NMR (DMSO- $d_{6}, 300$ MHz): $\delta=170.4(\mathrm{C}-1)$, 138.7 (C-16), 134.9 (CH-19), 133.21 (CH-21), 133.20 (CH-25), 133.0 (CH-23), 132.9 (CH-20), 132.8 (CH-22), 129.1 (CH-24), 127.1 (CH-17), 127.0 (CH-18), 78.1 (CH-15), 76.5 (CH-14), 73.1 (CH-27), 71.2 (CH-9), 71.1 (CH-5), 70.4 (CH-7), 70.3 (CH-3), 70.0 (CH-26), 69.7 (CH-1'), 69.6 (CH-11), 69.2 (CH-13), 58.7 (CH-2), 43.9 $\left(\mathrm{CH}_{2}-8\right), 43.8\left(\mathrm{CH}_{2}-6\right), 42.9\left(\mathrm{CH}_{2}-10\right), 40.1\left(\mathrm{CH}_{2}-4\right), 38.6\left(\mathrm{CH}_{2}-12\right), 34.2\left(\mathrm{CH}_{2}-2^{\prime}\right)$, $31.2\left(\mathrm{CH}_{2}-4^{\prime}\right), 24.5\left(\mathrm{CH}_{2}-3^{\prime}\right), 22.0\left(\mathrm{CH}_{2}-5^{\prime}\right), 17.7\left(\mathrm{CH}_{3}-29\right), 13.8\left(\mathrm{CH}_{3}-6^{\prime}\right), 11.6\left(\mathrm{CH}_{3}-\right.$ 28). - (+)-ESI MS: $m / z(\%)=671\left([\mathrm{M}+\mathrm{H}]^{+}, 63\right), 693\left([\mathrm{M}+\mathrm{Na}]^{+}, 100\right), 1363$ $\left([2 \mathrm{M}+\mathrm{Na}]^{+}, 90\right) .-(-)$-ESI MS: $m / z(\%)=669\left([\mathrm{M}-\mathrm{H}]^{-}, 38\right), 1339\left([3 \mathrm{M}-\mathrm{H}]^{-}, 96\right) .-$ (+)-HRESI MS: $m / z=671.40010\left([\mathrm{M}+\mathrm{H}]^{+}\right)\left(\right.$calc. 671.39984 for $\left.\mathrm{C}_{35} \mathrm{H}_{59} \mathrm{O}_{12}\right)$.

\subsection{Terrestrial Streptomyces sp. Ank248}

The well-grown agar plate of the terrestrial Streptomyces sp. Ank248 isolate was used for inoculation in $1 \mathrm{~L} \mathrm{M}_{2}$ medium and the culture was incubated for 3 days at 28 ${ }^{\circ} \mathrm{C}$ on linear shaker $(95 \mathrm{rpm})$. The culture broth was then extracted with ethyl acetate to afford the crude extract for pre-screening.

The biological activity assay resulted in weak antimicrobial activity against bacteria, but it showed strong activity against Artemia salina. 
Table 6: Antimicrobial activities of the crude extract of terrestrial Streptomyces sp. Ank248

Tested microorganisms

Inhibition zone $\varnothing[\mathrm{mm}]$

Bacillus subtilis

Staphylococcus aureus

Streptomyces viridochromogenes (Tü 57)

Escherichia coli

Artemia salina
15

11

12

11

$100 \%$

\subsubsection{Fermentation and isolation}

Well-developed colonies on agar plates were used for inoculation of $10 \mathrm{~L}$ of LB medium. The culture was incubated at $28{ }^{\circ} \mathrm{C}$ on linear shaker ( $95 \mathrm{rpm}$ ) for 4 days. The resulting brown culture broth was mixed with Celite and pressed through a filter press to afford the aqueous filtrate and the mycelial residue. The aqueous phase was extracted by Amberlite XAD-16 resin using methanol as the eluent. The biomass was extracted with ethyl acetate and acetone separately. Then, all three organic extracts were evaporated to dryness under reduced pressure. On TLC, all three crude extracts showed the same spot pattern and accordingly they were combined to obtain $1.23 \mathrm{~g}$ of brown crude extract. This extract was then dissolved in methanol and subjected to Sephadex LH-20 column using $\mathrm{MeOH} / \mathrm{CH}_{2} \mathrm{Cl}_{2}$ (4:6) solvent system to afford four fractions. Fraction II was purified on Sephadex LH-20 column using methanol as eluent and followed by RP-18 column using $\mathrm{MeOH} / \mathrm{H}_{2} \mathrm{O}$ as eluent system to isolate 1 -acetyl- $\beta$-carboline $(\mathbf{8 4}, 11.3 \mathrm{mg})$. Fraction III and fraction IV were subjected to Sephadex LH-20 columns with methanol as eluent to obtain tryptophol and 3hydroxy-acetylindole.

1-Acetyl- $\beta$-carboline (84): Brownish powder, UV absorbing band at $254 \mathrm{~nm}$, pale yellow with anisaldehyde/sulphuric acid. $-\boldsymbol{R}_{f}=0.31\left(\mathrm{CH}_{2} \mathrm{Cl}_{2} / 5 \% \mathrm{MeOH}\right) .-{ }^{\mathbf{1}} \mathbf{H}$ NMR $\left(\mathbf{C D C l}_{3}, 300 \mathrm{MHz}\right): \delta=10.28$ (br s, NH), $8.53\left(\mathrm{~d},{ }^{3} \mathrm{~J}\right.$<smiles>CC(=O)c1nccc2c1[nH]c1ccccc12</smiles>
$=4.9 \mathrm{~Hz}, 1 \mathrm{H}, 4-\mathrm{H}), 8.15-8.13(\mathrm{~m}, 2 \mathrm{H}, 3,5-\mathrm{H}), 7.58\left(\mathrm{dd},{ }^{4} J=1.1 \mathrm{~Hz},{ }^{3} J=6.7 \mathrm{~Hz}, 2 \mathrm{H}\right.$, 7,8-H), 7.32 (m, 1H, 6-H), 2.88 (s, 3H, $\left.\mathrm{COC}_{3}{ }_{3}\right)$. 


\subsection{Terrestrial Streptomyces sp. Ank181}

The marine Streptomyces sp. Ank 181 grew as white aerial mycelium colonies when cultivated on $\mathrm{M}_{2}^{+}$medium agar plates at $28{ }^{\circ} \mathrm{C}$ for 3 days. The chemical screening showed UV absorbing zones as colourless and strongly blue florescent (365 $\mathrm{nm})$ bands in the non polar region and absorbing zones $(254 \mathrm{~nm})$ in the middle polar region, which turned dark green, violet and red with anisaldehyde/sulphuric acid.

Table 7: Antimicrobial activities of the crude extract of terrestrial Streptomyces sp. Ank181

\section{Tested microorganisms}

Inhibition zone $\varnothing[\mathrm{mm}]$

Bacillus subtilis

0

Staphylococcus aureus

Streptomyces viridochromogenes (Tü 57)

0

Escherichia coli

0

Candida albicans

0

Mucor miehei (Tü 284)

Chlorella vulgaris

0

Chlorella sorokiniana

0

Scenedesmus subspicatus

0

Artemia salina

0

\subsubsection{Fermentation and isolation}

Well-grown agar plates of the terrestrial Streptomyces sp. Ank 181 were used to inoculate a shaker culture on $\mathrm{M}_{2}$ medium, which was cultivated for 6 days at $28{ }^{\circ} \mathrm{C}$ affording a faint yellow broth. The resulting culture was harvested and filtered over Celite using a filter press. The filtrate was extracted by XAD-16, and the absorbed metabolites were eluted with methanol. The methanol phase was evaporated and the resulting water residue was extracted by ethyl acetate. The biomass was extracted with acetone and the water residue obtained after concentration was extracted with ethyl acetate. The combined organic phases were evaporated under vacuum to afford a yellow-brown crude extract $(3.10 \mathrm{~g})$.

TLC of the strain extract showed mostly middle polar UV absorbing bands at $254 \mathrm{~nm}$, which stained to green, red and violet with anisaldehyde/sulphuric acid and 
heating. Additionally, a fast moving band was found, which turned dark green by spraying with anisaldehyde/sulphuric acid and heating.

The crude extract ( $3.10 \mathrm{~g})$ was subjected to column chromatography on silica gel and was eluted with a $\mathrm{CH}_{2} \mathrm{Cl}_{2} / \mathrm{MeOH}$ gradient system $\left(100 \% \mathrm{CH}_{2} \mathrm{Cl}_{2}, \mathrm{CH}_{2} \mathrm{Cl}_{2}\right.$ / $3 \% \mathrm{MeOH}, \mathrm{CH}_{2} \mathrm{Cl}_{2} / 5 \% \mathrm{MeOH}, \mathrm{CH}_{2} \mathrm{Cl}_{2} / 10 \% \mathrm{MeOH}, \mathrm{CH}_{2} \mathrm{Cl}_{2} / 20 \% \mathrm{MeOH}$, $\left.\mathrm{CH}_{2} \mathrm{Cl}_{2} / 40 \% \mathrm{MeOH}\right)$ to give four fractions, which were monitored by TLC. Fraction I contained fats and was not further continued for investigation. Fractions II, III, and IV were subjected to Sephadex LH-20 columns using methanol as the solvent system to obtain reductiomycin $(\mathbf{8 5}, 20.1 \mathrm{mg}), 2,3$-dihydroxybenzoic acid and indole-3carboxylic acid, respectively.

Reductiomycin (85): Middle polar yellow solid, UV absorbing band at $254 \mathrm{~nm}$, and green with anisaldehyde/sulphuric acid. - $\boldsymbol{R}_{\boldsymbol{f}}=0.30\left(\mathrm{CH}_{2} \mathrm{Cl}_{2} / 5 \% \mathrm{MeOH}\right) . \quad-{ }^{\mathbf{1}} \mathbf{H}$

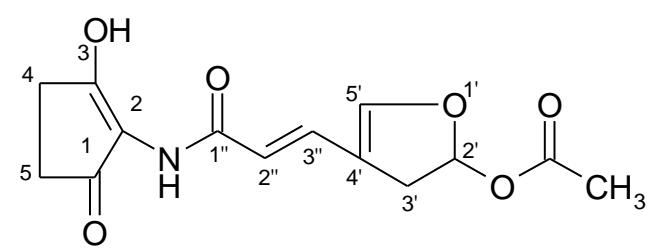
NMR (CDCl 3 , 300 MHz): $\delta=13.76$ (br s, 1 H, 3-OH), 7.68 (br s, 1 H, 2-NH), 7.50 (d, $\left.{ }^{3} J=15.1 \mathrm{~Hz}, 1 \mathrm{H}, \mathrm{H}-3^{\prime \prime}\right), 6.90$ (s, $\left.1 \mathrm{H}, \mathrm{H}-5^{\prime}\right), 6.70$ (dd, $\left.{ }^{3} J=7.5,2.3 \mathrm{~Hz}, 1 \mathrm{H}, \mathrm{H}-2^{\prime}\right)$, $5.81\left(\mathrm{~d},{ }^{3} \mathrm{~J}=15.1 \mathrm{~Hz}, 1 \mathrm{H}, \mathrm{H}-2 "\right), 3.05\left(\mathrm{~m}, 1 \mathrm{H}, \mathrm{H}_{\mathrm{A}}-3\right.$ '), 2.60 (m, $5 \mathrm{H}, 4 / 5-\mathrm{CH}_{2}, \mathrm{H}_{\mathrm{B}^{-}}$ 3'). - (-)-ESI MS: $m / z(\%)=607\left([2 \mathrm{M}-\mathrm{H}+\mathrm{Na}]^{-}, 100\right), 292\left([\mathrm{M}-\mathrm{H}]^{-}, 77\right)$.

\subsection{Ruminal Bacterium Enterobacter amnigenus ZIA}

The strain of Enterobacter amnigenus ZIA was isolated and identified by Imene Zendah from rumen cow in Tunisia. The strain was cultivated on LB agar medium at $37{ }^{\circ} \mathrm{C}$ for 24 hours. In the biological screening, the crude extract of the ruminal bacterium Enterobacter amnigenus ZIA did not give biological activity against testedmicroorganisms.

\subsubsection{Fermentation and isolation}

Pieces of well-gown agar plates were used to inoculate in 40 of $1 \mathrm{~L}$ Erlenmeyer flasks, each containing $250 \mathrm{~mL}$ of sterilized LB medium. The fermentation was carried out on linear shaker $(180 \mathrm{rpm})$ at $28{ }^{\circ} \mathrm{C}$ for 4 days. The culture broth was harvested and mixed with Celite. The Celite-mixture of the culture broth was then filtered with a filter press to obtain the water phase and biomass. The biomass was extracted with ethyl acetate (3 times) and acetone ( 2 times), respectively. The filtrate 
was passed on the XAD-16 resin column, washed with $15 \mathrm{~L}$ demineralised water, and then eluted with $15 \mathrm{~L}$ methanol. After evaporating the solvents, all the organic phases were combined together based on thin layer chromatogram.

The crude extract $(3.3 \mathrm{~g})$ was then chromatographed on silica gel (column $3 \times$ $60 \mathrm{~cm}, 150$ g) using a $\mathrm{CH}_{2} \mathrm{Cl}_{2} / \mathrm{MeOH}$ gradient $\left(1.5 \mathrm{~L} \mathrm{CH}_{2} \mathrm{Cl}_{2}, 1.0 \mathrm{~L} \mathrm{CH}_{2} \mathrm{Cl}_{2} / 2 \%\right.$ $\mathrm{MeOH}$ ), $1.0 \mathrm{~L} \mathrm{CH} \mathrm{Cl}_{2} / 3 \% \mathrm{MeOH}, 1.0 \mathrm{~L} \mathrm{CH}_{2} \mathrm{Cl}_{2} / 5 \% \mathrm{MeOH}, 0.5 \mathrm{~L} \mathrm{CH}_{2} \mathrm{Cl}_{2} / 10 \%$ $\mathrm{MeOH}, \mathrm{CH}_{2} \mathrm{Cl}_{2} / 20 \% \mathrm{MeOH}, 0.5 \mathrm{~L} \mathrm{CH}_{2} \mathrm{Cl}_{2} / 50 \% \mathrm{MeOH}$, and $0.5 \mathrm{~L} \mathrm{MeOH}$ ), which afforded four main fractions. The purification of fraction II with Sephadex LH-20 delivered poly-(hydroxybutyric acid) (PHB) (86, $8.8 \mathrm{mg}$ ) and indole-3-acetic acid. From fraction III, uracil and tyrosol were isolated through Sephadex LH-20 chromatography (methanol as eluent). Hypoxanthine and butyl glycoside $(\mathbf{8 7}, 1.0 \mathrm{mg}$ ) were purified from fraction IV by Sephadex LH-20 (MeOH) and RP-18 $\left(\mathrm{MeOH}: \mathrm{H}_{2} \mathrm{O}\right)$ chromatography columns, respectively.

Poly-(hydroxybutyric acid) (PHB) (86): White solid, no UV absorbing band, and pink with anisaldehyde/sulphuric acid. $-\boldsymbol{R}_{f}=0.57\left(\mathrm{CH}_{2} \mathrm{Cl}_{2} / 10 \% \mathrm{MeOH}\right)$. $-{ }^{1} \mathbf{H}$ NMR $\left(\mathrm{CDCl}_{3}, 300 \mathrm{MHz}\right): \delta=5.20(\mathrm{~m}, 1 \mathrm{H}, \mathrm{CH})$, $2.45\left(\mathrm{dd}, 1 \mathrm{H},{ }^{3} \mathrm{~J}=7.4 \mathrm{~Hz},{ }^{2} \mathrm{~J}=15.5 \mathrm{~Hz}, \mathrm{CH}_{2}\right), 1.22(\mathrm{~d}$, $\left.{ }^{3} J=6.3 \mathrm{~Hz}, \mathrm{CH}_{3}\right)$.

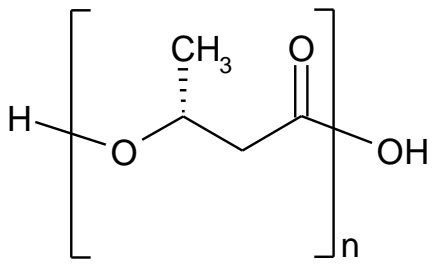

$\mathrm{n}=10-22$

Butyl glycoside (87): Pale yellow oil, no UV absorbing band, green with anisaldehyde/sulphuric acid. $-\boldsymbol{R}_{f}=0.11\left(\mathrm{CH}_{2} \mathrm{Cl}_{2} / 10 \%\right.$ $\mathrm{MeOH}) .-{ }^{1} \mathrm{H}$ NMR (CD $\left.\mathbf{O D}, 300 \mathrm{MHz}\right): \delta=$<smiles>CCCCO[C@H]1O[C@H](CO)C(O)C(O)C1O</smiles>
$4.72\left(\mathrm{~d},{ }^{3} \mathrm{~J}=1.5 \mathrm{~Hz}, 1 \mathrm{H}, 1^{\prime}-\mathrm{H}\right), 3.81-3.39$ (m, 9H, 1,1', 2', 3', $\left.4^{\prime}, 5^{\prime}, 6^{\prime}-\mathrm{H}\right), 1.56$ (m, 2H, 2- $\left.\mathrm{H}_{2}\right), 1.41\left(\mathrm{~m}, 2 \mathrm{H}, 3-\mathrm{H}_{2}\right), 0.93\left(\mathrm{t},{ }^{3} J=7.3 \mathrm{~Hz}, 3 \mathrm{H}, 4-\mathrm{H}_{3}\right) .-{ }^{\mathbf{1 3}} \mathbf{C}$ NMR $\left(\mathbf{C D}_{\mathbf{3}} \mathbf{O D}, 125\right.$ MHz): $\delta=101.5\left(\mathrm{CH}-1^{\prime}\right), 74.5\left(\mathrm{CH}-2^{\prime}\right), 72.6(\mathrm{CH}-3 '), 72.3\left(\mathrm{CH}-4{ }^{\prime}\right), 68.6\left(\mathrm{CH}-5^{\prime}\right)$, $68.2\left(\mathrm{CH}_{2}-1\right), 62.9\left(\mathrm{CH}_{2}-6\right), 32.7\left(\mathrm{CH}_{2}-2\right), 20.5\left(\mathrm{CH}_{2}-3\right), 14.3\left(\mathrm{CH}_{3}-4\right)$. - (+)-ESI MS: $m / z(\%)=259.1\left([\mathrm{M}+\mathrm{Na}]^{+}, 100\right), 495.2([2 \mathrm{M}+\mathrm{Na}]+, 17) .-(+)$-HRESI MS: $m / z=259.11536\left([\mathrm{M}+\mathrm{Na}]^{+}\right)\left(\right.$calc. 259.11521 for $\left.\mathrm{C}_{10} \mathrm{H}_{20} \mathrm{O}_{6} \mathrm{Na}\right)$. 


\subsection{Terrestrial Streptomyces sp. Ank75}

The terrestrial Streptomyces sp. Ank75 was grown on $\mathrm{M}_{2}$ agar medium for 3 days at $28^{\circ} \mathrm{C}$, forming a white aerial mycelium. This culture was used for $1 \mathrm{~L}$ fermentation in $\mathrm{M}_{2}$ medium at $28^{\circ} \mathrm{C}$ for three days on the round shaker. The crude extract of the strain presented activities against fungi Candida albicans and Mucor miehei (Tü 284) as shown in Table 8. The TLC exhibited several UV absorbing zones, which changed to red, and green with anisaldehyde/sulphuric acid.

Table 8: Antimicrobial activities of the crude extract of terrestrial Streptomyces sp. Ank75

Tested microorganisms

Inhibition zone $\varnothing$ [mm]

Bacillus subtilis

0

Staphylococcus aureus

Streptomyces viridochromogenes (Tü 57)

0

Escherichia coli

0

Candida albicans

Mucor miehei (Tü 284)

Chlorella vulgaris

Chlorella sorokiniana

Scenedesmus subspicatus

\subsubsection{Fermentation and Isolation}

The Ank75 strain was fermented in $25 \mathrm{~L}$ scale on $\mathrm{M}_{2}$ medium for 5 days at 28 ${ }^{\circ} \mathrm{C}$. The culture broth was filtered over Celite and the water phase was adsorbed on resin Amberlite XAD-16 column and eluted with methanol after washing with demineralised water prior to the elution with methanol. The biomass was exhaustively extracted with ethyl acetate and acetone. TLC of crude extract from each organic residues was identical, thus the three extracts $(5.4 \mathrm{~g})$ were combined together for further work-up procedure.

The separation of crude extract $(5.4 \mathrm{~g})$ was performed by silica gel column chromatography $(3 \times 75 \mathrm{~cm}, 200 \mathrm{~g})$ using $\mathrm{aCH}_{2} \mathrm{Cl}_{2} / \mathrm{CH}_{3} \mathrm{OH}$ gradient. Based on the spot pattern on the TLC, four fractions were selected for further purification. Fraction II was subjected to Sephadex LH-20 column with methanol as the eluent to afford benadrostin $(\mathbf{8 8}, 1.6 \mathrm{mg})$ and 2-O-methylpyrogallol $(\mathbf{8 9}, 1.1 \mathrm{mg})$. From fraction III, N-aminocarbonyl-2,3-dihydroxybenzamide (91, $1.2 \mathrm{mg})$, 2,3-dihydroxybenz- 
amide (92,1.3 $\mathrm{mg})$, and 2,3-dihydroxybenzoic acid $(1.5 \mathrm{mg})$ were isolated via Sephadex $\mathrm{LH}-20$ column using $\mathrm{CH}_{3} \mathrm{OH} / \mathrm{CH}_{2} \mathrm{Cl}_{2}$ (6:4) as the solvent system. Bandamycin (94,1.2 mg) and 3-[(1Z)-1-hexenyl-ONN-azoxyl]-1,2-butanediol (93,1.1 mg) were isolated from fraction IV after being subjected to Sephadex LH-20 (MeOH) and RP-18 (MeOH: $\left.\mathrm{H}_{2} \mathrm{O}\right)$ columns. Finally, the fraction $\mathrm{V}$ delivered $\mathrm{N}^{6}, \mathrm{~N}^{6}$-dimethyladenosine $(\mathbf{9 5}, 2.3 \mathrm{mg})$.

Benadrostin (88): Colourless solid. $-\boldsymbol{R}_{f}=0.20$ $\left(\mathrm{CH}_{2} \mathrm{Cl}_{2} / 5 \% \mathrm{MeOH}\right)$, strong $\mathrm{UV}$ absorbing band at $254 \mathrm{~nm}$, orange with anisaldehyde/sulphuric acid. - ${ }^{1}$ H NMR (DMSO$\left.d_{6}, 300 \mathrm{MHz}\right): \delta=11.17$ (br s, $\left.1 \mathrm{H}, 3-\mathrm{H}\right), 7.35\left(\mathrm{dd},{ }^{3} J=7.5 \mathrm{~Hz}\right.$, $\left.{ }^{4} J=1.7 \mathrm{~Hz}, 1 \mathrm{H}, 5-\mathrm{H}\right), 7.24\left(\mathrm{dd},{ }^{3} J=7.9 \mathrm{~Hz},{ }^{4} J=1.7 \mathrm{~Hz}, 1 \mathrm{H}\right.$,<smiles>O=c1[nH]c(=O)c2cccc(O)c2o1</smiles>
7-H), $7.18\left(\mathrm{t},{ }^{3} J=7.7 \mathrm{~Hz}, 1 \mathrm{H}, 6-\mathrm{H}\right)$. - (-)-ESI MS: $m / z(\%)=178\left([\mathrm{M}-\mathrm{H}]^{-}, 100\right)$, $356\left([2 \mathrm{M}-\mathrm{H}]^{-}, 80\right), 379([2 \mathrm{M}-\mathrm{H}+\mathrm{Na}])^{-} .-(-)$-HRESI MS: $m / z=178.01403\left([\mathrm{M}-\mathrm{H}]^{-}\right)$, (calculated 178.01348 for $\mathrm{C}_{8} \mathrm{H}_{4} \mathrm{NO}_{4}$ ).

2-O-Methylpyrogallol (89): Colorless oil, UV absorbing band at $254 \mathrm{~nm}$ and red with anisaldehyde/sulphuric acid. $-\boldsymbol{R}_{f}=$ $0.23\left(\mathrm{CH}_{2} \mathrm{Cl}_{2} / 5 \% \mathrm{MeOH}\right) .-{ }^{1} \mathbf{H}$ NMR (acetone- $\left.\boldsymbol{d}_{6}, 300 \mathrm{MHz}\right): \delta$ $=6.72\left(\mathrm{t},{ }^{3} J=8.1 \mathrm{~Hz}, 1 \mathrm{H}, 5-\mathrm{H}\right), 6.37\left(\mathrm{~d},{ }^{3} J=8.1 \mathrm{~Hz}, 2 \mathrm{H}, 4,6-\mathrm{H}\right)$,<smiles>COc1c(O)cccc1O</smiles>
$3.77\left(\mathrm{~s}, 3 \mathrm{H}, 7-\mathrm{H}_{3}\right) .-{ }^{13} \mathbf{C}$ NMR (acetone-d $\left.\boldsymbol{d}_{\mathbf{6}}, 125 \mathbf{~ M H z}\right): \delta=151.5(\mathrm{C}-1,2,3), 124.6$ (CH-5), $108.3(\mathrm{CH}-4,6), 60.5\left(\mathrm{CH}_{3}-7\right) .-(+)-\mathbf{E I} \mathrm{MS}(\mathbf{7 0} \mathbf{~ e V}) \mathrm{m} / \mathrm{z}(\%)=142$ $\left([\mathrm{M}+2 \mathrm{H}]^{+}, 12\right), 140\left([\mathrm{M}]^{+}, 92\right), 125(100), 97$ (36), 79 (8), 51 (12) . - (+)-HRESI MS: $m / z=140.04730\left([\mathrm{M}]^{+}\right)$, (calculated 140.04730 for $\left.\mathrm{C}_{7} \mathrm{H}_{8} \mathrm{O}_{3}\right)$.

N-Aminocarbonyl-2,3-dihydroxybenzamide (91): Brown solid. $-\boldsymbol{R}_{\boldsymbol{f}}=0.17\left(\mathrm{CH}_{2} \mathrm{Cl}_{2} / 5 \% \mathrm{MeOH}\right)$, strong UV absorbing band at $254 \mathrm{~nm}$, pink with anisaldehyde/sulphuric acid. $-{ }^{\mathbf{1}} \mathbf{H}$ NMR $\left(\mathrm{CD}_{3} \mathrm{OD}, 300 \mathrm{MHz}\right): \delta=7.45\left(\mathrm{dd},{ }^{3} J=8.1 \mathrm{~Hz},{ }^{4} J=1.5 \mathrm{~Hz}, 1 \mathrm{H}, 6-\right.$ H), $7.00\left(\mathrm{dd},{ }^{3} J=7.8 \mathrm{~Hz},{ }^{4} J=1.5 \mathrm{~Hz}, 1 \mathrm{H}, 4-\mathrm{H}\right), 6.79\left(\mathrm{t},{ }^{3} J=7.9\right.$ Hz, 1H, 5-H). - (+)-ESI MS: $m / z(\%)=197\left([\mathrm{M}+\mathrm{H}]^{+}, 68\right), 219$<smiles>NC(=O)NC(=O)c1cccc(O)c1O</smiles>
$\left([\mathrm{M}+\mathrm{Na}]^{+}, 100\right) . \quad-(+)-H R E S I$ MS: $m / z=197.05573\left([\mathrm{M}+\mathrm{H}]^{+}\right), \quad($ calculated 197.05568 for $\mathrm{C}_{8} \mathrm{H}_{9} \mathrm{~N}_{2} \mathrm{O}_{4}$ ). 
2,3-Dihydroxybenzamide (92): Strong UV absorbing band at $254 \mathrm{~nm}$ and no colour reaction with anisaldehyde/sulphuric acid. $\boldsymbol{R}_{f}=0.26\left(\mathrm{CH}_{2} \mathrm{Cl}_{2} / 5 \% \mathrm{MeOH}\right) .-$ UV/VIS (MeOH): $\lambda_{\max }(\log \varepsilon)=$ 214 (4.06), 319 (3.29) nm. $-{ }^{1}$ H NMR (CD $\mathbf{3}$ OD, 300 MHz ): $\delta=$ $7.33\left(\mathrm{dd},{ }^{3} J=7.9 \mathrm{~Hz},{ }^{4} J=1.5 \mathrm{~Hz}, 1 \mathrm{H}, 6-\mathrm{H}\right), 6.89\left(\mathrm{dd},{ }^{3} J=7.9 \mathrm{~Hz}\right.$,<smiles>NC(=O)c1cccc(O)c1O</smiles>
$\left.{ }^{4} J=1.5 \mathrm{~Hz}, 1 \mathrm{H}, 4-\mathrm{H}\right), 6.64\left(\mathrm{t},{ }^{3} J=7.9 \mathrm{~Hz}, 1 \mathrm{H}, 5-\mathrm{H}\right) .-{ }^{13} \mathbf{C}$ NMR $\left(\mathbf{C D}_{\mathbf{3}}\right.$ OD, 125 MHz ): $\delta=174.8\left(\mathrm{C}-1^{\prime}\right), 151.6(\mathrm{C}-2), 146.8$ (C-3), 122.0 (CH-6), 120.7 (CH-4), $119.2(\mathrm{CH}-5), 116.0(\mathrm{C}-1)$. - (-)-ESI MS: $m / z(\%)=152\left([\mathrm{M}-\mathrm{H}]^{-}, 50\right) .-(-)$-HRESI MS: $m / z=152.03535\left([\mathrm{M}-\mathrm{H}]^{-}\right)$, (calculated 152.03532 for $\left.\mathrm{C}_{7} \mathrm{H}_{6} \mathrm{NO}_{3}\right)$.

\section{3-[(1Z)-1-Hexenyl-ONN-azoxyl]-}

1,2-butanediol (93): Colourless oil, UV absorbing band at $254 \mathrm{~nm}$, yellow-green with anisaldehyde/sulphuric acid. $-\boldsymbol{R}_{f}=$<smiles>CCCCC=C[N+]([O-])=NC(C)C(O)CO</smiles>
$0.19\left(\mathrm{CH}_{2} \mathrm{Cl}_{2} / 5 \% \mathrm{MeOH}\right) .-{ }^{1} \mathbf{H}$ NMR (CD $\left.\mathbf{3} \mathbf{O D}, 300 \mathrm{MHz}\right): \delta=6.78\left(\mathrm{~d}, 1 \mathrm{H},{ }^{3} \mathrm{~J}=\right.$ $\left.9.1 \mathrm{~Hz}, 1^{\prime}-\mathrm{H}\right), 5.78$ (m, 1H, 2'-H), 4.16 (m, 1H, 3-H), 3.76 (m, 1H, 2-H), 3.56 (m, 2H, 1-H), 2.60 (m, 2H, 3'-H), 1.49-29 (m, 4H, 4',5'-H), 1.17 (d, 3H, $\left.{ }^{3} J=6.6 \mathrm{~Hz}, 4-\mathrm{H}\right)$, $0.92\left(\mathrm{t}, 3 \mathrm{H},{ }^{3} J=7.1 \mathrm{H}, 6^{\prime}-\mathrm{H}\right) .-(+)$-ESI MS: $m / z(\%)=239\left([\mathrm{M}+\mathrm{Na}]^{+}, 90.6\right), 455$ $\left([2 \mathrm{M}+\mathrm{Na}]^{+}, 100\right) .-(+)-H R E S I$ MS: $m / z=239.13680\left([\mathrm{M}+\mathrm{Na}]^{+}\right)$, (calculated 239.1366 for $\mathrm{C}_{10} \mathrm{H}_{20} \mathrm{~N}_{2} \mathrm{O}_{3} \mathrm{Na}$ ).

Bandamycin (94): Colourless oil, UV absorbing band at $254 \mathrm{~nm}$, green with anisaldehyde/sulphuric acid. $-\boldsymbol{R}_{\boldsymbol{f}}=0.16$ $\left(\mathrm{CH}_{2} \mathrm{Cl}_{2} / 5 \% \mathrm{MeOH}\right) .-$ UV/VIS (MeOH):<smiles>CCC(=O)CCC[N+]([O-])=NC(C)C(O)CO</smiles>
$\lambda_{\max }(\log \varepsilon)=222(3.64) \mathrm{nm} .-\mathbf{I R}(\mathbf{K B r}): v=3420,2937,1710,1505,1457,1376$, 1316, 1119, $1065 \mathrm{~cm}^{-1} .{ }^{1}{ }^{1} \mathbf{H}$ NMR (DMSO- $d_{6}, 300$ MHz ): $\delta=4.73$ (br s, $1 \mathrm{H}, \mathrm{OH}$ ), 4.48 (br s, 1H, OH), 4.10 (t, ${ }^{3} J=6.9 \mathrm{~Hz}, 2 \mathrm{H}, 1^{\prime}-\mathrm{H}_{2}$ ), 3.94 (quint, ${ }^{3} J=6.5 \mathrm{~Hz}, 1 \mathrm{H}, 3$ $\mathrm{H}), 3.55(\mathrm{~m}, 1 \mathrm{H}, 2-\mathrm{H}), 3.34\left(\mathrm{~m}, 2 \mathrm{H}, 1-\mathrm{H}_{2}\right), 2.49\left(\mathrm{~m}, 2 \mathrm{H}, 3^{\prime}-\mathrm{H}_{2}\right.$, overlapped by DMSO), 2.41 (q, ${ }^{3} J=7.38 \mathrm{~Hz}, 2 \mathrm{H}, 5^{\prime}-\mathrm{H}_{2}$ ), 2.00 (quintet, ${ }^{3} J=6.98 \mathrm{~Hz}, 2 \mathrm{H}, 2^{\prime}-\mathrm{H}_{2}$ ), $1.01\left(\mathrm{~d},{ }^{3} \mathrm{~J}=6.5 \mathrm{~Hz}, 3 \mathrm{H}, 4-\mathrm{H}_{3}\right), 0.92\left(\mathrm{t},{ }^{3} J=7.3 \mathrm{~Hz}, 3 \mathrm{H}, 6{ }^{\prime}-\mathrm{H}_{3}\right) .-{ }^{13}$ C NMR (DMSO$\left.d_{6}, 125 \mathrm{MHz}\right): \delta=209.7\left(\mathrm{C}^{-} 4^{\prime}\right), 73.0(\mathrm{CH}-2), 68.6\left(\mathrm{CH}_{2}-1^{\prime}\right), 63.3\left(\mathrm{CH}_{2}-1\right), 56.5$ (CH-3), $37.6\left(\mathrm{CH}_{2}-3^{\prime}\right), 34.9\left(\mathrm{CH}_{2}-5^{\prime}\right), 21.5\left(\mathrm{CH}_{2}-2^{\prime}\right), 11.6\left(\mathrm{CH}_{3}-4\right), 7.5\left(\mathrm{CH}_{3}-6^{\prime}\right)$. - 
(+)-ESI MS: $m / z(\%)=255\left([\mathrm{M}+\mathrm{Na}]^{+}, 100\right), 487\left([2 \mathrm{M}+\mathrm{Na}]^{+}, 6\right) .-(+)-H R E S I$ MS: $m / z=255.13159\left([\mathrm{M}+\mathrm{Na}]^{+}\right)\left(\right.$calc. 255.13153 for $\left.\mathrm{C}_{10} \mathrm{H}_{20} \mathrm{~N}_{2} \mathrm{O}_{4} \mathrm{Na}\right)$.

$\mathbf{N}^{6}, \mathbf{N}^{6}$-Dimethyl-adenosine (95): Pale yellow oil. $-\boldsymbol{R}_{f}=$ $0.12\left(\mathrm{CH}_{2} \mathrm{Cl}_{2} / 5 \% \mathrm{MeOH}\right), \mathrm{UV}$ absorbing band at $254 \mathrm{~nm}$, greenish yellow with anisaldehyde/sulphuric acid. - ${ }^{1} \mathbf{H}$ NMR $\left(\mathrm{CD}_{3} \mathrm{OD}, 300 \mathrm{MHz}\right): \delta=8.18(\mathrm{~s}, 1 \mathrm{H}, 8-\mathrm{H}), 8.15(\mathrm{~s}, 1 \mathrm{H}, 2-\mathrm{H})$, $5.94\left(\mathrm{~d},{ }^{3} J=6.5 \mathrm{~Hz}, 1^{\prime}-\mathrm{H}\right), 4.74\left(\mathrm{~m}, 1 \mathrm{H}, 2^{\prime}-\mathrm{H}\right), 4.31\left(\mathrm{dd},{ }^{3} J=\right.$ $\left.5.1,2.5 \mathrm{~Hz}, 1 \mathrm{H}, 3^{\prime}-\mathrm{H}\right), 4.16$ (q, $\left.{ }^{3} J=2.5 \mathrm{~Hz}, 1 \mathrm{H}, 4^{\prime}-\mathrm{H}\right), 3.87$ (m, $\left.2 \mathrm{H}, 5^{\prime}-\mathrm{H}\right), 3.47$ (br s, 3H, N-CH 3 ), 3.34 (s, 3H, N-CH $)_{3}$. - (+)-

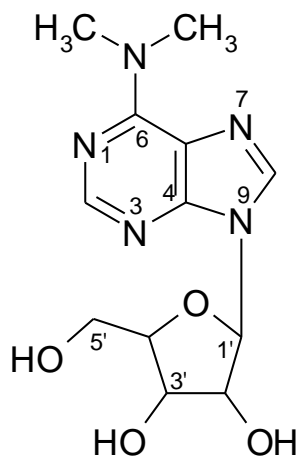
ESIMS $m / z=296\left([\mathrm{M}+\mathrm{H}]^{+}, 100\right) .-(+)-\operatorname{EIMS}(70 \mathrm{eV}) \mathrm{m} / z(\%)=295\left([\mathrm{M}]^{+}, 16\right)$, 206 (24), 192 (50), 163 (52), 148 (28), 134 (100).

\subsection{Marine Streptomyces sp. $\mathbf{B 5 7 9 8}$}

In the biological screening, the crude extract B5798 demonstrated 90\% activity against Artemia salina, but it did not show any activities against bacteria, fungi and algae.

\subsubsection{Fermentation and isolation}

The well-grown agar plates were inoculated into $25 \mathrm{~L}$ shaker culture of $\mathrm{M}_{2}{ }^{+}$medium and grown at $28^{\circ} \mathrm{C}$ for 5 days on linear shaker. After 5 days, the culture broth was harvested and mixed with Celite to separate mycelium and the water phase with the aid of filter press. The mycelium was extracted with ethyl acetate and acetone, while the water phase was passed through XAD-16 resin column and eluted with methanol. Due to the similar compositions of each crude extracts on the TLC, they were combined and the constituents were isolated using different chromatographic techniques.

The crude extract ( $6.4 \mathrm{~g})$ was subjected to silica gel chromatography column (3 $\times 75 \mathrm{~cm}, 150 \mathrm{~g})$ using gradient of $\mathrm{CH}_{2} \mathrm{Cl}_{2} / \mathrm{MeOH}$ solvent systems $\left(1.5 \mathrm{~L} \mathrm{CH}_{2} \mathrm{Cl}_{2}, 1.0\right.$ $\left.\mathrm{L} \mathrm{CH}_{2} \mathrm{Cl}_{2} / 2 \% \mathrm{MeOH}\right), 1.0 \mathrm{~L} \mathrm{CH}_{2} \mathrm{Cl}_{2} / 3 \% \mathrm{MeOH}, 1.0 \mathrm{~L} \mathrm{CH}_{2} \mathrm{Cl}_{2} / 5 \% \mathrm{MeOH}, 0.5 \mathrm{~L}$ $\mathrm{CH}_{2} \mathrm{Cl}_{2} / 10 \% \mathrm{MeOH}, \mathrm{CH}_{2} \mathrm{Cl}_{2} / 20 \% \mathrm{MeOH}, 0.5 \mathrm{~L} \mathrm{CH}_{2} \mathrm{Cl}_{2} / 50 \% \mathrm{MeOH}$, and $0.5 \mathrm{~L}$ $\mathrm{MeOH})$. Fractions II and III were purified separately with Sephadex LH-20 column and methanol as the eluent to isolate $p$-hydroxyphenylacetic acid $(\mathbf{9 6}, 1.4 \mathrm{mg})$, in- 
dole-3-carboxylic acid and indole-3-acetic acid, respectively. Finally, fraction IV was subjected to Sephadex LH-20 and RP-18 columns to obtain macrolactin-A (97, $2.46 \mathrm{mg}$ ) as oily compound.

p-Hydroxyphenylacetic acid (96): Colourless solid, UV absorbing band at $254 \mathrm{~nm}$, red with anisaldehyde/sulphuric acid. $-\boldsymbol{R}_{\boldsymbol{f}}=0.49\left(\mathrm{CH}_{2} \mathrm{Cl}_{2} / 5 \% \mathrm{MeOH}\right) .-{ }^{1} \mathbf{H}$<smiles>O=C(O)Cc1ccc(O)cc1</smiles>
NMR (CD $\left.{ }_{3} \mathrm{OD}, 300 \mathrm{MHz}\right): \delta=7.08(\mathrm{~d}, J=8.6 \mathrm{~Hz}, 2 \mathrm{H}, 2,6-\mathrm{H}), 6.73(\mathrm{~d}, J=8.6 \mathrm{~Hz}$, $2 \mathrm{H}, 3,5-\mathrm{H}), 3.47$ (s, 2H, 2'-H).

Macrolactin A (97): Reddish oil, UV absorbing band at $254 \mathrm{~nm}$, black with anisaldehyde/sulphuric acid. $-\boldsymbol{R}_{f}=0.25\left(\mathrm{CH}_{2} \mathrm{Cl}_{2} / 10 \%\right.$ $\mathrm{MeOH}) .-{ }^{\mathbf{1}} \mathrm{H}$ NMR $\left(\mathbf{C D C l}_{3}, 300 \mathrm{MHz}\right): \delta=$ $7.16\left(\mathrm{dd},{ }^{3} J=14.9,11.7 \mathrm{~Hz}, 1 \mathrm{H}, 4-\mathrm{H}\right), 6.56$ (dd, $\left.{ }^{3} J=11.4,10.9 \mathrm{~Hz}, 2 \mathrm{H}, 3,9-\mathrm{H}\right), 6.09(\mathrm{~m}, 4 \mathrm{H}$,

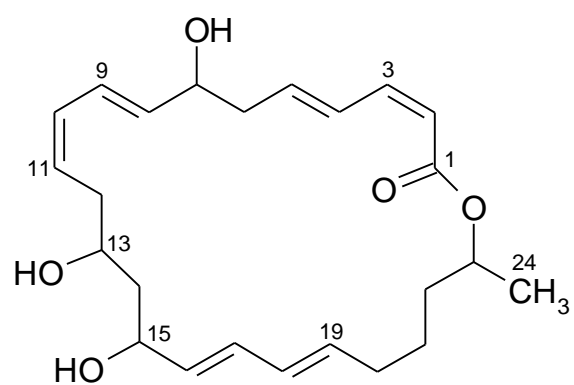
18,17,10,5-H), $5.75\left(\mathrm{dd},{ }^{3} J=15.1,5.1 \mathrm{~Hz}, 1 \mathrm{H}, 8-\mathrm{H}\right), 5.65-5.34$ (m, 4H, 19,16,11,2H), $5.01(\mathrm{~m}, 1 \mathrm{H}, 23-\mathrm{H}), 4.48(\mathrm{~m}, 1 \mathrm{H}, 15-\mathrm{H}), 4.32(\mathrm{~m}, 1 \mathrm{H}, 7-\mathrm{H}), 4.17$ (br s, 3H, 15,13,7-Oㅂ), 3.95 (m, 1H, 13-H), 2.43 (m, 4H, 12,6-H), 2.02 (m, 2H, 20-H), 1.62 (m, 4H, 22,14-H), 1.50 (m, 2H, 21-H), $1.26\left(\mathrm{~d},{ }^{3} J=6.3 \mathrm{~Hz}, 3 \mathrm{H}, 24-\mathrm{H}\right) .-{ }^{13} \mathbf{C}$ NMR $\left(\mathrm{CDCl}_{3} / \mathrm{CD}_{3} \mathrm{OD}, 125 \mathrm{MHz}\right): \delta=167.6$ (C-1), $144.4(\mathrm{CH}-3), 141.5$ (CH-5), 136.8 (CH-8), 134.6 (CH-16), 134.2 (CH-19), 131.0 (CH-18), 130.9 (CH-17), 130.6 (CH10), 129.6 (CH-4), 127.8 (CH-11), 125.3 (CH-9), 117.6 (CH-2), 71.9 (CH-23), 71.7 (CH-7), 69.5 (CH-15), 68.9 (CH-13), $42.4\left(\mathrm{CH}_{2}-14\right), 42.2\left(\mathrm{CH}_{2}-6\right), 35.8\left(\mathrm{CH}_{2}-12\right)$, $35.5\left(\mathrm{CH}_{2}-22\right), 32.5\left(\mathrm{CH}_{2}-20\right), 25.1\left(\mathrm{CH}_{2}-21\right), 20.1\left(\mathrm{CH}_{3}, \mathrm{C}-24\right) .-(+)-$ ESIMS: $m / z$ $(\%)=425\left([\mathrm{M}+\mathrm{Na}]^{+}, 100\right), 827\left([2 \mathrm{M}+\mathrm{Na}]^{+}, 86\right) .-(+)$-HRESIMS: $m / z, 425.22980$ $[\mathrm{M}+\mathrm{Na}]^{+}$, (calcd. 425.22970 for $\mathrm{C}_{24} \mathrm{H}_{34} \mathrm{O}_{5} \mathrm{Na}$ ).

\subsection{Terrestrial Streptomyces sp. GT-2005/049}

The culture of the terrestrial Streptomyces sp. GT-2005/049 exhibited white mycelium on $\mathrm{M}_{2}$ agar medium after incubation at $28{ }^{\circ} \mathrm{C}$ for four days. Fermentation of the strain in $1 \mathrm{~L}$ scale of $\mathrm{M}_{2}$ at $28^{\circ} \mathrm{C}$ and $95 \mathrm{rpm}$ for 5 days afforded a brown culture. After extraction of the filtrate and biomass with ethyl acetate, a brown crude extract was obtained. 
The crude extract was then tested antimicrobially and the activity result was presented in Table 9.

Table 9: Antimicrobial activities of the crude extract of terrestrial Streptomyces sp. GT-2005/049

\section{Tested microorganisms}

Inhibition zone $\varnothing[\mathrm{mm}]$

Bacillus subtilis 15

Staphylococcus aureus

22

Streptomyces viridochromogenes (Tü 57)

Escherichia coli

Candida albicans

Mucor miehei (Tü 284)

26

Chlorella vulgaris

0

Chlorella sorokiniana

0

Scenedesmus subspicatus

5
2
7
6
0
6
0
0
0

\subsubsection{Fermentation and isolation}

The well-grown agar plate of the terrestrial Streptomyces sp. GT-2005/049 was inoculated into 100 of $1 \mathrm{~L}$ Erlenmeyer flasks containing $300 \mathrm{ml}$ of $\mathrm{M}_{2}$ medium (pH 7.8 before sterilisation) in each flask. The culture was incubated on linear shaker culture at $28{ }^{\circ} \mathrm{C}$ for 6 days. The culture was harvested after 6 days and the resulting brown culture broth was filtered over Celite using a filter press. The filtrate and the mycelial cake were separately extracted by ethyl acetate and acetone. The combined extracts, prior to monitoring on TLC, were evaporated to dryness under reduced pressure to afford a brown crude extract (4.1 g).

The separation of the crude extract was performed on silica gel column chromatography $(3 \times 60 \mathrm{~cm}, 150 \mathrm{~g})$ by gradient solvent systems $\left(1.5 \mathrm{~L} \mathrm{CH}_{2} \mathrm{Cl}_{2}, 1.0 \mathrm{~L}\right.$ $\left.\mathrm{CH}_{2} \mathrm{Cl}_{2} / 2 \% \mathrm{MeOH}\right), 1.0 \mathrm{~L} \mathrm{CH}_{2} \mathrm{Cl}_{2} / 3 \% \mathrm{MeOH}, 1.0 \mathrm{~L} \mathrm{CH}_{2} \mathrm{Cl}_{2} / 5 \% \mathrm{MeOH}, 0.5 \mathrm{~L}$ $\mathrm{CH}_{2} \mathrm{Cl}_{2} / 10 \% \mathrm{MeOH}, \mathrm{CH}_{2} \mathrm{Cl}_{2} / 20 \% \mathrm{MeOH}, 0.5 \mathrm{~L} \mathrm{CH}_{2} \mathrm{Cl}_{2} / 50 \% \mathrm{MeOH}$, and $0.5 \mathrm{~L}$ $\mathrm{MeOH})$. According to the spot pattern on TLC, three fractions were selected for further investigation.

Lumichrome $(98,3.8 \mathrm{mg})$ and were isolated from fraction II by purification steps with Sephadex LH-20 (in MeOH system) and RP-18 column (using $\mathrm{MeOH}: \mathrm{H}_{2} \mathrm{O}$ eluent system). From fraction III, 1-acetyl- $\beta$-carboline (84) and indole3-carboxylic acid were obtained via Sephadex LH-20 column using methanol as the 
solvent. Finally, the purification of fraction IV with Sephadex LH-20 column ( $\mathrm{MeOH}$ as the mobile phase) resulted in uracil, thymine and 1-methyluracil.

Lumichrome (98): Pale yellow solid, yellow with anisaldehyde/sulphuric acid, UV absorbing band at $254 \mathrm{~nm} .-\boldsymbol{R}_{\boldsymbol{f}}=0.37\left(\mathrm{CH}_{2} \mathrm{Cl}_{2} / 10 \% \mathrm{MeOH}\right) .-{ }^{\mathbf{1}} \mathbf{H}$ NMR (pyridin- $\left.d_{5}, 300 \mathrm{MHz}\right): \delta=8.03(\mathrm{~s}, 1 \mathrm{H}, 6-\mathrm{H})$,

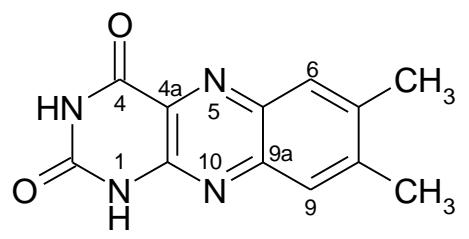
7.85 (s, 1H, 9-H), 2.33 (s, 3H, 8- $\mathrm{CH}_{3}$ ), 2.27 (s, 3H, 7- $\left.\mathrm{CH}_{3}\right) .-{ }^{13} \mathbf{C}$ NMR (pyridin- $d_{5}$, 125 MHz): $\delta=161.9$ (C-4), 151.7 (C-2), 147.6 (C-10a), 144.8 (C-8), 143.0 (C-9a), 139.9 (C-7), 139.1 (C-5a), 130.9 (C-4a), 129.8 (CH-6), 127.2 (CH-9), $20.4\left(\mathrm{CH}_{3}-8\right)$, $19.8\left(\mathrm{CH}_{3}-7\right) .-(+)$-ESI MS: $\mathrm{m} / z(\%)=265\left([\mathrm{M}+\mathrm{Na}]^{+}, 28\right), 507\left([2 \mathrm{M}+\mathrm{Na}]^{+}, 100\right) .-$ (-)-ESI MS: $m / z(\%)=241\left([\mathrm{M}-\mathrm{H}]^{-}, 100\right), 505\left([2 \mathrm{M}-\mathrm{H}]^{-}, 7\right) .-(+)$-HRESI MS: $m / z$ $=265.07014\left([\mathrm{M}+\mathrm{Na}]^{+}\right)$, (calculated 265.06970 for $\left.\mathrm{C}_{12} \mathrm{H}_{10} \mathrm{~N}_{4} \mathrm{O}_{2} \mathrm{Na}\right)$.

\subsection{Terrestrial Streptomyces sp. GW 4723}

The crude extract of Streptomyces sp. GW 4723 had been reported to show antibacterial, anti-algal and anti-fungal activities against Bacillus subtilis, Staphylococcus aureus, Escherichia coli, activity, Chlorella vulgaris, Chlorella sorokiniana and Scenedesmus subspicatus, Mucor miehei and Candida albicans. ${ }^{[148]}$

\subsubsection{Fermentation and isolation}

Well-grown agar subcultures of terrestrial Streptomyces sp. GW 4723 were used to inoculate a $50 \mathrm{~L}$ shaker culture for ten days at $28{ }^{\circ} \mathrm{C}$ on $\mathrm{E}$ medium. The culture broth was harvested after 10 days and mixed with Celite. The mixture was then filtered with the aid of filter press to separate water phase and biomass. The biomass was extracted with ethyl acetate and acetone. The TLC of ethyl acetate extract, in addition to fats, exhibited two yellow bands, where one of them turned violet with dilute sodium hydroxide while the other remained unchanged. The ethyl acetate extract $(2.4 \mathrm{~g})$ was then fractionated on silica gel column chromatography $(1.5 \times 40$ cm, 20 g) using a $\mathrm{CH}_{2} \mathrm{Cl}_{2} / \mathrm{MeOH}$ gradient $\left(\mathrm{CH}_{2} \mathrm{Cl}_{2}, 1 \mathrm{~L} ; 3 \% \mathrm{MeOH}, 0.5 \mathrm{~L} ; 5 \%\right.$ $\mathrm{MeOH}, 0.5 \mathrm{~L} ; 7 \% \mathrm{MeOH}, 0.5 \mathrm{~L} ; 10 \% \mathrm{MeOH}, 0.5 \mathrm{~L} ; 15 \% \mathrm{MeOH}, 0.5 \mathrm{~L} ; 20 \%$ $\mathrm{MeOH}, 0.5 \mathrm{~L} ; 50 \% \mathrm{MeOH}, 0.5 \mathrm{~L} ; 100 \% \mathrm{MeOH}, 1 \mathrm{~L})$. Under TLC control, four fractions were obtained. The PTLC $\left(10 \times 20 \mathrm{~cm}, \mathrm{CH}_{2} \mathrm{Cl}_{2} / 4 \% \mathrm{MeOH}\right)$ of fraction III 
delivered celastramycin B (103) and acetyl- $\beta$-carboline. Fraction IV was subjected to Sephadex LH-20 column using methanol to obtain tyrosol, uracil and adenosine.

Celastramycin B (103, $1.4 \mathrm{mg})$ : Yellow solid, UV absorbing, violet with $\mathrm{NaOH} 2 \mathrm{~N} .-R_{f}$ $=0.65\left(\mathrm{CH}_{2} \mathrm{Cl}_{2} / 5 \% \mathrm{MeOH}\right) .-{ }^{1} \mathrm{H} \mathrm{NMR}$ and ${ }^{13} \mathrm{C}$ NMR, see table Table 1.

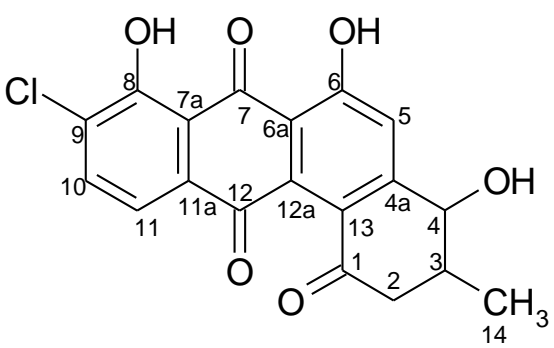

\section{Metabolites from selected endophytic fungi}

\subsection{Endophytic fungus LAF20}

\subsubsection{Fermentation and isolation}

The endophytic fungus LAF20 was cultivated on sterilized rice medium in $20 \mathrm{P}$ flasks and incubated for one month at $28{ }^{\circ} \mathrm{C}$. The resulting fermented rice was extracted with ethyl acetate (5 times) and was then concentrated to dryness under vacuum. The ethyl acetate extract (10.7 g) was chromatographed on silica gel (column 3 $\times 60 \mathrm{~cm}, 150 \mathrm{~g})$ using gradient solvent systems of $\mathrm{CH}_{2} \mathrm{Cl}_{2} / \mathrm{MeOH}$ to deliver four fractions. Fraction I contained fats and was not further worked up. Fraction II was subjected to Sephadex LH-20 column and methanol as eluent to give a mixture of stigmasterol and $\beta$-sitosterol. Isoclerone $(\mathbf{1 0 4}, 1.1 \mathrm{mg})$ was isolated from fraction III using Sephadex LH-20 (MeOH as solvent system). Finally, fraction IV delivered poly-(hydroxybutyric acid) (PHB) (86, $7.3 \mathrm{mg}$ ) and 4,6,8-trihydroxy-1-tetralone $(\mathbf{1 0 5}, 2.1 \mathrm{mg})$ after purification steps via silica gel and Sephadex LH-20 chromatography columns.

Isosclerone (104): White solid, grey with anisaldehyde/ sulphuric acid, UV absorbing band at $254 \mathrm{~nm}$. $-\boldsymbol{R}_{f}=0.41\left(\mathrm{CH}_{2} \mathrm{Cl}_{2} / 5 \%\right.$ $\mathrm{MeOH}),-{ }^{1} \mathbf{H}$ NMR $\left(\mathbf{C D C l}_{3}, 300\right.$ MHz): $\delta=12.40$ (s, 1H, 8-OH), $7.48\left(\mathrm{dd}, 1 \mathrm{H},{ }^{3} \mathrm{~J}=7.7\right.$ and $\left.8.2 \mathrm{~Hz}, 6-\mathrm{H}\right), 7.00\left(\mathrm{~d}, 1 \mathrm{H},{ }^{3} \mathrm{~J}=7.5 \mathrm{~Hz}, 5-\right.$

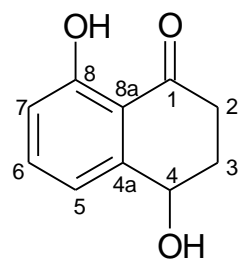
H), $6.91\left(\mathrm{~d}, 1 \mathrm{H},{ }^{3} J=8.4 \mathrm{~Hz}, 7-\mathrm{H}\right), 4.90(\mathrm{dd}, 1 \mathrm{H}, J=7.3$ and $3.9 \mathrm{~Hz}, 4-\mathrm{H})$, 2.96-2.64 (m, 2H, 2-H), 2.32-2.17 (m, 2H, 3-H). - ${ }^{\mathbf{1 3}} \mathbf{C}$ NMR $\left(\mathbf{C D C l}_{3}, 125 \mathbf{M H z}\right): \delta=204,1$ (C-1), 162.6 (C-8), 145.7 (C-4a), 136.9 (CH-6), 117.7 (CH-5), 117.3 (CH-7), 115.2 
(C-8a), $67.7(\mathrm{CH}-4), 34.6\left(\mathrm{CH}_{2}-2\right), 31.3\left(\mathrm{CH}_{2}-3\right)$. - EIMS $(70 \mathrm{eV}) \mathrm{m} / z=178\left([\mathrm{M}]^{+}\right.$, 100), $160\left(\left[\mathrm{M}-\mathrm{H}_{2} \mathrm{O}\right]^{+}, 15\right), 150\left(\left[\mathrm{M}-\mathrm{C}_{2} \mathrm{H}_{4}\right]^{+}, 45\right)$.

4,6,8-Trihydroxy-1-tetralone (105): Brown solid, grey with anisaldehyde/sulphuric acid, UV active at $254 \mathrm{~nm}$. $-\boldsymbol{R}_{f}=$ $0.35\left(\mathrm{CH}_{2} \mathrm{Cl}_{2} / 5 \% \mathrm{MeOH}\right) .-{ }^{1} \mathbf{H}$ NMR (300 MHz, $\left.\mathbf{C D}_{3} \mathrm{OD}\right): \delta$ $=6.52\left(\mathrm{~d}, 1 \mathrm{H},{ }^{4} \mathrm{~J}=2.3 \mathrm{~Hz}, 5-\mathrm{H}\right), 6.15\left(\mathrm{~d}, 1 \mathrm{H},{ }^{4} \mathrm{~J}=2.3 \mathrm{~Hz}, 7-\mathrm{H}\right)$,<smiles>O=C1CCC(O)c2cc(O)cc(O)c21</smiles>
$4.70(\mathrm{~m}, 1 \mathrm{H}, 4-\mathrm{H}), 2.75-2.58(\mathrm{~m}, 2 \mathrm{H}, 2-\mathrm{H}), 2.23-2.03(\mathrm{~m}, 2 \mathrm{H}$, 3-H). $-{ }^{13}$ C NMR (CD 3 OD, 125 MHz): $\delta=203.5$ (C-1), 167.3 (C-6), 166.7 (C-8), 150.9 (C-4a), 110.0 (C-8a), 107.6 (CH-5), 102.5 (CH-7), $68.5(\mathrm{CH}-4), 35.7\left(\mathrm{CH}_{2}-2\right)$, $32.6\left(\mathrm{CH}_{2}-3\right)$. - (-)-ESI MS: $m / z(\%)=193\left([\mathrm{M}-\mathrm{H}]^{+}, 60\right), 387\left([2 \mathrm{M}-\mathrm{H}]^{+}, 100\right) .-(-)-$ HRESI MS: $m / z=193.05008\left([\mathrm{M}-\mathrm{H}]^{+}\right)\left(\right.$calculated 193.04953 for $\left.\mathrm{C}_{10} \mathrm{H}_{10} \mathrm{O}_{4}\right)$.

\subsection{Endophytic fungus NP32-A}

Well-grown culture on agar plate of endophytic fungus NP32-A was inoculated in $1 \mathrm{~L}$ of $\mathrm{M}_{2}$ medium and incubated on a rotary shaker at $28^{\circ} \mathrm{C}$ for 5 days. The culture broth was extracted with ethyl acetate and the crude extract was used for the biological assay.

On the agar diffusion test, the crude extract of endophytic fungus NP32-A exhibited promising biological activities against Bacillus subtilis, Escherichia coli, Candida albicans, Mucor miehei (Tü 284), Rhizoctonia solani and Artemia salina.

Table 10: Antimicrobial activities of the crude extract of endophytic fungus NP32-A

Tested microorganisms

Inhibition zone $\varnothing$ [mm]

\begin{tabular}{lc}
\hline Bacillus subtilis & 15 \\
Escherichia coli & 12 \\
Candida albicans & 11 \\
Mucor miehei (Tü 284) & 14 \\
Rhizoctonia solani & 15 \\
Artemia salina & $100 \%$ \\
\hline
\end{tabular}

\subsubsection{Fermentation and isolation}

The endophytic fungus NP32-A was cultivated and fermented on $10 \mathrm{~L}$ of $\mathrm{M}_{2}$ medium in 10 P-flasks. Then, the culture was incubated at $28{ }^{\circ} \mathrm{C}$ for one month. The 
mycelium was extracted with ethyl acetate and the water phase was passed through XAD-16 resin column and followed by elution with methanol. Then, both these organic phases were evaporated to dryness under reduced pressure. Based on the TLC, both organic extracts were combined to afford $3.7 \mathrm{~g}$ of crude extract. This crude extract was then subjected to silica gel column $(3 \times 60 \mathrm{~cm}, 150 \mathrm{~g})$ with dichloromethane and methanol as solvent system (gradient condition) to give five fractions. Fraction I contained fats and was not further investigated. Fraction II was purified on Sephadex LH-20 column using $\mathrm{MeOH}$ as the eluent to isolate cerevisterol (106). Fraction III and IV were also subjected to Sephadex LH-20 column (MeOH as mobile phase) to obtain fusaproliferin $(\mathbf{1 0 7}, 6.1 \mathrm{mg})$ and beauvericin $(\mathbf{1 0 8})$ as white solids. Finally, fraction V was purified on silica gel column and followed by Sephadex LH-20 column (methanol as the solvent system) to afford trichosetin (109) and cerebroside B (110), respectively.

Cerevisterol (106): White solid, red with anisaldehyde/sulphuric acid. $-\boldsymbol{R}_{f}=$ $0.25\left(\mathrm{CH}_{2} \mathrm{Cl}_{2} / 7 \% \mathrm{MeOH}\right) . \quad-{ }^{1} \mathbf{H} \mathbf{N M R}$ $\left(\right.$ DMSO- $\left.d_{6}, 300 \mathrm{MHz}\right): \delta=5.23-5.08(\mathrm{~m}$, $3 \mathrm{H}, 7,22,23-\mathrm{H}), 4.44\left(\mathrm{~d},{ }^{3} J=5.3 \mathrm{~Hz}, 1 \mathrm{H}\right.$, 6-H), $3.78(\mathrm{~m}, 1 \mathrm{H}, 3-\mathrm{H}), 3.37(\mathrm{~m}, 2 \mathrm{H}, 4-$

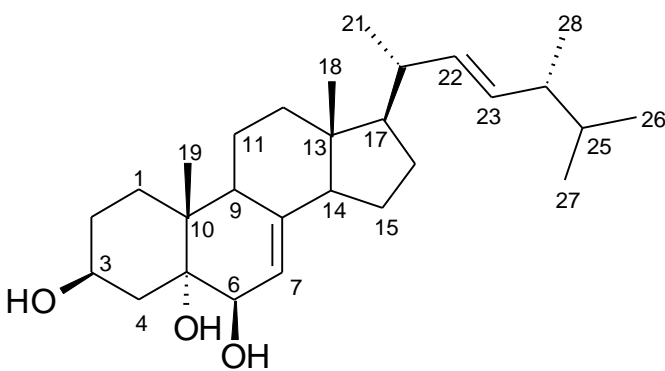
H), 2.02-1.22 (m, 18H, 1,2,9,11,12,14,15,16,17,20,24,25-H), $1.0\left(\mathrm{~d},{ }^{3} J=6.6 \mathrm{~Hz}, 3 \mathrm{H}\right.$, $21-\mathrm{H}), 0.89\left(\mathrm{~d},{ }^{3} J=6.8 \mathrm{~Hz}, 3 \mathrm{H}, 28-\mathrm{H}\right), 0.91$ (s, 3H, 19-H), $0.82\left(\mathrm{~d},{ }^{3} J=6.7 \mathrm{~Hz}, 3 \mathrm{H}\right.$, 27-H), $0.80\left(\mathrm{~d},{ }^{3} J=6.8 \mathrm{~Hz}, 3 \mathrm{H}, 26-\mathrm{H}\right), 0.55$ (s, 3H, 18-H). $-{ }^{13}$ C NMR (DMSO- $d_{6}$, 125 MHz): $\delta=139.6$ (Cq-8), 135.3 (CH-23), 131.3 (CH-22), 119.4 (CH-7), 74.4 (Cq-5), 72.1 (CH-6), 65.9 (CH-3), 55.3 (CH-17), 54.1 (CH-14), 42.9 (Cq-13), 42.2 (CH-9), 41.9 (CH-24), 39.8 (CH-20), $39.6\left(\mathrm{CH}_{2}-4\right), 38.9\left(\mathrm{CH}_{2}-12\right), 36.6(\mathrm{Cq}-10)$, $32.4\left(\mathrm{CH}_{2}-1\right), 32.4(\mathrm{CH}-25), 31.1\left(\mathrm{CH}_{2}-2\right), 27.6\left(\mathrm{CH}_{2}-16\right), 22.5\left(\mathrm{CH}_{2}-15\right), 21.3\left(\mathrm{CH}_{2}-\right.$ 11), $20.9\left(\mathrm{CH}_{3}-27\right), 19.7\left(\mathrm{CH}_{3}-26\right), 19.4\left(\mathrm{CH}_{3}-21\right), 17.6\left(\mathrm{CH}_{3}-19\right), 17.2\left(\mathrm{CH}_{3}-28\right)$, $11.9\left(\mathrm{CH}_{3}-18\right) .-(+)$-ESI MS: $m / z(\%)=471\left(\left[\mathrm{M}+\mathrm{Na}+\mathrm{H}_{2} \mathrm{O}\right]^{+}, 100\right), 883\left([2 \mathrm{M}+\mathrm{Na}]^{+}\right.$, 62). $-(+)$-HRESI MS: $m / z=471.34560\left(\left[\mathrm{M}+\mathrm{Na}+\mathrm{H}_{2} \mathrm{O}\right]^{+}\right)$, (calculated 471.34580 for $\left.\mathrm{C}_{28} \mathrm{H}_{48} \mathrm{O}_{4} \mathrm{Na}\right)$. 
Fusaproliferin (107): White solid, UV absorbing at $254 \mathrm{~nm}$, red with anisaldehyde/sulphuric acid. $\quad-\boldsymbol{R}_{\boldsymbol{f}}=0.30$ $\left(\mathrm{CH}_{2} \mathrm{Cl}_{2} / 7 \% \mathrm{MeOH}\right) .-{ }^{\mathbf{1}} \mathbf{H}$ NMR $\left(\mathbf{C D}_{3} \mathbf{O D}\right.$, $300 \mathrm{MHz}): \delta=5.38(\mathrm{~m}, 1 \mathrm{H}, 12-\mathrm{H}), 5.29$

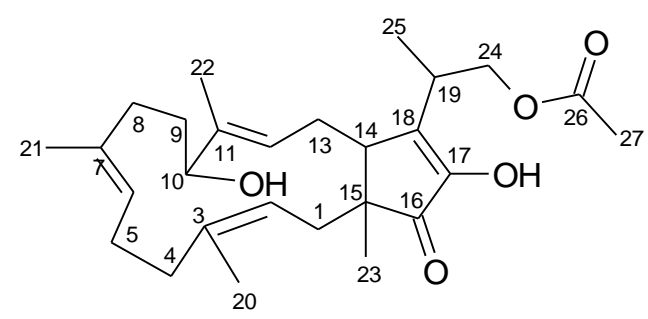
(m, 1H, 2-H), $5.16(\mathrm{~m}, 1 \mathrm{H}, 6-\mathrm{H}), 4.27(\mathrm{~m}, 2 \mathrm{H}, 24-\mathrm{H}), 3.96(\mathrm{~m}, 1 \mathrm{H}, 10-\mathrm{H}), 3.77(\mathrm{t}$, 1H, 14-H), 2.77 (m, 1H, 19-H), 2.45-2.04 (m, 8H, 4,5,8,9-H), 1.99 (s, 3H, 27-H), 1.79-1.71 (m, 4H, 1,13-H), $1.64(\mathrm{~s}, 6 \mathrm{H}, 20,21-\mathrm{H}), 1.28$ (d, 3H, 25-H), 1.55 (s, 3H, 22-H), 0.96 (s, 3H, 23-H). - ${ }^{13}$ C NMR (CD $\mathbf{O D D}, 125$ MHz): $\delta=209.9$ (C-16), 172.7 (C-26), 149.9 (C-17), 149.5 (C-18), 138.9 (C-3), 137.4 (C-11), 133.8 (C-7), 130.2 (CH-12), 125.3 (CH-6), 123.0 (CH-2), 77.1 (CH-10), 67.7 ( $\left.\mathrm{CH}_{2}-24\right), 50.7$ (CH-14), 50.2 (Cq-15), $41.4\left(\mathrm{CH}_{2}-4\right), 40.4\left(\mathrm{CH}_{2}-1\right), 35.9\left(\mathrm{CH}_{2}-8\right), 35.2(\mathrm{CH}-19), 30.8\left(\mathrm{CH}_{2}-9\right)$, $30.1\left(\mathrm{CH}_{2}-13\right), 24.8\left(\mathrm{CH}_{2}-5\right), 20.8\left(\mathrm{CH}_{3}-27\right), 16.9\left(\mathrm{CH}_{3}-23\right), 15.7\left(\mathrm{CH}_{3}-20\right), 15.5$ $\left(\mathrm{CH}_{3}-21\right), 14.7\left(\mathrm{CH}_{3}-25\right), 10.4\left(\mathrm{CH}_{3}-22\right)$. - (+)-ESI MS: $m / z(\%)=467\left([\mathrm{M}+\mathrm{Na}]^{+}\right.$, 100), $911([2 \mathrm{M}+\mathrm{Na}]+, 87) .-(+)$-HRESI MS: $m / z=467.27680\left([\mathrm{M}+\mathrm{Na}]^{+}\right)$, (calculated 467.27550 for $\mathrm{C}_{27} \mathrm{H}_{40} \mathrm{O}_{5} \mathrm{Na}$ ).

Beauvericin (108): White solid, UV absorbing band at $254 \mathrm{~nm}$, blue with chlorine/dianisidine reagent. $-\boldsymbol{R}_{f}=0.57$ $\left(\mathrm{CH}_{2} \mathrm{Cl}_{2} / 5 \% \mathrm{MeOH}\right) .-{ }^{1} \mathbf{H}$ NMR $\left(\mathbf{C D}_{3} \mathrm{OD}, \mathbf{3 0 0}\right.$ MHz ): $\delta=7.25\left(\mathrm{~m}, 5 \mathrm{H}, 5^{\prime}, 6^{\prime}, 7^{\prime}, 8^{\prime}, 9^{\prime}-\mathrm{H}\right), 5.79$ $\left(\mathrm{dd},{ }^{3} J=12.7\right.$ and $\left.4.6 \mathrm{~Hz}, 1 \mathrm{H}, 2-\mathrm{H}\right), 4.85(\mathrm{~m}$, $\left.1 \mathrm{H}, 2^{\prime}-\mathrm{H}\right), 3.41-3.36\left(\mathrm{~d},{ }^{3} \mathrm{~J}=4.6 \mathrm{~Hz}, 2 \mathrm{H}, 3^{\prime}-\mathrm{H}\right)$, $3.14\left(\mathrm{~s}, 3 \mathrm{H}, \mathrm{N}-\mathrm{CH}_{3}\right), 1.78(\mathrm{~m}, 1 \mathrm{H}, 3-\mathrm{H}), 0.84$ (d,

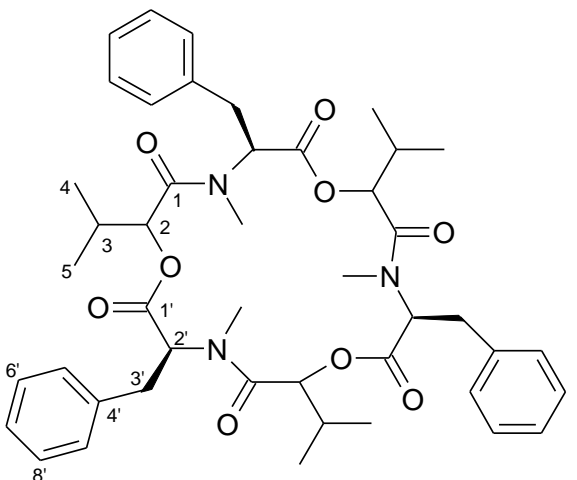
$\left.{ }^{3} J=6.6 \mathrm{~Hz}, 3 \mathrm{H}, 4-\mathrm{H}\right), 0.24\left(\mathrm{~d},{ }^{3} J=6.8 \mathrm{~Hz}, 3 \mathrm{H}, 5-\mathrm{H}\right) \cdot-{ }^{13} \mathbf{C}$ NMR (CD $\mathbf{O D}, 125$ MHz): $\delta=173.1(\mathrm{C}-1), 170.9\left(\mathrm{C}-1^{\prime}\right), 138.1(\mathrm{C}-4 '), 129.8\left(\mathrm{CH}-5^{\prime}, 9^{\prime}\right), 129.7(\mathrm{CH}-$ 6',8'), 127.9 (CH-7'), 77.2 (CH-2), $57.8\left(\mathrm{CH}-2^{\prime}\right), 35.4\left(\mathrm{CH}_{2}-3^{\prime}\right), 32.2\left(\mathrm{CH}_{3}-\mathrm{N}\right), 31.2$ $(\mathrm{CH}-3), 19.1\left(\mathrm{CH}_{3}-4\right), 17.2\left(\mathrm{CH}_{3}-5\right) .-(+)$-ESI MS: $m / z(\%)=806\left([\mathrm{M}+\mathrm{Na}]^{+}, 100\right)$, $1590([2 \mathrm{M}+\mathrm{Na}+\mathrm{H}], 9.4) .-(+)-H R E S I$ MS: $m / z=806.39831\left([\mathrm{M}+\mathrm{Na}]^{+}\right),($calculated 806.39870 for $\left.\mathrm{C}_{45} \mathrm{H}_{57} \mathrm{~N}_{3} \mathrm{O}_{9} \mathrm{Na}\right)$.

Trichosetin (109): White solid, UV absorbing band at $254 \mathrm{~nm}$, yellow with anisaldehyde/sulphuric

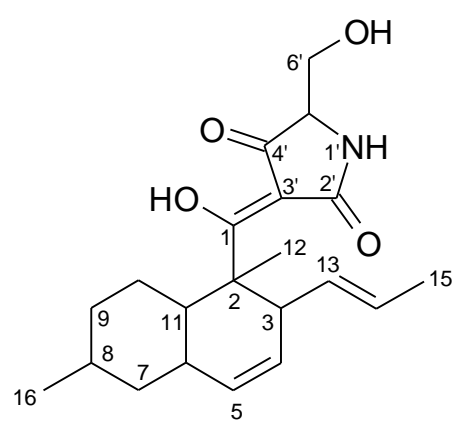


acid. $-\boldsymbol{R}_{\boldsymbol{f}}=0.34\left(\mathrm{CH}_{2} \mathrm{Cl}_{2} / 10 \% \mathrm{MeOH}\right) .-{ }^{\mathbf{1}} \mathbf{H}$ NMR $\left(\mathbf{C D}_{3} \mathbf{O D}, 300 \mathrm{MHz}\right): \delta=5.36$ $\left(\mathrm{dd},{ }^{4} J=2.4 \mathrm{~Hz},{ }^{3} J=5.0 \mathrm{~Hz}, 1 \mathrm{H}, 4-\mathrm{H}\right), 5.26-5.18(\mathrm{~m}, 3 \mathrm{H}, 13,14,5-\mathrm{H}), 4.0(\mathrm{~m}, 1 \mathrm{H}$, 3-H), 3.81-3.52 (dd, $\left.{ }^{4} J=2.6 \mathrm{~Hz},{ }^{3} J=10.0 \mathrm{~Hz}, 2 \mathrm{H}, 66^{\prime}-\mathrm{H}\right), 3.56\left(\mathrm{~d},{ }^{3} J=2.6 \mathrm{~Hz}, 1 \mathrm{H}, 5^{\prime}-\right.$ H), 1.98-1.94 (m, 2H, 6, 8-H), 1.69-1.08 (m, 6H, 7, 9, 10, 11-H), 1.49 (d, ${ }^{3} J=5.3$ $\mathrm{Hz}, 3 \mathrm{H}, 15-\mathrm{H}), 1.34$ (s, 3H, 12-H), $1.19\left(\mathrm{~d},{ }^{3} \mathrm{~J}=7.2 \mathrm{~Hz}, 3 \mathrm{H}, 16-\mathrm{H}\right) .-{ }^{\mathbf{1 3}} \mathbf{C}$ NMR (CD) ${ }_{3}$ OD, 125 MHz): $\delta=203.6$ (C-1), 192.7 (C-4'), 180.3 (C-2'), 134.9 (CH-13), 131.1 (CH-5), 129.7 (CH-4), $125.6(\mathrm{CH}-14), 104.9$ (C-3'), $65.0\left(\mathrm{CH}_{2}-6 '\right), 62.7(\mathrm{CH}-$ 5'), 52.7 (C-2), 44.9 (CH-3), 43.2 (CH-11), $41.1\left(\mathrm{CH}_{2}-7\right), 33.9\left(\mathrm{CH}_{2}-9\right), 33.7(\mathrm{CH}-6)$, 29.4 (CH-8), $23.3\left(\mathrm{CH}_{2}-10\right), 19.2\left(\mathrm{CH}_{3}-16\right), 18.2\left(\mathrm{CH}_{3}-15\right), 15.4\left(\mathrm{CH}_{3}-12\right)$. - (+)-ESI MS: $m / z(\%)=382\left([\mathrm{M}+\mathrm{Na}]^{+}, 100\right), 741\left([2 \mathrm{M}+\mathrm{Na}]^{+}, 50\right) .-(+)$-HRESI MS: $m / z=$ $382.19890\left([\mathrm{M}+\mathrm{Na}]^{+}\right)$, (calculated 382.19880 for $\left.\mathrm{C}_{21} \mathrm{H}_{29} \mathrm{NO}_{4} \mathrm{Na}\right)$.

\section{Cerebroside $\quad B$}

(110): White solid, not UV absorbing, violet with anisaldehyde/sulphuric acid. $-\boldsymbol{R}_{f}=$

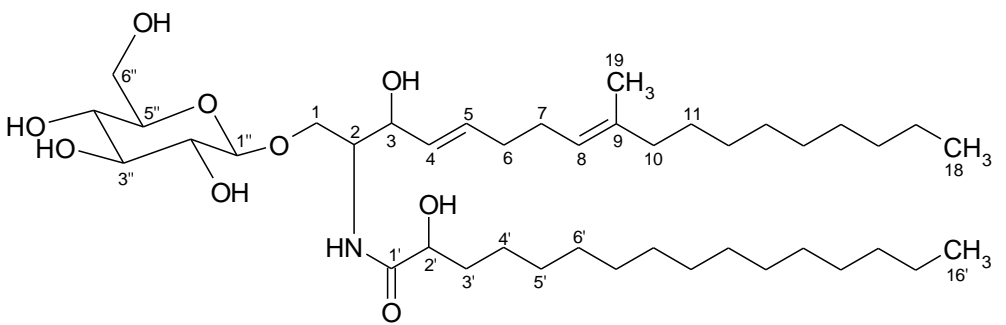
$0.15\left(\mathrm{CH}_{2} \mathrm{Cl}_{2} / 10 \% \mathrm{MeOH}\right) .-{ }^{1} \mathbf{H}$ NMR $\left(\mathbf{C D}_{3} \mathbf{O D} / \mathbf{C D C l}_{3}, 300 \mathrm{MHz}\right): \delta=7.31\left(\mathrm{~d},{ }^{3} \mathrm{~J}\right.$ $=7.6 \mathrm{~Hz}, \mathrm{NH}), 5.72(\mathrm{~m}, 1 \mathrm{H}, 5-\mathrm{H}), 5.45\left(\mathrm{dd},{ }^{3} J=7.3,15.4 \mathrm{~Hz}, 1 \mathrm{H}, 4-\mathrm{H}\right), 5.10(\mathrm{~m}$, 1H, 8-H), 4.25 (d, $\left.{ }^{3} J=7.8 \mathrm{~Hz}, 1 \mathrm{H}, 1 "-\mathrm{H}\right), 4.12-4.05$ (m, 2H, 2",3-H), 3.97 (m, 1H, 2H), $3.85\left(\mathrm{dd},{ }^{3} J=12.6 \mathrm{~Hz}, 1 \mathrm{H}, 1-\mathrm{H}_{\mathrm{B}}\right), 3.65\left(\mathrm{dd},{ }^{3} J=4.7 \mathrm{~Hz}, 1 \mathrm{H}, 1-\mathrm{H}_{\mathrm{A}}\right), 3.38-3.19$ (m, 5H, 3",4",5",6"-H), 1.56 (s, 3H, 19-H), 2.04 (m, 4H, 6,7-H), 1.93 (t, ${ }^{3} J=7.1 \mathrm{~Hz}$, 2H, 10-H), 1.25 (s, 38H, 3'-17-H), 0.86 (t, ${ }^{3} J=7.0 \mathrm{~Hz}, 6 \mathrm{H}, 16$ ' $\left.18-\mathrm{H}\right) .-{ }^{13} \mathbf{C}$ NMR $\left(\mathrm{CD}_{3} \mathrm{OD} / \mathrm{CDCl}_{3}, 125 \mathrm{MHz}\right): \delta=176.7$ (C-1'), 136.5 (C-9), $134.6(\mathrm{CH}-5), 130.3$ (CH-4), 124.3 (CH-8), 104.1 (CH-1"), 77.4 (CH-3",5"), 74.4 (CH-2'), 72.8 (CH-3), 72.6 (CH-2"), 71.0 (CH-4"), $69.2\left(\mathrm{CH}_{2}-1\right), 62.2\left(\mathrm{CH}_{2}-6 "\right), 54.1(\mathrm{CH}-2), 40.4\left(\mathrm{CH}_{2}-\right.$ 10), $35.4\left(\mathrm{CH}_{2}-3\right), 33.3\left(\mathrm{CH}_{2}-6\right), 32.7-30.0\left(\mathrm{CH}_{2}\right.$ of aliphatic chains $), 28.7\left(\mathrm{CH}_{2}-7\right)$, $28.3\left(\mathrm{CH}_{2}-11\right), 25.8\left(\mathrm{CH}_{2}-4{ }^{\prime}\right), 23.4\left(\mathrm{CH}_{2}-15 ', 17\right), 16.2\left(\mathrm{CH}_{3}-19\right), 14.4\left(\mathrm{CH}_{3}-16 ', 18\right)$. (+)-ESI MS: $m / z(\%)=750\left([\mathrm{M}+\mathrm{Na}]^{+}, 100\right), 1478\left([2 \mathrm{M}+\mathrm{Na}]^{+}, 7\right) .-(+)-H R E S I$ MS: $m / z=750.55030\left([\mathrm{M}+\mathrm{Na}]^{+}\right)\left(\right.$calculated 750.54910 for $\left.\mathrm{C}_{41} \mathrm{H}_{77} \mathrm{NO}_{9} \mathrm{Na}\right)$.

\subsection{Endophytic fungus FT44}

The endophytic fungus FT44 was isolated by Ferdinand Talontsi from the Cameroonian plant Endodesmia calophylloides (Clusiaceae). According to morphological 
characteristics, it was identified as Penicillium sp. at IRAD (Institute of Agricultural Research for Development), Yaoundé, Cameroon. A culture of this fungus was deposited in our group.

\subsubsection{Fermentation and isolation}

The Penicillium sp. FT44 was cultivated on sterilized rice medium in $10 \mathrm{P}$ flasks and incubated for one month at room temperature. The fermented rice was extracted with ethyl acetate (5 times) and was then concentrated to dryness under vacuum. The ethyl acetate extract (12.16 g) was chromatographed on silica gel (column $3 \times 75 \mathrm{~cm}, 200 \mathrm{~g}$ ) with a $\mathrm{CH}_{2} \mathrm{Cl}_{2} / \mathrm{MeOH}$ gradient to deliver four fractions. Fractions III and IV were subjected to Sephadex LH-20 column separately with methanol as the eluent to isolate 1233-A (111, $3.4 \mathrm{mg})$ and ergosterol peroxide (112, $5.8 \mathrm{mg})$, respectively.

1233-A (111): Colourless oil, UV absorbing band at $254 \mathrm{~nm}$, red with anisaldehyde/sulphuric acid. $\boldsymbol{R}_{\boldsymbol{f}}=0.54\left(\mathrm{CH}_{2} \mathrm{Cl}_{2} / 5 \% \mathrm{MeOH}\right) .-$

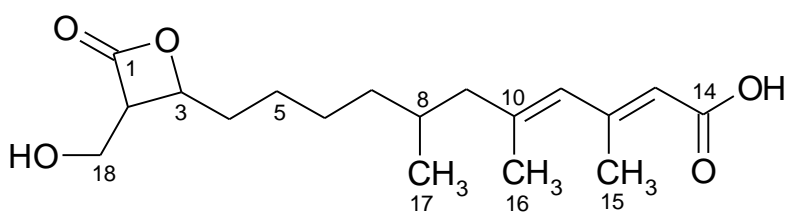
${ }^{1} \mathbf{H}_{\text {NMR }}\left(\mathbf{C D C l}_{3}, 300 \mathrm{MHz}\right): \delta=5.73$ (br s, 1H, 11-H), 5.68 (br s, $\left.1 \mathrm{H}, 13-\mathrm{H}\right), 4.59$ $(\mathrm{m}, 1 \mathrm{H}, 3-\mathrm{H}), 4.05\left(\mathrm{dd},{ }^{3} J=4.9 \mathrm{~Hz},{ }^{2} J=11.7 \mathrm{~Hz}, 1 \mathrm{H}, 18-\mathrm{H}_{\mathrm{A}}\right), 3.88\left(\mathrm{dd},{ }^{3} J=4.0 \mathrm{~Hz}\right.$, ${ }^{2} J=11.7 \mathrm{~Hz}, 1 \mathrm{H}, 18-\mathrm{H}_{\mathrm{B}}$ ), 3.41 (quartet, ${ }^{3} J=4.0$ and $\left.4.9 \mathrm{~Hz}, 1 \mathrm{H}, 2-\mathrm{H}\right), 2.24(\mathrm{~s}, 3 \mathrm{H}$, 15-H), 2.09 (dd, $\left.{ }^{3} J=6.2 \mathrm{~Hz},{ }^{2} J=13.2 \mathrm{~Hz}, 2 \mathrm{H}, 9-\mathrm{H}\right), 1.88$ (m, 2H, 4-H), 1.81 (s, 3H, $16-\mathrm{H}), 1.62(\mathrm{~m}, 1 \mathrm{H}, 8-\mathrm{H}), 1.39-1.14(\mathrm{~m}, 6 \mathrm{H}, 5,6,7-\mathrm{H}), 0.84\left(\mathrm{~d},{ }^{3} \mathrm{~J}=6.6 \mathrm{~Hz}, 3 \mathrm{H}, 17-\right.$

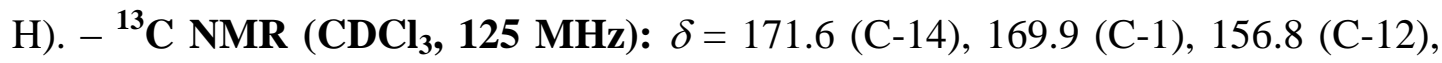
141.9 (C-10), 129.4 (CH-11), 116.7 (CH-13), 75.0 (CH-3), $58.6(\mathrm{CH}-2), 57.9\left(\mathrm{CH}_{2-}\right.$ 18), $49.0\left(\mathrm{CH}_{2}-9\right), 36.6\left(\mathrm{CH}_{2}-7\right), 34.0\left(\mathrm{CH}_{2}-4\right), 30.9(\mathrm{CH}-8), 26.6\left(\mathrm{CH}_{2}-5\right), 25.2$ $\left(\mathrm{CH}_{2}-6\right), 20.0\left(\mathrm{CH}_{3}-15\right), 19.5\left(\mathrm{CH}_{3}-17\right), 18.6\left(\mathrm{CH}_{3}-16\right)$. - (-)-ESI MS: $m / z(\%)=323$ ([M-H] $\left.]^{-}, 73\right) .-(-)-H R E S I$ MS: $m / z=323.18584[\mathrm{M}-\mathrm{H}]^{-}$(calc. 323.18529 for $\left.\mathrm{C}_{18} \mathrm{H}_{27} \mathrm{O}_{5}\right)$. - DCI MS $\left(\mathbf{N H}_{3}\right): m / z(\%)=342\left(\left[\mathrm{M}+\mathrm{NH}_{3}\right]^{+}, 48\right), 298\left(\left[\mathrm{M}-\mathrm{CO}_{2}\right]^{+}, 100\right)$, 251 (48), 230 (16), 144 (16), 101 (15).

Ergosterol peroxide (112): White solid, blue with anisaldehyde/sulphuric acid. $-\boldsymbol{R}_{\boldsymbol{f}}=0.43\left(\mathrm{CH}_{2} \mathrm{Cl}_{2} / 5 \% \mathrm{MeOH}\right)$. -

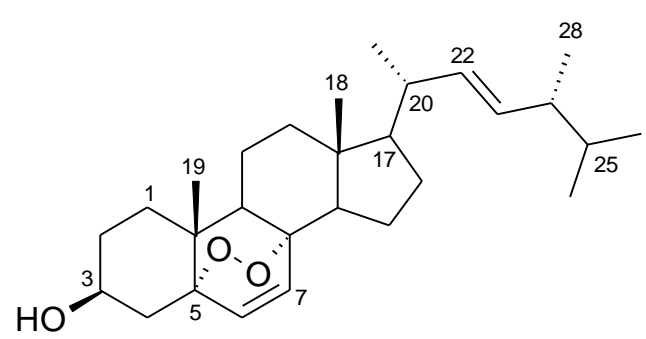


${ }^{1} \mathbf{H}$ NMR $\left(\mathbf{C D C l}_{3}, 300 \mathrm{MHz}\right): \delta=6.50\left(\mathrm{~d},{ }^{3} J=8.5 \mathrm{~Hz}, 1 \mathrm{H}, 6-\mathrm{H}\right), 6.24\left(\mathrm{~d},{ }^{3} J=8.5\right.$ $\mathrm{Hz}, 1 \mathrm{H}, 7-\mathrm{H}), 5.23\left(\mathrm{dd},{ }^{3} J=7.1,15.2 \mathrm{~Hz}, 1 \mathrm{H}, 22-\mathrm{H}\right), 5.14\left(\mathrm{dd},{ }^{3} J=7.8,15.2 \mathrm{~Hz}, 1 \mathrm{H}\right.$, 23-H), 3.97 (m, 1H, 3-H), 0.99 (d, $\left.{ }^{3} J=6.6 \mathrm{~Hz}, 3 \mathrm{H}, 28-\mathrm{H}\right), 0.91$ (d, ${ }^{3} J=6.6 \mathrm{~Hz}, 3 \mathrm{H}$, 21-H), 0.88 (s, 3H, 19-H), 0.82 (m, 9H, 18,26,27-H). - ${ }^{13} \mathbf{C}$ NMR (CDCl, 125 MHz): $\delta=135.3(\mathrm{CH}-6), 135.1$ (CH-22), 132.2 (CH-23), 130.6 (CH-7), 82.1 (C-5), 79.4 (C-8), 66.5 (CH-3), 56.2 (CH-17), 51.7 (CH-14), 51.1 (CH-9), 44.6 (Cq-13), 42.8 (CH-24), 39.8 (CH-20), 39.4 (C-12), 37.0 (C-10), $36.9\left(\mathrm{CH}_{2}-4\right), 34.7\left(\mathrm{CH}_{2}-1\right)$, 33.1 (CH-25), $30.1\left(\mathrm{CH}_{2}-2\right), 28.7\left(\mathrm{CH}_{2}-16\right), 23.5\left(\mathrm{CH}_{2}-11\right), 20.9\left(\mathrm{CH}_{3}-21\right), 20.7$ $\left(\mathrm{CH}_{2}-15\right), 20.0\left(\mathrm{CH}_{3}-27\right), 19.7\left(\mathrm{CH}_{3}-26\right), 18.2\left(\mathrm{CH}_{3}-19\right), 17.6\left(\mathrm{CH}_{3}-28\right), 12.95\left(\mathrm{CH}_{3}-\right.$ 18). - (+)-ESI MS: $m / z(\%)=451\left([\mathrm{M}+\mathrm{Na}]^{+}, 100\right), 879\left([2 \mathrm{M}+\mathrm{Na}]^{+}, 31\right) .-(+)-$ HRESI MS: $m / z=451.31830\left(\left[\mathrm{M}+\mathrm{Na}^{+}\right]\right)\left(\right.$calc. 451.31630 for $\left.\mathrm{C}_{28} \mathrm{H}_{44} \mathrm{O}_{3} \mathrm{Na}\right)$.

\subsection{Endophytic fungus FTM1}

The endophytic fungus FTM1 was isolated by Ferdinand Talontsi from the Cameroonian plant Endodesmia calophylloides (Clusiaceae). According to morphological characteristics, it was identified as Alternaria sp. at IRAD (Institute of Agricultural Research for Development), Yaoundé, Cameroon. A culture of this fungus was deposited at our Institute.

\subsubsection{Fermentation and isolation}

The endophytic fungus Alternaria sp. FTM1 was cultivated on sterilized rice medium in 10 P-flasks and incubated for one month at room temperature. The fermented rice was then extracted with ethyl acetate (5 times) and was then concentrated to dryness under vacuum. The ethyl acetate extract (10.06 g) was chromatographed on silica gel (column $3 \times 75 \mathrm{~cm}, 200 \mathrm{~g}$ ) with a $\mathrm{CH}_{2} \mathrm{Cl}_{2} / \mathrm{MeOH}$ gradient to deliver four fractions. Fractions III was subjected to PTLC with $\mathrm{CH}_{2} \mathrm{Cl}_{2} / 1 \% \mathrm{MeOH}$ to give 8-hydroxy-6,7-dimethoxy-3-methylisocoumarin $(\mathbf{1 1 3}, 1.5$ $\mathrm{mg}$ ) and 5-methylochracin $(\mathbf{1 1 4}, 2.1 \mathrm{mg})$. Fraction IV was subjected to Sephadex LH-20 column (methanol) to obtain alternariol-monomethylether $(\mathbf{1 1 5}, 1.7 \mathrm{mg})$.

\section{8-Hydroxy-6,7-dimethoxy-3-methylisocou-}

marin (113): White solid, grey with anisaldehyde/sulphuric acid, UV absorbing band at $254 \mathrm{~nm}$.

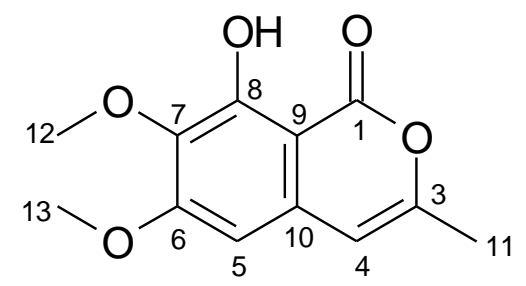


$-\boldsymbol{R}_{\mathbf{f}}=0.86(\mathrm{CHCl} / \mathrm{MeOH} ; 95: 5) .-{ }^{\mathbf{1}} \mathbf{H} \mathbf{N M R}\left(\mathbf{C D C l}_{\mathbf{3}}, \mathbf{3 0 0} \mathbf{~ M H z}\right): \delta=11.07(\mathrm{brs}$, 8-OH), 6.33 (s, 1H, 5-H), 6.19 (s, 1H, 4-H), 3.95 (s, 3H, 12-H), 3.91 (s, 3H, 13-H), 2.27 (s, 3H, 11-H). - ${ }^{13} \mathbf{C}$ NMR (CDCl 3,125 MHz): $\delta=166.3$ (C-1), 159.9 (C-6), 154.6 (C-8), 153.4 (C-3), 135.1 (C-7), 134.4 (C-10), 104.3 (CH-5), 100.8 (C-9), 97.9 (CH-4), $60.8\left(\mathrm{CH}_{3}-12\right), 56.2\left(\mathrm{CH}_{3}-13\right), 19.4\left(\mathrm{CH}_{3}-11\right)$. - (+)-ESI MS: $\mathrm{m} / \mathrm{z}(\%)=259$ $\left([\mathrm{M}+\mathrm{Na}]^{+}, 16\right), 495\left([2 \mathrm{M}+\mathrm{Na}]^{+}, 100\right) . \quad-(+)-H R E S I$ MS: $m / z=259.05780$ $\left([\mathrm{M}+\mathrm{Na}]^{+}\right)$(calculated 259.05770 for $\mathrm{C}_{12} \mathrm{H}_{12} \mathrm{O}_{5} \mathrm{Na}$ ).

5-Methylochracin (114): White solid, grey with anisaldehyde/sulphuric acid, UV absorbing band at $254 \mathrm{~nm}$. $-\boldsymbol{R}_{\mathbf{f}}=0.78\left(\mathrm{CHCl}_{3} / \mathrm{MeOH} ; 95: 5\right) .-{ }^{\mathbf{1}} \mathbf{H} \mathbf{N M R}\left(\mathbf{C D C l}_{3}, \mathbf{3 0 0}\right.$ MHz ): $\delta=10.96$ (brs, 8-OH), $7.24\left(\mathrm{~d}, 1 \mathrm{H},{ }^{3} \mathrm{~J}=8.5 \mathrm{~Hz}, 6-\right.$ H), $6.76\left(\mathrm{~d}, 1 \mathrm{H},{ }^{3} J=8.5 \mathrm{~Hz}, 7-\mathrm{H}\right), 4.64(\mathrm{~m}, 1 \mathrm{H}, 3-\mathrm{H}), 2.91$

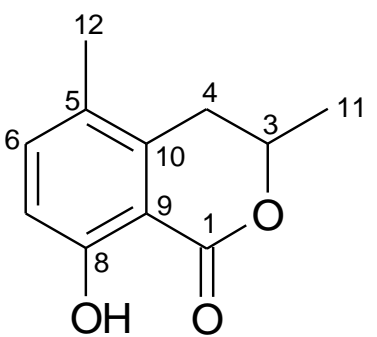
(dd, $1 \mathrm{H}, J=16.7,3.4 \mathrm{~Hz}, 4-\mathrm{Ha}), 2.67$ (dd, $1 \mathrm{H}, J=16.7,11.6 \mathrm{~Hz}, 4-\mathrm{Hb}), 2.16$ (s, 3H, 12-H), 1.51 (d, 3H, $\left.{ }^{3} J=6.3 \mathrm{~Hz}, 11-\mathrm{H}\right) .-{ }^{13} \mathbf{C ~ N M R}\left(\mathbf{C D C l}_{3}, \mathbf{1 2 5} \mathbf{M H z}\right): \delta=170.1$ (C-1), $160.3\left(\mathrm{C}_{\mathrm{q}}-8\right), 137.8$ (C-10), 136.9 (CH-6), 124.8 (C-5), 115.5 (C-7), 107.9 (C9), $75.4(\mathrm{CH}-3), 31.9\left(\mathrm{CH}_{2}-4\right), 20.9\left(\mathrm{CH}_{3}-11\right), 18.1\left(\mathrm{CH}_{3}-12\right) .-(+)$-ESI MS: $m / z$ $(\%)=215\left([\mathrm{M}+\mathrm{Na}]^{+}, 46\right), 407\left([2 \mathrm{M}+\mathrm{Na}]^{+}, 100\right) .-(+)$-HRESI MS: $m / z=215.2010$ $\left([\mathrm{M}+\mathrm{Na}]^{+}\right)$(calculated 215.20045 for $\mathrm{C}_{11} \mathrm{H}_{12} \mathrm{O}_{3} \mathrm{Na}$ ).

Alternariol-monomethyl ether (115): Reddishwhite solid, UV absorbing band at $254 \mathrm{~nm}$, violet with anisaldehyde/sulphuric acid. $-\boldsymbol{R}_{\mathbf{f}}=0.68\left(\mathrm{CHCl}_{3} /\right.$ $\mathrm{MeOH} ; 95: 5) .-{ }^{\mathbf{1}} \mathbf{H}$ NMR (DMSO- $d_{6}, 300 \mathrm{MHz}$ ): $\delta=$ $11.80(\mathrm{~s}, 3-\mathrm{OH}), 10.35\left(\mathrm{~s}, 4^{\prime}-\mathrm{OH}\right), 7.19\left(\mathrm{~d}, 1 \mathrm{H},{ }^{4} \mathrm{~J}=\right.$

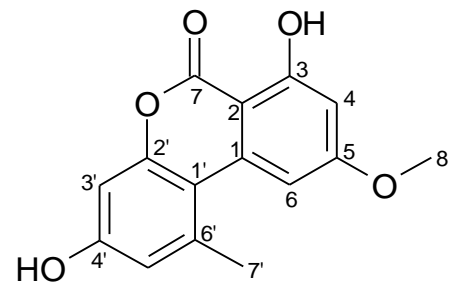
$2.0 \mathrm{~Hz}, 6-\mathrm{H}), 6.72\left(\mathrm{~d}, 1 \mathrm{H},{ }^{4} \mathrm{~J}=2.5 \mathrm{~Hz}, 5^{\prime}-\mathrm{H}\right), 6.63$ (d, 1H, $\left.{ }^{4} \mathrm{~J}=2.5 \mathrm{~Hz}, 3^{\prime}-\mathrm{H}\right), 6.59$ (d, $\left.1 \mathrm{H},{ }^{4} \mathrm{~J}=2.1 \mathrm{~Hz}, 4-\mathrm{H}\right), 3.90$ (s, 3H, 8-H), 2.72 (s, 3H, 7'-H). - ${ }^{13}$ C NMR (DMSO- $d_{6}$, 75 MHz): $\delta=166.1$ (C-5), 164.5 (C-7), 164.0 (C-3), 158.5 (C-4'), 152.5 (C-2’), 138.3 (C-6'), 137.8 (C-1), 117.5 (CH-5'), 108.7 (C-1'), 103.2 (CH-6), 101.5 (CH-3'), 99.1 (CH-4), 98.4 (C-2), $55.7\left(\mathrm{CH}_{3}-8\right), 24.8\left(\mathrm{CH}_{3}-7^{\prime}\right)$. - (-)-ESI MS: $m / z(\%)=271$ $\left([\mathrm{M}-\mathrm{H}]^{-}, 67\right), 543\left([2 \mathrm{M}-\mathrm{H}]^{-}, 100\right) .-(-)-H R E S I ~ M S: ~ m / z=271.06120\left([\mathrm{M}-\mathrm{H}]^{-}\right)(\mathrm{cal}-$ culated 271.06040 for $\mathrm{C}_{15} \mathrm{H}_{11} \mathrm{O}_{5}$ ). 


\section{Plant metabolites}

Garcinon D (116): Yellow solid, 10.1 mg. - ${ }^{1}$ H NMR (DMSO- $d_{6}, 300$ MHz $): \delta=$ 6. $78(\mathrm{~s}, 1 \mathrm{H}, 5-\mathrm{H}), 6.34(\mathrm{~s}, 1 \mathrm{H}, 4-\mathrm{H}), 5.18$ (t, $\left.1 \mathrm{H},{ }^{3} J=7.1 \mathrm{~Hz}, 11-\mathrm{H}\right), 3.75\left(\mathrm{~s}, 3 \mathrm{H}, \mathrm{CH}_{3}\right.$ O), $3.32\left(\mathrm{~m}, 2 \mathrm{H}, 16-\mathrm{CH}_{2}\right), 3.21\left(\mathrm{~d}, 2 \mathrm{H},{ }^{3} \mathrm{~J}=\right.$ 7.1 Hz, 10- $\left.\mathrm{CH}_{2}\right), 1.73\left(\mathrm{~s}, 3 \mathrm{H}, 14-\mathrm{CH}_{3}\right), 1.62$

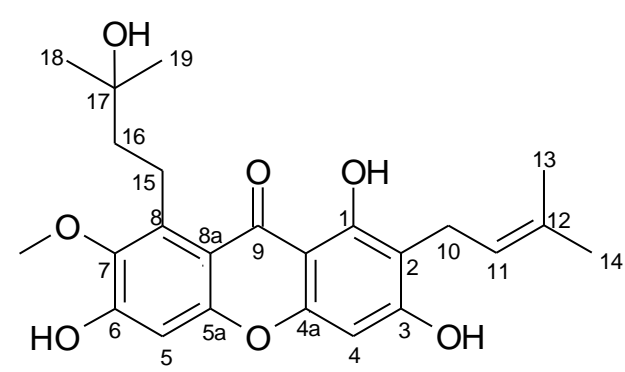
$\left(\mathrm{s}, 3 \mathrm{H}, 13-\mathrm{CH}_{3}\right), 1.57\left(\mathrm{~m}, 2 \mathrm{H}, 15-\mathrm{CH}_{2}\right), 1.22\left(\mathrm{~s}, 6 \mathrm{H}, 18,19-\mathrm{CH}_{3}\right) .-{ }^{13} \mathbf{C} \mathbf{N M R}$ (DMSO- $d_{6}, 125$ MHz): $\delta=181.9$ (C-9), 161.9 (C-3), 159.6 (C-1), 156.5 (C-5a), 154.3 (C-6), 153.8 (C-4a), 143.1 (C-7), 138.4 (C-8), 130.1 (C-12), 122.2 (CH-11), 109.8 (C-8a), 109.3 (C-2), 101.7 (C-9a), 101.3 (CH-5), 92.0 (CH-4), 69.0 (C-17), $60.3\left(7-\mathrm{OCH}_{3}\right), 44.7\left(\mathrm{CH}_{2}-16\right), 28.7\left(\mathrm{CH}_{3}-18,19\right), 25.4\left(\mathrm{CH}_{3}-13\right), 22.1\left(\mathrm{CH}_{2}-15\right)$, $20.9\left(\mathrm{CH}_{2}-10\right), 17.6\left(\mathrm{CH}_{3}-14\right) .-(+)$-ESI MS: $m / z=451\left([\mathrm{M}+\mathrm{Na}]^{+}\right) .-(+)$-HRESI MS: $m / z=451.17276\left([\mathrm{M}+\mathrm{Na}]^{+}\right)$, (calculated 451.17272 for $\left.\mathrm{C}_{24} \mathrm{H}_{28} \mathrm{O}_{7} \mathrm{Na}\right)$.

Rubraxanthone (117): Yellow solid, $9.8 \mathrm{mg}$. $-{ }^{1} \mathrm{H}$ NMR (CD $\mathbf{3}$ OD, $\left.300 \mathrm{MHz}\right): \delta=6.60(\mathrm{~s}, 1 \mathrm{H}$, $5-\mathrm{H}), 6.09\left(\mathrm{~d}, 1 \mathrm{H},{ }^{4} \mathrm{~J}=2.2 \mathrm{~Hz}, 4-\mathrm{H}\right), 6.03\left(\mathrm{~d}, 1 \mathrm{H},{ }^{4} \mathrm{~J}\right.$ $=2.2 \mathrm{~Hz}, 2-\mathrm{H}), 5.17(\mathrm{~m}, 1 \mathrm{H}, 15-\mathrm{H}), 4.97(\mathrm{~m}, 1 \mathrm{H}$, $11-\mathrm{H}), 4.00\left(\mathrm{~d}, 2 \mathrm{H},{ }^{3} \mathrm{~J}=6.3 \mathrm{~Hz}, 10-\mathrm{H}\right), 3.73(\mathrm{~s}, 3 \mathrm{H}$, $\left.\mathrm{CH}_{3}-\mathrm{O}\right), 1.99$ (m, 2H, 13-H), $1.95(\mathrm{~m}, 2 \mathrm{H}, 14-\mathrm{H})$, 1.78 (s, 3H, 19-H), 1.52 (s, 3H, 18-H), 1.49 (s, 3H, 17-H). $-{ }^{13}$ C NMR (CD3OD, 125 MHz): $\delta=182.8$

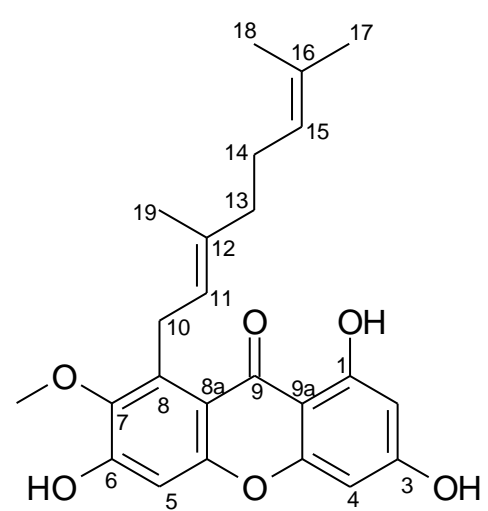
(C-9), 165.7 (C-3), 164.6 (C-1), 158.2 (C-5a), 157.9 (C-4a), 156.6 (C-6), 144.7 (C7), 138.5 (C-12), 135.3 (C-8), 131.8 (C-16), 125.3 (CH-11), 125.1 (CH-15), 112.1 (C-8a), 103.8 (C-9a), 102.8 (CH-5), 98.7 (CH-2), 93.9 (CH-4), $61.3\left(7-\mathrm{OCH}_{3}\right), 40.8$ $\left(\mathrm{CH}_{2}-13\right), 27.6\left(\mathrm{CH}_{2}-14\right), 27.0\left(\mathrm{CH}_{2}-10\right), 25.8\left(\mathrm{CH}_{3}-18\right), 17.7\left(\mathrm{CH}_{3}-17\right), 16.6\left(\mathrm{CH}_{3}-\right.$ 19). $-(+)$-ESI MS: $m / z=433\left([\mathrm{M}+\mathrm{Na}]^{+}\right) .-(+)$-HRESI MS: $m / z=433.162196$ $\left([\mathrm{M}+\mathrm{Na}]^{+}\right)$, (calculated 433.162159 for $\left.\mathrm{C}_{24} \mathrm{H}_{26} \mathrm{O}_{6} \mathrm{Na}\right)$.

Acetyl aleuritolic acid (118): White solid, $11.6 \mathrm{mg} .-{ }^{1} \mathbf{H}$ NMR $\left(\mathbf{C D C l}_{3}, 300 \mathrm{MHz}\right)$ : $\delta=5.20(1 \mathrm{H}, \mathrm{m}, 15-\mathrm{H}), 4.50(1 \mathrm{H}, \mathrm{m}, 3-\mathrm{H})$,

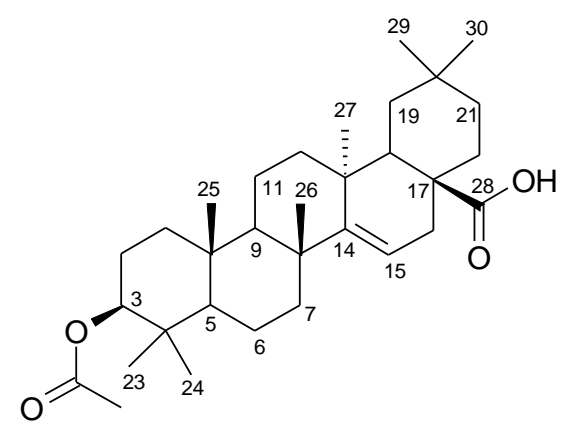


$2.80\left(2 \mathrm{H}, \mathrm{m}, 16-\mathrm{CH}_{2}\right), 1.90\left(3 \mathrm{H}, \mathrm{s}, \mathrm{COOCH}_{3}\right), 1.10\left(3 \mathrm{H}, \mathrm{s}, 26-\mathrm{CH}_{3}\right), 0.92(3 \mathrm{H}, \mathrm{s}, 30-$ $\left.\mathrm{CH}_{3}\right), 0.90\left(3 \mathrm{H}, \mathrm{s}, 29-\mathrm{CH}_{3}\right), 0.88\left(3 \mathrm{H}, \mathrm{s}, 23-\mathrm{CH}_{3}\right), 0.84\left(3 \mathrm{H}, \mathrm{s}, 24-\mathrm{CH}_{3}\right), 0,82(3 \mathrm{H}, \mathrm{s}$, 27- $\left.\mathrm{CH}_{3}\right), 0.70\left(3 \mathrm{H}, \mathrm{s}, 25-\mathrm{CH}_{3}\right) .-{ }^{13} \mathbf{C} \mathbf{N M R}\left(\mathbf{C D C l}_{3}, \mathbf{1 2 5} \mathbf{M H z}\right): \delta=184.12(\mathrm{C}-28$, $\underline{\mathrm{COOH}}), 170.86\left(\mathrm{C}_{\mathrm{q}}, \underline{\mathrm{COOCH}}_{3}\right), 143.47(\mathrm{C}-14), 122.44(\mathrm{CH}-15), 80.84(\mathrm{CH}-3)$, 55.29 (CH-5), 47.57 (C-17), 46.57 (CH-9), 45.85 (CH-18), $41.56\left(\mathrm{CH}_{2}-7\right), 40.92$ (C8), 39.31 (C-4), 38.09 (C-10), $37.72\left(\mathrm{CH}_{2}-1\right), 37.02$ (C-13), $33.82\left(\mathrm{CH}_{2}-19\right), 33.10$ $\left(\mathrm{CH}_{2}-12\right), 32.55\left(\mathrm{CH}_{2}-21\right), 32.48\left(\mathrm{CH}_{3}-29\right), 30.71\left(\mathrm{CH}_{2}-16\right), 28.09\left(\mathrm{CH}_{2}-22\right), 27.71$ (C-20), $25.96\left(\mathrm{CH}_{3}-30\right), 23.64\left(\mathrm{CH}_{3}-23\right), 23.58\left(\mathrm{CH}_{3}-26\right), 23.44\left(\mathrm{CH}_{2}-2\right), 22.91$ $\left(\mathrm{CH}_{3}-27\right), 21.36\left(\mathrm{COOCH}_{3}\right), 18.23\left(\mathrm{CH}_{2}-6\right), 17.25\left(\mathrm{CH}_{2}-11\right), 16.72\left(\mathrm{CH}_{3}-24\right), 15.45$ $\left(\mathrm{CH}_{3}-25\right) .-(+)$-ESI MS: $m / z=521\left([\mathrm{M}+\mathrm{Na}]^{+}\right) .-(+)$-HRESI MS: $m / z=521.36066$ $\left([\mathrm{M}+\mathrm{Na}]^{+}\right)$, (calculated 521.36032 for $\left.\mathrm{C}_{32} \mathrm{H}_{50} \mathrm{O}_{4} \mathrm{Na}\right)$.

Betulinic acid (119): White needles, 10.03 mg. - ${ }^{1}$ H NMR (DMSO- $d_{6}, 300$ MHz): $\delta=$ 12.0 (bs, 1H, 28-OH), 4.70 (s, 1H, 29-Ha), 4.56 (s, 1H, 29-Hb), 4.20 (bs, 1H, 3-OH), 2.98 (m, $1 \mathrm{H}, 3-\mathrm{H}), 2.22(\mathrm{dt}, J=14.1,3.6 \mathrm{~Hz}, 1 \mathrm{H}, 19-\mathrm{H})$, $1.80(\mathrm{~m}, 2 \mathrm{H}, 16-\mathrm{H}), 1.65\left(\mathrm{~s}, 3 \mathrm{H}, 30-\mathrm{CH}_{3}\right), 0.65-$ 1.85 (m, 1,2,6,7,11,12,15,21,22- $\left.\mathrm{CH}_{2}, \quad 5,9-\mathrm{H}\right)$,

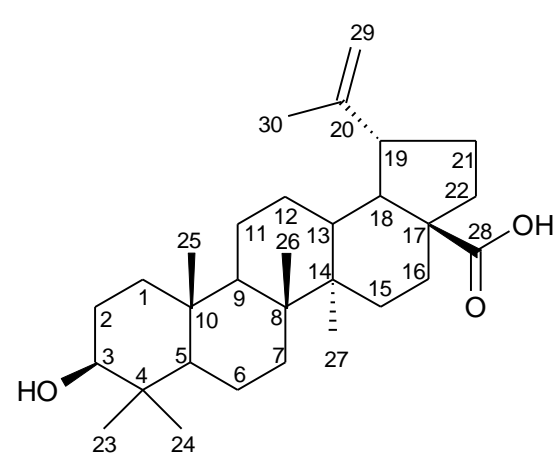
0.93 (s, 3H, 23- $\left.\mathrm{CH}_{3}\right), 0.87$ (s, 6H, 26,27- $\left.\mathrm{CH}_{3}\right), 0.77$ (s, 3H, 24- $\left.\mathrm{CH}_{3}\right), 0.65$ (s, 3H, 25$\mathrm{CH}_{3}$ ). $-{ }^{13} \mathbf{C}$ NMR (DMSO- $d_{6}, 125$ MHz): $\delta=176.9$ (C-28), 150.1 (C-20), 109.4 ( $\left.\mathrm{CH}_{2}-29\right), 76.7$ (CH-3), 55.3 (C-17), 54.8 (CH-5), 49.9 (CH-9), 48.5 (CH-18), 46.5 (CH-19), 41.9 (C-14), 40.2 (C-8), 39.0 (C-4), $38.4\left(\mathrm{CH}_{2}-1\right), 38.2$ (CH-13), 37.6 $\left(\mathrm{CH}_{2}-7\right), 36.7$ (C-10), $36.3\left(\mathrm{CH}_{2}-22\right), 31.7\left(\mathrm{CH}_{2}-6\right), 30.1\left(\mathrm{CH}_{2}-21\right), 29.2\left(\mathrm{CH}_{2}-15\right)$, $28.0\left(\mathrm{CH}_{3}-23\right), 27.1\left(\mathrm{CH}_{2}-2\right), 25.1\left(\mathrm{CH}_{2}-12\right), 20.4\left(\mathrm{CH}_{2}-11\right), 18.9\left(\mathrm{CH}_{3}-30\right), 17.9$ $\left(\mathrm{CH}_{2}-6\right), 15.9\left(\mathrm{CH}_{3}-26\right), 15.74\left(\mathrm{CH}_{3}-25\right), 15.71\left(\mathrm{CH}_{3}-24\right), 14.4\left(\mathrm{CH}_{3}-27\right)$. - (-)-ESI MS: $m / z=455\left([\mathrm{M}-\mathrm{H}]^{-}\right) .-(-)$-HRESI MS: $m / z=455.35309\left([\mathrm{M}-\mathrm{H}]^{-}\right),($calculated 455.353069 for $\mathrm{C}_{30} \mathrm{H}_{48} \mathrm{O}_{3}$ ).

5,7-Dimethoxyapigenin (121): White solid, $10.3 \mathrm{mg} .-{ }^{\mathbf{1}} \mathbf{H}$ NMR (DMSO- $d_{6}, \mathbf{3 0 0}$ MHz): $\delta=7.87\left(\mathrm{~d}, 2 \mathrm{H},{ }^{3} J=8.8 \mathrm{~Hz}, 2^{\prime}, 6^{\prime}-\mathrm{H}\right)$, $6.90\left(\mathrm{~d}, 2 \mathrm{H},{ }^{3} \mathrm{~J}=8.8 \mathrm{~Hz}, 3^{\prime}, 5^{\prime}-\mathrm{H}\right), 6.82(\mathrm{~d}, 1 \mathrm{H}$, $\left.{ }^{4} J=2.3 \mathrm{~Hz}, 8-\mathrm{H}\right), 6.56$ (s, 1H, 3-H), 6.49 (d,

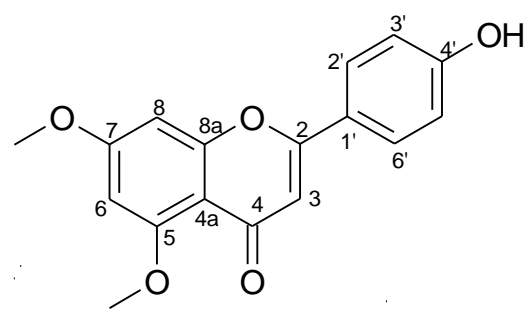


$\left.1 \mathrm{H},{ }^{4} \mathrm{~J}=2.3 \mathrm{~Hz}, 6-\mathrm{H}\right), 3.89\left(\mathrm{~s}, 3 \mathrm{H}, \mathrm{CH}_{3}-\mathrm{O}\right), 3.83\left(\mathrm{~s}, 3 \mathrm{H}, \mathrm{CH}_{3}-\mathrm{O}\right) .-{ }^{\mathbf{1 3}} \mathbf{C} \mathbf{N M R}$ (DMSO- $d_{6}, 125$ MHz): $\delta=175.3$ (C-4), 163.3 (C-7), 160.1 (C-4'), 160.0 (C-5), 159.8 (C-2), 158.8 (C-8a), 127.6 (CH-2', 6'), 121.0 (C-1'), 115.6 (CH-3', 5'), 108.1 (C-4a), $105.8(\mathrm{CH}-3), 96.0(\mathrm{CH}-6), 93.2(\mathrm{CH}-8), 55.9\left(5-\mathrm{OCH}_{3}\right), 55.8\left(7-\mathrm{OCH}_{3}\right)$. (+)-ESI MS: $m / z=297\left([\mathrm{M}-\mathrm{H}]^{+}\right) .-(+)$-HRESI MS: $m / z=299.09153\left([\mathrm{M}+\mathrm{H}]^{+}\right)$, (calculated 299.09140 for $\mathrm{C}_{17} \mathrm{H}_{15} \mathrm{O}_{5}$ ).

Ganschisandrine (122): White solid, $9.71 \mathrm{mg} .-{ }^{1} \mathbf{H}$ NMR $\left(\mathrm{CDCl}_{3}, \mathbf{3 0 0}\right.$ MHz $): \delta=6.83\left(\mathrm{~d}, 1 \mathrm{H},{ }^{3} J=8.2 \mathrm{~Hz}, 5-\right.$ $\mathrm{H}), 6.94\left(\mathrm{~d}, 1 \mathrm{H},{ }^{4} \mathrm{~J}=1.9 \mathrm{~Hz}, 2-\mathrm{H}\right), 6.95$ (dd, $\left.1 \mathrm{H},{ }^{3} J=8.2,{ }^{4} \mathrm{~J}=1.9 \mathrm{~Hz}, 6-\mathrm{H}\right), 4.51$

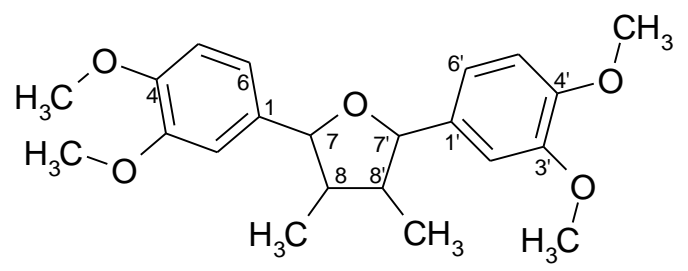
(d, $\left.1 \mathrm{H},{ }^{3} J=6.4 \mathrm{~Hz}, 7-\mathrm{H}\right), 3.86,3.85(2 \mathrm{~s}, 6 \mathrm{H}, 3,4-\mathrm{OMe}), 2.31$ (m, 1H, 8-H), 1.02 (d, $\left.3 \mathrm{H},{ }^{3} \mathrm{~J}=6.7 \mathrm{~Hz}, 8-\mathrm{CH}_{3}\right) .-{ }^{13} \mathbf{C} \mathbf{N M R}\left(\mathbf{C D C l}_{3}, 125 \mathbf{M H z}\right): \delta=148.8(\mathrm{C}-3), 148.3(\mathrm{C}-$ 4), 134.7 (C-1), 118.5 (CH-6), 110.8 (CH-5), 109.7 (CH-2), 87.2 (CH-7), 55.9 (4OMe), 55.8 (3-OMe), $44.4(\mathrm{CH}-8), 13.0\left(\mathrm{CH}_{3}-8\right) .-(+)$-ESI MS: $\mathrm{m} / z=395$ $\left([\mathrm{M}+\mathrm{Na}]^{+}\right) .-(+)$-HRESI MS: $m / z=395.18289\left([\mathrm{M}+\mathrm{Na}]^{+}\right),($calculated 395.18283 for $\mathrm{C}_{22} \mathrm{H}_{28} \mathrm{O}_{5} \mathrm{Na}$ ).

\section{Stigmasterol glucoside} (123): White solid, $11.1 \mathrm{mg}$. ${ }^{1} \mathrm{H}$ NMR (DMSO- $d_{6}, \quad 300$ MHz ): $\delta=5.32($ br s, 6-H), 5.4 (m, 22-H), $5.01(\mathrm{~m}, 23-\mathrm{H}), 4.80$ (m, 1'-H), $4.34\left(\mathrm{t},{ }^{3} \mathrm{~J}=7.6 \mathrm{~Hz}\right.$, $\left.6 \mathrm{~b}^{\prime}-\mathrm{H}\right), 4.22\left(\mathrm{~d},{ }^{3} \mathrm{~J}=5.6 \mathrm{~Hz}\right.$, 6a'-H), 3.64 (m, 3, 3'-H), 3.44

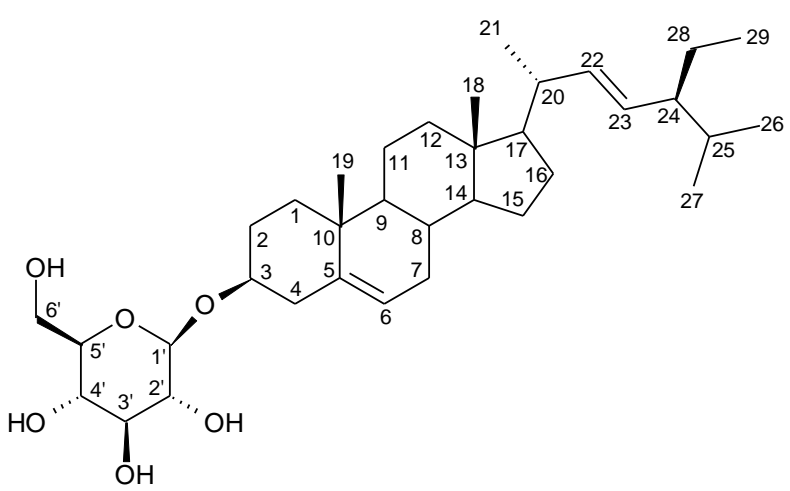
(m, 4'-H), 3.08 (m, 5'-H), 2.91 (m, 2'-H), 2.36 (m, 4-H), 2.15-0.65 (m, 1, 2, 7, 8, 9, 11, 12, 14, 15, 16, 17, 20, 21, 24, 25, 26, 27, 28, 29-H). - ${ }^{\mathbf{1 3}}$ C NMR (DMSO-d $d_{6}, 125$ MHz ): $\delta=36.8\left(\mathrm{CH}_{2}-1\right), 29.2\left(\mathrm{CH}_{2}-2\right), 70.0(\mathrm{CH}-3), 41.7\left(\mathrm{CH}_{2}-4\right), 140.2(\mathrm{C}-5)$, 120.9 (CH-6), $31.3\left(\mathrm{CH}_{2}-7\right), 31.4(\mathrm{CH}-8), 49.5$ (CH-9), 36.2 (C-10), $22.6\left(\mathrm{CH}_{2}-11\right)$, $38.1\left(\mathrm{CH}_{2}-12\right), 41.8$ (C-13), $56.1(\mathrm{CH}-14), 23.8\left(\mathrm{CH}_{2}-15\right), 28.7\left(\mathrm{CH}_{2}-16\right), 55.4(\mathrm{CH}-$ 17), $11.7\left(\mathrm{CH}_{3}-18\right), 19.6\left(\mathrm{CH}_{3}-19\right), 38.3(\mathrm{CH}-20), 20.8\left(\mathrm{CH}_{3}-21\right), 137.7(\mathrm{CH}-22)$, 128.6 (CH-23), 50.5 (CH-24), 33.3 (CH-25), $19.0\left(\mathrm{CH}_{3}-26\right), 20.5\left(\mathrm{CH}_{3}-27\right), 25.5$ 
( $\left.\mathrm{CH}_{2}-28\right), 12.0\left(\mathrm{CH}_{3}-29\right), 100.7$ (CH-1'), $76.6\left(\mathrm{CH}-2^{\prime}\right), 76.7(\mathrm{CH}-3 '), 73.3\left(\mathrm{CH}-4{ }^{\prime}\right)$, $76.8\left(\mathrm{CH}-5^{\prime}\right), 61.0\left(\mathrm{CH}_{2}-6{ }^{\prime}\right) .-(+)$-ESI MS: $\mathrm{m} / z=597\left([\mathrm{M}+\mathrm{Na}]^{+}\right) .-(+)$-HRESI MS: $m / z=597.41256\left([\mathrm{M}+\mathrm{Na}]^{+}\right)$, (calculated 597.41244 for $\left.\mathrm{C}_{35} \mathrm{H}_{58} \mathrm{O}_{6} \mathrm{Na}\right)$.

Oleanolic acid (124): Pale yellow solid, $10.0 \mathrm{mg} .-{ }^{1} \mathrm{H}$ NMR $\left(\mathrm{CDCl}_{3}, 300 \mathrm{MHz}\right): \delta=$ $5.23\left(\mathrm{t}, 1 \mathrm{H},{ }^{3} \mathrm{~J}=3.5 \mathrm{~Hz}, 12-\mathrm{H}\right), 3.14(\mathrm{dd}, 1 \mathrm{H}$, $\left.{ }^{3} J=10.7,6.1 \mathrm{~Hz}, 3-\mathrm{H}\right), 2.82\left(\mathrm{dd}, 1 \mathrm{H},{ }^{3} J=\right.$ 13.1 and $4.4 \mathrm{~Hz}, 18-\mathrm{H}), 2.01-1.28(1,2,5,6$, $7,11,15,16,19,21,22-\mathrm{H}), 1.15-0.77(23,24$, 25, 26, 27, 29, 30-Me). - (+)-ESI MS: $\mathrm{m} / \mathrm{z}=$

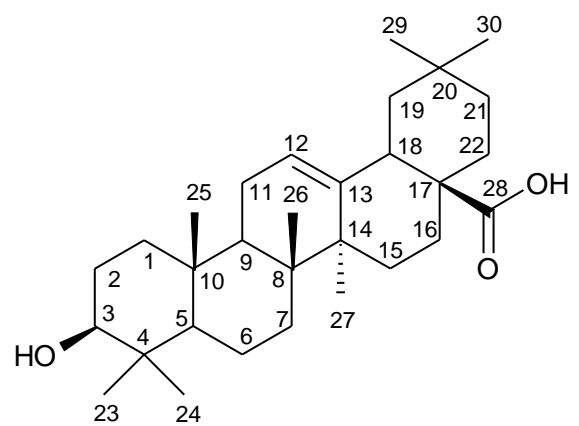
$479\left([\mathrm{M}+\mathrm{Na}]^{+}\right) . \quad-(+)$-HRESI MS: $m / z=479.34961\left([\mathrm{M}+\mathrm{Na}]^{+}\right)$, (calculated 479.34956 for $\mathrm{C}_{30} \mathrm{H}_{48} \mathrm{O}_{3} \mathrm{Na}$ ).

Betulin (120): White solid, $8.9 \mathrm{mg} .-{ }^{\mathbf{1}} \mathbf{H}$ NMR ( $\left.\mathbf{C D C l}_{3}, 300 \mathrm{MHz}\right): \delta=4.66,4.56(2 \mathrm{~d}$, $2 \mathrm{H}, 29-\mathrm{H}), 3.78,3.53\left(2 \mathrm{xd}, 2 \mathrm{H},{ }^{2} \mathrm{~J}=10.8 \mathrm{~Hz}\right.$, 28-H), 3.21 (m, 1H, 3-H), 2.37 (m, 1H, 19-H), $1.67\left(\mathrm{~s}, 3 \mathrm{H}, 30-\mathrm{CH}_{3}\right)$. - (+)-ESI MS: $\mathrm{m} / z=465$ $\left([\mathrm{M}+\mathrm{Na}]^{+}\right) .-(+)$-HRESI MS: $m / z=465.37043$ $\left([\mathrm{M}+\mathrm{Na}]^{+}\right), \quad$ (calculated 465.37030 for

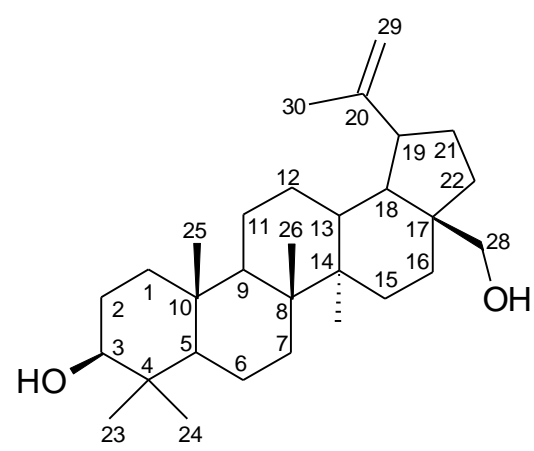
$\left.\mathrm{C}_{30} \mathrm{H}_{50} \mathrm{O}_{2} \mathrm{Na}\right)$.

2-Methylanthraquinone (125): Yellow solid, $9.6 \mathrm{mg}$. $-{ }^{\mathbf{1}} \mathbf{H}$ NMR $\left(\mathbf{C D C l}_{\mathbf{3}}, \mathbf{3 0 0} \mathbf{M H z}\right): \delta=8.28(\mathrm{~m}, 2 \mathrm{H}, 5-\mathrm{H}$ and $8-\mathrm{H}), 8.18\left(\mathrm{~d},{ }^{3} \mathrm{~J}=7.9 \mathrm{~Hz}, 1 \mathrm{H}, 4-\mathrm{H}\right), 8.08\left(\mathrm{~d},{ }^{4} \mathrm{~J}=1.1 \mathrm{~Hz}\right.$, $1 \mathrm{H}, 1-\mathrm{H}), 7.77(\mathrm{~m}, 2 \mathrm{H}, 6-\mathrm{H}$ and $7-\mathrm{H}), 7.58\left(\mathrm{dd}, 1 \mathrm{H},{ }^{3} J=\right.$

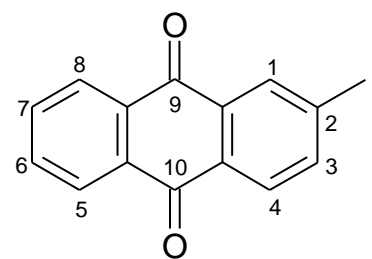
$\left.7.9 \mathrm{~Hz},{ }^{4} J=1.1 \mathrm{~Hz}, 3-\mathrm{H}\right), 2.52\left(\mathrm{~s}, 3 \mathrm{H}, 2-\mathrm{CH}_{3}\right) .-(+)$-ESI MS: $m / z=245\left([\mathrm{M}+\mathrm{Na}]^{+}\right)$. - (+)-HRESI MS: $m / z=245.05735\left([\mathrm{M}+\mathrm{Na}]^{+}\right)$, (calculated 245.05730 for $\mathrm{C}_{15} \mathrm{H}_{10} \mathrm{O}_{2} \mathrm{Na}$ ).

Scopoletin (126): Yellow solid, $9.0 \mathrm{mg} .-{ }^{\mathbf{1}} \mathbf{H}$ NMR $\left(\mathbf{C D C l}_{3}, 300 \mathrm{MHz}\right): \delta=7.58\left(\mathrm{~d}, 1 \mathrm{H},{ }^{3} \mathrm{~J}=9.5 \mathrm{~Hz}, 4-\mathrm{H}\right)$, $6.89(\mathrm{~s}, 1 \mathrm{H}, 5-\mathrm{H}), 6.82(\mathrm{~s}, 1 \mathrm{H}, 8-\mathrm{H}), 6.25\left(\mathrm{~d}, 1 \mathrm{H},{ }^{3} J=9.5\right.$<smiles>COc1cc2ccc(=O)oc2cc1O</smiles>
Hz, 3-H), 3.93 (s, 3H, OMe). - (+)-ESI MS: $m / z=215\left([\mathrm{M}+\mathrm{Na}]^{+}\right) .-(+)$-HRESI MS: $m / z=215.03148\left([\mathrm{M}+\mathrm{Na}]^{+}\right),\left(\right.$calculated 215.03147 for $\left.\mathrm{C}_{10} \mathrm{H}_{8} \mathrm{O}_{4} \mathrm{Na}\right)$. 


\section{References}

[1] Farnsworth, N. R.; Akerele, O.; Bingel, A. S.; Soejarto, D. D.; Guo, Z. Bull. WHO 1985, 63, 965-981.

[2] Newman, D. J.; Cragg, G. M.; Snader, K. M. Nat. Prod. Rep. 2000, 17, 215234.

[3] Butler, M. S. Nat. Prod. Rep. 2005, 22,162-195.

[4] Strobel, G.; Daisy, B. Micrbiol. Mol.Biol. Rev. 2003, 67, 491-502.

[5] Saleem, M.; Ali, M. S.; Hussain, S.; Jabbar, A.; Ashraf, M.; Lee, Y. S. Nat.Prod. Rep. 2007, 24, 1142-1152

[6] Faulkner, D. J. Nat. Prod. Rep. 2002, 19, 1.

[7] Newman, D. J.; Cragg, G. M. Curr. Med. Chem. 2004, 11, 1689.

[8] Andersen, R. J.; Williams, D. E. In Chemistry in the Marine Environment; Hester, R. E.; Harrison, R. M. Ed.; The Royal Society of Chemistry, Cambridge, UK, 2000, 55-79.

[9] WHO. Combating Emerging Infectious Diseases in the South-East Asia Region; World Health Organization: New Delhi, 2005, p 1.

[10] Dharmaraj, S. World J. Microbiol. Biotechnol., 2010, 26, 2123-2139.

[11] Strobel, G.; Daisy, B.; Castillo, U.; Harper, J. J. Nat. Prod. 2004, 67, $257-$ 268.

[12] Grabley, S.; Thiericke, R. The impact of natural products on drug discovery. p. 1-37; In: Drugs discovery from Nature; Grabley, S., Thiericke, R., Ed.; Springer-Verlag Berlin Heidelberg, 1999, p. 1-37.

[13] Li, J. W.-H.; Vederas, J. C. Science 2009, 325, 161-165.

[14] Baxter, R. L.; Blake, A. J. Tetrahedron Lett. 1988, 29, 2101-2102.

[15] Bentley, R. Chem. Rev. 2000, 100, 3801-3825.

[16] G. Minotti, P. Menna, E. Salvatorelli, G. Cairo, L. Gianni, Pharmacol. Rev. 2004, 56, 185-229. 
[17] Fischbach, M. A.; Wals, C. T. Science 2009, 325, 1089-1093.

[18] Lautru, S.; Deeth, R. J.; Bailey, L. M.; Challis, G. L. Nature Chem. Biol. 2005, 1, 265-269.

[19] Schatz, A.; Bugie, E.; Waksman, S. A. Proc. Soc. Exp. Biol. Med. 1944, 55, p 66.

[20] A. D. Buss, ; Butler, M. S., Ed.; Natural Product Chemistry for Drug Discovery; RCS Publishing, Cambridge, UK, 2010, p 215.

[21] Watve, M. G.; Tickoo, R.; Jog, M. M.; Bhole, B. D. Arch. Microbiol. 2001, $176,386-390$.

[22] Lancini, G.; Parenti, F.; Gallo, G. G. Antibiotics: A Multidisciplinary Approach; Third Edition, Plenum Press, New York, 1995, p 3.

[23] Clardy, J.; Fishbach, M. A.; Walsh, C. T. Nature Biotechnol. 2006, 24, 15411550 .

[24] Shiomi, K.; Omura, S. In: Macrolide Antibiotics, Chemistry, Biology and Practice; Omura, S., Ed.; Second Edition, Elsevier Science, San Fransisco, 2002, p 8.

[25] Beuther, D. A.; Martin, R. J. Current Allergy and Atshma 2004, 4, 132-138.

[26] Wright, A. E.; Botelho, J. C.; Guzman, E.; Harmody, D.; Linley, P.; McCarthy, P. J.; Pitts, T. P.; Pomponi, S. A.; Reed, J. K. J. Nat. Prod. 2007, 70, 412-416.

[27] Williams, P. G.; Miller, E. D.; Asolkar, R. N.; Jensen, P. R.; Fenical, W. J. Org. Chem. 2007, 72, 5025-5034.

[28] Aparicio, J. F.; Colina, A. J.; Ceballos, E.; Martin, J. F. J. Biol. Chem. 1999, 274, 10133-10139.

[29] Komaki, H.; Izumikawa, M.; Ueda, J.; Nakashima, T.; Khan, S. T.; Takagi, M.; Shin-Ya, K. Appl. Microbiol. Biotechnol. 2009, 83, 127-133. 
[30] Nachtigall, N.; Kulik, A.; Helaly, S.; Bull, A. T.; Goodfellow, M.; Asenjo, J. A.; Maier, A.; Wiese, J.; Imhoff, J. F.; Süssmuth, R. D.; Fiedler, H. P. J. Antibiot. 2011, 64, 775-780.

[31] Wang, J.; Soisson, S. M.; Young, K.; Shoop, W.; Kodali, S.; Galgoci, A.; Painter, R.; Parthasarathy, G.; Tang, Y. S.; Cummings, R.; Ha, S.; Dorso, K.; Motyl, M.; Jayasuriya, H.; Ondeyka, J.; Herath, K.; Zang, C.; Hernandez, L.; Allocco, J.; Basilio, A.; Tormo, J. R.; Genilloud, O.; Vicente, F.; Pelaez, F.; Colwell, L.; Lee, S. H.; Michael, B.; Falcetto, T.; Gill, C.; L. L. Silver, Hermes, J. D.; Bartizal, K.; Barret, J.; Schmatz, D.; Becker, J. W.; Cully, D.; Singh, S. B. Nature 2006, 441, 358-361.

[32] Abdelfatah, M. New Secondary Metabolites from Bacteria: Seitomycin with high Anti-Helicobacter pylori Activity, Exfoliazone B, new Steffimycinones, Espicufolin B, Flavomarine A and B, and BS-46 with a Novel Carbon Skeleton. PhD Thesis, University of Goettingen, Germany, 2003.

[33] Dwight, D. B.; Min, C.; Uma, O.; Vineet, R. Nat. Prod. Rep. 2007, 24, 1225 1244.

[34] Voet, D.; Voet, J. G. Biochemistry; Third Edition, John Wiley \& Sons Inc., 2004, pp 730-732.

[35] Nikodinovic, J.; Dinges, J. M.; Bergmeier, S. C.; McMills, M. C.; Wright, D. L.; Priestley, N. D. Org. Lett. 2006, 8, 443-445.

[36] Nelson, M. E.; Priestley, N. D. J. Am. Chem. Soc. 2002, 124, 2894-2902.

[37] A. J. K.-Karamyan, M. T. Hamann, Chem. Rev. 2010, 110, 4489-4497.

[38] S. W. Yang, G. A. Cordell, J. Nat. Prod. 1997, 60, 230.

[39] Soengas, R. G.; Anta, C.; Espada, A.; Paz, V.; Ares, I. R.; Balado, Rodriguez, M.; J.; Lemos, M. L.; Jimenez, C. Tetrahedron Lett. 2006, 47, 7113-7116.

[40] Blunt J. W., ; Copp, B. R.; Hu, W.-P.; Munro, M. H. G.; Northcote, P. T.; Prinsep, M. R. Nat. Prod. Rep. 2009, 26, 170-244. 
[41] Rajamani, S.; Bauer, W. D.; Robinson, J. B.; Farrow, J. M.; Pesci, E. C.; Teplitski, M.; Gao, M.; Sayre, R. T.; Phillips, D. A. MPMI 2008, 2, 1184-1192.

[42] Powers, H. J. Am. J. Clin. Nutr. 2003, 77, 1352-1360.

[43] Zendah, I.; Riaz, N.; Nasr, H.; Frauendorf, H.; Schüffler, A.; Raies, A.; Laatsch, H. J. Nat. Prod. 2012, 75, 2-8.

[44] Ren, X.; Lu, X.; Ke, A.; Zheng, Z.; Lin, J.; Hao, W.; Zhu, J.; Fan, Y.; Ding, Y.; Jiang, Q.; Zhang, H. J. Antibiot. 2011, 64, 339-343.

[45] Tang, G.-L.; Cheng, Y.-Q.; Shen, B. J. Nat. Prod. 2006, 69, 387-393.

[46] Dewick, P. M. In Medicinal Natural Product: A Biosynthetic Approach; Second Edition, John Wiley \& Sons Ltd.: Weinheim, Germany, 2001, p 421.

[47] Oh, D.-C.; Strangman, W. K.; Kauffman, C. A.; Jensen, P. R.; Fenical, W. Org. Lett. 2007, 9, 1525-1528.

[48] Zang, C.; Occi, J.; Masurekar, P.; Barret, J. F.; Zink, D. L.; Smith, S.; Onishi, R.; Ha, S.; Salazar, O.; Genilloud, O.; Basilio, A.; Vicente, F.; Gill, C.; Hickey, E. J.; Dorso, K.; Motyl, M.; Singh, S. B. J. Am. Chem. Soc. 2008, 130, 12102-12110.

[49] Sajid, I.; Shaaban, K. A.; Frauendorf, H.; Hasnain, S.; Laatsch, H. Z. Naturforsch. 2008, 63b, 1223-1230.

[50] Rogers, M. J.; Cundliffe, E.; McCutchan, T. F. Antimicrob. Agents Chemother. 1998, 42, 715-716.

[51] Maskey, R. P.; Fotso, S.; Sevvana, M.; Usón, I.; Wollny, I. G.; Laatsch, H. J. Antibiot. 2006, 59, 309-314.

[52] Parry, R. J.; Li, Y.; Lini, F.-L. J. Am. Chem. Soc. 1992, 114, 10062-10064.

[53] Garg, R. P.; Alemany, L. B.; Moran, S.; Parry, R. J. J. Am. Chem. Soc. 2009, $131,9608-9609$.

[54] Parry, R. J.; Rao, H. S. P.; Mueller, J. J. Am. Chem. Soc. 1982, 104, 339-340.

[55] Takahashi, Y.; Nakayama, M.; Watanabe, I.; Deushi, T.; Ishiwata, H.; Shiratsuchi, M.; Otani, G. J. Antibiot. 1989, 42, 1541-1546. 
[56] Nakayama, M.; Takahashi, Y.; Itoh, H.; Kamiya, K.; Shiratsuchi, M.; Otani, G. J. Antibiot. 1989, 42, 1535-1540.

[57] Kim, B. Y.; Willbold, S.; Kulik, A., Helaly, S. E.; Zinecker, H.; Wiese, J.; Imhoff, J. F.; Goodfellow, M.; Süssmuth, R. D.; Fiedler, H. P. J. Antibiot. 2011, 64, 595-597.

[58] Nakayama, M.; Takahashi, Y.; Itoh, H.; Kamiya, K.; Shiratsuchi, M.; Otani, G. J. Antibiot. 1989, 42, 1535-1540.

[59] Rosemeyer, H. Chem. Biodivers. 2005, 2, 977-1063.

[60] Brémond, P.; Audran, G.; Aubin, Y.; Monti, H. Synlett 2007, 1124-1126.

[61] Jiang, M. X.-W.; Jin, B.; Gage, J. L.; Priour, A.; Savela, G.; Miller, M. J. J. Org. Chem. 2006, 71, 4164-4169.

[62] Kehr, J. C.; Picchi, D. G.; Dittmann, E. Beilstein J. Org. Chem. 2011, 7, $1622-1635$.

[63] Malloy, K. L.; Suyama, T. L.; Engene, N.; Debonsi, H.; Cao, Z.; Matainaho, T.; Spadafora, C.; Murray, T. F.; Gerwick, W. H. J. Nat. Prod. 2012, 75, 6066.

[64] Kjer, J.; Debbab, A.; Aly, A. H.; Proksch, P. Nature 2010, 5, 479-490.

[65] Rateb, M. E.; Ebel, R. Nat. Prod. Rep. 2011, 28, 290-334.

[66] Süssmuth, R.; Müller, J.; Döhren, H. V.; Molnar, I. Nat. Prod. Rep. 2011, 28, 99-124.

[67] Shiono, Y.; Tsuchinari, M.; Shimanuki, K.; Miyajima, T.; Murayama, T.; Koseki, T.; Laatsch, H.; Funakoshi, T.; Takanami, K.; Suzuki, K. J. Antibiot. 2007, 60, 309-316.

[68] Matasyoh, J. C.; Dittrich, B.; Schueffler, A.; Laatsch, H. Parasitol. Res. 2010, pp 1-6.

[69] Isaka, M.; Chinthanom, P.; Supothina, S.; Tobwor, P.; Jones, N. L. H. J. Nat. Prod. 2010, 73, 2057-2060. 
[70] Laurent, D.; Guella, G.; Mancini, I.; Roquebert, M.-F.; Farinole, F.; Pietra, F. Tetrahedron 2002, 58, 9163-9167.

[71] Strobel, G.; Daisy, B. Microbiol. Mol. Biol. Rev. 2003, 67, 491-502.

[72] Li, J. Y.; Strobel, G. A. Phytochemistry 2001, 57, 261-265.

[73] Amagata, T.; Doi, M.; Tohgo, M.; Minoura, K.; Numata, A. Chem. Comm. 1999, 1321-1322.

[74] Wang, W.; Wang, Y.; Tao, H.; Peng, X.; Liu, P.; Zhu, W. J. Nat. Prod. 2009, $72,1695-1698$.

[75] Gunatilaka, A. A. L. J. Nat. Prod. 2006, 69, 509-526.

[76] H. Greve, P. J. Schupp, E. Eguereva, S. Kehraus, G. M. König, J. Nat. Prod., 2008, 71, 1651-1653.

[77] Verma, V. J.; Labkovsky, E.; Gange, A. C.; Singh, S. K.; Prakash, S. J. Antibiot. 2011, 64, 427-431.

[78] Tan, N.; Tao, Y.; Pan, J.; Wang, S.; Xu, F.; She, Z.; Lin, Y.; Jones, E. B. G. Chem. Nat. Compd. 2008, 44, 296-300.

[79] Jang, J. H.; Asami, Y.; Jang, J. P.; Kim, S. O.; Moon, D. O.; Shin, K. S.; Hashizume, D.; Muroi, M.; Saito, T.; Oh, H.; Kim, B. Y.; Osada, H.; Ahn, J. S. J. Am. Chem. Soc. 2011, 133, 6865-6867.

[80] Dictionary of Natural Products on DVD, Chapman and Hall/CRC, Chemical Database, Version 19.1, 2010.

[81] Kerr, R.G.; Kerr, S. S. Expert Opin. Ther. Pat. 1999, 9,1207.

[82] Manly, S. P.; Padmanabha, R.; Lowe, S. E.; Janzen, W. P., Ed.; High Throughput Screening; Humana Press, Tatowa, New York, 2002, pp 153168.

[83] Mohamed, M.A.A. ent- Homoabyssomicins A and B, Two New Spirotetronates, Khatmiamycin, a Zoosporicidal Napththoquinone, and Further New Biologically Active Secondary Metabolites from Marine and Terrestrial Streptomyces spp. PhD Thesis, University of Göttingen, Germany, 2010. 
[84] Laatsch, H.; Mikroorganismen als biologische Quelle neuer Wirkstoffe, In: Pharmazeutische Biotechnologie; Kayse, O.; Müller, R. H., Ed.; Wissenschaftliche Verlagsges., Stuttgart, 2004, pp 13-43.

[85] Dinan, L.; In Natural Product Isolation : Dereplication and Partial Identification of Compounds; Second Edition, Humana Press Inc., Tatowa, New Jersey, 2006, p 297.

[86] Füllbeck, M.; Michalsky E., ; Dunkel, M.; Preissner, R. Nat. Prod. Rep. 2006, $23,347-356$.

[87] Middleswotrh, F. v.; Cannel, R. J. P.; In Natural Product Isolation: Method in Biotechnology; Cannel, R. J. P., Ed.; Humana Press Inc., Tatowa, New Jersey, 1998, pp 279-327.

[88] Laatsch, H.; AntiBase, A Database for Rapid Dereplication and Structure Determination of Microbial Natural Products. 2011, Wiley-VCH Verlag $\mathrm{GmbH}$ and Co., Weinheim, Germany: see Internet http://wwwuser.gwdg.de/ hlaatsc/Antibase.htm.

[89] Shaaban, K.A. Nafisamycin, Cyclisation Product of a New Enediyne Precursor, Highly Cytotoxic Mansouramycins, Karamomycins Possesing a Novel Heterocyclic Skeleton and Further Unusual Secondary Metabolites from Terrestrial and Marine Bacteria. PhD Thesis, University of Göttingen, Germany, 2008.

[90] Zhang, Q.; Yadav, P. Personal comm. University of Göttingen.

[91] Jizba, J ; Sedmera, P. ; Zima, J.; Blumauerova, M.; Kandybin, N. V. Folia. Microbiol. 1991, 36, 437-443.

[92] Prikrylova, V.; Beran, M.; Sedmera, P.; Jizba, J. Folia. Microbiol. 1994, 39, 191-196.

[93] Findlay, J. A. ; Li, G.; Miller, J. D.; Womiloju, T. O. Can. J. Chem. 2003, 81, 284-292.

[94] Brockmann, H. Angew. Chem. 1960, 72, 939-947. 
[95] Cravens, S. L.; Navapanich, A. C.; Geierstanger, B. H.; Tahmassebi, D. C.; Dwyer, T. J. J. Am. Chem. Soc. 2010, 132, 17588-17598.

[96] Green, D.M. Eur. J. Cancer. 1997, 33, 409-418.

[97] Womer, R.B. J. Am. Med. Soc. 1997, 33, 2230-2234.

[98] Gou, J.; Wu, T.; Bess, J.; Henderson, L.E.; Levin, J.G. J. Virol. 1998, 72, 716-724.

[99] Shaaban, M.A. Bioactive Secondary Metabolites from Marine and Terrestrial Bacteria: Isoquinolinequinone, Bacterial Compounds with a Novel Pharmacophor. PhD Thesis, University of Göttingen, Germany, 2004.

[100] Noguchi, H.; Harrison, P.H.; Arai, K.; Nakashima, T. T.; Trimble, L. A.; Vederas, J. C. J. Am.Chem. Soc. 1988, 110, 2938-2945.

[101] Yu Win, H. Bioactive Mandalarpyrones, their Derivatives and Further Novel Secondary Metabolites from Marine and Terrestrial Bacteria. PhD Thesis, University of Göttingen, Germany, 2009.

[102] Shaaban, M.; Schröder, D.; Shaaban, K.A.; Helmke, E.; Wollny, I. G.; Döbler, I. W.; Laatsch, H. Rev. Latinoamer. Quim. 2008, 35, 58-67.

[103] Allen, J.R.F.; Holmstedt, B.R. Phytochemistry 1980, 19, 1573-1582

[104] Husson, H.-P. In The Alkaloids; Brossi, A., Ed.; Elsevier Science; 1985, 26, pp 1-51.

[105] Bracher, F.; Hildebrand, D. Liebigs Ann. Chem. 1992, 1315-1319.

[106] Ohmoto, T.; Koike, K. Shoyakugaku Zasshi 1988, 42, 160-162.

[107] Barker, M. D.; Woodward, P. R.; Lewis, J. R. Patent GB 2155462 A119850925, 1985.

[108] Ikeda, R.; Iwaki, T.; Iida, T.; Okabayashi, T.; Nishi, E.; Kurosawa, ; M. Sakai, N.; Konakahara, T. Eur. J. Med. Chem. 2011, 46, 636-646.

[109] Shimizu, K.; Tamura, G. J. Antibiot. 1981, 34, 649-53. 
[110] Cho, H.; Beale, J. M.; Graff, C.; Mocek, U.; Nakagawa, A.; Ömura, S.; Floss, H. G. J. Am. Chem. Soc. 1993, 115, 12296-12304.

[111] Shimizu, K.; Tamura, G. J. Antibiot. 1981, 34, 654-657.

[112] Pearce, P. D.; Bauchop, T. Appl. Environ. Microbiol. 1985, 49, 1265-1269.

[113] Williams, K. P.; Gillespie, J. J.; Sobral, B. W. S.; Norberg, E. K.; Snyder, E. E.; Shallom, J. M.; Dickerman, A. W. J. Bacteriol. 2010, 192, 2305-2314.

[114] Maskey, R.P. Neuartige Wirkstoffe aus marinen Streptomyceten: Sagunamycine, Parimycin, Himalomycine, Gottingamycin, Dhanyabadomycin, Akashine und stark cytotoxische Trioxacarcine mit hoher AntimalariaAktivität. PhD Thesis, University of Göttingen, 2001.

[115] Maskey, R.P.; Kock, I.; Shaaban, M.; Wollny, I. G.; Helmke, E.; Mayer, F.; Döbler, I. W.; Laatsch, H. Polymer Bull. 2002, 49, 87-93.

[116] Zendah, I. Isolation, Purification and Structure Elucidation of New Secondary Metabolites from Terrestrial, Marine, and Ruminal Microorganisms. PhD Thesis, University of Göttingen, Germany, 2012.

[117] Castro, M. J. L.; Kovensky, J.; Cirelli, A. F. Molecules 2000, 5, 608-609.

[118] Ismail, A.; Soultani, S.; Ghoul, M. Biotechnol. Prog. 1998, 14, 874-878.

[119] Ayer, W. A.; Miao, S. J. Nat. Prod. 1993, 56, 2046-2052.

[120] Yoshida, S.; Naganawa, H.; Aoyagi, T.; Takeuchi, T.; Umezawa, H. J. Antibiot. 1988, XLI, 1015-1018.

[121] Aoyagi, T.; Yoshida, S.; Harada, S.; Okuyama, A.; Nakayama, C.; Yoshida, T.; Hamada, M.; Takeuchi, T.; Umezawa, H. J. Antibiot. 1988, XLI, 10091018.

[122] Sugimura, T. Prog. Nucleic Acid Res. Mol. Biol. 1973, 13, 127-151.

[123] Hilz, H.; Stone, P. Rev. Physiol. Biochem. Pharmacol. 1976, 76, 1-58.

[124] Hayaishi, O.; Ueda, K. Annu. Rev. Biochem. 1977, 46, 95-116. 
[125] Durkacz, B.W.; Omidiji, O.; Gray, D.A.; Shall, S. Nature 1980, 283, 593596.

[126] Aoyagi, T.; Wada, T.; Kojima, F.; Nagai, M.; Yoshida, S.; Sato, T.; Takeuchi, T. Biochem. Int. 1989, 18, 391-398.

[127] Aoyagi, T.; Wada, T.; Kojima, F.; Sasakawa, S.; Tamura, M.; Umezawa, H. Biochem. Int. 1989, 18, 383-389.

[128] Green, K. J. Org. Chem. 1991, 56, 4325-4326.

[129] Yasuda, T.; Inaba, A.; Ohmori, M.; Endo, T.; Kubo, S.; Ohsawa, K. J. Nat. Prod. 2000, 63, 1444-1446.

[130] Dudkin, M. S.; Skornyakova, N. S.; Bazilevskii, A. S.; Tul'chinskii, V. P.; Mishnaevskii, M. S. Odesskii Tekh. Inst. Pishchevoi Promyshlennosti im. M. V. Lomonosova, USSR 1976, 1, 30-34.

[131] Bianchi, G.; Dallavalle, S.; Merlini, L.; Nasini, G.; Quaroni, S. Planta Med. 2003, 69, 574-576.

[132] Yamato, M.; Iinuma, H.; Naganawa, H.; Yamagishi, Y.; Hamada, M.; Masuda, T.; Umezawa, H.; Abe, Y.; Hori, M. J. Antibiot. 1986, 39, 184-191.

[133] Lindel, T.; Junker, J.; Köck, M. WEBCOCON, see internet http://cocon.nmr.de.

[134] Hecht, S. S.; Greene, F. D. J. Am. Chem. Soc. 1976, 89, 6761-6762.

[135] Taylor, K. G.; Isaac, S. R.; Swigert, J. L. J. Org. Chem. 1976, 41, 1146-1152.

[136] Helaly, S. E.; Pesic, A.; Fiedler, H. P.; Süssmuth, R. D. Org. Lett. 2011, 13, 1052-1055.

[137] Chang, C. F., Liu, S. T. J. Mol. Catal. A 2009, 299, 121-126.

[138] Bigelow, H. E. Chem. Rev. 1931, 9, 117-167.

[139] Naureen, H. Dehydrorabelomycin-1-O- $\alpha$-L-rhamnopyranoside, Actinofuranone C and Further New Bioactive Secondary Metabolites from Terrestrial Streptomyces spp. PhD Thesis, University of Göttingen, Germany, 2011. 
[140] Ayer, W. A.; Trifonov, L. S. Phytochemistry 1995, 38, 371-372.

[141] O'connor, K. E. ; Witholt, B.; Duetz, W. J. Bacteriol. 2001, 183, 928-933.

[142] Kim, H.-H.; Kim, W.-G.; Ryoo, I.-J.; Kim, C.-J.; Suk, J.-E.; Han, K.-H.; Hwang, S.-Y.; Yoo, I.-D. J. Microbiol. Biotechnol. 1997, 7, 429-434.

[143] Choi, S.-W.; Bai, D.-H.; Yu, J.-H.; Shin, C.S. Can. J. Microbiol. 2003, 49, 663-668.

[144] Gustafson, K.; Roman, M.; Fenical, W. J. Am. Chem. Soc. 1989, 111, 75197524.

[145] Schneider, K.; Chen, X.-H.; Vater, J.; Franke, P.; Nicholson, G.; Borriss, R.; Süssmuth, R. D. J. Nat. Prod. 2007, 70, 1417-1423.

[146] Kurl, R. N.; Villee, C. A. Pharmacology 1985, 30, 241-4.

[147] Ströch, K.; Zeeck, A.; Antal, N.; Fiedler, H. P. J. Antibiot. 2005, 58, 103-110.

[148] Fotso, F. Y. Aqabamycins, Rare Nitro Maleimides and other Novel Metabolites from Microorganisms; Generation and Application of an HPLC-UV-ESI MS/MS Database. PhD Thesis, University of Göttingen, Germany, 2007

[149] Laatsch, H.; Fotso, S. Topics Curr. Chem. 2008, 282, 3-74.

[150] Pullen, C.; Schmitz, P.; Maurer, K; Bamberg, D. D.v.; Lohmann, S.; França, S. De C.; Groth, I.; Schlegel, B.; Möllmann, U.; Gollmick, F.; Gräfe, U.; Leistner, E. Planta 2002, 216, 162-167.

[151] Wu, Y. H.; Hao, B. J.; Shen, E.; Zhang, X. M.; Zhao, Y. J. Med. Plant. Res. 2011, 12, 2494-2498.

[152] Rodriguez, R. J.; White Jr, J. F.; Arnold, A. E.; Redman, R. S. New Phytologist 2009, 182, 314-330.

[153] Fujimoto, Y.; Yokoyama, E.; Takahashi, T.; Uzawa, J.; Morooka, N.; Tsunoda, H.; Tatsuno, T. Chem. Pharm. Bull. 1986, 34, 1497-1500.

[154] Morita, T.; Aoki, H. Agr. Biol. Chem. 1974, 38, 1501-1505

[155] Venkatasubbaiah, P.; Chilton, W. S. J. Nat. Prod. 1991, 54, 1293-1297. 
[156] Dong, J. Y.; Song, H. C.; Li, J. H.; Tang, Y. S.; Sun, R.; Wang, L.; Zhou, Y. P.; Wang, L. M.; Shen, K. Z.; Wang, C. R.; Zhang, K. Q. J. Nat. Prod. 2008, 71, 952-956.

[157] Jinming, G.; Lin, H.; Jikai, L. Steroids 2001, 66, 771-775.

[158] Cafieri, F.; Fattorusso, E. J. Nat. Prod. 1985, 48, 944-947.

[159] Honeywell, E. M.; Bills, C. E. J. Biol. Chem. 1932, 99, 71-78.

[160] Kawagishi, H.; Katsumi, R.; Sazawa, T.; Mizuno, T.; Hagiwara, T.; Nakamura, T. Phytochemistry 1988, 27, 2777-2779.

[161] Mizushina, Y.; Takahashi, N.; Hanashima, L.; Koshino, H.; Esumi, Y.; Uzawa, J.; Sugawara, F.; Sakaguchi, K. Bioorg. Med. Chem. 1999, 7, 2047-2052.

[162] Randazzo, G.; Fogliano, V.; Ritieni, A.; Mannina, L.; Rossi, E.; Scarallo, A.; Segre, A. L. Tetrahedron 1993, 49, 10883-10896.

[163] Ritieni, A.; Fogliano, V.; Randazzo, G.; Scarallo, A.; Logrieco, A.; Moretti, A.; Mannina, L.; Bottalico, A. Natural Toxins 1995, 3, 17-20.

[164] Jestoi, M. Critical Review in Food Science and Nutrition 2008, 48, 21-49

[165] Logrieco, A.; Moretti, A.; Fornelli, F.; Fogliano, V.; Ritieni, A.; Caiaffa, M. F.; Randazzo, G.; Bottalico, A.; Macchia, L. App. Env. Microbiol. 1996, 62, 3378-3384.

[166] Gupta, S.; Montllor, C.; Hwang, Y.-S. J. Nat. Prod. 1995, 58, 733-738.

[167] Shimada, A.; Fujioka, S.; Koshino, H.; Kimura, Y. Z. Naturforsch. 2010, 65c, 207-210.

[168] Nilanonta, C.; Isaka, M.; Kittakoop, P.; Trakulnaleamsai, S.; Tanticharoen, M.; Thebtaranonth, Y. Tetrahedron 2002, 58, 3355-3360.

[169] Hamill, R. L.; Higgens, C. E.; Boaz, H. E.; Gorman, M. Tetrahedron Lett. 1969, 49, 4255-4258.

[170] Zhan, J.; Burns, A.M.; Liu, M.X.; Faeth, S.H.; Gunatilaka, A.A.L. J. Nat. Prod. 2007, 70, 227-232. 
[171] Marfori, E. C.; Kajiyama, S.; Fukusaki, E.; Kobayashi, A. Z. Naturforsch. 2002, 57c, 465-470.

[172] Marfori, E. C.; Bamba, T.; Kajiyama, S. Tetrahedron 2002, 58, 6655-6658.

[173] Batrakov, S. G.; Konova, I. V.; Sheichenko, V. I.; Esipov, S. E.; Galanina, L. A.; Istratova, L. N. Chem. Phys. Lipids 2002, 117, 45-51.

[174] Umemura, K.; Ogawa, N.; Koga, J.; Iwata, M.; Usami, H. Plant Cell Physiol. 2002, 43, 778-784.

[175] Sitrin, R. D.; Chan, G.; Dingerdissen, J.; deBrosse, C.; Mehta, R.; Roberts, G.; Rottschaefer, S.; Staiger, D.; Valenta, J.; Snader, K. M.;. Stedman, R. J; Hoover, J. R. E. J. Antibiot. 1988, 41, 469-480.

[176] Ngouamegne, E. T.; Fongang, R. S. ; Ngouela, S.; Fabrice Fekam Boyom, F. F.; Rohmer, M.; Tsamo, E.; Gut, J.; Rosenthal, P. J. Chem. Pharm. Bull. 2008, 56, 374-377.

[177] Koseki, K.; Takahashi, Y.; Shimazaki, K.; Ebata, T.; Chuman, T.; Mori, K. Biosci. Biotech. Biochem. 1992, 56, 1728-1731

[178] Aldridge D. C.; Giles, D.; Turner, W. B. J. Chem. Soc. 1971, 23, 3888-3891

[179] Pascale, G. D.; Nazi, I.; Harrison, P. H. M.; Wright, G. D. J. Antibiot. 2011, 64, 483-487.

[180] Saepudin, E.; Harrison, P. Can. J. Chem. 1995, 73, 1-5

[181] Kim, D. H.; Jung, S. J.; Chung, I. S.; Lee, Y. H.; Kim, D. K.; Kim, S. H.; Kwon, B. M.; Jeong, T. S.; Park, M. H.; Seoung, N. S.; Baek, N. I. Arch. Pharm. Res. 2005, 28, 541-545.

[182] Nam, K.S.; Jo, Y.S.; Kim, Y.H.; Hyun, J.W.; Kim, H.W. Life Sci. 2001, 69, 229-237.

[183] Duerte, N.; Ferreira, M. J. U.; Martins, M.; Viveiros, M.; Amaral, L. Phytother. Res. 2007, 21, 601-604.

[184] Windaus, A.; Brunken, J. Liebigs Ann. Chem. 1928, 460, 225-235. 
[185] Yongcheng, L.; Jun, W.; Shining, Z; Jones E B, G. Chem. J. on Internet 2001, 7, 1-5: http://www.chemistrymag.org/cji/2001/037030ne.htm.

[186] Eaton, M. A. W.; Hutchinson, D. W. Tetrahedron Lett. 1971, 18, 1337-1340.

[187] Boonlarppradab, C.; Suriyachadkun, C.; Suphothina, S. ; Tobwor, P. J. Antibiot. 2011, 64, 267-270.

[188] Saeed, A.; Ehsan, S. Chem. Heterocycl. Comp. 2005, 41, 1381-1385.

[189] Bi, Y.-M; Bi, X.-B; Fang, A; Zhao, Q.-R. Arch. Pharm. Res. 2007, 30, 267269

[190] Ballio, A.; Barcellona, S.; Santurbano, B. Tetrahedron Lett. 1966, 31, 37233726.

[191] Ballio, A.; Casinovi, C. G.; Framondino, M.; Marino, G.; Nota, G.; Santurbano, B. Biochim. Biophys. Acta 1979, 573, 51-60.

[192] Luis, S. M.; Chrigo, L.; Spadafora, C.; Gerwick, W. H.; Rios, L. C. B. Rev. Latinoamer. Quím. 2009, 37, 104-114.

[193] Kokubun, T.; Veitch, N. C.; Bridge, P. D.; Simmonds, M. S. J. Phytochemistry 2003, 62, 779-782.

[194] Tan, N.; Tao, Y.; Pan, J.; Wang, S.; Xu, F.; She, Z.; Lin, Y.; Jones, E. B. G. Chem. Nat. Comp. 2008, 44, 296-300.

[195] Aly, A. H.; Ebel, R. A. E.; Indriani, I. D.; Wray. V.; Müller, W. E. G.; Totzke, F.; Zirrgiebel, U.; Schächtele, C.; Kubbutat, M. H. G.; Lin, W. H.; Procksch, P.; Ebel, R. J. Nat. Prod. 2008, 71, 972-980.

[196] Caluwé, E. D.; Halamavá, K.; Damme, P. V. Afrika Focus 2010, 23, 11-51.

[197] Shahat, A. A. Pharm. Biol. 2006, 44, 445-450.

[198] Campaoré, W. R., Nikièma, P. A.; Bssaolé, H. I. N.; Savadago, A.; Mouecoucou, J.; Hounhouigan, D. J.; Traoré, S. A. Curr. Res. J. Biol. Sci. 2011, 3, 6472.

[199] Han, A. R.; Kim, J. A.; Lantvit, D. D.; Kardono, L. B. S.; Riswan, S.; Chai, H. J. Nat. Prod. 2009, 72, 2028-2031 
[200] Kikuchi, H.; Ohtsuki, T.; Koyano, T.; Kowithayakorn, T.; Sakai, T.; Ishibashi, M. J. Nat. Prod. 2010, 73, 452-455.

[201] Ampofo, S. A.; Waterman, P. G. Phytochemistry 1995, 25, 2351-355.

[202] Lee, H. H. ; Chan, H. K. Phytochemistry 1977, 16, 2038-2039.

[203] Ha, L. D.; Hansen, P. E.; Vang, O.; Duus, F.; Pham, H. D.; Nguyen, L. H. D. Chem. Parm. Bull. 2009, 57, 830-834.

[204] Jantan, I.; Pisar, M. Md.; Idris, M. S.; Taher, M.; Ali, R. M. Planta Med. 2002, 68, 1133-1134

[205] Prachayasittikul, S.; Suphapong, S.; Worachartcheewan, A.; Lawung, R.; Ruchirawat, S.; Prachayasittikul, V. Molecules 2009, 14, 850-867

[206] Misra, D. R.; Khastgir, H. N. Tetrahedron 1970, 26, 3017-3021.

[207] Woo, W. S.; Wagner, H. Phytochemistry 1977, 16, 1845-1846.

[208] Ramos, M. P. O.; Silva, G. D. F. ; Duarte, L. P. ; Miranda, R. R. S. ; Souza, G. H. B. ; Filho, S. A. V. J. Chem. Pharm. Res. 2010, 2, 265-274.

[209] Campos, M. C. O.; Salamão, K.; Pinto, D. B. C.; Leon, L. L.; Barbosa, H. S.; Maciel, M. A. M.; Castro, S. L. Parasitol. Res. 2010, 107, 1193-1204.

[210] Macahig, R. A. S.; Matsunami, K.; Otsuka, H. Chem. Pharm. Bull. 2011, 59, 397-401.

[211] Banerji, N.; Majumder, P.; Dutta, N. L. Phytochemistry 1975, 14, 1447-1448.

[212] Haque, M. E.; Islam, M. N.; Hossain, M.; Mohamad, A. U.; Karim, M. F.; Rahman, M. A. J. Pharm. Sci. 2008, 71, 103-105.

[213] Rahman, H. Unusual Sesquiterpenes: Gorgonenes and Further Bioactive Secondary Metabolites Derived from Marine and Terrestrial Bacteria. Ph.D Thesis, University of Göttingen, 2008.

[214] Siddiqui, S. ; Hafeez, F. ; Begum, S. ; Siddiqui, B. S. J. Nat. Prod. 1988, 51, 229-233. 
[215] Abyshev, A. Z.; Agaev, E. M.; Guseinov, A. B. Pharm.Chem. J. 2007, 41, 419-423.

[216] Gauthier, C.; Legault, J.; Gauthier, M. P.; Pichette, A. Phytochem. Rev. 2011, $10,521-544$.

[217] Muceniece, R.; Saleniece, K.; Riekstina, U.; Krigere, L.; Tirzitis, G.; Ancans, J. Cell Biochem. Funct. 2007, 25, 291-296.

[218] Azuma, T.; Tanaka, Y.; Kikuzaki, H. Phytochemistry 2008, 69, 2743-2748

[219] Patanasethanont, D.; Nagai, J.; Yumoto, R.; Murakami, T.; Sutthanut, K.; Sripanidkulchai, B. O.; Yenjai, C.; Takano, M. J. Pharm. Sci. 2007, 96, 223233.

[220] Sirat, H. M. ; Jamil, S. ; Siew, L. W. ACGC Chem. Res. Comm. 2001, 13, 4854.

[221] Pancharoen, O.; Tuntiwachwuthkul, P.; Taylor, W. C. Phytochemistry 1989, 28, 1143-1148.

[222] Kaneko, T.; Sakamoto, M.; Ohtani, K.; Ito, A.; Kasai, R.; Yamasaki, K.; Padorina, W.G. Phytochemistry 1995, 39, 115-120.

[223] Azuma, T., Kayano, S.; Matsumura, Y.; Konishi, Y.; Tanaka, Y.; Kikuzaki, H. Food Chemistry 2011, 125, 471-475.

[224] Liu, H. T.; Xu, L. J.; Peng, Y.; Yang, X. W.; Xiao, P. G. Chem. Pharm. Bull. 2009, 57, 405-407.

[225] Yue, J. M.; Chen, Y. Z. ; Hua, S. M.; Cheng, J. L.; Cui, Y. X. Phytochemistry 1989, 28, 1774-1776.

[226] Da Silva Filho, A. A.; Albuquerque, S.; e Silva, M. L. A.; Eberlin, M. N.; Tomazela, D. M.; Bastos, J. K. J. Nat. Prod. 2004, 67, 42-45.

[227] Fonseca, S. F.; Barata, L. E. S.; Rúveda, E. A. Can. J. Chem. 1979, 57, 441443.

[228] Prachayasittikul, S.; Suphapong, S.; Worachartcheewan, A.; Lawung, R.; Ruchirawat, S.; Prachayyasittikul, V. Molecules 2009, 14, 850-867. 
[229] Sha, Y.; Yan, M. C.; Liu, J.; Liu, Y.; Cheng, M. S. Molecules 2008, 13, 14721486.

[230] Niesen, A.; Barthel, A.; Kluge, R.; Köwitzsch, A.; Ströhl, D.; Schwarz, S.; Csuk, R. Arch. Pharm. Chem. Life Sci. 2009, 342, 569-576

[231] Fotso, S. Highly Cytotoxic Kettapeptine, Bhimamycins Possesing Unusual Chromophores and Further New Secondary Metabolites from Terrestrial and Marine Bacteria. PhD Thesis, University of Göttingen, Germany, 2005.

[232] Berger, Y.; Deguée, M. B. Org. Magn. Reson. 1981, 15, 244-247

[233] Feng, S. X.; Hao, J.; Chen, T.; Qiu, S. X. S. Helv. Chim. Acta 2011, 94, $1843-$ 1849

[234] Kudha-Bukhsh, A. R.; Bhattacharyya, ; S. S. S. Paul, ; Baujedaini, N. J. Chinese Integrative Med. 2010, 8, 853-862.

[235] Jirge, S. S.; Chaudhary, Y. S. IJRAP 2010, 1, 55-62.

[236] Attaway, D. A.; Zaborsky, O. R., Eds.; Marine Biotechnology; Plenum Press, New York, 1993, p 327. 


\section{Spectra}

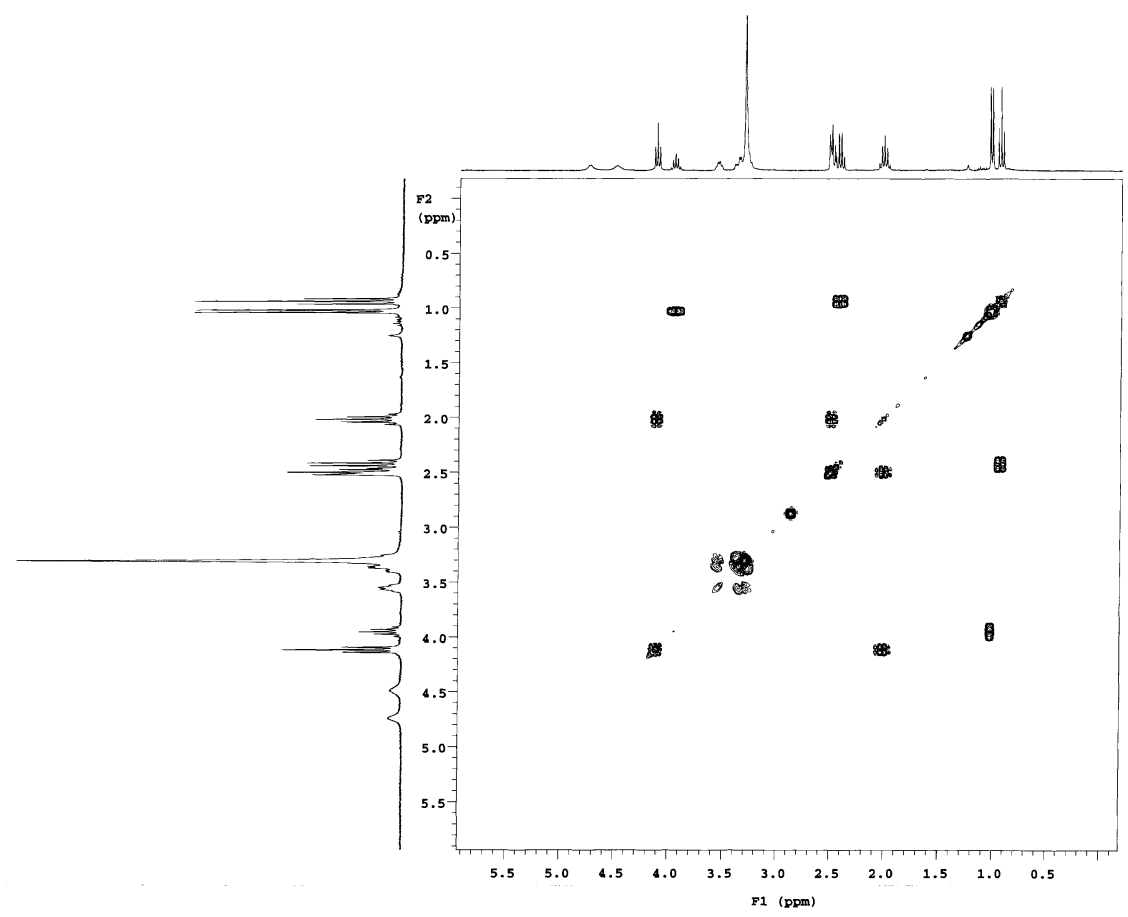

Figure 111: H, H COSY spectrum (DMSO- $d_{6}, 600 \mathrm{MHz}$ ) of bandamycin (94)

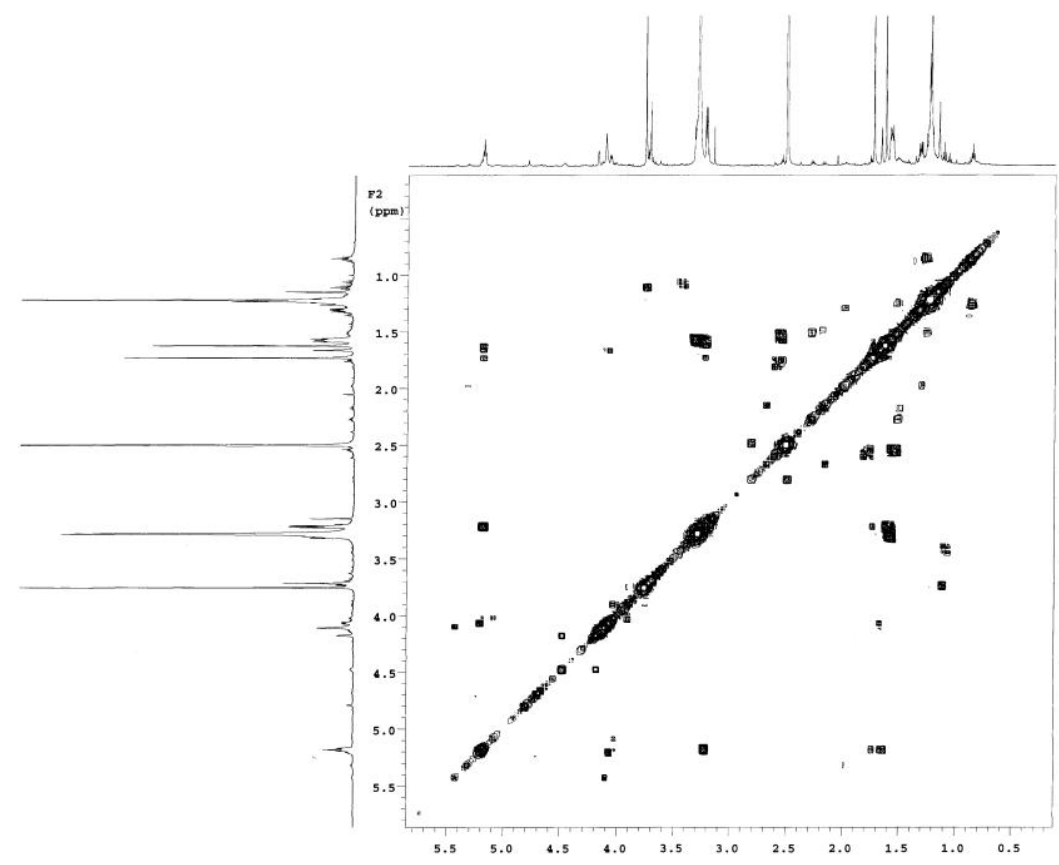

Figure 112: COSY spectrum (DMSO- $d_{6}, 300 \mathrm{MHz}$ ) of garcinon D (116) 


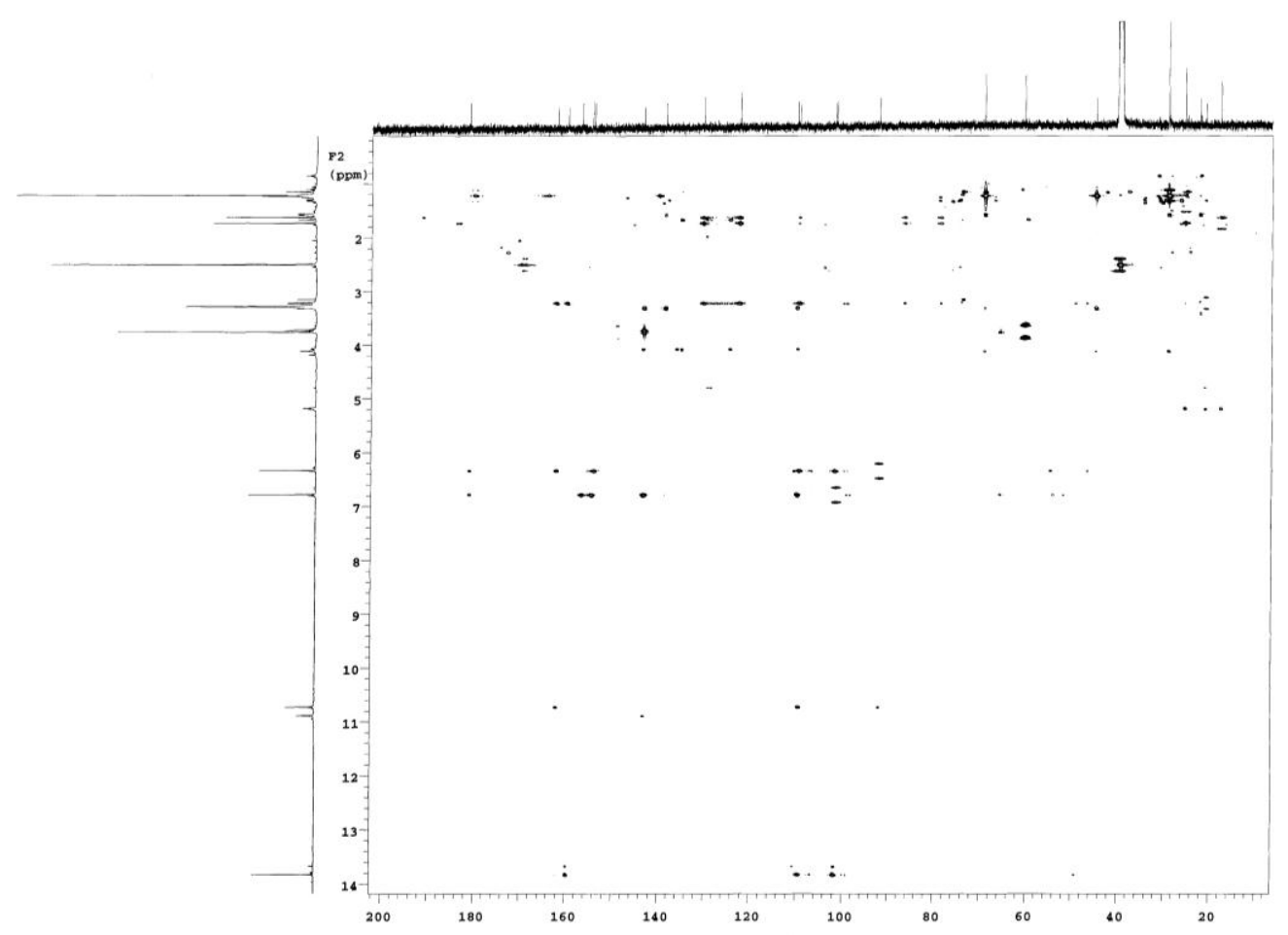

Figure 113: $\mathrm{HMBC}$ spectrum $\left(\mathrm{DMSO}-d_{6}, 300 \mathrm{MHz}\right)$ of garcinon $\mathrm{D}(116)$

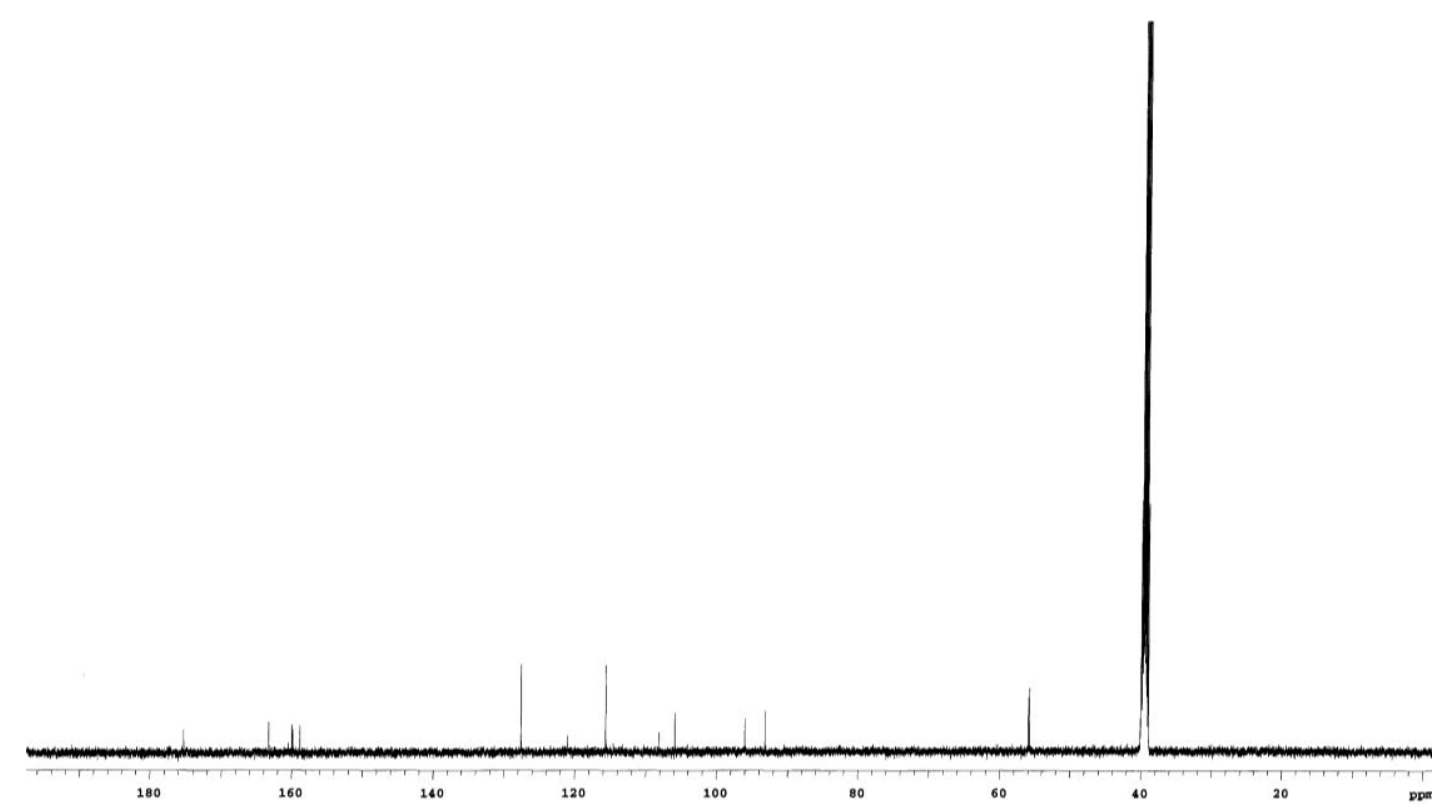

Figure 114: ${ }^{13} \mathrm{C}$ NMR spectrum (DMSO- $d_{6}, 125 \mathrm{MHz}$ ) of 5,7-dimethoxyapigenin (121) 


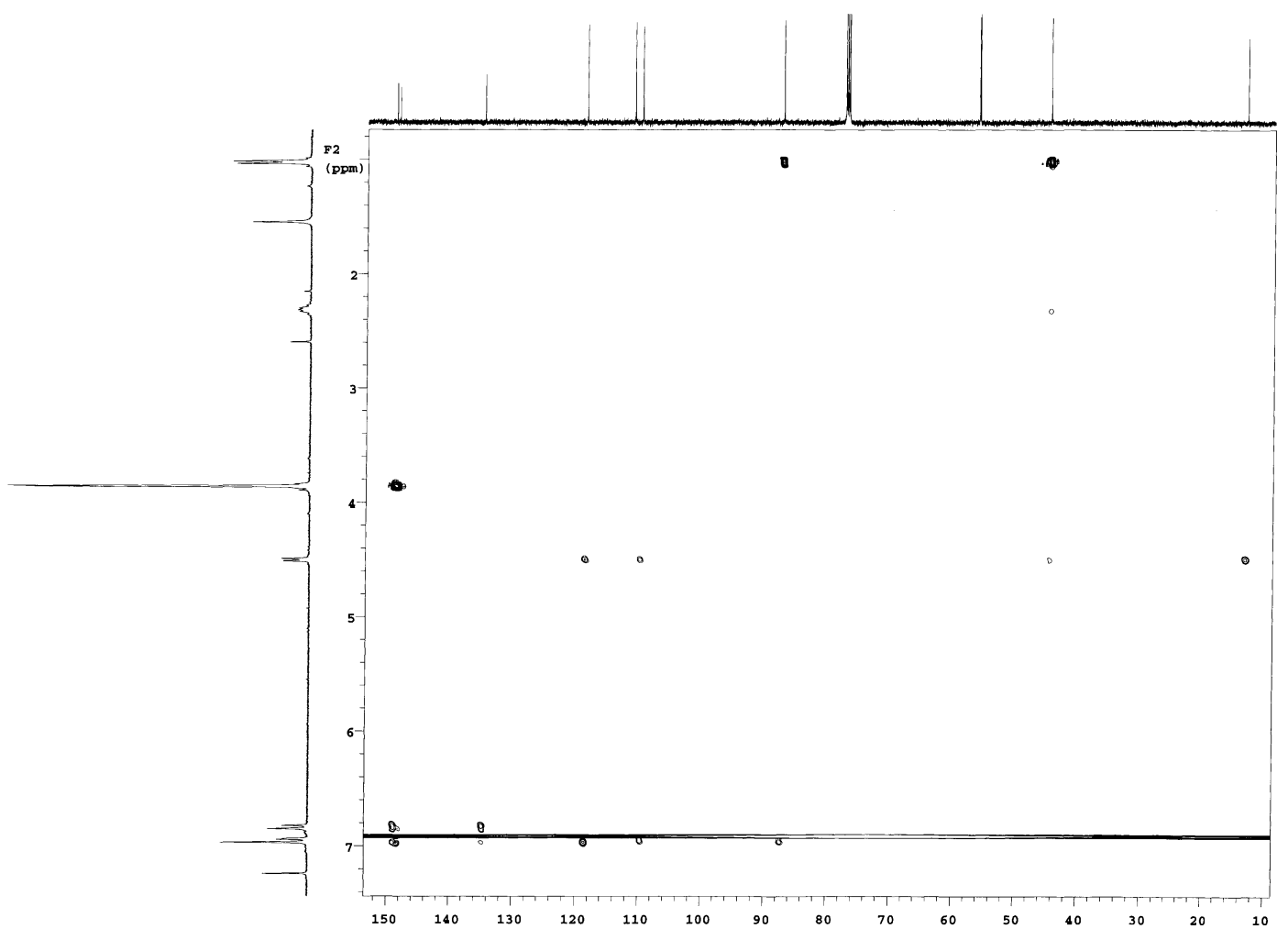

Figure 115: $\mathrm{HMBC}$ spectrum $\left(\mathrm{CDCl}_{3}, 300 \mathrm{MHz}\right)$ of ganschisandrine (122) 


\section{Acknowledgements}

First of all, I would like to express my sincere gratitude and respect to my supervisor Prof. Dr. H. Laatsch, Institute of Organic and Bio-molecular Chemistry, University of Göttingen, Germany. I deeply appreciate of his sincere support, valuable and constructive discussions, guidance and fruitful advices during my study. It is my pleasure to be one of his students and I am grateful for the provision of both excellent scientific and working facilities.

I am also thankful to Prof. Dr. A. Zeeck for his acceptance to read this work and for being my co-referent.

After all, I am deeply thankful to my dear colleagues and employees in the Prof. Laatsch group for their friendships, the warm atmosphere during my study and the good times we had together. My appreciation goes to Ferdinand Talontsi, Imene Zendah, Michael Kongue, Wang Hong Peng and Nelum Piyasena for their support and collaboration during my stay in this group.

My special thanks go to all the members of the Institute of Organic and Biomolecular Chemistry, especially to Dr. F. Frauendorf and Mrs. G. Udvarnoki for the mass spectrometry and Dipl. Chem. R. Machinek, Mr. M. Weitemeyer, Mr. U. Leonhardt, Mrs. C. Zolke and C. Siebert in the NMR department.

Also, my special appreciation goes to Dr. P. Facey, Dr. M. Shaaban and Dr. J. Matasyoh for fruitful collaboration throughout my study and scientific discussions. I am also indebted to Dipl.-Geol. Mrs. F. Lissy for the microbiological works, and Mr. Andreas Kohl for technical assistance.

I owed my gratitute to the "German Academic Exchange Service (DAAD)" for financing and offering a chance to pursue my study in Germany.

Lastly but not the least, my precious gratefulness goes to my wife (Anizar) and my children (Fiqhan, Amirul and Syauqal) for their supports and understanding spiritually and physically. I am also indebt to my parent and my brothers/sisters and words are inadequate to express my feelings for their encouragements and moral support. Their loves, prayers and encouragements have helped me in every stage of my life. Finally, I will keep all of them with gratitude in my heart. 


\section{CURRICULUM VITAE}

Name

: Muhammad Bahi

Place and date of birth $\quad$ : Banda Aceh, 28 September 1972

Sex

: Male

Nationality

: Indonesia

Religion

: Islam

Marital status

: Married (with 3 sons)

Language provenienc- : Indonesian (mother language); English (foreign language) es

Email address

: bahi.usk@gmail.com

\section{Current Employment:}

Assistant lecture at Chemistry Dept., Faculty of Mathematics and Natural Sciences, Syiah Kuala University, Banda Aceh, Indonesia (from 1998 till present)

\section{Educational Background}

B. Sc., in Chemistry, University of Syiah Kuala, Banda Aceh, Indonesia, 1996

M. Sc., in Chemistry, University of North Sumatera, Medan, Indonesia, 2005

Ph.D student, Institute of Organic and Biomolecular Chemistry, Georg-August University of Göttingen, Germany, March 2007 to April 2012 (Natural Product Chemistry from microorganisms)

\section{Scientific Publications}

\section{Posters}

[1] Muhammad BAHI, Mahmoud Al REFAI, Muna A. Abdalla, Heidrun ANKE, and Hartmut LAATSCH (2008), A Bioactive Oxydiaziridine or $\mathrm{N}$ Oxydiazenyl derivative together with other New Phenolic Compounds Isolated from a Terrestrial Streptomyyces sp., Chemie Forum, Institute of Organic 
and Biomolecular Chemistry, Georg-August University of Göettingen, Tammannstrasse 2, 37077 Göttingen, Germany.

[2] Mahmoud Al REFAI, Muhammad BAHI, Muna A. Abdalla, Hafizur RAHMAN, E. HELMKE, and Hartmut LAATSCH (2008), Marine-derived Streptomyces sp. B8041, a source of new Butanolides, Chemie Forum, Institute of Organic and Biomolecular Chemistry, Georg-August University of Göttingen, Tammannstrasse 2, 37077 Göttingen, Germany.

[3] Imene Zendah, Khaled A. SHAABAN, Muhammad BAHI, Mohamed SHAABAN, Aly RAIES, and Hartmut LAATSCH (2008), A new microbial Butylglucoside from the Ruminal Bacterium Enterobacter amnigenus, XVIII $^{\text {èmes }}$ Journées Nationales de Biologie de la SSNT << Génie Biologiques et Environnement>>, Yasmine Hammamet, Tunisie.

[4] Dhafer Saber ZINAD, Khaled A. SHAABAN, Muhammad BAHI, Muna A. Abdalla, Hamdi, A. NASR, Heidrun ANKE, and Hartmut LAATSCH (2009), New Isocoumarins from a Terrestrial Streptomyces sp., Chemie Forum, Institute of Organic and Biomolecular Chemistry, Georg-August University of Göttingen, Tammannstrasse 2, 37077 Göttingen, Germany.

[5] K. FANDI, M. BAHI, M.A. Abdalla, M. MASSADEH, H. LAATSCH (2010), Screening Metabolites of Thermophiles from Thermal Springs Jordanian Bacteria by LC-MS/MS Profile, The American Society for Microbiology (ASM) $\mathbf{1 1 0}^{\text {th }}$ General Meeting, May 23-27, in San Diego, California, USA. 